\title{
THERM 2.1 NFRC Simulation Manual
}

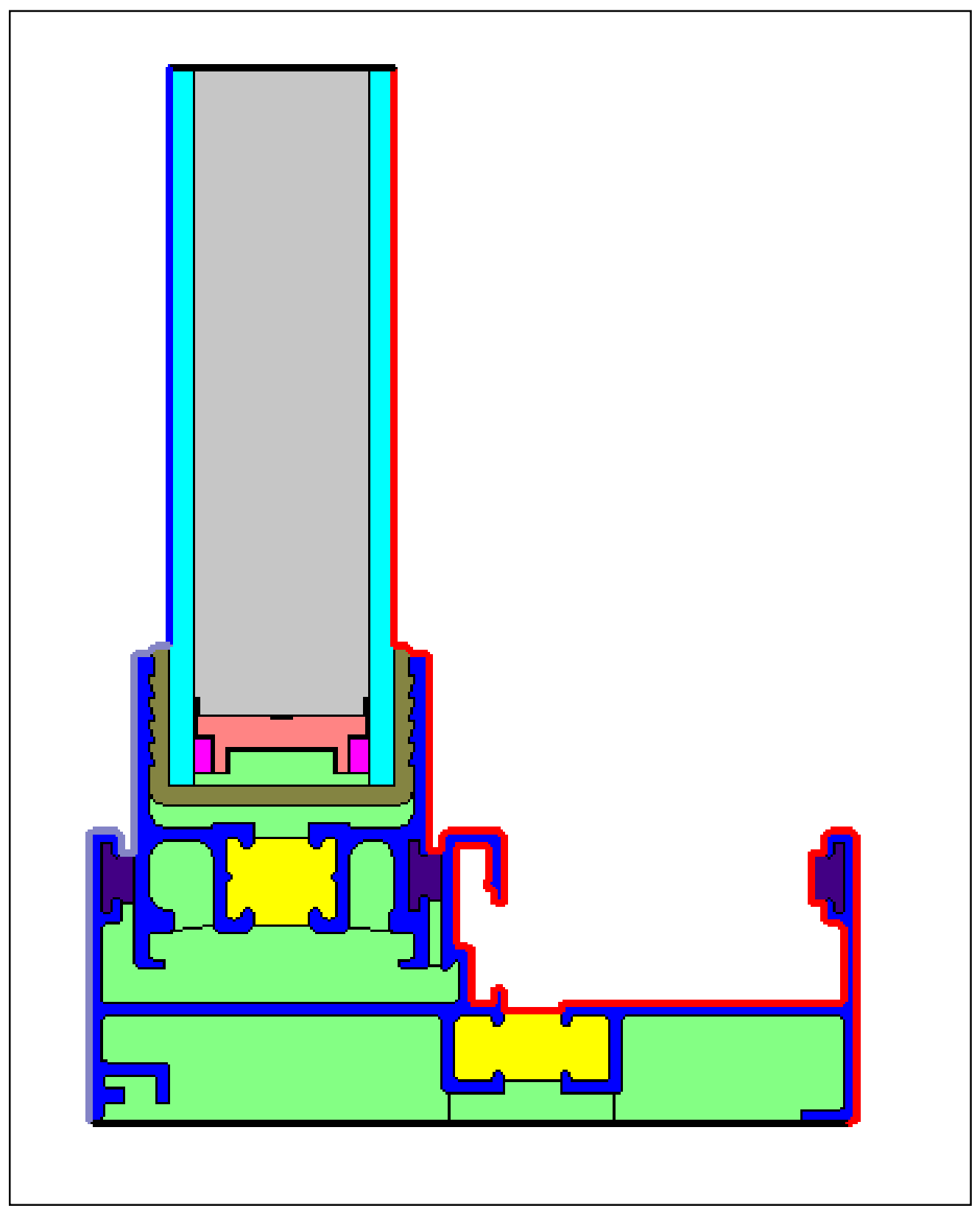

Lawrence Berkeley National Laboratory

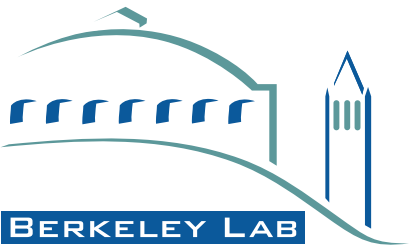

JULY 2000 
DISCLAIMER

This document was prepared as an account of work sponsored by the United States Government. While this document is believed to contain correct information, neither the United States Government nor any agency thereof, nor The Regents of the University of California, nor any of their employees, makes any warranty, express or implied, or assumes any legal responsibility for the accuracy, completeness, or usefulness of any information, apparatus, product, or process disclosed, or represents that its use would not infringe privately owned rights. Reference herein to any specific commercial product process, or service by its trade name, trademark, manufacturer, or otherwise, does not necessarily constitute or imply its endorsement, recommendation, or favoring by the United States Government or any agency thereof, or The Regents of the University of California. The views and opinions of authors expressed herein do not necessarily state or reflect those of the United States Government or any agency thereof, or The Regents of the University of California. 
PUB-3147

\title{
THERM 2.1 NFRC Simulation Manual
}

\author{
Robin Mitchell, Christian Kohler, Dariush Arasteh, and Elizabeth Finlayson \\ Windows and Daylighting Group \\ Building Technologies Department \\ Environmental Energy Technologies Division \\ Lawrence Berkeley National Laboratory \\ Berkeley, California 94720 \\ http://windows.lbl.gov/software/software.html \\ Charlie Huizenga \\ Center for Environmental Design Research \\ University of California \\ Berkeley, California \\ Dragan Curcija \\ Carli, Inc \\ Amherst, Massachusetts \\ John Carmody \\ College of Architecture and Landscape Architecture \\ University of Minnesota \\ Minneapolis, Minnesota
}

JULY 2000

(C) Regents of the University of California

This work was supported by the Assistant Secretary for Energy Efficiency and Renewable Energy, Office of Building Technology, State and Community Programs, Office of Building Systems of the U.S. Department of Energy under Contract No. DE-AC03-76SF00098. 



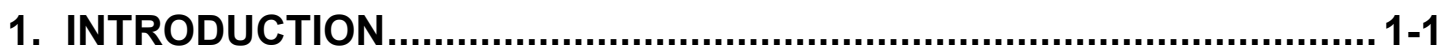

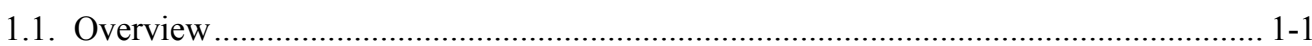

2. FENESTRATION PRODUCTS ….......................................................... 2-1

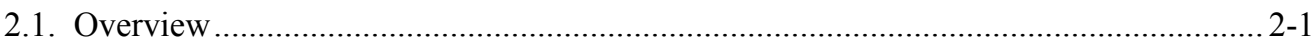

2.2 Fenestration Product Sash Operation ........................................................................... 2-1

2.2.1. Projected or Hinged Windows ................................................................ 2-2

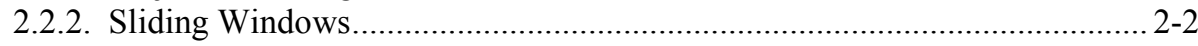

2.2.3. Sliding Glass Doors ............................................................................... 2-2

2.2.4. French Doors and Folding Patio Doors......................................................... 2-2

2.2.5. Skylights and Roof Windows ................................................................... 2-2

2.2.6. Greenhouse (Garden) Windows ................................................................ 2-3

2.3 Performance Implications of Basic Fenestration Types ................................................. 2-3

2.3.1. Hinged Windows ................................................................................ 2-3

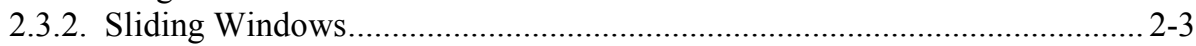

2.3.3. Sliding Glass and French Doors.............................................................. 2-4

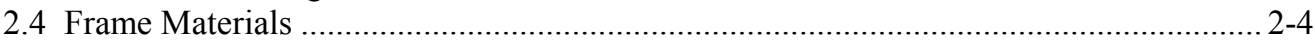

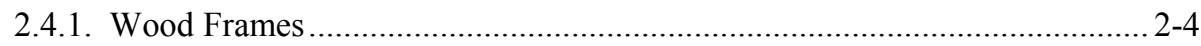

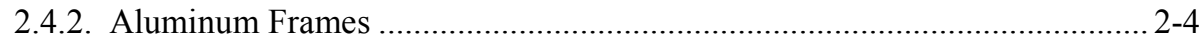

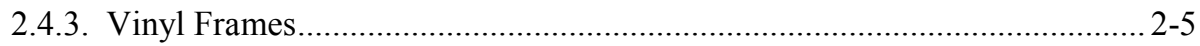

2.4.4. Fiberglass and Engineered Thermoplastics.............................................. 2-5

2.4.5. Wood Composites ...................................................................................... 2-5

2.4.6. Hybrid and Composite Frames ……….................................................. 2-5

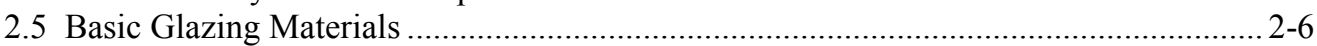

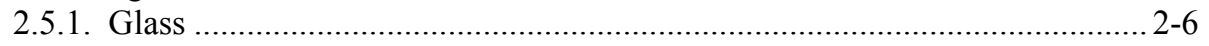

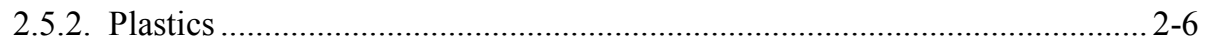

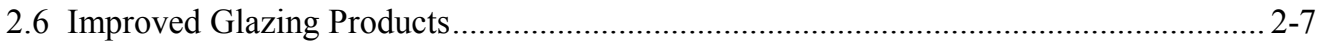

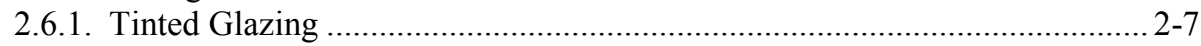

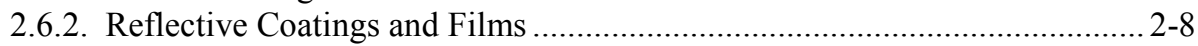

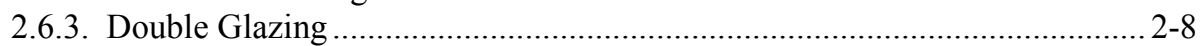

2.6.4. Glass Coatings and Tints in Double Glazing ............................................ 2-8

2.6.5. Gap Width in Multiple Glazed Units ......................................................... 2-9

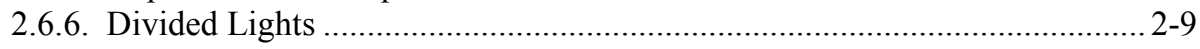

2.6.7. Special Products............................................................................... 2-10

2.6.8. Multiple Panes or Films .................................................................... 2-10

2.6.9. Low-Emittance Coatings ......................................................................... 2-11

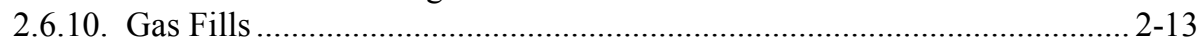

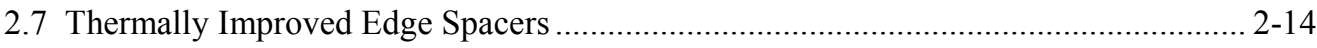

3. FENESTRATION HEAT TRANSFER BASICS.................................... 3-1

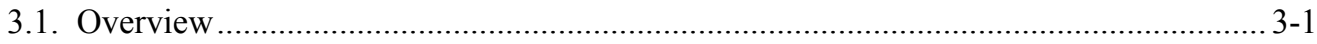

3.2 Energy Flow Mechanisms ................................................................................ 3-1

3.3 Temperature Driven Heat Transfer ....................................................................... 3-2

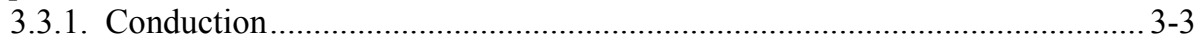

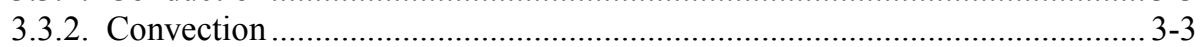

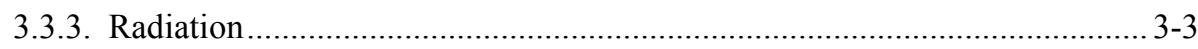

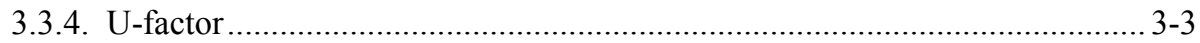


3.4 Solar Heat Gain and Visible Transmittance ............................................................. 3-4

3.4.1. Determining Solar Heat Gain.................................................................... 3-5

3.4.2. Solar Heat Gain Coefficient (SHGC) ........................................................ 3-6

3.4.3. Visible Transmittance ........................................................................ 3-6

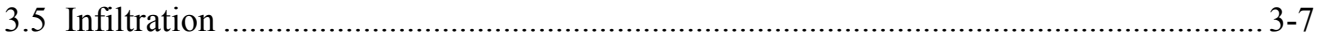

3.6 Solar Properties of Glazing Materials ..................................................................... 3-7

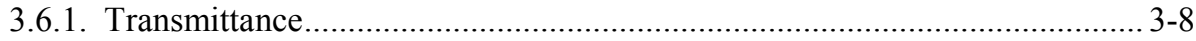

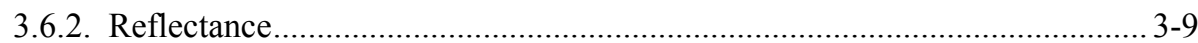

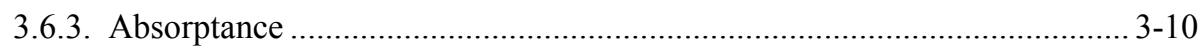

3.7 Infrared Properties of Glazing Materials (Emittance) …………................................. 3-10

4. SUMMARY OF ALGORITHMS ........................................................ 4-1

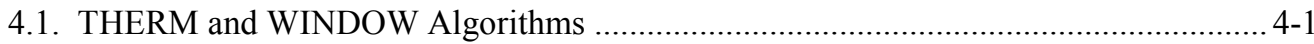

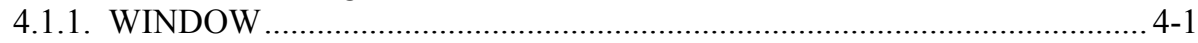

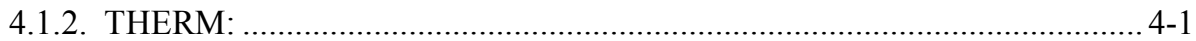

4.2 WINDOW Computational Method …………….................................................... 4-1

4.3 THERM Computational Methods ............................................................................ 4-3

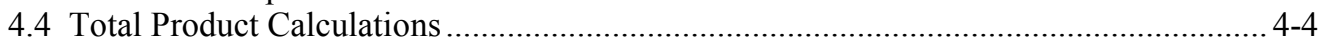

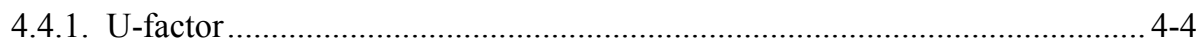

4.4.2. Solar Heat Gain Coefficient (SHGC) ...................................................... 4-5

4.4.3. Visible Transmittance ……….............................................................. 4-6

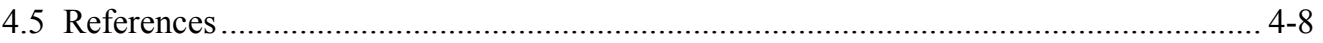

5. MODELING CENTER OF GLASS WITH WINDOW ............................. 5-1

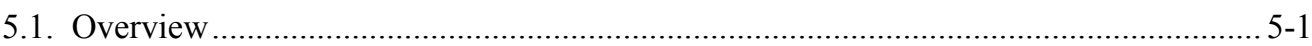

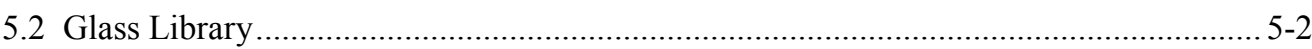

5.3 Glazing System Library -- Center-of-Glass U-factor ................................................ 5-3

5.3.1. Environmental Conditions …….............................................................. 5-4

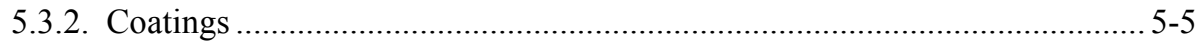

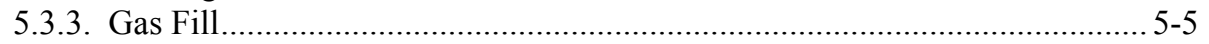

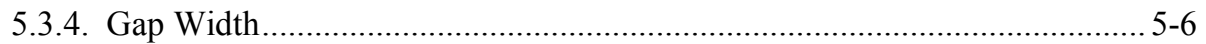

5.3.5. Laminated Glass / Applied Films................................................................ 5-6

5.4 Solar Heat Gain Coefficient and Visible Transmittance .................................................. 5-7

5.4.1. Define the Product Line .............................................................................. 5-7

5.4.2. Simplify the Product Line …………………........................................... 5-8

5.4.3. Calculate the Center-of-Glass SHGC and VT ............................................ 5-9

5.4.4. Determine the Product Groupings................................................................. 5-9

5.4.5. Determine Divider and Frame U-factors for SHGC and VT ..................... 5-10

5.4.6. Calculate the whole product SHGC and VT ....................................... 5-10

5.4.7. Specialty Products Table......................................................................... 5-10

\section{MODELING FRAME AND EDGE HEAT TRANSFER WITH THERM .... 6-1}

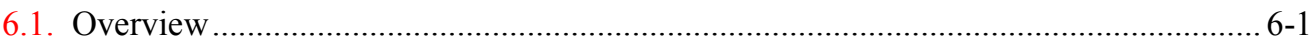

6.2 Cross-Sections to be Modeled ................................................................................ 6-1

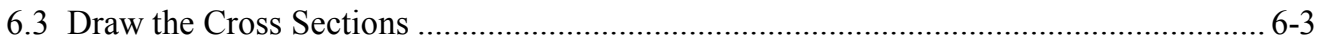

6.3.1. Getting Started - Drawings and DXF Files .............................................. 6-3

6.3.2. Cross-Section Orientation ................................................................ 6-3

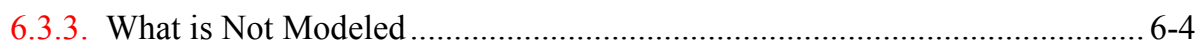

6.3.4. Deformable Parts ............................................................................................ 6-4

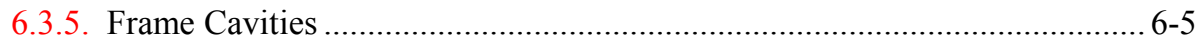

6.3.6. Vented Exterior Cavities........................................................................... 6-9

6.3.7. Modeling Sloped and Curved Surfaces.................................................. 6-10

6.3.8. Modeling Sloped Sills .......................................................................... 6-11 


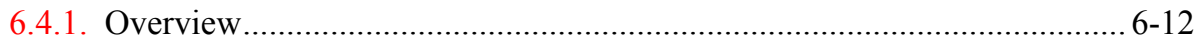

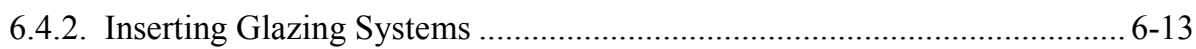

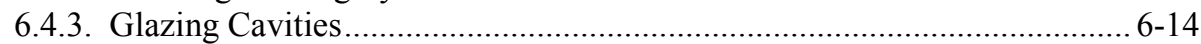

6.4.4. Multiple Glazing Options ....................................................................... 6-14

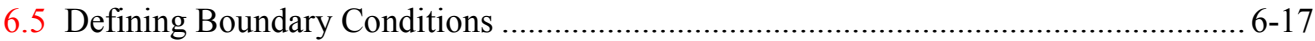

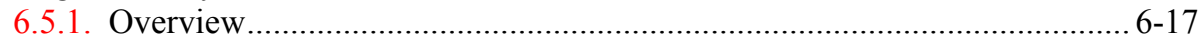

6.5.2. Assigning Boundary Conditions and U-factor Surface Tags ...................... 6-17

6.5.3. Voids, Overlaps and Bad Points .............................................................. 6-19

6.5.4. Examples of Bad Points ............................................................................. 6-21

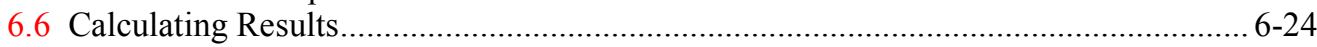

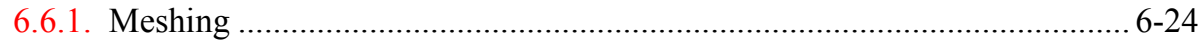

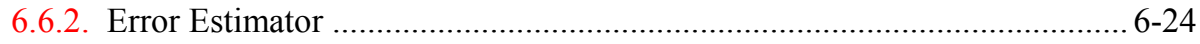

6.6.3. Required Settings for NFRC Simulations .............................................. 6-25

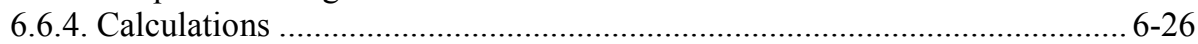

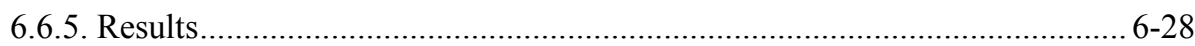

6.6.6. Custom Frame Length........................................................................... 6-29

6.6.7. Exporting Results to WINDOW Files................................................... 6-30

7. TOTAL PRODUCT CALCULATIONS ….............................................. 7-1

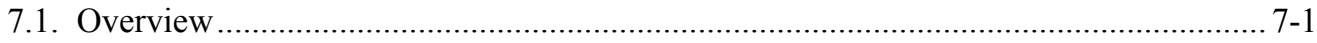

7.2 Frame and Edge U-factors from THERM .................................................................... 7-1

7.2.1. Importing T2W Files into WINDOW .................................................... $7-1$

7.3 Center-of-Glass U-factors from WINDOW ............................................................. $7-3$

7.4 Overall Product U-factor, SHGC, and VT Calculations ................................................ 7-4

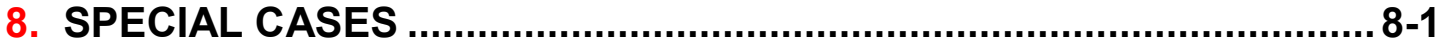

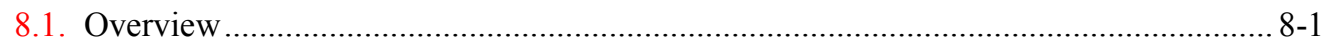

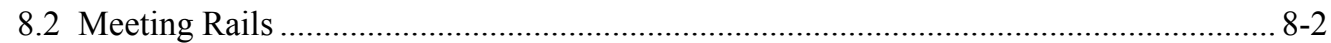

8.2.1. Modeling Meeting Rails ....................................................................... 8-2

8.2.2. Steps for Meeting Rail Calculation ........................................................... 8-3

8.3 Internal Dividers (Suspended Grilles) ..................................................................... 8-8

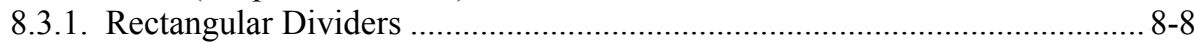

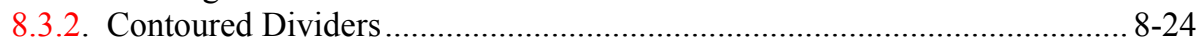

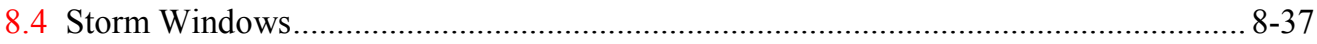

8.4.1. Modeling Steps ...................................................................................... 8-37

8.4.2. Example Storm Window Problem ………….......................................... 8-38

8.5 Projecting Products (Skylights / Greenhouse Windows) ............................................. 8-44

8.5.1. Skylight Modeling Steps ........................................................................ 8-44

8.5.2. Skylight Mounting Details ....................................................................... 8-45

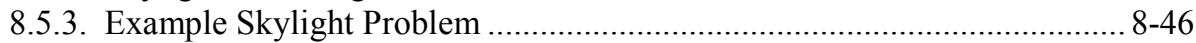

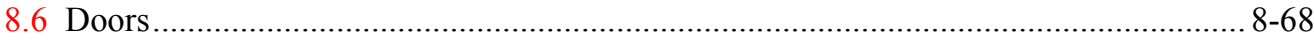

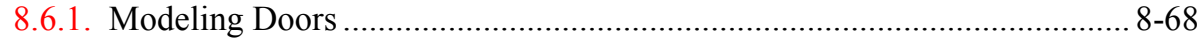

8.6.2. Door Cross Sections................................................................................. 8-68

8.6.3. Stile and Rail, and Embossed Door Glazing Options .................................. 8-69

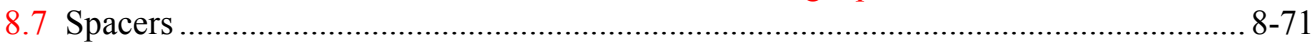

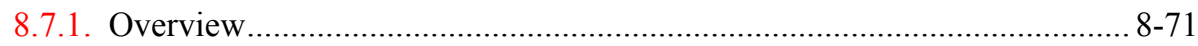

8.7.2. Modifying Glazing Cavities for Open Spacers ........................................... 8-72

9. SAMPLE PROBLEMS

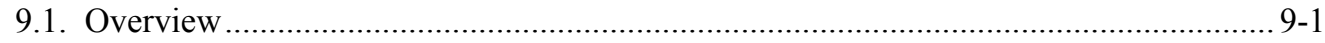

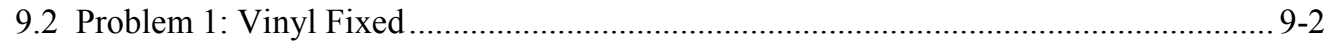

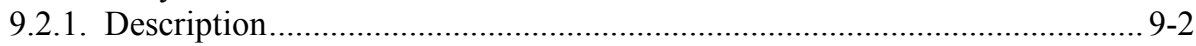

9.2.2. Glazing Matrix ......................................................................................... 9-2

9.2.3. Center-of-Glass Modeling (WINDOW) ....................................................... 9-2 
9.2.4. Edge-of-Glass and Frame Modeling (THERM)........................................... 9-3

9.2.5. Total Product U-Factor .............................................................................. 9-7

9.2.6. Total Product Solar Heat Gain Coefficient and Visible Transmittance ........ 9-8

9.2.7. Drawings Vinyl Fixed Window ................................................................ 9-9

9.3 Problem 2: Aluminum Horizontal Slider Window ..................................................... 9-11

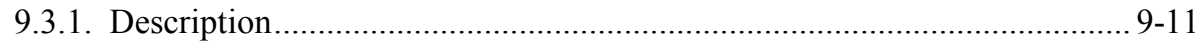

9.3.2. Glazing Matrix ……………….......................................................... 9-11

9.3.3. Center-of-Glass Modeling (WINDOW) ................................................ 9-12

9.3.4. Edge-of-Glass and Frame Modeling (THERM).......................................... 9-13

9.3.5. Total Product U-Factor ............................................................................ 9-23

9.3.6. Total Product Solar Heat Gain Coefficient and Visible Transmittance ...... 9-25

9.3.7. Drawings for Aluminum Horizontal Slider Window .................................. 9-25

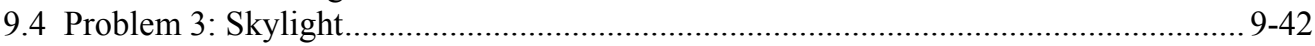

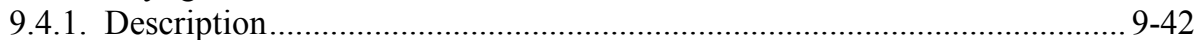

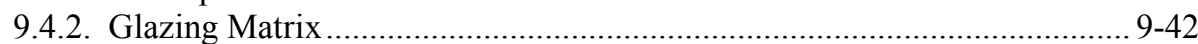

9.4.3. Center-of-Glass Modeling (WINDOW) ……............................................ 9-43

9.4.4. Edge-of-Glass and Frame Modeling (THERM)......................................... 9-44

9.4.5. Total Product U-Factor ............................................................................. 9-47

9.4.6. Total Product Solar Heat Gain Coefficient and Visible Transmittance ...... 9-48

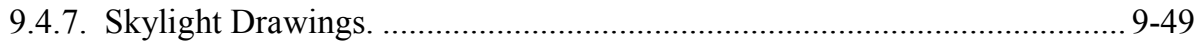

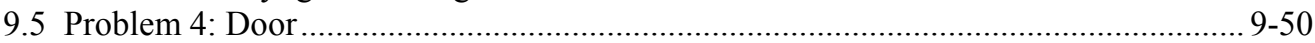

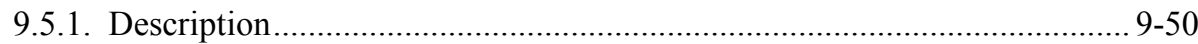

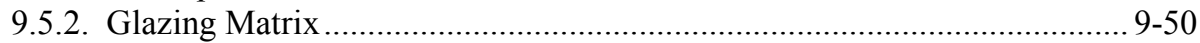

9.5.3. Center-of-Glass Modeling (WINDOW) ……......................................... 9-51

9.5.4. Edge-of-Glass and Frame Modeling (THERM)....................................... 9-52

9.5.5. Total Product U-Factor ....................................................................... 9-56

9.5.6. Total Product Solar Heat Gain Coefficient and Visible Transmittance ...... 9-58

9.5.7. Wood Stile and Rail Door Drawings. ...................................................... 9-59

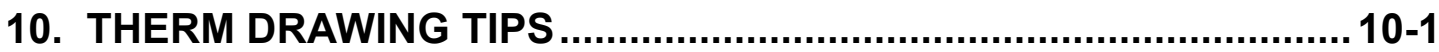

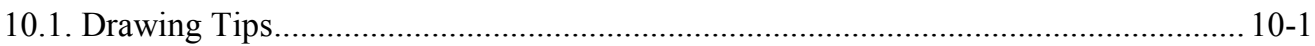

10.1.1. Drawing Methods..................................................................................

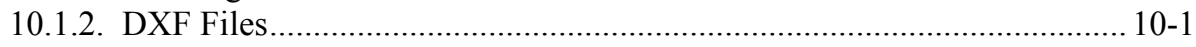

10.1.3. Representing Curves with Line Segments .............................................. 10-2

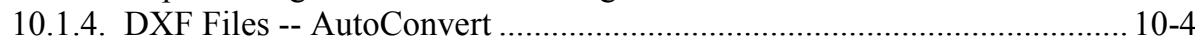

10.1.5. Sticky Distance and Snapping............................................................ 10-6

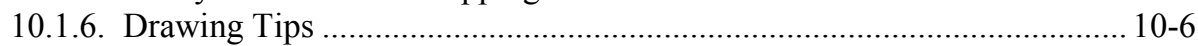

10.1.7. Drawing with the Keyboard ............................................................... 10-9

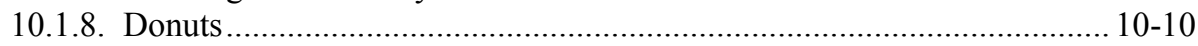

10.1.9. Breaking Up Extrusions into Polygons ............................................... 10-11

10.1.10. Drawing Extrusions from DXF Files -- False Die Mold Method ......... 10-13

10.1.11. Cuttins, Relocating, Flipping and Rotating Discussion ....................... 10-15

11. AKNOWLEDGEMENTS …........................................................... 11-1

Appendix A: THERM Material Library....................................................-1 


\section{INTRODUCTION}

\subsection{Overview}

This document, the THERM 2.1 NFRC Simulation Manual, discusses how to use THERM to model products for NFRC certified simulations and assumes that the user is already familiar with the THERM program. In order to learn how to use THERM, it is necessary to become familiar with the material in the THERM User's Manual.

In general, this manual references the THERM User's Manual rather than repeating the information.

If there is a conflict between the THERM User's Manual and the THERM 2.1 NFRC Simulation Manual, the THERM 2.1 NFRC Simulation Manual takes precedence.

The CD that is included with the manual includes all sample files that are referenced in the manual as well as some additional samples. The following cases include a full WINDOW library and WINDOW glazing systems files:

\begin{tabular}{|l|l|l|}
\hline Section & Title & Directory on CD \\
\hline $8.2 \& 9.3$ & Aluminum Horizontal Slider Window & \Aluminum Slider \\
\hline 8.3 & Internal Dividers & |Divider \\
\hline 8.4 & Storm Windows & IStormwindows \\
\hline $8.5 \& 9.4$ & Skylights & ISkylights \\
\hline $8.6 \& 9.5$ & Doors & |Doors \\
\hline 9.2 & Vinyl Fixed & IVinyl Fixed \\
\hline
\end{tabular}

Each of these folders has a subdirectory that starts with "w4xxxxx" (eg w4door, w4divider). These subdirectories should be copied to the W4 directory on the harddrive (usually c: $|w 4|$ ). When the files are copied to the harddrive, they will preserve the Read-Only attribute from the CD. Once all the necessary WINDOW and THERM files have been copied from the CD, copy the NotReadOnly.bat file from the $\backslash \mathrm{W} 4$ directory on the CD to the W4 directory on your harddrive. Double-clicking on NotReadOnly will run the batch file, which will remove the Read-Only attribute from all the files in directories under the current one (eg running it in c: $\mid w 4$, will also remove the Read-Only attribute in c: $|w 4| w 4 s k y$, c: $|w 4| w 4$ door etc). Also run the NotReadOnly.bat file from the directory where the THERM files were installed.

The Doc directory on the CD contains the THERM 2 and WINDOW4.1 users manual in PDF format, as well as a PDF version of this document. The SpectralData directory contains a current spectral data library. The directories RoundRobin and ExtraDxfs contain sample files for practice. 


\section{FENESTRATION PRODUCTS}

\subsection{Overview}

Much of the information in this chapter is taken from Residential Windows: A Guide to New Technologies and Energy Performance by John Carmody, Stephen Selkowitz, and Lisa Heschong.

Although glazing materials are the focus of much of the innovation and improvement in fenestration products, the overall performance of any unit is determined by the complete fenestration product assembly. The assembly includes the operating and fixed parts of the product frame as well as associated hardware and accessories. These are defined and illustrated at the beginning of this section. The next two sections address the different options available for sash operation and new advances in frame materials designed to improve product energy efficiency. Proper installation is an important aspect of their performance as well. The final section of this chapter discusses other installation issues.

\subsection{Fenestration Product Sash Operation}

There are numerous operating types available for fenestration products. Traditional operable types include the projected or hinged types such as casement, awning, and hopper, and the sliding types such as doubleand single-hung and horizontal sliding. In addition, the current market includes storm windows, sliding and swinging patio doors, skylights and roof-mounted (i.e., sloping) windows, and systems that can be added to a house to create bay or bow windows, miniature greenhouses, or full sun rooms.

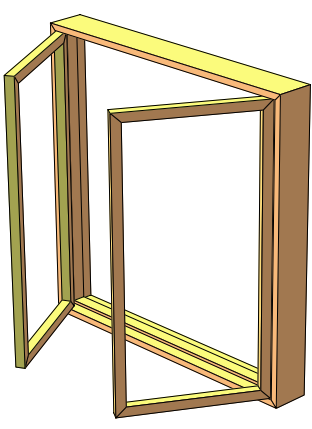

Casement

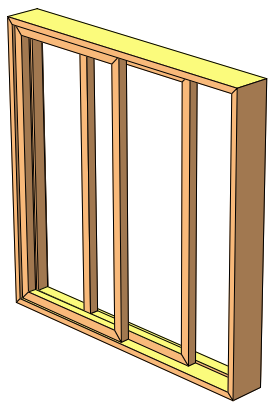

Horizontal slider
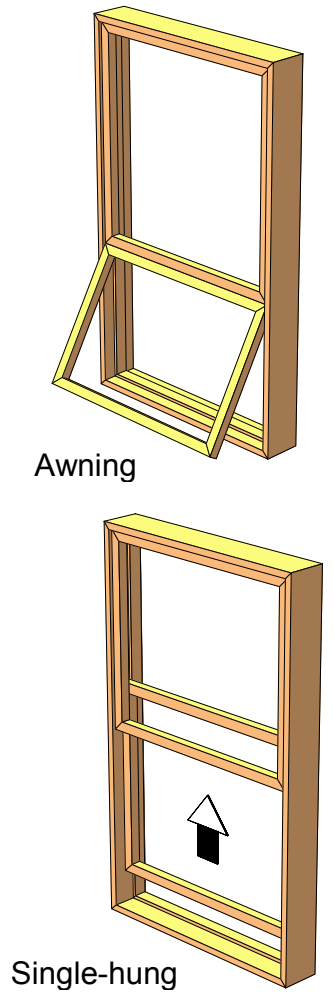

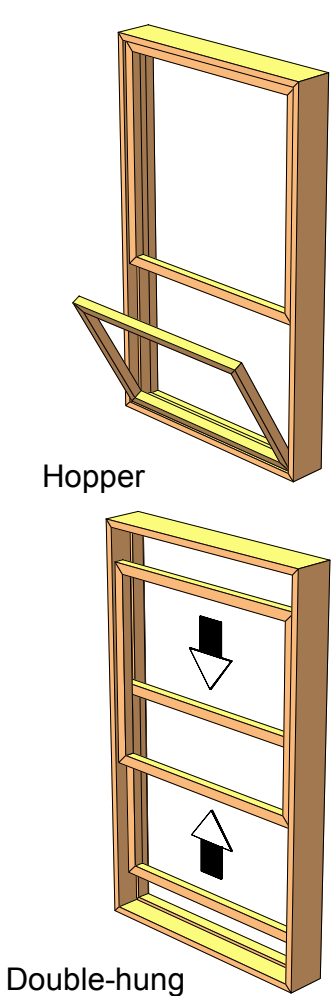

Figure 2-1. Fenestration Operator Types 


\subsubsection{Projected or Hinged Windows}

Hinged windows include casements, awnings, and hoppers - hinged at the side, top, and bottom, respectively. Some manufacturers also make pivoting and combination windows that allow for easier cleaning of the exterior surfaces. Hinged windows, especially casements, project outward, providing significantly better ventilation than sliders of equal size. Because the sash protrudes from the plane of the wall, it can be controlled to catch passing breezes, but screens must be placed on the interior side. Virtually the entire casement window area can be opened, while sliders are limited to less than half of the window area.

\subsubsection{Sliding Windows}

Sliders are the most common type of windows and include horizontal sliders and single-hung and doublehung windows. Ventilation area can vary from a small crack to an opening of one-half the total glass area. Screens can be placed on the exterior or interior of the window unit.

In double-hung or double-sliding units, both sashes can slide. In double-sliding units, the same net amount of glass area can be opened for ventilation as in single sliders, but it can be split between the top and bottom or two ends of the window for better control of the air flow.

\subsubsection{Sliding Glass Doors}

Sliding glass doors (patio doors) are essentially big sliding windows. As extremely large expanses of glass, patio doors exaggerate all of the issues related to comfort and energy performance. Since the proportion of glass to frame is very high for a glass sliding door, the selection of high-performance glass can have significant benefits.

\subsubsection{French Doors and Folding Patio Doors}

French doors and folding glazed doors are growing in popularity. A basic double French door consists of two hinged doors with no center mullion, resulting in a 5- to 6-foot-wide (1.5 to $1.8 \mathrm{~m}$ ) opening. Folding doors are typically made of pairs of hinged doors, so that a double folding door with two pairs of doors can create an opening of 12 feet $(3.7 \mathrm{~m})$ or more.

\subsubsection{Skylights and Roof Windows}

The vast majority of skylights are permanently fixed in place, mounted on a curb above a flat or sloped roof. However, hatch-style skylights that can be opened with an extended crank, push latch, or remote control motor are becoming more common. Some skylights have a domed profile made of one or two layers of tinted or diffusing plastic.

A roof window is a hybrid between a skylight and a standard window. They have become increasingly popular as homeowners and designers seek to better utilize space in smaller houses by creating habitable rooms under sloping roofs. They are glazed with glass rather than plastic and are available with most of the glazing and solar control options of standard windows. Both fixed and operable versions are available, and the operable roof windows can be opened manually or by a motorized system. In addition, some manufacturers offer special venting mechanisms that allow some ventilation air flow without actually opening the window. Operable skylights or vents allow hot air that rises to the ceiling level to be effectively exhausted from the space.

Skylights and roof windows present a special case for insulating around windows because they are typically set into the thickest, most heavily insulated framing in the house, and they must also meet much more 
stringent conditions for shedding water. In order to create a positive water flow around them, skylights are commonly mounted on "curbs" set above the roof plane. These curbs, rising 6 to 12 inches (15 to 30 centimeters) above the roof, create additional heat loss surfaces, right where the warmest air of the house tends to collect. Ideally, they should be insulated to the same level as the roof. In practice, it is often difficult to achieve insulation levels much above R-11. Some manufacturers provide curbs prefabricated out of a rigid insulating foam, which can be further insulated at the site.

Roof windows, mounted in a sloping roof, often include a metal flashing system. If this metal flashing is in contact with a metal window frame, it can create additional surfaces for conducting heat. Thus, as with thermally broken aluminum windows, care should be taken to ensure a thermal separation between the cold outer metal surfaces and metal parts of the window frame that are exposed to the warm interior air.

\subsubsection{Greenhouse (Garden) Windows}

Greenhouse windows, also known as garden windows, are typically prefabricated frame and glass kits that can be inserted into a new or existing window opening. They may include shelves for plants or simply be used as a means of creating a greater sense of spaciousness. Greenhouse windows generally have higher heat loss and heat gain than a regular window of the same size because they contain more glazing than a conventional window that fills the same wall opening.

\subsection{Performance Implications of Basic Fenestration Types}

There are subtle performance differences between a fixed and operable fenestration product that fills an identical rough opening. The fixed unit will typically have a smaller fraction of frame and proportionately more glass than the similar operable unit. Thus, fixed products with high-performance glass will have a better, lower U-factor, but a higher SHGC due to a smaller frame area and larger glass area. Fixed products have very low infiltration rates, but then they also do not provide natural ventilation and do not satisfy building code requirements for fire egress.

For operating fenestration products, the type of operation has little direct effect on the U-factor or SHGC of the unit, but it can have a significant effect on the air infiltration and ventilation characteristics. Operation can be broken into two basic types: sliding products and hinged products. The comments below are a general characterization of American fenestration products; however, they may not apply to a specific product made by a given manufacturer.

\subsubsection{Hinged Windows}

Hinged windows such as casements, awnings, and hoppers generally have lower air leakage rates than sliding windows from the same manufacturer because the sash closes by pressing against the frame, permitting the use of more effective compression-type weatherstripping. In most types, the sash swings closed from the outside, so that additional external wind pressure tends to push the sash more tightly shut. Hinged windows require a strong frame to encase and support the projecting sash. Also, because projectingtype sashes must be strong enough to swing out and still resist wind forces, the stiffer window units do not flex as readily in the wind. In addition, hinged windows have locking mechanisms that force the sash against the weatherstripping to maximize compression. These design details tend to reduce air infiltration of hinged windows in comparison to sliders.

\subsubsection{Sliding Windows}

Sliding windows, whether single-hung, double-hung, or horizontal sliders, generally have higher air leakage rates than projecting or hinged windows. Sliding windows typically use a brush-type weatherstripping that 
allows the sash to slide past. This type is generally less effective than the compression gaskets found in projecting windows. The weatherstrip effectiveness also tends to be reduced over time due to wear and tear from repeated movement of the sliding sash. The frames and sashes of sliding units can be made with lighter, less rigid frame sections since they only need to support their own weight. This lightness may permit the sliding frames to flex and can allow more air leakage under windy conditions. Manufacturers can choose to engineer greater stiffness in their products by design and material selection.

Slider window performance can also be improved with latching mechanisms that compress the sash to the fixed frame and by the addition of compression weatherstripping at the head and sill of double-hung windows or the end jamb of horizontal sliders.

\subsubsection{Sliding Glass and French Doors}

As previously noted, sliding doors are essentially big sliding windows. However, they are more complicated because of their size and weight and because the sill is also a door threshold, which must keep water out while allowing easy passage of people and objects. The threshold is typically the most difficult part of the frame to weatherstrip effectively.

French doors benefit from being much more like traditional doors than sliding doors. French doors can use weatherstripping and operating hardware designed for similar nonglazed doors. However, when there are large openings with multiple hinged doors, it is more difficult to positively seal the joints between door leaves and to create the stiffness that will resist infiltration.

\subsection{Frame Materials}

The material used to manufacture the frame governs the physical characteristics of the fenestration product, such as frame thickness, weight, and durability, but it also has a major impact on the thermal characteristics of the product. Increasingly, manufacturers are producing hybrid or composite sash and frames, in which multiple materials are selected and combined to best meet the overall required performance parameters. Thus, a simple inspection of the inner or outer surface of the frame is no longer an accurate indicator of the total material or its performance. Since the sash and frame represent from 10 to 30 percent of the total area of the fenestration unit, the frame properties will significantly influence the total product performance.

\subsubsection{Wood Frames}

Wood fenestration products are manufactured in all configurations, from sliders to swinging windows. Wood is favored in many residential applications because of its appearance and traditional place in house design.

A variation of the wooden product is to clad the exterior face of the frame with either vinyl or aluminum, creating a permanent weather-resistant surface. Clad frames thus have lower maintenance requirements, while retaining the attractive wood finish on the interior.

From a thermal point of view, wood-framed products perform well. The thicker the wood frame, the more insulation it provides. Wood-framed fenestration products typically exhibit low heat loss rates.

However, metal cladding, metal hardware, or the metal reinforcing often used at corner joints can degrade the thermal performance of wood frames. If the metal extends through the fenestration product from the cold side to the warm side of the frame, it creates a thermal short circuit, conducting heat more quickly through that section of the frame. 


\subsubsection{Aluminum Frames}

The biggest disadvantage of aluminum as a fenestration product frame material is its high thermal conductance. It readily conducts heat, greatly raising the overall U-factor of a fenestration unit. Because of its high thermal conductance, the thermal resistance of an aluminum frame is determined more by the amount of surface area of the frame than by the thickness or the projected area, as with other frame materials. Thus, an aluminum frame profile with a simple compact shape will perform much better than a profile with many fins and undulations.

In cold climates, a simple aluminum frame can easily become cold enough to condense moisture or frost on the inside surfaces of fenestration product frames. Even more than the problem of heat loss, the condensation problem spurred development of a more insulating aluminum frame.

The most common solution to the heat conduction problem of aluminum frames is to provide a "thermal break" by splitting the frame components into interior and exterior pieces and use a less conductive material to join them. There are many designs available for thermally broken aluminum frames. The most prevalent technique used in residential fenestration products is called "pouring and debridging." The frame is first extruded as a single piece with a hollow trough in the middle. This is filled with a plastic that hardens into a strong intermediate piece. The connecting piece of aluminum is then milled out, leaving only the plastic to join the two halves of aluminum. Functionally, the resulting piece is cut, mitered, and assembled like a simple aluminum extrusion. Thermally, the plastic slows the heat flow between the inside and outside. There are other manufacturing techniques for producing a thermal break, but the thermal results are similar.

\subsubsection{Vinyl Frames}

Plastics are relative newcomers as fenestration product frame materials in North America. Vinyl, also known as polyvinyl chloride (PVC), is a versatile material with good insulating value.

The thermal performances of vinyl frames is roughly comparable to wood. Large hollow chambers within the frame can allow unwanted heat transfer through convection currents. Creating smaller cells within the frame reduces this convection exchange, as does adding an insulating material. Most manufacturers are conducting research and development to improve the insulating value of their vinyl fenestration product assemblies.

\subsubsection{Fiberglass and Engineered Thermoplastics}

In addition to vinyl fenestration products two other polymer-based technologies have become available, fiberglass and thermoplastics. Frames can be made of glass-fiber-reinforced polyester, or fiberglass, which is pultruded into lineal forms and then assembled into fenestration products. These frames are dimensionally stable and have good insulating value by incorporating air cavities (similar to vinyl).

\subsubsection{Wood Composites}

Most people are familiar with composite wood products, such as particle board and laminated strand lumber, in which wood particles and resins are compressed to form a strong composite material. The wood fenestration product industry has now taken this a step further by creating a new generation of wood/polymer composites that are extruded into a series of lineal shapes for frame and sash members. These composites are stable, and have the same or better structural and thermal properties as conventional wood, with better moisture resistance and more decay resistance. They can be textured and stained or painted much like wood. They were initially used in critical elements, such as sills and thresholds in sliding patio doors, but are now being used for entire units. This approach has the added environmental advantage of reusing a volume of sawdust and wood scrap that would otherwise be discarded. 


\subsubsection{Hybrid and Composite Frames}

Manufacturers are increasingly turning to hybrid frame designs that use two or more of the frame materials described above to produce a complete fenestration product system. The wood industry has long built vinyland aluminum-clad products to reduce exterior maintenance needs. Vinyl manufacturers and others offer interior wood veneers to produce the finish and appearance that many homeowners desire. Split-sash designs may have an interior wood element bonded to an exterior fiberglass element.

\subsection{Basic Glazing Materials}

Two basic materials are used for fenestration product glazing: glass, which is by far the most common, and plastics, which have many specialized applications.

\subsubsection{Glass}

Traditionally, fenestration products have been made of clear glass. Most residential-grade clear glass today is produced with the float technique in which the glass is "floated" over a bed of molten tin. This provides extremely flat surfaces, uniform thicknesses, and few if any visual distortions. The glass has a slight greenish cast, due to iron impurities, but this is generally not noticeable except from the edge. An even higher-quality glass with reduced iron content eliminates the greenness and also provides a higher solar energy transmittance. This is commonly called "water-white glass."

Obscure glasses still transmit most of the light but break up the view in order to provide privacy. This effect is generally achieved either with decorative embossed patterns or with a frosted surface that scatters the light rays.

By adding various chemicals to glass as it is made, glass can be produced in a wide variety of colors. Glass colors are typically given trade names, but the most frequently used colors can be generally described as clear, bronze, gray, and blue-green. After clear glass, the gray glasses are most commonly used in residential construction, as they have the least effect on the perceived color of the light. Tinted glass is discussed later in this chapter.

The mechanical properties of glass can be altered, as well as its basic composition and surface properties. Heat-strengthening and tempering make glass more resistant to breakage. Heat-strengthened glass is about twice as strong as standard glass. Tempered glass is produced by reheating and then quickly chilling the glass. It breaks into small fragments, rather than into long, possibly dangerous shards. Laminated glass is a sandwich of two outer layers of glass with a plastic inner layer that holds the glass pieces together in the event of breakage. Fully tempered and laminated glass is required by building codes in many door and fenestration product applications.

\subsubsection{Plastics}

Several plastic materials have been adapted for use as glazing materials. Their primary uses are fenestration products with special requirements and skylights.

The following list of plastic glazing materials covers the major types of plastic glazing materials and compares their general properties:

- Clear acrylic is widely available and relatively inexpensive. It is available in various tints and colors. It has excellent visible light transmittance and longevity. However, it is softer than glass, which makes it vulnerable to scratching.

- Frosted acrylic is like clear acrylic, except that it diffuses light and obscures the view. It comes in varying degrees of light transmittance. Most bubble skylights are made of frosted acrylic. 
- Clear polycarbonate is like acrylic sheet, but it is harder and tougher, offering greater resistance to scratching and breakage. It is more expensive than acrylic.

- Fiber-reinforced plastic is a tough, translucent, flexible sheet material with good light-diffusing properties. Short lengths of fiberglass are embedded in a polymer matrix to form flat or ribbed sheets. Stiff, insulating, translucent panels are created by bonding double layers to a metal frame and adding fiberglass insulation. It is also formed into corrugated sheets as a translucent roofing material. Surface erosion may shorten its useful life.

- Extruded multicell sheet, usually made with acrylic or polycarbonate plastic, is a transparent or tinted plastic extruded into a double- or triple-wall sheet with divider webs for stiffness, insulating value, and light diffusion.

- Polyester is a thin film used to carry specialized coatings and/or to divide the air space between two layers of glass into multiple air spaces. Highly transparent, it is protected from abuse and weathering by the two exterior glass layers. It can also be used in tinted or coated forms as film that is glued to the inner surface of existing fenestration products for retrofitting applications.

\subsection{Improved Glazing Products}

There are three fundamental approaches to improving the energy performance of glazing products:

1. Alter the glazing material itself by changing its chemical composition or physical characteristics. An example of this is tinted glazing. The glazing material can also be altered by creating a laminated glazing.

2. Apply a coating to the glazing material surface. Reflective coatings and films were developed to reduce heat gain and glare, and more recently, low-emittance and spectrally selective coatings have been developed to improve both heating and cooling season performance.

3. Assemble various layers of glazing and control the properties of the spaces between the layers. These strategies include the use of two or more panes or films, low-conductance gas fills between the layers, and thermally improved edge spacers.

Two or more of these approaches may be combined. Each of these improvements to the glazing is discussed below

\subsubsection{Tinted Glazing}

Both plastic and glass materials are available in a large number of tints. The tints absorb a portion of the light and solar heat. Tinting changes the color of the fenestration product.

Tinted glazings retain their transparency from the inside, so that the outward view is unobstructed. The most common colors are neutral gray, bronze, and blue-green, which do not greatly alter the perceived color of the view and tend to blend well with other architectural colors. Many other specialty colors are available for particular aesthetic purposes.

Tinted glass is made by altering the chemical formulation of the glass with special additives. Its color changes with the thickness of the glass and the addition of coatings applied after manufacture. Every change in color or combination of different glass types affects transmittance, solar heat gain coefficient, reflectivity, and other properties. Glass manufacturers list these properties for every color, thickness, and assembly of glass type they produce.

Tinted glazings are specially formulated to maximize their absorption across some or all of the solar spectrum and are often referred to as "heat-absorbing." All of the absorbed solar energy is initially transformed into heat within the glass, thus raising the glass temperature. Depending upon climatic conditions, up to 50 
percent of the heat absorbed in a single layer of tinted glass may then be transferred via radiation and convection to the inside. Thus, there may be only a modest reduction in overall solar heat gain compared to other glazings.

There are two categories of tinted glazing: the traditional tints that diminish light as well as heat gain, and spectrally selective tints that reduce heat gain but allow more light to be transmitted to the interior. The traditional tinted glazing often forces a trade-off between visible light and solar gain. For these bronze and gray tints, there is a greater reduction in visible light transmittance than there is in solar heat gain coefficient. This can reduce glare by reducing the apparent brightness of the glass surface, but it also reduces the amount of daylight entering the room.

To address the problem of reducing daylight with traditional tinted glazing, glass manufacturers have developed new types of tinted glass that are "spectrally selective." They preferentially transmit the daylight portion of the solar spectrum but absorb the near-infrared part of sunlight. This is accomplished by adding special chemicals to the float glass process. Like other tinted glass, they are durable and can be used in both monolithic and multiple-glazed fenestration product applications. These glazings have a light blue or green tint and have visible transmittance values higher than conventional bronze- or gray-tinted glass, but have lower solar heat gain coefficients. Because they are absorptive, they are best used as the outside glazing in a double-glazed unit. They can also be combined with Low-E coatings to enhance their performance further.

\subsubsection{Reflective Coatings and Films}

As the solar heat gain is lowered in single-pane tinted glazings, the visible light transmission drops even faster, and there are practical limits on how low the solar heat gain can be made using tints. If larger reductions are desired, a reflective coating can be used to lower the solar heat gain coefficient by increasing the surface reflectivity of the material. These coatings usually consist of thin metallic layers. The reflective coatings come in various metallic colors (silver, gold, bronze), and they can be applied to clear or tinted glazing (the substrate). The solar heat gain of the substrate can be reduced a little or a lot, depending on the thickness and reflectivity of the coating, and its location on the glass.

As with tinted glazing, the visible light transmittances of reflective glazings are usually reduced substantially more than the solar heat gain.

\subsubsection{Double Glazing}

Storm windows added onto the outside of window frames during the stormy winter season were the first double-glazed fenestration products. They reduce infiltration from winter winds by providing a seal around all the operating sash and they improve the insulating value of the glazing as well.

When manufacturers began to experiment with factory-sealed, double-pane glass to be installed for yearround use, they encountered a number of technical concerns, such as how to allow for different thermal movement between the two panes, how to prevent moisture from forming between the panes and condensing on an inaccessible surface, and how to allow for changes in atmospheric pressure as the assembly was moved from factory to installation site. These issues have been successfully addressed over the years with a variety of manufacturing techniques and material selections.

When double-glass units first came on the market, the two glass layers were often fused around the perimeter to make a permanently sealed air space. In recent years, however, spacers and polymer sealants have largely replaced glass-to-glass seals, and have proven sufficiently durable for residential applications. The layers of glass are separated by and adhere to a spacer, and the sealant, which forms a gas and moisture barrier, is applied around the entire perimeter. Normally, the spacer contains a desiccant material to absorb any residual moisture that may remain in the air space after manufacture. Sealed insulating glass units are now a 
mature, well proven technology. Designs utilizing high-quality sealants and manufactured with good quality control should last for decades without seal failure.

\subsubsection{Glass Coatings and Tints in Double Glazing}

Both solar reflective coatings and tints on double-glazed fenestration products are effective in reducing summer heat gain; however, only certain coatings contribute to reducing winter heat loss, and tints do not affect the heat loss rates at all. It is possible to provide reflective coatings on any one of the four surfaces, although they are usually located on the outermost surface or on the surfaces facing the air space. Coating location can also depend on the type of coating. Some vacuum-deposited reflective coatings must be placed in a sealed air space because they would not survive exposure to outdoor elements, finger prints, or cleaning agents. Pyrolytic coatings that are created with a high-temperature process as the glass is formed are extremely hard and durable and can be placed anywhere. Each location produces a different visual and heat transfer effect. Other advanced coatings such as low-emittance and spectrally selective coatings are normally applied to double-glazed or triple-glazed fenestration products. These applications are discussed later in this chapter.

Double-pane units can be assembled using different glass types for the inner and outer layers. Typically, the inner layer is standard clear glass, while the outer layer can be tinted, reflective, or both. The solar heat gain coefficient is reduced because the tinted glass and clear glass both reduce transmitted radiation. In addition, this design further reduces solar heat gain because the inner clear glass, the gas fill, and any Low-E coating keep much of the heat absorbed by the outer glass from entering the building interior.

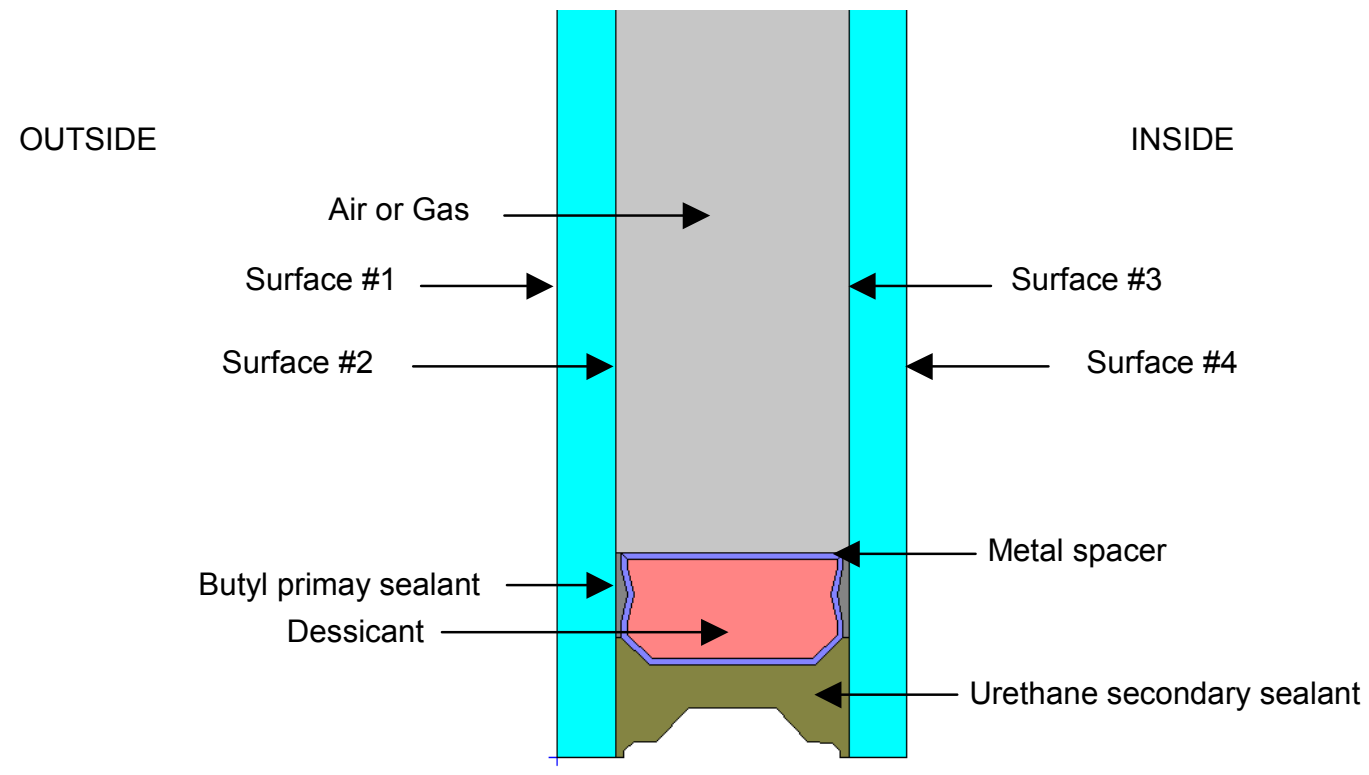

Figure 2-2. Insulating Glass Unit (IGU).

\subsubsection{Gap Width in Multiple Glazed Units}

Fenestration product manufacturers have some flexibility to reduce heat transfer by selecting the best gap width between two or more glazings. The air space between two pieces of glass reaches its optimum insulating value at about 1/2-inch $(12 \mathrm{~mm})$ thickness when filled with air or argon. As the gap gets larger, convection in the gap increases and slowly increases heat transfer. Below $3 / 8$ inch $(9 \mathrm{~mm})$, conduction through the air gap increases and the U-factor rises more rapidly. Krypton gas has its optimum thickness at about $1 / 4$ inch $(6 \mathrm{~mm})$, so that if smaller air gaps are required, for example in a three-layer fenestration 
product whose overall exterior dimensions are limited, krypton may be the best selection, although it is also more costly.

\subsubsection{Divided Lights}

Manufacturers have been struggling with the problem of many homeowners' preference for traditional, divided light fenestration products, which have many small panes separated by thin bars called muntins. With single-pane glass, true divided lights actually improved the thermal performance of the fenestration product because the wood muntins had a higher insulating value than the glass. Some manufacturers have introduced "true divided light" insulated units, in which traditional-looking muntins hold small, individual, insulated panes. However, these are expensive and difficult to fabricate with insulated glass and have greater thermal losses due to the number of edges, which now have metal in them.

A second option is to produce a single, large sealed glass unit with "muntins" glued to the inside and outside surfaces, while a grid is placed in the middle of one large insulated unit, giving the visual effect of divided lights. This reduces fabrication costs but does not reduce resistance to heat flow if the muntins in the middle are metal and if they touch both lights of glass.

A third option, which is more energy efficient, is to build a large-pane insulated unit that has snap-on or glued-on grilles to simulate the traditional lights.

The energy performance of the simple snap-on grid will be similar to a unit without any mullions; however, the true divided lights will result in greater heat transfer because of the additional edges.

\subsubsection{Special Products}

Glass blocks present a very special case of double glazing. They provide light with some degree of visual privacy. Plastic blocks, which have a lower U-factor than glass, are also available. However, when installed, the necessary grouting reduces the energy efficiency. Also, metal mesh and steel reinforcing bars, used between blocks to provide structural stability, provide thermal bridges which also reduce energy efficiency.

Plastic glazings are available in a number of configurations with double layers. Double-glazed acrylic bubble skylights are formed with two layers separated by an air space of varying thickness, ranging from no separation at the edges to as much as 3 inches $(7.6 \mathrm{~cm})$ at the top of the bubble. The average separation is used to calculate the effective U-factor.

Multicell polycarbonate sheets, which can be mounted with the divider webs running vertically or horizontally, are available. The divider webs increase the effective insulating value of the glazing by reducing convection exchange within the cells, especially when they are mounted horizontally.

\subsubsection{Multiple Panes or Films}

By adding a second pane, the insulating value of the fenestration product glass alone is doubled (the U-factor is reduced by half). As expected, adding a third or fourth pane of glass further increases the insulating value of the fenestration product, but with diminishing effect.

Triple- and quadruple-glazed fenestration products became commercially available in the 1980s as a response to the desire for more energy-efficient products. There is a trade-off with this approach, however. As each additional layer of glass adds to the insulating value of the assembly, it also reduces the visible light transmission and the solar heat gain coefficient, thereby reducing the fenestration product's value for providing solar gains or daylighting. In addition, other complications are encountered. Additional panes of glass increase the weight of the unit, which makes mounting and handling more difficult and transportation more expensive. 
Because of the difficulties discussed above, it is apparent there are physical and economic limits to the number of layers of glass that can be added to a fenestration product assembly. However, multiple-pane units are not limited to assemblies of glass. One popular innovation is based on substituting an inner plastic film for the middle layer of glass. The plastic film is very lightweight, and because it is very thin, it does not increase the thickness of the unit. The glass layers protect the inner layer of plastic from scratching, mechanical abuse, corrosion, weathering, and visual distortions caused by wind pressure. Thus, the strength and durability of plastic as a glazing material are no longer issues when the plastic is protected from physical abuse and weathering by inner and outer layers of glass. The plastic films are specially treated to resist UV degradation and they are heat shrunk so they remain flat under all conditions.

The plastic inner layer serves a number of important functions. It decreases the U-factor of the fenestration product assembly by dividing the inner air space into multiple chambers. Units are offered with one or two inner layers of plastic. Secondly, a Low-E coating can be placed on the plastic film itself to further lower the U-factor of the assembly. Also, the plastic film can be provided with spectrally selective coatings to reduce solar gain in hot climates without significant loss of visible transmittance. The performance of multiple-pane fenestration product assemblies with low-emittance coatings and gas fills is described in the following sections.

\subsubsection{Low-Emittance Coatings}

The principal mechanism of heat transfer in multilayer glazing is thermal radiation from a warm pane of glass to a cooler pane. Coating a glass surface with a low-emittance material and facing that coating into the gap between the glass layers blocks a significant amount of this radiant heat transfer, thus lowering the total heat flow through the fenestration product. The improvement in insulating value due to the Low-E coating is roughly equivalent to adding another pane of glass to a multipane unit.

The solar spectral reflectances of Low-E coatings can be manipulated to include specific parts of the visible and infrared spectrum. A glazing material can then be designed to optimize energy flows for solar heating, daylighting, and cooling.

With conventional clear glazing, a significant amount of solar radiation passes through the fenestration product, and then heat from objects within the house is reradiated back through the fenestration product. For example, a glazing design for maximizing solar gains in the winter would ideally allow all of the solar spectrum to pass through, but would block the reradiation of heat from the inside of the house. The first LowE coatings were designed to have a high solar heat gain coefficient and a high visible transmittance to transmit the maximum amount of sunlight into the interior while reducing the U-factor significantly.

A glazing designed to minimize summer heat gains but allow for some daylighting would allow visible light through, but would block all other portions of the solar spectrum, including ultraviolet light and nearinfrared, as well as long-wave heat radiated from outside objects, such as paving and adjacent buildings, as shown in Figure 2-3. These second-generation Low-E coatings were designed to reflect the solar nearinfrared, thus reducing the total solar heat gain coefficient while maintaining high levels of light transmission . Variations on this design (modified coatings and/or glazings) can further reduce summer solar heat gain and control glare.

There are three basic types of Low-E coatings available on the market today:

1. High-transmission Low-E:

These Low-E glass products are often referred to as pyrolitic or hard coat Low-E glass, due to the glass coating process. The properties presented here are typical of a Low-E glass product designed to reduce heat loss but admit solar gain.

2. Moderate-transmission Low-E:

These Low-E glass products are often referred to as sputtered (or soft-coat products) due to the glass 
coating process. (Note: Low solar gain Low-E products are also sputtered coatings.) Such coatings reduce heat loss and let in a reasonable amount of solar gain.

3. Low-transmission Low-E:

These Low-E products are often referred to as sputtered (or soft-coat) due to the glass coating process. (Note: Moderate solar gain Low-E products are also sputtered coatings.) This type of Low-E product, sometimes called spectrally selective Low-E glass, reduces heat loss in winter but also reduces heat gain in summer. Compared to most tinted and reflective glazings, this Low-E glass provides a higher level of visible light transmission for a given amount of solar heat reduction.

The type and quality of Low-E coating will affect not only the U-factor, but also the transmittance and solar heat gain coefficient of a glass. All these properties (U-factor, VT, and SHGC) need to be taken into

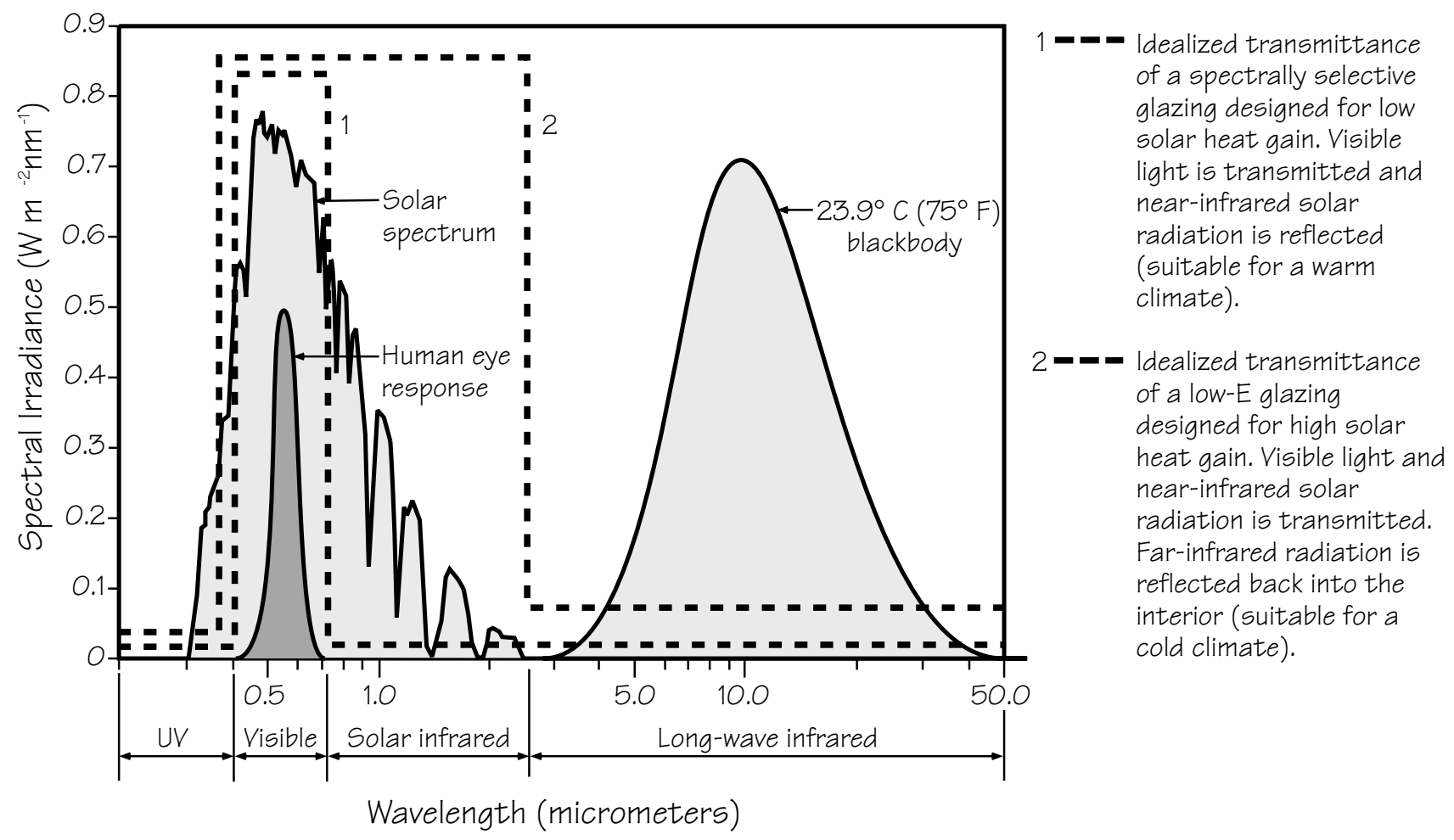

consideration in characterizing a particular glazing product.

Figure 2-3. Ideal spectral transmittance for glazings in different climates. (Source: "Sensitivity of Fenestration Solar Gain to Source Spectrum and Angle of Incidence." ASHRAE Transactions 10, R. McCluney, June 1996).

\subsubsection{Coating Placement}

The placement of a Low-E coating within the air gap of a double-glazed fenestration product does not significantly affect the U-factor but it does influence the solar heat gain coefficient (SHGC). That is why, in heating-dominated climates, placing a Low-E coating on the \#3 surface (outside surface of the inner pane) is recommended to maximize winter passive solar gain at the expense of a slight reduction in the ability to control summer heat gain. In cooling climates, a coating on the \#2 surface (inside surface of the outer pane) is generally best to reduce solar heat gain and maximize energy efficiency. Manufacturers sometimes place the coatings on other surfaces (e.g., \#2 surface in a heating climate) for other reasons, such as minimizing the potential for thermal stress. Multiple Low-E coatings are also placed on surfaces within a triple-glazed fenestration product assembly, or on the inner plastic glazing layers of multipane assemblies referred to as superwindows (discussed later in this chapter), with a cumulative effect of further improving the overall Ufactor. 


\subsubsection{Coating Types}

There are two basic types of Low-E coatings - sputtered and pyrolytic, referring to the process by which they are made. The best of each type of coating is colorless and optically clear. Some coatings may have a slight hue or subtle reflective quality, particularly when viewed in certain lighting conditions or at oblique angles.

A sputtered coating is multilayered (typically, three primary layers, with at least one layer of metal) and is deposited on glass or plastic film in a vacuum chamber. The total thickness of a sputtered coating is only $1 / 10,000$ of the thickness of a human hair. Sputtered coatings often use a silver layer and must be protected from humidity and contact. For this reason they are sometimes referred to as "soft coats." Since sputtering is a low-temperature process, these coatings can be deposited on flat sheets of glass or thin plastic films. While sputtered coatings are not durable in themselves, when placed into a sealed double- or triple-glazed assembly they should last as long as the sealed glass unit. Sputtered coatings typically have lower emittances than pyrolytic coatings. They are available commercially with emittance ratings of $\mathrm{e}=0.20$ to as low as $\mathrm{e}=0.04(\mathrm{e}=$ 0.20 means that 80 percent of the long-wavelength radiant energy received by the surface is reflected, while e $=0.04$ means 96 percent is reflected). For uncoated glass, $\mathrm{e}=0.84$, which means only 16 percent of the radiant energy received by the surface is reflected.

A typical pyrolytic coating is a metallic oxide, most commonly tin oxide with some additives, which is deposited directly onto a glass surface while it is still hot. The result is a baked-on surface layer that is quite hard and thus very durable, which is why this is sometimes referred to as a "hard coat." A pyrolytic coating can be ten to twenty times thicker than a sputtered coating but is still extremely thin. Pyrolytic coatings can be exposed to air, cleaned with normal cleaning products, and subjected to general wear and tear without losing their Low-E properties.

Because of their greater durability, pyrolytic coatings are available on single-pane glass and separate storm windows, but not on plastics, since they require a high-temperature process. In general, though, pyrolytic coatings are used in sealed, double-glazed units with the Low-E surface inside the sealed air space. While there is considerable variation in the specific properties of these coatings, they typically have emittance ratings in the range of $\mathrm{e}=0.20$ to $\mathrm{e}=0.15$.

A laminated glass with a spectrally selective Low-E sputtered coating on plastic film sandwiched between two layers of glass offers the energy performance of single-pane, spectrally selective glass and the safety protection of laminated glass. However, in this configuration, since the Low-E surface is not exposed to an air space, there is no effect on the glazing U-factor.

\subsubsection{Gas Fills}

Another improvement that can be made to the thermal performance of insulating glazing units is to reduce the conductance of the air space between the layers. Originally, the space was filled with air or flushed with dry nitrogen just prior to sealing. In a sealed glass insulating unit air currents between the two panes of glazing carry heat to the top of the unit and settle into cold pools at the bottom. Filling the space with a less conductive gas minimizes overall transfer of heat between two glass layers.

Manufacturers have introduced the use of argon and krypton gas fills, with measurable improvement in thermal performance. Argon is inexpensive, nontoxic, nonreactive, clear, and odorless. The optimal spacing for an argon-filled unit is the same as for air, about $1 / 2$ inch $(12 \mathrm{~mm})$. Krypton has better thermal performance, but is more expensive to produce. Krypton is particularly useful when the space between glazings must be thinner than normally desired, for example, $1 / 4$ inch $(6 \mathrm{~mm})$. A mixture of krypton and argon gases is also used as a compromise between thermal performance and cost.

Filling the sealed unit completely with argon or krypton presents challenges that manufacturers continue to work on. A typical gas fill system adds the gas into the cavity with a pipe inserted through a hole at the edge of the unit. As the gas is pumped in, it mixes with the air, making it difficult to achieve 100 percent purity. 
Recent research indicates that 90 percent is the typical concentration achieved by manufacturers today. Some manufacturers are able to consistently achieve better than 95 percent gas fill by using a vacuum chamber. An uncoated double-pane unit filled with 90 percent argon gas and 10 percent air yields a slightly more than 5 percent improvement in the insulating value at the center of the glass, compared to the same unit filled with air. However, when argon and krypton fills are combined with Low-E coatings and multipane glazings, more significant reductions of 15 to 20 percent can be achieved. Since the Low-E coating has substantially reduced the radiation component of heat loss, the gas fill now has a greater proportional effect on the remaining heat transfer by convection and conduction.

\subsection{Thermally Improved Edge Spacers}

The layers of glazing in an insulating glass (IG) unit must be held apart at the appropriate distance by spacers. The spacer system must provide a number of additional functions in addition to keeping the glass units at the proper dimension:

- accommodate stress induced by thermal expansion and pressure differences;

- provide a moisture barrier that prevents passage of water or water vapor that would fog the unit;

- provide a gas-tight seal that prevents the loss of any special low-conductance gas in the air space;

- create an insulating barrier that reduces the formation of interior condensation at the edge.

Older double-pane wood fenestration products used a wood spacer that could not be hermetically sealed and thus was vented to the outside to reduce fogging in the air gap. Modern versions of this system function well but, because they are not hermetically sealed, cannot be used with special gas fills or some types of Low-E coatings. Early glass units were often fabricated with an integral welded glass-to-glass seal. These units did not leak but were difficult and costly to fabricate, and typically had a less-than-optimal narrow spacing. The standard solution for insulating glass units (IGUs) that accompanied the tremendous increase in market share of insulating glass in the 1980s was the use of metal spacers, and sealants. These spacers, typically aluminum, also contain a desiccant that absorbs residual moisture. The spacer is sealed to the two glass layers with organic sealants that both provide structural support and act as a moisture barrier. There are two generic systems for such IGUs: a single-seal spacer and a double-seal system.

In the single-seal system, an organic sealant, typically a butyl material, is applied behind the spacer and serves both to hold the unit together and to prevent moisture intrusion. These seals are normally not adequate to contain special low-conductance gases.

In a double-seal system, a primary sealant, typically butyl, seals the spacer to the glass to prevent moisture migration and gas loss, and a secondary backing sealant, often silicone, provides structural strength. When sputtered Low-E coatings are used with double-seal systems, the coating must be removed from the edge first ("edge deletion") to provide a better edge seal.

Since aluminum is an excellent conductor of heat, the aluminum spacer used in most standard edge systems represented a significant thermal "short circuit" at the edge of the IGU, which reduces the benefits of improved glazings. As the industry has switched from standard double-glazed IGUs to units with Low-E coatings and gas fills, the effect of this edge loss becomes even more pronounced. Under winter conditions, the typical aluminum spacer would increase the U-factor of a Low-E, gas fill unit slightly more than it would increase the U-factor of a standard double-glazed IGU. The smaller the glass area, the larger the effect of the edge on the overall product properties. In addition to the increased heat loss, the colder edge is more prone to condensation.

Fenestration product manufacturers have developed a series of innovative edge systems to address these problems, including solutions that depend on material substitutions as well as radically new designs. One approach to reducing heat loss has been to replace the aluminum spacer with a metal that is less conductive, e.g., stainless steel, and change the cross-sectional shape of the spacer. Another approach is to replace the metal with a design that uses materials that are better insulating. The most commonly used design 
incorporates spacer, sealer, and desiccant in a single tape element. The tape includes a solid, extruded thermoplastic compound that contains a blend of desiccant materials and incorporates a thin, fluted metal shim of aluminum or stainless steel. Another approach uses an insulating silicone foam spacer that incorporates a desiccant and has a high-strength adhesive at its edges to bond to glass. The foam is backed with a secondary sealant. Both extruded vinyl and pultruded fiberglass spacers have also been used in place of metal designs.

There are several hybrid designs that incorporate thermal breaks in metal spacers or use one or more of the elements described above. Some of these are specifically designed to accommodate three- and four-layer glazings or IGUs incorporating stretched plastic films. All are designed to interrupt the heat transfer pathway at the glazing edge between two or more glazing layers.

Warm edge spacers have become increasingly important as manufacturers switch from conventional double glazing to higher-performance glazing. For purposes of determining the overall fenestration product Ufactor, the edge spacer has an effect that extends beyond the physical size of the spacer to a band about 2-1/2 inches $(64 \mathrm{~mm})$ wide. The contribution of this 2-1/2-inch-wide "glass edge" to the total fenestration product U-factor depends on the size of the product. Glass edge effects are more important for smaller fenestration products, which have a proportionately larger glass edge area. For a typical residential-size window (3 by 4 feet, 0.8 by 1.2 meters), changing from a standard aluminum edge to a good-quality warm edge will reduce the overall fenestration product $\mathrm{U}$-factor by 0.01 to $0.02 \mathrm{Btu} / \mathrm{hr}-\mathrm{sq} \mathrm{ft}-{ }^{\circ} \mathrm{F}$. 


\section{FENESTRATION HEAT TRANSFER BASICS}

\subsection{Overview}

Much of the information in this chapter is taken from the book Residential Windows: A Guide to New Technologies and Energy Performance by John Carmody, Stephen Selkowitz, and Lisa Heschong. Additional information can also be found in the ASHRAE 1997 Handbook of Fundamentals.

\subsection{Energy Flow Mechanisms}

Overall energy flow through a fenestration product is a function of:

- Temperature Driven Heat Transfer: When there is a temperature difference between inside and outside, heat is lost or gained through the fenestration product frame and glazing by the combined effects of conduction, convection, and radiation. This is indicated in terms of the U-factor of a fenestration assembly.

- Solar Gain: Regardless of outside temperature, heat can be gained through fenestration products by direct or indirect solar radiation. The amount of heat gain through products is measured in terms of the solar heat gain coefficient (SHGC) of the glazing.

- Infiltration: Heat loss and gain also occur by infiltration through cracks in the fenestration assembly. This effect is measured in terms of the amount of air (cubic feet or meters per minute) that passes through a unit area of fenestration product (square foot or meter) under given pressure conditions. In reality, infiltration varies with wind-driven and temperature-driven pressure changes. Infiltration also contributes to summer cooling loads in some climates by raising the interior humidity level.

The 1997 ASHRAE Handbook of Fundamentals contains the following equation for calculating the energy flow through a fenestration product (assuming no humidity difference):

$$
q=U_{t} * A_{p f}\left(t_{o u t}-t_{\text {in }}\right)+\left(S H G C_{t} * A_{p f} * E_{t}\right)+\left(60\left(A I^{*} A_{p f}\right) \rho c_{p}\left(t_{\text {out }}-t_{\text {in }}\right)\right)
$$

Where:

$$
\begin{array}{ll}
q & =\text { instantaneous energy flow, } \mathrm{Btu} / \mathrm{h} \\
U_{0} & =\text { overall coefficient of heat transfer (U-factor), Btu } / \mathrm{h}-\mathrm{ft}^{2}-\mathrm{o} \mathrm{F} \\
t_{i n} & =\text { interior air temperature, }{ }^{\circ} \mathrm{F} \\
t_{\text {out }} & =\text { exterior air temperature, }{ }^{\circ} \mathrm{F} \\
A_{p f} & =\text { Total projected area of fenestration, } \mathrm{ft}^{2} \\
S H G C_{o} & =\text { overall solar heat gain coefficient, non-dimensional } \\
E_{t} & =\text { incident total irradiance, Btu } / \mathrm{h}-\mathrm{ft}^{2} \\
A I & =\text { Air infiltration rate at current conditions in } \mathrm{cfm} / \mathrm{ft}^{2}, \text { not test conditions } \\
\rho & =\text { Air density, } \mathrm{lb} \mathrm{m} / \mathrm{ft}^{3}(\mathrm{about} 0.075) \\
c_{p} & =\text { Specific heat of air, Btu/lb-o } \mathrm{F}(\mathrm{about} 0.24)
\end{array}
$$


This equation shows that the properties of U-factor, SHGC, and infiltration are major factors which determine the energy flow through a fenestration product. For this reason, the NFRC rating system rates the U-factor and SHGC of products, and the NFRC 100, NFRC 200 and NFRC 400 documents define the procedures for calculating these values for the total product.

\subsection{Temperature Driven Heat Transfer}

Fenestration product heat loss/gain due to temperature is a combination of three modes of heat transfer:

1. Conduction (heat traveling through a solid material, the way a frying pan warms up) through glazing, frame elements, and air or other gases in the glazing gap

2. Convection (the transfer of heat by the movement of gases or liquids, like warm air rising from a candle flame) through air layers on the exterior and interior fenestration product surfaces and between glazing layers

3. Radiative heat transfer (the movement of heat energy through space without relying on conduction through the air or by movement of the air, the way you feel the heat of a fire) between glazing layers, or between IG units and interior or exterior spaces.

Solar radiation absorbed by glazing layers will influence these processes, while solar radiation transmitted by the glazing system serves as a heat gain to the space. These three modes of heat transfer are shown schematically in Figure 3-1. Heat flows from warmer to cooler bodies, thus from inside to outside in winter, and reverses direction in summer during periods when the outside temperature is greater than indoors.

The amount of heat transfer due to these three processes in quantified by its $\mathrm{U}$-factor $\left(\mathrm{Btu} / \mathrm{h}-\mathrm{ft}^{2}-\mathrm{o} F\right.$ or $\mathrm{W} / \mathrm{m}^{2}-$ $\left.{ }^{\circ} \mathrm{C}\right)$. The inverse of heat flow, or resistance to heat transfer, is expressed as an R-value. NFRC's rating system quantifies and predicts U-factors.

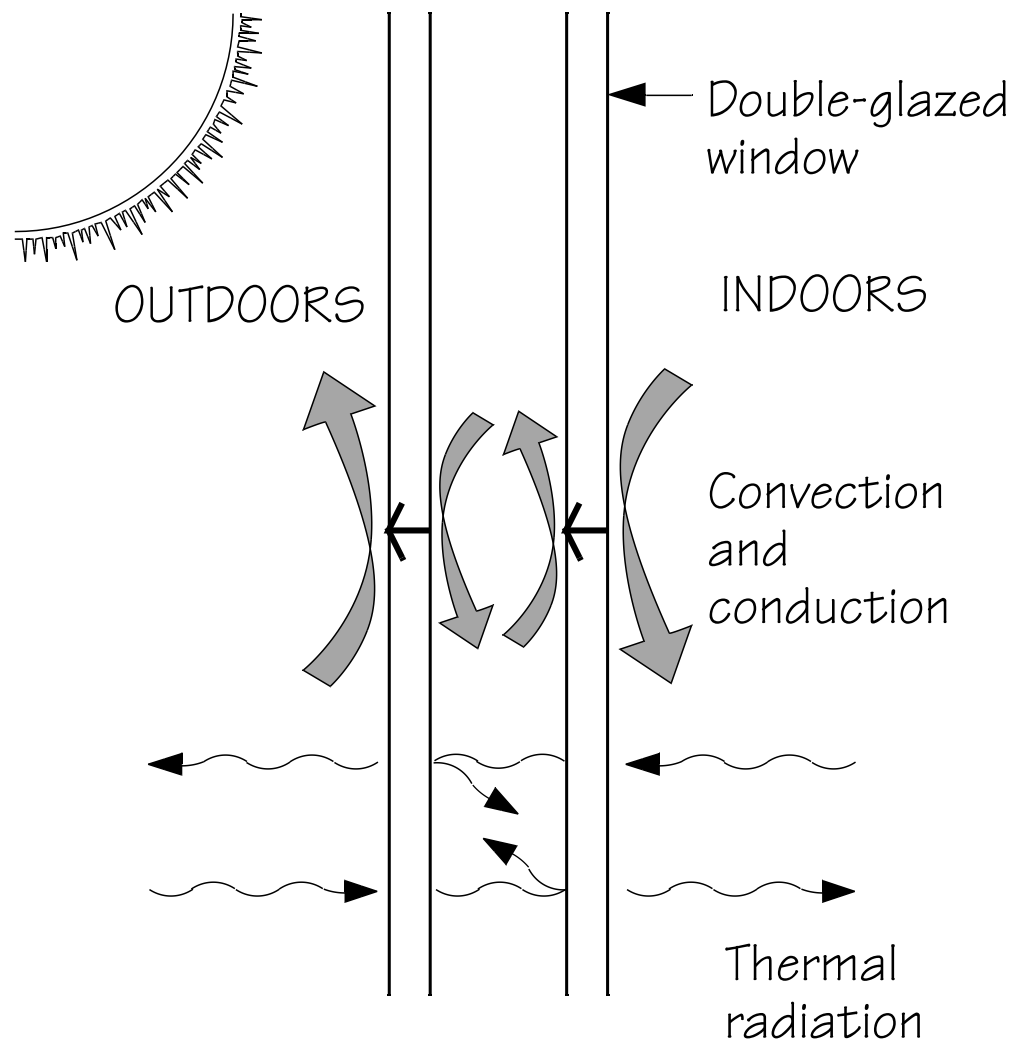


Figure 3-1. Mechanisms of heat transfer in a fenestration product.

\subsubsection{Conduction}

Compared to a well-insulated wall, heat transfer through a typical older fenestration product is generally much higher. A single-glazed fenestration product has roughly the same insulating qualities as a sheet of metal-most of the insulating value comes from the air layer on each surface of the glass. Such a product can be considered a thermal hole in a wall and typically has a heat loss rate ten to twenty times that of the wall. A product with such a poor insulating value allows heat to flow out of a space almost unimpeded. If the temperature inside is $70^{\circ} \mathrm{F}$ and outside $0^{\circ} \mathrm{F}\left(20^{\circ} \mathrm{C}\right.$ and $\left.-18^{\circ} \mathrm{C}\right)$, the glass surface of a single-glazed product would be about $17^{\circ} \mathrm{F}\left(-8^{\circ} \mathrm{C}\right)$ - cold enough to form frost on the inside of the glass.

\subsubsection{Convection}

Convection affects the heat transfer in many places in the assembly: the inside glazing surface, the outside glazing surface, inside frame cavities, and inside any air spaces between glazings. A cold interior glazing surface chills the air adjacent to it. This denser cold air then falls to the floor, starting a convection current which is typically perceived as a "draft" caused by leaky fenestration products. One remedy for this situation is to install a product with lower heat loss rates that provides a warmer glass surface.

On the exterior, a component of the heat transfer rate of a fenestration product is the air film against the glazing surface. As wind blows across the product (causing convection), and the insulating value of this air film diminishes which contributes to a higher rate of heat loss. Finally, when there is an air space between layers of glazing, convection currents can facilitate heat transfer through this air layer. By adjusting the space between the panes of glass, as well as choosing a gas fill that insulates better than air, double-glazed fenestration products can be designed to minimize this effect.

\subsubsection{Radiation}

All objects emit invisible thermal radiation, with warmer objects emitting more than colder ones. Hold your hand in front of an oven window and you will feel the radiant energy emitted by that warm surface. Your hand also radiates heat to the oven window, but since the window is warmer than your hand, the net balance of radiant flow is toward your hand and it is warmed. Now imagine holding your hand close to a singleglazed window in winter. The window surface is much colder than your hand. Each surface emits radiant energy, but since your hand is warmer, it emits more toward the window than it gains and you feel a cooling effect. Thus, a cold glazing surface in a room chills everything else around it.

\subsubsection{U-factor}

The U-factor (also referred to as U-value) is the standard way to quantify insulating value. It indicates the rate of heat flow through the fenestration product. The U-factor is the total heat transfer coefficient of the fenestration system (in Btu $/ \mathrm{hr}_{-} \mathrm{ft}^{2}-^{\circ} \mathrm{F}$ or $\mathrm{W} / \mathrm{m}^{2}{ }^{\circ} \mathrm{C}$ ), which includes conductive, convective, and radiative heat transfer for a given set of environmental conditions. It therefore represents the heat flow per hour (Btus per hour or watts) through each square foot (or square meter) of fenestration product for a $1^{\circ} \mathrm{F}\left(1^{\circ} \mathrm{C}\right)$ temperature difference between the indoor and outdoor air temperature. The smaller the U-factor of a material, the lower the rate of heat flow. The R-value, which measures thermal resistance, is the reciprocal of the total U-factor $(\mathrm{R}=1 / \mathrm{U})$.

The U-factor depends on the thermal properties of the materials in the fenestration product assembly, as well as the weather conditions, such as the temperature differential between indoors and out, and wind speed. NFRC has standardized the exterior conditions (called environmental conditions) for U-factor calculations for 
product ratings using the ASHRAE Winter Conditions, which have the following temperatures and wind speeds: $15 \mathrm{mph}(25 \mathrm{~km} / \mathrm{hr})$ wind, $70^{\circ} \mathrm{F}\left(20^{\circ} \mathrm{C}\right)$ indoors, $0^{\circ} \mathrm{F}\left(-18^{\circ} \mathrm{C}\right)$ outdoors. Skylights and roof fenestration products are currently rated at a vertical slope.

\subsubsection{Total Product U-factor}

The U-factor of a total fenestration assembly is a combination of the insulating values of the glazing aseembly itself, the edge effects that occur in the insulated glazing unit, and the insulating value of the frame and sash.

\subsubsection{Center-of-Glass U-factor}

The U-factor of the glazing portion of the fenestration unit is affected primarily by the total number of glazing layers, the dimension separating the various layers of glazing, the type of gas that fills the separation, and the characteristics of coatings on the various surfaces. The U-factor for the glazing alone is referred to as the center-of-glass U-factor.

\subsubsection{Edge Effects}

A U-factor calculation assumes that heat flows perpendicular to the plane of the fenestration product. However, fenestration products are complex three-dimensional assemblies, in which materials and cross sections change in a relatively short space.

For example, metal spacers at the edge of an insulating glass unit have much higher heat flow than the center of the insulating glass, which causes increased heat loss along the outer edge of the glass. The relative impact of these "edge effects" becomes more important as the insulating value of the rest of the assembly increases.

\subsubsection{Frames and Sashes}

The heat loss through a fenestration product frame can be quite significant: in a typical four-foot by three-foot ( 1.2 by $0.9 \mathrm{~m}$ ) double-hung wood frame product, the frame and sash can occupy approximately 30 percent of the product area.

In a frame with a cross section made of one uniform, solid material, the U-factor is based on the conduction of heat through the material. However, hollow frames and composite frames with various reinforcing or cladding materials are more complex. Here, conduction through materials must be combined with convection of the air next to the glazing and radiant exchange between the various surfaces.

Furthermore, frames rarely follow the same cross section around a fenestration product. For example, a horizontal slider has seven different frame cross sections, each with its own rate of heat flow.

\subsubsection{Overall U-factor}

Since the U-factors are different for the glass, edge-of-glass zone, and frame, it can be misleading to compare U-factors if they are not carefully described. In order to address this problem, the concept of a total fenestration product U-factor is utilized by the National Fenestration Rating Council (NFRC). A specific set of engineering assumptions and procedures must be followed to calculate the overall U-factor of a fenestration unit using the NFRC method. In most cases, the overall U-factor is higher than the U-factor for the glass alone, since the glass remains superior to the frame in insulating value.

The U-factor of a product is calculated with the product in a vertical position. A change in mounting angle can affect its U-factor. 


\subsection{Solar Heat Gain and Visible Transmittance}

The second major energy performance characteristic of fenestration products is the ability to control solar heat gain through the glazing. Solar heat gain through fenestration products tends to be the single most significant factor in determining the air-conditioning load of a residential building. The intensity of heat gain from solar radiation can greatly surpass heat gain from other sources, such as outdoor air temperature or humidity.

Visible transmittance (VT) is an optical property that indicates the amount of visible light transmitted through the glass. Although VT does not directly affect heating and cooling energy use, it is used in the evaluation of energy-efficient fenestration products and therefore is discussed following the solar heat gain section.

The origin of solar heat gain is the direct and diffuse radiation coming directly from the sun and the sky or reflected from the ground and other surfaces. Some radiation is directly transmitted through the glazing to the space, and some may be absorbed in the glazing and then indirectly admitted to the space. Sunlight is composed of electromagnetic radiation of many wavelengths, ranging from short-wave invisible ultraviolet, to the visible spectrum, to the longer, invisible near-infrared waves. About half of the sun's energy is visible light; the remainder is largely infrared with a small amount of ultraviolet. This characteristic of sunlight makes it possible to selectively admit or reject different portions of the solar spectrum. While reducing solar radiation through fenestration products is a benefit in some climates and during some seasons, maximizing solar heat gain can be a significant energy benefit under winter conditions. These often conflicting directives can make selection of the "best" product a challenging task. See Section 2.6.9 for a more detailed discussion of

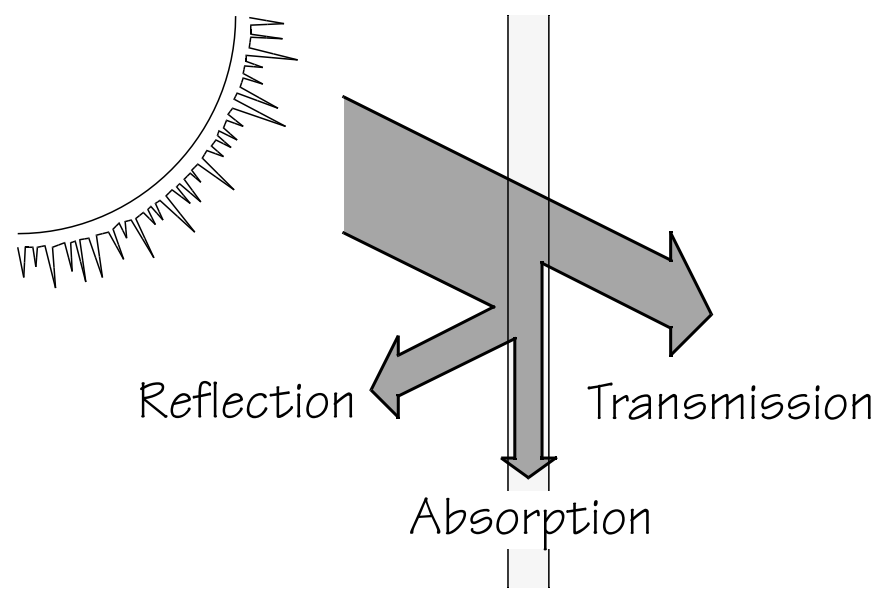

these properties of fenestration products.

Figure 3-2. A glazing systems properties of reflection, transmission and absorption determine what happens to solar gain.

\subsubsection{Determining Solar Heat Gain}

There are two means of indicating the amount of solar radiation that passes through a fenestration product. These are solar heat gain coefficient (SHGC) and shading coefficient (SC). In both cases, the solar heat gain is the combination of directly transmitted radiation and the inward-flowing portion of absorbed radiation (Figure 3-3). However, SHGC and SC have a different basis for comparison or reference. The SHGC value is calculated for NFRC rating and certification. SHGC replaces SC because the shading coefficient (SC) is only defined for the glazing portion of the fenestration product and does not include frame effects. SC represents the ratio of solar heat gain through the system relative to that through 1/8-inch $(3 \mathrm{~mm})$ clear glass at normal incidence. 


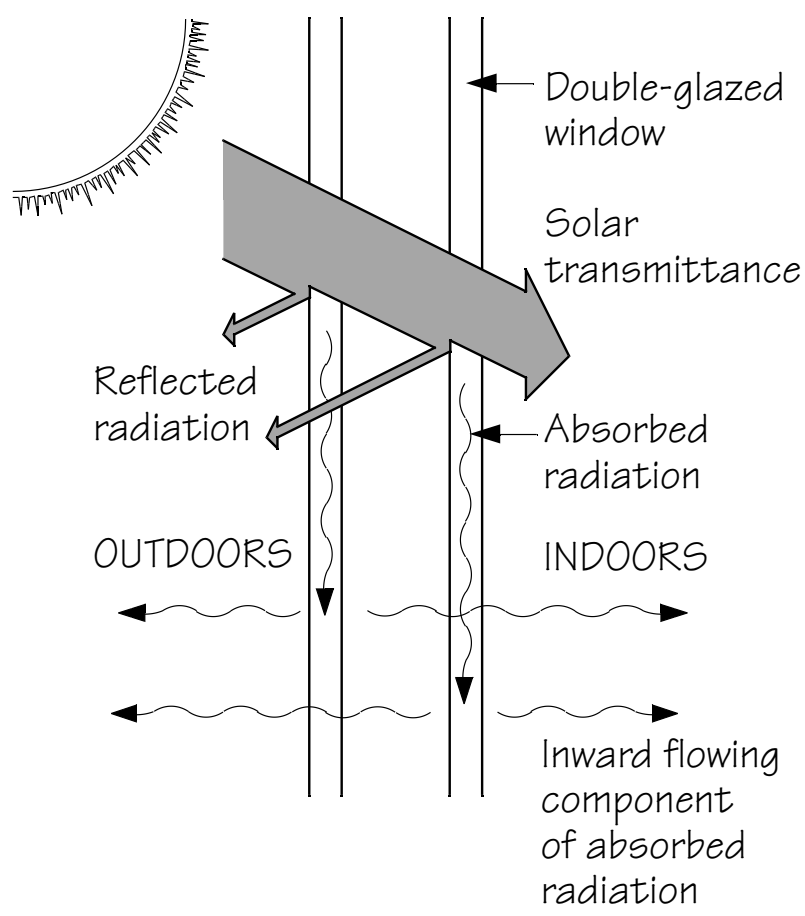

Figure 3-3. A glazing systems properties of reflection, transmission and absorption determine what happens to solar gain.

\subsubsection{Solar Heat Gain Coefficient (SHGC)}

The solar heat gain coefficient (SHGC represents the solar heat gain through the fenestration system relative to the incident solar radiation. Although SHGC can be determined for any angle of incidence, the default and most commonly used reference is normal incidence solar radiation. NFRC rated SHGC's are at $0^{\circ}$ incidence. The SHGC refers to total fenestration product system performance and is an accurate indication of solar gain under a wide range of conditions. SHGC is expressed as a dimensionless number from 0 to 1.0. A high SHGC value signifies high heat gain, while a low value means low heat gain.

\subsubsection{Visible Transmittance}

Visible transmittance is the amount of light in the visible portion of the spectrum that passes through a glazing material. This property does not directly affect heating and cooling loads in a building, but it is an important factor in evaluating energy-efficient fenestration products. Transmittance is influenced by the glazing type, the number of layers, and any coatings that might be applied to the glazings. These effects are discussed in more detail later in this chapter in conjunction with a review of various glazing and coating technologies. Visible transmittance of glazings ranges from above 90 percent for water-white clear glass to less than 10 percent for highly reflective coatings on tinted glass.

Visible transmittance is an important factor in providing daylight, views, and privacy, as well as in controlling glare and fading of interior furnishings. These are often contradictory effects: a high light transmittance is desired for view out at night, but this may create glare at times. These opposing needs are often met by providing glazing that has high visible transmittance and then adding attachments such as shades or blinds to modulate the transmittance to meet changing needs.

NFRC reports visible transmittance as a rating on label. Note that NFRC's rating is a whole product rating that combines the effect of both glazing and frame. There are many cases where the transmittance of glazing alone will be required, so it is important to make sure that the appropriate properties are being compared. 
In the past, products that reduced solar gain (with tints and coatings) also reduced visible transmittance. However, new spectrally selective tinted glasses and selective coatings have made it possible to reduce solar heat gain with little reduction in visible transmittance.

\subsection{Infiltration}

Infiltration is the leakage of air through cracks in the building envelope. Infiltration leads to increased heating or cooling loads when the outdoor air entering the building needs to be heated or cooled. Fenestration products and doors are typically responsible for a significant amount of the infiltration in homes. In extreme conditions, depending on the type and quality, infiltration can be responsible for as much heat loss or gain as the rest of the product. The level of infiltration depends upon local climate conditions, particularly wind conditions and microclimates surrounding the house. Typically, U-factor and SHCG effects far outweigh infiltration effects. Tight sealing and weatherstripping of the fenestration product, including sash, and frames is of paramount importance in controlling infiltration.

High quality fixed fenestration products help to reduce infiltration because they are easier to seal and keep tight. Operable fenestration products are necessary for ventilation, but they are also more susceptible to air leakage. Operable units with low air leakage rates are characterized by good design and high-quality construction and weatherstripping. They also feature mechanical closures that positively clamp the product shut against the wind. For this reason, compression-seal products such as awning, hopper, and casement designs are generally more effectively weatherstripped than are sliding-seal products. Sliding products rely on wiper-type weatherstripping, which is more subject to wear over time and can be bypassed when it flexes under wind pressure.

\subsection{Solar Properties of Glazing Materials}

Figure 2.3 shows the wavelength ranges of the solar spectrum. Three things happen to solar radiation as it passes through a glazing material. Some is transmitted, some is reflected, and the rest is absorbed. These are the three components of solar that determine many of the other energy performance properties of a glazing material, such as the solar heat gain coefficient and shading. Manipulating the proportion of transmittance, reflectance, and absorptance for different wavelengths of solar radiation has been the source of much recent innovation in fenestration energy performance.

Visible light is a small portion of the electromagnetic spectrum (see Figure 2-3). Beyond the blues and purples lie ultraviolet radiation and other higher-energy short wavelengths, from $X$ rays to gamma rays. Beyond red light are the near-infrared, given off by very hot objects, the far-infrared, given off by warm roomtemperature objects, and the longer microwaves and radio waves.

Glazing types vary in their transparency to different parts of the spectrum. On the simplest level, a glass that appears to be tinted green as you look through it toward the outside will transmit more sunlight from the green portion of the visible spectrum, and reflect/absorb more of the other colors. Similarly, a bronze-tinted glass will absorb the blues and greens and transmit the warmer colors. Neutral gray tints absorb most colors equally.

This same principle applies outside the visible spectrum. Most glass is partially transparent to at least some ultraviolet radiation, while plastics are commonly more opaque to ultraviolet. Glass is opaque to far-infrared radiation but generally transparent to near-infrared. Strategic utilization of these variations has made for some very useful glazing products.

The basic properties of glazing that affect solar energy transfer are:

- Visible transmittance

- Reflectance 
- Absorptance

Each is described below.

\subsubsection{Transmittance}

Transmittance refers to the percentage of radiation that can pass through glazing. Transmittance can be defined for different types of light or energy, e.g., "visible light transmittance," "UV transmittance," or "total solar energy transmittance." Each describes a different characteristic of the glazing. Visible transmittance is the total fenestration product system's transmittance across the visible portion of the solar spectrum.

Although VT can be determined for any angle of incidence, the default and most commonly used reference is normal incidence solar radiation. Transmission of visible light determines the effectiveness of a type of glass in providing daylight and a clear view through the fenestration product. For example, tinted glass has a lower visible light transmittance than clear glass.

With the recent advances in glazing technology, manufacturers can control how glazing materials behave in these different areas of the spectrum. The basic properties of the substrate material (glass or plastic) can be altered, and coatings can be added to the surfaces of the substrates. For example, a product optimized for daylighting and for reducing heat gains should transmit an adequate amount of light in the visible portion of the spectrum, while excluding unnecessary heat gain from the near-infrared part of the electromagnetic spectrum.

On the other hand, a product optimized for collecting solar heat gain in winter should transmit the maximum amount of visible light as well as the heat from the near-infrared wavelengths in the solar spectrum, while blocking the lower-energy radiant heat in the far-infrared range that is an important heat loss component. These are the strategies of spectrally selective and low-emittance coatings, described later in the chapter. 

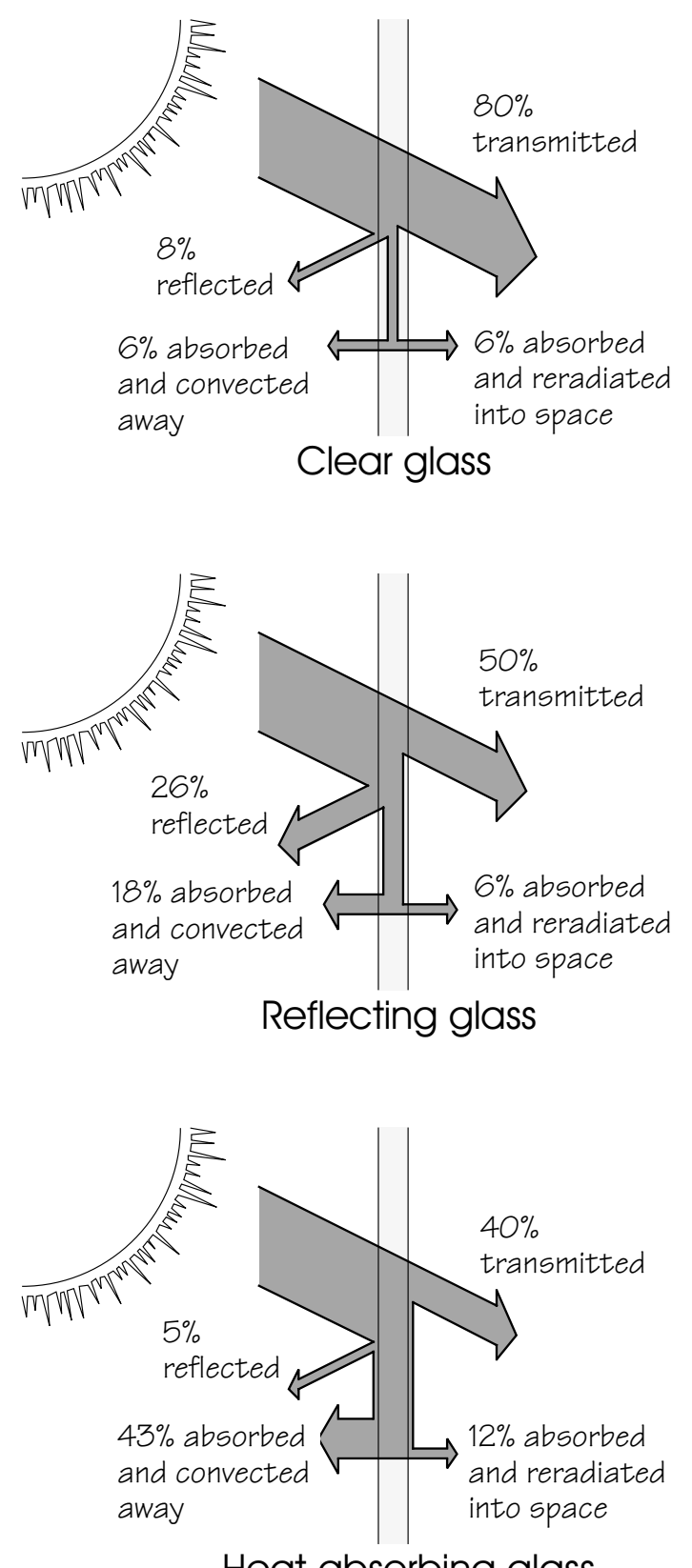

Heat-absorbing glass

Figure 3-3. Different glass types have different characteristics for the amount of solar radiation reflected, transmitted, absorbed, and re-radiated.

\subsubsection{Reflectance}

Just as some light reflects off of the surface of water, some light will always be reflected at every glass surface. A specular reflection from a smooth glass surface is a mirror-like reflection similar to when you see an image of yourself in a store window. The natural reflectivity of glass is dependent on the quality of the glass surface, the presence of coatings, and the angle of incidence of the light. Today, virtually all glass manufactured in the United States is float glass and has a very similar quality with respect to reflectance. The sharper the angle at which the light strikes, however, the more the light is reflected rather than transmitted or absorbed . Even clear glass reflects 50 percent or more of the light striking it at incident angles greater than about 70 degrees. (The incident angle is formed with respect to a line perpendicular to the glass surface.) 
Coatings can often be detected by careful examination of a reflected bright image, even if the coating is a transparent low-E coating. Hold a match several inches from a fenestration product at night and observe the reflections of the match in the glass. You will see two closely spaced images for each layer of glass, since the match reflects off the front and back surface of each layer of glass. A wider spacing between the two sets of pairs of images occurs with a wider air space between the glass panes. A subtle color shift in one of the reflected images normally indicates the presence of a low-E coating.

The reflectivity of various glass types becomes especially apparent during low light conditions. The surface on the brighter side acts like a mirror because the amount of light passing through the fenestration product from the darker side is less than the amount of light being reflected from the lighter side. This effect can be noticed from the outside during the day and from the inside during the night. For special applications when these surface reflections are undesirable (i.e., viewing merchandise through a store window on a bright day), special coatings can virtually eliminate this reflective effect.

The reflectivity of glass can be increased by applying various metallic coatings to the surface. Early processes used a liquid alloy of mercury and tin to create mirrors. A silvering process developed in 1865 improved the performance of mirrors. Today, mirror-like surfaces can be created by using vacuum-deposited aluminum or silver, or with a durable pyrolytic coating applied directly to the glass as it is manufactured. Thick coatings can be fully reflective and virtually opaque; a thinner coating is partially reflective and partially transmitting.

Most common coatings reflect all portions of the spectrum. However, in the past twenty years, researchers have learned a great deal about the design of coatings that can be applied to glass and plastic to reflect only selected wavelengths of radiant energy. Varying the reflectance of far-infrared and near- infrared energy has formed the basis for low-emittance coatings for cold climates, and for spectrally selective low-emittance coatings for hot climates.

\subsubsection{Absorptance}

Energy that is not transmitted through the glass or reflected off of its surfaces is absorbed. Once glass has absorbed any radiant energy, the energy is transformed into heat, raising the temperature of the glass.

Typical 1/8-inch ( $3 \mathrm{~mm}$ ) clear glass absorbs only about 4 percent of incident sunlight. The absorptance of glass is increased by adding to the glass chemicals that absorb solar energy. If they absorb visible light, the glass appears dark. If they absorb ultraviolet radiation or near-infrared, there will be little or no change in visual appearance. Clear glass absorbs very little visible light, while dark tinted glass absorbs a considerable amount. The absorbed energy is converted into heat, warming the glass. Thus, when these "heat-absorbing" glasses are in the sun, they feel much hotter to the touch than clear glass. They are generally gray, bronze, or blue-green and are used primarily to lower the solar heat gain coefficient and to control glare. Since they block some of the sun's energy, they reduce the cooling load placed on the building and its air-conditioning equipment. Absorption is not the most efficient way to reduce cooling loads, as discussed later.

\subsection{Infrared Properties of Glazing Materials (Emittance)}

Figure 2-3 shows the wavelength range of the electromagnetic spectrum. Glass and most plastic materials do not transmit any energy in this portion of the spectrum; hence the only physical phenomena of concern are the surface properties.

When heat or light energy is absorbed by glass, it is either convected away by moving air or reradiated by the glass surface. This ability of a material to radiate energy is called its emissivity. Fenestration products, along with all other household objects, typically emit, or radiate, heat in the form of long-wave far-infrared energy. This emission of radiant heat is one of the important heat transfer pathways for a fenestration product. Thus, reducing the product's emission of heat can greatly improve its insulating properties. 
Standard glass has an emittance of 0.84 over the long wavelength portion of the spectrum, meaning that it emits 84 percent of the energy possible for an object at its temperature. It also means that for long-wave radiation (where there is no transmittance) striking the surface of the glass, 84 percent is absorbed and only 16 percent is reflected. By comparison, low-E glass coatings have an emittance as low as 0.04 . This glazing would emit only 4 percent of the energy possible at its temperature, and thus reflect 96 percent of the incident longwave infrared radiation. 


\section{SUMMARY OF ALGORITHMS}

\subsection{THERM and WINDOW Algorithms}

This section is a brief overview of the calculational algorithms found in THERM and WINDOW. Much more detailed documentation is available and is referenced in the appropriate parts of this discussion.

\subsubsection{WINDOW}

The WINDOW program calculates:

- Center-of-glass properties of a glazing system

- Total product area-weighted properties (based on previously calculated center-of-glass properties, and frame, edge-of-glass, divider, and divider-edge properties calculated in THERM)

WINDOW algorithms are documented in two publications:

- "WINDOW 4.1: Program Description, A PC Program for Analyzing the Thermal Performance of Fenestration Products", D.K. Arasteh, E. U. Finlayson, and C. Huizenga, LBL-35298, 1994;

- "WINDOW 4.0: Documentation of Calculation Procedures", E. U. Finlayson, D.K. Arasteh, C. Huizenga, M. D. Rubin, and M. S. Reilly, LBL-33943, 1993.

\subsubsection{THERM:}

The THERM program calculates:

- Frame and edge-of-glass properties, the results of which are exported into WINDOW for the total product properties.

THERM algorithms are documented in:

- $\quad$ "THERM 2.0: Program Description, A PC Program for Analyzing Two-Dimensional Heat Transfer Through Building Products", E. U. Finlayson, R. Mitchell, D. Arasteh, C. Huizenga, D. Curcija, LBL37371 Rev.2, TA-338.

\subsection{WINDOW Computational Method}

Heat transfer across a fenestration product is a function of both the temperature difference between the inside and outside and the incident solar radiation on the product. In order to evaluate heat transfer through a specific product, its configuration and physical dimensions must be specified. This includes the glazing properties (visible, total solar and infrared optical properties, and thermal conductivity), the gap gas (air or low-conductivity gas) thermophysical properties, spacer and frame characteristics, and environmental conditions.

Fenestration product heat transfer through the center-of-glass area is primarily a one-dimensional process. It is analyzed by breaking down the glazing system cross section into an assembly of nodes and calculating the heat transfer between each node. Under steady-state conditions, the temperatures of the nodes are such that the net energy flux entering each node is equal to that leaving each node. To perform the energy balance, 
WINDOW models the user-defined glazing system as a one-dimensional, steady-state resistance network, shown in Figure 4-1. A finite-difference method is then used to converge upon the correct temperature

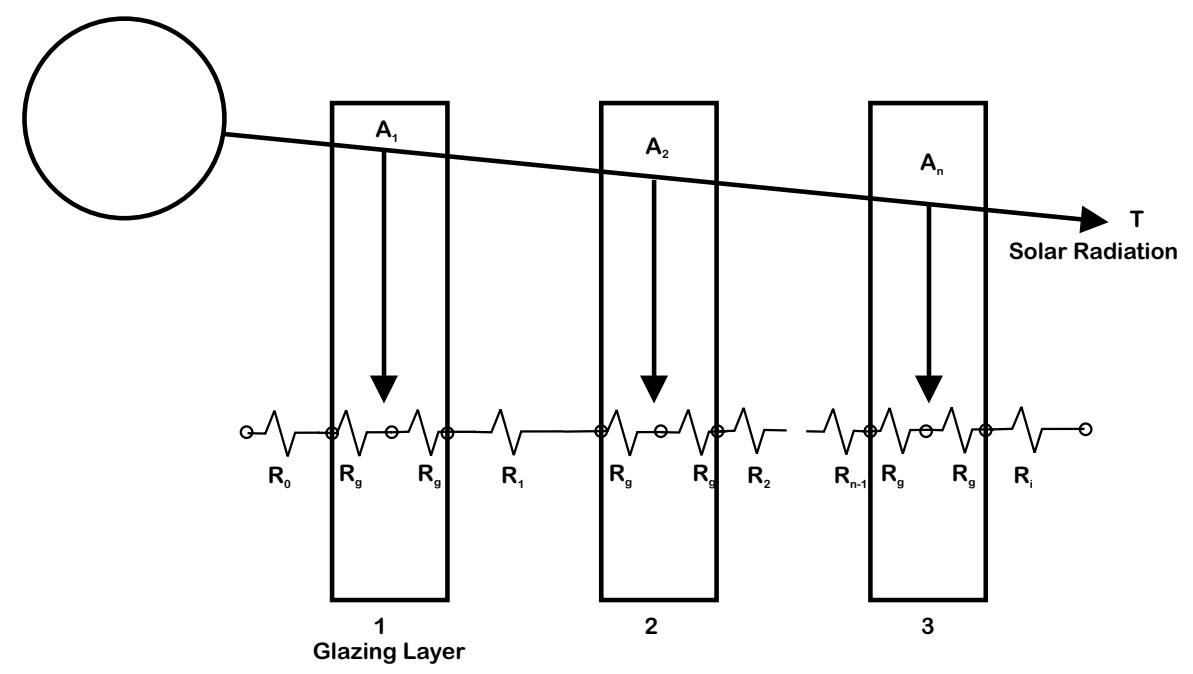

distribution. From this temperature distribution, any desired performance index can be calculated.

Figure 4-1. Resistance network used to model center-of-glass heat transfer in WINDOW.

Three temperature nodes are assigned to each glazing layer (center, and exterior and interior surface), along with outside air and inside air temperature nodes. The resistance between each node equals the inverse of the sum of the radiative and conductive/convective heat transfer coefficients. The temperature-dependent conduction/convection and effective-radiation heat transfer coefficients for the outward-facing and inwardfacing surfaces and for the gas-filled gaps are calculated from the temperature distribution. The heat transfer coefficients between the nodes within the solid materials simply depend on the conductivity of the materials.

Conductive/convective heat transfer coefficients are calculated based on empirical relationships. The outside film coefficient depends on the windspeed, the direction from which the wind is blowing, and the temperature difference between the outdoors and the outward-facing surface. The inside film coefficient is a function of the difference between the inward-facing surface temperature and the inside temperature. Gap heat transfer coefficients are computed from empirical equations for the Nusselt number. The Nusselt number relates the temperature difference between the surfaces bounding the gap, and width of the gap, and the gas viscosity, density, conductivity, and thermal diffusivity. Fenestration product tilt is also accounted for in all conductive/convective correlations.

The radiative energy flux leaving each surface is calculated from the Stephan-Boltzmann law using the surface infrared hemispherical emissivity and temperature. The net radiative flux between radiating nodes divided by the associated temperature difference gives an effective radiation heat transfer coefficient.

To accurately model glazing systems with multiple spectrally selective glazings (i.e., glazings with solaroptical properties which vary by wavelength, such as many low-emissivity coatings), a multi-band model is used in WINDOW. In this model, WINDOW calculates the transmittance and reflectance for the glazing layer or the glazing system wavelength by wavelength, and then weights the properties by the appropriate weighting functions to obtain the total solar, visible, thermal infrared properties, as well as the damageweighted transmittance and the transmittance between 0.30 and 0.38 microns. To use the multi-band model, WINDOW needs a spectral data file for each glazing layer. These data files are updated and maintained by LBNL and available from NFRC. If some of the glazing layers in a glazing system do not have a spectral data file, WINDOW assumes a flat spectral behavior of the glazings without the spectral data files, based on their stated visible and solar properties. For NFRC certification simulation, the NFRC-approved spectral data files must be used (indicated by .a \# symbol in the WINDOW Glass Library). These algorithms are documented in 
great detail in "WINDOW 4.0: Documentation of Calculation Procedures", E. U. Finlayson, D.K. Arasteh, C. Huizenga, M. D. Rubin, and M. S. Reilly, LBL-33943, 1993.

\subsection{THERM Computational Methods}

Figure 4-2 shows the steps involved in a THERM analysis. A more detailed summary is found in Appendix C of "THERM 2.0: Program Description, A PC Program for Analyzing Two-Dimensional Heat Transfer Through Building Products", E. U. Finlayson, R. Mitchell, D. Arasteh, C. Huizenga, D. Curcija, LBL-37371 Rev.2, TA338.

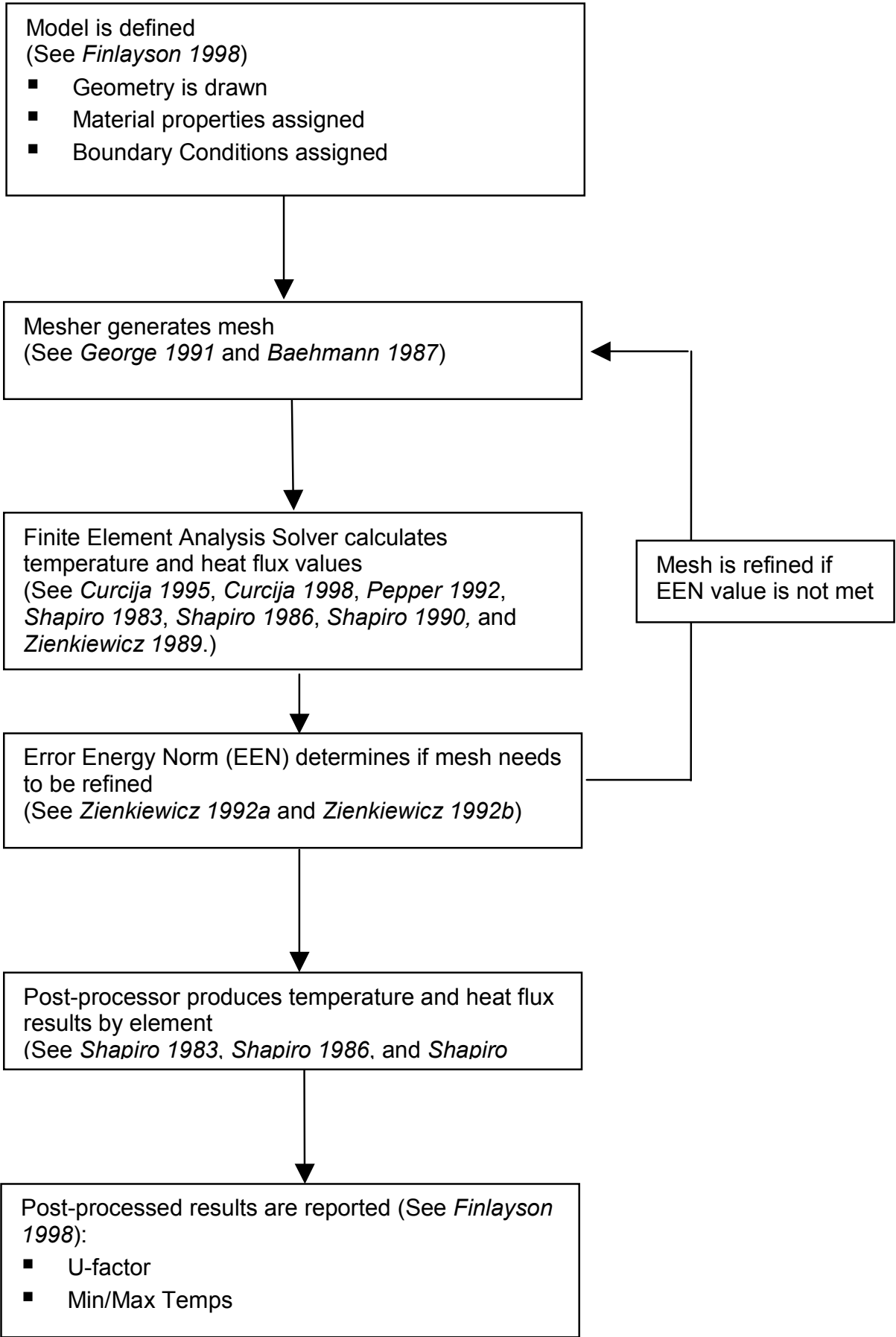


Figure 4-2. THERM program calculation procedures flow chart. 


\subsection{Total Product Calculations}

The total fenestration product properties for U-factor, SHGC and VT are based on an area-weighted average of the product's component properties which are:

- the center-of-glass properties of the glazing system

- the frame

- edge-of-glass

- divider

- divider edge-of-glass

The frame edge and divider edge properties depend on the center-of-glass properties of the associated glazing system.

This area-weighted total product value can be calculated using the WINDOW program, or other calculation tool such as a spreadsheet. This procedure for this area-weighted calculation is:

1. Multiply the component property by the component area

2. Sum these area-weighted component properties

3. Divide the area-weighted sum by the total projected area of the product

The operator types (fixed, vertical slider, horizontal slider, casement) determine which components (head, jamb, sill and meeting rail) are required to calculate the whole product area-weighted values.

\subsubsection{U-factor}

The whole-product area weighted U-factor calculation, shown below, is documented in Equation 4 in "NFRC 100: Procedures for Determining Fenestration Product U-factors".

$$
U_{t}=\frac{\left[\sum\left(U_{f} * A_{f}\right)+\sum\left(U_{d} * A_{d}\right)+\sum\left(U_{e} * A_{e}\right)+\sum\left(U_{d e} * A_{d e}\right)+\sum\left(U_{c} * A_{c}\right)\right]}{A_{p f}}
$$

Where:

$$
\begin{aligned}
& \mathrm{U}_{\mathrm{t}} \quad=\text { Total product } \mathrm{U}-\text { factor in Btu/hr-ft} \mathrm{ft}^{2} \mathrm{o} F\left(\mathrm{~W} / \mathrm{m}^{2}{ }^{2} \mathrm{o}\right) \text {. } \\
& \mathrm{A}_{\mathrm{pf}} \quad=\text { Projected fenestration product area in } \mathrm{ft}^{2}\left(\mathrm{~m}^{2}\right) \text {. } \\
& \mathrm{U}_{\mathrm{f}} \quad=\text { Frame U-factor in Btu/hr-ft'2-oF }\left(\mathrm{W} / \mathrm{m}^{2}-\mathrm{o} \mathrm{C}\right) \text {. } \\
& \mathrm{A}_{\mathrm{f}} \quad=\text { Frame area in } \mathrm{ft}^{2}\left(\mathrm{~m}^{2}\right) \text {. } \\
& \mathrm{U}_{\mathrm{d}} \quad=\text { Divider U-factor in Btu/hr-ft'2-oF }\left(\mathrm{W} / \mathrm{m}^{2}{ }^{\circ} \mathrm{C}\right) \text {. } \\
& \mathrm{A}_{\mathrm{d}} \quad=\text { Divider area in } \mathrm{ft}^{2}\left(\mathrm{~m}^{2}\right) . \\
& \mathrm{U}_{\mathrm{e}} \quad=\text { Edge-of-glass U-factor in Btu/hr-ft²-o F }\left(\mathrm{W} / \mathrm{m}^{2}{ }^{2} \mathrm{o}\right) \text {. } \\
& \mathrm{A}_{\mathrm{e}} \quad \text { = Edge-of-glass area in } \mathrm{ft}^{2}\left(\mathrm{~m}^{2}\right) \text {. } \\
& \mathrm{U}_{\mathrm{de}} \quad=\text { Divider edge U-factor in Btu/hr- } \mathrm{ft}^{2}{ }^{\circ} \mathrm{o} F\left(\mathrm{~W} / \mathrm{m}^{2}-^{\circ} \mathrm{C}\right) \text {. }
\end{aligned}
$$




$$
\begin{array}{ll}
\mathrm{A}_{\mathrm{de}} & =\text { Divider Edge Area in } \mathrm{ft}^{2}\left(\mathrm{~m}^{2}\right) . \\
\mathrm{U}_{\mathrm{c}} & =\text { Center-of-glass U-factor in Btu/hr-ft'-o } \mathrm{F}\left(\mathrm{W} / \mathrm{m}^{2}{ }^{\circ} \mathrm{C}\right) . \\
\mathrm{A}_{\mathrm{c}} & =\text { Center-of-glass area in } \mathrm{ft}^{2}\left(\mathrm{~m}^{2}\right) .
\end{array}
$$

\subsubsection{Solar Heat Gain Coefficient (SHGC)}

The total solar heat gain coefficient is determined by an area-weighted average of contributions from the transparent and the opaque elements in the fenestration product. The SHGC is a function of the solar transmittance, the solar absorptances of each layer and the inward flowing fraction of thermal energy. The SHGC is calculated for each component of the product separately. (See Finlayson 1993 for detailed algorithm documentation).

The SHGC of the frame or divider element is defined in NFRC 200 as:

$$
\frac{k * a * U}{h}
$$

Where:

$\mathrm{k}=$ factor to account for the differences between the surface area and projected area of the frame or divider; equal to 1.0 is the standard

a $\quad=$ frame or divider absorptance

$\mathrm{U} \quad=$ frame or divider U-factor

$\mathrm{h}=$ exterior film coefficient $\left(2.94 \mathrm{Btu} / \mathrm{hr}-\mathrm{ft}^{2}{ }^{2} \mathrm{o} F\right)$

All the transparent regions (center-of-glass, edge-of-glass, and divider edge) have the same SHGC. Once the SHGC of the opaque elements is determined the total SHGC is calculated as the area-weighted average of the SGHC through the transparent and the opaque portions of the fenestration product as shown below.

$$
S H G C_{t}=\frac{\left[\left(S H G C_{f} * A_{f}\right)+\left(S H G C_{d} * A_{d}\right)+\left(S H G C_{e} * A_{e}\right)+\left(S H G C_{d e} * A_{d e}\right)+\left(S H G C_{c} * A_{c}\right)\right]}{A_{p f}}
$$

Where:

$$
\begin{aligned}
& \mathrm{SHGC}_{\mathrm{t}}=\text { Total product SHGC in Btu/hr-ft} \mathrm{ft}^{2} \mathrm{~F}\left(\mathrm{~W} / \mathrm{m}^{2}{ }^{\circ} \mathrm{C}\right) \text {. } \\
& \mathrm{A}_{\mathrm{pf}} \quad=\text { Projected fenestration product area in } \mathrm{ft}^{2}\left(\mathrm{~m}^{2}\right) . \\
& \mathrm{SHGC}_{\mathrm{f}} \quad=\text { Frame SHGC (dimensionless). } \\
& \mathrm{A}_{\mathrm{f}} \quad=\text { Frame area in } \mathrm{ft}^{2}\left(\mathrm{~m}^{2}\right) \text {. } \\
& \mathrm{SHGC}_{\mathrm{d}} \quad=\text { Divider SHGC (dimensionless). } \\
& \mathrm{A}_{\mathrm{d}} \quad=\text { Divider area in } \mathrm{ft}^{2}\left(\mathrm{~m}^{2}\right) \text {. } \\
& \mathrm{SHGC}_{\mathrm{e}} \quad=\text { Edge-of-glass SHGC (dimensionless). } \\
& \text { SHGC }_{\mathrm{e}} \quad=\text { Edge-of-glass area in } \mathrm{ft}^{2}\left(\mathrm{~m}^{2}\right) \text {. } \\
& \mathrm{SHGC}_{\mathrm{de}}=\text { Divider edge SHGC }(\text { dimensionless }) \text {. }
\end{aligned}
$$


SHGC $_{\mathrm{de}}=$ Divider Edge Area in $\mathrm{ft}^{2}\left(\mathrm{~m}^{2}\right)$.

$\mathrm{SHGC}_{\mathrm{c}} \quad=$ Center-of-glass SHGC (dimensionless) .

$\mathrm{A}_{\mathrm{c}} \quad=$ Center-of-glass area inft ${ }^{2}\left(\mathrm{~m}^{2}\right)$.

\subsubsection{Visible Transmittance}

The whole-product area weighted visible transmittance calculation is shown below.

$$
V T_{t}=\frac{\left[\left(V T_{f} * A_{f}\right)+\left(V T_{d} * A_{d}\right)+\left(V T_{e} * A_{e}\right)+\left(V T_{d e} * A_{d e}\right)+\left(V T_{c} * A_{c}\right)\right]}{A_{p f}}
$$

Where:

$$
\begin{array}{ll}
\mathrm{VT}_{\mathrm{t}} & =\text { Total product VT (dimensionless) } \\
\mathrm{A}_{\mathrm{pf}} & =\text { Projected fenestration product area in } \mathrm{ft}^{2}\left(\mathrm{~m}^{2}\right) . \\
\mathrm{VT}_{\mathrm{f}} & =\text { Frame VT (dimensionless). } \\
\mathrm{A}_{\mathrm{f}} & =\text { Frame area in } \mathrm{ft}^{2}\left(\mathrm{~m}^{2}\right) . \\
\mathrm{VT}_{\mathrm{d}} & =\text { Divider VT (dimensionless). } \\
\mathrm{A}_{\mathrm{d}} & =\text { Divider area in } \mathrm{ft}^{2}\left(\mathrm{~m}^{2}\right) . \\
\mathrm{VT}_{\mathrm{e}} & =\text { Edge-of-glass } \mathrm{VT}(\text { dimensionless }) . \\
\mathrm{VT}_{\mathrm{e}} & =\text { Edge-of-glass area in } \mathrm{ft}^{2}\left(\mathrm{~m}^{2}\right) . \\
\mathrm{VT}_{\mathrm{de}} & =\text { Divider edge VT }(\text { dimensionless }) . \\
\mathrm{VT}_{\mathrm{de}} & =\text { Divider Edge Area in } \mathrm{ft}^{2}\left(\mathrm{~m}^{2}\right) . \\
\mathrm{VT}_{\mathrm{c}} & =\text { Center-of-glass VT }(\text { dimensionless }) . \\
\mathrm{A}_{\mathrm{c}} & =\text { Center-of-glass area in } \mathrm{ft}^{2}\left(\mathrm{~m}^{2}\right) .
\end{array}
$$

For opaque components (all known frames and dividers) the component visible transmittance $\left(\mathrm{VT}_{\mathrm{f}}, \mathrm{VT}_{\mathrm{d}}\right)$ are zero. Also note that, as defined by NFRC 200, visible transmittance $\mathrm{VT}_{\mathrm{c}}=\mathrm{VT}_{\mathrm{e}}=\mathrm{VT}_{\mathrm{de}}$ 


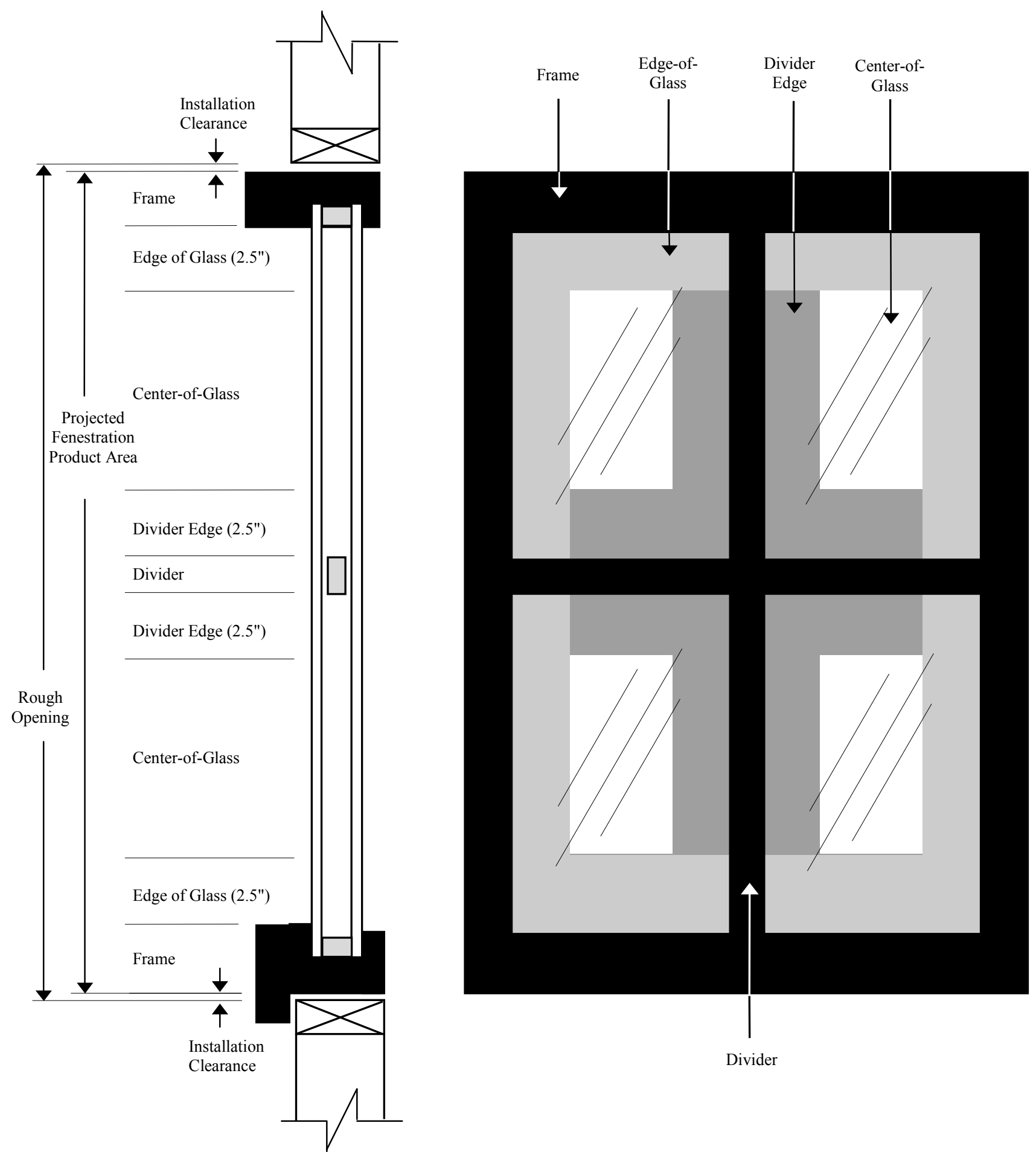

Figure 4-3. Components for the whole product area-weighted calculation. The view on the left is a section, and the view on the right is an elevation. 


\subsection{References}

1997 ASHRAE Handbook of Fundamentals, American Society of Heating, Refrigerating and Air-Conditioning Engineers, Inc., Atlanta, GA.

Arasteh, D. K. Finlayson, E. U., and Huizenga, C. 1994. "WINDOW 4.1: A PC Program for Analyzing Window Thermal Performance in Accordance with Standard NFRC Procedures." LBL Report 35298. Berkeley, CA.

ASHRAE Standard 142P, Standard Method for Determining and Expressing the Heat Transfer and Total Optical Properties of Fenestration Products, Public Review Draft, October 1996, American Society of Heating, Refrigerating and Air-Conditioning Engineers, Inc., Atlanta, GA.

Baehmann, P. L. Wittchen, S.L., Shephard, M.S., Grice, K.R. and Yerry, M.A., 1987. “Robust, Geometrically Based, Automatic Two-Dimensional Mesh Generation." International Journal for Numerical Methods in Engineering. 24, 1043 - 1078.

Curcija, D.; Power, J. P.; and Goss, W. P. 1995. "CONRAD: A Finite Element Method Based Computer Program Module for Analyzing 2-D Conductive and Radiative Heat Transfer in Fenestration Systems". Draft Report, University of Massachusetts at Amherst.

Curcija; D., and Goss, W. P. The 'Variable-h' Model For Improved Prediction of Surface Temperatures in Fenestration Systems, DRAFT June 1998 University of Massachusetts Report.

Finlayson, E. U., Arasteh, D. K., Huizenga, C., Rubin, and M. D., Reilly, M. S., 1993. "WINDOW 4.0: Documentation of Calculation Procedures." LBL Report 33943. Berkeley CA.

Finlayson, E.U., Mitchell, R., Arasteh, D., Huizenga, C., Curcija, D.. 1998. "THERM 2.0: Program Description, A PC Program for Analyzing the Two-Dimensional Heat Transfer Through Building Products". LBL-37371 Rev 2.

George, P.L. Automatic Mesh Generation, Application to Finite Element Methods. John Wiley \& Sons.

Pepper, P. W. and Heinrich, J. C. 1992. The Finite Element Method Basic Concepts and Applications. Hemisphere Publishing Corporation, Washington.

Rohsenow, W. M., Harnett, J. P., and Ganic, E. N., 1985. Handbook of Heat Transfer Fundamentals, 2nd Edition, McGraw Hill.

Shapiro, A. B. 1986. "TOPAZ2D - A Two-Dimensional Finite Element Code for Heat Transfer Analysis, Electrostatic, and Magnetostatic Problems" Lawrence Livermore National Laboratory, Rept. UCID-20824.

Shapiro, A. B. 1990. "TOPAZ2D Heat Transfer Code Users Manual and Thermal Property Data Base", Lawrence Livermore National Laboratory, Rept. UCRL-ID-104558, May 1990.

Shapiro, A. B., 1983. FACET - A Radiation View Factor Computer Code for Axisymetric, 2D Planar, and 3D Geometries with Shadowing. Lawrence Livermore National Laboratory, Rept. UCID-19887.

Zhao, Y., Curcija, D., and Goss, W. P., 1996, Condensation Resistance Validation Project - Detailed Computer Simulations Using Finite - Element Methods. PP 508-515, ASHRAE Transactions, Vol 102, Part 2.

Zienkiewicz, O. C.; and Taylor, R. L. 1989. The Finite Element Method. 4th ed. Vol. 1. McGraw Hill, Maidenhead, UK.

Zienkiewicz, O.C. and Zhu, J.Z. 1992a. "The Superconvergent Patch Recovery and A Posteriori Error Estimates. Part 1: The Recovery Technique." International Journal for Numerical Methods in Engineering. Vol 33. pp. 1331-1364. 
Zienkiewicz, O.C. and Zhu, J.Z., 1992b. “The superconvergent patch recovery and a posteriori error estimates. Part 2. The error estimates and adaptivity." International Journal for Numerical Methods in Engineering. Vol 33. pp. 1365-1382. 


\section{MODELING CENTER-OF-GLASS WITH WINDOW}

\subsection{Overview}

The WINDOW program calculates the center-of-glass U-factor $\left(\mathrm{U}_{c}\right)$, Solar Heat Gain Coefficient $\left(\mathrm{SHGC}_{\mathrm{c}}\right)$ and Visible Transmittance $\left(\mathrm{VT}_{\mathrm{c}}\right)$ according to the NFRC 100: Procedure for Determining Fenestration Product Thermal Properties and NFRC 200: Procedure for Determining Fenestration Product Solar Heat Gain Coefficients at Normal incidence and NFRC 300: Procedure for Determining Solar Optical Properties of Simple Fenestration Product.

Read the WINDOW program user's manual, WINDOW 4.1: Program Description, A PC Program for Analyzing the Thermal Performance of Fenestration Products to become familiar with the program before reading this manual.

The basic procedure for calculating the center-of-glass U-factor in WINDOW is to:

- Make sure the Glass Library entries are from the currently approved spectral data file

- Create a glazing system for your product composed of entries from the Glass and Gas Libraries

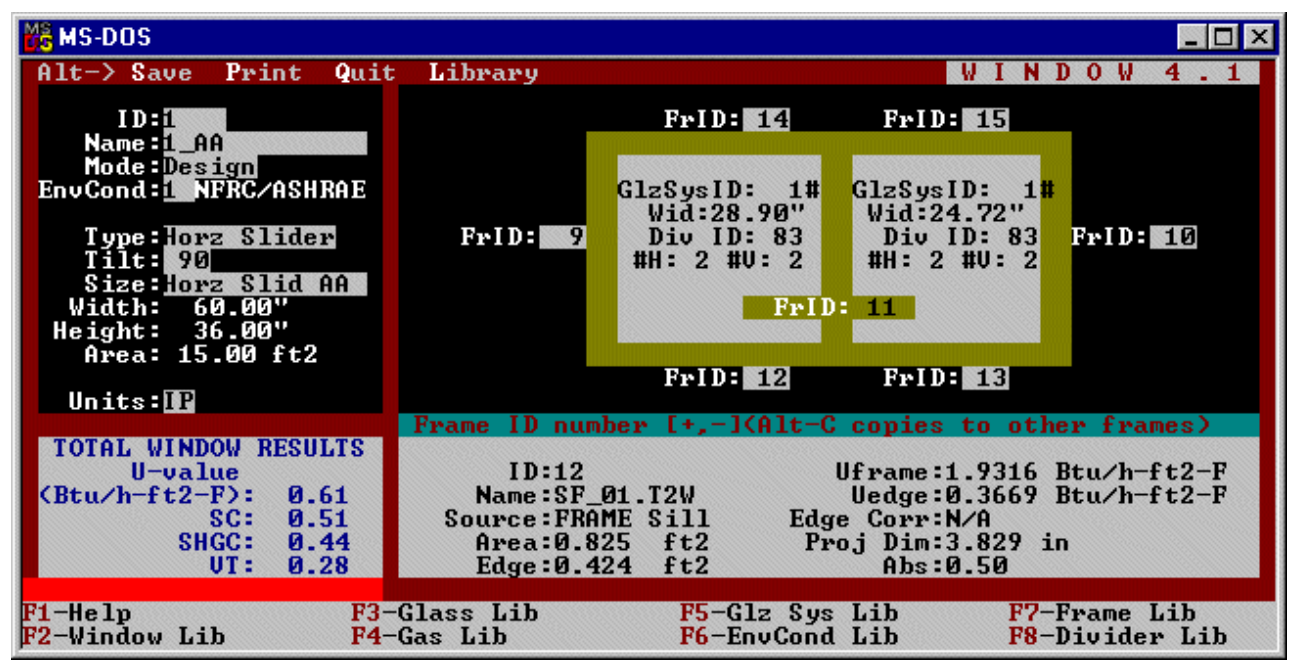

Figure 5-1. WINDOW Main Screen. 


\subsection{Glass Library}

The Glass Library in WINDOW is where individual glass products are defined. For NFRC simulations, NFRC approved spectral data files must be used for glass layers. These spectral data files are regularly updated and can be obtained from NFRC or downloaded from the web at

http://www.nfrc.org/nfrc_spectral_data_library.htm. These spectral data files use approved optical property data determined according to NFRC 300 and NFRC 301. When the approved glass data is used, a \# symbol appears at the end of the glass name in the Name field of the Glass Library (shown in Figure 5-2), and before the number of glass layers in the \# Lay field in the Glazing System Library (shown in Figure 5-3). Any product recertification simulations must use the most current NFRC-approved spectral data files.

In addition to the glass products of specific manufacturers, the spectral data files used to create the Glass Library contain several entries for generic uncoated products. The values for these entries are not measured properties from any specific glass products, but are averaged spectral data from at least two samples. The NFRC Spectral Data Manual has a table that lists what specific glass products these generic files may be substituted for.

The optical properties defined for each glass entry are listed below:

\begin{tabular}{|l|l|}
\hline Variable & Description \\
\hline Thick & Glass thickness. Units: inches (IP); mm (SI). \\
\hline Tsol & Solar transmittance of the glazing layer. \\
\hline Rsol1 & Solar reflectance of the glazing layer, exterior-facing side. \\
\hline Rsol2 & Solar reflectance of the glazing layer, interior-facing side. \\
\hline Tvis & Visible transmittance of the glazing layer. \\
\hline Rvis1 & Visible reflectance of the glazing layer, exterior-facing side. \\
\hline Rvis2 & Visible reflectance of the glazing layer, interior-facing side. \\
\hline Tir & Thermal infrared (longwave) transmittance of the glazing layer. \\
\hline Emis1 & Infrared (longwave) emittance of the glazing layer, exterior-facing side \\
\hline Emis2 & Infrared (longwave) emittance of the glazing layer, interior-facing side \\
\hline
\end{tabular}

The \# symbol indicates that these glass entries came from the NFRCapproved spectral data files, and can be used for

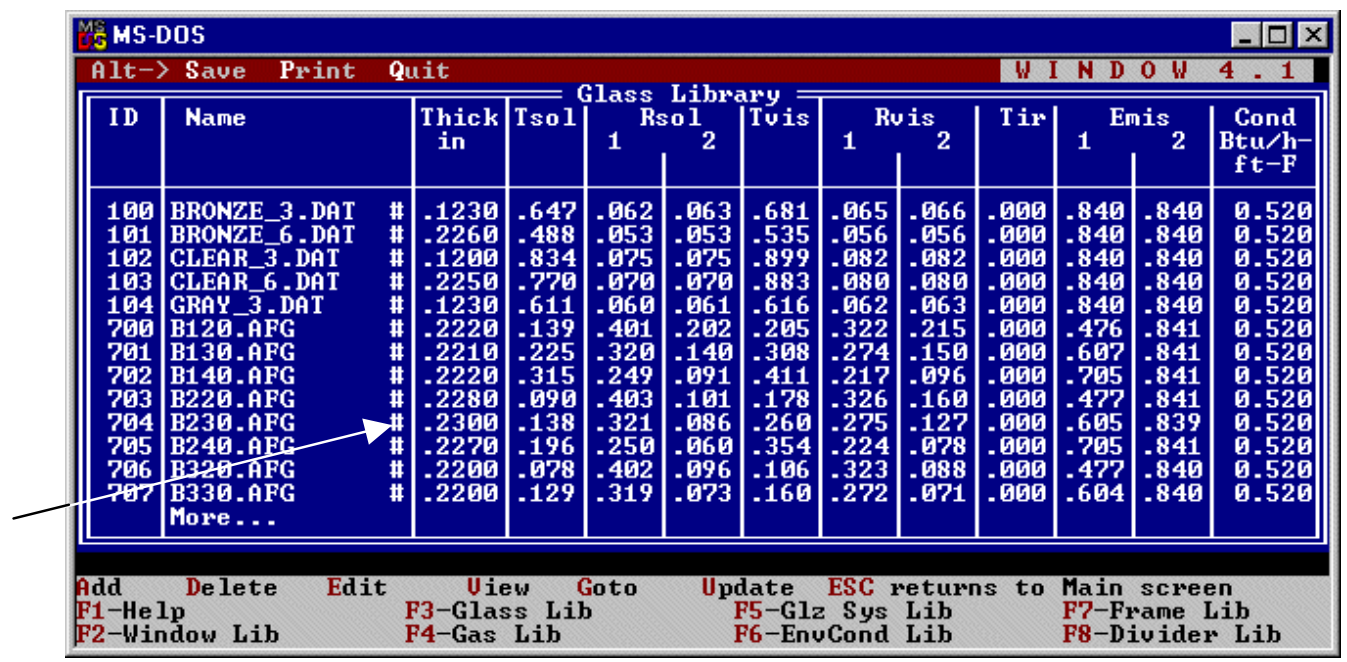
NFRC simulations.

Figure 5-2. WINDOW Glass Library. 


\subsection{Glazing System Library -- Center-of-Glass U-factor}

The center-of-glass U-factor is determined in the WINDOW Glazing System Library. A glazing system is created by defining layers of glass from the Glass Library, as well as defining the material between the layers, such as air or argon. Detailed instructions about creating a glazing system can be found in the WINDOW User's Manual. A sample glazing system entry is shown in Figure 5-3.

When defining a glazing system, several inputs are specified, including the number of glass layers (\# Lay), the Tilt, and the Environmental Conditions (Env Cond). For NFRC certification calculations the

Environmental Conditions have restricted values, as discussed in the next section. Also, the glass layers must be from the approved spectral data library, indicated by the \# symbol at the end of the glass layer name, as shown in Figure 5-3.

Note: to see the $U_{c}$ value to four decimal places, press Alt-F4 in the Glazing System Library.

When the glass and gap layers have been defined, press Spacebar and the center-of-glass $U$-factor $\left(U_{c}\right)$ will be displayed. This value is used when the glazing system is imported into THERM.

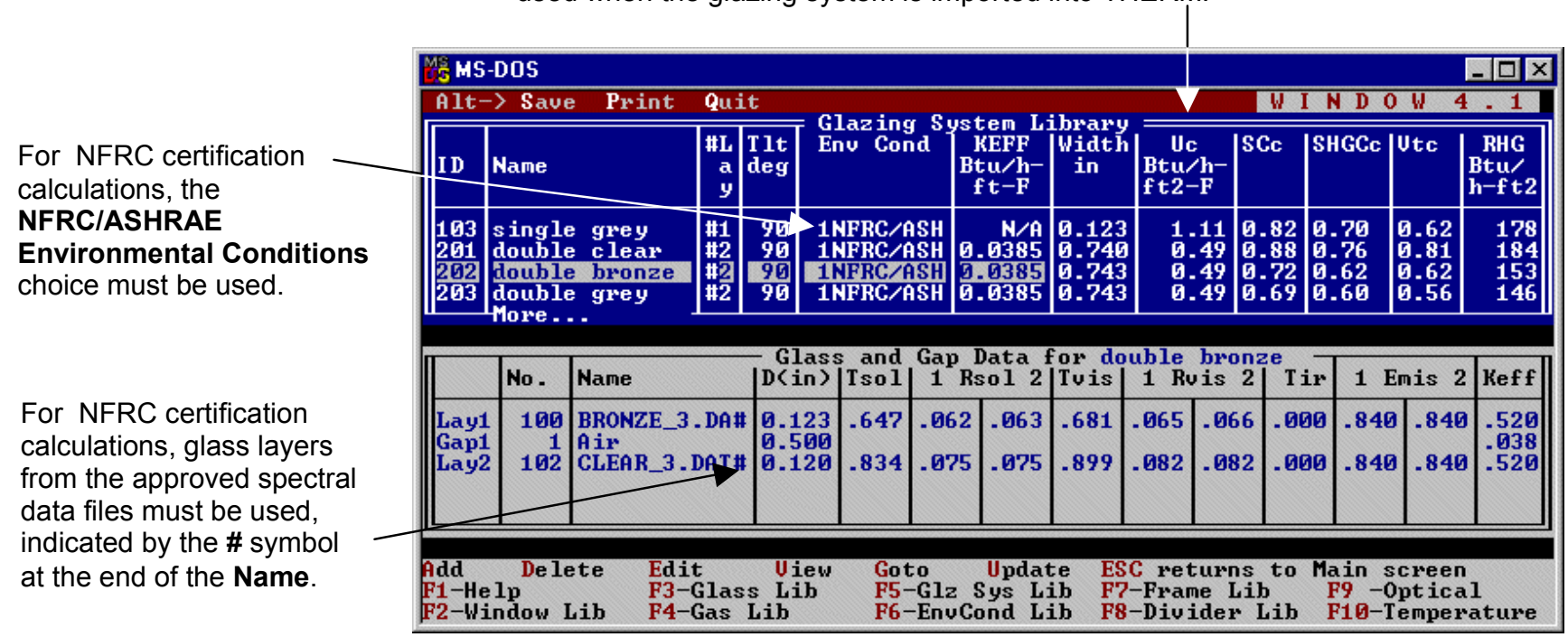

Figure 5-3. WINDOW Glazing Systems Library. 


\subsubsection{Environmental Conditions}

When defining the glazing system for use in an NFRC certified simulation, the NFRC/ASHRAE choice must be used for the Environmental Conditions (Env Cnd) parameter, which contains the parameters defined by NFRC 100. Figure 5-4 shows the NFRC/ASHRAE choice in the WINDOW Environmental Conditions

Library. Table 5-1 lists the values for the U-factor calculation and Table 5-2 lists the values for the Solar Heat Gain Coefficient calculation.

Use the NFRC/ASHRAE Environmental Conditions Library in WINDOW for NFRC center-of-glass simulations

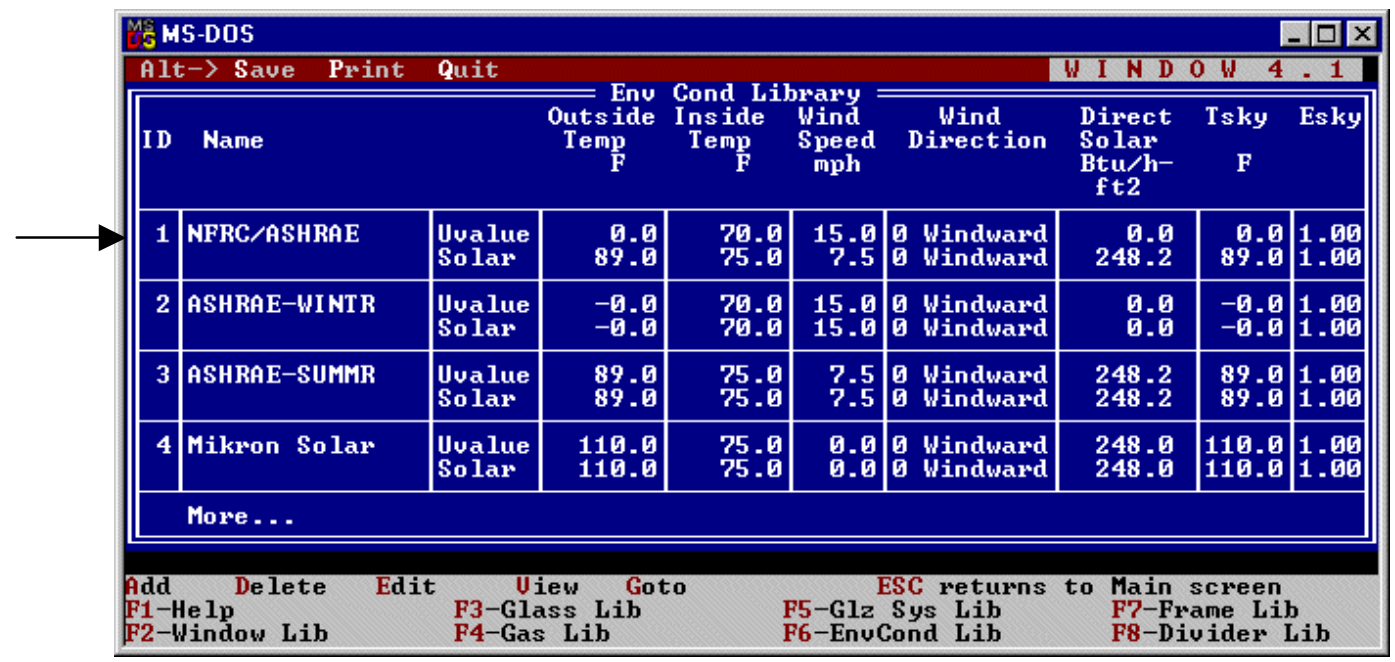

Figure 5-4. WINDOW Environmental Conditions Library.

Table 5-1. Environmental Conditions for NFRC Simulations for U-factor calculations.

\begin{tabular}{|l|l|l|}
\hline Variable & IP Units & SI Units \\
\hline Outside Temperature & $0.0^{\circ} \mathrm{F}$ & $-17.8^{\circ} \mathrm{C}$ \\
\hline Inside Temperature & $70.0 \circ \mathrm{F}$ & $21.1^{\circ} \mathrm{C}$ \\
\hline Wind Speed & $15.0 \mathrm{mph}$ & $6.7 \mathrm{~m} / \mathrm{s}$ \\
\hline Wind Direction & Windward & Windward \\
\hline Direct Solar & $0.0 \mathrm{Btu} / \mathrm{hr}-\mathrm{ft}^{2}$ & $0.0 \mathrm{~W} / \mathrm{m}^{2}$ \\
\hline Sky Temperature (Tsky) & $0.0 \circ \mathrm{F}$ & $-17.8^{\circ} \mathrm{C}$ \\
\hline Sky Emissivity (Esky) & 1.00 & 1.00 \\
\hline
\end{tabular}

Table 5-2. Environmental Conditions for NFRC Simulations for SHGC and VT calculations.

\begin{tabular}{|l|l|l|}
\hline Variable & IP Units & SI Units \\
\hline Outside Temperature & $89.0 \circ \mathrm{F}$ & $31.7 \mathrm{C}^{\circ}$ \\
\hline Inside Temperature & $75.0 \circ \mathrm{F}$ & $23.9^{\circ} \mathrm{C}$ \\
\hline Wind Speed & $7.5 \mathrm{mph}$ & $3.4 \mathrm{~m} / \mathrm{s}$ \\
\hline Wind Direction & Windward & Windward \\
\hline Direct Solar & $248.2 \mathrm{Btu} / \mathrm{hr}-\mathrm{ft}^{2}$ & $783.0 \mathrm{~W} / \mathrm{m}^{2}$ \\
\hline Sky Temperature (Tsky) & $89.0 \circ \mathrm{F}$ & $31.7 \circ \mathrm{C}$ \\
\hline Sky Emissivity (Esky) & 1.00 & 1.00 \\
\hline
\end{tabular}


It is possible to make new environmental conditions with specific conditions specified, in order to evaluate the design of a product. However, only the pre-defined NFRC/ASHRAE Environmental Conditions can be used for NFRC rating purposes.

\subsubsection{Coatings}

The location of coatings on a glass layer can affect the center-of-glass U-factor and therefore the whole product calculation, so it is important to specify it correctly. When using a glass entry from the WINDOW Glass Library, if the coating is not on the correct surface, the glass layer shall be flipped. To flip a glass layer, while on a glass layer in Edit mode, press the $\mathbf{F}$ key and the glass surfaces will be flipped. An $\mathbf{F}$ will appear after the glass layer name to indicate that it is flipped.

\subsubsection{Gas Fill}

The gas used to fill the gap between the glass layers makes a significant contribution to the center-of-glass Ufactor, although it does not have much affect on the Solar Heat Gain Coefficient. For NFRC certified simulations, the gas to be shipped in the window is what must be modeled. When creating a glazing system in WINDOW, the gas fills are chosen from the Gas Library, shown in Figure 5-5.

When a gas is used to fill the gap between glass layers, there is always a mixture of the gas and air. The amount of air mixed in is dependent on the method used to fill the gap, either evacuated chamber filling, two-probe filling with a concentration sensor, or single-probe timed filling. Table 5-3 shows the maximum gas concentrations that can be achieved with each method. For NFRC certification simulations, the simulator must request the gas-filling technique and the gas concentration for their product from the manufacturer, and this information shall be reported in the simulation report.

Table 5-3. Gas Concentrations based on Filling Technique.

\begin{tabular}{|l|l|}
\hline Filling Technique & Maximum Gas Concentrations Achieved \\
\hline Evacuated Chamber Filling & $97 \%$ \\
\hline Two-Probe Filling with a Concentration Sensor & $\begin{array}{l}95 \% \text { for Argon filled } \\
90 \% \text { for Krypton filled }\end{array}$ \\
\hline Single-Probe Timed Filling & $60-90 \%$ \\
\hline
\end{tabular}




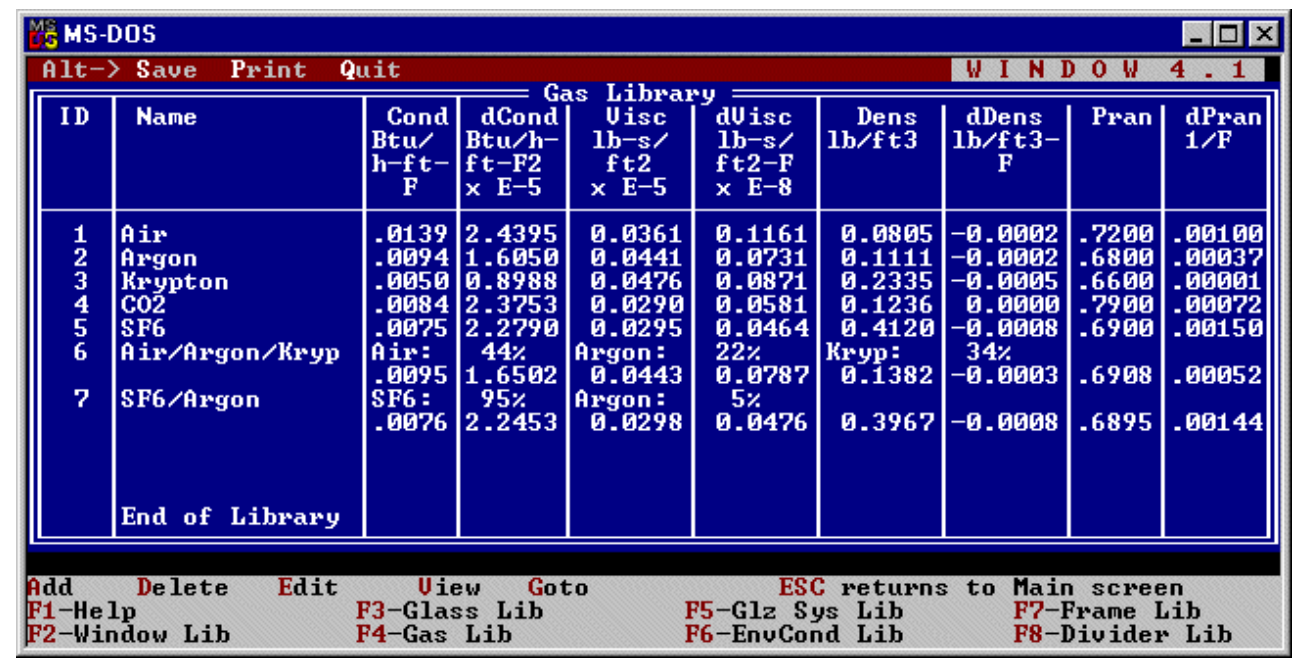

Figure 5-5. WINDOW Gas Library.

\subsubsection{Gap Width}

In the WINDOW Glazing System Library, the width of the gap between the glass layers must be defined. The width of this gap has a great influence on the center-of-glass U-factor, although the Solar Heat Gain Coefficient is not affected by the width. The pane spacing affects the convection and conduction heat transfer through the gas between the glass layers, but does not affect the radiative heat transfer across the gap.

When the gap between glass layers is large, such as storm windows which might have gaps from 1.0 inches $(25.40 \mathrm{~mm})$ to 4.0 inches $(101.60 \mathrm{~mm})$, the assumptions made about heat transfer for smaller gaps can not be used. Research has shown that the U-factor for air-filled gaps, with or without low-E coatings, are nearly constant for glass layer gaps between 1.374 inches $(34.93 \mathrm{~mm})$ and 4.0 inches $(101.60 \mathrm{~mm})$. However, WINDOW results show a small increase (typically $2 \%$ to $4 \%$ ) in U-factors for fenestration products with gap widths greater than 1.375 inches $(34.93 \mathrm{~mm})$. Therefore, for gap widths greater than 1.375 inches, it is necessary to calculate a new effective conductivity using a width-weighted calculation between 1.375 inches and the actual width. This is explained in detail in Section 8.4, "Storm Windows". Briefly, the technique is to make two glazing systems in WINDOW, one with a width of 1.375 inches, and one with the actual width. Then a Keff value is calculated from the following equation:

$$
\operatorname{Keff} x=\frac{(\operatorname{Keff} 1.375) * X}{1.375}
$$

Where:

$$
\begin{aligned}
& X=\text { actual distance of the gap in inches } \\
& \text { Keff }_{x}=\text { Keff of the gap at the actual gap width } X \\
& \text { Keff }_{1.375}=\text { Keff of the gap at a gap width of } 1.375 \text { inches }(34.93 \mathrm{~mm})
\end{aligned}
$$

\subsubsection{Laminated Glass / Applied Films}

To model laminated glass in WINDOW, the spectral data must be known for the laminated product so that the glass layer can be created in the Glass Library. However, glass layers cannot be combined in WINDOW to create the laminated layer. Using a gap width of zero in WINDOW is inaccurate and will not work.

The Optics5 program, currently under development, is designed to calculate the optical properties of multiple glazing layers, and will be used for creating laminated glass layers for the WINDOW program. 


\subsection{Solar Heat Gain Coefficient and Visible Transmittance}

The document NFRC 200: Procedure for Determining Fenestration Product Solar heat Gain Coefficients at Normal Incidence contains the rules and definitions for calculating the Solar Heat Gain Coefficient for products.

The following steps are used to determine the SHGC of a product:

1. Define the Product Line matrix of individual products.

2. Simplify the Product Line.

3. Calculate the center-of-glass SHGC and VT.

4. Determine Product Groupings.

5. Determine Divider and Frame U-factors for SHGC and VT.

6. Calculate the whole product SHGC and VT.

7. Use the Specialty Products Alternative to develop a SHGC and VT Specialty Products Table for a Product Line.

\subsubsection{Define the Product Line}

The following list of fenestration product features must be accounted for when defining the SHGC product line matrix include:

- number of glazing layers

- nominal glass thickness 0.125 inch $(3 \mathrm{~mm})$ and 0.25 inch $(6 \mathrm{~mm})$ glass $)$

- low-e coatings and their location

- $\quad$ tints (tints need not be accounted for under NFRC 100)

- spacer type

- dividers

- frame color

The following list of fenestration product features do not have to be accounted for when defining the SHGC product line (some of these features have to be accounted for when defining the NFRC $100 \mathrm{U}$-factor product line) include:

- gap thickness between the glazings

- fill gas type 


\subsubsection{Simplify the Product Line}

The number of individual products in a product line can be greatly reduced by using some or all of the options available in NFRC 200 to simplify a product line. The following is a list of the variations that can be simplified:

- glass thickness

- frame color

- spacer type and frame/divider thermal properties

- divider types

- pane spacing and gas fill

\section{Glass Thickness}

NFRC 200 states that only two thicknesses of glass are to be rated, nominal 0.125 inch $(3 \mathrm{~mm})$ glass in the residential size and nominal 0.25 inch $(6 \mathrm{~mm})$ glass in the non-residential size. All other glass thicknesses must be represented by these two thicknesses.

\section{Frame Color}

The color of the frame has an effect on the total-product SHGC. The frame solar absorptance varies with the frame color. Manufacturers can choose to rate every color of frame that a product is manufactured in, or they can choose to rate all their product frames with a default solar absorptance of 0.5 .

\section{Spacer Type and Frame/Divider Thermal Properties}

The spacer type and frame/divider thermal properties can also effect the total product SHGC. The spacer type and frame/divider thermal properties effect the thermal conductance through the product and this has a small effect on the SHGC. Manufacturers can choose to rate every spacer option and frame/divider type offered in a product line, or they can select a group or entire matrix representative of the frame and the divider for a group or entire matrix of products within the product line (the grouping of products within a product line is discussed in Step 3). The representative frame and divider for a group or entire matrix of products within the product line is the frame and divider for the product in the grouping with the lowest center-of-glass U-factor (if there are several individual products with the lowest center-of-glass U-factor, use the product with the highest frame and edge-of-glass heat loss).

\section{Divider Types}

A manufacturer may offer many divider options within one product line. The height of the divider has a significant effect on the product SHGC. Manufacturers can rate every divider type offered in a product line, or they can group the dividers into two groups: For all dividers less than 1.00 inch $(25.40 \mathrm{~mm})$ in width [PDD $<1.00$ inch $(25.40 \mathrm{~mm})$ ], the representative $\mathrm{SHGC}_{\mathrm{d}}$ shall be used with a divider width (PDD) of 0.75 inch $(19.05 \mathrm{~mm})$. For all dividers greater than or equal to 1.00 inch $(25.40 \mathrm{~mm})$ in width [PDD $\geq 1.00$ inch $(25.40)]$, the representative $\mathrm{SHGC}_{\mathrm{d}}$ shall be used with a divider width (PDD) of 1.50 inch $(38.10 \mathrm{~mm})$. All true divided lites, internal grilles, mutin bars, and other dividers may be grouped together for the purposes of calculating SHGC and VT.

\section{Pane Spacing and Gas-fill}

Gap-width and gas-fill are not factors in determining individual products under NFRC 200 (section 4.3). For the purpose of simplification, simulators may use 0.50 inch $(12.7 \mathrm{~mm})$ gap with air-fill for all double-glazed 
products, and 0.25 inch $(6.35 \mathrm{~mm})$ gap with air-fill for all other products (all triple or quad products). However, simulators are free to use actual gap widths. 


\subsubsection{Calculate the Center-of-Glass SHGC and VT}

The center-of-glass SHGC and VT, shown in Figure 5-6, must be calculated for all the products in the product line using the most current NFRC-approved spectral data files with the WINDOW 4.1 program.

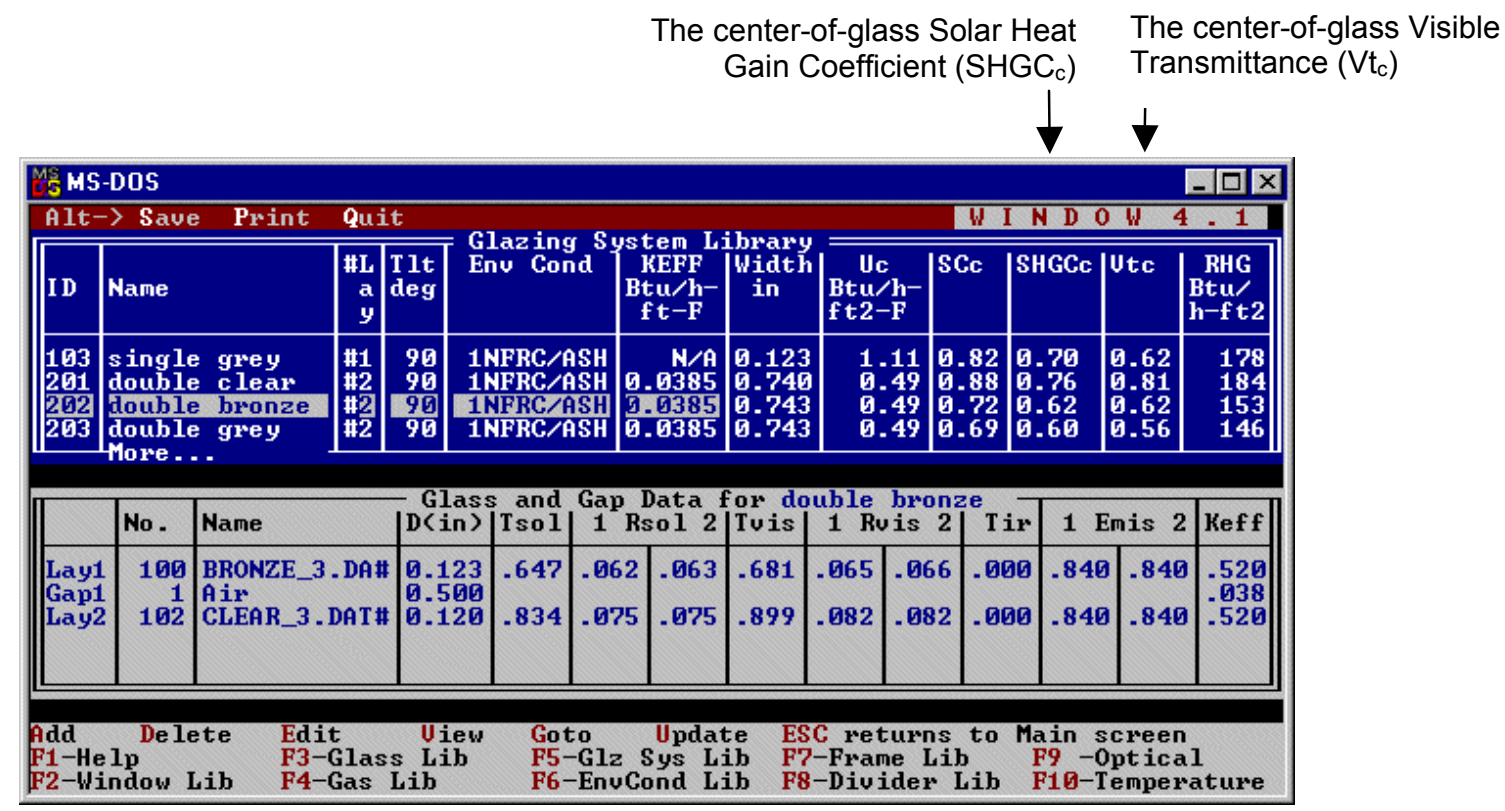

Figure 5-6. The center-of-glass SHGC and VT are calculated in the Glazing System Library.

\subsubsection{Determine the Product Groupings}

Once all the individual products have been identified and the center-of-glass SHGC and VT determined for each product, the products can be grouped to reduce the number of products that must be reported in the Product Line. Two or more glazing systems when grouped together shall meet the following conditions:

(1) The $\mathrm{SHGC}_{\mathrm{c}}$ of all the glazing systems [using nominal 0.125 inch $(3 \mathrm{~mm})$ glass] are within a 0.05 band.

(2) The $\mathrm{SHGC}_{\mathrm{c}}$ of all the glazing systems [using nominal 0.25 inch $(6 \mathrm{~mm})$ glass] are within a 0.05 band.

(3) $\mathrm{The}_{\mathrm{VT}}$ of all the glazing systems [using nominal 0.125 inch $(3 \mathrm{~mm})$ glass] are within a 0.05 band.

(4) The $\mathrm{VT}_{\mathrm{c}}$ of all the glazing systems (using nominal 0.25 inch $(6 \mathrm{~mm})$ glass) are within a 0.05 band.

(5) The glazing systems within a group all include the same number of layers and each layer is generically the same (i.e. clear, tinted, low-e on clear, low-e on tinted, reflective on clear, reflective on tinted).

(6) The individual product chosen to be the group representative is at the midpoint, or within 0.01 of the midpoint, for all four properties $\left(\mathrm{SHGC}_{\mathrm{c}} 0.125 \mathrm{inch}(3 \mathrm{~mm})\right.$ nominal thickness; $\mathrm{SHGC}_{\mathrm{c}} 0.25 \mathrm{inch}(6 \mathrm{~mm})$ nominal thickness, $\mathrm{Vt}_{\mathrm{c}} 0.125$ inch $(3 \mathrm{~mm})$ nominal thickness, $\mathrm{Vt}_{\mathrm{c}} 0.25$ inch $(6 \mathrm{~mm})$ nominal thickness. Each group is then represented by the individual product in the group with the center-of-glass SHGC and VT closest to the mid-point of the group (the Group Representative). If this approach is used, the totalwindow SHGC and VT for the Group Representative shall be used to represent the total-window SHGC and VT for all individual products within the group. The center-of-glass SHGC and VT grouping only allows variation in the glazing thickness, glazing tint, and low-emissivity coating. 


\subsubsection{Determine Divider and Frame U-factors for SHGC and VT}

If no grouping is desired, all the frame U-factors and divider U-factors for all the individual products in the Product Line have to calculated. If grouping is performed, a simulator can determine the frame representative and divider representative U-factors by modeling each center-of-glass group representative (lowest center-of-glass U-factor in the group) or center-of-glass matrix representative (lowest center-of-glass U-factor in the entire matrix). Therefore Frame grouping and Divider grouping may encompass all or part of a Product Line and may or may not, at the simulator's discretion, correspond to frame groups previously defined under NFRC 100. Frame groupings may include more than one 0.05 center-of-glass group.

For developing Specialty Products table one shall use the center-of-glass matrix representative (lowest centerof-glass U-factor in the entire matrix).

\subsubsection{Calculate the whole product SHGC and VT}

Once the center-of-glass SHGC and VT have been determined, the divider U-factor, and the frame U-factor have been established, the total-product SHGC can be calculated using the equation in NFRC 200, section 5.4 .3 (b) (3), and VT can be calculated using equation 5.1. The example problems in Chapter 9 include solar heat gain calculations and visible transmittance.

$$
\begin{array}{ll}
V T_{t}=\frac{\left(V T_{c}{ }^{*} A_{c}+V T_{e}{ }^{*} A_{e}+V T_{d e}{ }^{*} A_{d e}+V T_{d} * A_{d}+V T_{f} * A_{f}\right)}{A_{p f}} \\
\boldsymbol{V} \boldsymbol{T}_{\boldsymbol{f}} & \text { Frame visible transmittance (dimensionless) } \\
\boldsymbol{A}_{\boldsymbol{f}} & \text { Frame Area in } \mathrm{m}^{2} \text { or } \mathrm{ft}^{2} \\
\boldsymbol{V} \boldsymbol{T}_{\boldsymbol{d}} & \text { Divider visible transmittance (dimensionless) } \\
\boldsymbol{A}_{\boldsymbol{d}} & \text { Divider Area in } \mathrm{m}^{2} \text { or } \mathrm{ft}^{2} \\
\boldsymbol{V} \boldsymbol{T}_{\boldsymbol{e}} & \text { Edge-of-Glass visible transmittance (dimensionless) } \\
\boldsymbol{A}_{\boldsymbol{e}} & \text { Edge-of-Glass Area in } \mathrm{m}^{2} \text { or } \mathrm{ft}^{2} \\
\boldsymbol{V} \boldsymbol{T}_{\boldsymbol{d e}} & \text { Divider Edge visible transmittance (dimensionless) } \\
\boldsymbol{A}_{\boldsymbol{d}} & \text { Divider Edge Area in } \mathrm{m}^{2} \text { or } \mathrm{ft}^{2} \\
\boldsymbol{V} \boldsymbol{T}_{\boldsymbol{c}} & \text { Center-of-glass visible transmittance (dimensionless) } \\
\boldsymbol{A}_{\boldsymbol{c}} & \text { Center-of-glass Area in } \mathrm{m}^{2} \text { or } \mathrm{ft}^{2} \\
\boldsymbol{A}_{\boldsymbol{p} \boldsymbol{f}} & \text { is the total projected fenestration product area in } \mathrm{m}^{2} \text { or } \mathrm{ft}^{2}
\end{array}
$$

\subsubsection{Specialty Products Table}

It is not necessary to calculate the SHGC and VT values for all the individual products within a product line if a Specialty Products Table for both SHGC and VT is created for the product line. In summary, the SHGC is calculated for two glazings, one with a SHGC of 0 and another with an SHGC of 1.0. When these two values have been determined, any SHGC value can be calculated in the center-of-glass SHGC is known.

A simulator need not precalculate the SGHC and VT for all individual products within a product line. The manufacturer may elect not to have the simulator precalculate SHGC and VT for some or all of the individual products within a product line. If a manufacturer elects not to precalculate SHGC and VT for one or more individual products within a product line, the simulator will have to use the Specialty Products Procedure, described under this step, in order to develop a "SHGC Specialty Products Table" and a "VT Specialty Products Table" for a given Product Line. 
Under the Specialty Products procedure, for a give Product Line, the simulation laboratory shall first determine the SHGC values for individual products with two "fictitious" glazings; one with a SHGCc=0 and one with a SHGCc=1.0. These two SHGC values are defined as SHGC0 and SHGC1 respectively. SHGC0 is calculated according to NFRC 200, setting SHGCc $=$ SHGCe $=$ SHGCde $=0.00$. SHGC1 is calculated according to NFRC 200, setting SHGCc $=$ SHGCe $=$ SHGCde $=1.00$ If the product line includes dividers, the simulation laboratory shall include the SHGC0 and SHGC1 values for cases with no dividers, dividers less than or equal 1.00 inch $(25.40 \mathrm{~mm})$ and greater than or equal to 1.00 inch $25.40 \mathrm{~mm})$ wide. Once the SHGC0 and SHGC1 values have been determined, any SHGC value can be calculated if the SHGCc value is known. The SHGC for any specialty product is calculated using the following equation:

$$
\mathrm{SHGC}=\mathrm{SHGCO}+\mathrm{SHGCC} *(\mathrm{SHGC1}-\mathrm{SHGC0})
$$

Table 5.4a lists the $\mathrm{SHGC}_{0}$ and $\mathrm{SHGC}_{1}$ values for an example casement window Product Line. The casement window Product Line has the following properties:

\section{Casement Window:}

- Frame Height

- $\quad$ Divider $<1$ " Height

- $\quad$ Divider $\geq 1$ " Height

- Vertical Divider Bars

- Horizontal Divider Bars
3.00" Frame U-factor $0.60 \mathrm{BTU} / \mathrm{hr}^{\prime}-\mathrm{ft}^{2}-\mathrm{F}$

0.75" Divider U-factor $0.50 \mathrm{BTU} / \mathrm{hr}-\mathrm{ft}^{2}-\mathrm{F}$

$1.50^{\prime \prime}$

1

2

Table 5-4. SHGC $C_{0}$ and $S H G C_{1}$ Values For an Example Product Line

\begin{tabular}{|l|l|l|l|l|l|l|}
\hline & \multicolumn{2}{|l|}{ No Dividers } & \multicolumn{2}{l|}{ Dividers <25.40 $\mathbf{~ m m ~ ( 1 . 0 0 ~ i n ) ~}$} & \multicolumn{2}{l|}{ Dividers $\mathbf{2 5 . 4 0} \mathbf{~ m m ~ ( 1 . 0 0 ~ i n ) ~}$} \\
\hline & Res. & Nonres. & Res. & Nonres. & Res. & Nonres. \\
\hline SHGC $_{0}$ & 0.035 & 0.028 & 0.039 & 0.032 & 0.043 & 0.035 \\
\hline SHGC $_{1}$ & 0.691 & 0.748 & 0.645 & 0.710 & 0.602 & 0.673 \\
\hline
\end{tabular}

Note: Values in this table shall be reported to three decimal places. The WINDOW program displays three decimal places in the WINDOW 4.0 report format.

Using the values calculated in Table 5.4 and Equation 5.2, for this example, the simulation laboratory can produce the Specialty Product SHGC table. Table 5.5 is a Specialty Products SHGC table for the example casement window above. The Specialty Products SHGC table is to have values for SHGC with $\mathrm{SHGC}_{\mathrm{c}}$ values varying from 0.00 to 1.00 in 0.01 increments. (note that, due to space limitation in this manual, Table 5.5 does not include all the values that are needed for a Specialty Products SHGC table). 
Table 5-5. SHGC Specialty Products Table for an Example Product

\begin{tabular}{|c|c|c|c|c|c|c|}
\hline \multicolumn{7}{|c|}{ Total Product Solar Heat Gain Coefficient $\left(\right.$ SHGC $\left._{t}\right)$} \\
\hline & \multicolumn{2}{|c|}{ No Dividers } & \multicolumn{2}{|c|}{ Dividers $<25.40 \mathrm{~mm}(1.00 \mathrm{in})$} & \multicolumn{2}{|c|}{ Dividers $\geq 25.40 \mathrm{~mm}(1.00 \mathrm{in})$} \\
\hline $\mathrm{SHGC}_{\mathrm{c}}$ & Res. & Nonres. & Res. & Nonres. & Res. & Nonres. \\
\hline 0.00 & 0.04 & 0.03 & 0.04 & 0.03 & 0.04 & 0.04 \\
\hline 0.01 & 0.04 & 0.04 & 0.05 & 0.04 & 0.05 & 0.04 \\
\hline 0.02 & 0.05 & 0.04 & 0.05 & 0.05 & 0.05 & 0.05 \\
\hline 0.03 & 0.06 & 0.05 & 0.06 & 0.05 & 0.06 & 0.05 \\
\hline 0.04 & 0.06 & 0.06 & 0.06 & 0.06 & 0.07 & 0.06 \\
\hline$:$ & : & : & : & : & : & : \\
\hline$:$ & : & : & : & : & : & : \\
\hline 0.98 & 0.68 & 0.73 & 0.63 & 0.70 & 0.59 & 0.66 \\
\hline 0.99 & 0.69 & 0.74 & 0.64 & 0.70 & 0.60 & 0.67 \\
\hline 1.00 & 0.69 & 0.75 & 0.65 & 0.71 & 0.60 & 0.67 \\
\hline
\end{tabular}

Note: Value in this table shall be reported to two decimal places.

The development of a VT Specialty Products Table following the same method. $\mathrm{VT}_{0}$ is calculated according to Equation 5.1, setting $\mathrm{VT}_{\mathrm{c}}=\mathrm{VT}_{\mathrm{e}}=\mathrm{VT}_{\mathrm{de}}=0.00 . \mathrm{VT}_{1}$ is calculated according to Equation 5.1, setting $\mathrm{VT}_{\mathrm{c}}=$ $\mathrm{VT}_{\mathrm{e}}=\mathrm{VT}_{\mathrm{de}}=1.00$. If the Product Line includes dividers, the simulation laboratory shall include the $\mathrm{VT}_{0}$ and $\mathrm{VT}_{1}$ values for cases with no dividers, dividers less than or equal $1.00 \mathrm{inch}(25.40 \mathrm{~mm})$ and greater than or equal to 1.00 inch $(25.40 \mathrm{~mm})$ wide. Once the $\mathrm{VT}_{0}$ and $\mathrm{VT}_{1}$ values have been determined, any VT value can be calculated if the $\mathrm{VT}_{\mathrm{c}}$ value is known. The VT is calculated using the following equation:

$$
\mathrm{VT}=\mathrm{VT}_{0}+\mathrm{VT}_{\mathrm{c}} *\left(\mathrm{VT}_{1}-\mathrm{VT}_{0}\right)
$$

Table 5.6 lists the $\mathrm{VT}_{0}$ and $\mathrm{VT}_{1}$ values for an example casement window Product Line. The casement window Product Line as the following properties:

Casement Window

- Frame Height

- Divider $<1$ " Height

- $\quad$ Divider $\geq 1$ " Height

- Vertical Divider Bars

- Horizontal Divider Bars
3"

$0.75^{\prime \prime}$

$1.50^{\prime \prime}$

1

2

Table 5-6. VT $T_{0}$ and $V T_{1}$ Values For an Example Product

\begin{tabular}{|l|l|l|l|l|l|l|}
\hline & \multicolumn{2}{|l|}{ No Dividers } & \multicolumn{2}{l|}{ Dividers $<\mathbf{2 5 . 4 0 ~} \mathbf{~ m m ~ ( 1 . 0 0 ~ i n ) ~}$} & \multicolumn{2}{l|}{ Dividers $\mathbf{2 5 . 4 0} \mathbf{~ m m ~ ( 1 . 0 0 ~ i n ) ~}$} \\
\hline & Res. & Nonres. & Res. & Nonres. & Res. & Nonres. \\
\hline $\mathrm{VT}_{0}$ & 0.000 & 0.000 & 0.000 & 0.000 & 0.000 & 0.000 \\
\hline $\mathrm{VT}_{1}$ & 0.656 & 0.720 & 0.606 & 0.678 & 0.559 & 0.638 \\
\hline
\end{tabular}


Note: Values in this table shall be reported to three decimal places. The WINDOW program displays three decimal places in the WINDOW 4.1 report format.

Using the values calculated in Table 5.6 and Equation 5.2, the simulation laboratory can produce the Specialty Products VT table. Table 5.7 is a Specialty Products VT table for the example casement Product Line above. The Specialty Products VT table is to have values for VT with $\mathrm{VT}_{\mathrm{c}}$ values varying from 0.00 to 1.00 in 0.01 increments. (note that, due to space limitation in this manual, Table 5.7 does not include all the values that are needed for a Specialty Products VT table).

Table 5-7. VT Specialty Products Table for an Example Product

\begin{tabular}{|c|c|c|c|c|c|c|}
\hline \multicolumn{7}{|c|}{ Total Product Visible Transmittance (VT) } \\
\hline \multirow[b]{2}{*}{$\mathbf{V} T_{c}$} & \multicolumn{2}{|c|}{ No Dividers } & \multicolumn{2}{|c|}{ Dividers $<25.40 \mathrm{~mm}$ (1.00 in) } & \multicolumn{2}{|c|}{ Dividers $\geq 25.40 \mathrm{~mm}$ (1.00 in) } \\
\hline & Res. & Nonres. & Res. & Nonres. & Res. & Nonres. \\
\hline 0.00 & 0.00 & 0.00 & 0.00 & 0.00 & 0.00 & 0.00 \\
\hline 0.01 & 0.01 & 0.01 & 0.01 & 0.01 & 0.01 & 0.01 \\
\hline 0.02 & 0.01 & 0.01 & 0.01 & 0.01 & 0.01 & 0.01 \\
\hline 0.03 & 0.02 & 0.02 & 0.02 & 0.02 & 0.02 & 0.02 \\
\hline 0.04 & 0.03 & 0.03 & 0.02 & 0.03 & 0.02 & 0.03 \\
\hline : & : & : & & : & : & $:$ \\
\hline : & : & : & : & : & : & : \\
\hline 0.98 & 0.64 & 0.71 & 0.59 & 0.67 & 0.55 & 0.63 \\
\hline 0.99 & 0.65 & 0.71 & 0.60 & 0.67 & 0.55 & 0.63 \\
\hline 1.00 & 0.66 & 0.72 & 0.61 & 0.68 & 0.56 & 0.64 \\
\hline
\end{tabular}

Note: Value in this table shall be reported to two decimal places.

The manufacturer, or any other user of the Certified Products Directory (CPD), may then take these two tables the SHGC Specialty Products Table and the VT Specialty Products Table) and the list of SHGC $\mathrm{C}_{\mathrm{C}}$ and $\mathrm{VT}_{\mathrm{c}}$ found in the CPD and use them in combination to determine the individual product SHGC for any glazing system for that Product Line. 


\section{MODELING FRAME AND EDGE HEAT TRANSFER WITH THERM}

\subsection{Overview}

In order to calculate the total product U-factor, the U-factor must be determined for the following components, and then these values must be area-weighted:

- Center-of-glass values from WINDOW

- Frame and edge values from THERM

The NFRC 100: Procedures for Determining Fenestration Product $U$-factors is the definitive source for the methodology for calculating U-factors, and should be used for situations not covered in this manual. In addition, many NFRC Technical Interpretations have been created which apply to the NFRC 100 document, and these should also be consulted when questions arise.

\subsection{Cross-Sections to be Modeled}

The fenestration product being modeled must be divided into the cross-sections which completely define the heat transfer through the product. THERM is used to calculate the U-factor values for the edge-of-glass as well as the frame components (sill, head, jamb, meeting rail, and divider).

The operator type of the fenestration product, as well as the configuration of each component of the frame, will determine the number of cross sections that must be modeled. Table 6-1 lists the minimum and maximum cross sections that must be modeled for each operator type, and Figure 6-1 illustrates where the cross sections must be taken. The maximum assumes that each frame cross section is unique and therefore must be modeled. (Note that significant operating hardware may require more cross sections to be simulated. See Section 6.3.3, "What is Not Modeled"). If there are some cross sections of the frame which are identical, such as a fixed window with the same cross section for the head, jamb and sill, then only one cross section needs to be modeled.

Table 6-1. Cross sections to be modeled

\begin{tabular}{|c|c|c|c|}
\hline \multirow[b]{2}{*}{ Operator Type } & \multicolumn{2}{|c|}{ Number of Cross Sections to Model } & \multirow[b]{2}{*}{ Cross Sections } \\
\hline & Minimum & Maximum & \\
\hline $\begin{array}{l}\text { Fixed, Picture, } \\
\text { Transom, etc }\end{array}$ & 1 & 4 & $\begin{array}{ll}- & \text { Head } \\
: & \text { Jamb (1 or } 2)\end{array}$ \\
\hline Casement, Awning & 1 & 4 & $\begin{array}{ll}- & \text { Head } \\
& \text { Jamb (1 or } 2) \\
& \text { Sill }\end{array}$ \\
\hline Vertical Slider & $\begin{array}{l}3 \\
\text { If the Head and Upper Jambs are } \\
\text { the same and the Sill \& Lower } \\
\text { Jambs are the same }\end{array}$ & 7 & $\begin{array}{ll}\text { - } & \text { Head Fixed \& Vented } \\
\text { - } & \text { Jamb Fixed \& Vented } \\
\text { - } & \text { Meeting Rixed \& Vented }\end{array}$ \\
\hline $\begin{array}{l}\text { Horizontal Slider, } \\
\text { Sliding Patio Door }\end{array}$ & $\begin{array}{l}3 \\
\text { If fixed Head, Jamb, and Sill are } \\
\text { all the same and the Vented Head, } \\
\text { Jamb, and Sill are all the same.. }\end{array}$ & 7 & $\begin{array}{ll} & \text { Head Fixed \& Vented } \\
\text { - } & \text { Jamb Fixed \& Vented } \\
\text { - } & \text { Sill Fixed \& Veeting Rail }\end{array}$ \\
\hline
\end{tabular}


In Section 9, "Sample Problems", Problem 2 is an example of a horizontal slider where all seven cross sections must be simulated because the construction of each component of the frame is unique. 


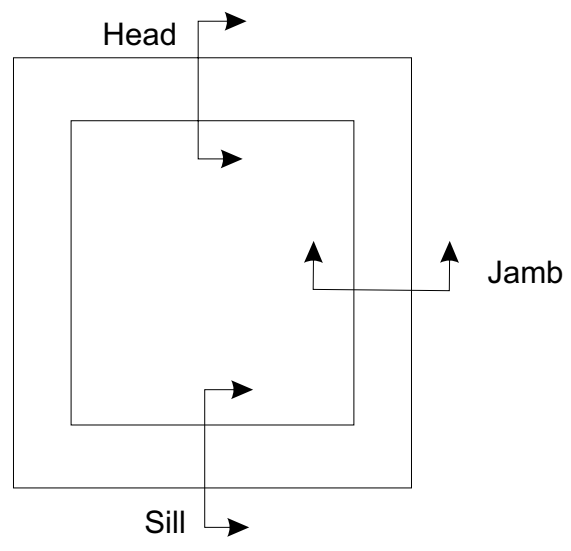

Fixed

(Max. 4, Min. 1 cross sections)

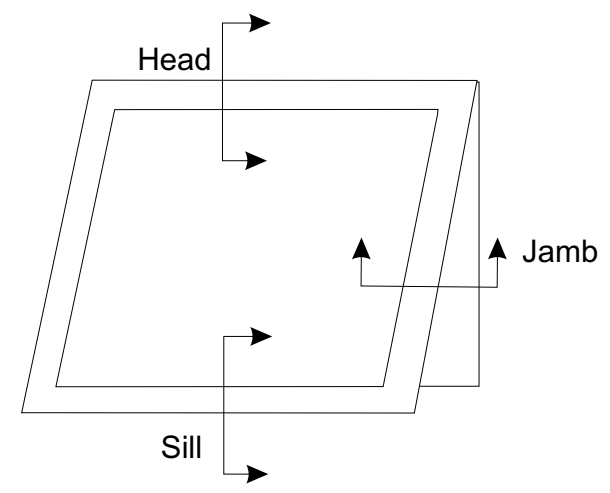

Projecting / Awning

(Max. 4, Min. 1 cross sections)

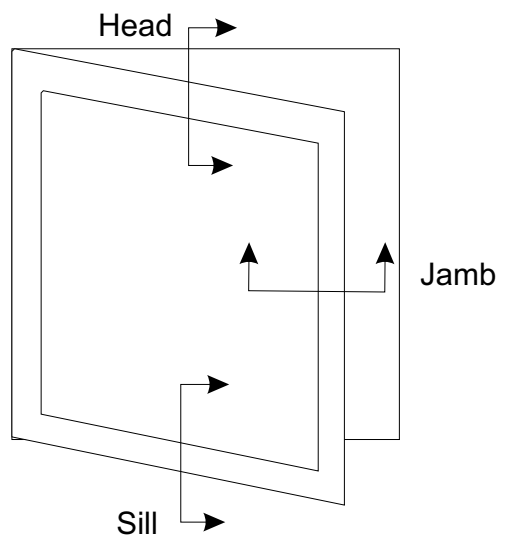

Casement

(Max. 4, Min 1 cross sections)

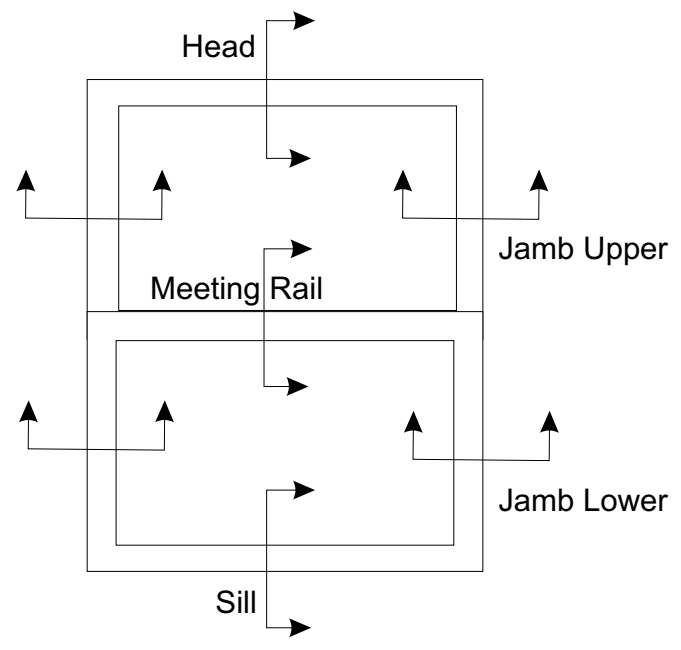

Vertical Slider

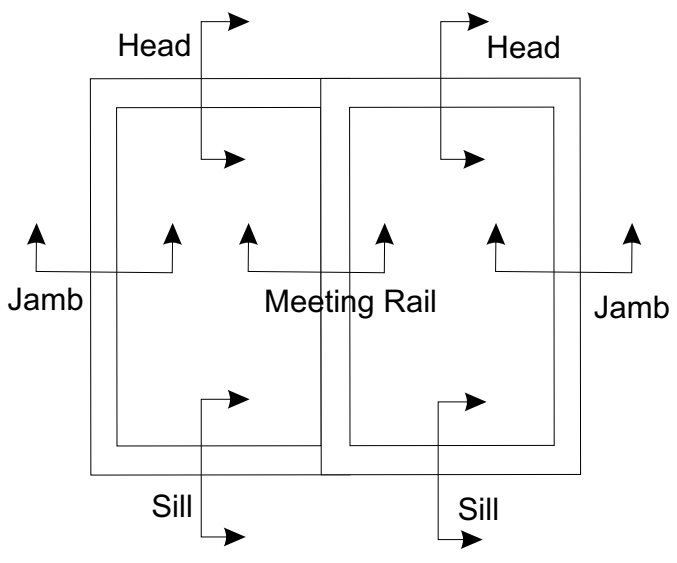

Horizontal Slider (Max. 7, Min. 3 cross sections)

Figure 6-1. Minimum and maximum cross sections to be modeled based on operator type. 


\subsection{Draw the Cross Sections}

When the number of cross sections to be modeled has been determined, it is time to begin drawing them in THERM. The Section 5, "Drawing Cross-Section Geometry" in the THERM User's Manual contains detailed instructions about how to draw cross sections. This section of the THERM NFRC Simulation Manual contains information pertaining specifically to the NFRC procedures. Section 10, "Drawing Tips" of this manual contains information about drawing in THERM that is not included in the THERM User's Manual.

\subsubsection{Getting Started - Drawings and DXF Files}

In order to reproduce accurate cross sections in THERM, it is necessary to use dimensioned drawings and/or DXF files. Section 5.2, "Importing a DXF or Bitmap File as an Underlay" in the THERM User's Manual, and Section 10 in this manual, contain detailed information about DXF files.

If only dimensioned drawings are available (assembly drawings and die drawings), and there are no DXF files, it is still relatively easy to create a cross-section in THERM. Section 5.5, "Drawing Using the Keyboard (Numeric Cursor Positioning)" of the THERM User's Manual, and Section 10 of this manual discuss various methods for efficiently creating cross sections from dimensioned drawings.

\subsubsection{Cross-Section Orientation}

When the THERM model is simulated, all the cross sections must be oriented in a vertical direction, i.e., all the glazing systems must be pointing either up or down, not horizontally. When creating the cross section, the frame components may be drawn horizontally if that is easiest (i.e., the DXF file is drawn that way), but before importing the glazing system, rotate the cross section so that the glazing will come in either up or down. (Note: the only exception to this may be future NFRC procedure for simulating skylights at a tilt, but as of the writing of this manual, that method is not approved).

So, for example, jamb cross sections could be drawn horizontally, but they must be rotated into a vertical position for modeling. Figure 6-2 shows how a DXF file might be drawn. The horizontal jamb and meeting rail sections could be drawn horizontally as in the DXF file until the point of importing the glazing system, at which time they must be rotated 90 degrees. It is not important whether the glass is facing up or down, but for consistency, the standard method is to have the glass pointing up for units with one glazing system, with the inside surfaces of the model facing to the right and the exterior surfaces facing to the left.

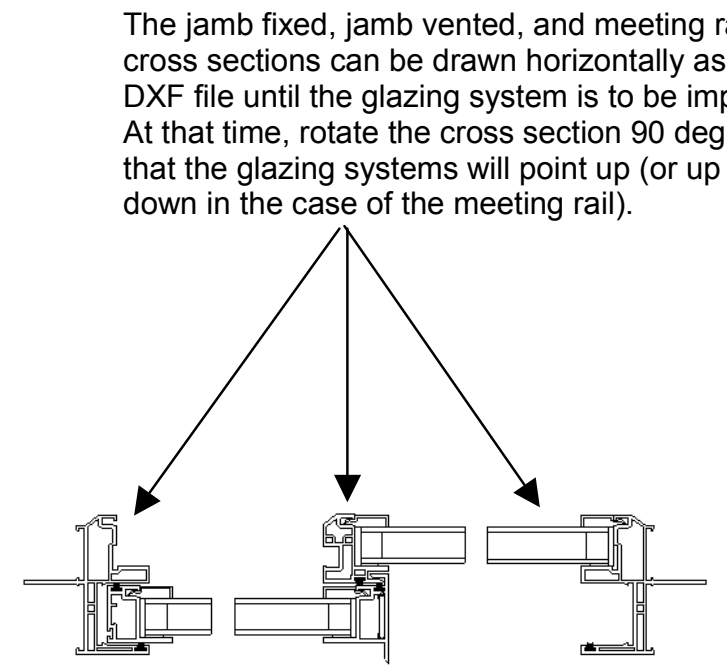

Figure 6-2. DXF file with horizontal cross sections that must be rotated 90 degrees before importing glazing systems and simulating. 


\subsubsection{What is Not Modeled}

NFRC 100 specifically excludes certain options on fenestration products which are not modeled in THERM. These options include:

- Screens

- Interior trim

- Grilles applied to the interior or exterior surface of the glass (i.e., snap-on grilles). Grilles or dividers between glass layers must be modeled under some circumstances, as discussed in Section 8.3, "Modeling Internal Dividers."

- Optional jamb extensions

- Optional sill extensions

- Interior or exterior shading devices

- Nailing flanges which can be removed from a fenestration product, and which are removed for testing. These may be vinyl flanges on wood windows, or flanges on vinyl or aluminum windows designed to be removed for some installations. Permanent nailing flanges that would be in place during a test shall be modeled.

In addition, as stated in NFRC 100, "including but not liminted to screws and bolts in curtainwalls and pourand-debridge thermal breaks which are not full debridged, shall be simulated". For the time being, components that do not have to be modeled include:

- Hinges

- Locks

- Balances

- Non-continuous Operator Hardware

- Weep Holes

- Setting Blocks

\subsubsection{Deformable Parts}

Because deformable parts, such as glazing clips, weatherstripping and other snap-in parts, are often drawn in the undeformed states in DXF files and assembly drawings (many times in the DXF files they overlap other parts), simulator judgement is still required to ensure that these parts are modeled in a way that results in accurate heat transfer results. For example, there are several instances when small air gaps can be replaced with solid materials, such as in sweeps and seals.

When making decisions about how to model such components, take the most conservative approach (the case with the highest U-factor) in terms of the thermal performance. For example, replace very small air cavities with a material that has a higher conductivity than air. So in a vinyl extrusion replace small air cavities with vinyl and similarly in a wood window.

Files included on the CD, Lowhoriz.dxf, example1.dxf, and example2.dxf are examples of files which have deformable parts. 


\subsubsection{Frame Cavities}

THERM has two cavity models for NFRC modeling:

- NFRC Simplified

- NFRC Detailed

The NFRC Simplified frame cavity uses a method consistent with NFRC 100, and it is the only frame cavity model that can be used for NFRC simulations. The Material Library provided with THERM contains three NFRC Simplified entries, which represent different emissivity conditions:

- Frame Cavity (0.9/0.9) NFRC Simplified: used to model frame cavities where both sides of the cavity are next to non-exposed metallic surfaces with emissivity of 0.9 (the standard default value).

- Frame Cavity (0.9/1.0) NFRC Simplified: used to model frame cavities where one side of the cavity is next to non-exposed metalic surfaces with emissivity of 0.9 and the other side is next to air.

- Frame Cavity (0.2/0.2) NFRC Simplified: used to model frame cavities where both sides of the cavity have an emissivity of 0.2 , which represents an exposed metallic surface.

- Frame Cavity (0.2/0.9) NFRC Simplified: used to model frame cavities where one side of the cavity has an emissivity of 0.2 and one side has an emissivity of 0.9 . The program does not distinguish which side the emissivity values are on.

- Frame Cavity (0.2/1.0) NFRC Simplified: used to model frame cavities where one side of the cavity is next to exposed metalic surfaces with emissivity of 0.9 and the other side is next to air.

THERM bases the convection in the frame cavity on the bounding rectangle of the cavity. The current technical NFRC interpretation is to break up cavities that are separated by a connection, or "throat", less than or equal to 0.25 inches $(6.35 \mathrm{~mm})$. However, if a frame cavity has a Nusselt number less than or equal to 1.20, it is not necessary to break it up into smaller cavities. The Nusselt number can be displayed by double clicking on the frame cavity, which displays the Properties dialog box. The horizontal and vertical dimensions of the bounding rectangle, which determine the Nusselt number, are also shown in this dialog box. 


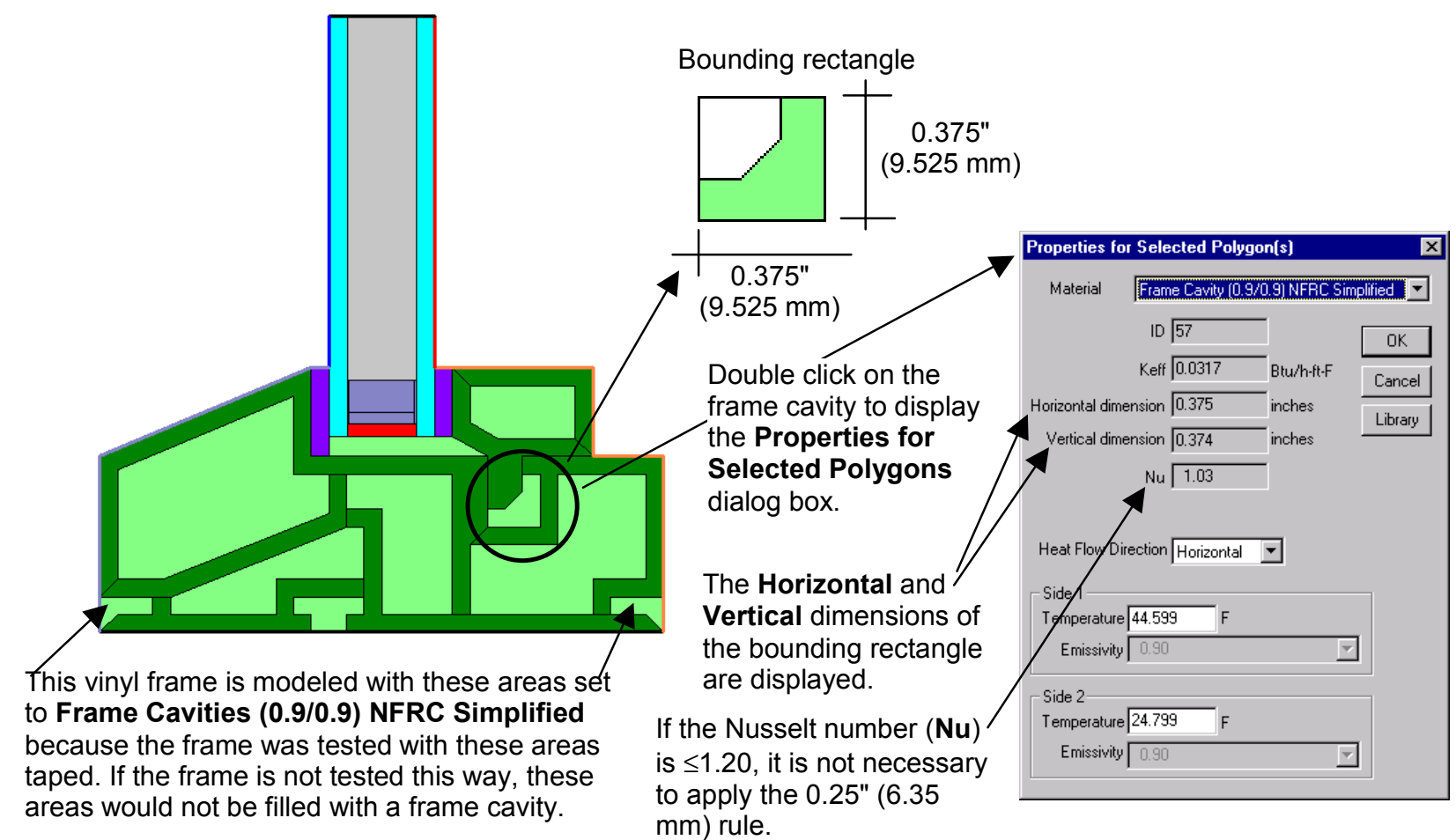

Figure 6-3. THERM uses bounding rectangles to model the thermal characteristics of cavities. 
The best method for determining whether a frame cavity with a $0.25 "(6.35 \mathrm{~mm})$ throat will have to be divided up, and how to divide it if necessary, is the following:

1. Verify that there are throats (constrictions) in the cavity less than or equal to 0.25 inches $(6.35 \mathrm{~mm})$. A throat is defined as any place in a frame cavity where two walls of the cavity are at or within 0.25 inches of each other.

2. Check the Nusselt Number.

3. If the Nusselt Number is less than or equal to 1.20, the simulator shall not break the cavity into smaller polygons.

4. If the Nusselt Number is greater than 1.20, the simulator shall:

a) First, identify all cavities that are less than or equal to 0.25 inches $(6.35 \mathrm{~mm})$ in both the horizontal and vertical direction, such as screw bosses, and define them as separate cavities.

b)Next, break-up the cavity at the first throat's inflection point where the larger part of the cavity joins the smaller part of the cavity:

- working from the largest part of the cavity to the smallest

- starting in a horizontal direction and then in a vertical direction.

5. After breaking the cavity at the throat, if either dimension of the bounding rectangle is less than or equal to 0.25 inches $(6.35 \mathrm{~mm})$, there is no need to subdivide the cavity further.

6. Each time a cavity is divided, check the newly created cavities for throats (constrictions) less than or equal to 0.25 inches $(6.35 \mathrm{~mm}$ ). If there are throats (constrictions) in any of these new cavities, go back to Step 2 and begin the process for further breaking up these cavities.

Step 1:

For Cavity 1 , identify throats (constrictions) equal to or less than 0.25 inches $(6.35 \mathrm{~mm})$. The areas marked 2-9 are such throats.

Step 2:

Check the Nusselt Number of Cavity 1 by double clicking on the cavity.

Step 3:

The Nusselt Number for cavity 1 is greater than 1.20 so go to Step 4.

Step 4a:

Identify all cavities less than or equal to 0.25 inches ( 6.35 $\mathrm{mm}$ ), in this example the screw bosses at areas marked $3,5,7$, and 8 .

Step 4b:

In this case the Nusselt Number is greater than 1.20, so the cavity must be further subdivided at each throat (constriction). This will result in new cavities being created at the throats marked 2, 4, 6, and 9 in this example.

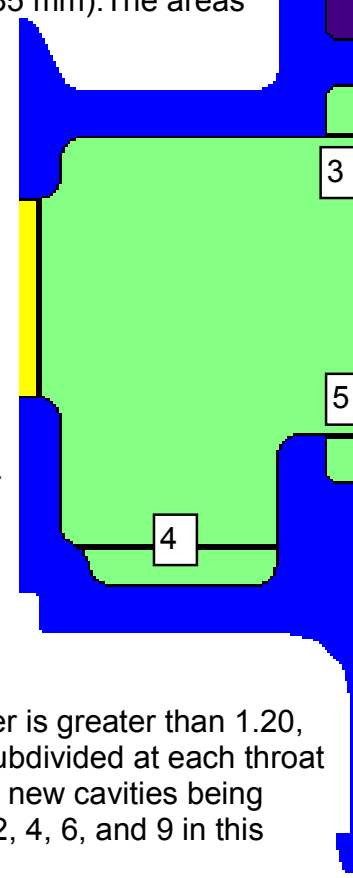

Step 5:

Check the bounding rectangle dimensions (by double clicking on the polygon) of the new cavity. If either dimension is $<=0.25$ inches $(6.35 \mathrm{~mm})$ do not subdivide further.

Figure 6-4. Breaking up frame cavities. 
The frame cavity model default assumption is that the heat flow is horizontal, as shown in Figure 6-5. By rotating horizontal cross-sections to a vertical position before importing the glazing system and doing a simulation there is no need to change the default heat flow setting.

Select the Frame Cavity choice from the Material Library pulldown list that matches the emissivity values for each side of frame cavities. The emissivity should be set to that of the most dominant material for each side. The emissivity values do not have to match the warm and cold sides of the cavity.

The emissivity values are associated with the material, so they cannot be changed here. For materials with emissivities different than the default Frame Cavity entries, go to the Material Library and make a new Frame Cavity.

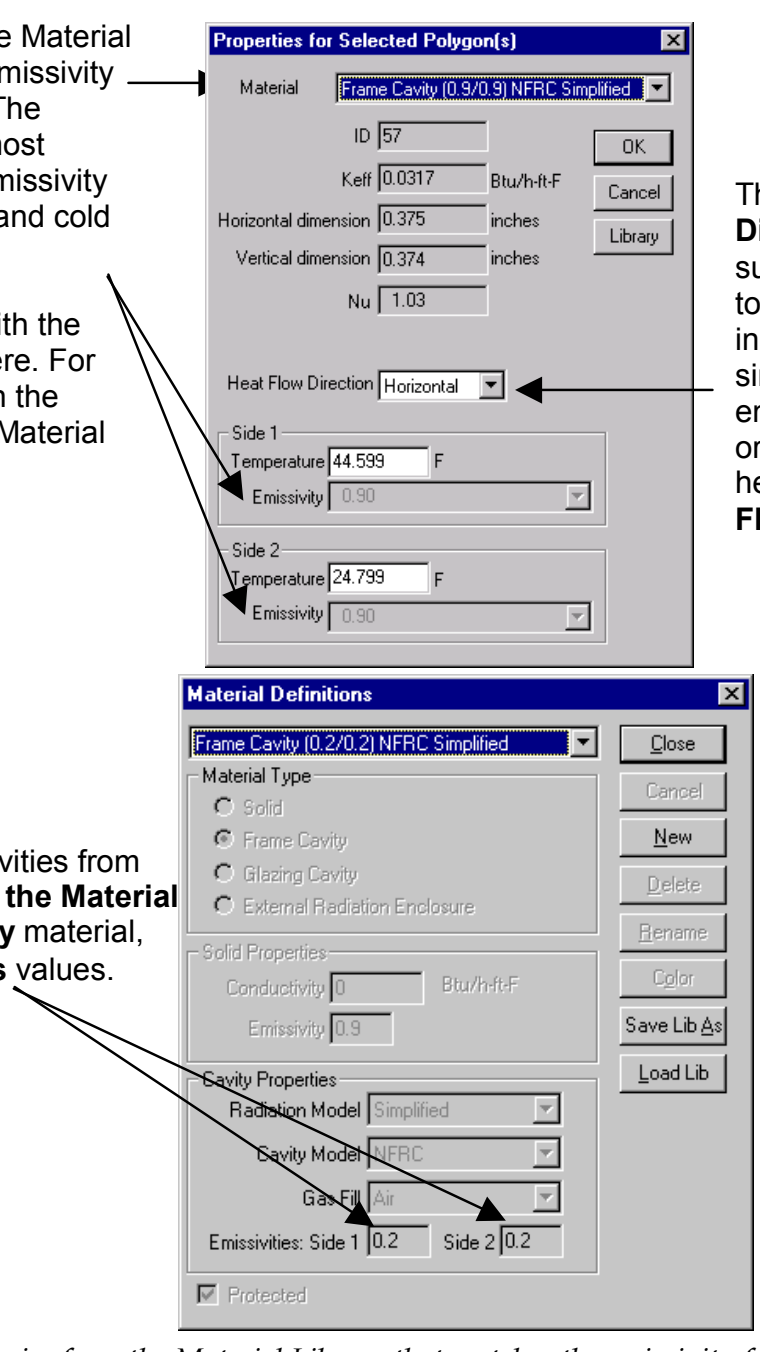

For frame cavities with different emissivities from the default Frame Cavity entries, go to the Materia Library and make a new Frame Cavity material, and enter the appropriate Emissivities values.

Figure 6-5. Select the Frame Cavity choice from the Material Library that matches the emissivity for both sides of each frame.

The emittance of frame cavity walls depends on the neighboring material, and must be specified for each cavity separately. To do this, double click on a cavity and select the Frame Cavity choice from the default list that correctly describes the cavity being modeled. The emissivities cannot be changed in this dialog box. If the appropriate choice is not in the pulldown list, a new Frame Cavity entry can be created in the Material Library, and the appropriate emissivities entered there. The following values are to be used for NFRC simulations:

- 0.20 for exposed metal

- 1.0 for air next to air

- 0.90 for all other cases, including painted metals 
For NFRC simulations, the following frame cavity wall temperatures, which are the THERM default values for the three NFRC frame cavity entries in the Material Library, must be used (Note: it is easy to check that the correct temperatures are set in all the frame cavities after the simulation has been completed by looking at the THERM report, which prints the temperatures of each frame cavity.):

- $24.8^{\circ} \mathrm{F}\left(-4^{\circ} \mathrm{C}\right)$ for the cold side

- $44.6^{\circ} \mathrm{F}\left(7^{\circ} \mathrm{C}\right)$ for the warm side

\subsubsection{Vented Exterior Cavities}

The modeling rules for air cavities that are open to the exterior air within a frame section, such as the air cavity under the sash of a casement window that is vented to the exterior, can be open to interpretation. The issue is how far to extend the exterior boundary condition into the open cavity. The exterior boundary can be extended, along the centerline of the cavity, into the open cavity for a certain distance, and any area of the open cavity beyond the distance is filled with an internal air cavity. For NFRC modeling, simulators shall use five times the distance of the opening to model this. Figure 6-6 illustrates this method.

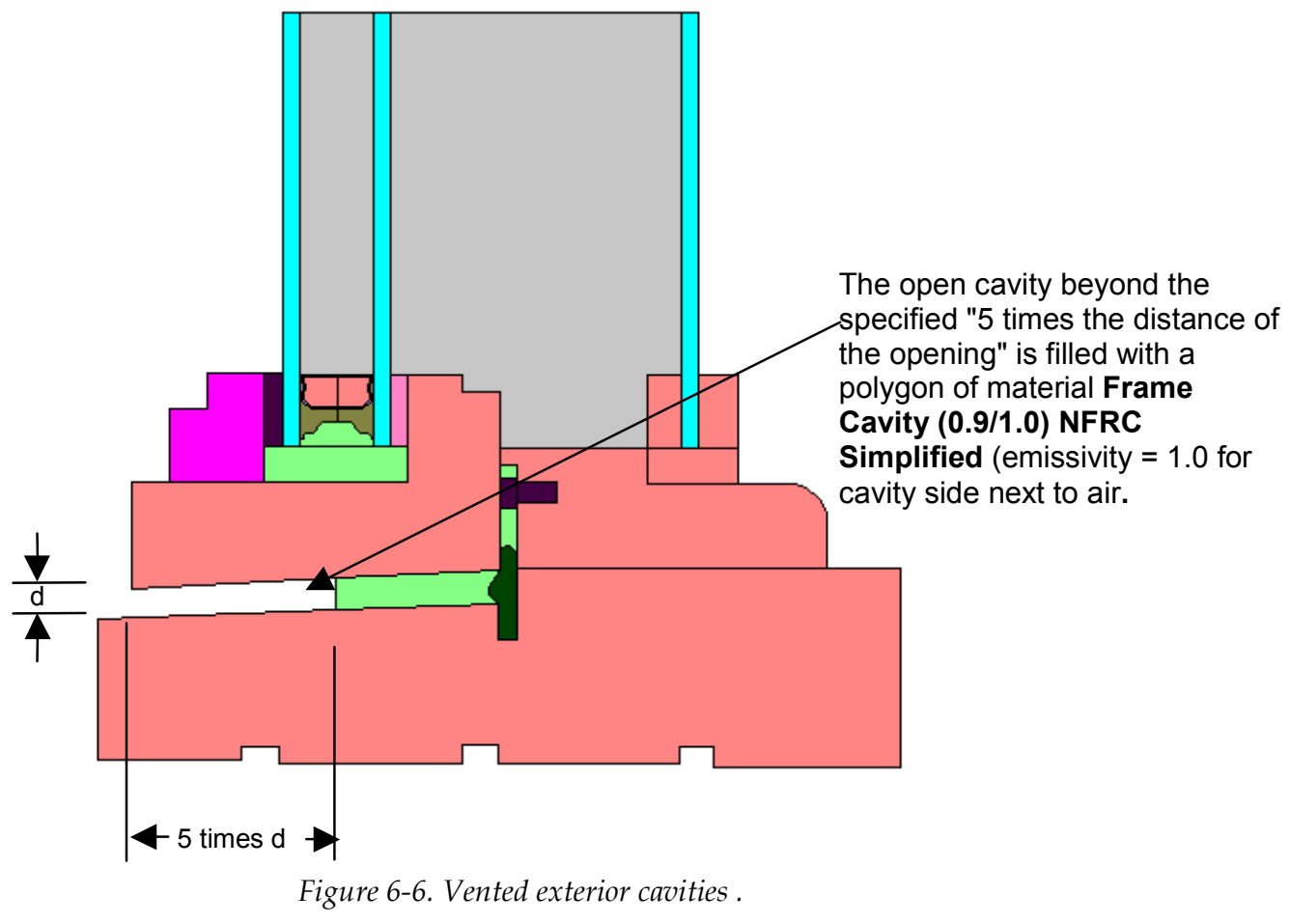

Air cavities that are open to the interior are not treated with the "five- times rule". During testing, the product is sealed at the inside surface, and these gaps are usually sealed with tape. All the air gaps which are sealed during a test must be simulated with the appropriate NFRC frame cavity material choice (see Section 6.3.5, "Frame Cavities"), filling the air spaces flush to the inside surface of the product to match the way it was tested. (Make sure to set the emissivity on the warm side to 1.0 where there is the air-to-air condition). The example file vinylp.thm on the CD shows how cavities that are open to the interior of the room are filled with the material Frame Cavity (0.9/0.9) NFRC Simplified to model the product as it was tested. The emissivity of the cavity next to aid is 0.9 rather than 1.0 because during testing the opening is taped. 


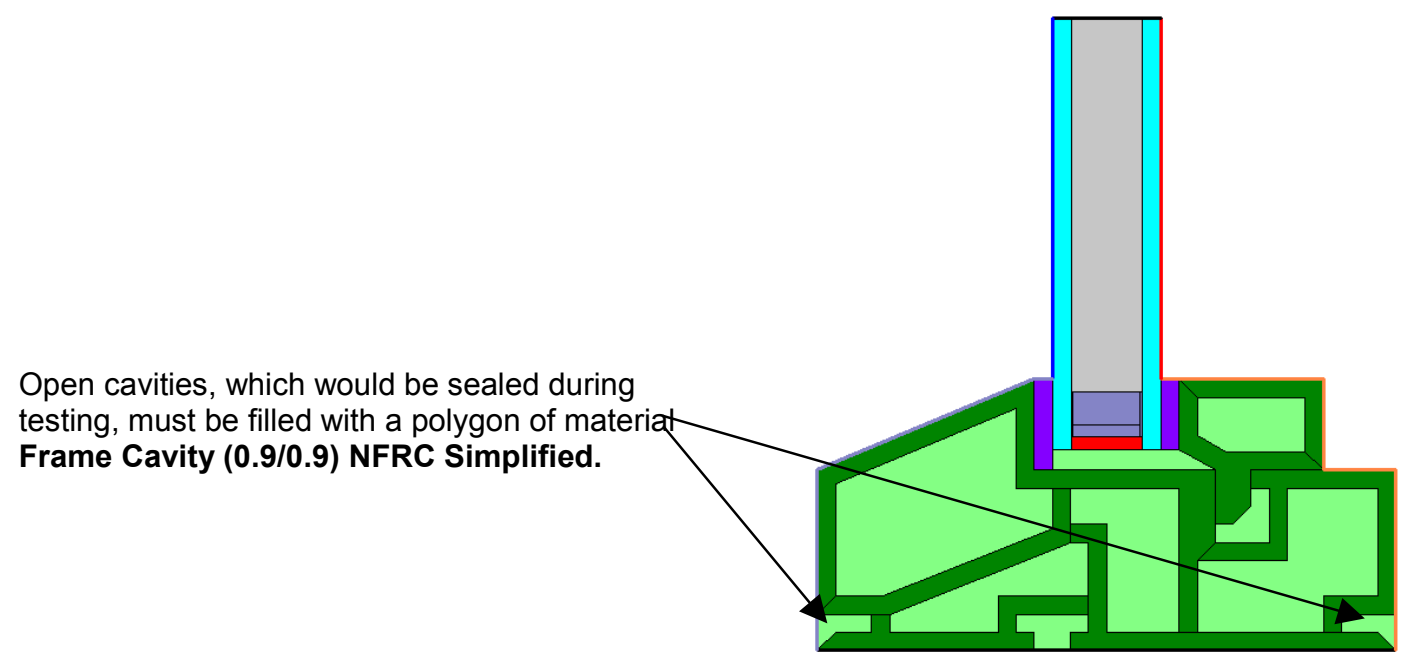

Figure 6-7. Cavities open to the interior are filled with Frame Cavity (0.9/0.9) NFRC Simplified material .

\subsubsection{Modeling Sloped and Curved Surfaces}

Because the exact geometry of sloped surfaces can be modeled in THERM, there is no need to change the boundary conditions for sloped surfaces.

For curved surfaces, THERM approximates curves with line segments. The number of line segments used in the curve will determine how close the final model is to the original geometry of the product. When importing a DXF file, the number of line segments is set using the Arc to Polygon setting in the Options menu. See Section 10, "Interpreting Arcs" in this manual for a detailed discussion of this procedure. In general, an Arc to Polygon setting of 45 degrees for small rounds and fillets in extrusions will give great enough accuracy without introducing unnecessary detail. For larger curved details, all points on the represented line must be within $5 \mathrm{~mm}$ of the actual line or curve. The averaged distance (for all points) between the represented line and the actual line or curve must be $2.5 \mathrm{~mm}$ (ISO 15099 Standard Draft \#3 April 1999). 


\subsubsection{Modeling Sloped Sills}

A product with a sloped sill must be modeled in the same way that it was tested. When a vertical sliding or fixed window is tested, the air space underneath the sloped portion of the sill is filled with an insulating material. For the simulation of the sill section this window must have the insulating material modeled underneath the sloped portion of the sill as well, as shown in the figure below. The bottom surface of the insulating material will have an adiabatic boundary condition applied. The surface of the insulating material that faces to the interior will have a boundary condition applied that is the same as the condition applied to the frame above the insulating material. The U-factor surface tag for the interior facing surface of the insulating material will be defined as Frame, which ensures the correct projected frame dimension will be used in the area weighting of the total window U-factor.

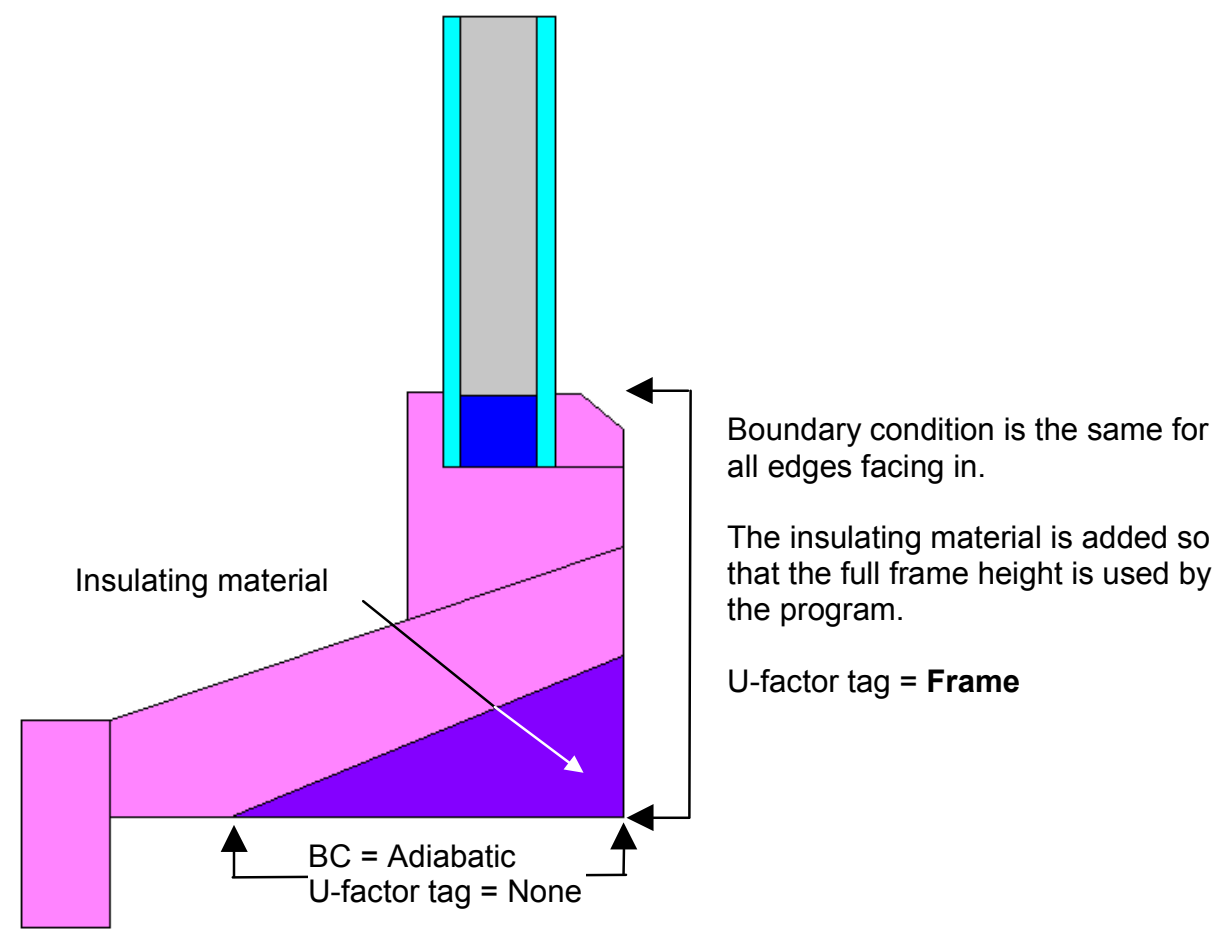

Figure 6-8. Model the sloped sill as it was tested, with insulating material under the sill. 
- 6.4 Importing Glazing Systems

When the frame cross section has been drawn, and rotated into a vertical orientation if necessary, the next step is to import a glazing system. Section 5.9, "Inserting a Glazing System" in the THERM User's Manual contains detailed information about this step. The discussion in this manual is added information about glazing systems.

\subsubsection{Overview}

The recommended method for defining a glazing system in THERM is to import it from the Glazing System Library in the WINDOW program. When THERM imports the glazing system from the WINDOW library, it obtains the emittance of the glazing, the effective conductivity of the glazing cavity, and the interior and exterior boundary conditions of the glazing system. (NOTE: THERM reads the WINDOW files named glass.dat and glzsys.w4 when importing a glazing system. Any directory can be specified for the location of these files, but make sure that both files are in the same directory.) While it is possible to obtain all this information from WINDOW by hand, it is not easy and not recommended under most circumstances.

As discussed in Section 6.5, glazing systems should always be imported into the cross section in either a vertical (up or down) orientation; it may be necessary to rotate the cross section in order to do this. Figure 6-9 shows where the Locator should be positioned based on the orientation of the glazing system when it is imported.

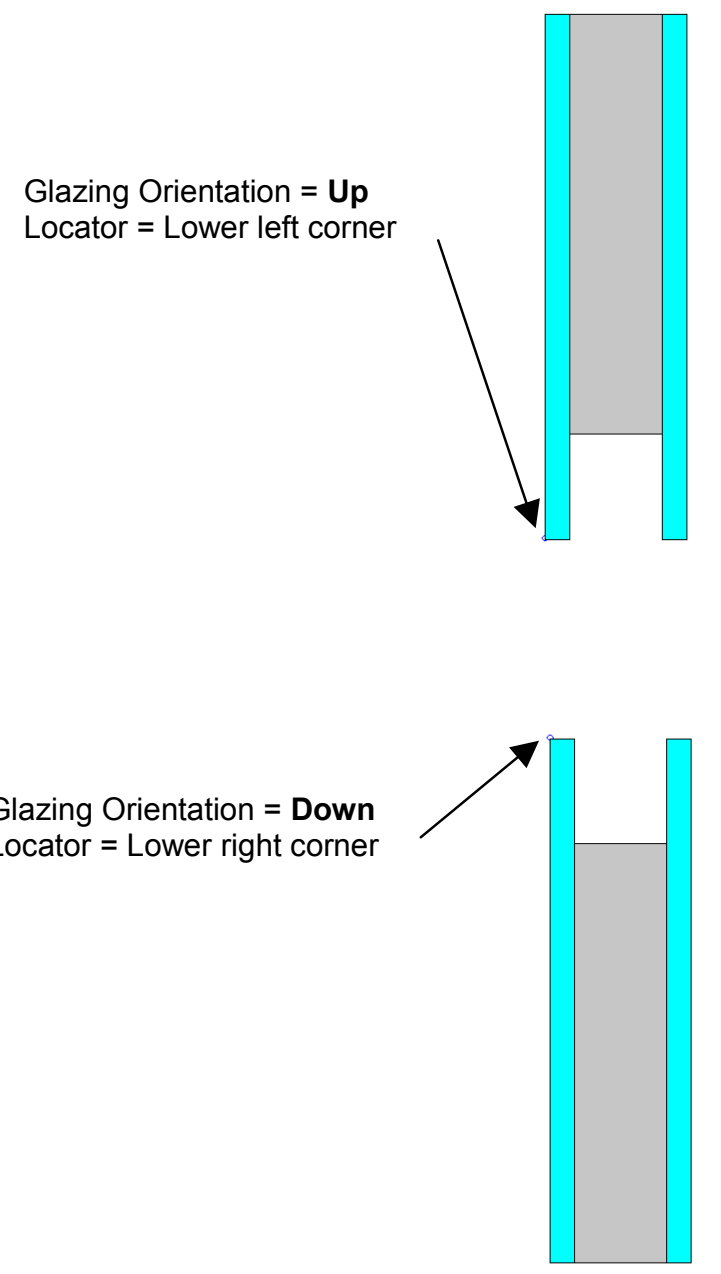

Figure 6-9. The Locator position when importing glazing systems in different orientations. 
If the lower left corner of the glazing is not a conveniently defined location, import the glazing away from the cross section, and then cut and paste it into the desired location using the Locator. (See Section 3.5, "Adding a Custom Spacer" in the THERM User's Manual for more details about cut and paste.)

If the cross section needs to be flipped after the glazing system has been inserted, THERM will keep track of the interior and exterior side of the glazing system, which will become important when boundary conditions are defined.

\subsubsection{Inserting Glazing Systems}

The THERM User's Manual contains a detailed description about how to insert glazing systems into a THERM file. The figure below is a brief overview of the steps. The two NFRC modeling requirements are:

- Exterior Boundary Condition: Set to "Exterior Surface" from the Boundary Conditions Library

- Interior Boundary Condition: Set to "Use interior U-factor BC imported with glazing system" (except for boundary conditions inside a radiation enclosure. See Section 8.5, "Projecting Products (Skylight / Greenhouse Windows in this manual for more details.)

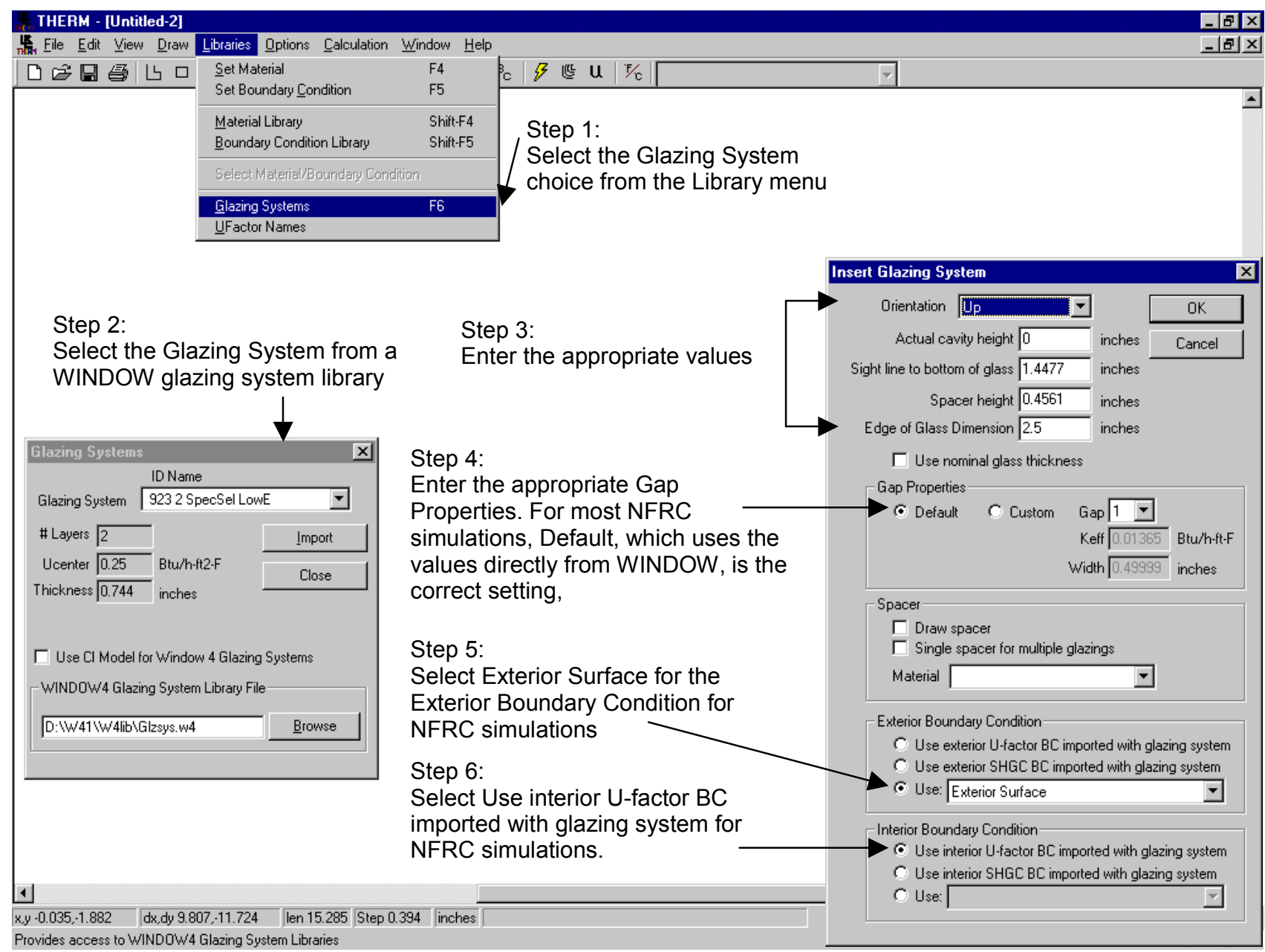

Figure 6-10. Inserting a Glazing System into a frame. 


\subsubsection{Glazing Cavities}

In general, the properties and geometry of the glazing cavity as it is defined in WINDOW is correct when the glazing system is imported into THERM, and no changes are necessary.

However, there are a few circumstances when the glazing systems will need to be edited, and these include:

- Open spacers, discussed in Section 8.7, "Spacers"

- Internal Grilles or Dividers, discussed in Section 8.3, "Modeling Internal Dividers"

The default condition in THERM is that glazing cavities cannot be edited. Therefore it is necessary to change that setting in the Options/Preferences menu. In the Drawing Options tab, there is a checkbox labeled Allow editing of IG polygons. If this box is checked, the glazing cavity can be edited. See the sections listed above for the specific circumstances when this can be done.

It is also possible to specify a different effective conductivity (Keff) value for each glazing cavity at the time it is exported from WINDOW into THERM. See Section 8.4, "Storm Windows" for a specific use of this feature.

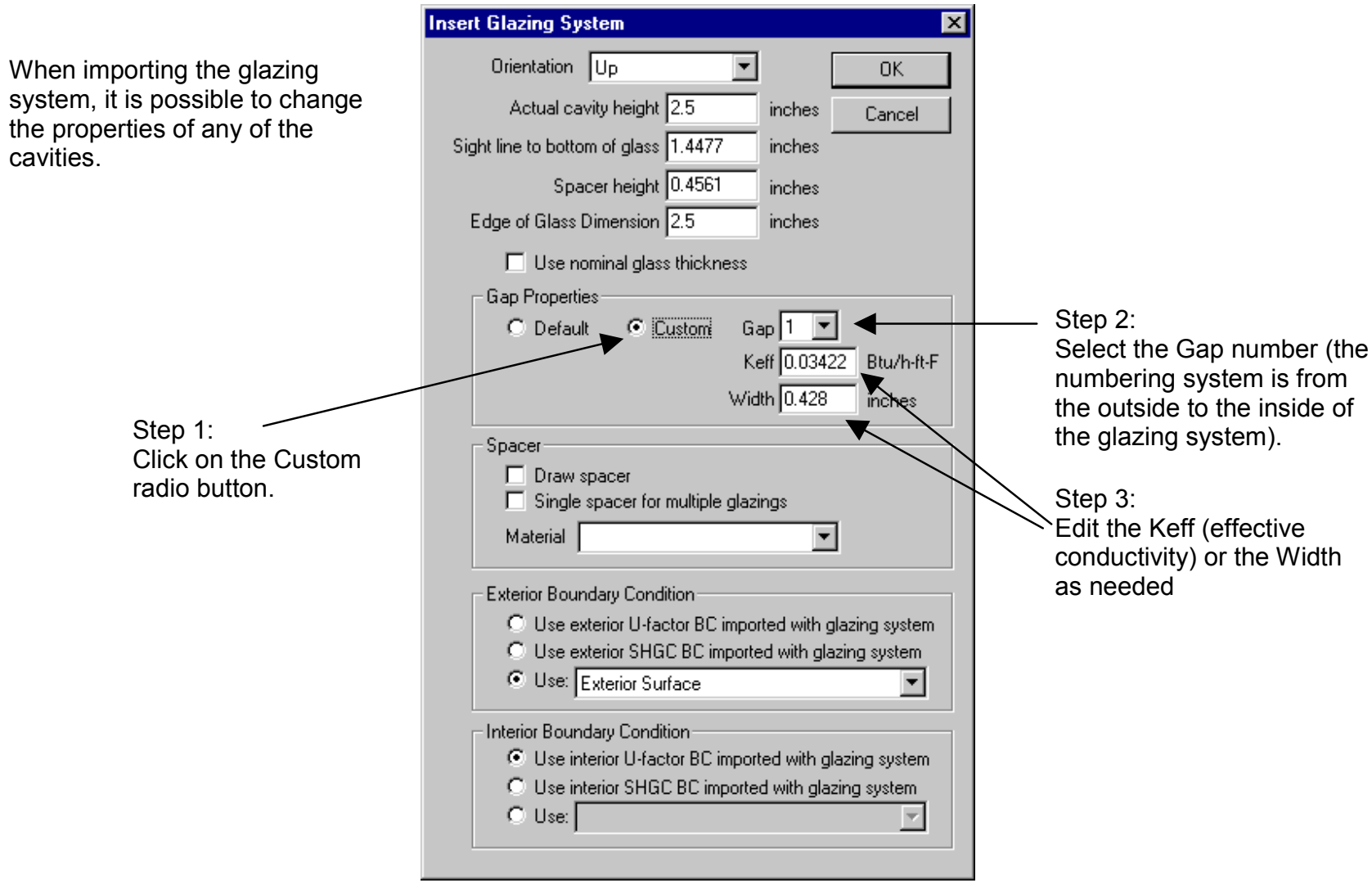

Figure 6-11. The Glazing System Gap Keff and Width can be changed upon import.

\subsubsection{Multiple Glazing Options}

THERM allows multiple glazing options to be associated with a glazing system. This feature can be useful for simulations where many different glazing systems are to be modeled in the same frame cross section.

To use this feature, follow these steps:

1. Determine the frame cross sections to be modeled: The glazing options for a frame cross section must be identical in their geometry, including overall thickness, cavity thickness, spacer height, and sight line dimension. 
Therefore, the first step is to determine the number of frame cross sections that must be defined in THERM for each set of glazing options.

2. Create and Simulate the Base Case File: For each frame/glazing option set, create a THERM file with the complete frame cross section and one of the glazing options. Create the boundary conditions for this model, and simulate it to make sure that the geometry is correct. This will be the "base case" file, and will contain no results once the multiple glazing options have been defined and simulated.

3. Define the glazing options: There are two ways to define the glazing options in a file:

- From the Calculation menu, select the Glazing Options menu choice.

OR

- Double click on the glazing system geometry, and click on the Glazing Options button in the Glazing System Info dialog box.

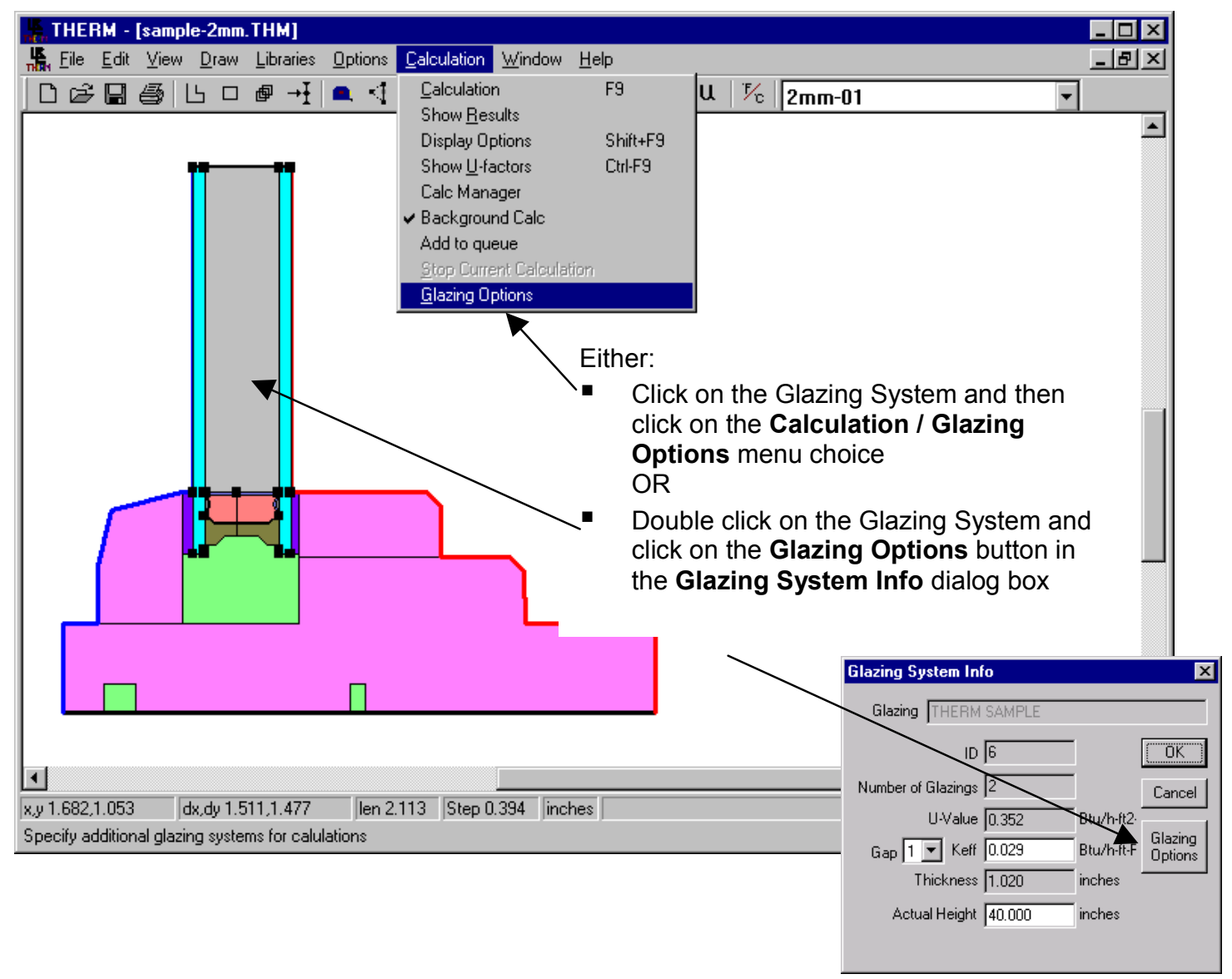

Figure 6-12. Define multiple glazing options.

- Add glazing options: From the Glazing System Options dialog box, click on the Add button to see a list of all the glazing systems in the currently selected Glazing System Library that have the same overall thickness and glass layer thickness as the base case glazing system. Click on the glazing systems in the Add Glazing Options dialog box that are to be associated with this cross section (use Shift-click or Ctrl-click to select multiple glazing systems) Click OK when all are selected.

THERM will make a separate file for each glazing option, and so in the Glazing System Options dialog box, click on the radio button choice to determine how the program will automatically name each file. The options are either that the program will append the Glazing System ID (as a 3 character number, such as 001, 002) or Name to the base case filename.

Note: WINDOW 4.1 needs 8 character file names for T2W files, so think about naming the THERM files with four 
characters. When indicating the file naming convention for the multiple glazing runs, choosing the Glazing System ID is preferable over the Glazing System Name, in order to reduce THERM filename size.

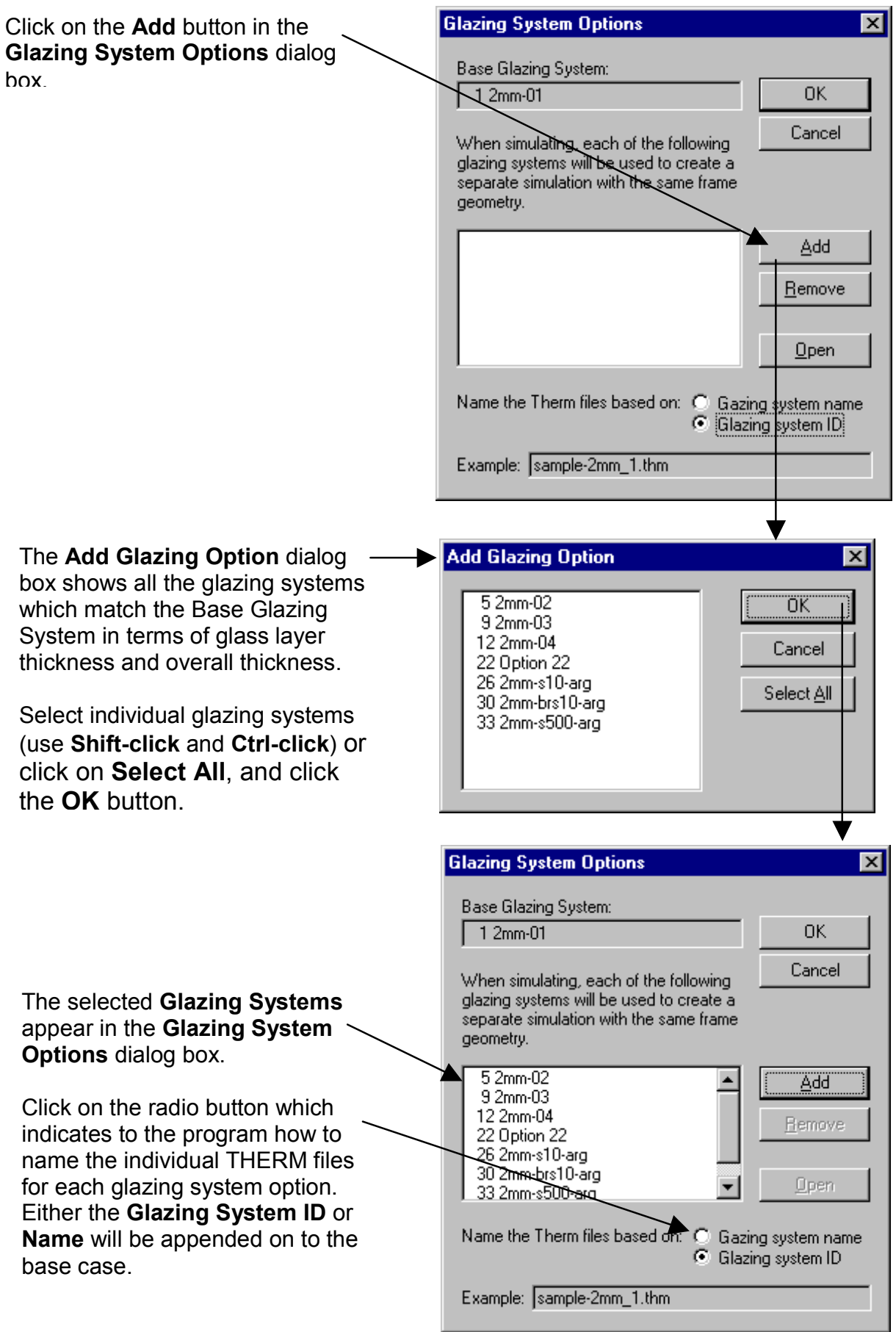

Figure 6-13. Select the glazing systems to be associated with the base case file.

1. Calculate the Results: the file can now be simulated, or more files can be created and all the files simulated through the Calc Manager. This is explained in more detail in Section 6.6, "Calculating the Results". 


\subsection{Defining Boundary Conditions}

Chapter 6 of the THERM User's Manual contains a detailed explanation of how to define boundary conditions in a model. The information found in this manual is supplemental to that discussion.

\subsubsection{Overview}

Boundary conditions must be defined for all the surfaces on the perimeter of the model. These boundary conditions define the temperatures and film coefficients for each element of the perimeter. Different boundary conditions are defined for the surfaces on the interior and the exterior of the cross section, and for different materials such as vinyl, wood, or metal frames. Surfaces which are assumed to have no heat transfer are assigned Adiabatic boundary conditions.

There are three main categories of boundary conditions:

- Interior: An interior boundary condition is used for all interior surfaces, and assumes that the surface is exposed to natural convection, and the heat transfer coefficient used depends on the temperature of the surface, which is a function of the material. Therefore, NFRC has defined different boundary conditions for general classes of frame materials, as listed in Table 6-2: Aluminum Frame, Thermally Broken Frame, and Interior Wood/Vinyl Frame. WINDOW calculates the center-of-glass surface temperatures, and THERM uses these values to automatically calculate the edge-of-glass boundary conditions for glazing systems imported from WINDOW.

- Exterior: The exterior boundary condition is used for all exterior surfaces, and assumes that the surface is exposed to a $15 \mathrm{mph}(6.70 \mathrm{~m} / \mathrm{sec})$ wind, which corresponds to a film coefficient of $5.11 \mathrm{Btu} / \mathrm{h}-\mathrm{ft}^{2} \mathrm{o}^{\circ} \mathrm{F}(29.03$ $\left.\mathrm{W} / \mathrm{m}^{2}{ }^{\circ} \mathrm{C}\right)$.

- Adiabatic: The adiabatic boundary condition is used for any surface assumed to have no heat flow. This is used for the top of the glazing system at the boundary between the edge-of-glass and the center-ofglass, because the assumption is that the heat transfer between the two sections are independent of each other. Adiabatic is also used for the bottom of the frame that would sit in the mask wall of the thermal chamber during testing.

THERM has a Boundary Condition Library (accessed from the Library/Boundary Conditions menu, or by double clicking on a boundary condition) which has the standard boundary conditions defined by NFRC, shown in Table 6-2, as well as Adiabatic. By default, the exterior boundary conditions are blue, interior boundary conditions are red, and the adiabatic boundary condition is black.

Table 6-2. Boundary condition definitions

\begin{tabular}{|l|r|r|r|r|}
\hline \multirow{2}{*}{ Boundary Condition } & \multicolumn{2}{|c|}{ Film Coefficient } & \multicolumn{2}{c|}{ Temperature } \\
\cline { 2 - 5 } & $\mathbf{W} / \mathbf{m}^{\mathbf{2}} \mathbf{}^{\mathbf{0}} \mathbf{C}$ & $\mathbf{B t u} / \mathbf{h}-\mathbf{f t}^{\mathbf{2}}-{ }^{\mathbf{}} \mathbf{F}$ & \multicolumn{1}{c|}{${ }^{\mathbf{}} \mathbf{C}$} & ${ }^{\mathbf{0}} \mathbf{F}$ \\
\hline Exterior Surface & 29.03 & 5.11 & -17.78 & 0 \\
\hline Interior Aluminum Frame & 8.30 & 1.46 & 21.11 & 70 \\
\hline Interior Thermally Broken Frame & 7.90 & 1.39 & 21.11 & 70 \\
\hline Interior Wood/Vinyl Frame & 7.61 & 1.34 & 21.11 & 70 \\
\hline
\end{tabular}

\subsubsection{Assigning Boundary Conditions and U-factor Surface Tags}

Boundary conditions for a cross section are automatically created in THERM by pressing the Boundary

Condition toolbar button, clicking on the Draw/Boundary Conditions menu option, or pressing the F10 key. 
THERM will automatically assign boundary conditions, both interior and exterior, to a glazing system imported from WINDOW, as discussed above. However, the frame boundary conditions must be assigned in most cases -- Exterior for the entire exterior surface of the frame, Adiabatic for bottom of the frame, and one of the Interior choices from Table 6.2 depending on the frame material and construction for the interior frame surfaces. The technique for doing this is described in detail in Chapter 6, "Defining Boundary Conditions" of the THERM User's Manual. The boundary condition for the interior surface of the frame must be the same for all segments.

In THERM, in addition to assigning boundary conditions to a boundary segment, the U-factor Surface Tags must also be assigned for each boundary condition. The U-factor Surface Tags, which are selected from the U-factor Names library, are used by THERM to calculate the total U-factor. For a THERM U-factor calculation that will be used in WINDOW, it is necessary to use the following U-factor Surface Tags, with the same capitalization (WINDOW will not recognize any other values):

- Frame: Use this tag for all interior boundary conditions that are part of the frame U-factor calculation, including the boundaries of the glazing system below the sight line. See NFRC 100, "Figure 2,

Fenestration Product Schematic -- Vertical Section". THERM will automatically assign the U-factor tag of Frame to the portion of the glazing system that is below the sight line, based on the Site Line to Bottom of Glass value entered when the glazing system is inserted from WINDOW.

- Edge: Use this tag for all interior boundary conditions that are to be used in the edge-of-glass calculation. This includes the boundaries of the glazing system above the sight line up to the center-of-glass boundary (2.5 inches, or $6.35 \mathrm{~mm}$, up from the sight line). THERM will automatically assign the U-factor tag of Edge to the portion of the glazing system from the sight line up 2.5 inches $(6.35 \mathrm{~mm})$ to the center-of-glass boundary.

- None: Use this tag for all exterior boundary conditions. This is the default U-factor tag automatically assigned by THERM for all surfaces except the glazing system.

Always assign the U-factor tags of Frame and Edge to the interior surface of the model, which usually are the red boundary conditions.

To change the boundary condition for one boundary segment, double click on the segment, or single click and press Enter, and the Boundary Condition Type dialog box will appear. This dialog box allows specification of both the boundary condition and the U-factor tag at the same time. To change the boundary condition for multiple boundary segments, click on the first segment and double click (or single and press Enter) on the last segment in a counterclockwise direction. The choices made in the Boundary Condition Type dialog box will then be applied to all the selected boundary segments. It is also possible to change the boundary condition definition for boundary segments using the Select Material/BC toolbar button. Click on the eye dropper tool, click on a boundary segment whose definition is to be duplicated to other segments, then click on the boundary segment(s) which need that boundary condition definition. The program will assign both the boundary condition and U-factor tag from the copied segment. There isalso one level of "undo" for boundary conditions, accessed through the Edit/Undo file menu choice

When boundary conditions are generated for a model, the program keeps them in the model until they are deleted by the user. Therefore it is important to check to make sure that the boundary conditions in the final model are correctly defined. All the boundary conditions can be deleted by deleting one boundary segment.

Boundary conditions and U-factor tags are stored in libraries, and new entries can be defined for both using the Libraries menu choice. See Section 6.2, "Assigning Boundary Condition Definitions" in the THERM User's Manual for more detailed information.

Occasionally THERM loses track of the glazing system boundary conditions. If this happens they can be reassigned from the Boundary Condition Library. 


\subsubsection{Voids, Overlaps and Bad Points}

In order to perform a simulation, the geometry of the cross section must be correctly defined, i.e., there must not be any voids, overlapping polygons, or points that cannot be resolved by THERM. See Section 6.3, "Error Detection in THERM" in the THERM User's Manual.

While drawing, there are a number of features in THERM to help avoid creating voids, overlapping polygons, and bad points. The Always Check for Overlapping Polygons feature (in

Options/Preferences/Drawing Options) is the main tool used to avoid drawing overlaps and voids. This option can be turned off, but this is NOT recommended for most situations. The View Voids and Overlaps (from the Draw menu) feature is also a good tool for visual inspection to find voids and overlapping polygons in a cross section.

In addition to the features that attempt to prevent the creation of bad geometry during the drawing process, a number of error checks are performed when THERM calculates the boundary conditions in order to make sure the model geometry is correct.

For example, when the boundary conditions are calculated THERM identifies all points within 0.004 inches $(0.1 \mathrm{~mm})$ and 0.0004 inches $(0.01 \mathrm{~mm}$ ) of each other (points closer together than $0.01 \mathrm{~mm}$ are automatically merged). A message box appears saying that there are points closer than the program tolerance, as shown in Figure 6-10. Choose the Mark the points but don't adjust them option, which causes THERM to draw red circles in the problem areas. Look at all the circled points and fix them if appropriate. If the marked points are actual details in the drawing that need to be kept, they do not have to be fixed, although they might cause meshing problems during the simulation because of too much detail. If they are mistakes, fix them. Once all the circled areas in the drawing have been examined, press the Boundary Conditions toolbar again, and if there are still "bad points", select the Automatically adjust points within tolerance option, in which case THERM will fix all of the points automatically. (Having THERM adjust the points is an "all or nothing" proposition -- which is why all the points should be examined first before having the program adjust them. Also keep in mind that the automatic fixing of points cannot be reversed by THERM.) It is especially important when modeling heat mirror products not to let the program automatically adjust the points.

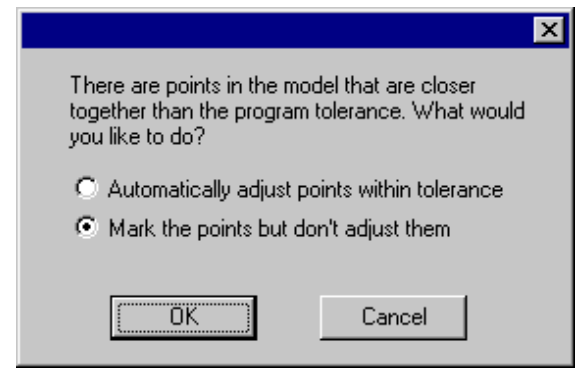

Figure 6-14. Message when trying to define boundary conditions indicating that there is a problem in the geometry.

The best way to fix points that are too close together is to delete or move the offending point. Many times, the point in question is in all the adjacent polygons, so it is best to delete the point in all the polygons at the same time by selecting all the polygons using the Shift key, then delete the point; it will be deleted from all the selected polygons.

THERM also marks Voids and Overlaps by checking for "lonely edges" -- two adjacent edges with a different number of points on each edge. THERM marks voids and overlapping polygons with red circles also. One way to eliminate these two conditions is by "jiggling" the points -- moving the points away from the adjacent polygon, then moving them back within the sticky distance and letting the program "snap" them to the polygon. Another way is to delete the offending polygon, and use the Fill Void toolbar button to create it again, which means that THERM will automatically align all points and ensure that no voids are created. 
THERM can also find invalid polygons caused by points collapsing on each other, creating a zero-area extension. In this case both the point at the tip of the extension and the extra point at its base must be deleted.

Turning off the Material Color and the Boundary Conditions from the View menu can sometimes help to find the problems.

THERM will simulate a problem with bad points, because in some cases details are drawn that give bad points but which need to be modeled.

If changes are made to polygons on the boundary of the cross section, the boundary conditions will be automatically deleted by the program and must be generated again. If all the corrections are on internal polygons, the boundary conditions will not be automatically deleted. However, in this case, it is still a good idea to press the Boundary Conditions toolbar button again, to ensure that all points on all surfaces have boundary segments defined between them.

There are a series of files on the CD (bad1.thm, bad2.thm and bad4.thm) that have geometry problems such as voids and points that are too close together. The following discussion illustrates what is wrong with each file, with hints about how to fix them. It is good practice to try to fix the problems in these files. 


\subsubsection{Examples of Bad Points}

The CD included with this manual contains several samples files which contain bad points. These files can be used to learn how to fix bad points when they are encountered.

\section{Bad1.thm}

Problem 1: points are closer together than 0.004 inches $(0.1 \mathrm{~mm})$

Solution: move the cavity point up to the top of the glazing, so the 2 points become the same point

Problem 2: Void in the drawing.

Solution: Move one of the points (on all the adjacent polygons) so there is no gap and the 2 points become 1 point.

Problem 3: points are closer together than 0.004 inches $(0.1 \mathrm{~mm})$

Solution: Delete or move one of the points (on all the adjacent polygons) so that there is only 1 point.

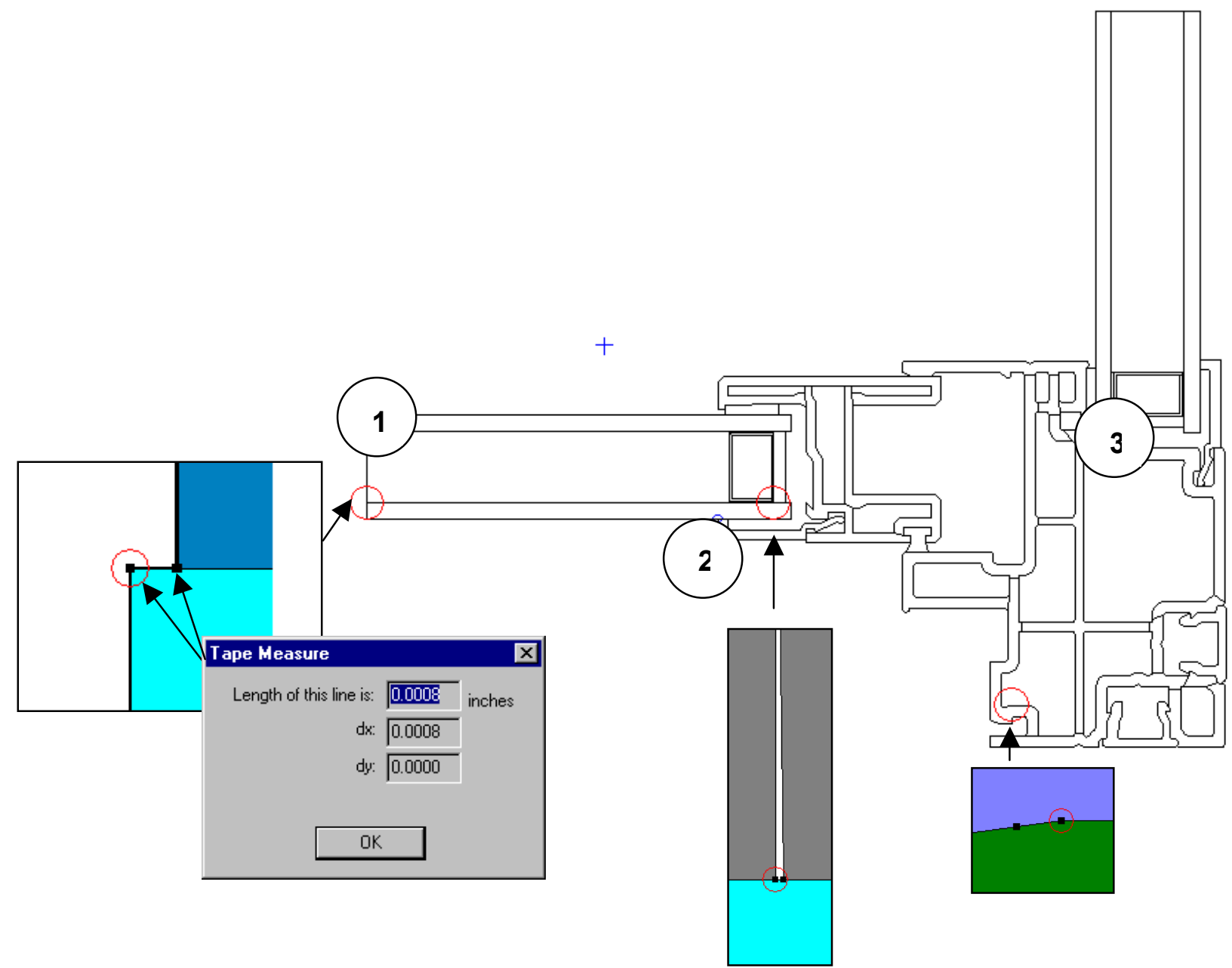

Figure 6-15. Bad1.thm has several problems including a void in the geometry and two sets of points that are too close together. 


\section{Bad2.thm}

Problem 1: Void in the drawing.

Solution: Move one of the points (on all the adjacent polygons) so there is no gap and the 2 points become 1 point.

Problem 1: points are closer together than 0.004 inches $(0.1 \mathrm{~mm})$

Solution: move the cavity point up to the top of the glazing, so the 2 points become the same point

Problem 3; points are closer together than 0.004 inches $(0.1 \mathrm{~mm})$

Solution: Delete or move one of the points (on all the adjacent polygons) so that there is only 1 point.

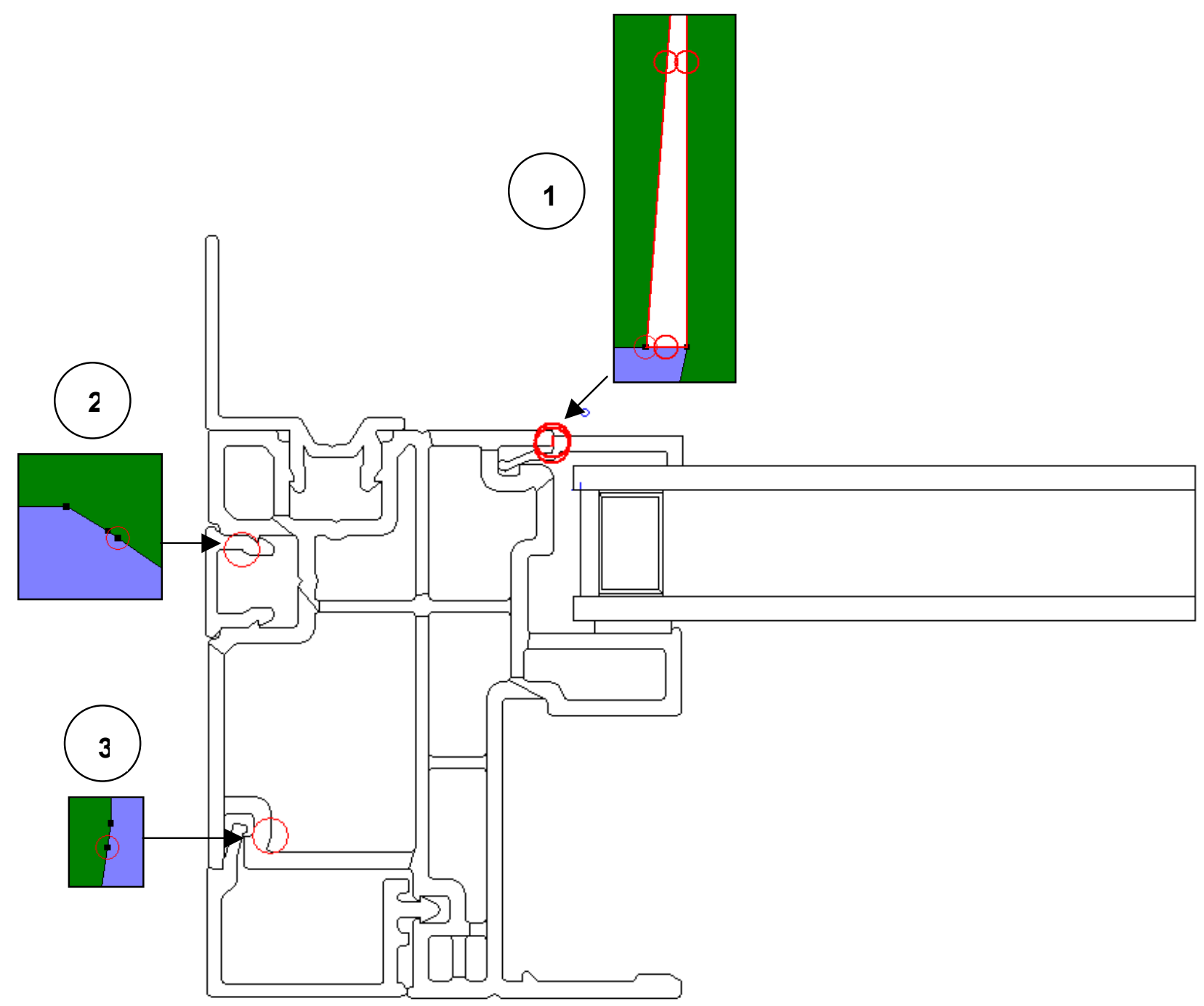

Figure 6-16. Bad2.thm has several problems including a void in the geometry and two sets of points that are too close together. 


\section{Bad4.thm}

Some of the problems in this file are in the glazing system (cavity and glass) so in order to fix those, either turn on the Allow Editing of IG unit and fix the problems by hand, or use the Automatically Adjust.

Problem 1: Void in the drawing.

Problem 2: Points too close together.

Problem 3: Void in the drawing.

Problem 4: Void in the drawing.

Problem 5: not obvious. Try jiggling the points or delete the polygon and user the fill void to recreate it. Or let the program automatically adjust the points.

Problem 6: not obvious. Try jiggling the points or delete the polygon and user the fill void to recreate it. Or let the program automatically adjust the points.

\section{Problem 7:}

Problem 8: Points too close together.

Problem 9: Points too close together. Solution: Delete some of the polygons, move points, and use the Fill Void tool to recreate polygons.

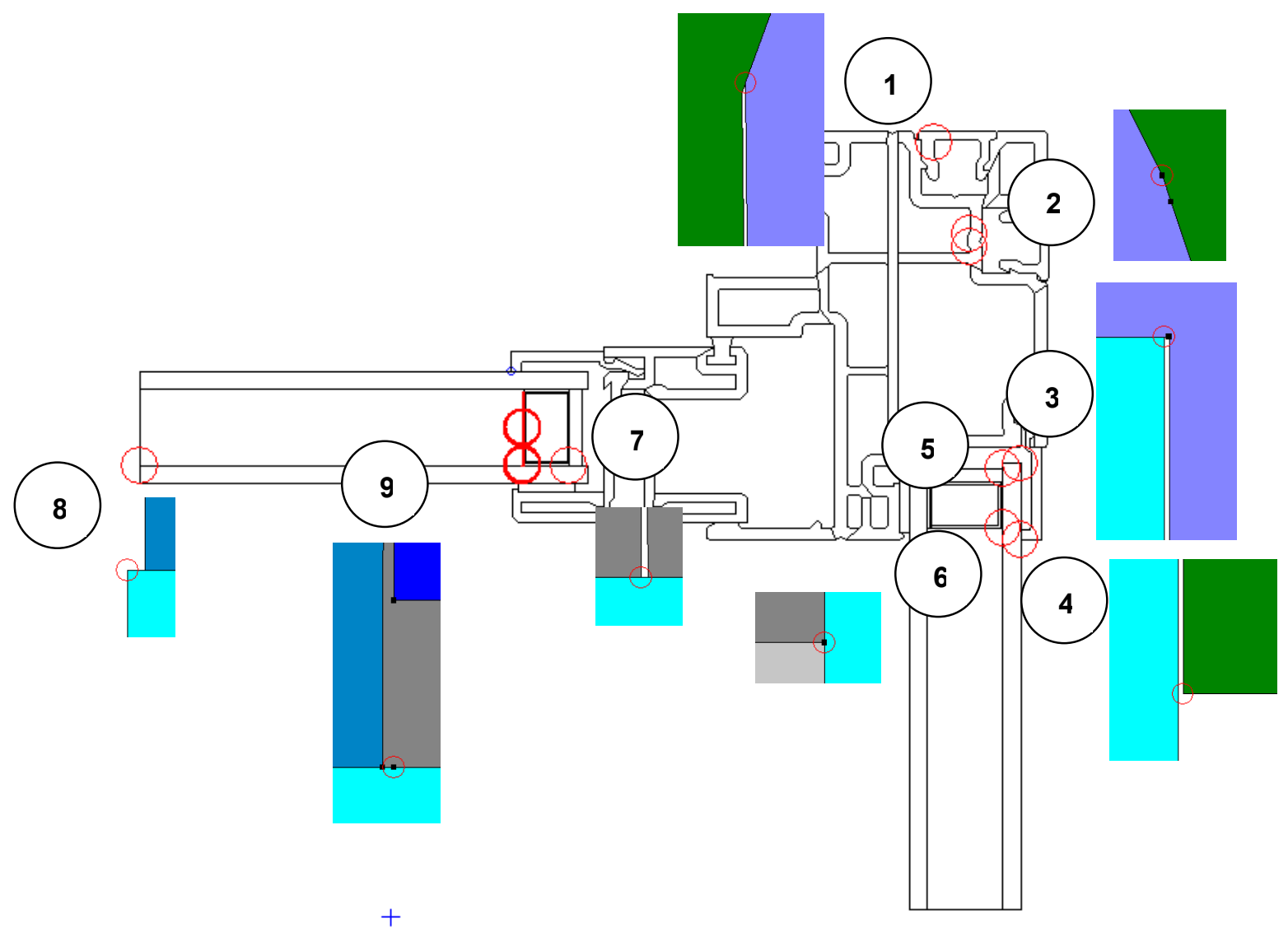


Figure 6-17. Bad3.thm.

\subsection{Calculating Results}

When the boundary conditions have been correctly defined for the cross section, the model can be simulated. Begin the calculation by either clicking on the Lightning Bolt toolbar button, selecting the Calculation/Calculation menu option, or pressing F9.

See Chapter 7 in the THERM User's Manual for detailed information about the calculation procedures, and the results produced by THERM.

\subsubsection{Meshing}

The first step performed by THERM in the simulation is to generate a mesh from the geometry on which the thermal analysis is based. This mesh is generated automatically, and a summary of how it works is found in "Appendix C" in the THERM User's Manual.

The one variable in the mesh generation that the user can control is the Quadtree Mesh Parameter, which determines how fine the mesh is. The larger the mesh parameter value, the smaller the largest element in the mesh is. The NFRC required default value for the mesh parameter is 6, as shown in Table 6-3.

Occasionally the mesh generator cannot create a closed mesh with the geometry that has been drawn, usually created because of extremely fine detail in the cross section. THERM circles the point(s) where the mesher failed. If this happens, first:

- Rerun the problem with a higher mesh parameter value. This causes the program to generate a finer mesh, and results in longer run times. The default mesh parameter value is 6 . Mesh parameter values greater than 8 are rarely needed for single cross sections, but may be needed for full height product simulations required for skylights and greenhouse windows.

If this doesn't work:

- Simplify any unnecessarily complicated details. Keep in mind that the circled point often lies on the boundary between two polygons; the detail that is causing the problem could be anywhere in those two polygons and is often not at the place that is circled. One method to try is to break the problem polygon into multiple polygons, while keeping the geometry otherwise unchanged.

\subsubsection{Error Estimator}

THERM has a built-in error estimator, and automatically refines the mesh in the areas where it is needed. The details of the error estimation algorithm are found in "Appendix C" of the THERM User's Manual. The error estimator returns the Percent Error Energy Norm, which is related to the gradient of heat flux (energy). If the returned value is greater than the target value, THERM refines the mesh in areas with a high rate of change in the heat flux.

The default value for the Percent Error Energy Norm in THERM to 10\%, as shown in Figure 6-16. This setting forces THERM to do the error estimator calculation and return a value for the error estimate (recorded with the U-factor results) and the program may or may not refine the mesh, depending on the result of the Error Energy Norm. If an error message is displayed saying that the error energy norm cannot be reached, increase the Maximum Iterations value. 


\subsubsection{Required Settings for NFRC Simulations}

In the THERM File Options tab found in the Options/Preferences menu, the following values must be set:

Table 6-3. Options/Preferences settings for THERM NFRC modeling

\begin{tabular}{|l|l|}
\hline Setting & Value for NFRC Modeling \\
\hline Quad Tree Mesh Parameter & 6 or greater \\
\hline Maximum \% Error Energy Norm & $10 \%$ \\
\hline Run Error Estimator & must be checked \\
\hline
\end{tabular}

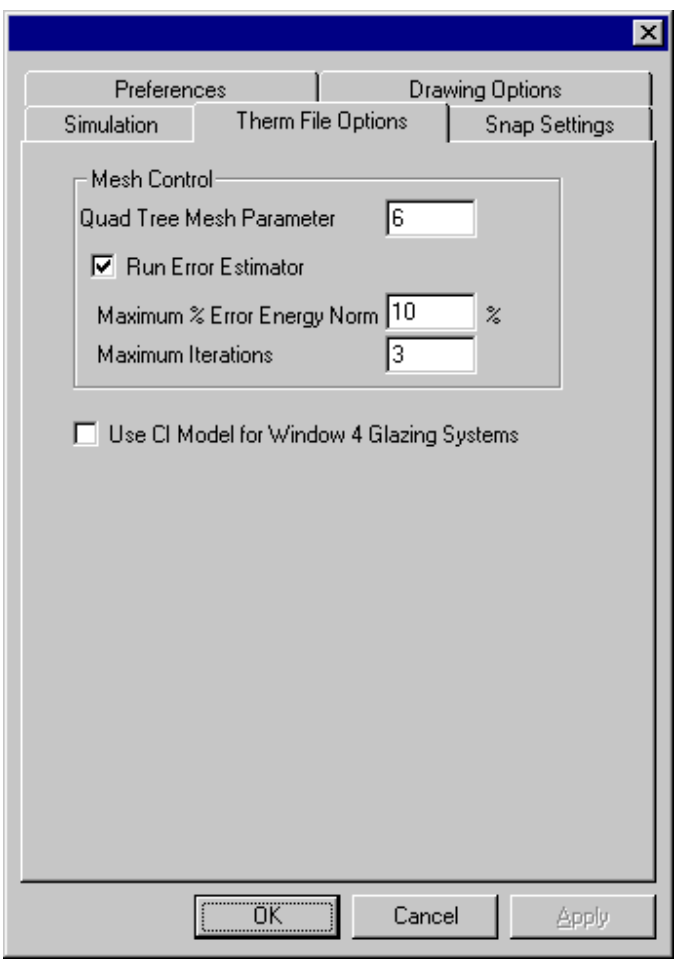

Figure 6-18. NFRC required THERM File Options settings. 


\subsubsection{Calculations}

When a calculation is started, THERM can do the calculations in the background while another model is being worked on. There is also a Calculation Manager, accessed from the Calculation/Calc Manager menu option that allows the submittal of many files at one time to be calculated in the order they are added. The Calc Manager Log shows whether the jobs ran or not. Section 7.2, "Calculations," in the THERM User's Manual contains detailed information about the Calc Manager.

\section{Calculating Multiple Glazing Options}

If a base case file has been created with multiple glazing options (see Section 6.4.3, "Multiple Glazing Options"), there will be extra options when the file is simulated using the Calc toolbar, the Calculation/Calculation menu option, or the $\mathbf{F 9}$ shortcut key. Using these methods, a Glazing Option Simulation dialog box will appear, allowing the following three options, as shown in Figure 6-17:

- Create the glazing option files and perform all simulations: This choice is the default, and it creates and runs all the THERM files for the multiple glazing options defined in the base case file.

- Simulate the current file (base case) only: This choice is useful when setting up the base case file to make sure there are no geometry errors before running the entire multiple glazing options.

- Create the glazing option files but do not perform the simulations: This choice will cause the program to make individual THERM files for each of the glazing options associated with the base case file, with either the Glazing System ID or Name appended on to the base case filename, but it will not simulate the files. These files can then be opened and simulated individually, or simulated through the Calc Manager.

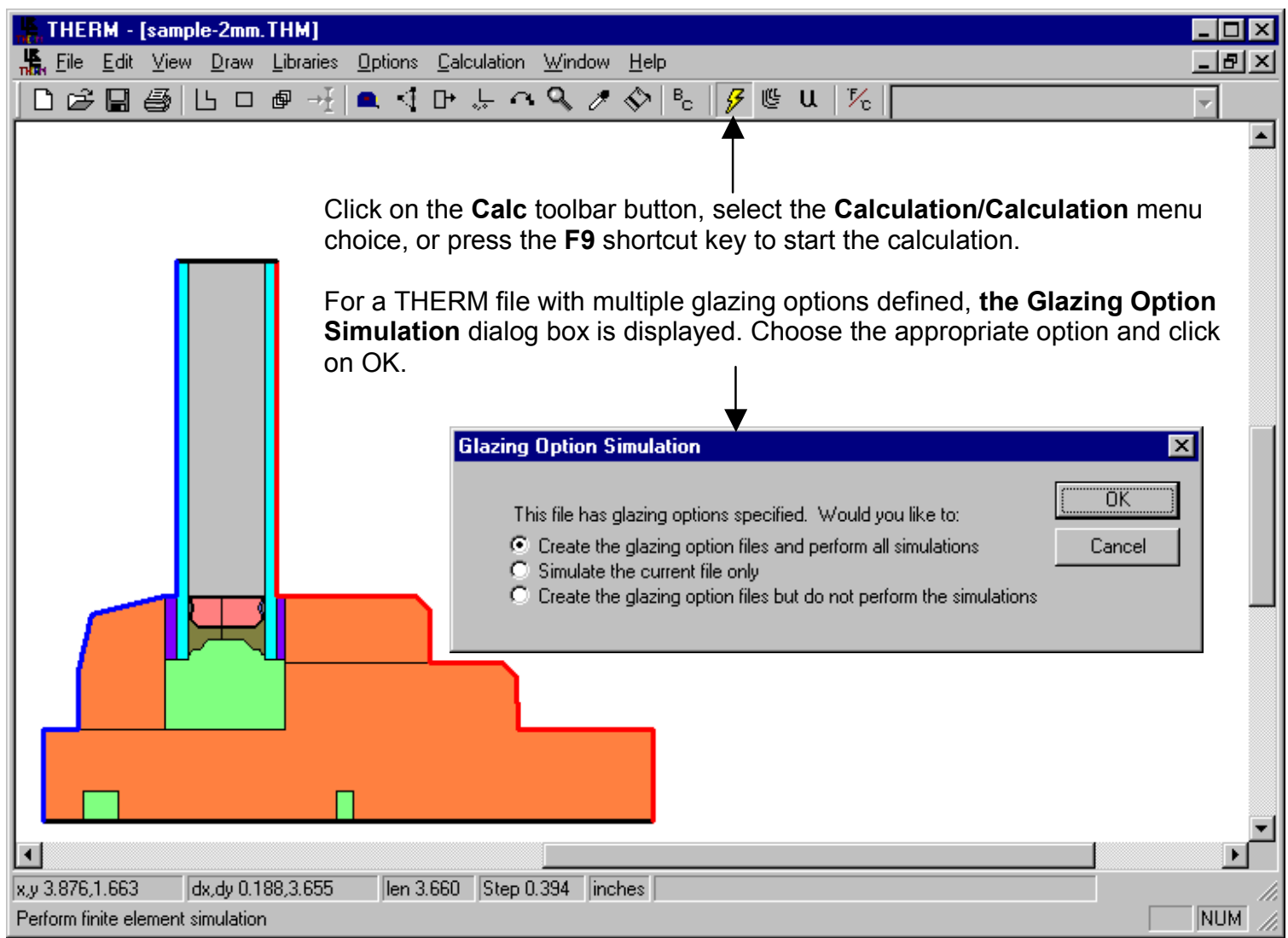

Figure 6-19. When a file with multiple glazing options is calculated, THERM gives several calculation options. 
Multiple base case files with multiple glazing options in each file can be added to the Calc Manager, as shown in Figure 6-18. The Calc Manager does not ask the three questions shown in Figure 6-17; it implements the first choice, which is that to make all the files and then simulate them. The Calculation Log can be viewed to see the status of the runs. The information in the Calculation log can be sorted by clicking on a column.

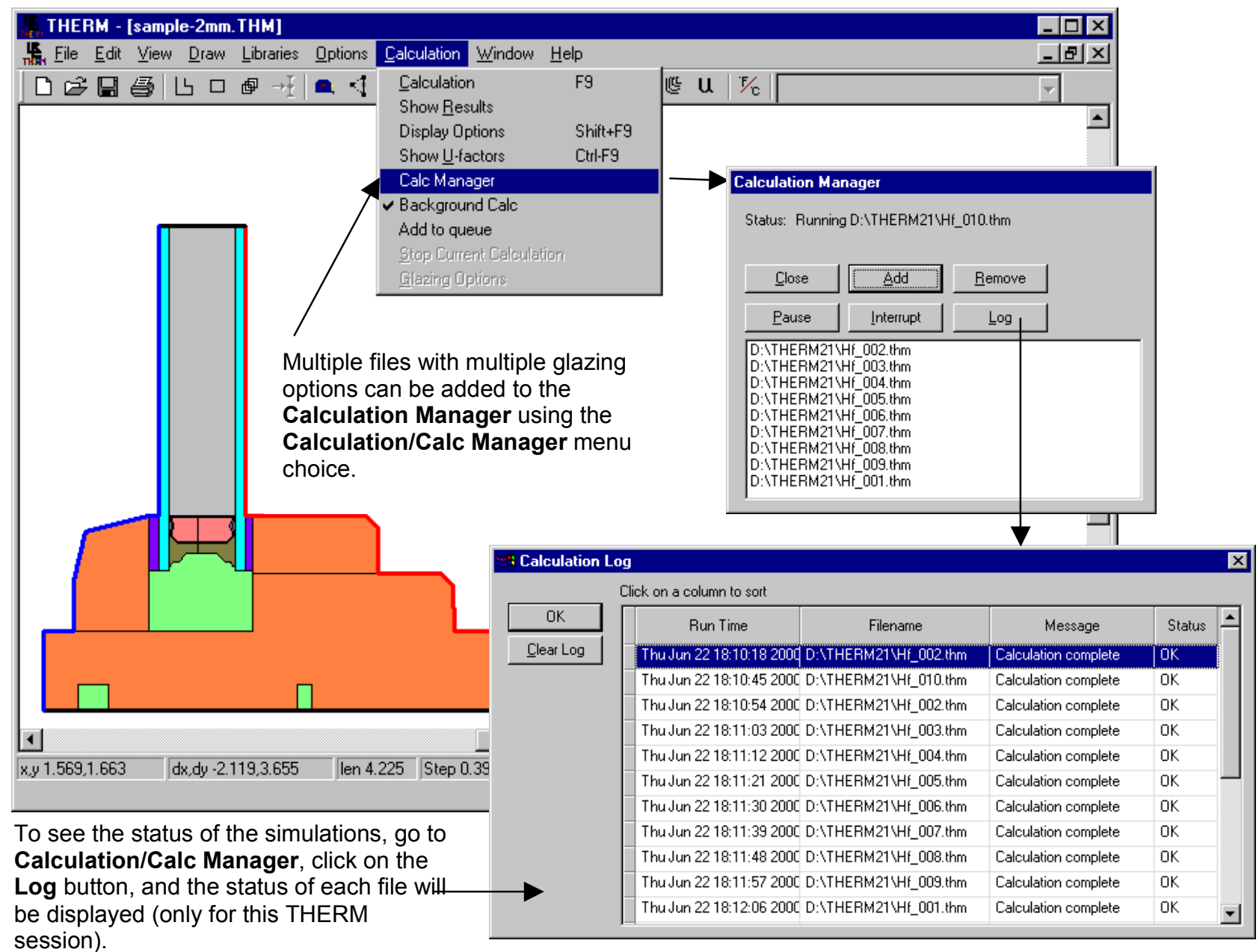

Figure 6-20. The Calc Manager can be used to simulate multiple files with multiple glazing options in each file. 


\subsubsection{Results}

When the calculation is finished, the U-factors (shown in Figure 6-19) can be viewed using the Calculation/Show U-factor menu choice or the Show U-factor toolbar button. $u$

In addition, the graphic display of the results can be controlled using either the Calculation/Show Results

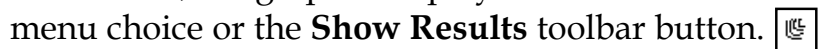

The U-factors can be viewed for Projected X, Projected Y, Total Surface Length, or a Custom Frame Length. U-factors are generally calculated in the Projected $\mathbf{Y}$ direction, but there are a few special cases where the Custom Frame Length feature must be used for an NFRC simulation. (See the following discussion in Section 6.6.6, "Custom Frame Length" for an example using Custom Frame Length.)

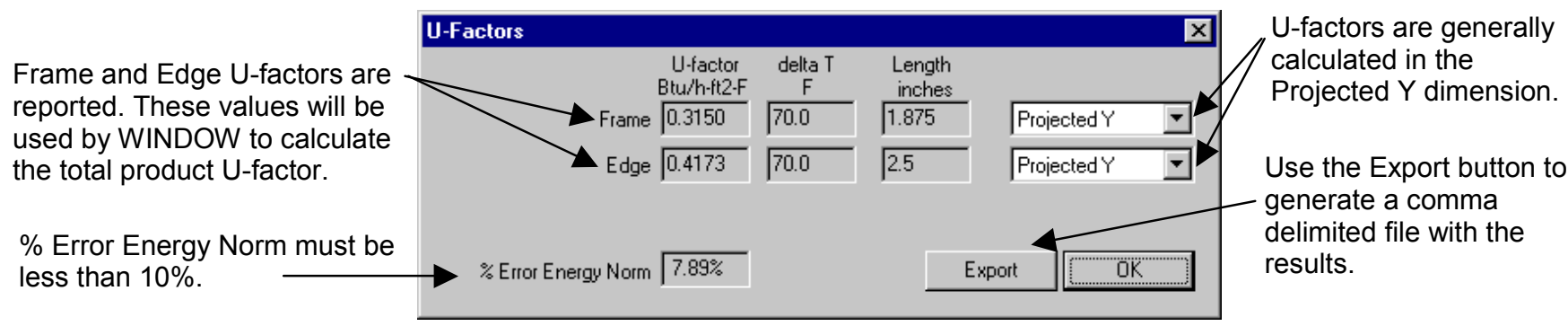

Figure 6-21. U-factor results from the Calculation/Show U-factors menu choice.

Local temperatures can be displayed at the cursor (from the View/Temperature at Cursor menu choice) and average temperatures can be displayed with the tape measure (from the Options/Preferences menu, Drawing Options tab, check the Tape Measure Average Temperature checkbox).

THERM has a report which summarizes all the details of a THERM file, including the polygon ID numbers for all cavities. This report can be viewed and printed from the File/Report menu choice.

THERM files (*.THM) can be saved without the detailed temperature data by selecting the Options/Preferences menu, going to the Simulation tab, and unchecking Save Simulation results in THM files. The THERM file will then contain only the geometry and boundary conditions used to run the simulation, the U-factor results, and the report, thus making the file much smaller. 


\subsubsection{Custom Frame Length}

There is a case where THERM will not calculate the project frame length correctly. This happens when a section of an adiabatic boundary "overlaps" a boundary with a U-factor tag in the projected dimension over which the length is being calculated. The figure below illustrates this. This incorrect frame length will cause the U-factor over the projected length to be calculated incorrectly. The U-factors results dialog box has a feature which allows a custom frame length to be entered, in order for THERM to calculate the correct Ufactor. This situation happens in some skylight files, although it potentially could happen in any model. The figure below describes the steps required to use the Custom Frame Length feature.

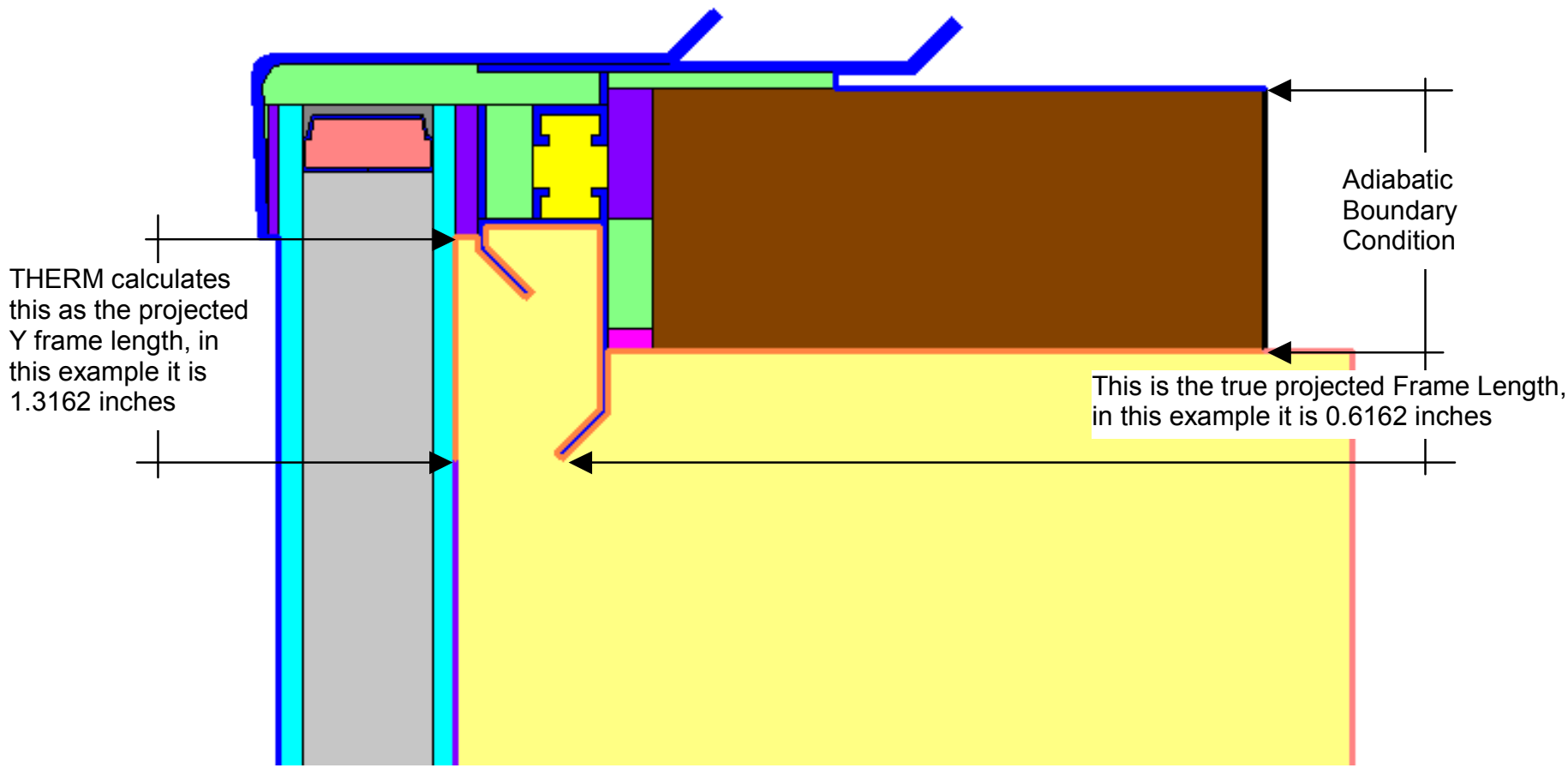

Figure 6-22 Example of a case where THERM does not calculate the projected $Y$ frame length correctly because of an adiabatic boundary condition that "overlaps" an interior boundary condition.

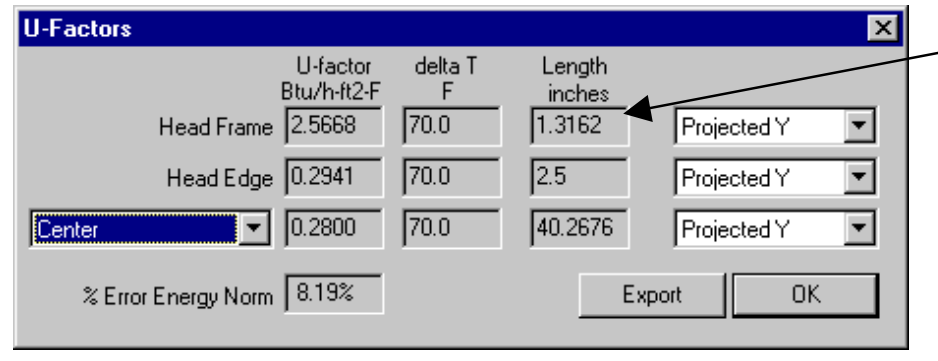

Step 3:

THERM recalculates the $U$-factor based on the custom frame length.

\section{U-Factors}

the
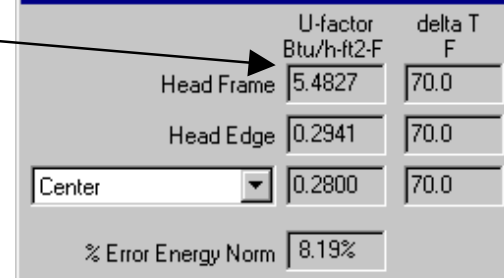
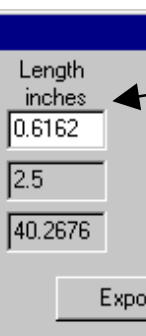

Step 2:

The Length field will become editable. Enter the correct length

Step 1:

Select Custom from the pulldown

Figure 6-23 The Custom Frame Length feature allows the correct frame length to be entered. 


\subsubsection{Exporting Results to WINDOW Files}

Because the U-factors from individual THERM cross sections will be used for the whole product calculations in WINDOW, it is necessary to export the U-factor results into a file format that WINDOW can read. This is explained in detail in Section 7.4.12, "Export to WINDOW" in the THERM User's Manual. There are two ways to do the export:

- Export each cross section by hand using the File/Export menu choice

- Set THERM to export the results automatically each time the file is saved, by selecting the

Options/Preferences menu choice, selecting the Preferences tab, and checking the Automatic WINDOW 4 Export on Save option, as shown in Figure 6-16. In this mode, the directory where the file is to be saved cannot be defined, but THERM automatically saves the T2W files in the same directory as the THM files. So the best strategy is to create all the THM files in the correct WINDOW FRAMEDAT directory, and then the T2W files will also get saved there.

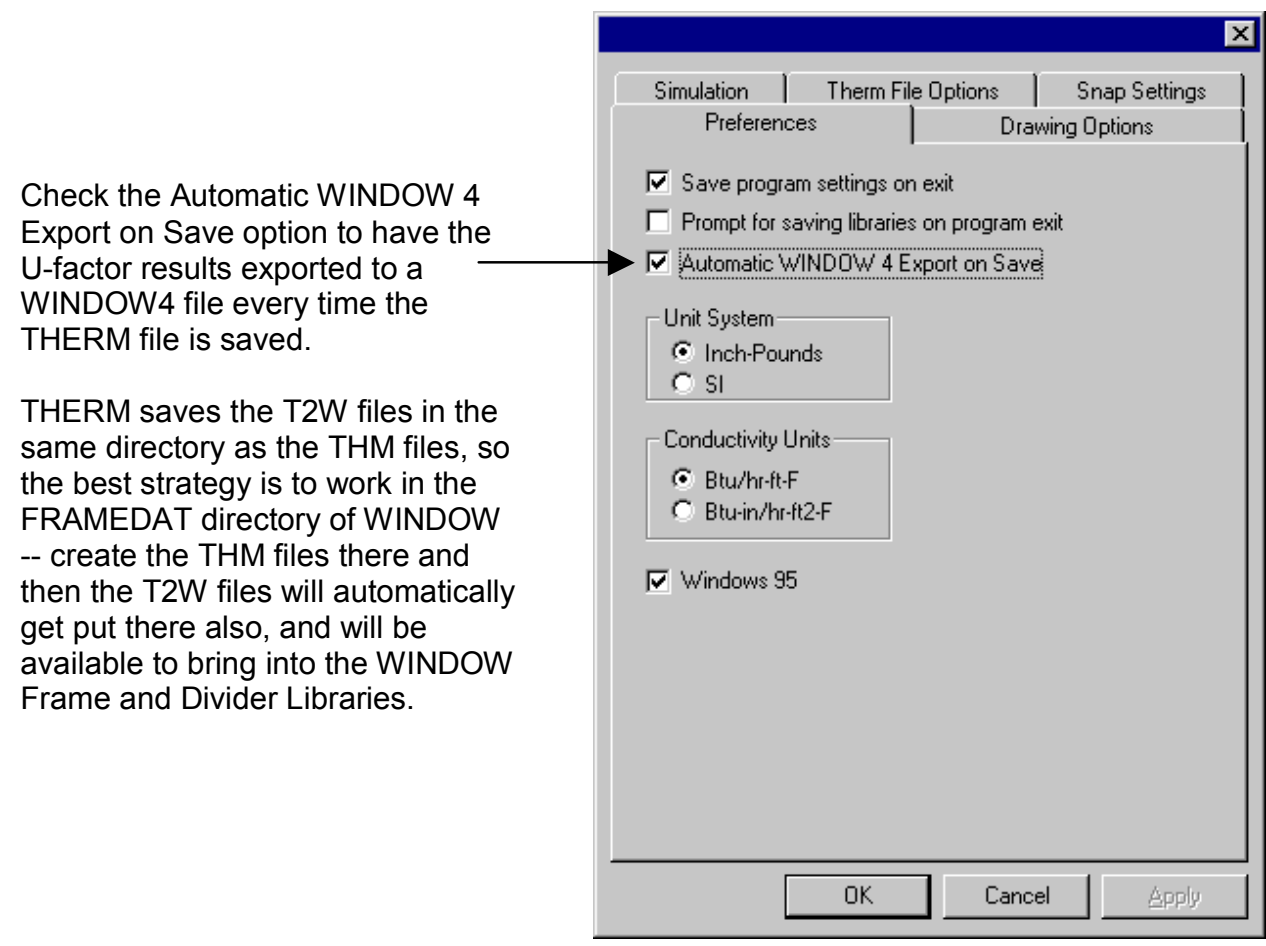

Figure 6-24. Check the Automatic WINDOW 4 Export on Save to have the results exported automatically.

Before executing the final export from THM to T2W, go to the File/Properties menu choice, and select the Cross Section Type that describes the model. This type will then appear in the WINDOW Frame Library under the Source column, and will help keep track of the type of cross section. 


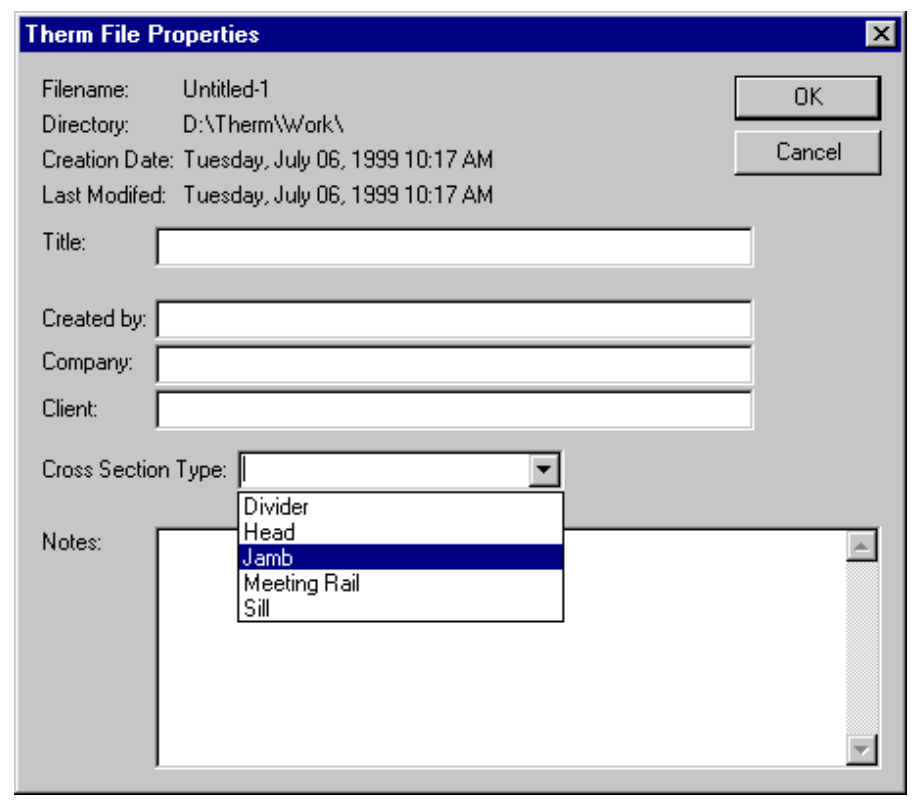

Figure 6-25. Set the Cross Section Type in File/Properties and this description will be imported into the WINDOW Frame Library.

THERM will save each cross section results file into a file with a T2W extension, which can then be imported into WINDOW, as discussed in Section 7 of this manual.

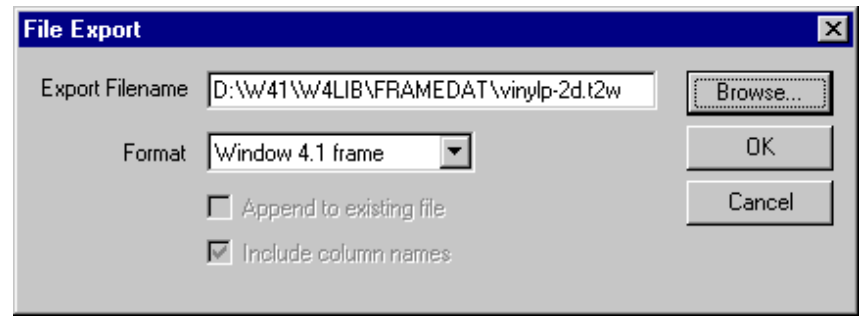

Figure 6-26. Check the Automatic WINDOW 4 Export on Save to have the results exported automatically. 


\section{TOTAL PRODUCT CALCULATIONS}

\subsection{Overview}

In order to calculate the total product U-factor, an area-weighted average of the U-factors of the following components must be calculated:

- Frame and edge values for each cross section calculated in THERM: The U-factors are area-weighted based on the projected area on a plane parallel to the glass (the Projected Y in THERM if the cross section is modeled vertically), not the total surface area of the frame and edge.

- Center-of-glass values from WINDOW

This area-weighted average can be calculated using a spreadsheet (see NFRC 100, Section 5.4 .3 for the equations), or with the WINDOW program. WINDOW calculates the total fenestration product areaweighted U-factor according to the NFRC 100 procedures, and the total product solar heat gain coefficient and visible transmittance according to the NFRC 200 procedures. If the frame and edge results from THERM are exported to T2W files (see Section 6.6.5, "Exporting Results to WINDOW Files" in this manual), then those results can be imported into the WINDOW Frame and Divider libraries and can be used with the center-ofglass U-factors to calculate the total product U-factor, solar heat gain coefficient, and visible transmittance.

\subsection{Frame and Edge U-factors from THERM}

As discussed in Section 6.6.5, "Exporting Results to WINDOW Files", the THERM results can be exported to a file that WINDOW can read. These files should be in the FRAMEDAT directory of the WINDOW program.

\subsubsection{Importing T2W Files into WINDOW}

Import the THERM T2W files either into the Frame or Divider Library, depending on what type of cross section it is. The following steps illustrate how to add a new record to the Frame Library, but the exact same technique would be used for the Divider Library.

1. Open the WINDOW program and select the Frame Library (F7), shown in Figure 7-1.

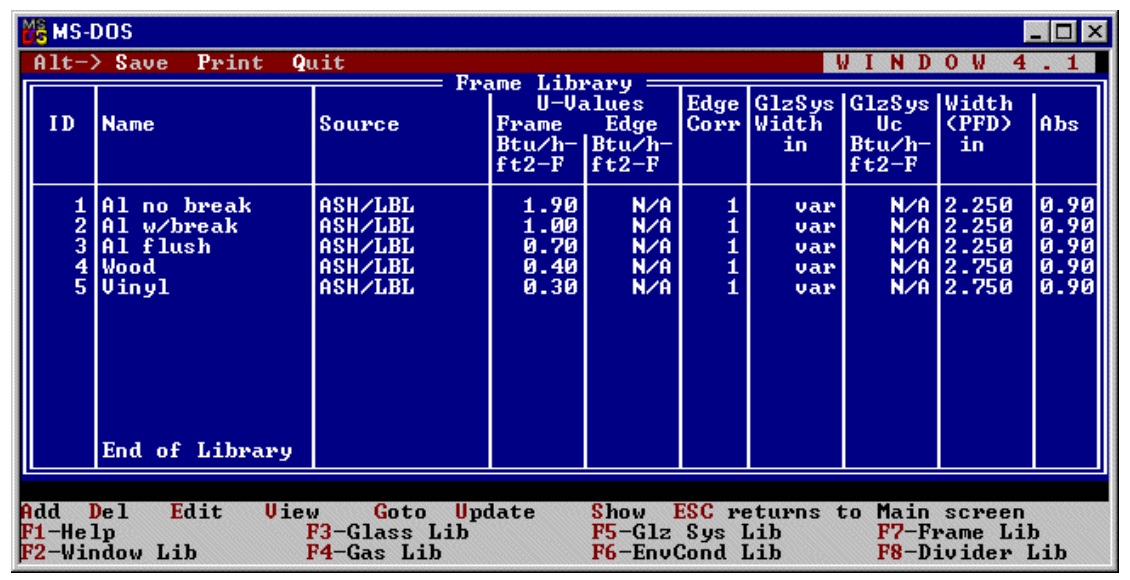

Figure 7-1. WINDOW Frame Library . 
2. Add a new frame to the library by typing A to select the Add choice.

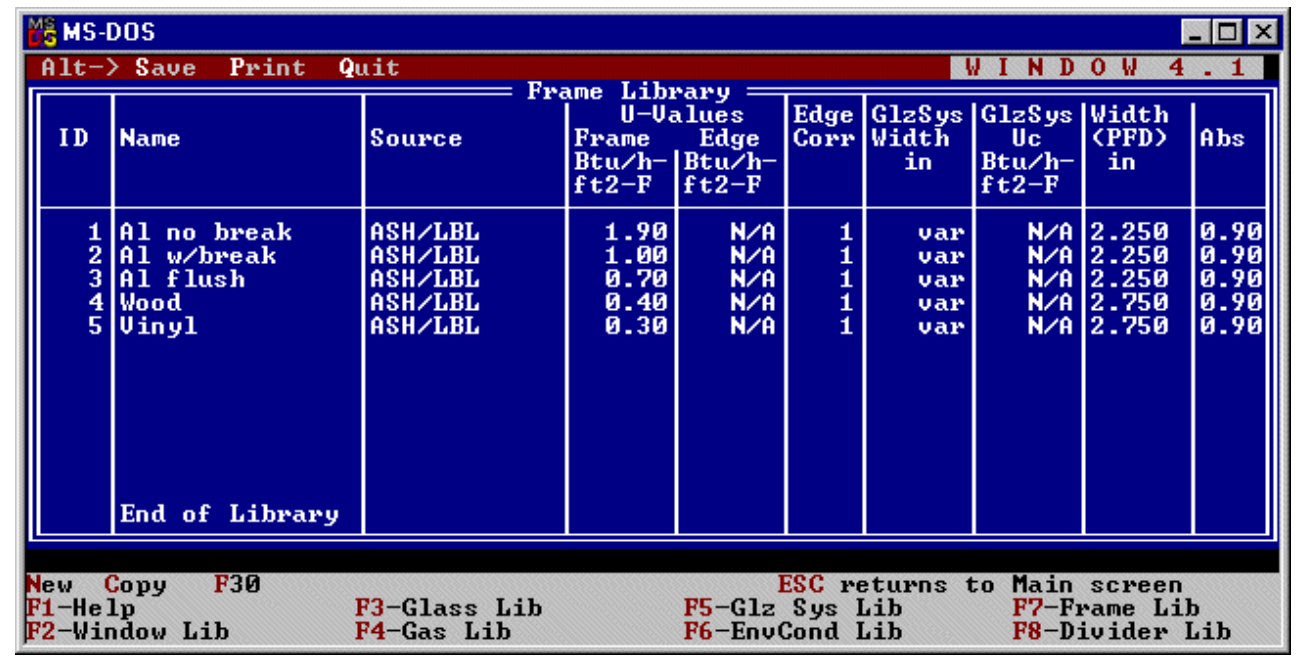

Figure 7-2. After choosing to add a new frame, select the F30 option.

3. Type $\mathbf{F}$ to select the $\mathbf{F} \mathbf{3 0}$ choice, and a Search for: *.f30 message will come up. Change the *.f30 to *.t2w, so that WINDOW will look for the THERM results files that have the extension T2W.

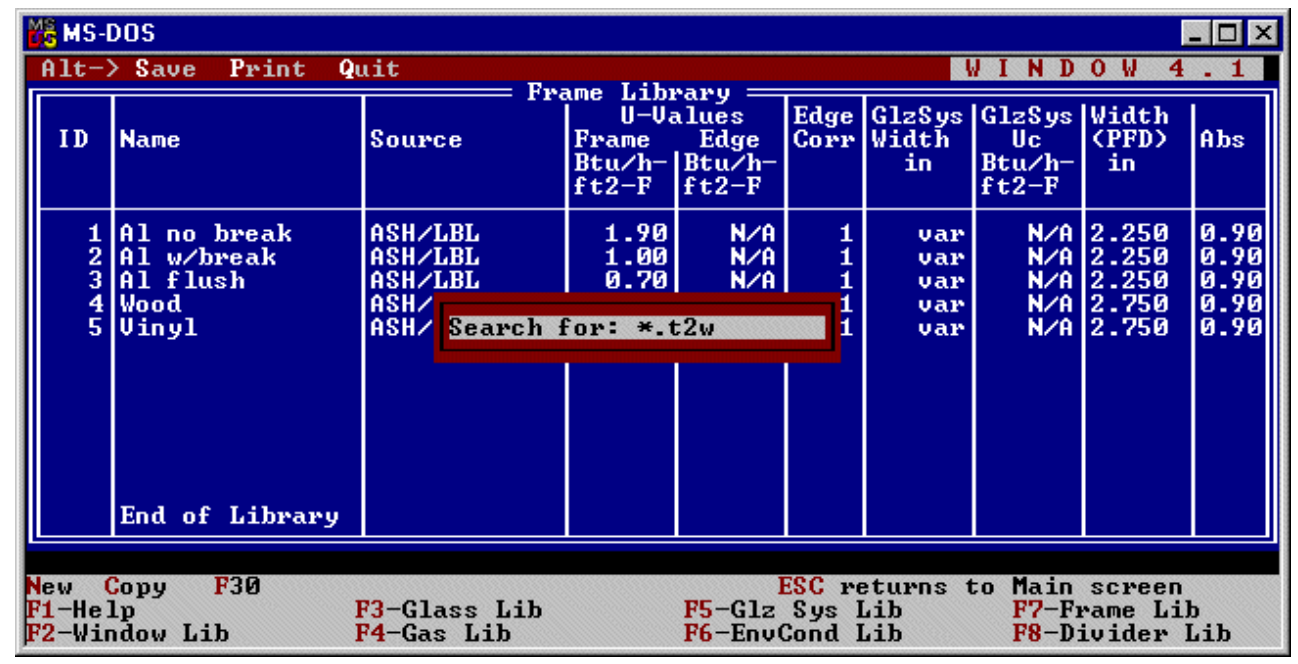

Figure 7-3. Change the search from ${ }^{*} . f 30$ to ${ }^{*} . t 2 w$.

4. A list of all the files with a T2W extension in the current library's FRAMEDAT directory will be listed, and the desired file can be selected. This step can be repeated for each file or the Select All option can be used to import all of the files currently displayed.

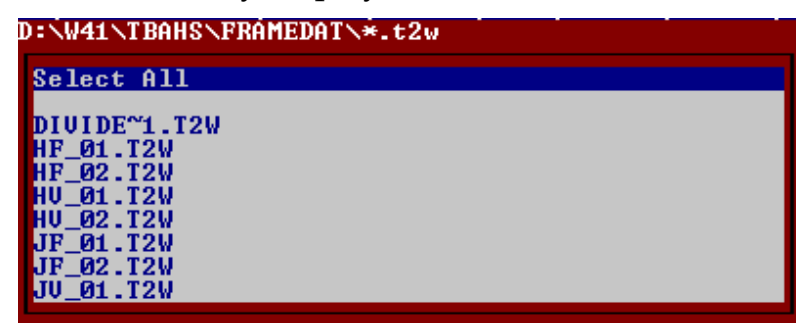

Figure 7-4. List of T2W files in the WINDOW FRAMEDAT directory . 
5. When a T2W file is selected, it will be added as a new record in the Frame Library, and the results from THERM will be displayed in the columns. If the File/Properties menu was used to set the Cross Section Type, that will be displayed in the Source column. Use the T2W file the way as any other frame library entry. (Note, however, that in order to use the T2W Frame Library entries from the Main Screen, the Mode must be set to Design rather than NFRC).

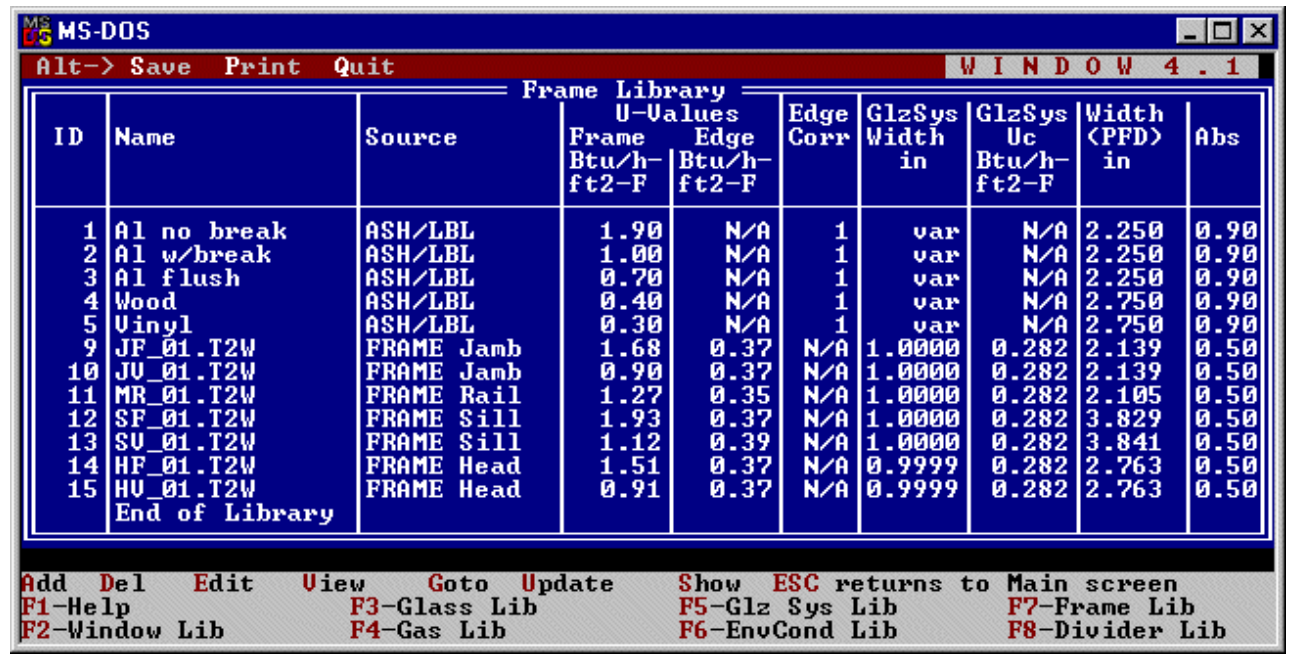

Figure 7-5. WINDOW Frame Library .

\subsection{Center-of-Glass U-factors from WINDOW}

The WINDOW 4.1 User's Manual contains detailed information about calculating the center-of-glass Ufactors.

For an NFRC simulation, create glazing systems that represent all the glazing types to be modeled from the glass matrix provided by the manufacturer. Access the Glazing System Library by pressing F5 in WINDOW, as shown in Figure 7-6.

\begin{tabular}{|c|c|c|c|c|c|c|c|c|c|c|c|c|c|c|c|c|c|}
\hline \multicolumn{5}{|c|}{ MS MS-DOS } & & & & & & & & & & & & & - \\
\hline \multicolumn{3}{|c|}{ Alt $->$ Save } & \multicolumn{2}{|c|}{ Quit } & & & & \multicolumn{5}{|c|}{$\begin{array}{lllllllll}W & \text { I } & N & D & 0 & W & 4 \cdot 1\end{array}$} \\
\hline I D & \multicolumn{2}{|l|}{ Name } & \begin{tabular}{r|}
$\# L$ \\
$a$ \\
$y$
\end{tabular} & $\begin{array}{l}\text { T1t } \\
\text { deg }\end{array}$ & \multicolumn{3}{|c|}{$\begin{array}{l}\text { Glazing } \$ y \\
\text { Enu Cond }\end{array}$} & $\begin{array}{r}\text { yst } \\
\mathrm{K} \\
\mathrm{Bt} \\
\mathrm{f}\end{array}$ & $\begin{array}{l}\text { em } L i \\
\text { EFF } \\
u / h- \\
t-F\end{array}$ & $\begin{array}{c}\text { ibrary } \\
\text { Width } \\
\text { in }\end{array}$ & $\begin{array}{l}\text { Uc } \\
\text { Btur } \\
\text { ft2 }\end{array}$ & -F & SCc & & |SHGCc | & $\overline{|U t c|}$ & \begin{tabular}{|c|} 
RHG \\
Btu/ \\
h-f t 2
\end{tabular} \\
\hline \multirow[t]{2}{*}{\begin{tabular}{||l|}
1 \\
2 \\
3 \\
4
\end{tabular}} & \multicolumn{2}{|c|}{$\begin{array}{l}3 \mathrm{~mm} \text { CIG } \\
4 \mathrm{~mm} \text { CIG } \\
5 \mathrm{~mm} \text { CIG } \\
6 \mathrm{~mm} \text { CI G }\end{array}$} & $\begin{array}{l}\# 2 \\
\# 2 \\
\# 2 \\
\# 2\end{array}$ & \begin{tabular}{|l|}
90 \\
90 \\
90 \\
90
\end{tabular} & \multicolumn{3}{|c|}{$\begin{array}{l}\text { 1NFRC/ASH } \\
1 \text { NFRC/ASH } \\
1 \text { NFRC/ASH } \\
1 \text { NFRC/ASH }\end{array}$} & $\begin{array}{l}0.1 \\
0.1 \\
0.1\end{array}$ & $\begin{array}{l}.0249 \\
0217 \\
0197 \\
0172\end{array}$ & $\begin{array}{l}1.090 \\
1.000 \\
1.009 \\
1.000\end{array}$ & 田. & $\begin{array}{l}28 \\
.28 \\
27 \\
27\end{array}$ & \multicolumn{2}{|c|}{$\begin{array}{l}0.68 \\
0.66 \\
0.65 \\
0.64\end{array}$} & \multicolumn{2}{|c|}{\begin{tabular}{l|l|}
0.58 & 0.45 \\
0.57 & 0.45 \\
0.56 & 0.44 \\
0.55 & 0.44 \\
\end{tabular}} & $\begin{array}{l}140 \\
137 \\
135 \\
131\end{array}$ \\
\hline & No . & & 10 & & S $\mathrm{T}$ sol & Gap & P D & $\cot ^{2} \mathbf{f}$ & $\begin{array}{l}\text { for } 3 \mathrm{mr} \\
\mid \mathrm{Tu} \text { is }\end{array}$ & $\ln _{1} \mathrm{CIC}$ & is & & T ir & \multicolumn{2}{|c|}{1 Emis 2} & Keff \\
\hline $\begin{array}{l}\text { Lay1 } \\
\text { Gap1 } \\
\text { Lay2 }\end{array}$ & $\left|\begin{array}{r}2001 \\
6\end{array}\right|$ & $\begin{array}{l}\text { CLR-3 - C } \\
\text { Air/Arg } \\
\text { SUN45-3 }\end{array}$ & $\begin{array}{l}\mathrm{G} \\
\mathrm{n} / \mathrm{Kr} \\
\mathrm{CI} \mathrm{GH}\end{array}$ & $\begin{array}{l}0.1 \\
0.7 \\
0.1\end{array}$ & $\begin{array}{l}17 \\
66 \\
17\end{array}$ & $\begin{array}{l}844 \\
\text { Air: } \\
-338\end{array}$ & $\begin{array}{r}.07 \\
5 \\
-26\end{array}$ & & $\begin{array}{r}.075 \\
.241\end{array}$ & $\begin{array}{l}-904 \\
\text { Ar: } \\
-496\end{array}$. & $\begin{array}{r}.082 \\
95 \% \\
.671\end{array}$ & $\begin{array}{r}.08 \\
.13\end{array}$ & 82 & $\begin{array}{l}\text { Kag日 } \\
\text { Kr: } \\
.000\end{array}$ & $\begin{array}{r}-837 \\
0 \% \\
-100\end{array}$ & $\begin{array}{l}.837 \\
.837\end{array}$ & $\begin{array}{l}-520 \\
-525 \\
-520\end{array}$ \\
\hline $\begin{array}{l}\text { Add } \\
\text { F1-He } \\
\text { F2-Wi }\end{array}$ & $\begin{array}{l}\text { Dele } \\
\text { lp } \\
\text { indow } L\end{array}$ & te & $\begin{array}{l}\text { las } \\
\text { as }\end{array}$ & Lib & & $\begin{array}{l}\text { Go } \\
\text { F5 } \\
\text { F6 }\end{array}$ & -Glz & & $\begin{array}{l}\text { Updat } \\
\text { ys Li } \\
\text { ond Li }\end{array}$ & & $\begin{array}{l}\mathrm{C} \mathbf{r} \\
-\mathbf{F r} \\
-\mathrm{Di}\end{array}$ & ne $L$ & & & $\begin{array}{c}\text { Main sc } \\
\text { F9-0y } \\
\text { F10-Te }\end{array}$ & $\begin{array}{l}\text { creen } \\
\text { ptica } \\
\text { emper }\end{array}$ & 1 \\
\hline
\end{tabular}

Figure 7-6. WINDOW Glazing System Library .

For NFRC certified simulations, use the current certified Spectral Data Library. 


\subsection{Overall Product U-factor, SHGC, and VT Calculations}

When the THERM results have been imported into the WINDOW Frame and Divider Libraries, and the needed glazing systems have been defined in the Glazing System Library, the whole product values can be calculated.

From the main screen in WINDOW, set the appropriate values on the left-hand side of the screen, which depend on the type of fenestration product. For NFRC simulations, model both the AA (Residential) and BB (Non-residential) sizes of the product.

On the right-hand side of the screen, for each cross section component, select the appropriate T2W file from the Frame and Divider libraries. When the frame cross sections and the glazing systems are specified, press the Spacebar and WINDOW will calculate the total product U-factor, Solar Heat Gain Coefficient (SHGC) and the Visible Transmittance (VT), shown in the lower left corner of the main screen.

When using the THERM T2W files for Frame and Dividers, set the Mode to Design.

Set the EnvCond field to NFRC/ASHRAE and select a Size corresponding to either AA or BB.

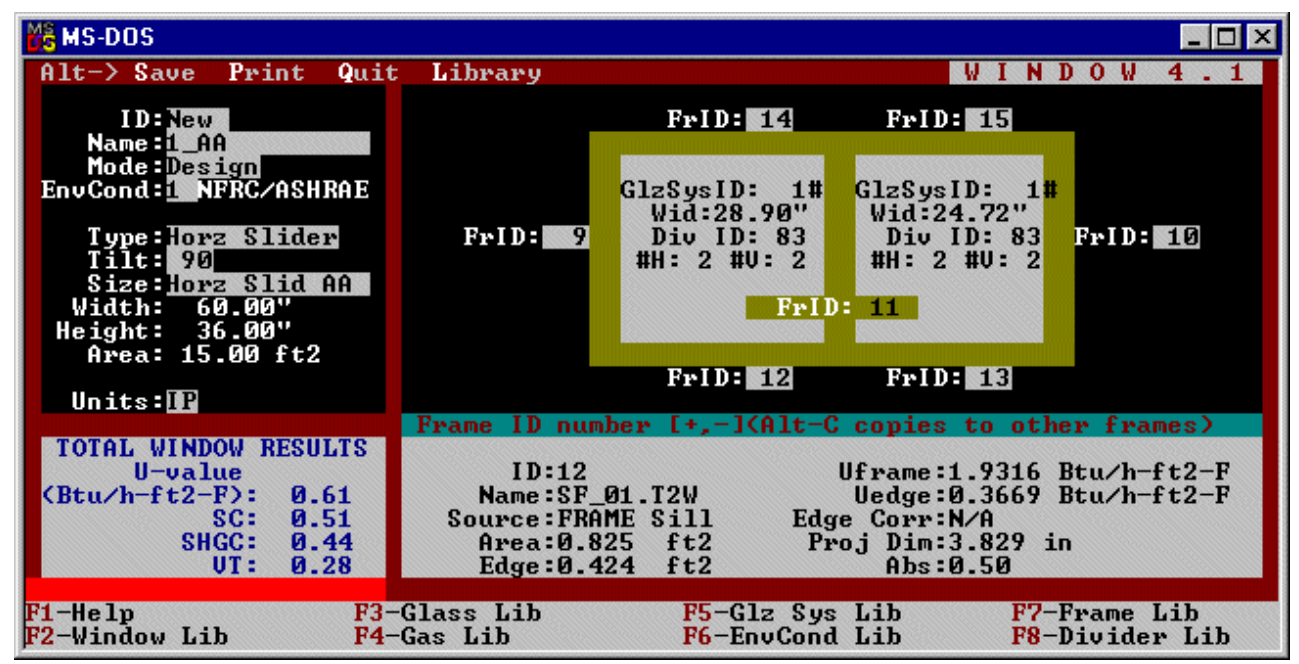

Figure 7-7. Main WINDOW screen showing whole product values .

To see U-factors to three decimal places, go to the Window Library (F2) and press Alt-F4, as shown in Figure 7-8.

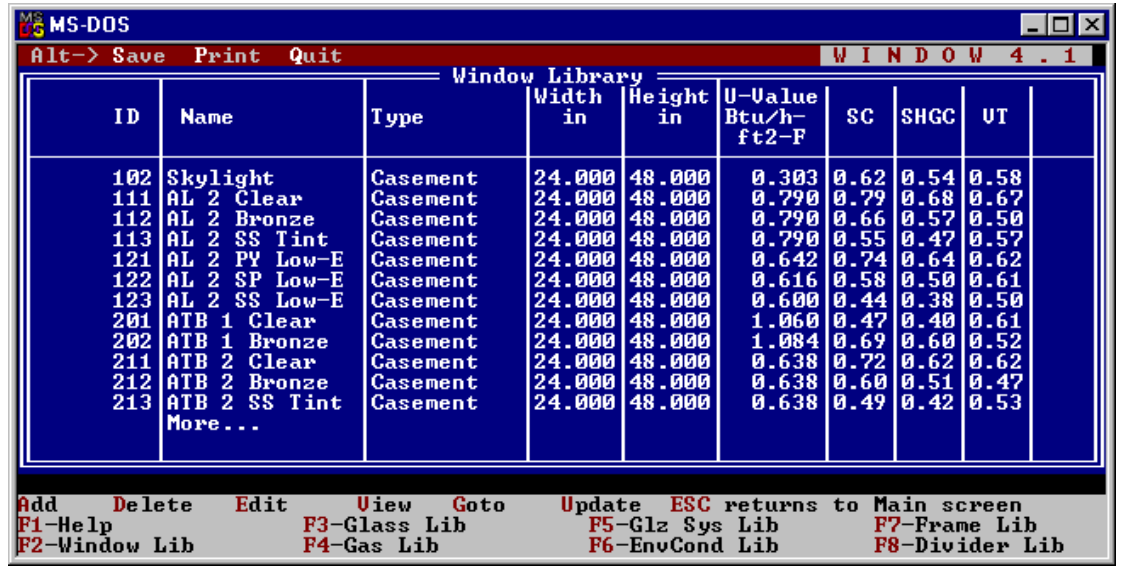

Figure 7-8. Window Library with U-factors to three decimal places (Alt-F4) . 


\section{SPECIAL CASES}

\subsection{Overview}

The following special cases are covered in this section:

8.2. Meeting Rails

page $8-2$

8.3. Dividers

page $8-8$

8.4. Storm Windows

page 8-37

8.5. Projecting Products (Skylights/Greenhouse Windows)

page $8-43$

8.6. Doors

page 8-67

8.7. Spacers

page $8-70$ 


\subsection{Meeting Rails}

Meeting rail cross sections are the stiles or rails that meet in the middle of a sliding window. In this manual, the term "meeting rail' is used generically to describe meeting rails, meeting stiles, interlock stiles, interlocking stiles, sliding stiles, check rails, and check stiles.

\subsubsection{Modeling Meeting Rails}

When modeling a meeting rail, both the sashes and their associated glazing systems are modeled. Figure 8-1 shows an example of the meeting rail from a horizontal aluminum slider.

Creating the cross section for a meeting rail is no different than any other model in THERM. A few things to keep in mind are:

- Two glazing systems are imported, one facing up and one facing down

- Interior boundary conditions for each of the glazing systems are labeled with the Edge U-factor tag, and the program averages the values for both to derive one Edge U-factor.

- Model the meeting rail with the glazing systems facing up and down (see Section 6.3.2, "Cross Section Orientation" in this manual). If the DXF file is drawn with them in a horizontal position, draw the frame cross section, and then rotate it before inserting the glazing system.

The following discussion lists the steps for making a cross section with two glazing systems and assigning the correct boundary conditions.

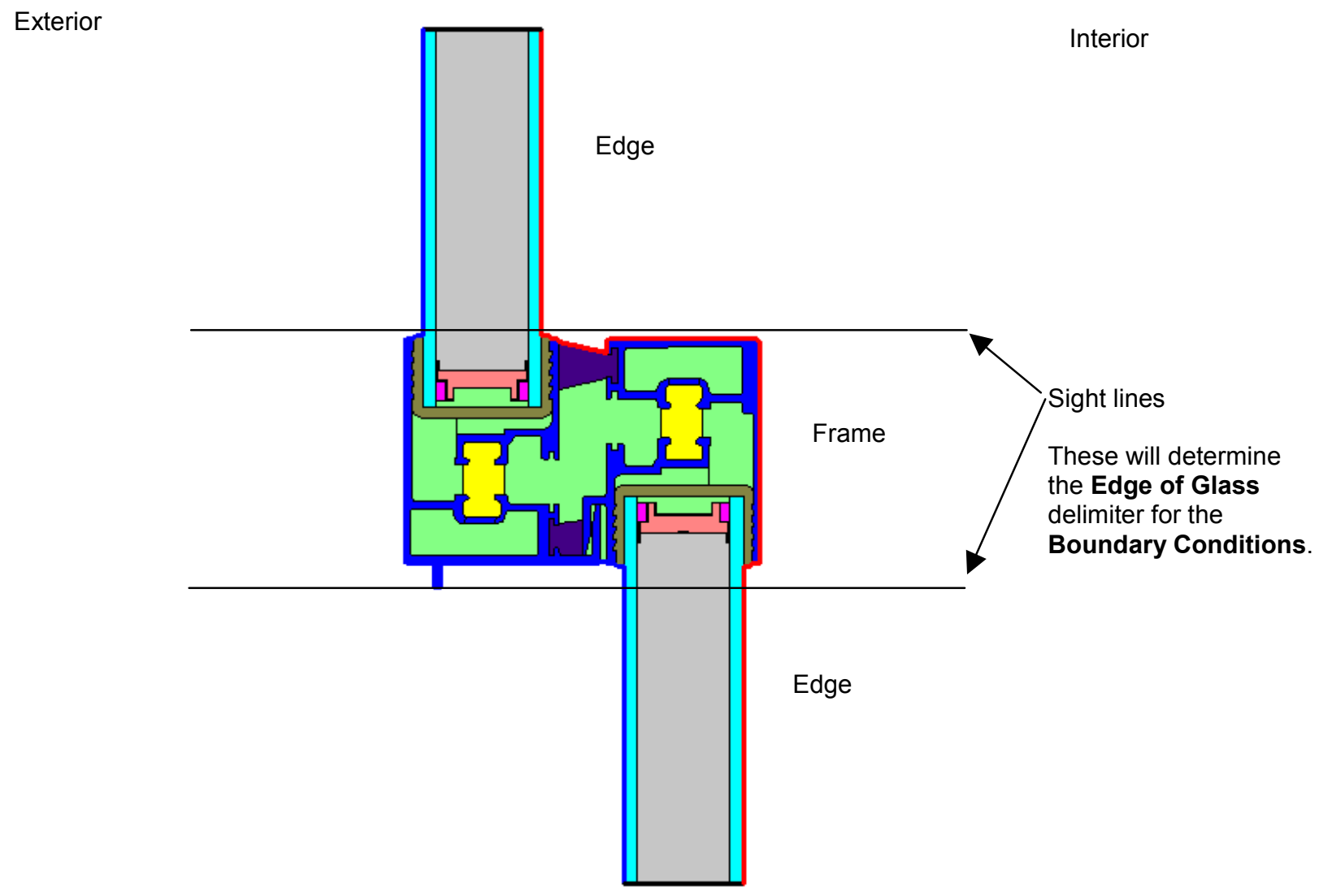

Figure 8-1. Meeting rail cross section. 


\subsubsection{Steps for Meeting Rail Calculation}

1. Using dimensioned drawings or a DXF file, create the cross section for the frame portion of the meeting rail. In Figure 8-2, the frame for the horizontal aluminum slider meeting rail has been created.

Step 1:

Draw the frame portion of the meeting rail cross section, including both sash elements, and the sweeps between them.

Define the air between the sashes as Frame Cavity (0.2/0.2) NFRC Simplified because the predominant surface emissivites are exposed metal

Sash 1

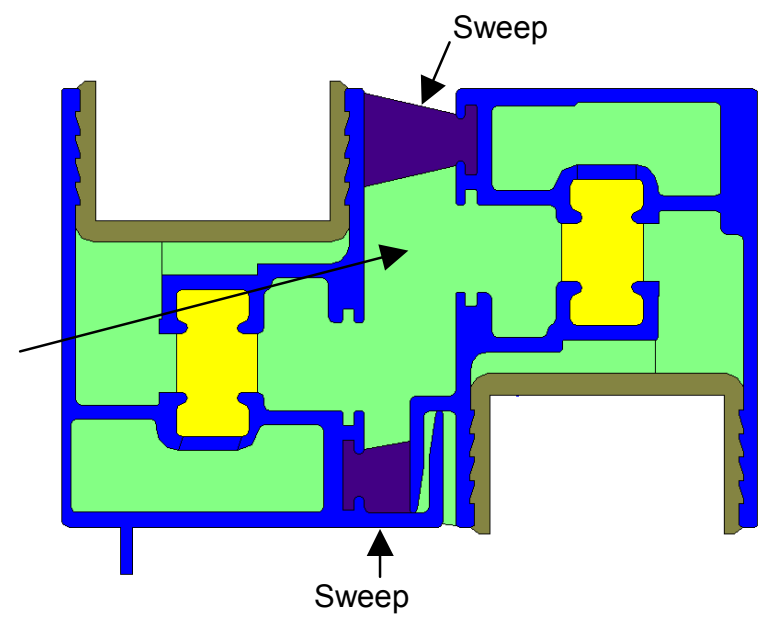

Sash 2

Figure 8-2. Frame portion of meeting rail cross section.

2. Position the Locator (using the Draw/Locator menu choice, or pressing Shift F2) in the lower left corner of the frame where the first glazing system will be inserted, as shown in Figure 8-3.

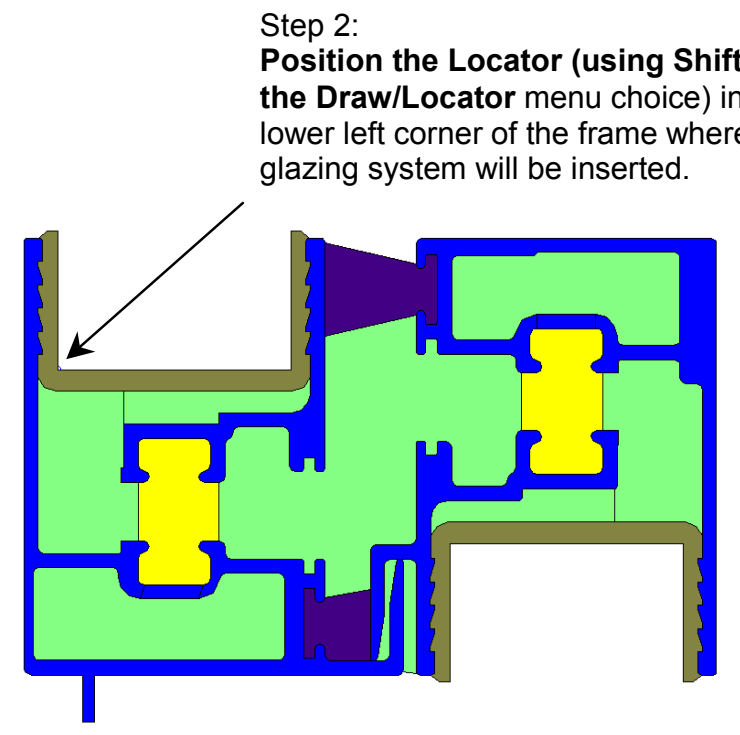

Figure 8-3. Position the Locator for the first glazing system.

3. Using the Libraries/Glazing Systems menu option (or the F6 key), insert the upper glazing system, as shown in Figure 8-4. In this example, the spacer will be copied and pasted into the cross section later, but a generic spacer of a continuous material can also be drawn automatically at the time the glazing system is inserted by checking the Draw Spacer box in the Insert Glazing System dialog box. 


\section{THERM - [Mr_01.thm]}

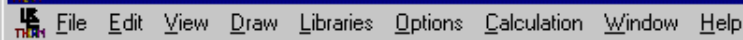

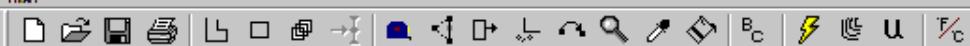

\section{Insert Glazing System}

Orientation $1 \mathrm{Wp}$

Actual cavity height 2.5

Site line to bottom of glass 0.5955

$$
\text { Spacer height } 0.3851 \text { inches }
$$

Edge of Glass Dimension $\longdiv { 2 . 5 }$ inches

ГUse nominal glass thickness

- Gap Properties

C Default C Custom Gap 1 . Keff $\longdiv { 0 . 0 2 4 9 1 }$ Btu/h-ft-F Width 0.766 inches

- Spacer

$\Gamma$ Draw spacer

Г Single spacer for multiple glazings

Material Fiberglass (PE Resin) $\quad$

Exterior Boundary Condition

$\mathrm{C}$ Use exterior $\mathrm{U}$-factor $\mathrm{BC}$ imported with glazing system

C Use exterior SHGC BC imported with glazing system

(c) Use: Exterior Surface

- Interior Boundary Condition

C Use interior U-factor BC imported with glazing system

$C$ Use interior SHGC BC imported with glazing system

C Use: Interior Aluminum Frame

Step 3:

Insert the glazing system using the Libraries/Glazing Systems menu choice or the F6 key. Specify the appropriate values in the dialog boxes (such as Orientation $=\mathbf{U p}$ ) and then click on the OK button and the glazing system will be imported.

4

\begin{tabular}{|l|l|l|l|l|}
\hline$x, y 2.156 .2 .041$ & $d x, d y 1.563,0.105$ & len 1.567 & Step 0.394 & inches \\
\hline
\end{tabular}

Ready

$+1$

Figure 8-4. Insert the first glazing system.

4. Reposition the locator to the upper left corner for the $2^{\text {nd }}$ glazing system.

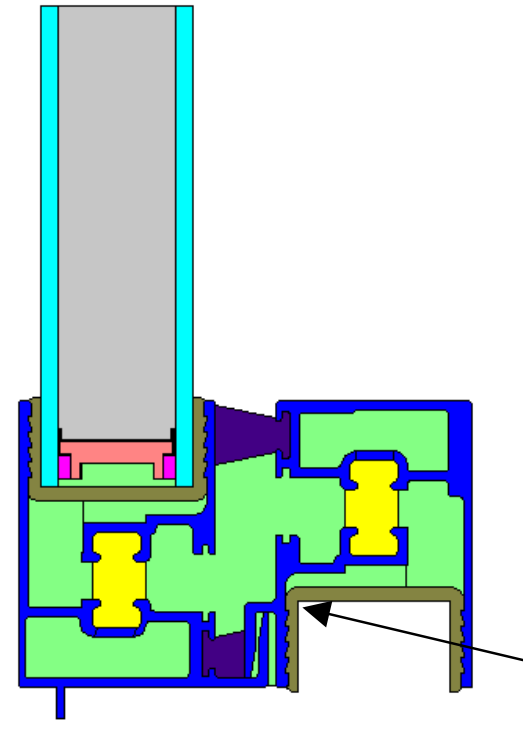

Step 4:

Reposition the Locator in the upper left corner of the frame for the $2^{\text {nd }}$ glazing

Figure 8-5. Reposition the Locator for the $2^{\text {nd }}$ glazing system. 
5. Insert the $2^{\text {nd }}$ glazing system, setting the Orientation to Down, and entering the correct values for Sight line to bottom of glass and Spacer height.

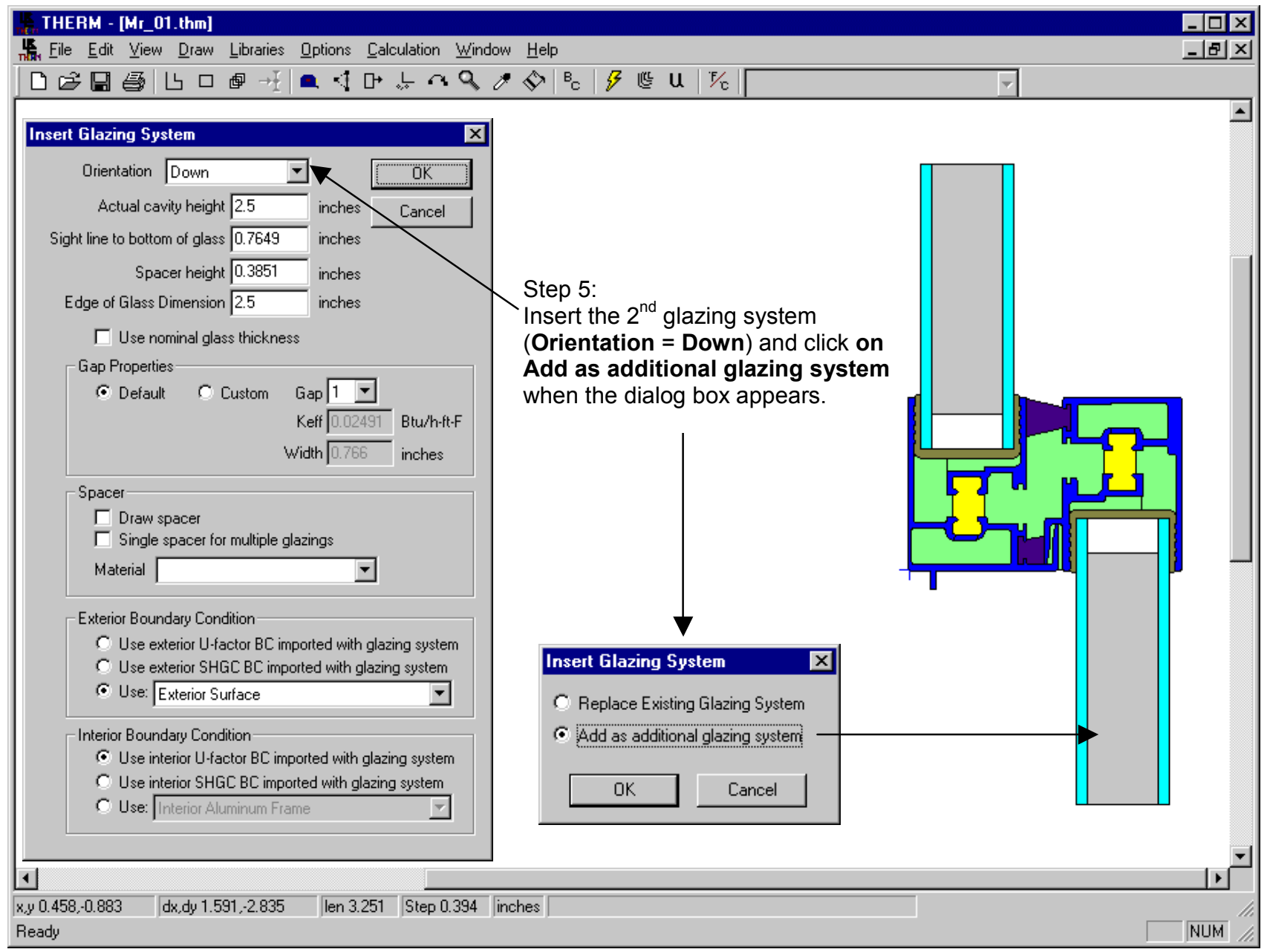

Figure 8-6. Insert the 2nd glazing system.

6. Add the spacers if they were not drawn automatically when the glazing systems were inserted.

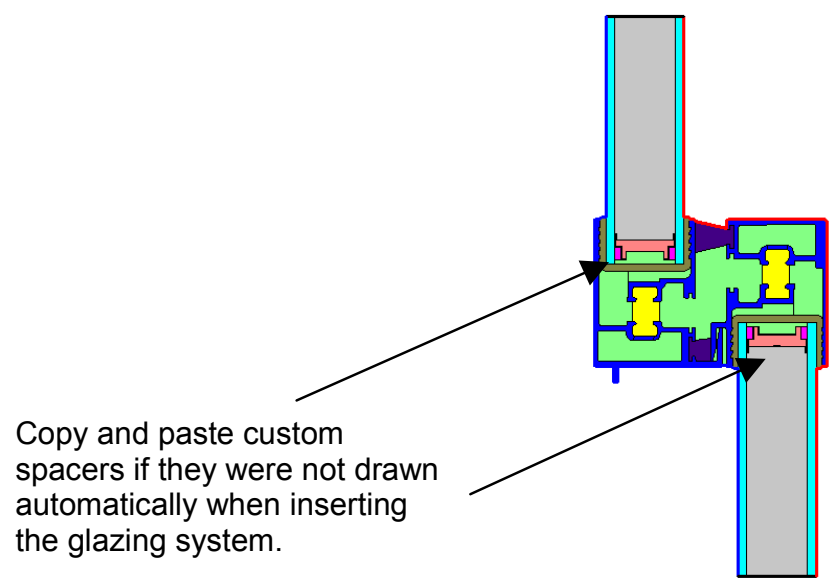

Figure 8-7. Add custom spacers if they were not drawn automatically when the glazing systems were inserted. 
7. Define the boundary conditions by pressing the Boundary Conditions toolbar button, or clicking on the Draw/Boundary Conditions menu choice, or pressing the F10 key. Assign the Edge U-factor tag to each of the interior glazing system boundary conditions, as shown in Figure 8-8.

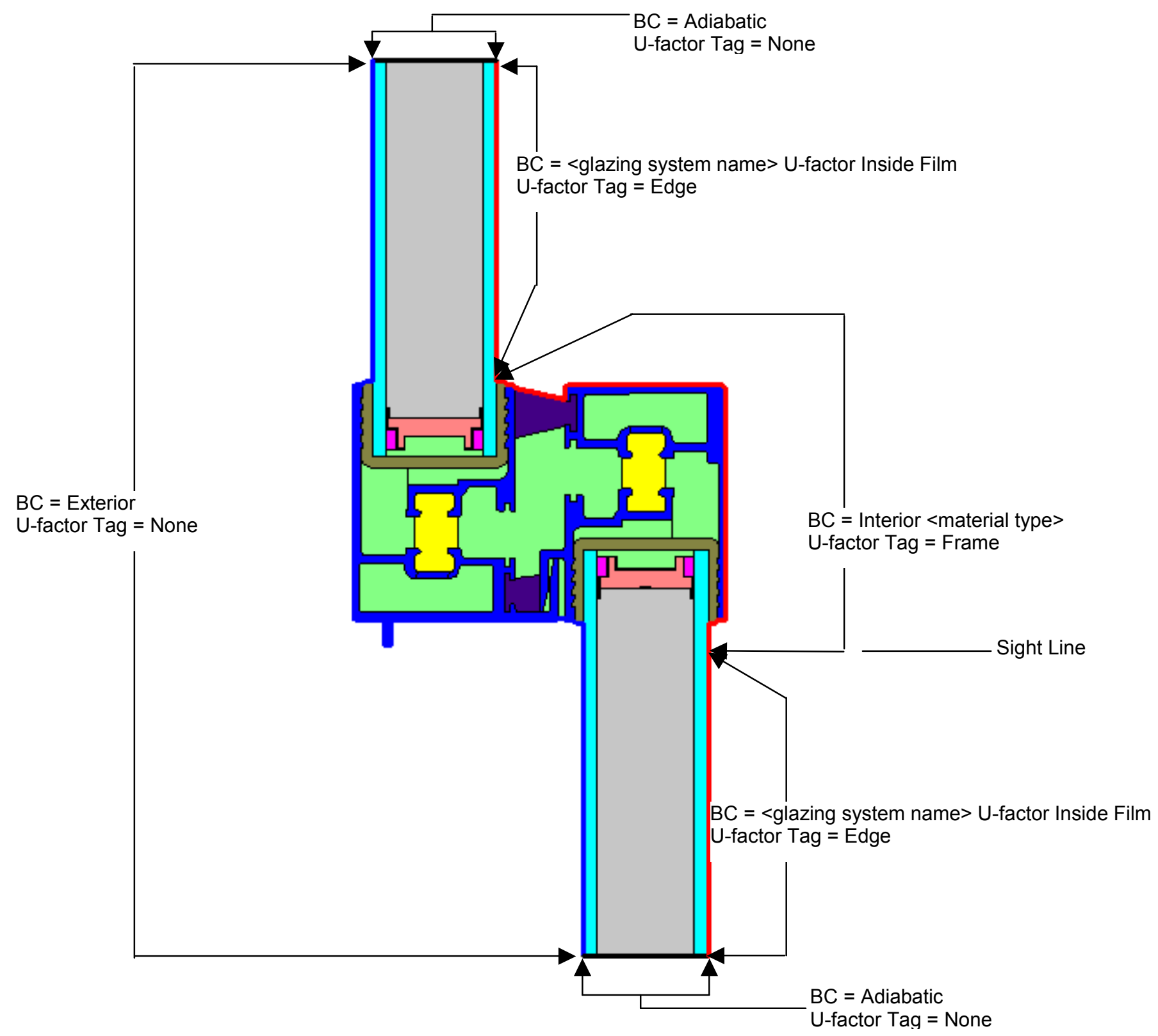

Figure 8-8. Define the Boundary Conditions for the meeting rail. 
8. Run the simulation, by pressing the Calc toolbar button, clicking on the Calculation/Calculation menu choice, or pressing the $\mathbf{F 9}$ key. The U-factor results are calculated for the Frame and Edge U-factor tags, as shown in Figure 8-9.

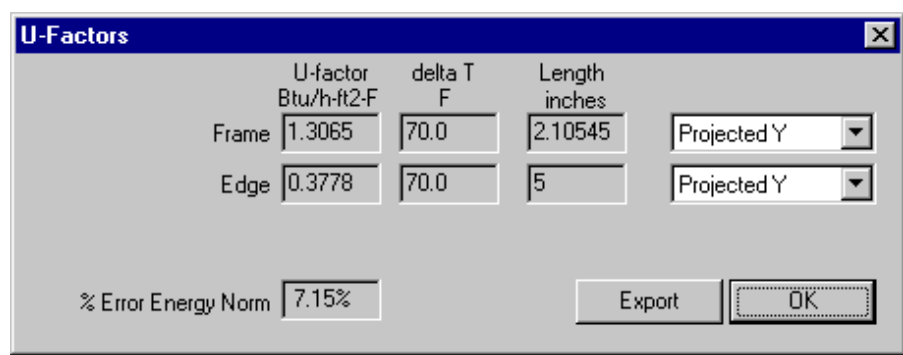

Figure 8-9. Calculate the results.

9. Export the U-factors to a T2W file so they can be used in the whole product calculation in WINDOW. 


\subsection{Internal Dividers (Suspended Grilles)}

Internal dividers can be modeled in WINDOW and THERM using the methods described below. The technique used will depend on whether the divider is rectangular or contoured. The discussion that follows is broken into two major sections, one section for rectangular dividers and one section for contoured dividers.

Internal dividers may not be modeled if there is a 0.125 inch $(3.18 \mathrm{~mm})$ or greater clearance between the glazing divider and the glass on both sides of the glazing divider. If there is less than this clearance, the dividers shall be modeled. (See NFRC 100, Section 6.2, "Simplifications to a Product Line"). The examples included in this example have less than 0.125 inch $(3.18 \mathrm{~mm})$ clearance, and so shall be modeled.

\subsubsection{Rectangular Dividers}

The modeling technique for the case of a rectangular divider, inside either an air-filled or a gas-filled glazing unit,is discussed in this section. The basic steps are the following:

In WINDOW:

- Make a glass layer to represent the aluminum material in the divider. Make sure to correctly define the emissivities of the aluminum, i.e., 0.2 for the unpainted metal, and 0.90 for painted metal. This layer is used twice and one layer may need to be flipped in order to get the correct emissivity $(0.90)$ facing the glass layers.

- Make a glazing system to represent the part of the glazing system which contains the divider, referred to in this manual as Divider. This is usually a 4 layer glazing system, with the following layers:

- Outer glass layer

- Gap between the outer glass layer and the outer side of the divider surface

- Divider layer (represented by a glass layer)

- Gap between the outer divider surface and the inner divider surface

- Divider layer (represented by a glass layer)

- Gap between the inner divider surface and the inner glass layer

- Inner glass layer

- Make a glazing system to represent the part of the glazing system which does not contains the divider, referred to in this manual as DividerEdge.

In THERM:

- Insert the glazing system which includes the divider, and then insert the glazing system without the divider above and below the first glazing system, one facing up, and the other facing down with the spacer height set to zero for both.

- Edit the glazing cavity in the divider glazing system to include the upper and lower aluminum components of the divider.

- Simulate the results 


\section{Example of Rectangular Divider}

The following example uses the method described above for an air-filled glazing system with a rectangular divider. The technique is the same for a gas-filled rectangular divider. For this example, assume the internal divider has the following characteristics:

- Width: 0.375 inches

- Height: 1.00 inches

- Interior Dimension: 0.343 inches

- Material: White Painted Aluminum on the exterior, unpainted on the interior, 0.016 inches thick

- Spacing in glazing cavity: 0.10 " space between divider and glass on each side.

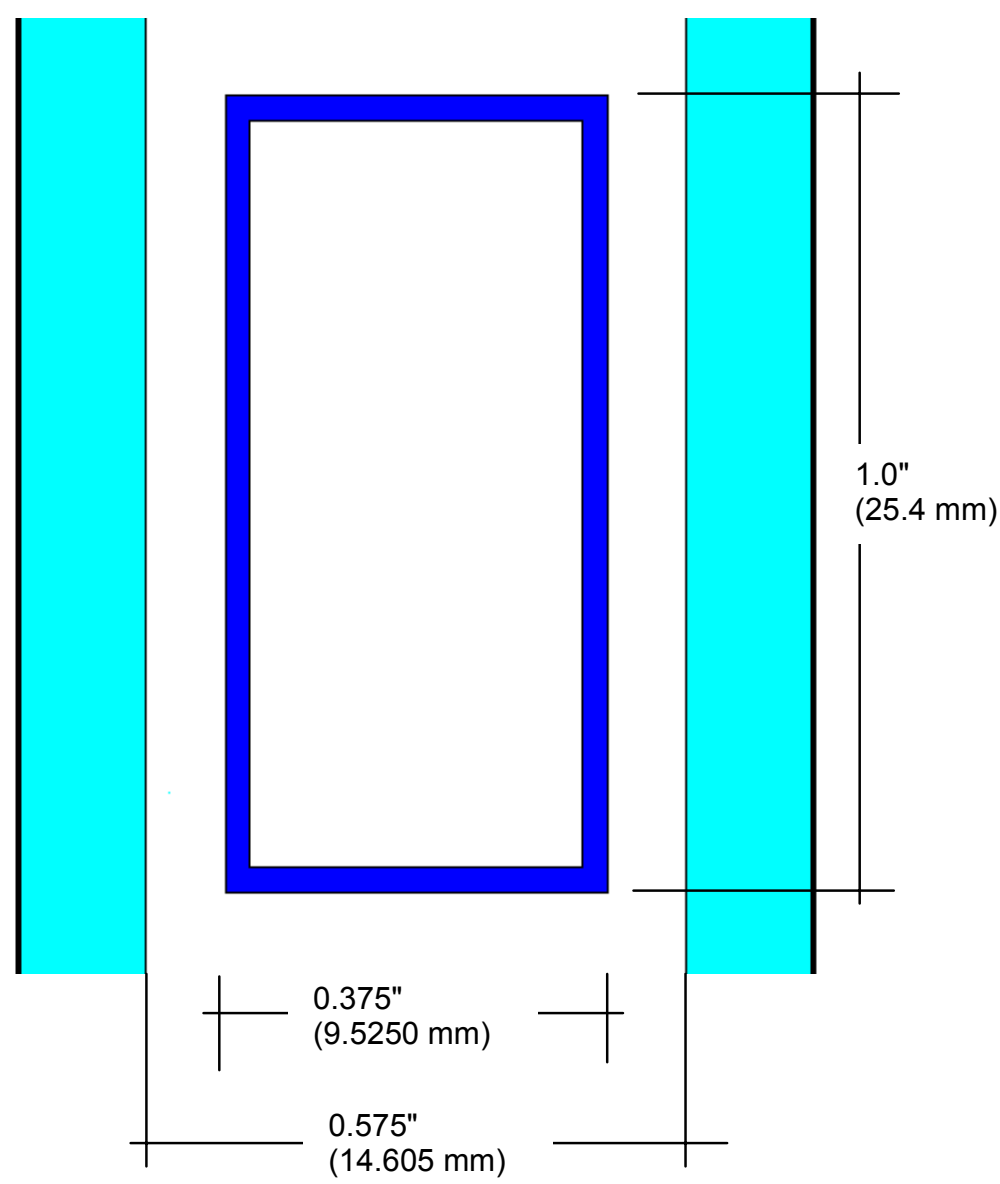

Figure 8-10. Rectangular divider. 


\section{In WINDOW 4.1:}

1. Make a glass layer to represent the aluminum material in the divider. Because this example is for an aluminum divider that is painted on the outside and unpainted on the inside, the emissivity of one side is 0.2 for the unpainted metal, and the other side is 0.90 for painted metal. This layer is used twice and one layer must be flipped in order to get the correct emissivity facing the glass layers.

Glass Library: To represent the aluminum divider, create a glass layer in the Glass Library called DividerAlum with the following characteristics:

- $\quad$ Thickness $=0.016(0.4 \mathrm{~mm})$

- Transmittances $=0$

- Reflectances $=0$ (not used in the calculations)

- Emissivity $=0.9$ for the painted metal side, and 0.20 for the unpainted metal side

- Conductivity $=92.448 \mathrm{Btu} / \mathrm{h}-\mathrm{ft}-\mathrm{F}$.

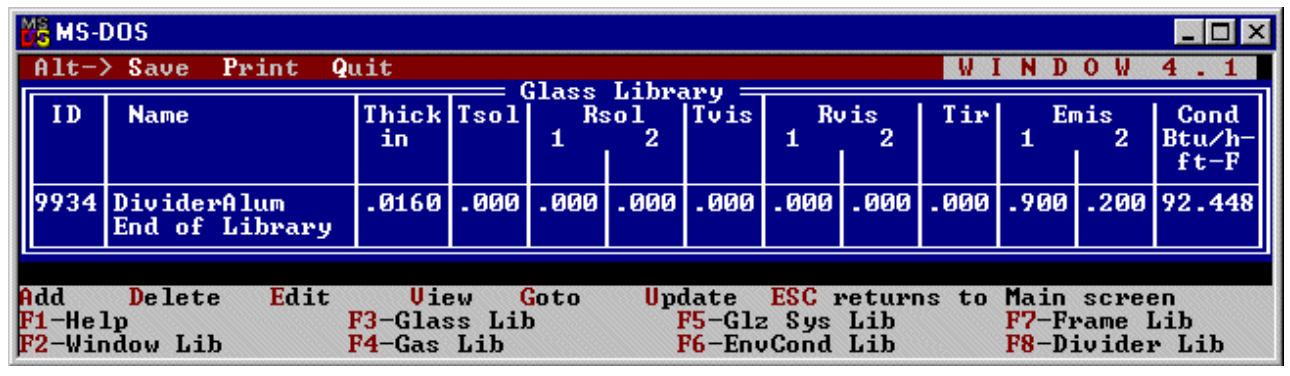

Figure 8-11 In the WINDOW Glass Library, define a glass layer to represent the aluminum divider.

2. Make a Divider Edge and Divider glazing system to represent the glazing system with and without the divder. In this case the glazing system cavities are air-filled but a gas-filled case is modeled the same way.

Glazing System Library: Create two different glazing systems, Divider Edge and Divider.

\section{- Divider Edge:}

Make a glazing system which represents the cross section of the product that does not have the divider. For this example, it is a two-layer model with the following construction:

- Layer 1 = Glass \#102, Clear_3 (3 mm)

- Gap 1 = Air cavity, $0.575 "$ thick $(14.6 \mathrm{~mm})$

- Layer 2 = Glass \#102, Clear_3 $(3 \mathrm{~mm})$

\begin{tabular}{|c|c|c|c|c|c|c|c|c|c|c|c|c|c|c|}
\hline$\frac{15}{5}$ MS- & -DOS & & & & & & & & & & & & & $-10 x$ \\
\hline A1t- & $>$ Save & Print & Qui & & & & & & & & WII & NDO & W & 1 \\
\hline I D & Name & & \begin{tabular}{r|}
$\# L$ \\
$a$ \\
$y$
\end{tabular} & $\begin{array}{l}\text { T1t } \\
\text { deg }\end{array}$ & $\begin{array}{l}\text { Giazing } \\
\text { Env Cond }\end{array}$ & 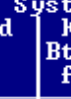 & $\begin{array}{l}\text { tem } \mathbf{i} \\
\text { KEFF } \\
\text { tu/h- } \\
\text { ft-F }\end{array}$ & $\begin{array}{l}\text { Wrary } \\
\text { Width } \\
\text { in }\end{array}$ & $\begin{array}{r}\text { Uc } \\
\text { Btu } \\
\mathrm{ft2} 2\end{array}$ & $\mathrm{~h}^{\mathrm{h}-}$ & Ce $|\mathrm{s}|$ & SHGCe |U & Utc & $\left|\begin{array}{c}\text { RHG } \\
\text { Btu } \\
h-f t 2\end{array}\right|$ \\
\hline 934 & Divide & rEdge & $\# 2$ & 90 & 1NFRC/AS & $\mathrm{SH} 0$. & .0440 & 0.815 & & $\begin{array}{lll}49 & 0 . \\
\end{array}$ & \begin{tabular}{l|l}
.88 & 0 \\
\end{tabular} & 0.76 & 0.81 & 184 \\
\hline & No . & Name & & $\begin{array}{l}\text { Gla } \\
\text { |b<in }\end{array}$ & $\begin{array}{l}\text { ass and } \\
\text { n) Tsol }\end{array}$ & $1 \mathrm{Rs}$ & $\begin{array}{l}\operatorname{Data} f \\
\operatorname{sol} 2\end{array}$ & Tuis & $1 \mathrm{Ru}$ & is 2 & Tir & $1 \mathrm{Em}$ & mis 2 & Keff \\
\hline $\begin{array}{l}\text { Lay1 } \\
\text { Gap1 } \\
\text { Lay2 }\end{array}$ & $\begin{array}{r}102 \\
1 \\
102\end{array}$ & $\begin{array}{l}\text { CLEAR_3. } \\
\text { Air } \\
\text { CLEAR_3. }\end{array}$ & $\begin{array}{l}\text { DAT\# } \\
\text { DAT\# }\end{array}$ & $\begin{array}{l}0.12 \\
0.57 \\
0.12\end{array}$ & \begin{tabular}{l|l|l}
20 & .834 &. \\
75 & & \\
20 & .834 &.
\end{tabular} & $\begin{array}{l}.075 \\
.075\end{array}$ & $\begin{array}{l}.075 \\
.075\end{array}$ & \begin{tabular}{|l|}
.899 \\
.899
\end{tabular} & $\begin{array}{l}.082 \\
.082\end{array}$ & $\begin{array}{l}.082 \\
.982\end{array}$ & $\begin{array}{l}.000 \\
.000\end{array}$ & \begin{tabular}{|l|}
.840 \\
.840
\end{tabular} & $\begin{array}{l}.840 \\
.840\end{array}$ & \begin{tabular}{|l|}
.520 \\
.644 \\
.520
\end{tabular} \\
\hline $2-w$ & $\begin{array}{l}\text { Del } \\
\text { ow }\end{array}$ & $\begin{array}{l}\text { Ed } \\
\text { F3- } \\
\text { F4- }\end{array}$ & $\begin{array}{l}\text { Glas: } \\
\text { Gas }\end{array}$ & sib & & & $\begin{array}{l}\text { Updat } \\
\text { sys Li } \\
\text { ond Li }\end{array}$ & & 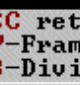 & $\begin{array}{l}\text { Lib } \\
\text { P L }\end{array}$ & Lib & $\begin{array}{l}\text { Main sc } \\
\text { F9-0p } \\
\text { F10-Te }\end{array}$ & $\begin{array}{l}\text { ptica. } \\
\text { emperc }\end{array}$ & $\begin{array}{l}\text { al } \\
\text { ratu }\end{array}$ \\
\hline
\end{tabular}


Figure 8-12 In the WINDOW Glazing System Library, define Divider Edge. 


\section{- Divider:}

Make a glazing system to represent the cross section in the product where the divider is, as shown in Figures 8-13. For this example, it is a four-layer model with 2 glass layers and 2 middle layers set to DividerAlum:

- $\quad$ Layer 1 = Glass \#102, Thickness of 0.120" (3 mm)

- Gap $1=$ Air, Thickness = 0.10" $(2.5 \mathrm{~mm})$

- Layer 2 = DividerAlum, Thickness $=0.016 "(0.4 \mathrm{~mm})$

- Gap $2=$ Air, Thickness $=0.343 "(8.7 \mathrm{~mm})$

- Layer $3=$ DividerAlum, FLIPPED to get the correct emissivities on each surface, Thickness = $0.016 "(0.4 \mathrm{~mm})$

- Gap $3=$ Air, Thickness $=0.10 "(2.5 \mathrm{~mm})$

- Layer 4 = Glass \#102, Thickness of 0.120" (3 mm)

\begin{tabular}{|c|c|c|c|c|c|c|c|c|c|c|c|c|c|c|}
\hline MS & & & & & & & & & & & & & & $-\square x$ \\
\hline$\overline{1 t->}$ & $>$ Save & Print & Qui & & & & & & & & WI & $\mathrm{INDO}$ & W 4 & 1 \\
\hline I D & Name & & \begin{tabular}{|r|r}
$\# L$ & \\
$a$ & \\
$y$ &
\end{tabular} & $\begin{array}{l}\text { T 1t } \\
\text { deg }\end{array}$ & $\begin{array}{l}\text { Ela } \\
\text { Env }\end{array}$ & $d^{5} \begin{array}{r}k \\
B t \\
f\end{array}$ & $\begin{array}{l}\text { tem } \\
\text { KEFF } \\
\text { tu/h- } \\
\text { ft }-F\end{array}$ & $\begin{array}{l}\text { Width } \\
\text { in }\end{array}$ & \begin{tabular}{|l} 
Uc \\
Btud \\
ft2-
\end{tabular} & $\left.\mathbf{h}^{-\mathrm{F}}\right|^{\mathbf{S}}$ & SCc & |SHGC |U & Utc & $\begin{array}{c}\text { RHG } \\
\text { Btu/ } \\
\text { h-ft } 2\end{array}$ \\
\hline $\begin{array}{l}17 \\
18\end{array}$ & Divide & er & \begin{tabular}{|l|}
4 \\
\end{tabular} & \begin{tabular}{|l|}
90 \\
90 \\
\end{tabular} & $\begin{array}{l}\text { 1NFRC/A } \\
1 \mathrm{NFRC} / \mathrm{A}\end{array}$ & $\begin{array}{ll}\text { SH } \\
\text { SH } \\
\text { SH. }\end{array}$ & \begin{tabular}{|l|}
.0183 \\
.0167
\end{tabular} & \begin{tabular}{|l|}
0.815 \\
0.815
\end{tabular} & & \begin{tabular}{l|l}
.28 & 0 \\
.26 & 0 \\
\end{tabular} & $\begin{array}{l}0.20 \\
0.21\end{array}$ & \begin{tabular}{|l|l}
0.17 & 0 \\
0.18 & 0 \\
\end{tabular} & 0.00 & 44 \\
\hline & No : & Name & & D<in & n) $\mid T$ sol 1 & $1 \mathrm{Rs}$ & $\mid \begin{array}{lll}\operatorname{soc} 1 & 2\end{array}$ & Tuis & $1 \mathrm{Rv}$ & is 2 & $\mathbf{T i}_{1}$ & ir $1 \mathrm{Em}$ & $\operatorname{mis} 2$ & Keff \\
\hline $\begin{array}{l}\text { Lay1 } \\
\text { Gap1 } \\
\text { Lay2 } \\
\text { Gap2 } \\
\text { Lay3 } \\
\text { Gap3 } \\
\text { Lay4 }\end{array}$ & $\begin{array}{r}102 \\
1 \\
9930 \\
1 \\
9930 \\
1 \\
102\end{array}$ & $\begin{array}{l}\text { CLEAR_3.D } \\
\text { Air } \\
\text { DividerAl } \\
\text { Air } \\
\text { FDividerAl } \\
\text { Air } \\
\text { CLEAR_3.D }\end{array}$ & $\begin{array}{l}\mathrm{DAT} \# \\
\operatorname{lum} \\
\mathrm{IuT} \#\end{array}$ & $\begin{array}{l}0.12 \\
0.16 \\
0.01 \\
0.34 \\
0.01 \\
0.16 \\
0.12\end{array}$ & \begin{tabular}{l|c}
20 & .834 \\
00 & \\
16 & .000 \\
43 & \\
16 & .000 \\
20 & .834
\end{tabular} & $\begin{array}{l}.075 \\
.000 \\
.090 \\
.075\end{array}$ & $\begin{array}{l}.075 \\
.000 \\
.000 \\
.075\end{array}$ & $\begin{array}{r}.899 \\
.090 \\
.090 \\
.899\end{array}$ & $\begin{array}{l}.082 \\
.000 \\
.000 \\
.082\end{array}$ & $\begin{array}{l}.082 \\
.000 \\
.000 \\
.082\end{array}$ & \begin{tabular}{|l}
2 \\
.000 \\
.000 \\
.000 \\
.00
\end{tabular} & \begin{tabular}{c|c}
-840 \\
.900 \\
.200 \\
.840
\end{tabular} & $\begin{array}{l}.840 \\
.200 \\
.900 \\
.840\end{array}$ & $\begin{array}{l}.520 \\
.018 \\
92-4 \\
.017 \\
92.4 \\
.020 \\
.520\end{array}$ \\
\hline & & $\begin{array}{ll} & \mathrm{F} 3 \\
\text { b } \quad \mathrm{F} 4 \\
\end{array}$ & ss & Li & F6 & 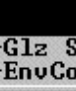 & sys & & 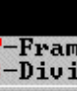 & der 1 & Lib & F10- & 1 & \\
\hline
\end{tabular}

Figure 8-13 In the WINDOW Glazing System Library, define Divider. 


\section{In THERM:}

1. Import the Divider glazing system with the following settings (Note: make sure that the glzsys.w4 and the glass.dat files are in the same directory when importing the glazing system libraries from WINDOW into THERM):

- Actual cavity height $=0.625$ " (height of the divider)

- $\quad$ Sight line to bottom of glass $=0 "$

- $\quad$ Spacer height $=0 "$

- $\quad$ Edge of Glass Dimension $=0.625$ " (height of divider)

- Draw spacer $=$ not checked

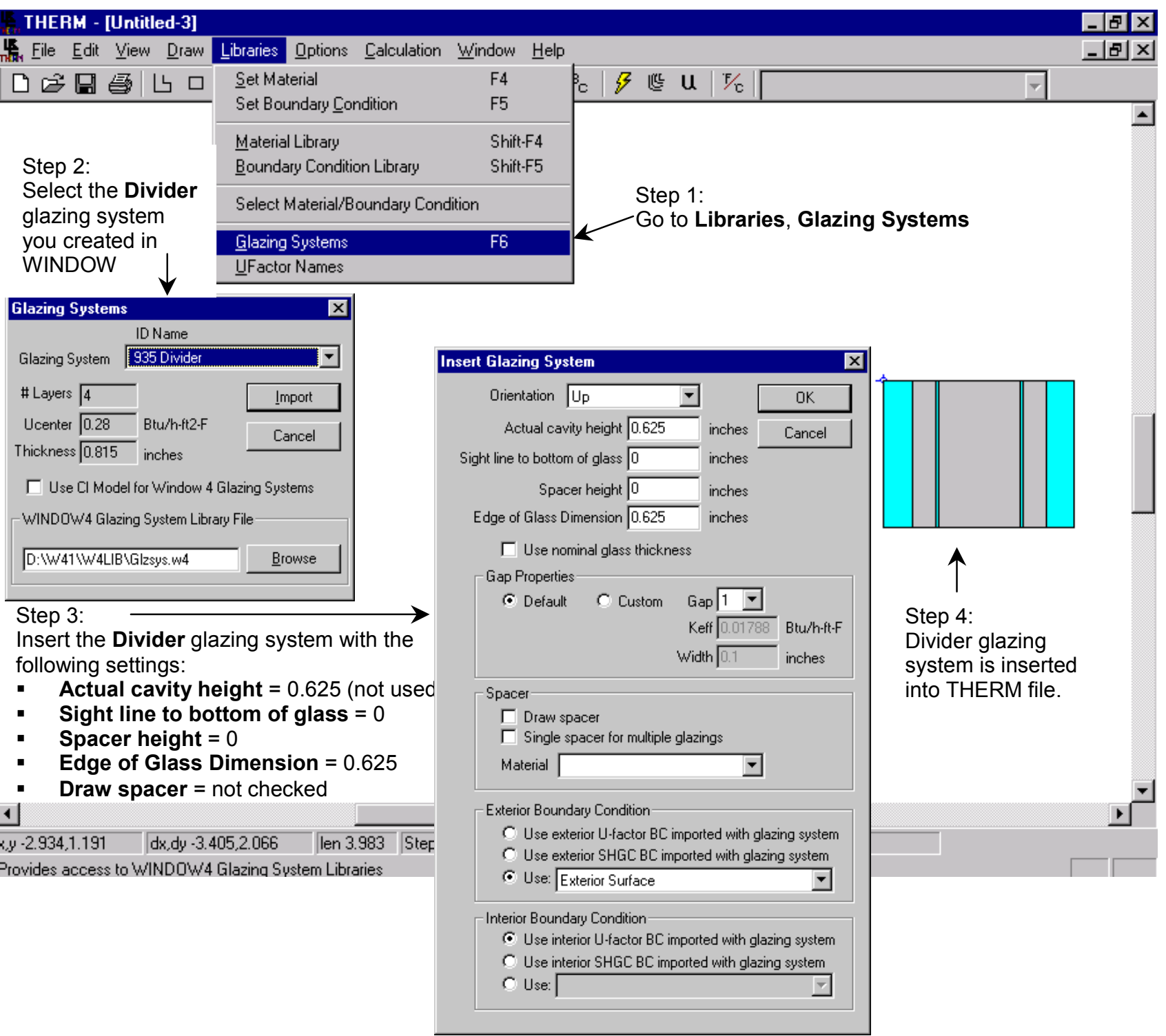

Figure 8-14 In THERM, insert the glazing system that represents the Divider. 
2. Add the glazing system Divider Edge as an additional glazing system above the Divider glazing system:

- Reposition the Locator to the upper left corner of the Divider glazing system.

- Import glazing system Divider Edge.

- In the Insert Glazing System screen, edit the following settings

Orientation $=\mathrm{Up}$

Actual cavity height $=2.5$ inches

Sight line to bottom of glass $=0$ inches

Spacer height $=0$ inches

Edge of Glass Dimension $=2.5$ inches

Draw spacer $=$ not checked

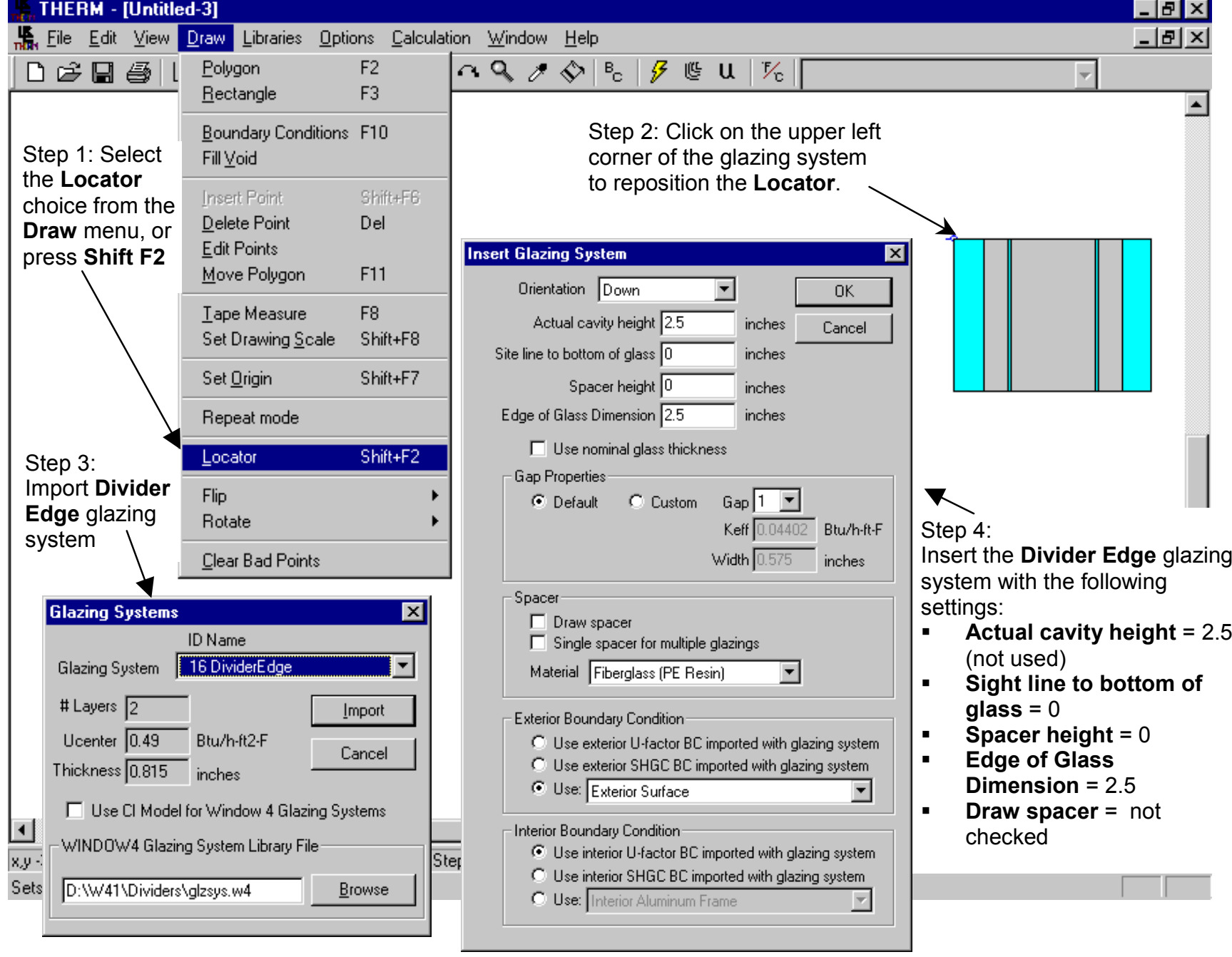

Figure 8-15 In THERM, insert the glazing system that represents the DividerEdge. 
- Click on the Add as additional glazing system radio button when asked

- The $2^{\text {nd }}$ glazing system is inserted on top of the Divider Edge glazing system.

\section{THERM - [Untitled-1]}

辑 File É

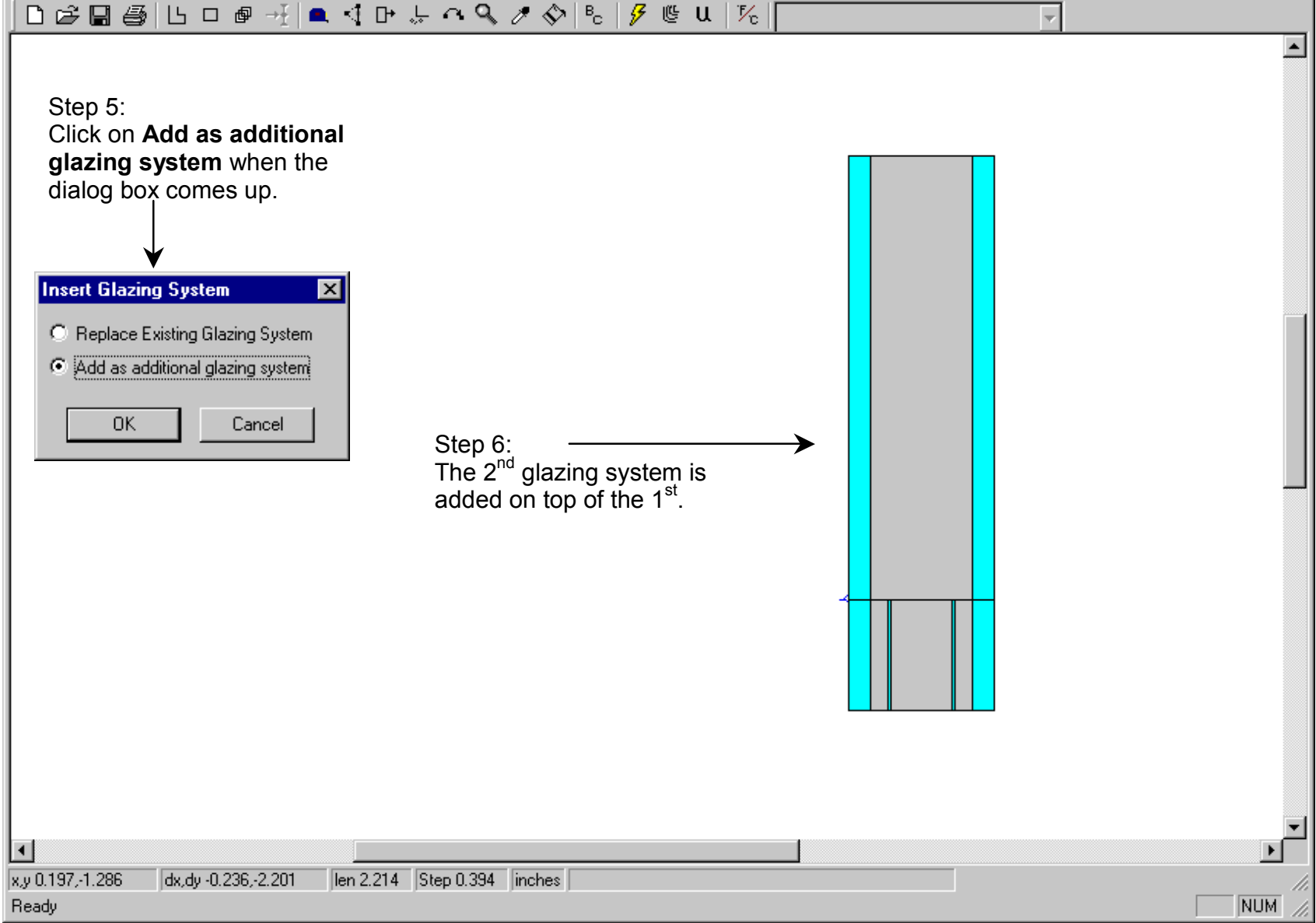

Figure 8-16 In THERM, add the Divider Edge glazing system as a second glazing system. 
3. Add the Divider Edge glazing system as a 3rd glazing system below the first Divider glazing system:

- Reposition the Locator to the lower left corner of the Divider glazing system.

- Import the Divider Edge glazing system a 2nd time.

- In the Insert Glazing System screen, edit the following settings

Orientation $=$ Down

Actual cavity height $=2.5$ inches

Sight line to bottom of glass $=0$ inches

Spacer height $=0$ inches

Edge of Glass Dimension $=2.5$ inches

Draw spacer $=$ not checked

THERM - [Untitled-1]

㒼 File Edit View Draw Libraries Qpptions Calculation Window Help

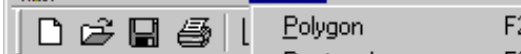

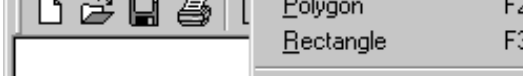

F3

Boundary Conditions F10

Fill Void

Insert Point Shift+FG

Delete Point Del

Edit Points

Move Polygon F11

Step 3

Import Divider

Edge glazing

system

Iape Measure F8

Set Drawing Scale Shift+F8

Set Drawing Scale Shift+F8

$\downarrow$

Set Qrigin

Shift+F7

Repeat mode

\section{Locator}

Shift+F2

Flip

Rotate

Clear Bad Points

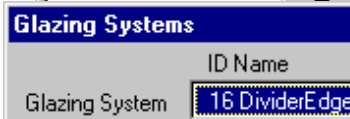

Step 4

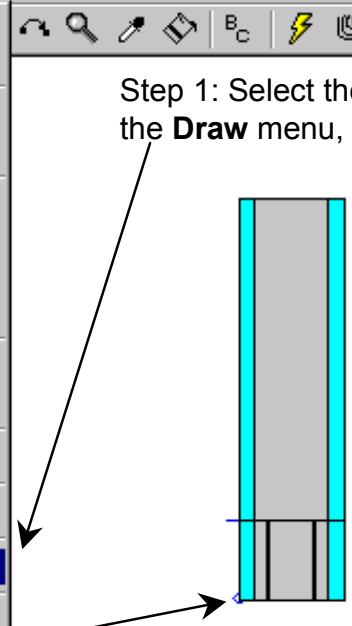

Step 2: Click on the lower left corner of the glazing system to reposition the Locator.

\begin{tabular}{|c|c|c|}
\hline \# Layers 2 & & Import \\
\hline Ucenter $\longdiv { 0 . 4 9 }$ & $\mathrm{Btu} / \mathrm{h}-\mathrm{ft} 2-\mathrm{F}$ & \\
\hline Thickness 0.815 & & \\
\hline
\end{tabular}

ᄃ Use Cl Model for Window 4 Glazing Systems

-WINDOW4 Glazing System Library File

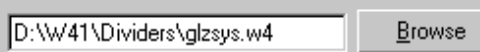

Insert the Divider Edge glazing

system with the following settings

- $\quad$ Orientation = Down

- Actual cavity height $=2.5$ (not used)

- $\quad$ Sight line to bottom of glass $=0$

- $\quad$ Spacer height $=0$

- $\quad$ Edge of Glass Dimension $=2.5$

- Draw spacer $=$ not checked

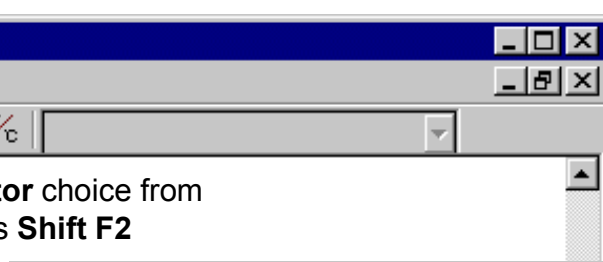


- Click on the Add as additional glazing system radio button when asked.

- The $3^{\text {rd }}$ glazing system is inserted below the Divider Glass glazing system.

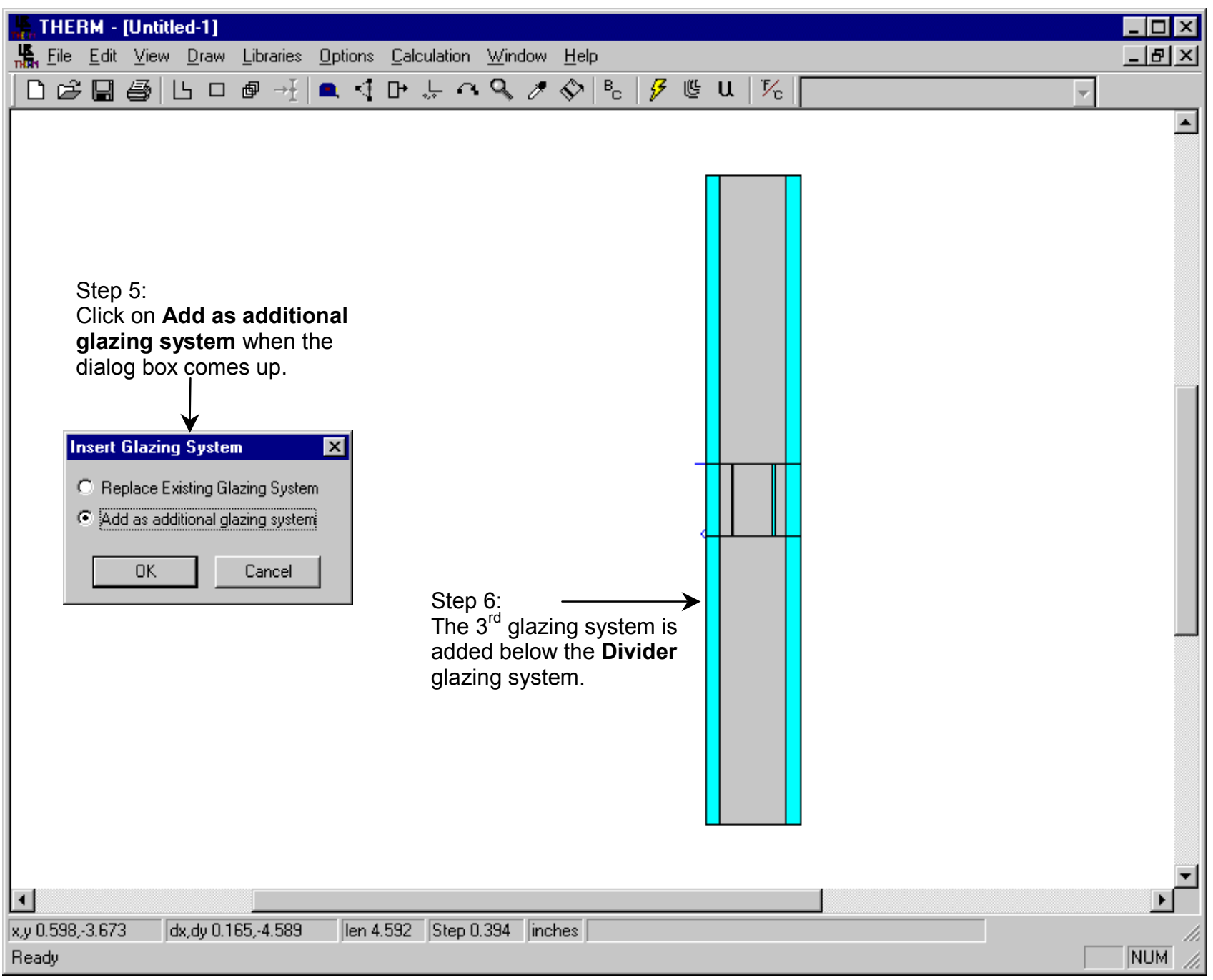

Figure 8-18 In THERM, add the Divider Edge glazing system again as a third glazing system. 
4. Add the top and bottom elements of the aluminum spacer.

- In the Options menu, Preferences menu choice, Drawing Options tab, make sure the Allow editing of IG polygons is checked.

- Click on the Divider air gap to select that polygon.

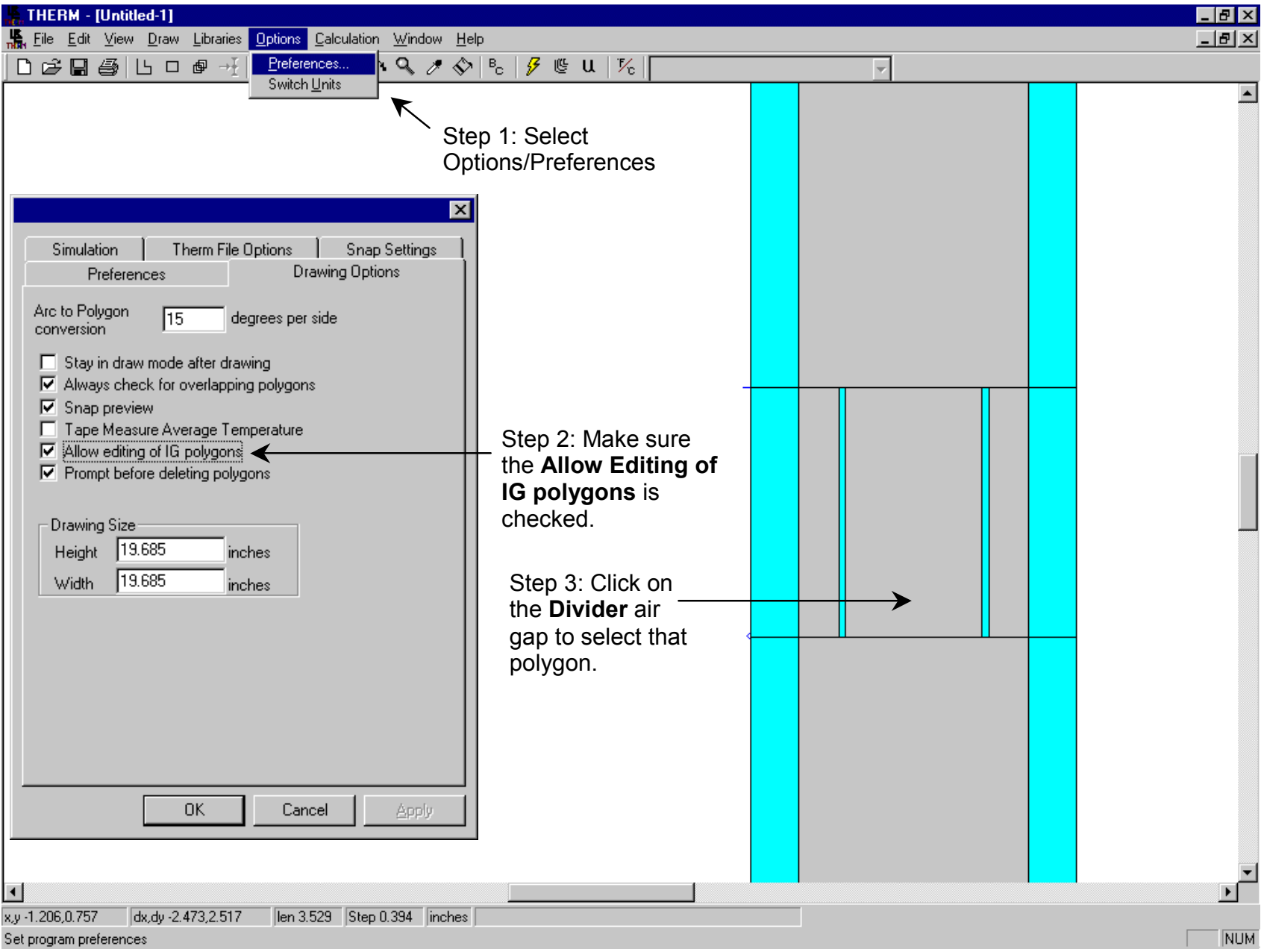

Figure 8-19 In THERM, check the Allow editing of IG polygons Drawing Option in order to edit the cavity inside the divider.. 
- Edit the air space in the divider:

Click on the air gap polygon to select it

Click on Edit Points toolbar button

Set the Step Size to $0.016 "$

Move the top and bottom edges of the air space the distance of the Step Size using the arrow keys.

Figure 8-20 Edit the divider cavity in order to place the horizontal aluminum pieces. 
- Use the Fill Void tool to fill the top and bottom voids with polygons of material = Aluminum Alloys.

THERM - [Untitled-1]

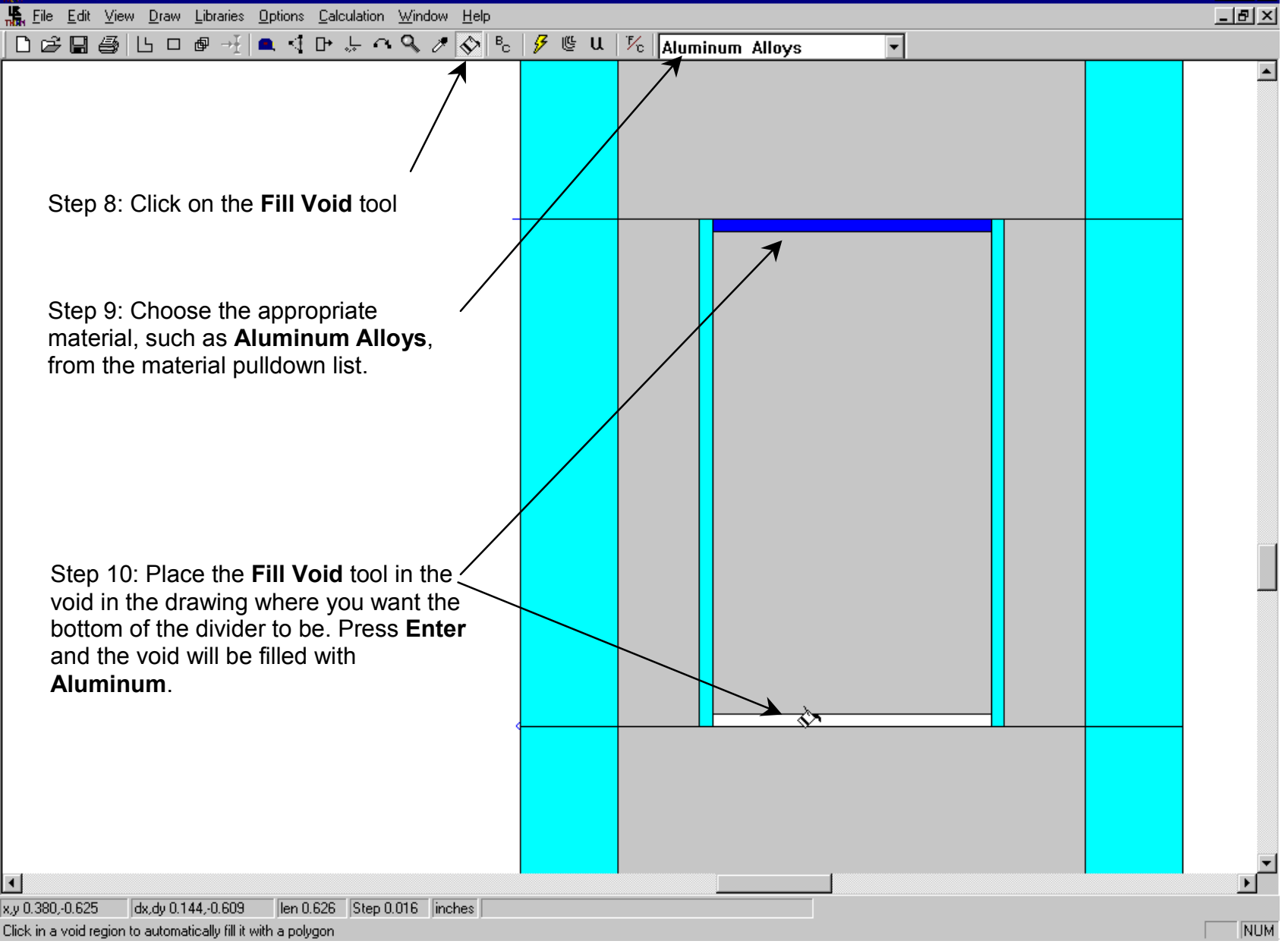

Figure 8-21 Use the Fill Void toolbar button to add the Aluminum divider element. 
5. Assign Boundary Conditions (BC)

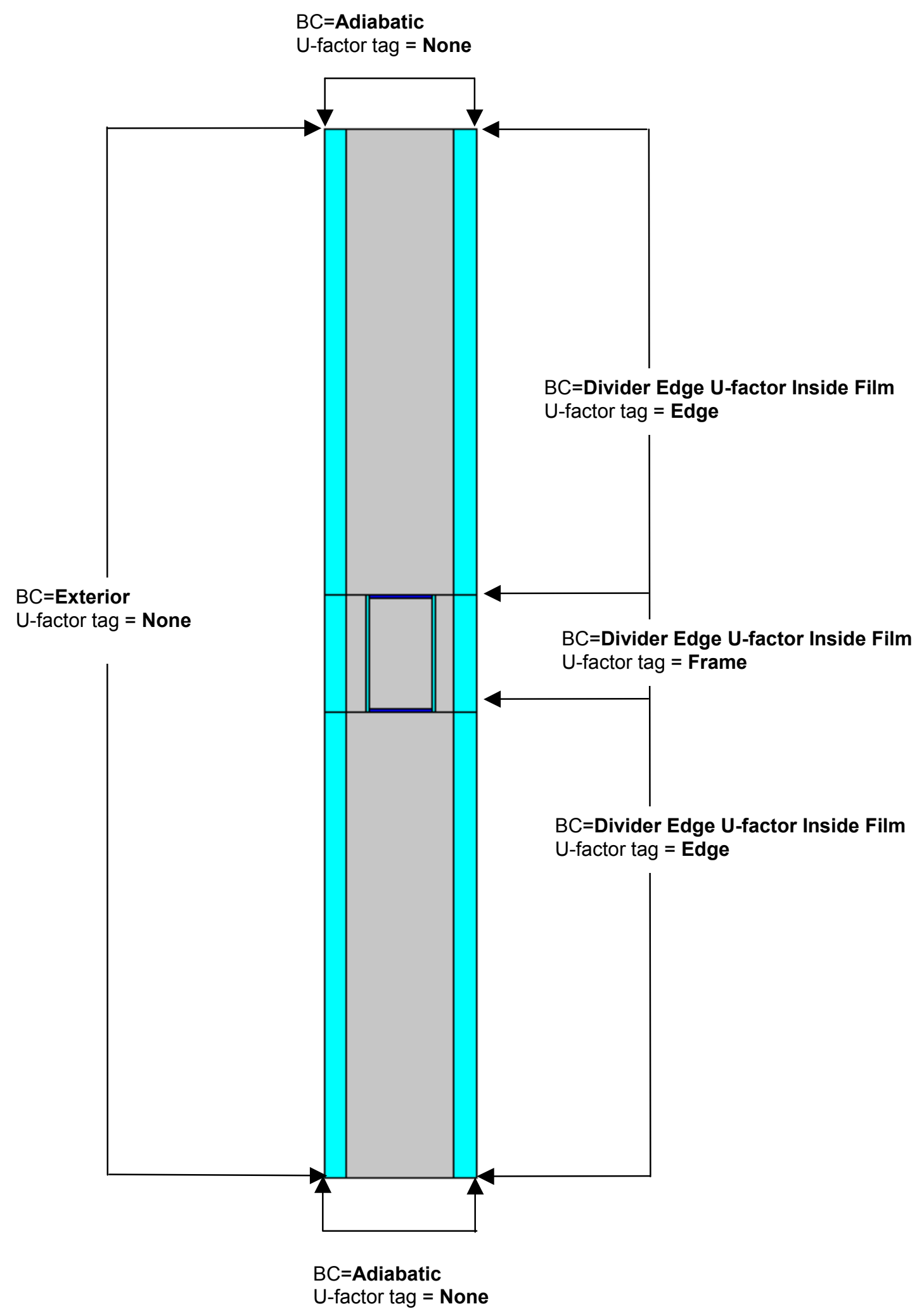

Figure 8-22 Assign the boundary conditions. 
6. Calculate the results

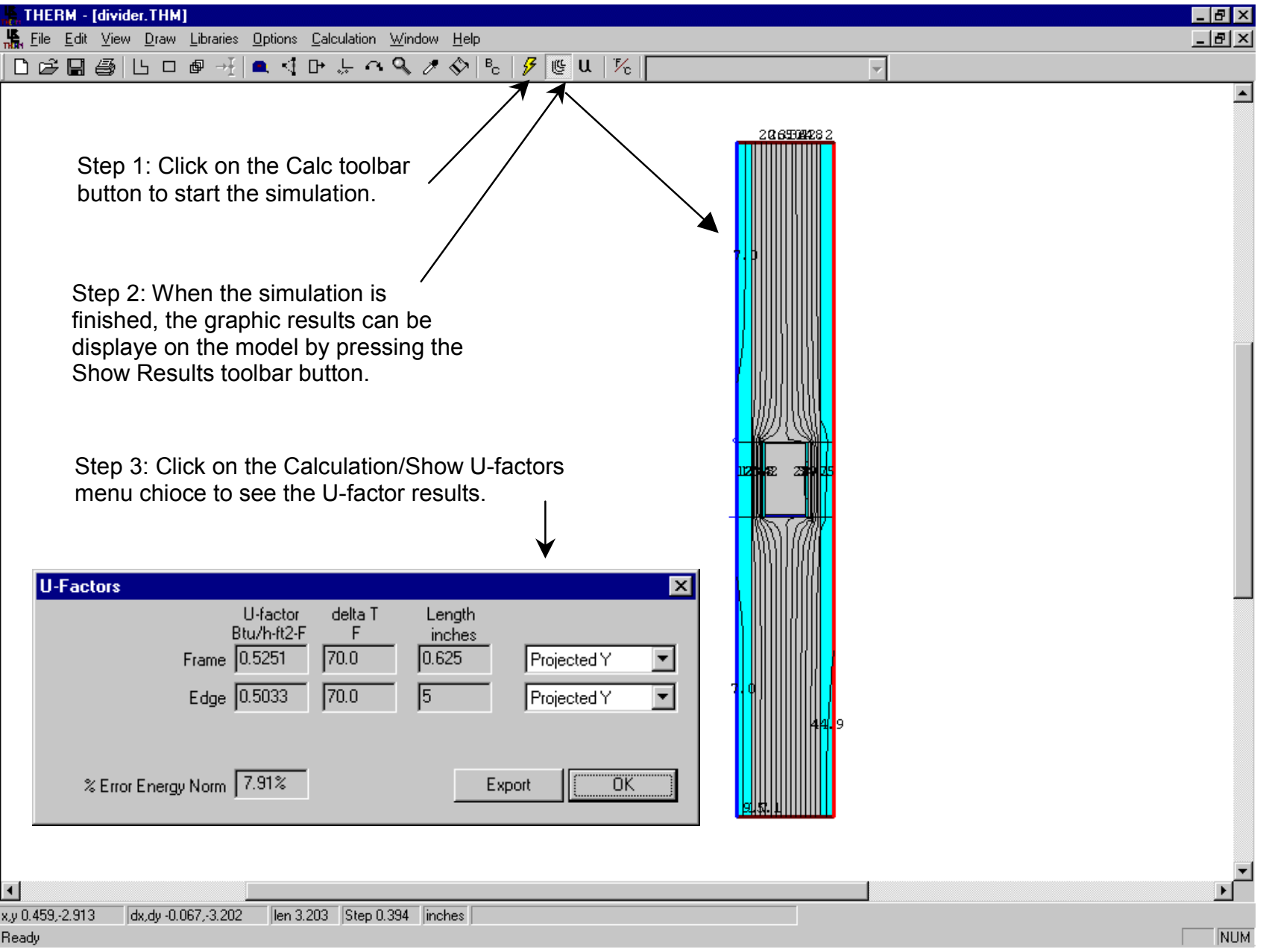

Figure 8-23 Calculate the results.

7. Save the file using the File/Save As menu choice

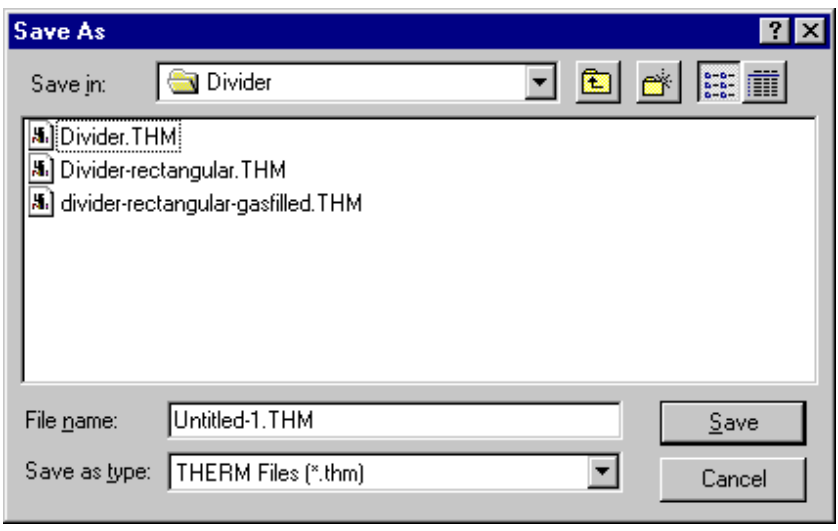

Figure 8-24 Save the THERM file. 
8. Export the results to WINDOW 4.1 as ${ }^{*} . \mathbf{T 2 W}$.

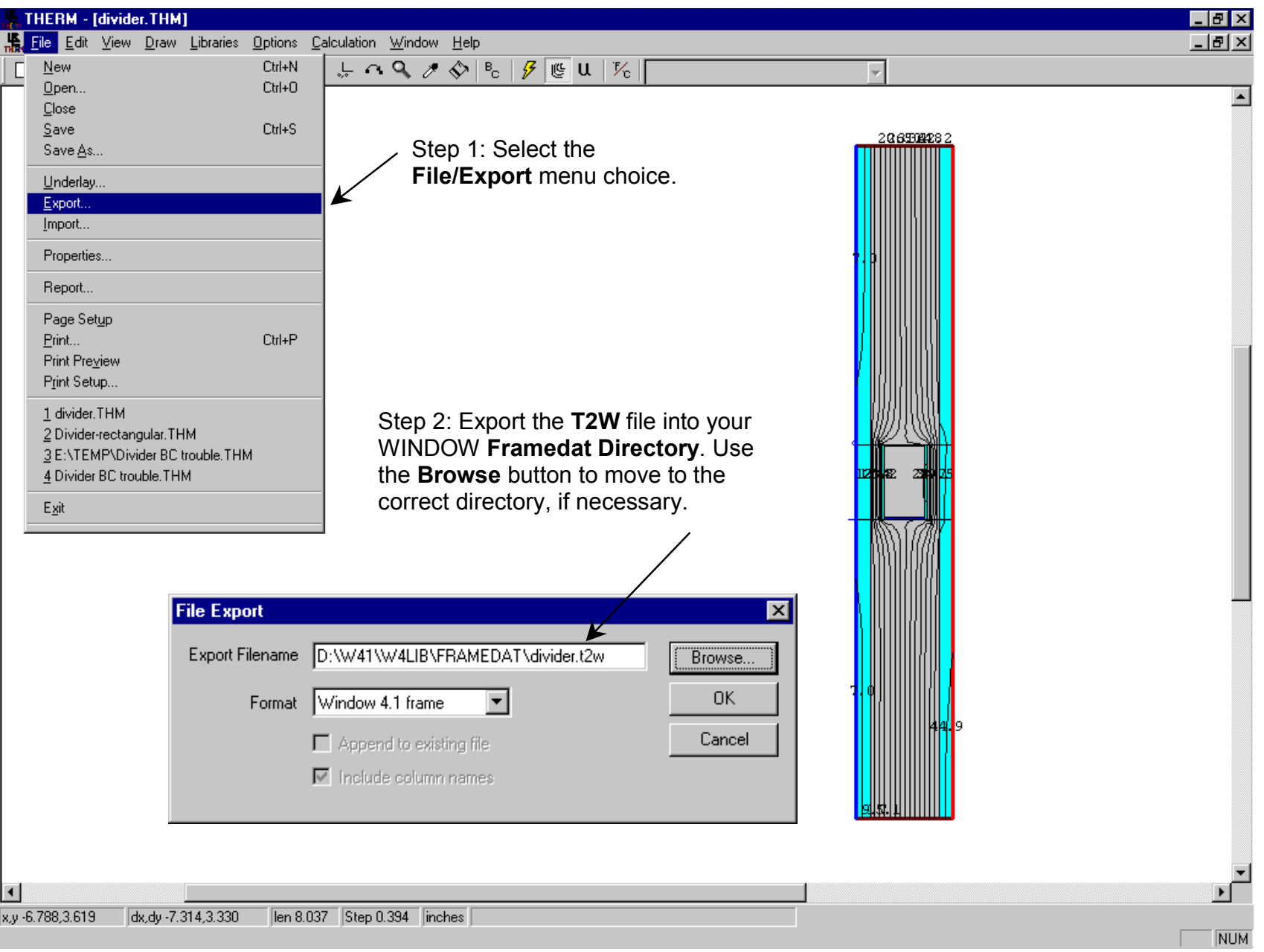

Figure 8-25 Save the THERM file.

THERM will always write the T2W file when a thm file is saved if the Options/Preferences option Automatic WINDOW 4 Export on Save is checked

Check the Automatic WINDOW 4 Export on Save option to create the T2W file each time a thm file is saved and after a calculation is completed.

This causes the $\mathbf{T} \mathbf{2} \mathbf{W}$ file to be saved in the same directory as the thm file, so the best place to work on these files is in the W4/Framedat directory.

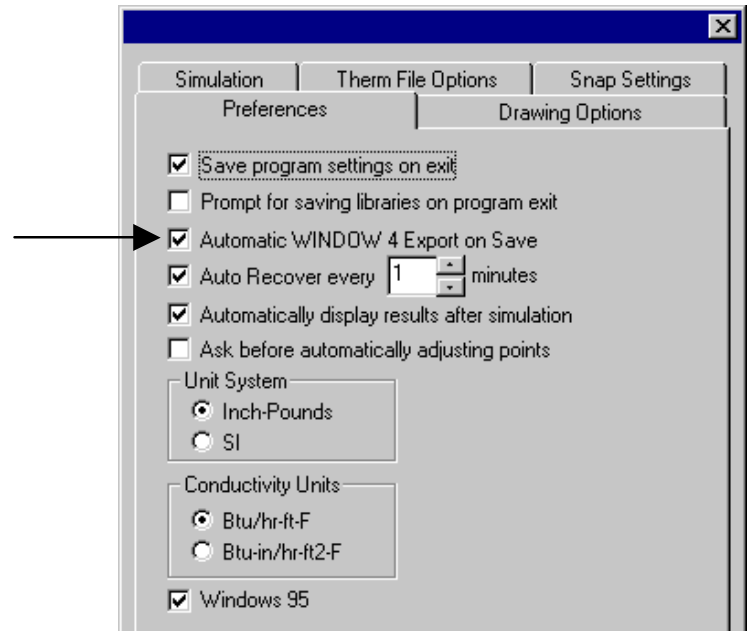

Figure 8-26 Check the Automatic WINDOW 4 Export on Save option to have the T2W files made automatically. 
9. Import the *.T2W results into WINDOW 4.1 in the Divider Library. Make sure the absorptivity (Abs field) is set to 0.50 for the dividers, per NFRC

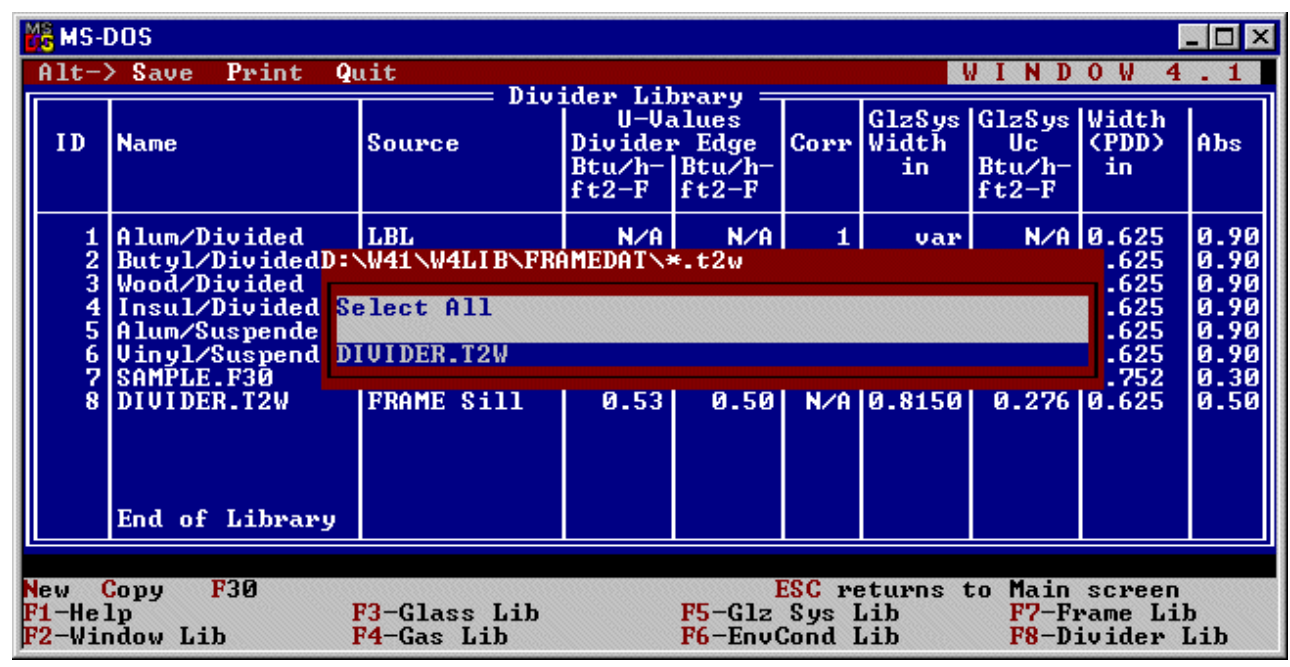

Figure 8-27 Import the divider T2W file into WINDOW.

10. Use the new divider in the calculation of the complete product values in the main screen of WINDOW. 


\subsubsection{Contoured Dividers}

For contoured dividers, when true geometry is being modeled, it is necessary to model the divider as a glazing system in WINDOW in order to obtain effective conductivity (Keff) values for the glazing system cavities (between the glass layers on each side of the divider as well as the cavity inside the divider) as shown in Figure 8-28. These Keff values are used to make new materials in THERM which are then assigned to cavities 1, 2 and 3. Use the half-way point between the largest and smallest gap width to determine how to model the Divider glazing system in WINDOW, as shown in Figure 8-28.

The technique for the contoured divider is as follows:

- In THERM, draw the true geometry of the divider

- In WINDOW, create a glazing system for the divider case (see the previous discussion about WINDOW) to get Keff values for Cavities 1, 2 and

- Include the appropriate air or gas fill for each cavity. For gas-filled glazing systems, assume the divider cavity (Cavity 2 ) is also filled with the same gas mixture.

- In THERM, make new materials with these Keff values.

- Fill Cavities 1, 2 and 3 with these new materials, using the correct Keff for the corresponding cavity.

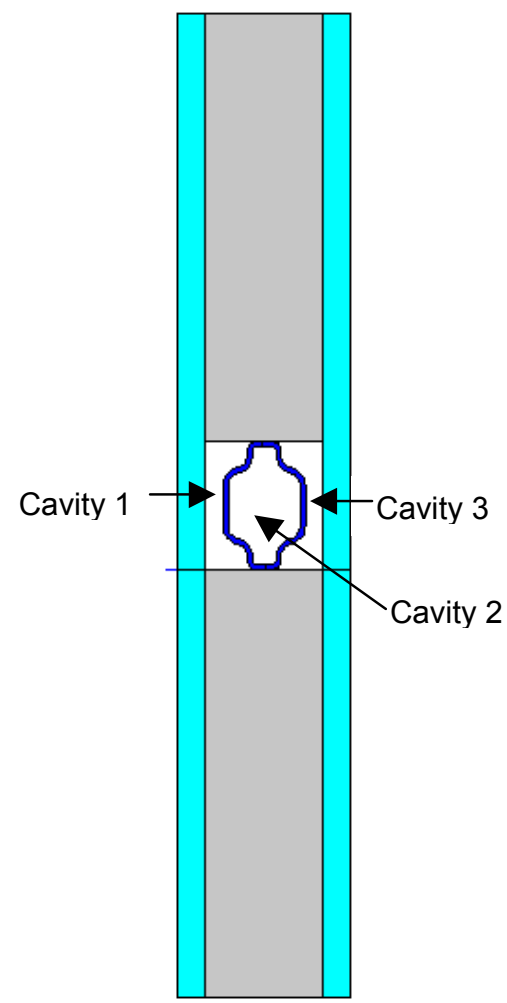

Figure 8-28 For a contoured divider, the true divider geometry is drawn and effective conductivities from WINDOW are used to define the properties of Cavities 1, 2, and 3.

The modeling steps, explained in detail in the following pages, are:

In WINDOW 4.1:

- Make a glass layer to represent the aluminum material in the divider. Make sure to correctly define the emissivities of the aluminum, i.e., 0.20 for the unpainted metal, and 0.90 for painted metal.

- Make a glazing system to represent the part of the glazing system which contains the divider, referred to in this manual as Divider. This is usually a 4 layer glazing system, with the following layers:

- Outer glass layer

- Gap between the outer glass layer and the outer side of the divider surface

- Divider layer (represented by a glass layer) 
- Gap between the outer divider surface and the inner divider surface

- Divider layer (represented by a glass layer)

- Gap between the inner divider surface and the inner glass layer

- Inner glass layer

- Write down the Keff of Gap1, Gap 2, and Gap3.

- Make a glazing system to represent the part of the glazing system which does not contains the divider, referred to in this manual as DividerEdge.

In THERM:

- Make three new materials in the Material Library with the Keff values from WINDOW of Gap1, Gap 2, and Gap3.

- Assign the new materials to the appropriate cavities in the THERM model.

- Generate the boundary conditions and simulate the results. 


\section{Example of Contoured Divider}

Figure 8-29 shows an example of a contoured divider in the same glazing system used in the previous example. When defining the divider in WINDOW, use the mid-point between the largest and smallest gap widths in each cavity to determine the gap widths in the WINDOW glazing system.

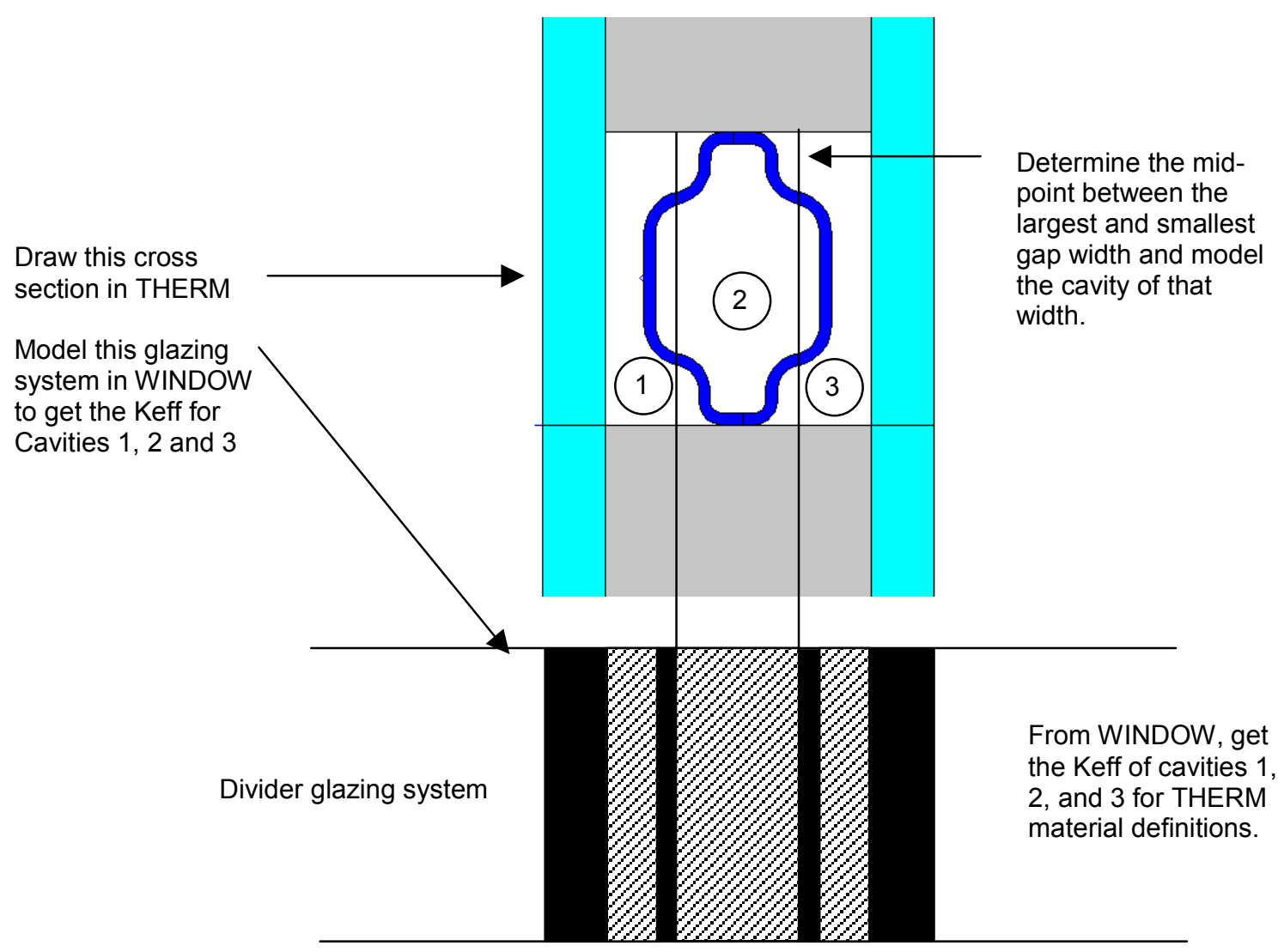

Figure 8-29 Use the same technique as in 8.3.1 for contoured dividers in air filled glazing cavities. 


\section{In WINDOW 4.1:}

1. Glass Library: To represent the aluminum divider, create a glass layer in the Glass Library called DividerAlum with the following characteristics:

- Thickness $=0.016(0.4 \mathrm{~mm})$

- Transmittances $=0$

- Reflectances $=0$ (not used by the calculation)

- Emissivity $=0.90$ for the painted metal side, and 0.20 for the unpainted metal side

- Conductivity $=92.448 \mathrm{Btu} / \mathrm{h}-\mathrm{ft}-\mathrm{F}$.

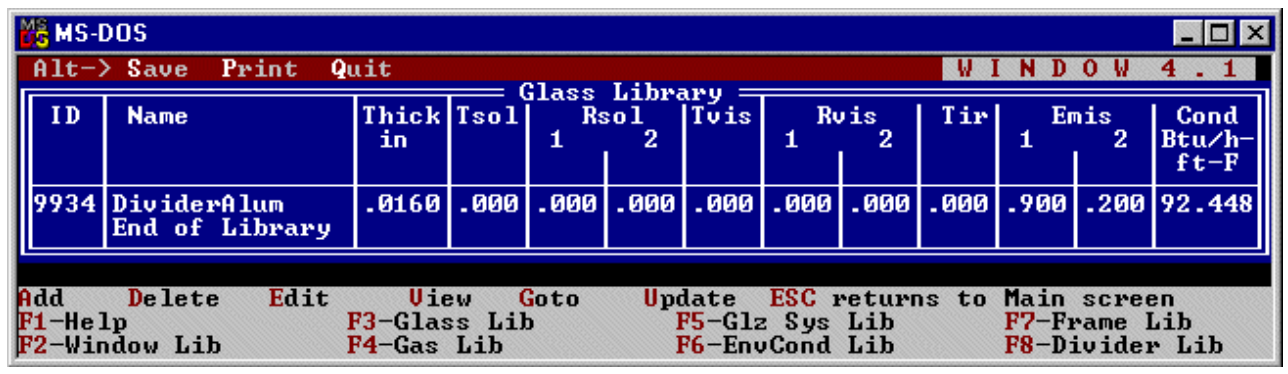

Figure 8-30 In the WINDOW Glass Library, define a glass layer to represent the aluminum divider.

2. Glazing System Library: Make a glazing system that represents the Divider Edge (the section without the divider) and Divider (the section with the divider), as shown in Figures 8-31 and 8-32. The Keff values for Gap1, Gap2, and Gap3 will be used to make THERM materials with those properties. In this example, the glazing systems are filled with air but the techique is exactly the same if they are filled with a gas mixture.

- Divider Edge:

Make a glazing system which represents the cross section of the product that does not have the divider. For this example, it is a two-layer model with the following construction:

- Layer 1

- Gap 1

- Layer 2

$$
\begin{aligned}
& =\text { Glass \#102,Clear_3 }(3 \mathrm{~mm}) \\
& =\text { Air cavity, 0.575" thick }(14.6 \mathrm{~mm}) \\
& =\text { Glass \#102, Clear_3 }(3 \mathrm{~mm})
\end{aligned}
$$

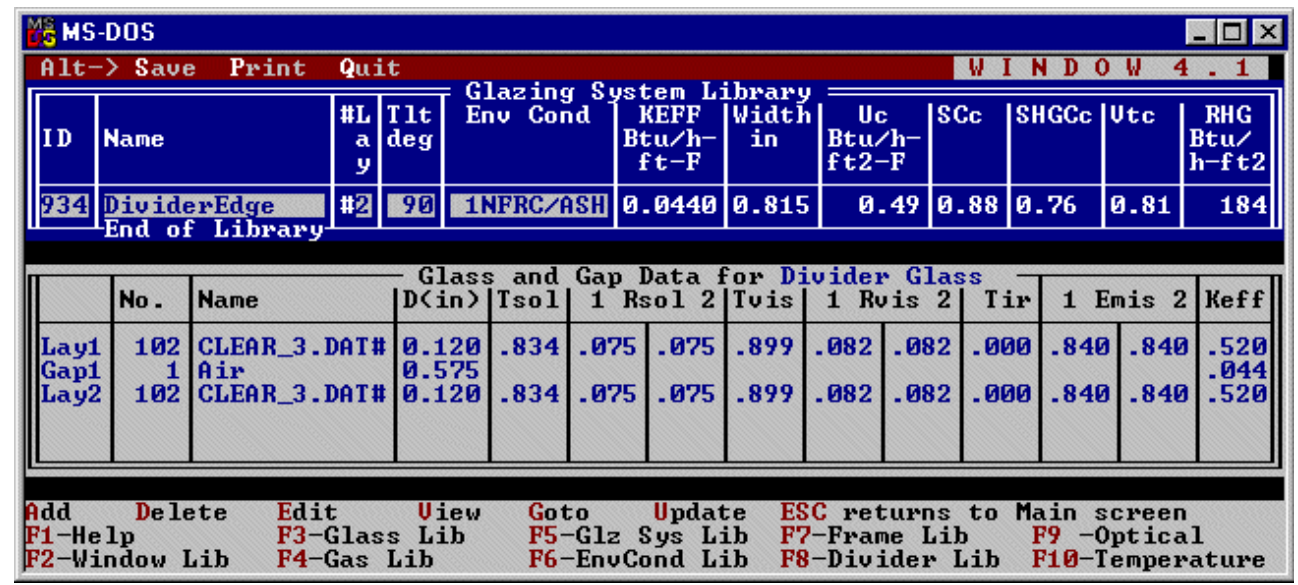

Figure 8-31 In the WINDOW Glazing System Library, define Divider Edge. 


\section{- Divider:}

Make a glazing system to represent the divider portion of the glazing system, as shown in Figures 8-30 and 8-32. For this example, it is a four-layer model with 2 glass layers and 2 middle layers set to DividerAlum:

- Layer $1=$ Glass \#102, Thickness of 0.120" (3 mm)

- Gap $1=$ Air, Thickness $=0.10^{\prime \prime}(2.5 \mathrm{~mm})$

- Layer 2 = DividerAlum, Thickness $=0.016 "(0.4 \mathrm{~mm})$

- Gap $2=$ Air, Thickness $=0.343^{\prime \prime}(8.7 \mathrm{~mm})$

- Layer 3 = DividerAlum, FLIPPED to get the correct emissivities on each surface, Thickness $=0.016^{\prime \prime}(0.4 \mathrm{~mm})$

- Gap $3=$ Air, Thickness $=0.10^{\prime \prime}(2.5 \mathrm{~mm})$

- Layer 4 = Glass \#102, Thickness of 0.120" (3 mm)

- Write down the resulting Keff for Gap1, Gap2 and Gap3, as shown in Figure 8-32. These values will be used to make materials with these properties in THERM:

- $\quad$ Keff for Gap1 $=0.031$

- Keff for Gap2 = 0.029

- $\quad$ Keff for Gap3 $=0.035$

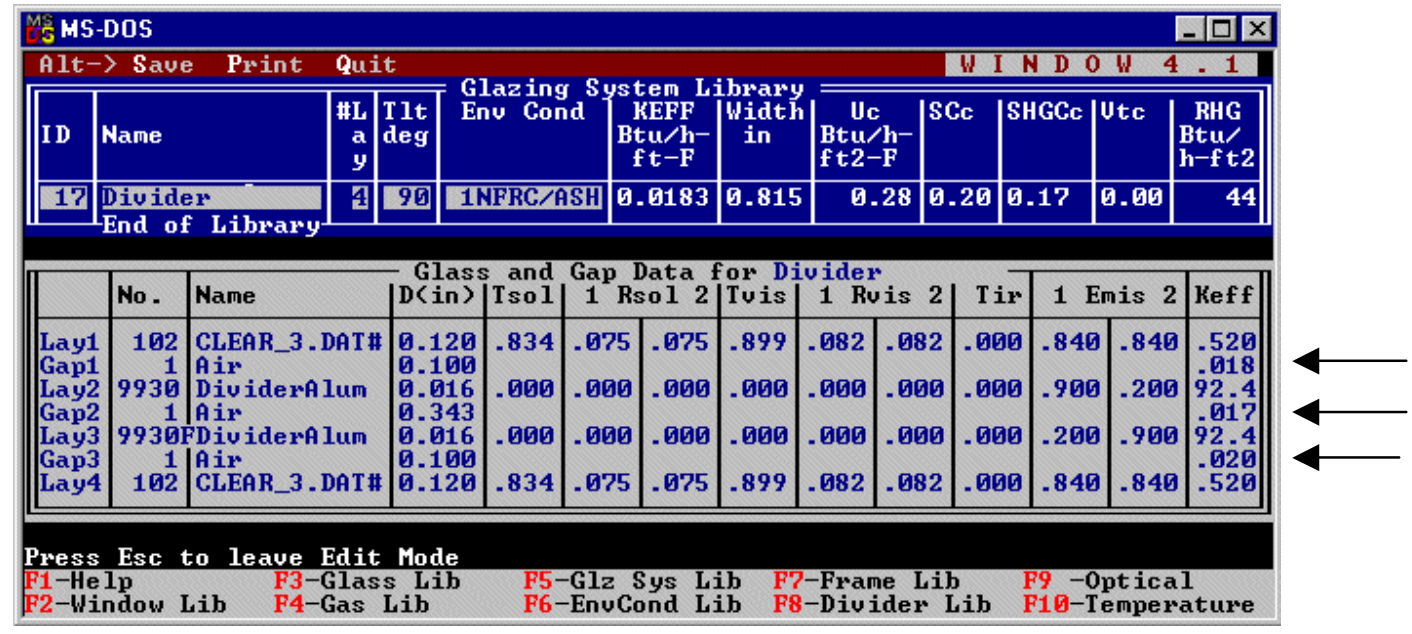

Write down the Keff values for Gap1, Gap2, and Gap3. These will be used to make materials in THERM with these properties.

Figure 8-32 In the WINDOW Glazing System Library, define a the Divider glazing system. 
In THERM:

1. Import the Divider Edge glazing system made in WINDOW, with the following settings:

- $\quad$ Orientation $=\mathbf{U p}$

- $\quad$ Cavity height $=2.5$

- Sight line to bottom of glass = height of the divider (in this example it is 0.750 )

- $\quad$ Spacer height $=$ height of the divider (in this example it is 0.75 )

- $\quad$ Edge of glass $=\mathbf{2} .5$

- $\quad$ Draw spacer $=$ Not checked

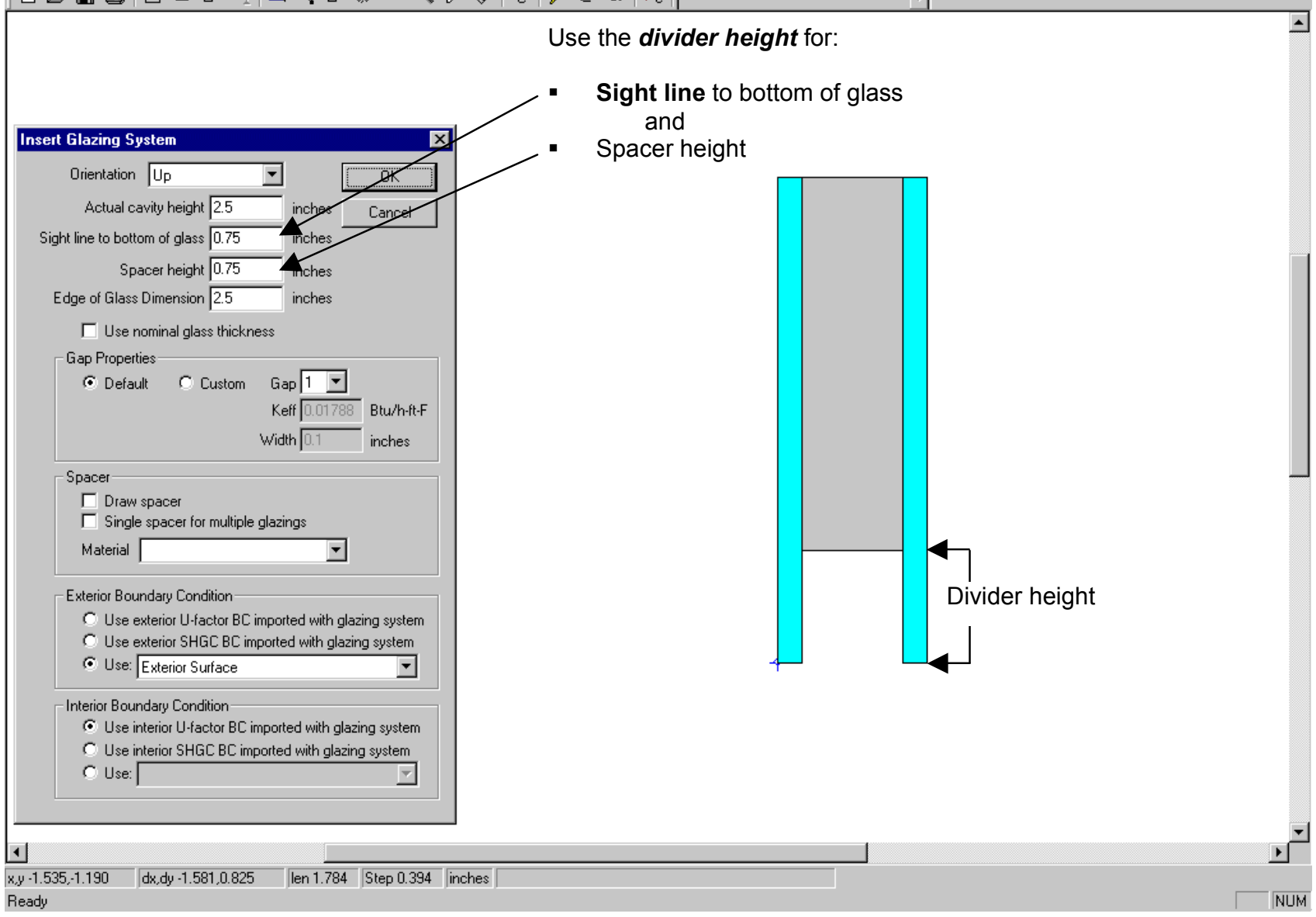

Figure 8-33. Import the first glazing system. 
2. Import the Divider Edgeglazing system again as an additional glazing system, below the first one; the locator does not have to be moved. Use the following settings for this glazing system:

- Orientation $=$ Down

- $\quad$ Cavity height $=\mathbf{2 . 5}$

- Sight line to bottom of glass $=0$

- Spacer height $=\mathbf{0}$

- $\quad$ Edge of glass $=\mathbf{2} .5$

- $\quad$ Draw spacer $=$ Not checked

Insert the glazing system as an Additional Glazing.

Figure 8-34. Import the glazing system again facing down. 
3. Draw (or copy and paste from another THERM file) the polygons in the cavity that represent the contoured divider. Figure 8-35 shows the divider for this example drawn with the material set to Aluminum Alloy

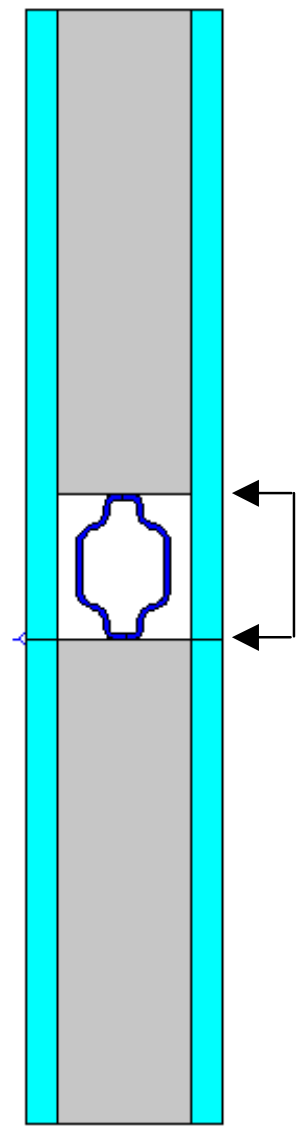

Draw the contoured divider in the cavity between the two glazing systems.

Figure 8-35. Draw the polygons to represent the contoured divider. 
4. Define three new materials in the Material Library, one each for the Keff of Gap1, Gap2, and Gap3 from WINDOW, as shown in Figure 8-36. Because these materials are defined as solids, the emissivity value is irrelevant, and therefore it is not necessary to change the default value of 0.90 .
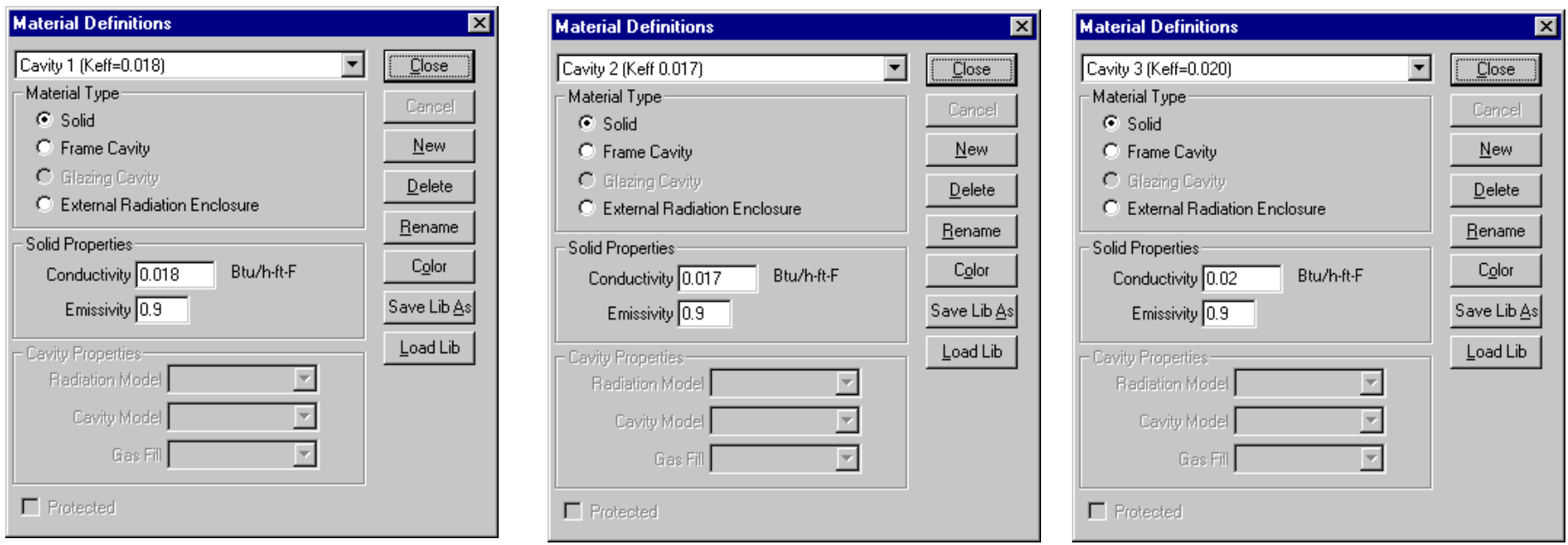

Figure 8-36 In the THERM Material Library, add three new materials, one for each Keff value for Gap1, Gap2, and Gap3.

5. Fill cavities 1, 2, and 3 in the THERM cross section with the material with the appropriate Keff values. In this example, Cavity 1 would have the material set to Keff $=0.018$, Cavity 2 would have thematerial set to Keff $=0.017$, and Cavity 3 would have the material set to Keff $=0.020$, as shown in Figure 8-37.

Use the Fill Void tool to fill Cavity 1 with the Material defined for this cavity from WINDOW; for this example, it is named Cavity 1 (Keff=0.018).

Fill Void tool to fill Cavity 2 with the Material defined for this cavity from WINDOW; for this example, it is named Cavity 2 (Keff=0.017).

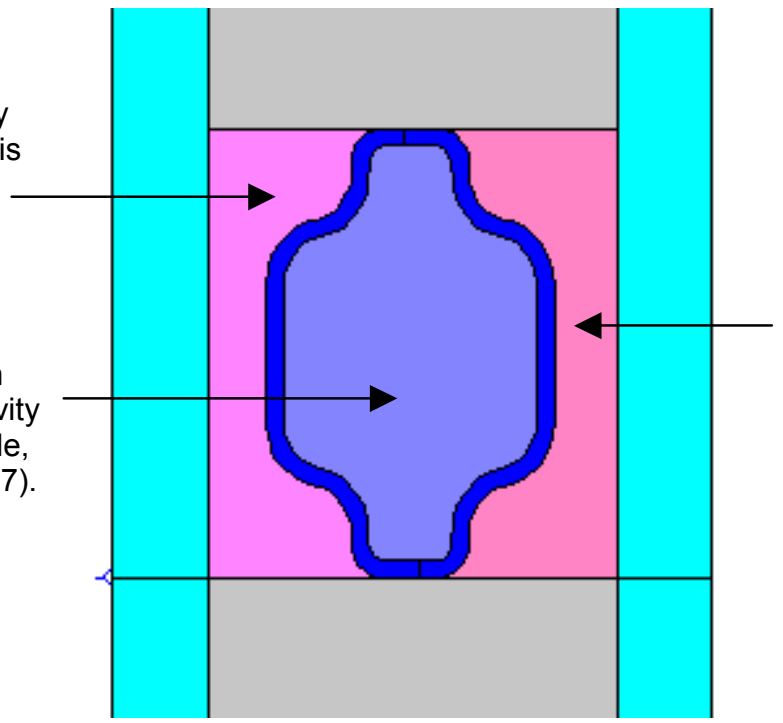

Fill Void tool to fill Cavity 3 with the Material defined for this cavity from WINDOW; for this example, it is named Cavity 3 (Keff=0.020).

Figure 8-37 In THERM, use the Fill Void tool to fill Cavities 1, 2, and 3 with the materials defined with the Keff values from WINDOW. 
6. Assign Boundary Conditions (BC)

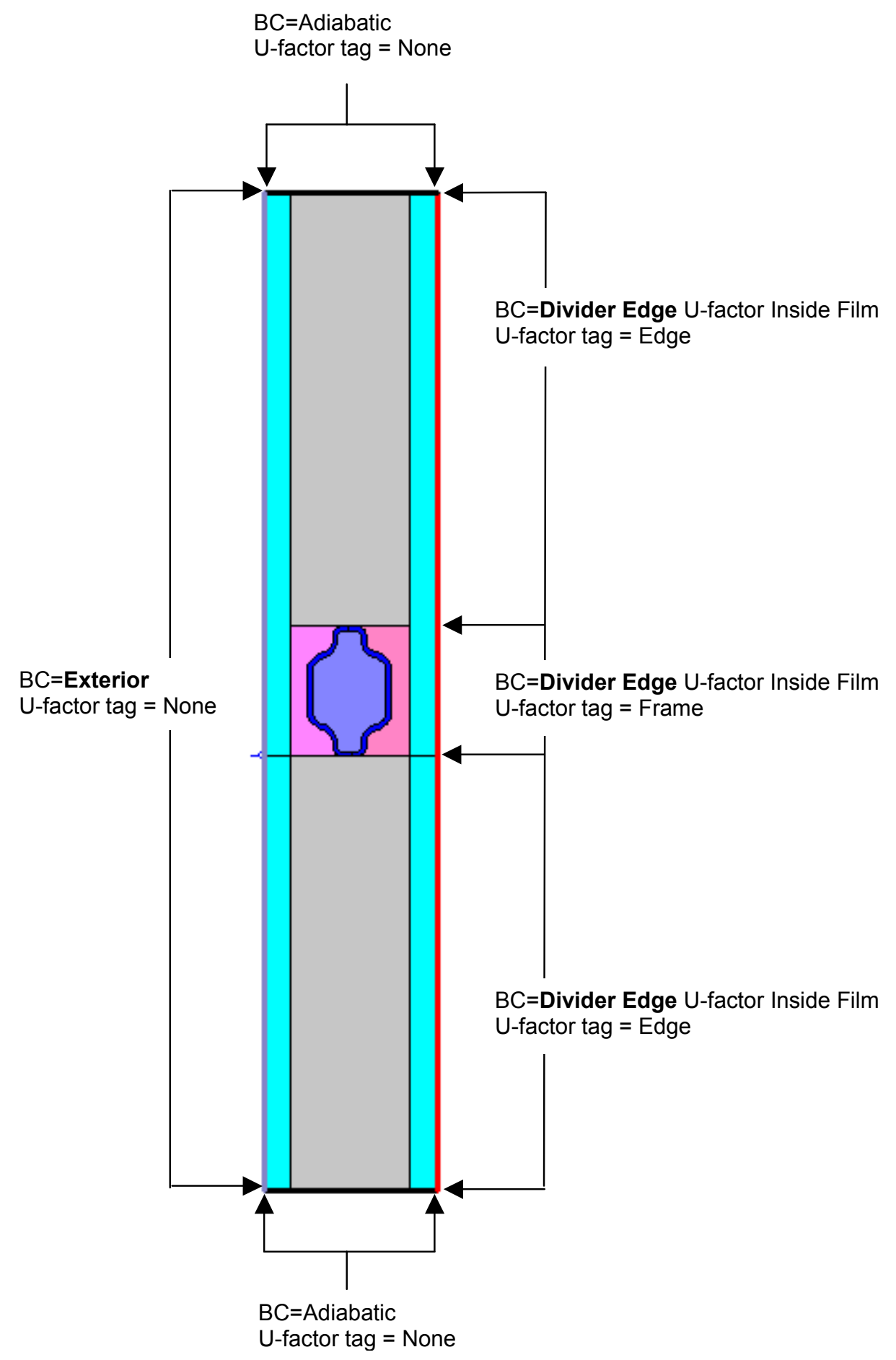

Figure 8-38 Assign the boundary conditions. 


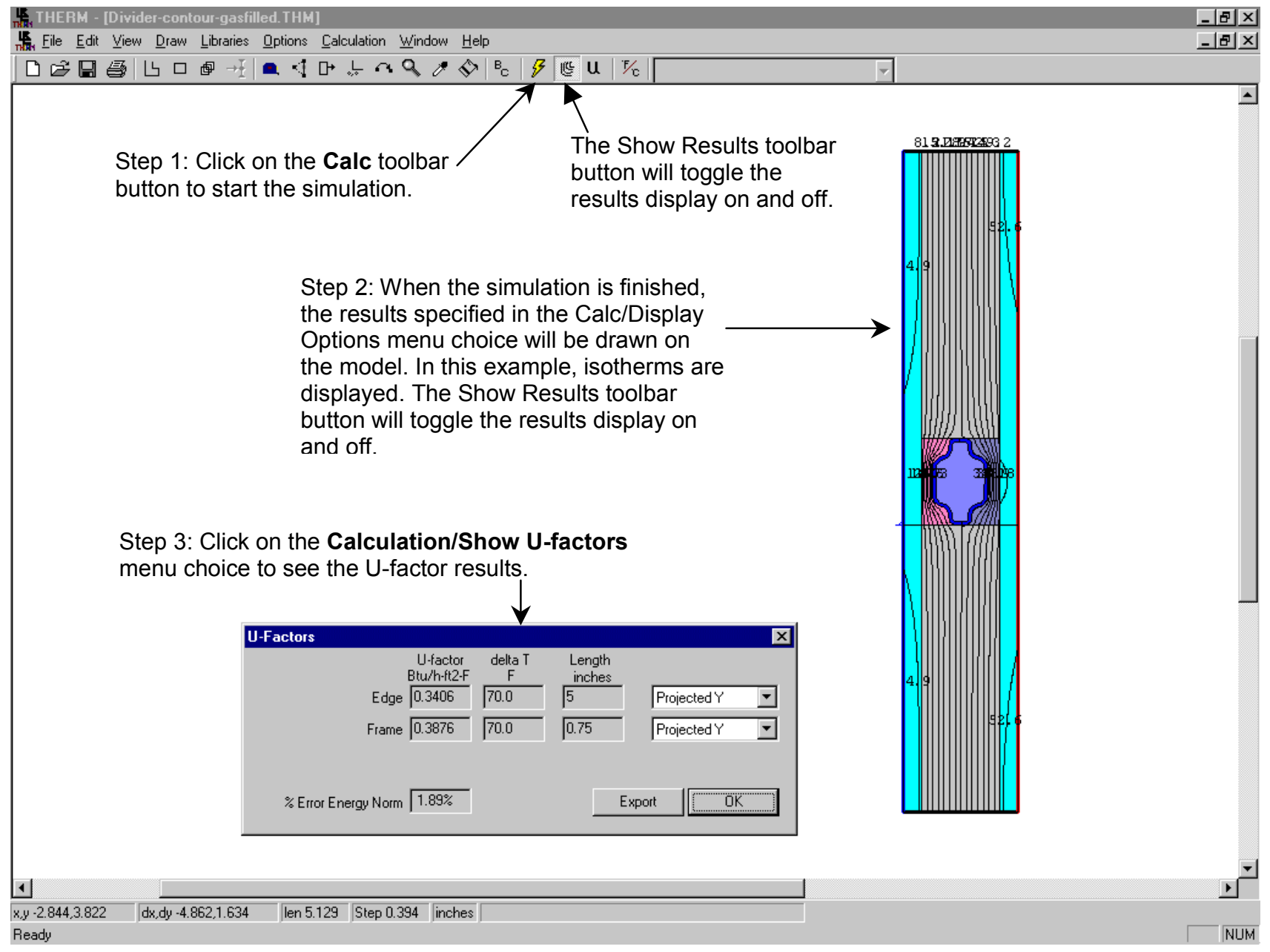

7. Calculate the results

Figure 8-39 Calculate the results.

8. Save the file using the File/Save As menu choice

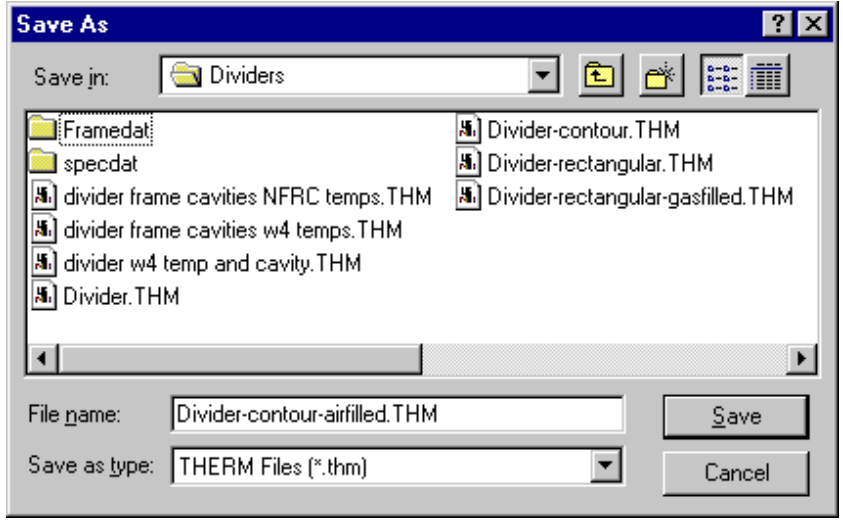

Figure 8-40 Save the THERM file. 
8. Export the results to WINDOW 4.1 as T2W files, as shown in Figure 8-41. See Section 7.4.12, "Export to WINDOW" in the THERM User's Manual for more information about exporting THERM files.

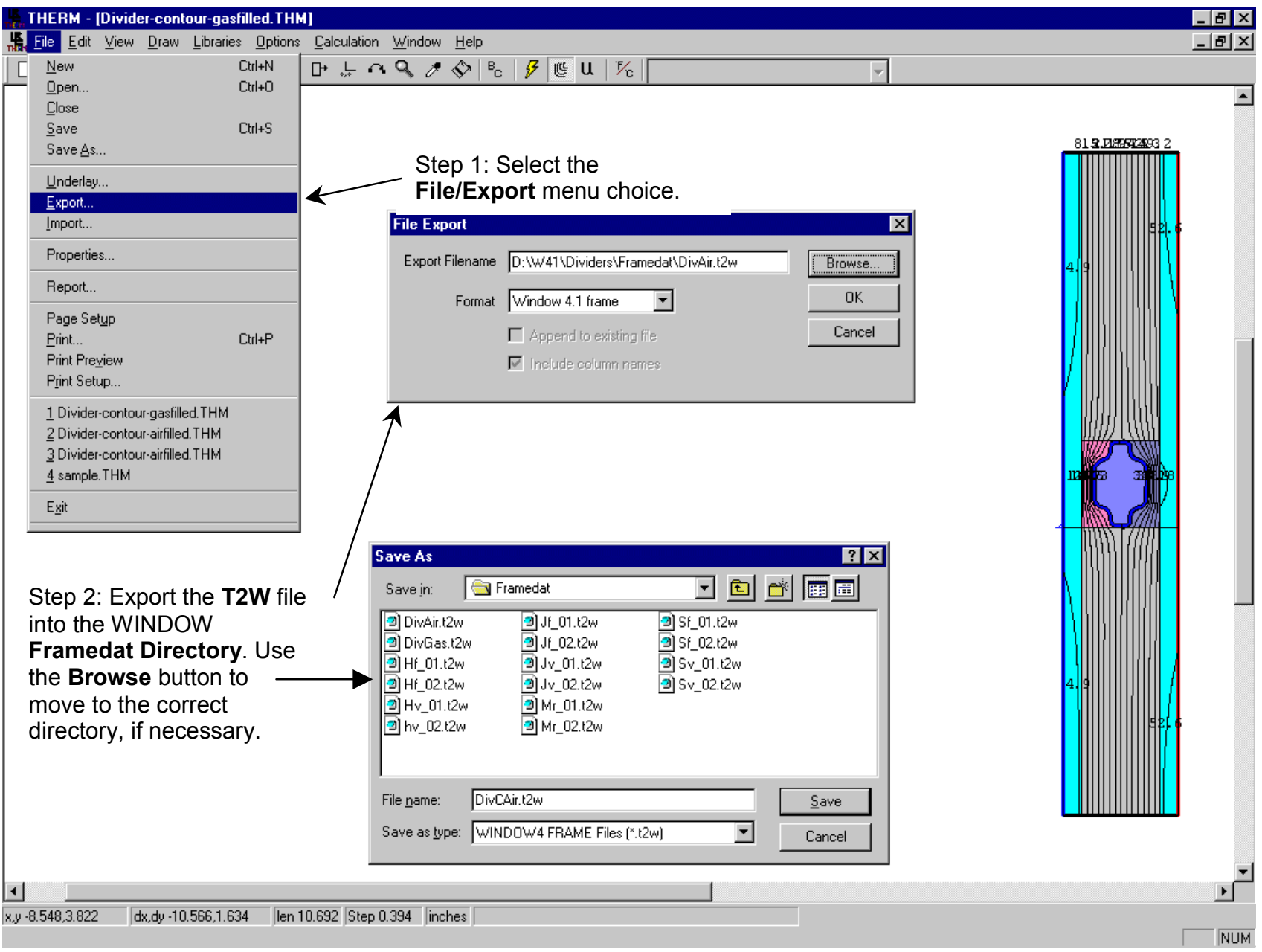

Figure 8-41 Export the results to a T2W file which can be imported into the WINDOW Divider Library.

THERM will always write the T2W when a thm file is saved if the Options/Preferences option Automatic WINDOW 4 Export on Save is checked

Check the Automatic WINDOW 4 Export on Save option to create the T2W file each time a thm file is saved.

This causes the $\mathbf{T} \mathbf{2} \mathbf{W}$ file to be saved in the same directory as the thm file, so the best place to work on these files is in the W4/Framedat directory.

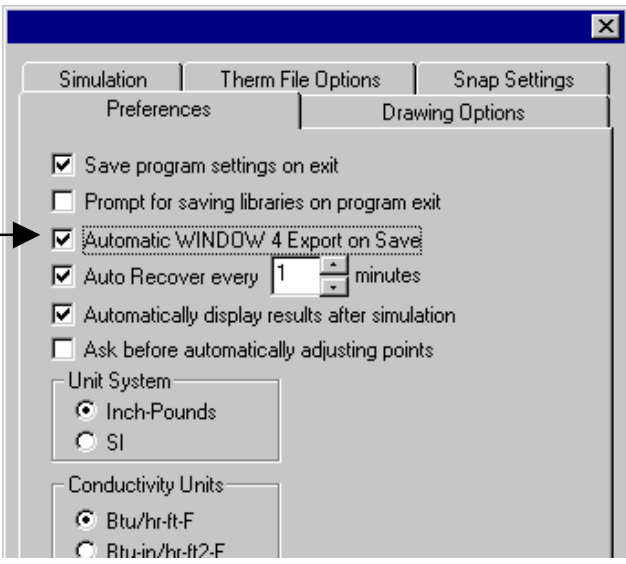

Figure 8-42 Check the Automatic WINDOW 4 Export on Save option to have the T2W files made automatically. 
9. Import the T2W results into WINDOW 4.1 in the Divider Library (F8). Make sure the absorptivity (Abs field) is set to 0.50 for the dividers, per NFRC procedures guidelines. See NFRC 200 for further information.

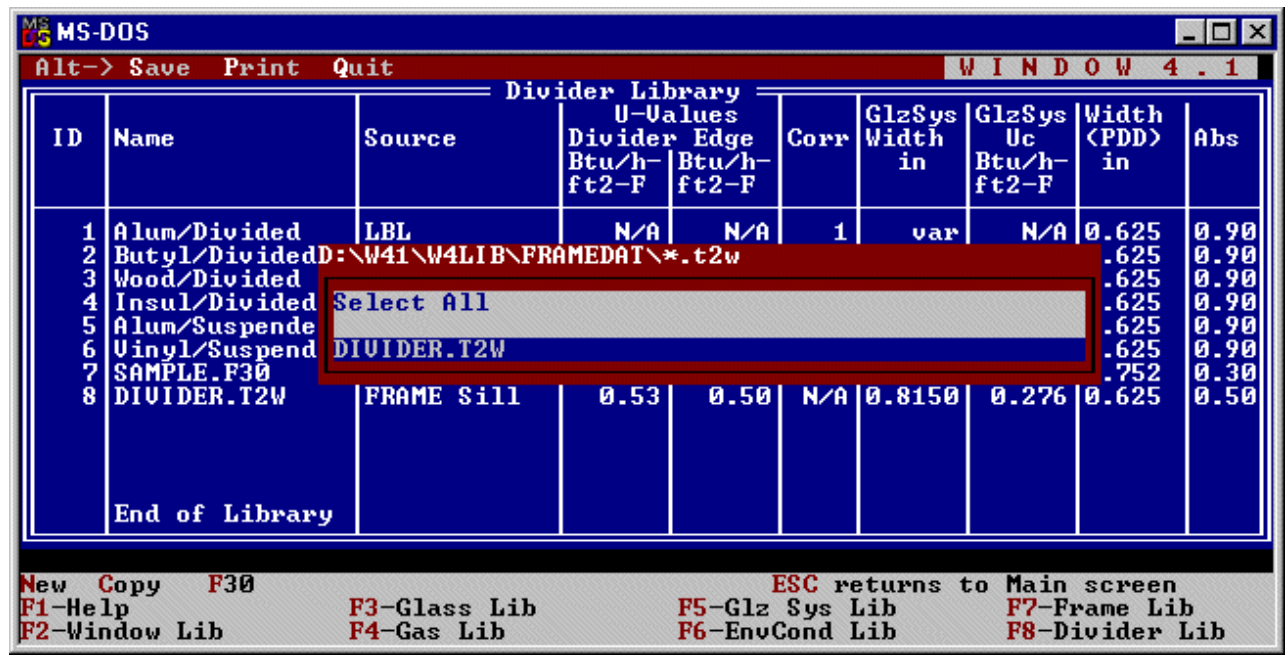

Figure 8-43 Import the $\mathbf{T} 2 \mathrm{~W}$ file into the WINDOW Divider Library .

9. Use the new divider in the calculation of the complete product values in the main screen of WINDOW. 


\subsection{Storm Windows}

Storm windows present a modeling problem different from most insulated glass (IG) units, because the

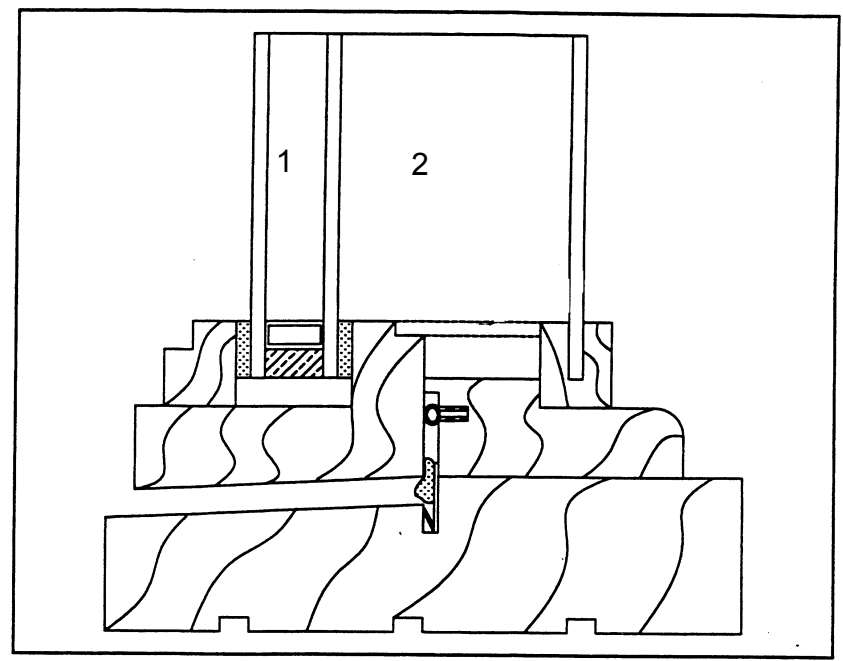

spacing between the IG unit and the storm window is usually quite large, as shown in Figure 8-44.

Figure 8-44 Product with an interior storm window.

\subsubsection{Modeling Steps}

The following steps can be used to model storm windows.

- Create a three-layer glazing system with the correct spacing between each of the glass layers.

- Create a second three-layer glazing system in the WINDOW Glazing System Library with a space of $1.375 "(34.93 \mathrm{~mm})$ between the IG and the storm window. Write down the Keff of the $1.375^{\prime \prime}$ cavity.

- It is necessary to calculate a weighted-average value for the effective conductivity (Keff) of the actual gap spacing if the gap is more than 1.375" when using values from WINDOW, using the

$$
\operatorname{Keff} d 2=\frac{d 2 *\left(\operatorname{Keff}_{1.375}\right)}{1.375}
$$

following formula:

where:

$\operatorname{Keff}_{\mathrm{d} 2}=$ effective conductivity (Keff) of the actual storm window cavity (cavity 2 in Figure 844)

Keff $_{1.375}=$ effective conductivity (Keff) of the storm window cavity $1.375 "$ thick.

- Bring the glazing system that represents the actual geometry into THERM, after drawing the frame for the product.

- When inserting the glazing, use the Custom Gas Properties feature and set the Keff of Gap 2 to the calculated value.

- Assign the boundary conditions 
- Simulate the problem

\subsubsection{Example Storm Window Problem}

The following example problem, based on the product in Figure 8-44, is explained in detail in the following discussion.

\section{In WINDOW:}

1. Make a glazing system consisting of three layers of glass, with the dimensions of the glazing cavity for the first gap, and the correct dimension from the glass to the storm window for the second gap.

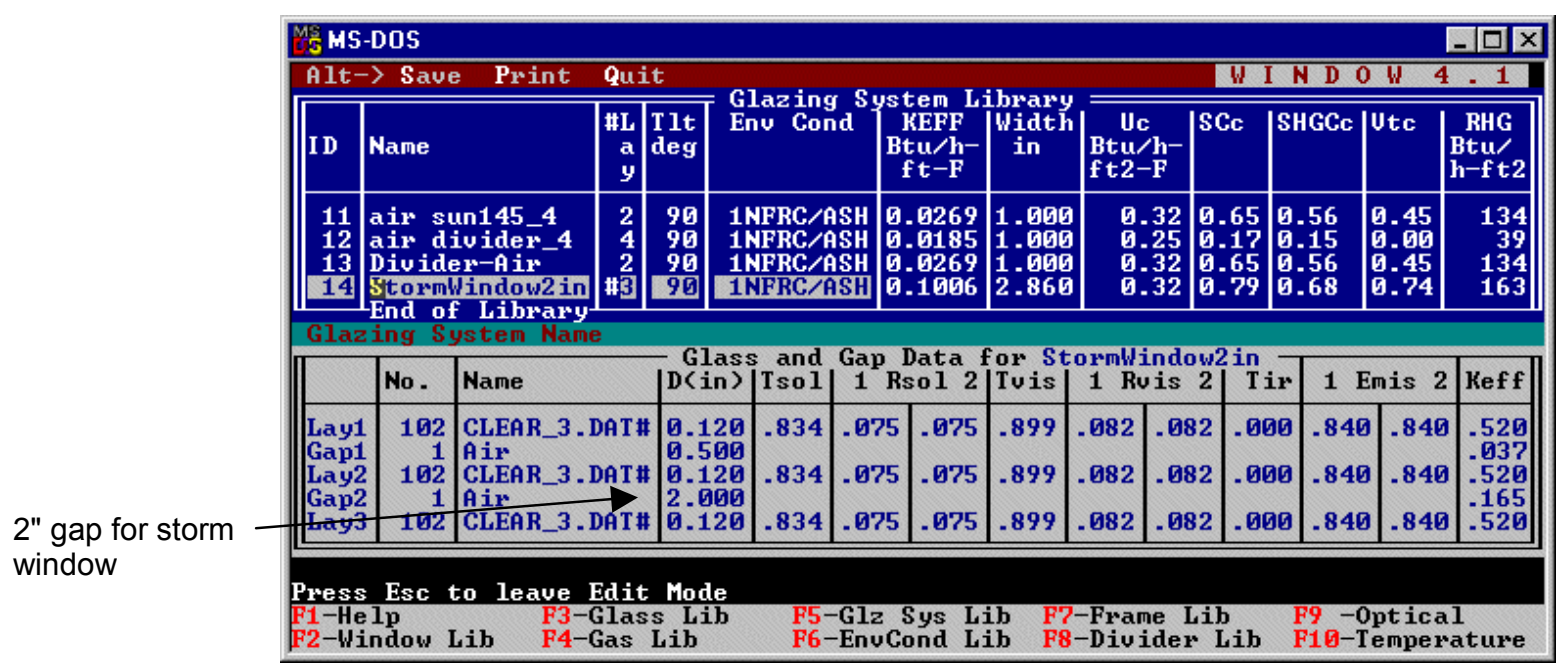

Figure 8-45 Make a triple glazed glazing system with a large gap width between the IG and the storm window.

2. Make a second glazing system like the first, except make the width of Gap 2 equal to 13/8". Write down the Keff value for Gap 2.

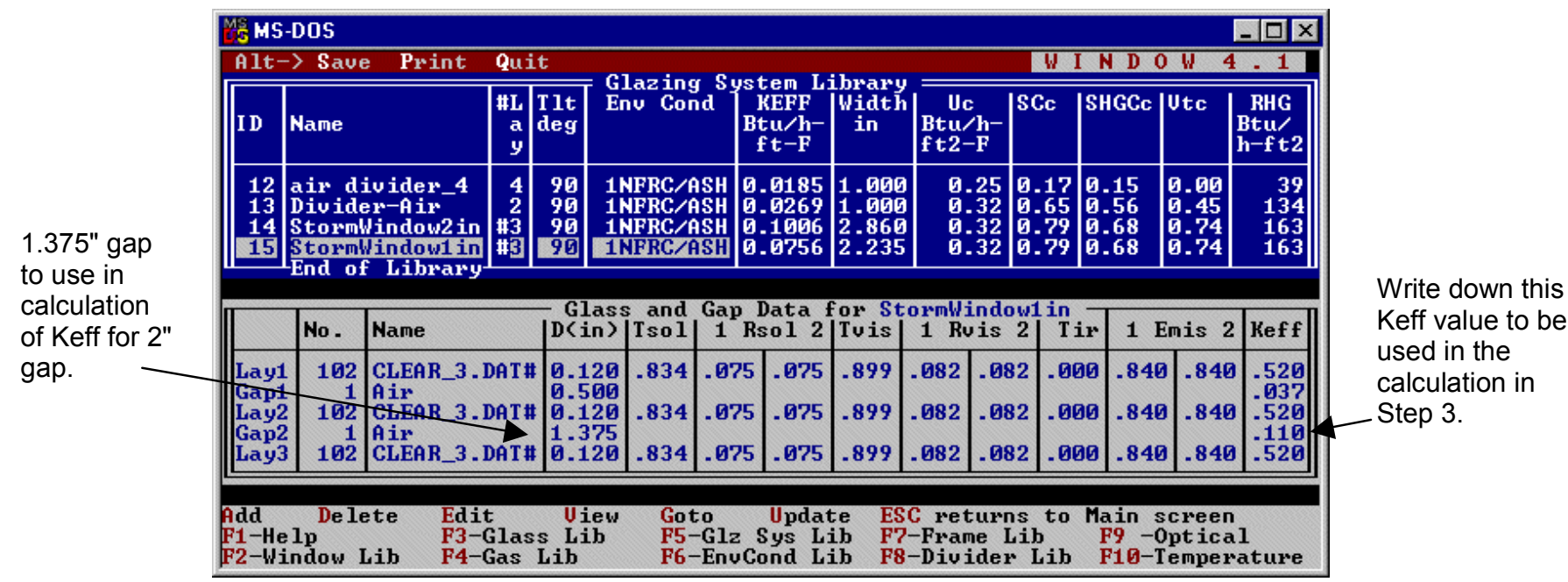

Figure 8-46 Make a triple glazed glazing system with a large gap width between the IG and the storm window.

3. Calculate a new Keff from the second glazing system using the following formula:

$$
\operatorname{Keff} 2^{\prime \prime}=\frac{2 *(\operatorname{Keff} 1.375)}{1.375}=\frac{2 *(.110)}{1.375}=\mathbf{0 . 1 6 0}
$$




\section{In THERM:}

1. After drawing the frame cross section, import the glazing system with the correct storm window cavity dimensions (created in WINDOW), in this case the glazing system with the 2" gap. Stretch glass layer 2 down to the frame if necessary. (Note: to edit the glazing system glass layers or cavities, check the box for Allow editing of IG polygons in the Options/Preferences menu, Drawing Options tab.

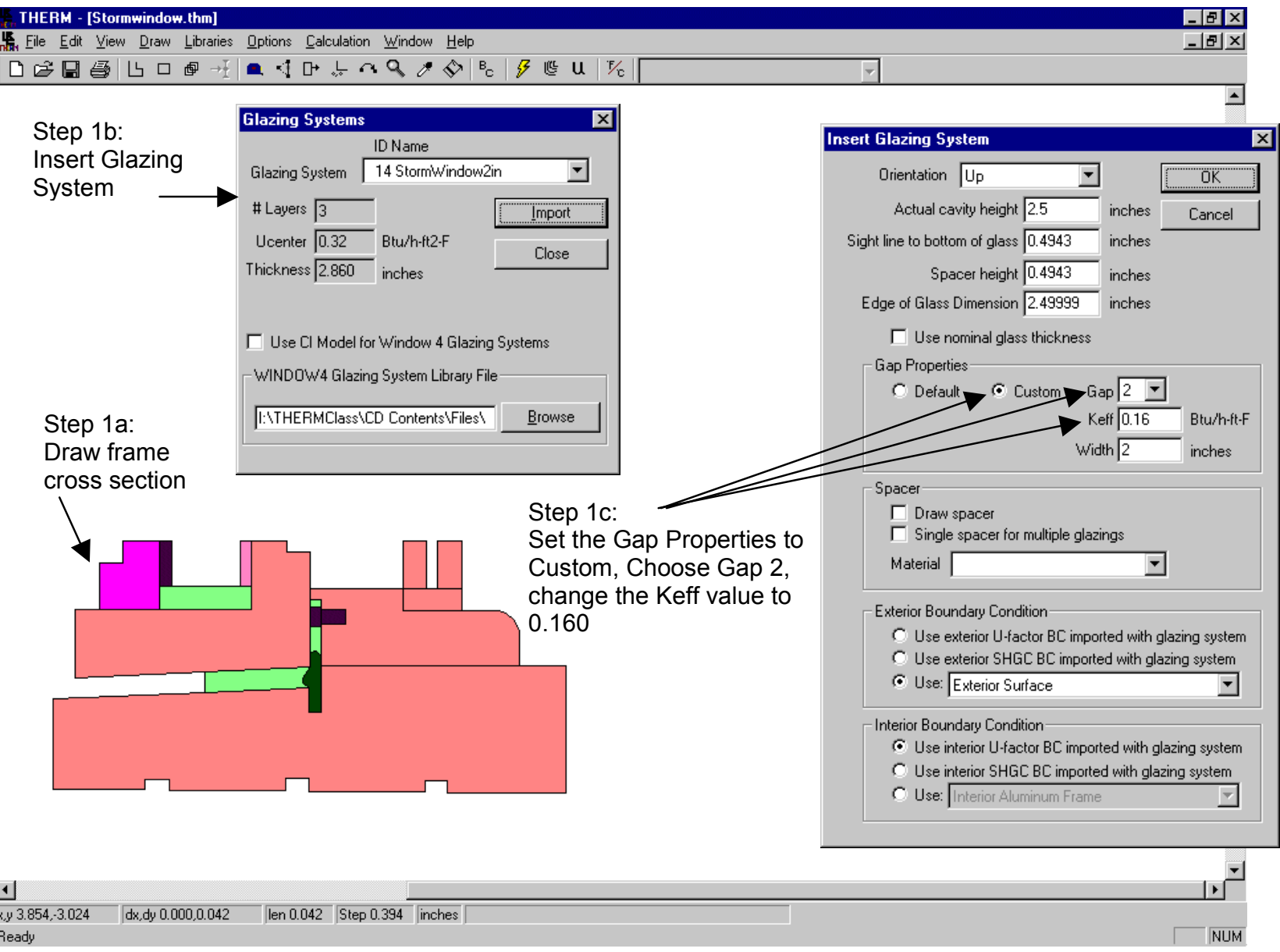

Figure 8-47 Insert the glazing system and edit the Keff of Gap 2. 
2. If necessary (as in this example), turn on the Allow editing of IG polygon, insert points into the glazing system gap, and pull the points down to the frame. (See Section 8.7.2, "Modifying Glazing Cavities (imported from WINDOW for Open Spacers" for an example of editing the glazing cavity down to the frame.)

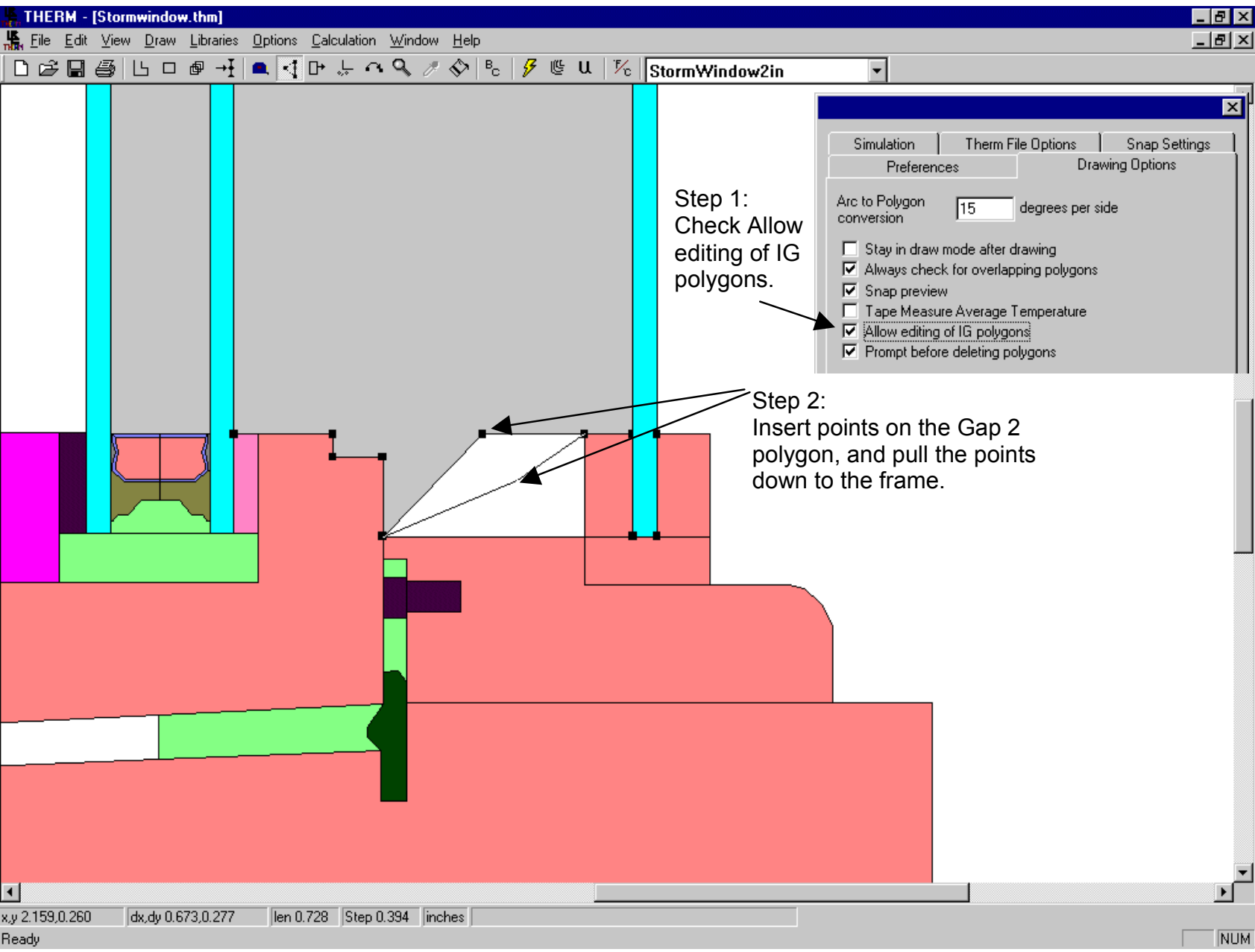

Figure 8-48 If needed, edit the geometry of the glazing system cavity. 
3. To check the Keff of each gap, double click on the glazing system. The Glazing System Info dialog box will be displayed. The Keff for each gap can be viewed (and edited if desired). In addition, the Actual Height value can be changed if needed (this is only used for Condensation Index calculations).

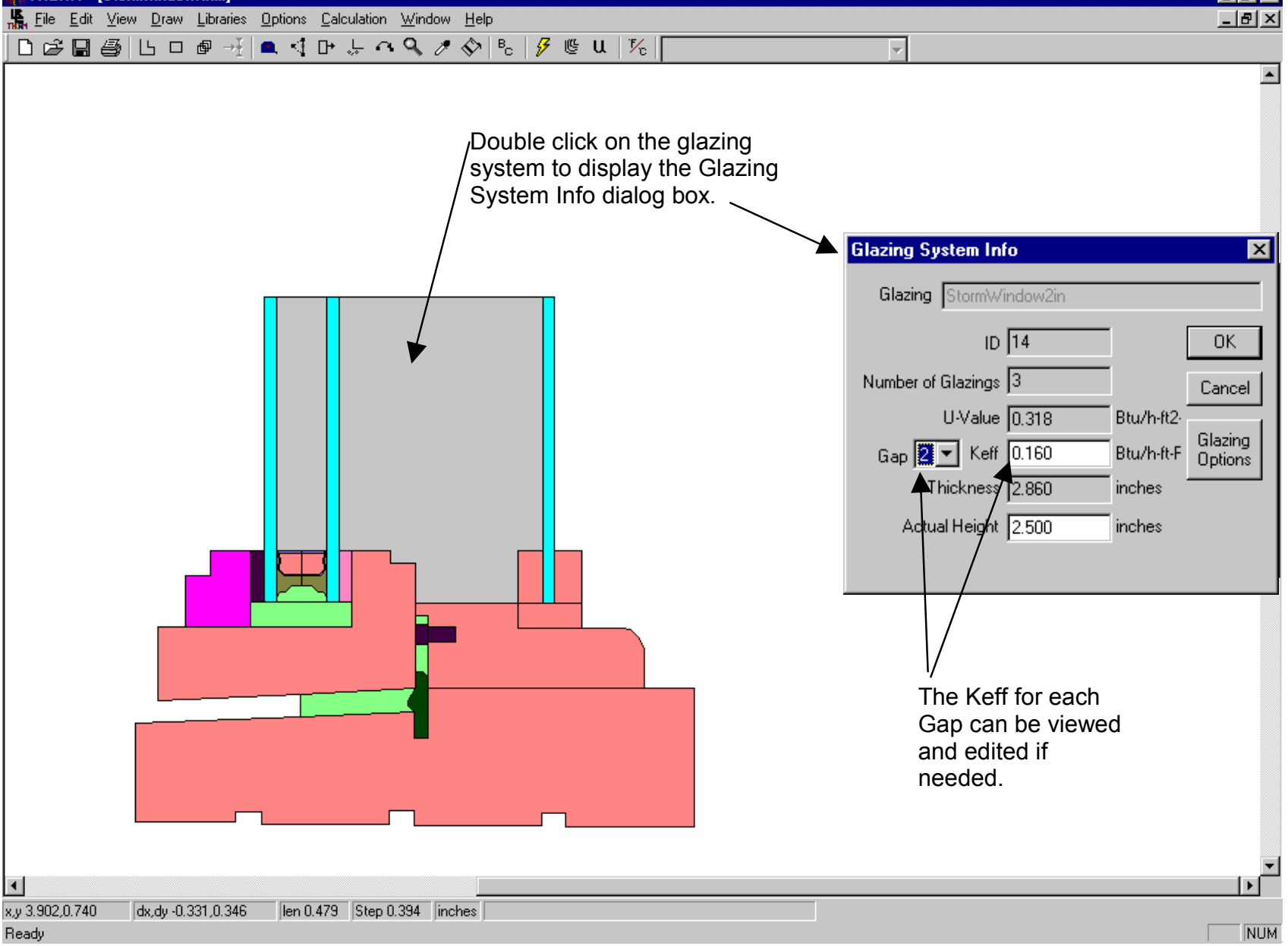

Figure 8-49 Double click on the glazing system to check the Keff of the gaps. 
4. Generate the Boundary Conditions by pressing the BC toolbar button. Assign the renamed boundary condition from Step 3 to the glazing system inside boundary.

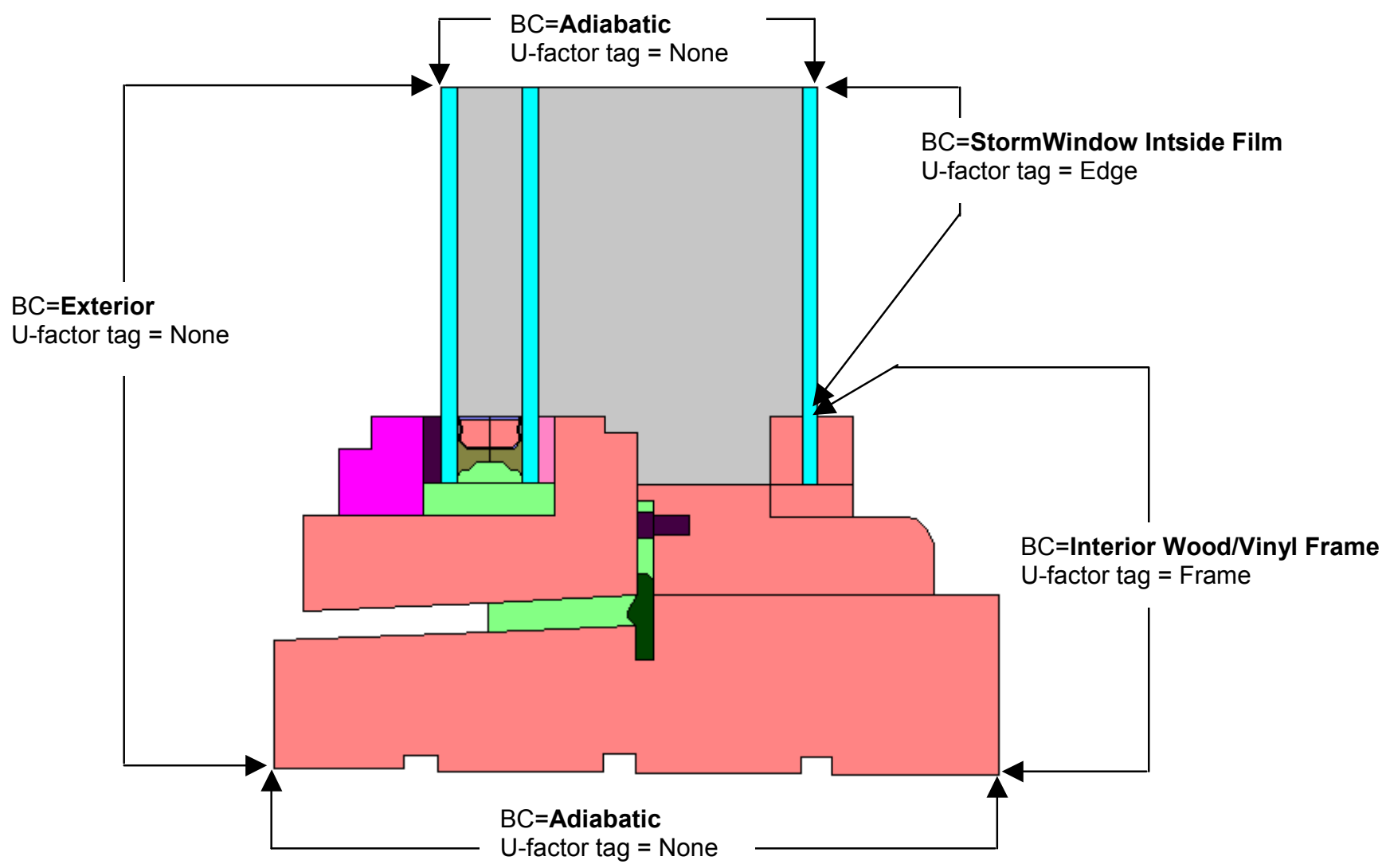

Figure 8-50 Boundary conditions for storm window.

5. Simulate the problem.

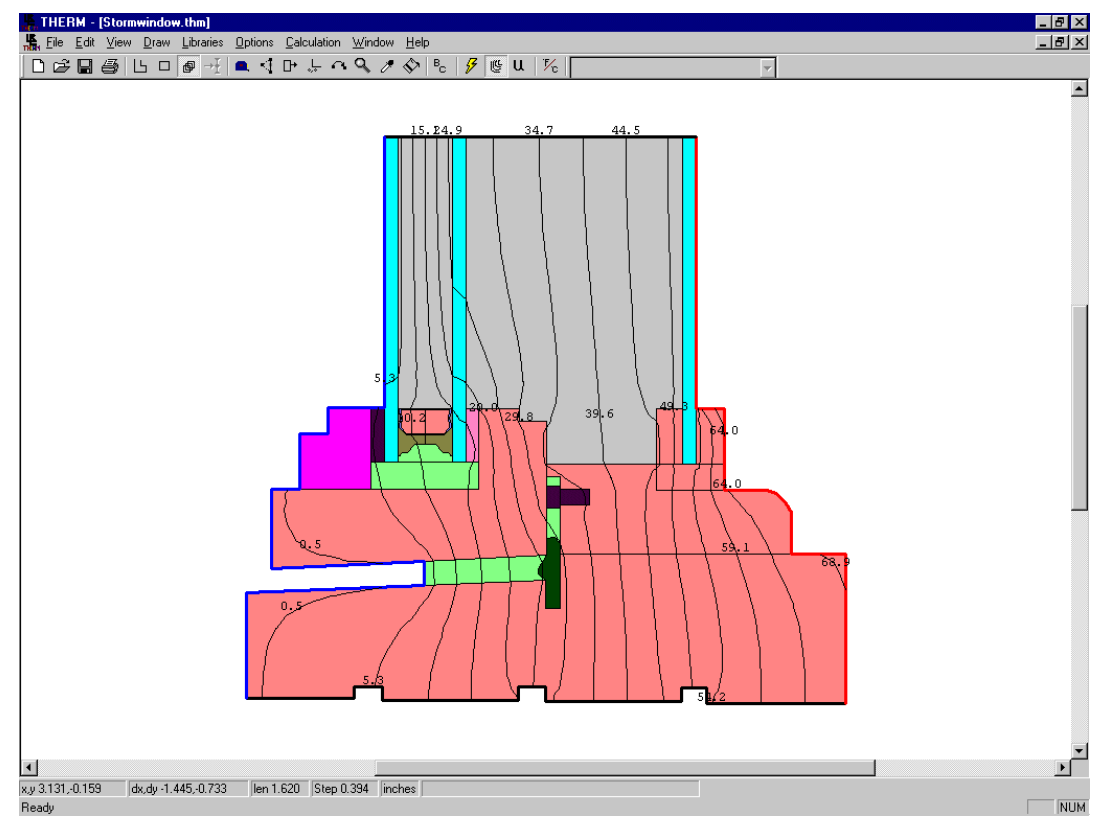

Figure 8-51 Simulate the file. 


\subsection{Projecting Products (Skylights / Greenhouse Windows)}

The THERM radiation model allows more accurate simulations of projecting products. In this section, the discussion centers around skylights, which are one of the more common examples of projecting products. The principles presented here can be applied to any other projecting product. Projecting products are modeled as full-height products in order to properly account for radiative heat transfer effects.

\subsubsection{Skylight Modeling Steps}

Although skylights are sometimes installed at a tilt, and it is possible to model tilted products in THERM, for NFRC certified simulations skylights are modeled in the vertical direction in order to match the conditions under which they were tested. In THERM, skylights are modeled as full-height products with a radiation enclosure surrounding the interior of the product in order to take full advantage of the modeling capabilities of the radiation enclosure feature. The steps for modeling a skylight a full-height product with a radiation enclosure are as follows:

- Draw the frame cross sections in THERM to make a full height cross section, for example a head and sill spaced the appropriate distance apart to insert the full height of the glass

- Create the appropriate glazing system for the skylight in WINDOW

- Insert the glazing system into a cross section three times

- Once with orientation up and the glass height $=2.5^{\text {" for the sill }}$

- Once with orientation down and the glass height $=2.5^{\prime \prime}$ for the head

- Once at the length remaining for the center-of-glass to finish defining the cross section with a fullheight glazing system

- Create a Radiation Enclosure on the inside of the full-height product

- Set the boundary conditions

- Define new boundary conditions in the Boundary Condition Library for the interior convectiononly edge-of-glass and center-of-glass interior film coefficients, using Equation 1 discussed on page 8-57.

- Define new boundary conditions in the Boundary Condition Library for the interior convectiononly frame components, based on the frame type from, Table 8-1.

- Simulate the product twice, once with U-factor surface tags defined as Frame and Edge for the head and once for the sill. (This is in order to bring the frame U-factors into WINDOW. If a spreadsheet is used for the area-weighted U-factor calculation, only one simulation is needed, and the Head and Sill can have their own uniquely named U-factor tags).

- After the simulation, use the Options/Drawing Options to set the Tape Measure Average Temperature feature, and then measure the average glass inside surface temperature over the full height of the product. If the temperatures are not within $5^{\circ} \mathrm{F}$ of the original surface temperatures, then use the THERM temperature to recalculate the convection-only interior glass film coefficient (in the boundary conditions) and rerun the problem. 


\subsubsection{Skylight Mounting Details}

There are two ways that skylights can be mounted into a roof system, either flush-mounted or curb-mounted. Figure 8-52 and 8-53 show these two different mounting styles. Each mounting style has a slightly different definition of the adiabatic boundary condition, and each will have a different projected frame length. The rules for modeling can be found in NFRC 100 and the NFRC Technical Interpretations. To model curb mounted skylights, if the projected frame height is zero, define a Frame U-factor Surface Tag 0.01 inches up the interior of the glass, which will result in a non-zero frame height.

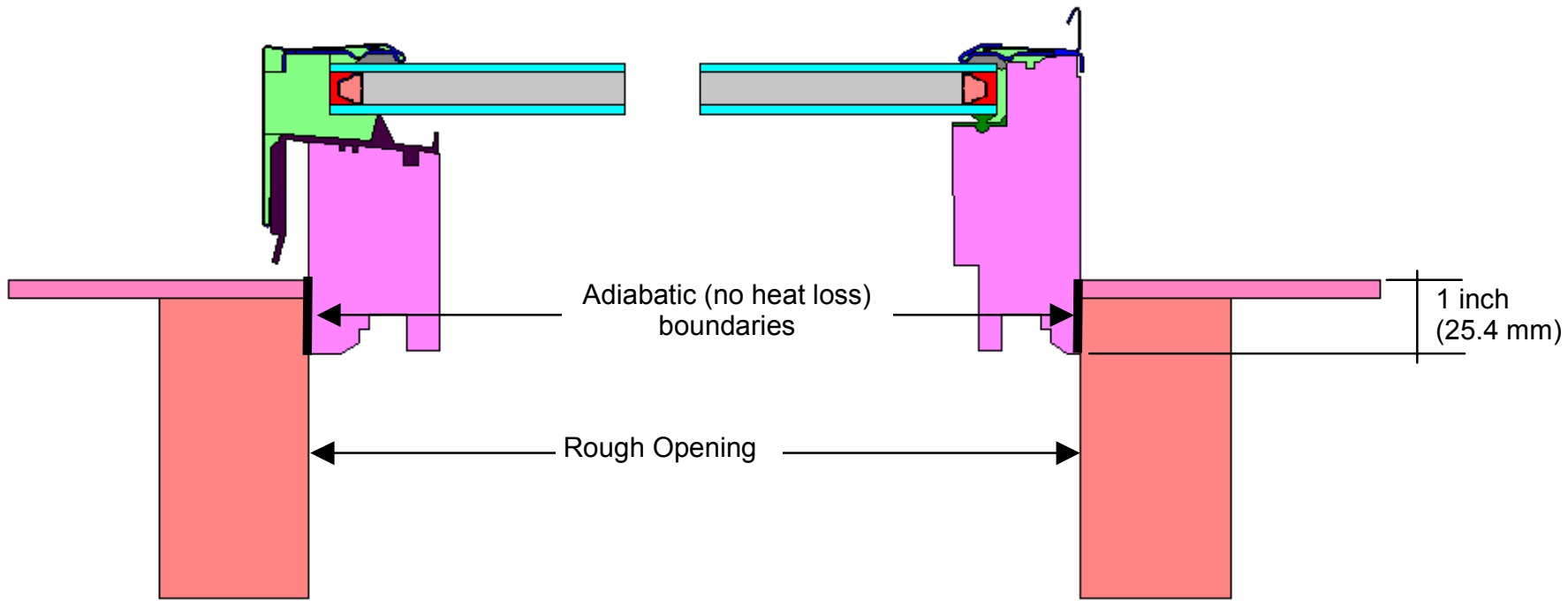

Figure 8-52 A flush-mounted skylight.

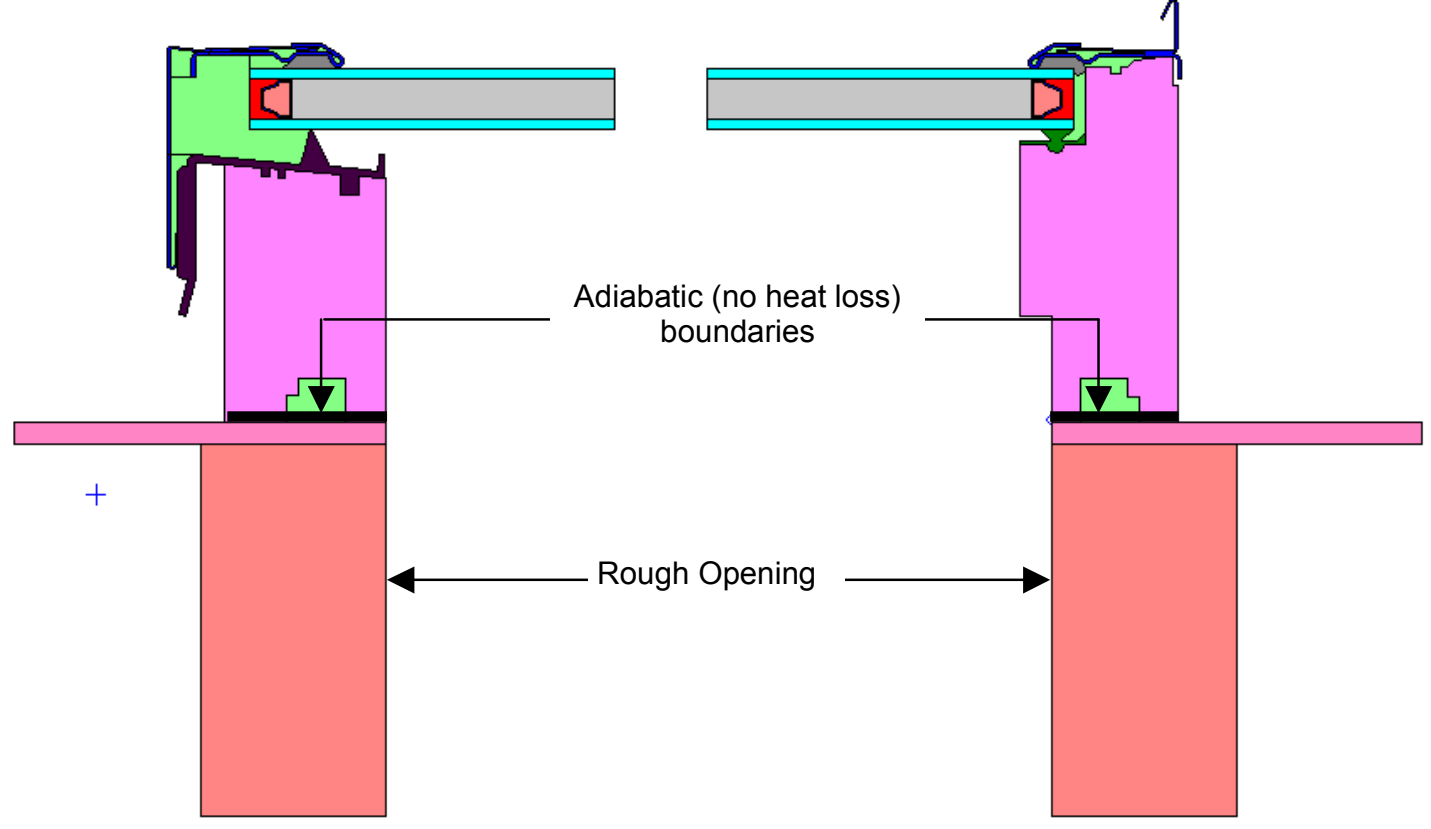

Figure 8-53 A curb-mounted skylight. 


\subsubsection{Example Skylight Problem}

This example assumes a flush-mounted skylight simulated in a vertical direction. This example only explains modeling the cross section for the head and sill. A similar technique is used for the jamb if the cross-section geometry is different from the head and sill. The jamb results would be imported into WINDOW in a similar manner as explained here for the head and sill.

In WINDOW:

1. Glazing System Library: Make a glazing system (with the default tilt of $90^{\circ}$ ) if needed. In this example, the glazing system is called Skylight-2Glz and is made up of generic glass layers.

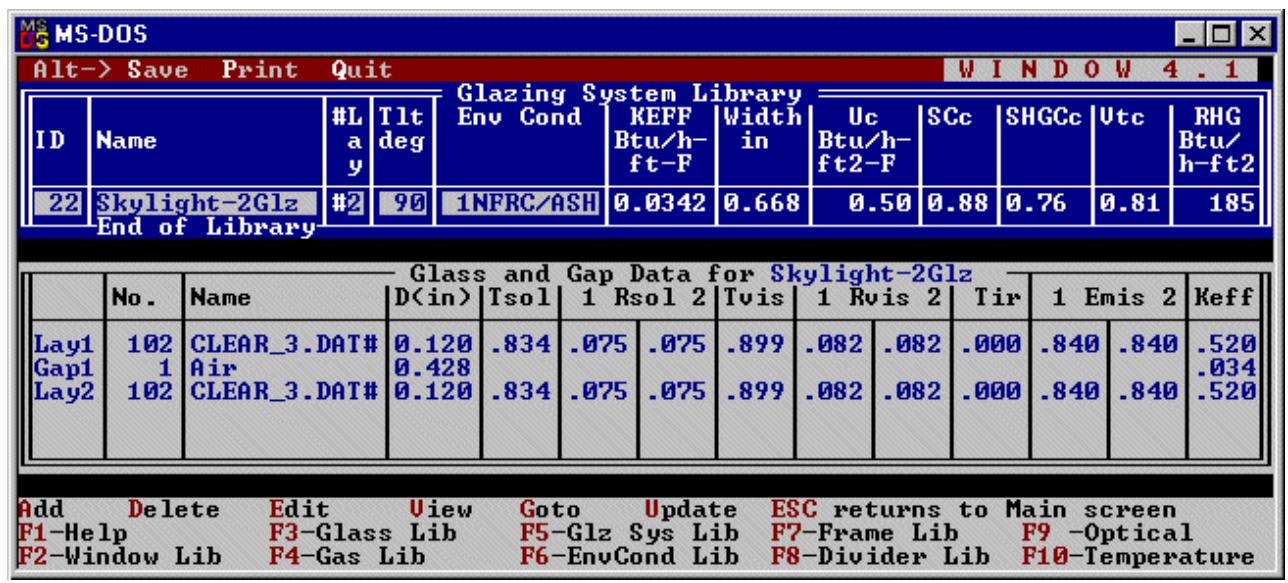

Figure 8-54 Make new glazing system in the Glazing System Library if necessary.

2. Press F10 and write down the center-of-glass temperature, as shown in Figure 8-55.

Write down the Layer 2 inner surface U-value temperature, in this example $=44.9^{\circ} \mathrm{F}$.

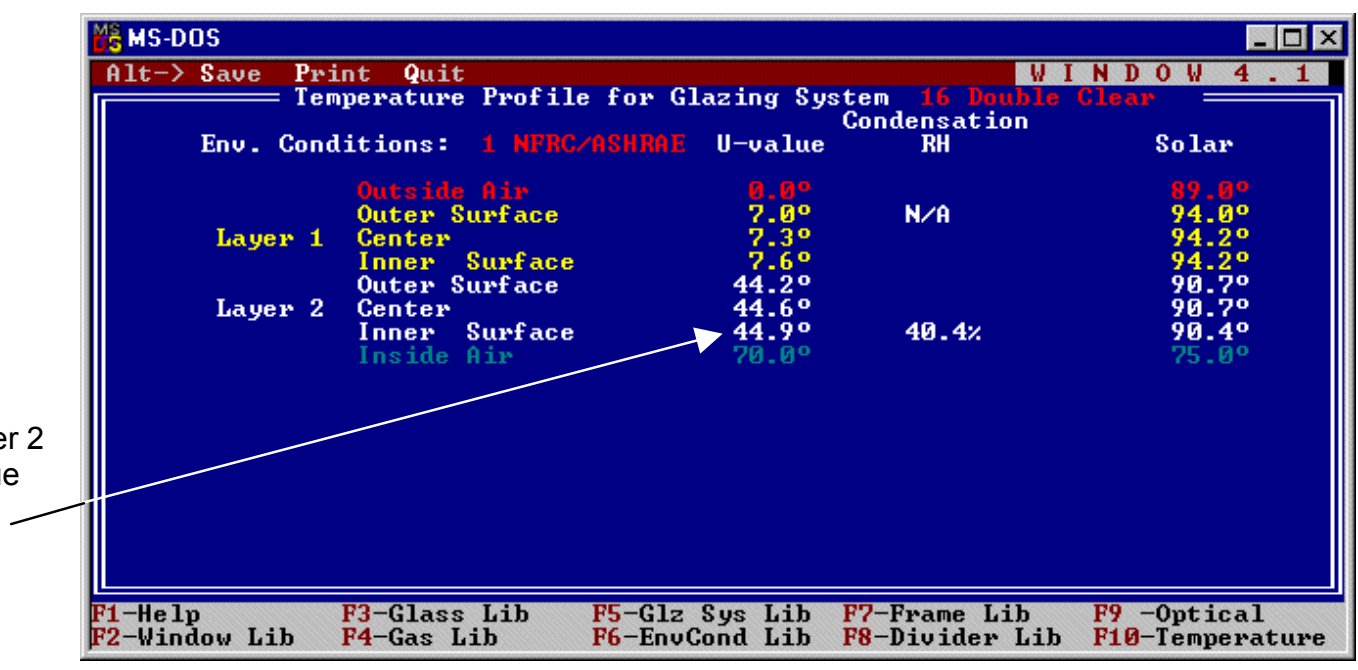

Figure 8-55 Press F10 from the Glazing System Library to see the center-of-glass temperature.

3. Save the file: Make sure to save the glazing system (Alt-S). 


\section{In THERM:}

1. There is more than one way to draw the cross section of a full-height product. For example, as described below, the head and sill can be drawn and the glazing systems inserted between them. Alternatively, the sill could be drawn, the glazing systems inserted, and then the head drawn at the top of the glazing systems. The method used will depend on the type of information available about the product as well as the availability and type of DXF files. In this example, the head and sill are drawn first, and the glazing system is inserted between them.

2. Draw the frame cross section for the skylight vertically, with the correct distance between the sill and the head of the skylight (using the Step Size feature), as shown in the figure below.

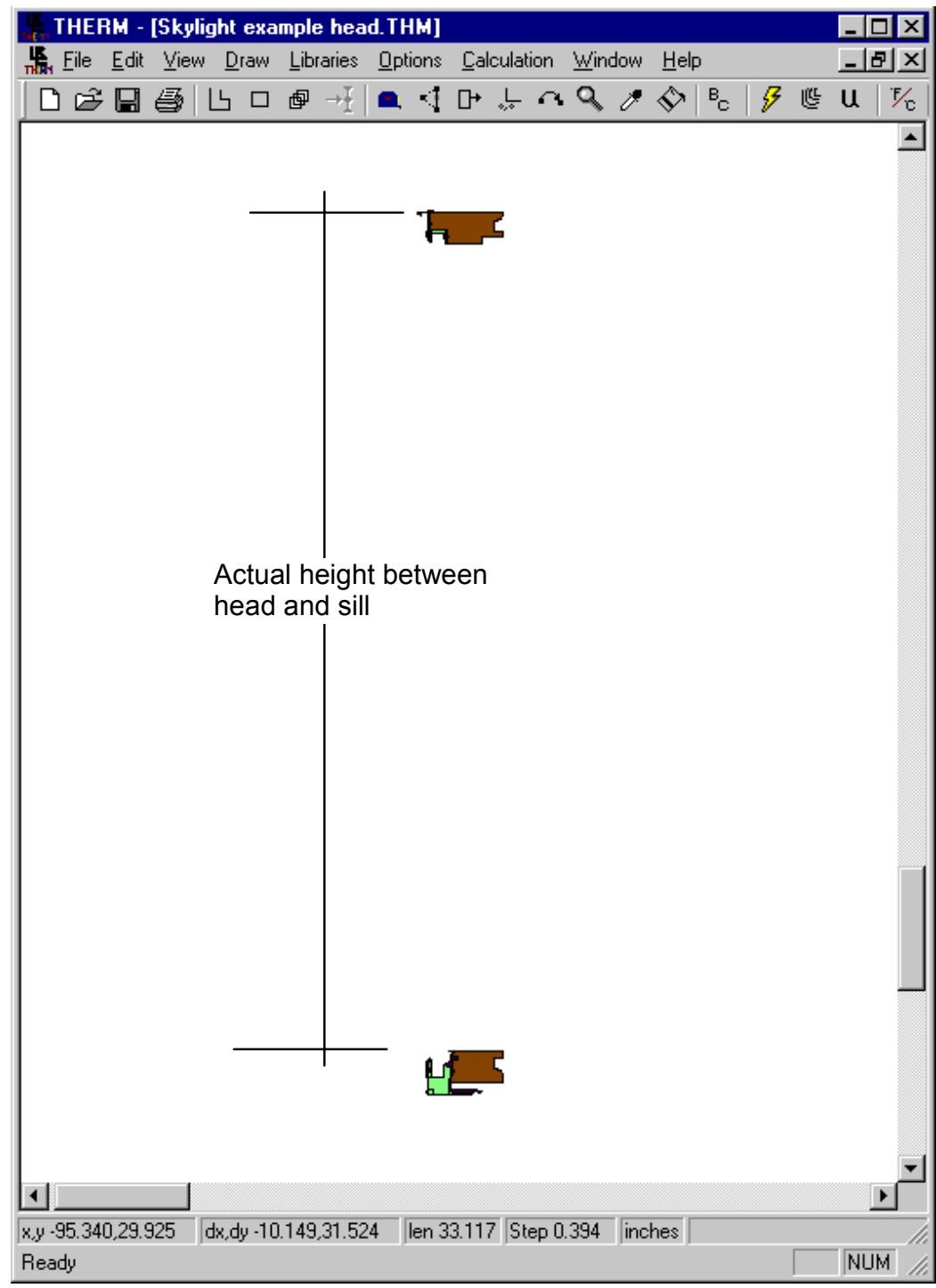

Figure 8-56 Skylight sill and head cross sections drawn to the full height of the product. 
3. Import the glazing systems: When modeling the full-height product, import the glazing system in three sections using the following steps. Position the Locator (using the Draw/Locator menu) in the lower left orner of the frame where the glazing system is to be inserted.

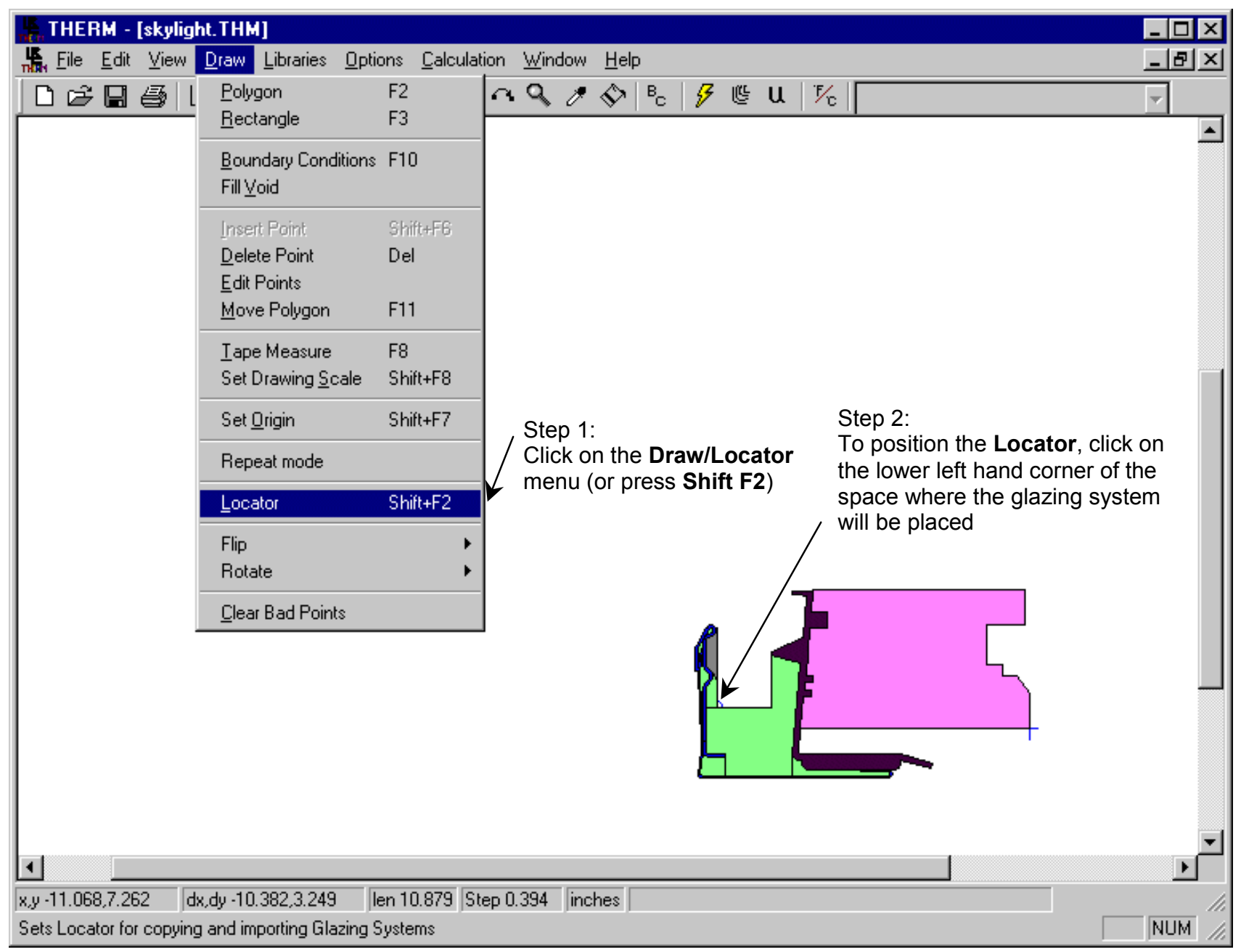

Figure 8-57 Position the locator so that the first glazing section can be inserted. 
4. Insert the first glazing system with the following settings:

- Orientation $=\mathrm{Up}$

- Cavity height $=0$ (this value is only used for CI modeling, so it can be set to anything).

- Sight line to bottom of glass = measure this value with the tape measure or get from dimensioned drawings

- Spacer height = measure this value with the tape measure or get from dimensioned drawings

- $\quad$ Edge of Glass Dimension $=2.5$

- Draw spacer $=$ not checked

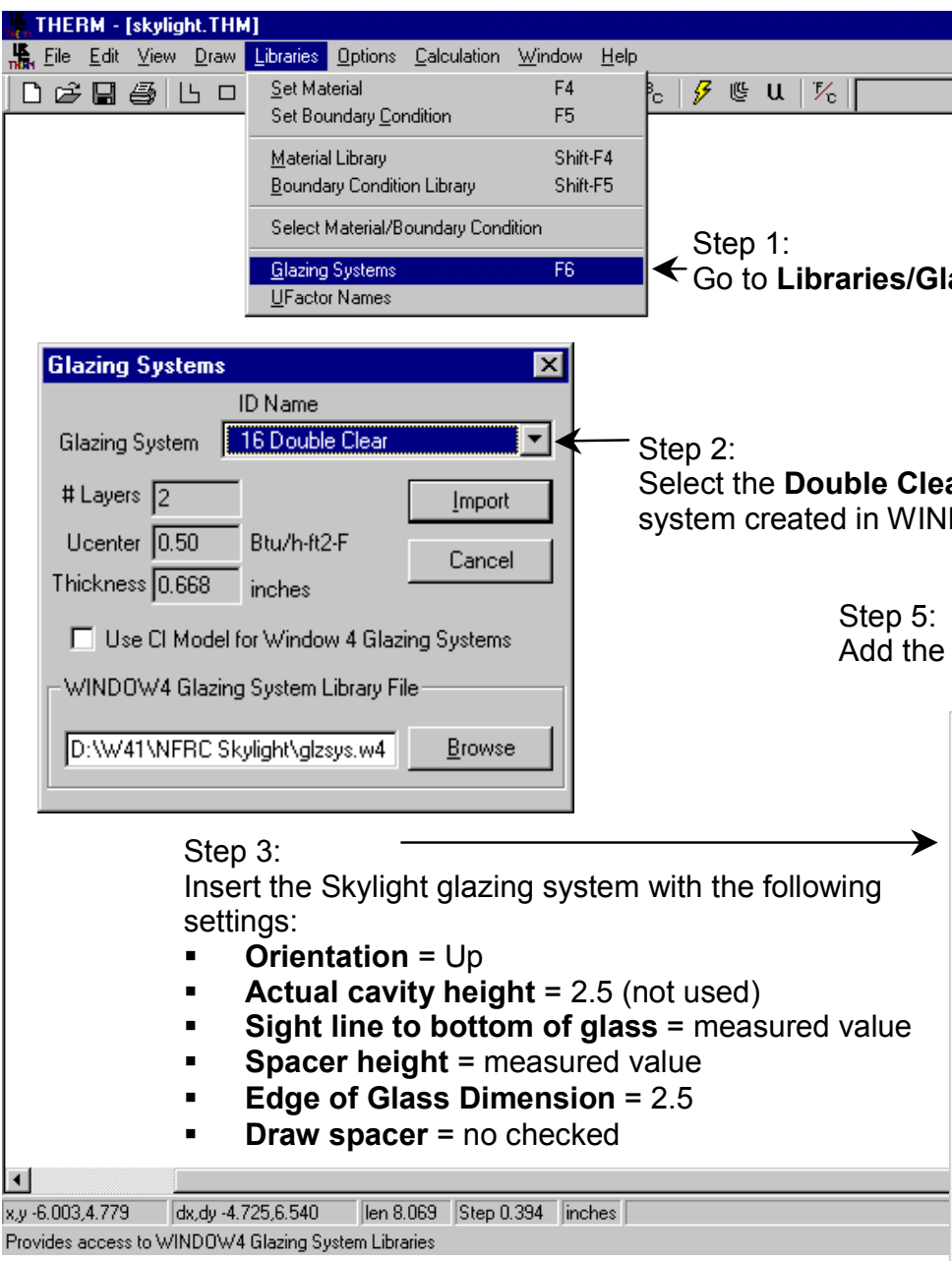

Figure 8-58 Insert the glazing system. 
5. Insert the glazing system section a second time, with the following settings, at "head" or top of skylight:

- Position the Locator (Shift F2) in the upper left corner of the opening where the glazing system will be inserted.

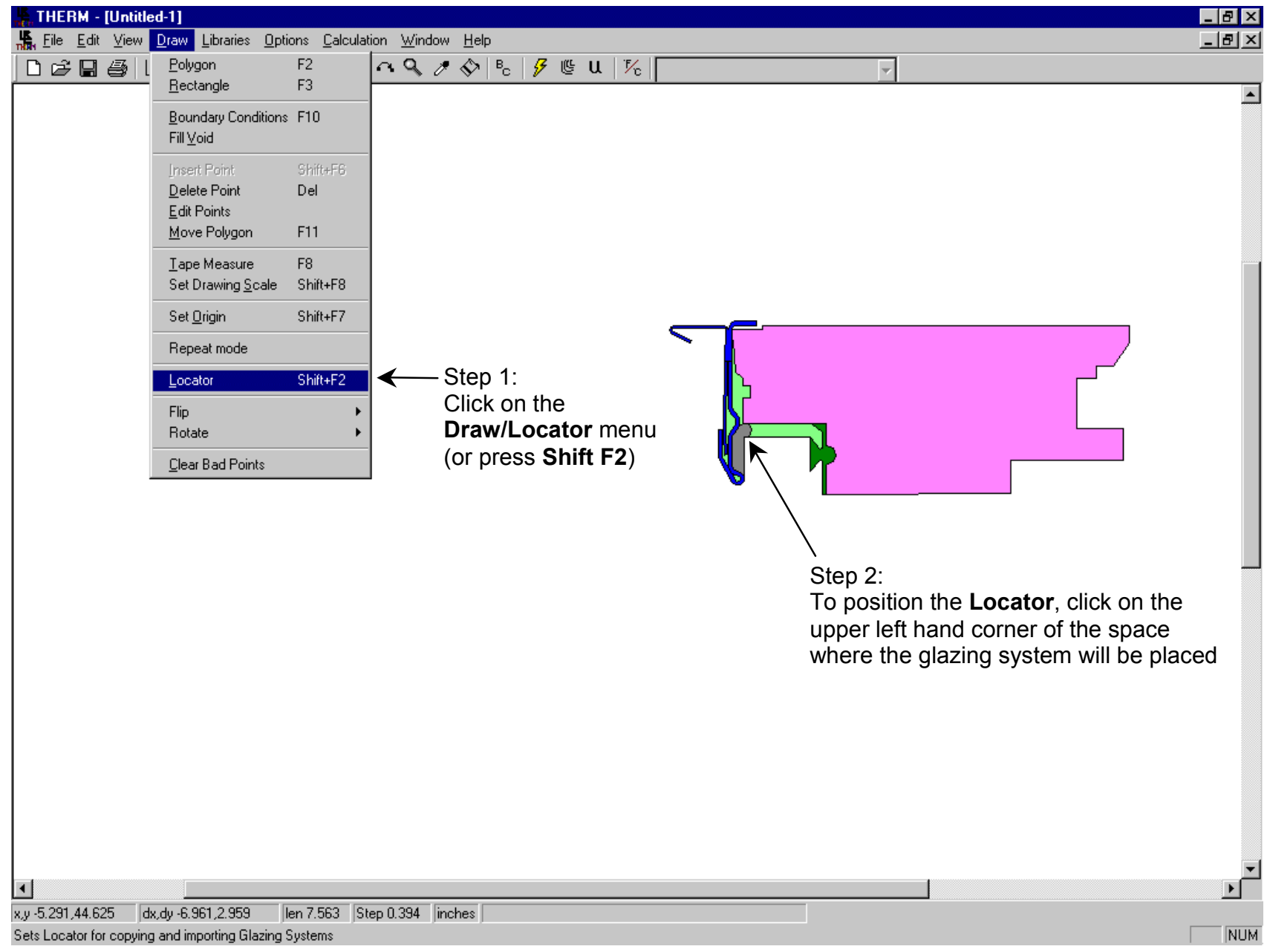

Figure 8-59 Position the locator to specify the location for the glazing next to the head cross section. 
6. Insert the glazing system:

- Orientation $=$ Down

- Cavity height $=0$ (this value is only used for CI modeling, so it's value can be anything).

- Sight line to bottom of glass = measure this value with the tape measure or get from dimensioned drawings

- Spacer height $=$ measure this value with the tape measure or get from dimensioned drawings

- $\quad$ Edge of Glass Dimension $=2.5$

- Draw spacer $=$ not checked

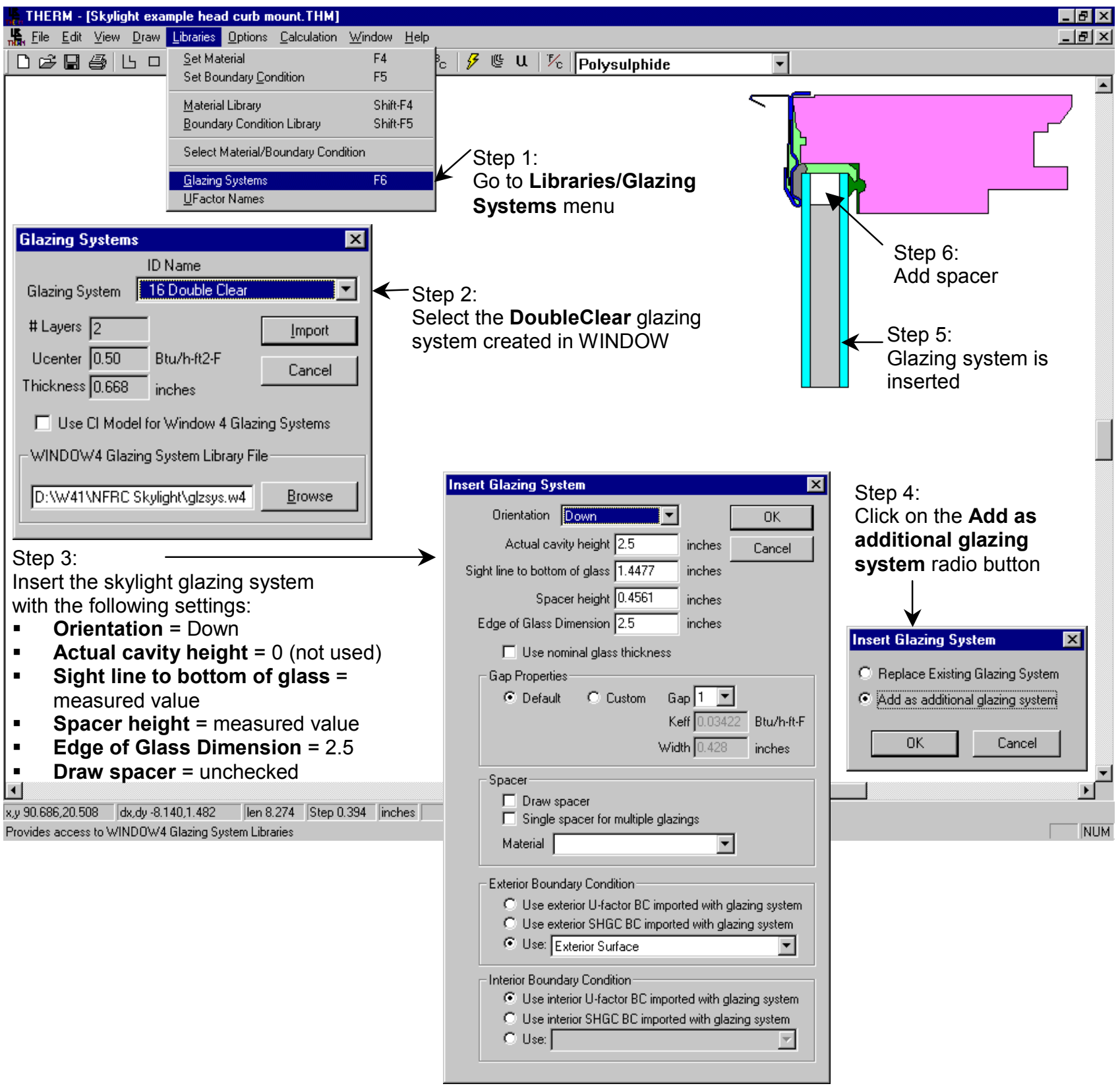

Figure 8-60 Insert the glazing system in the head cross section . 
7. Insert the glazing system a third time for the middle section:

- Position the Locator at the upper left corner of the bottom glazing system

- Measure the distance between two existing glazing systems

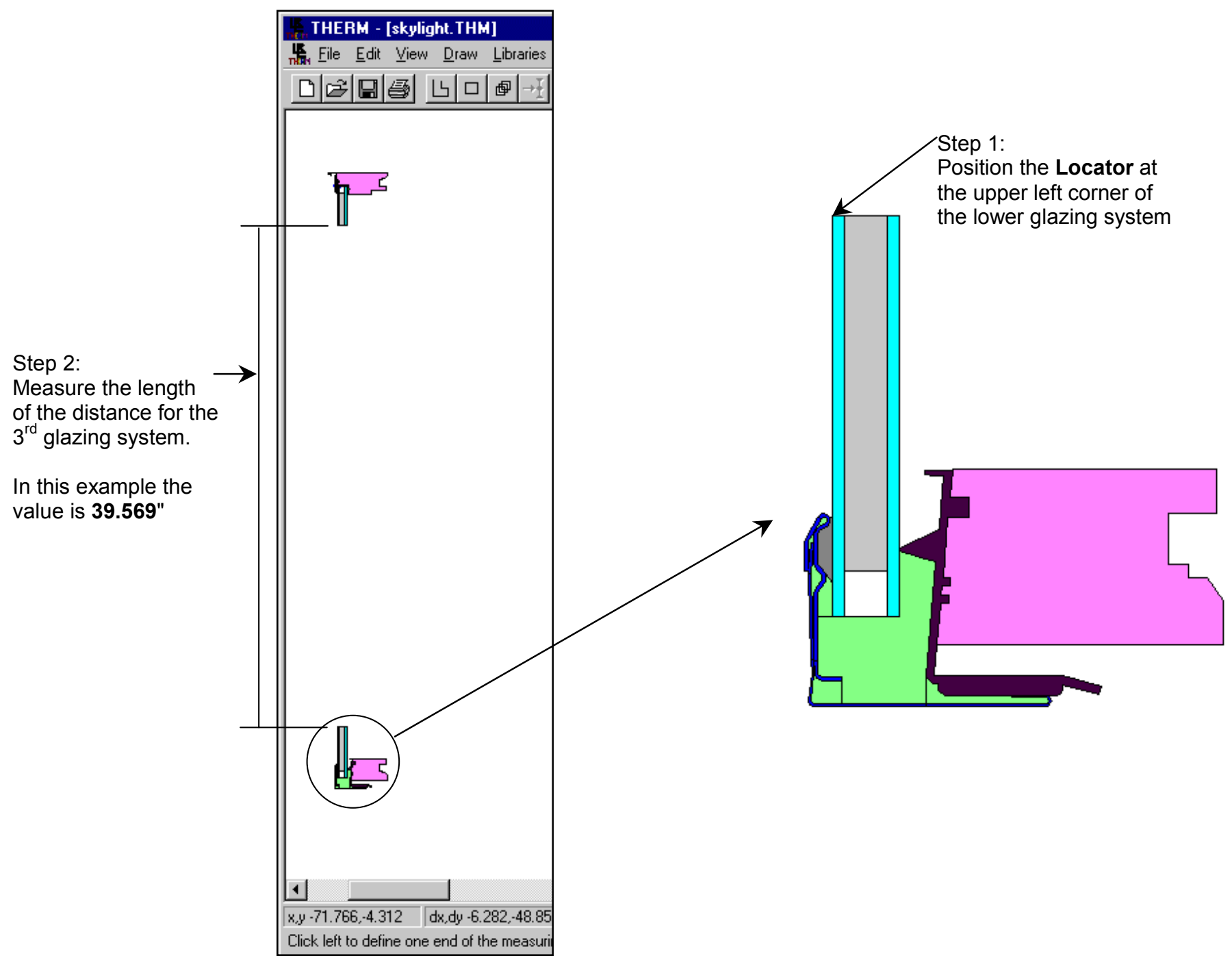

Figure 8-61 Position the locator to insert the middle glazing system section . 
8. Insert the glazing system as an additional glazing system with the following settings:

- Orientation $=\mathrm{Up}$

- Cavity height $=0$ (this value is only used for CI modeling, so it's value can be anything).

- Sight line to bottom of glass $=0$

- $\quad$ Spacer height $=0$

- Edge of Glass Dimension = measured height between top and bottom glazing systems, in this example equal to 39.569"

- Draw spacer $=$ not checked

THERM - [Skylight example head curb mount. THM]

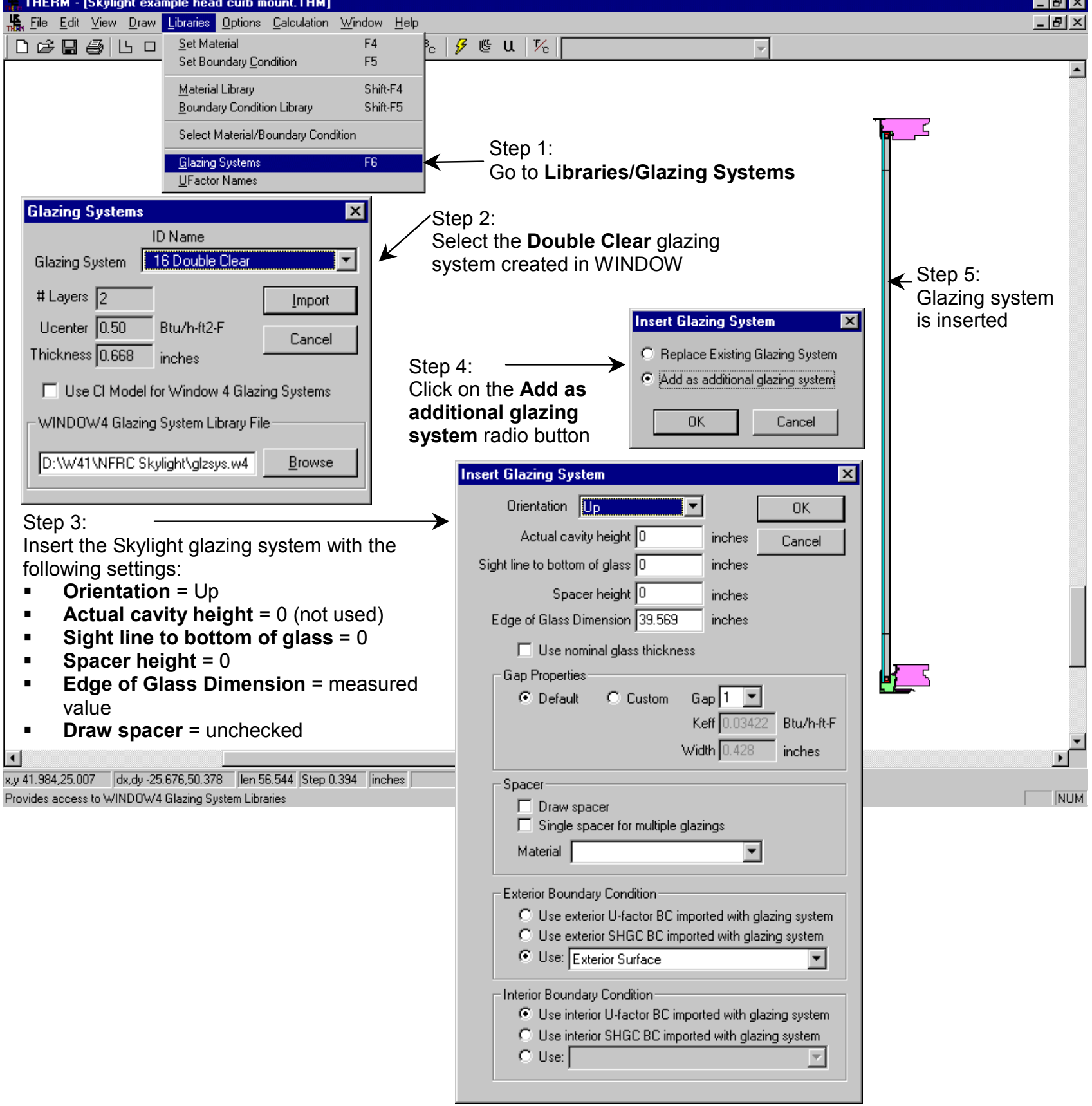

Figure 8-62 Insert the middle glazing system section. 
9. Add the Radiation Enclosure. The radiation enclosure should include all the components of the fullheight product that can "see" each other and should be 0.5 inches $(12.7 \mathrm{~mm})$ from the inner most frame component. See Section 6.5.2, "External Radiation Enclosures" in the THERM User's Manual for complete details about defining Radiation Enclosures.

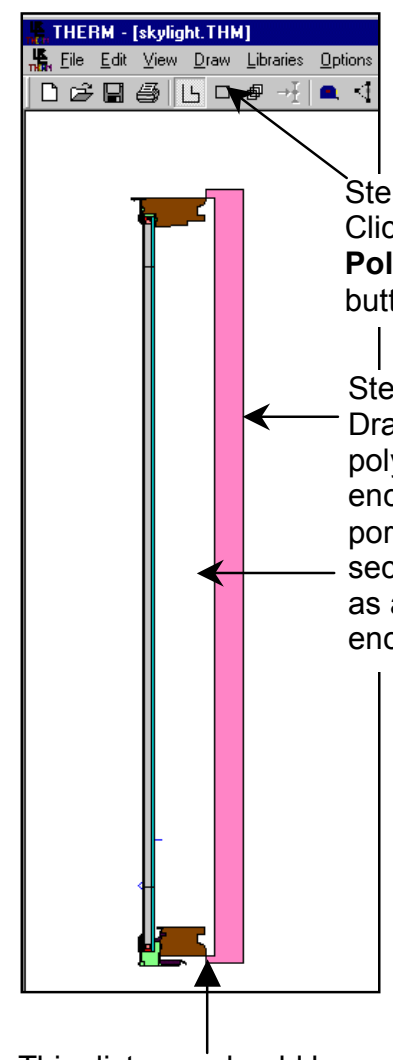

This distance should be 0.5 inches $(12.7 \mathrm{~mm})$
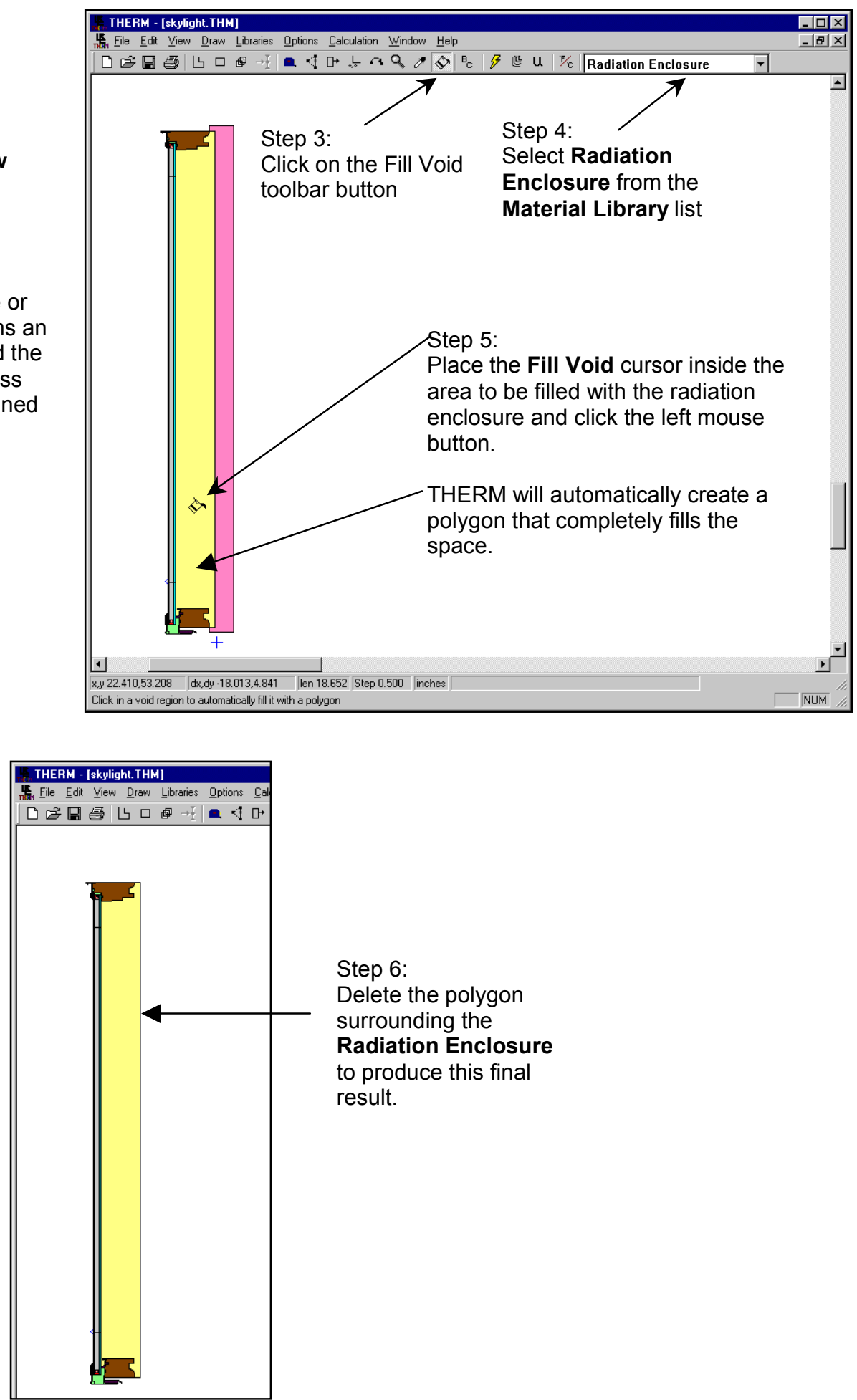

Step 6:

Delete the polygon

surrounding the

Radiation Enclosure

to produce this final

result.

Figure 8-63 Create the radiation enclosure. 


\section{Add Boundary Conditions and U-factor Tags to Libraries}

Because the standard boundary conditions in THERM include both radiation and convection effects, and the radiation enclosure is used to model radiation effects, the radiation component shall be eliminated from the boundary conditions within the radiation enclosure. Therefore special boundary conditions shall be defined for the "convection-only" heat transfer component in the radiation enclosure. To do this, two new boundary conditions shall be made in the Boundary Conditions Library, one for frame convection-only case and one for glazing convection-only.

\section{Frame convection-only boundary conditions:}

Define a new boundary condition (from the Libraries/Boundary Conditions Library menu choice) with the following attributes:

- $\quad$ Model = Comprehensive

- Convection $=$ checked

- $\quad$ Temperature $=70^{\circ} \mathrm{F}\left(21.11^{\circ} \mathrm{C}\right)$

- $\quad$ Film Coefficient $=$ value from Table 8-1. For this example, a wood frame skylight, the value would be $0.44 \mathrm{Btu} / \mathrm{hr}^{-\mathrm{ft}^{2}-\mathrm{o}} \mathrm{F}\left(2.52 \mathrm{~W} / \mathrm{m}^{2}{ }^{\circ} \mathrm{C}\right)$.

- $\quad$ Radiation $=$ checked

- Enclosure Model = selected

- Color: Click on the Color button to change the colors from the default blue value to distinguish these boundaries from the normal boundaries.

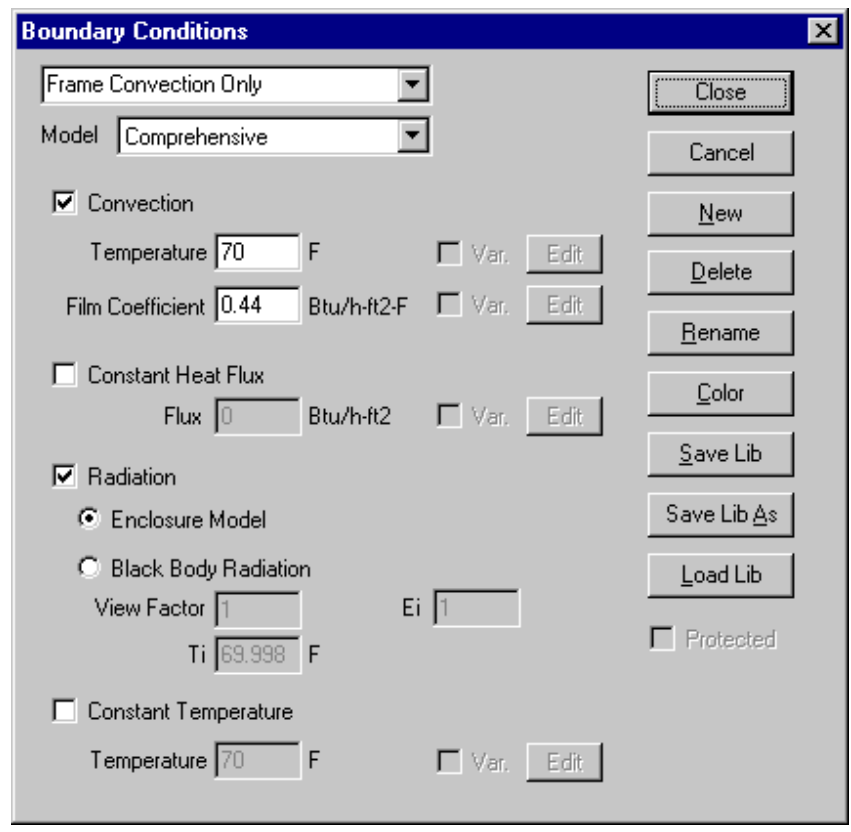

Figure 8-64 Define the frame convection-only boundary condition. 
Table 8-1 lists convection-only interior frame film coefficients to be used for the boundary conditions on the interior frame components that are inside the radiation enclosure.

Table 8-1 Interior convection-only frame film coefficients for radiation enclosures based on frame type.

\begin{tabular}{|c|c|c|}
\hline Frame Type & $\begin{array}{l}\text { Film Coefficient (hc) } \\
\left.\text { (Btu/h- } \mathrm{ft}^{2}-{ }^{\circ} \mathrm{F}\right)\end{array}$ & $\begin{array}{l}\text { Film Coefficient (hc) } \\
\left(\mathrm{W} / \mathrm{m}^{2}-{ }^{\circ} \mathrm{C}\right)\end{array}$ \\
\hline Aluminum Frame & 0.61 & 3.48 \\
\hline Thermally Broken Frame & 0.51 & 2.89 \\
\hline Wood/Vinyl Frame & 0.44 & 2.52 \\
\hline
\end{tabular}

\section{Glazing convection-only boundary conditions:}

Define a new boundary condition (from the Libraries/Boundary Conditions Library menu choice) with the following attributes:

- $\quad$ Model $=$ Comprehensive

- $\quad$ Convection $=$ checked

- $\quad$ Temperature $=70^{\circ} \mathrm{F}\left(21.11^{\circ} \mathrm{C}\right)$

- Film Coefficient $=\mathrm{hc}$ value from Equation 1 below. For this example, $\Delta \mathrm{T}=70^{\circ} \mathrm{F}-44.9^{\circ} \mathrm{F}=25.1^{\circ} \mathrm{F}$; so $h_{c}=0.27^{*}(25.1)^{0.25}=0.604$

- $\quad$ Radiation $=$ checked

- Enclosure Model $=$ selected

- Color: Click on the Color button to change the colors from the default blue value to distinguish these boundaries from the normal boundaries. 


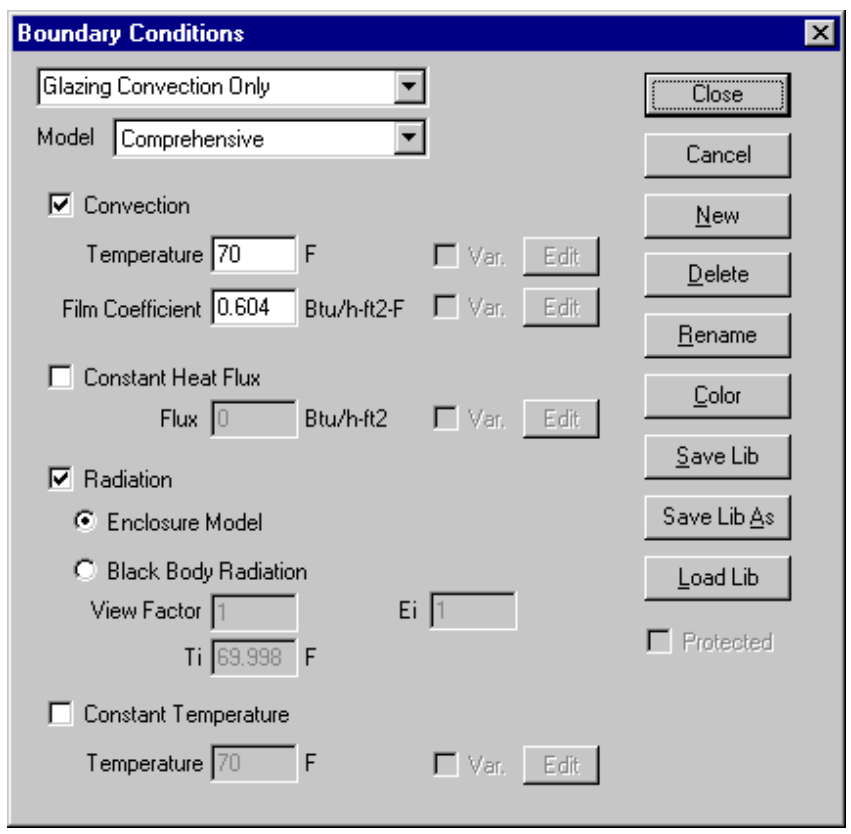

Figure 8-65 Define the glass convection-only boundary condition.

Use the equation below to calculate the interior convection-only film coefficient for the glass (to be used for both the center-of-glass and edge-of-glass boundary conditions). This equation is from page 27.4 of the 1993 ASHRAE Handbook of Fundamentals.

$$
h_{c}=A(\Delta \mathrm{T})^{0.25}
$$

where:

$\mathrm{h}_{\mathrm{c}}=\quad$ film coefficient for convection only

$\mathrm{A}=\quad 0.27$ (for IP units) or 1.77 (for SI units)

$\Delta \mathrm{T}=$ interior temperature $\left(70^{\circ} \mathrm{F}, 21.11^{\circ} \mathrm{C}\right)$ minus center-of-glass temperature from WINDOW (use F10 in WINDOW to obtain this value)

In this example, the result would be:

$h_{c}=0.27(70-44.9)^{0.25}=0.604$ 
11. Define the Boundary Conditions and Perform the Simulation

There are two methods for calculating the total product U-factor for skylights, and the method chosen will determine how the Boundary Conditions are defined in THERM. The two methods and their associated Boundary Conditions are:

- Spreadsheet: If the total product U-factor is calculated with a spread sheet, the U-factor Surface Tags associated with the Boundary Conditions for each cross section in THERM can be named in a manner that corresponds to the area of the product, and THERM will calculate a separate U-factor for every U-factor Surface Tag defined in one simulation. For example, for the sill/head full-height cross section, the U-factor surface tags could be the following (shown in Figure 8-67), and the spreadsheet would be set up to area-weight each of these U-factors:

- Sill Frame

- Sill Edge

- Center-of-glass

- Head Edge

- Head Frame

- WINDOW: If the total product U-factor is calculated with WINDOW, two simulations are calculated, the first with the U-factor Surface Tags of Frame and Edge applied to the head section of the product, and the second with those same tags of Frame and Edge applied to the sill section. This is shown in Figure 8-68. In addition, a center-of-glass U-factor is obtained from THERM and a generic glass layer and glazing system is constructed in WINDOW with this U-factor for the total product calculation, rather than using the skylight glazing system originally created in WINDOW.

\section{Steps Common to Both Methodologies}

The first step, which is common to both methodologies, is to click on the Boundary Conditions (BC) toolbar button and correct any problems encountered with the geometry (see Section 6.5.3, "Voids, Overlaps, and Bad Points" in this manual). When assigning the boundary also check the following:

- Check the Blocking Surfaces checkbox on all the Boundary Conditions associated with the Radiation Enclosure (this doesn't mean that the boundary conditions are blocking, it just tells THERM to verify whether they are when performing the calculation).

- $\quad$ Make sure the Emissivity is set to "0.84" for interior \& exterior glazing surfaces.

- Tag the center-of-glass section with a unique U-factor tag, such as Center, in order to get the THERM value for center-of-glass U-factor, which can be used to make a generic glazing system in WINDOW when modeling the total product. 


\section{Spreadsheet Method}

1. If the total product $U$-factor is going to be calculated with a spreadsheet, first define the U-factor Surface Tags needed for the product in the U-factor Names Library. For example, the skylight head/sill fullheight product cross section in Figure 8-67 has the following U-factor Surface Tags which were added to the library:

- Sill Frame

- Sill Edge

- Center-of-glass

- Head Edge

- Head Frame
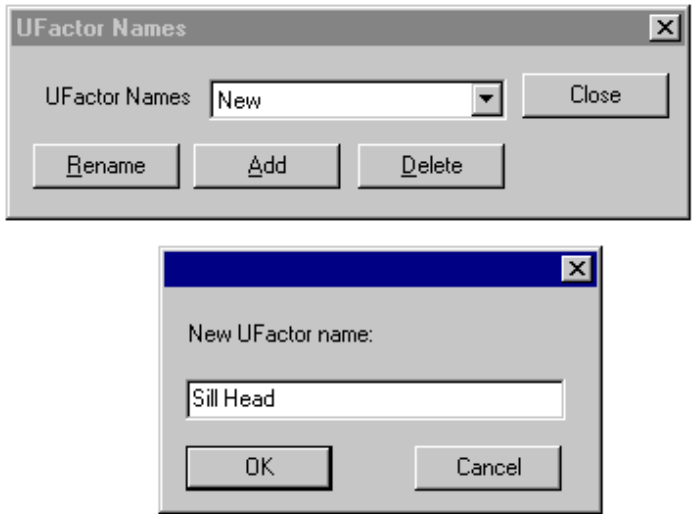

Figure 8-66 Make new records in the U-factor Names Library.

2. Assign the Boundary Conditions and U-factor Surface tags as shown in Figure 8-67, and then the simulation can be performed. During the meshing step of the simulation, if an error message appears saying that the geometry is too complicated, reset the mesh parameter (Options menu, THERM File Options choice) to a higher value. See Section 6.6, "Calculating Results" for more information about mesh parameters. 


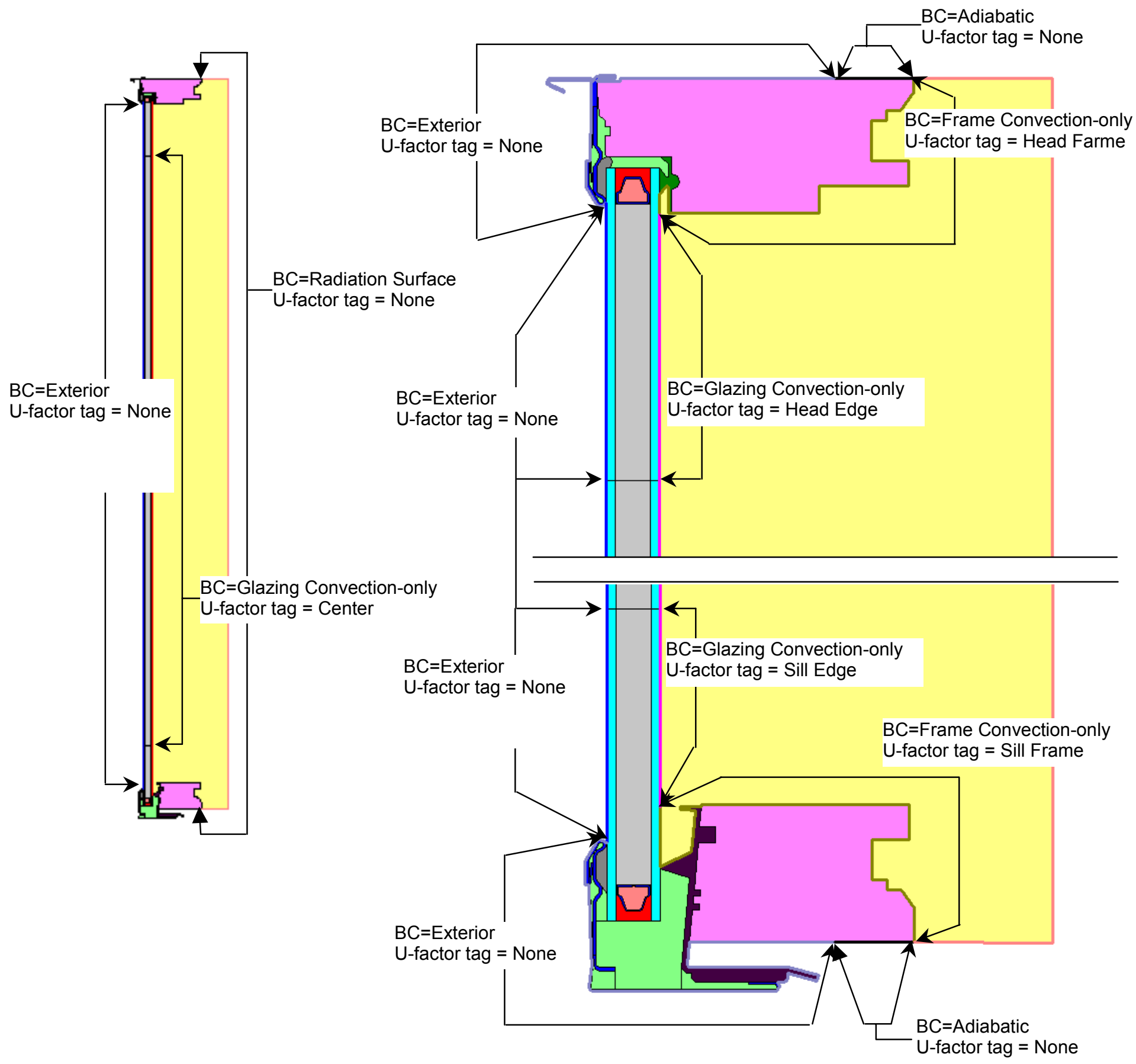

Figure 8-67 Boundary condition and U-factor tag settings for inset skylight example when using a spreadsheet for the total product U-factor calculation. 


\section{WINDOW Method}

If the total product U-factor is to be calculated in WINDOW, two simulations must be done for each fullheight cross section if the Frame and Edge U-factors are going to be imported into the WINDOW Frame Library. (If the jamb geometry was drawn in a different THERM file, another set of simulations would be performed for that cross section.) The Boundary Condition and U-factor Surface tags for each of these runs is shown in Figure 8-68.

1. Simulation \#1 (Head section of the full-height cross section):

- Assign the correct U-factor tags to all the components (Frame and Edge) associated with the Head

- Set the U-factor tags for the Sill to None

- Simulate the problem

- Export the results to a WINDOW T2W file

2. Simulation \#2 (Sill section of the full-height cross section):

- Assign the correct U-factor tags to all the components (Frame and Edge) associated with the Sill

- Set the U-factor tags for the Head to None

- Simulate the problem

- Export the results to a WINDOW T2W file 
First simulation

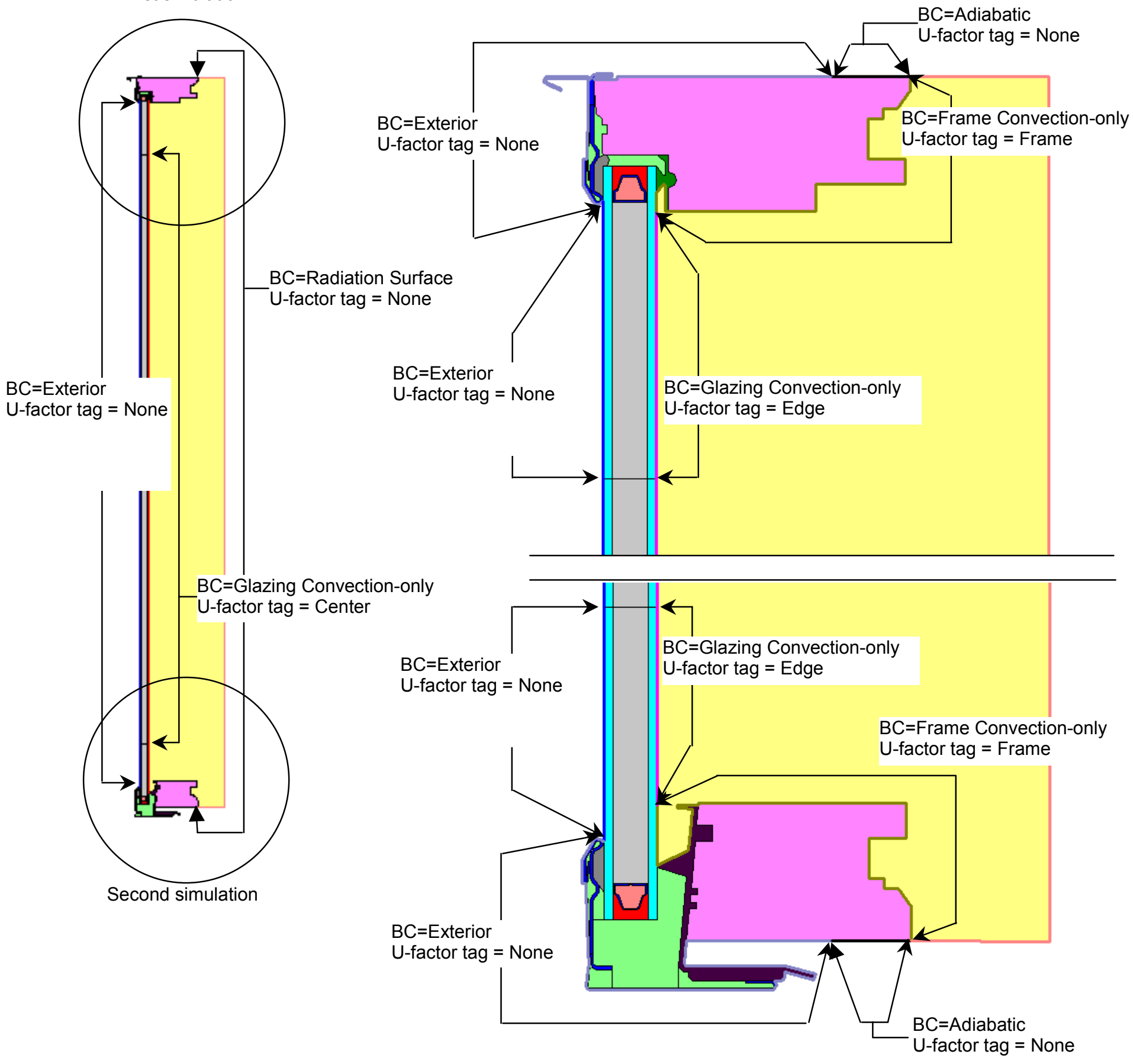

Figure 8-68 Boundary condition and U-factor tag settings for inset skylight example when using WINDOW to calculate the total product U-factor. 
3. During the meshing step of the simulation, if an error message appears saying that the geometry is too complicated, reset the mesh parameter (Options menu, THERM File Options choice) to a higher value. See Section 6.6, "Calculating Results" for more information about mesh parameters.

4. Figures 8-69 and 8-70 show the U-factors for each separate simulation. The results for each simulation would be saved to separate T2W files and imported as two separate frames into the WINDOW Frame Library as discussed previously.

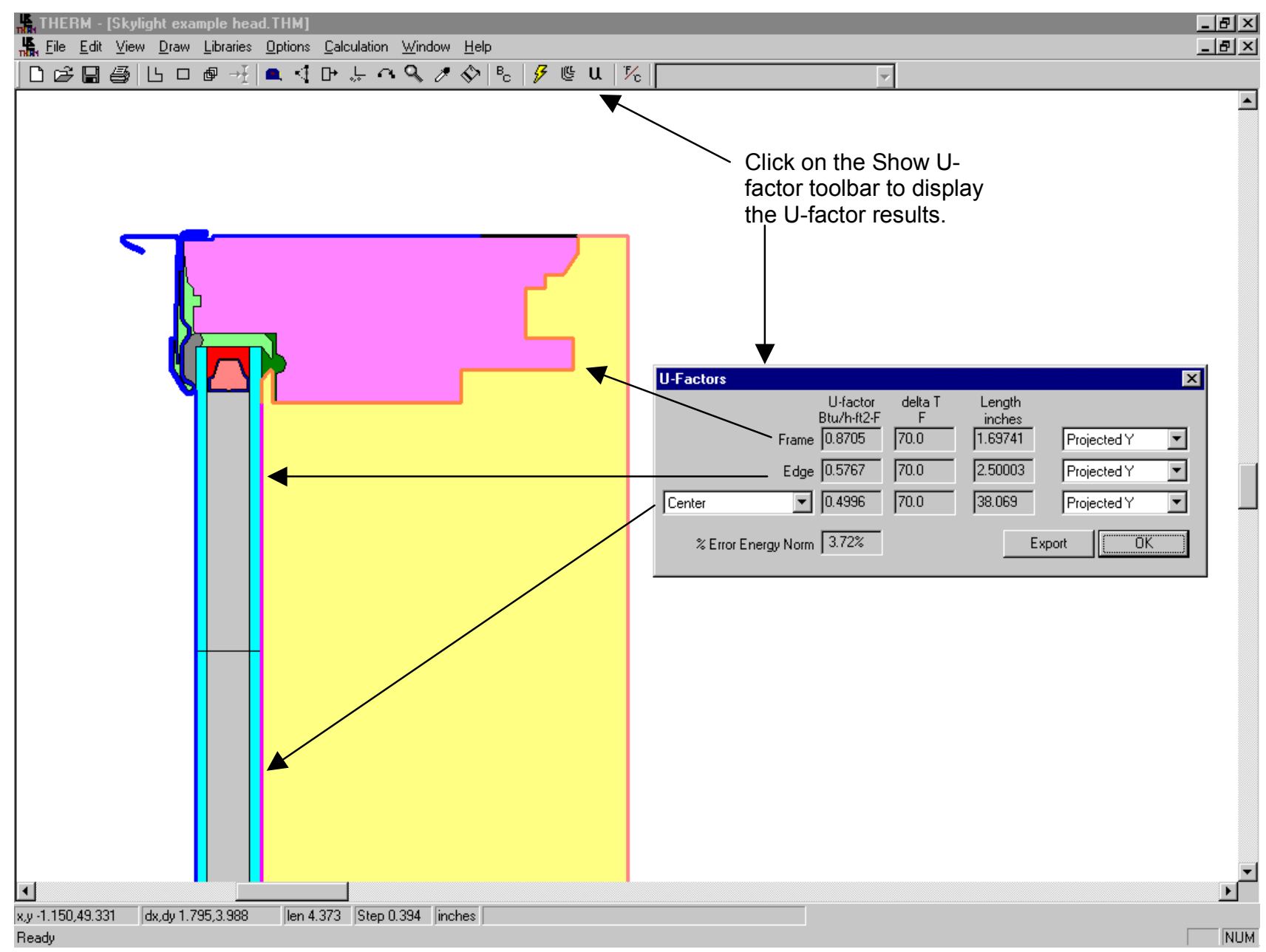

Figure 8-69 THERM U-factor results for the Head simulation. 


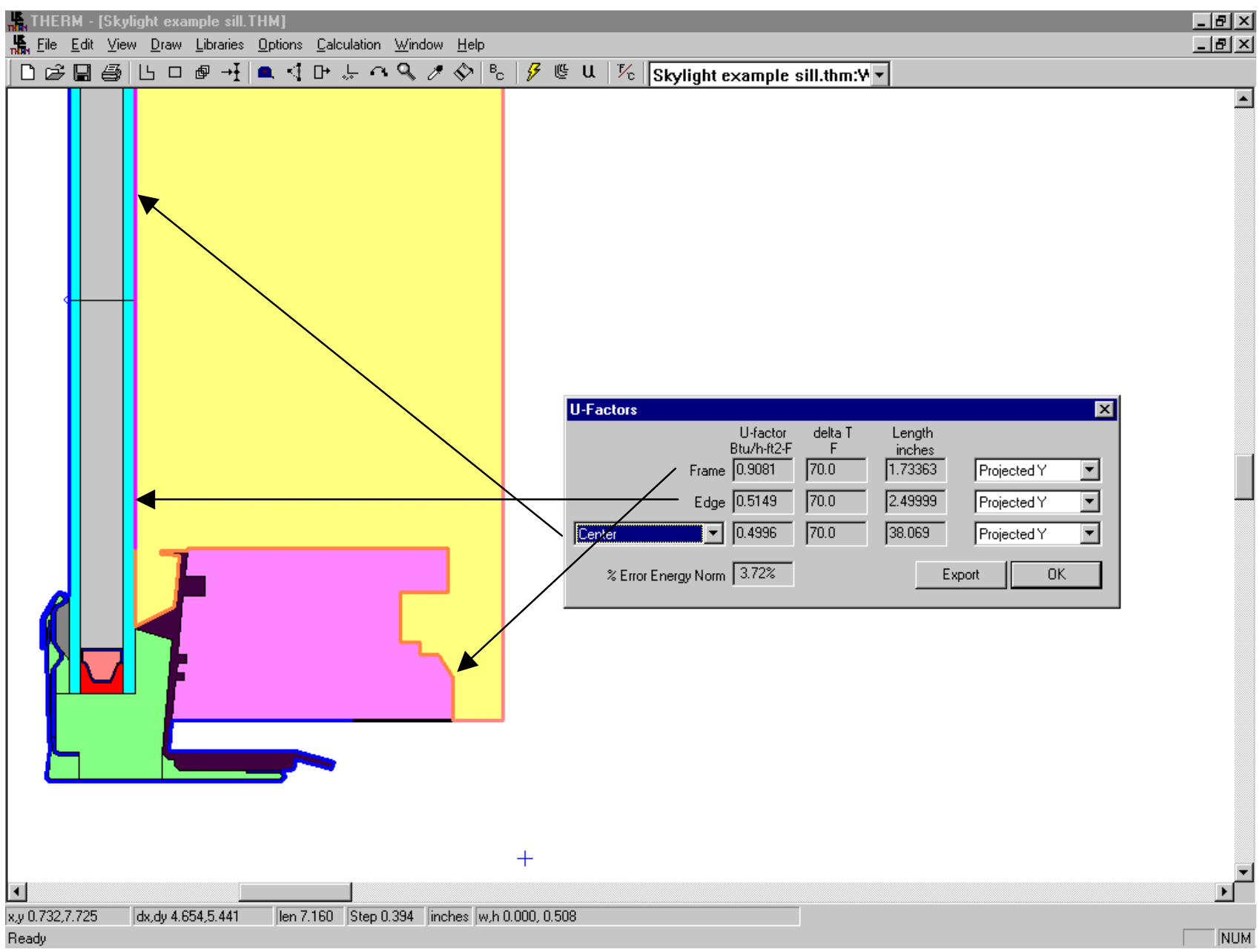

Figure 8-70 THERM U-factor results for the Sill simulation.

5. Check the glass temperature

Using the tape measure cursor (Options/Drawing Options menu, check Tape Measure Average Temperature) determine the average temperature of the glass from the sill to the head. If this temperature is not within $5^{\circ} \mathrm{F}$ of the inner surface glass temperature from WINDOW (in this example it was $44.9^{\circ} \mathrm{F}$ ), recalculate the glass convection-only film coefficient (using equation 1 ), make a new boundary condition record in the Boundary Condition Library with this new film coefficient, assign this boundary condition to the interior of the glazing system, and re-simulate the problem.

6. In WINDOW, import the THERM frame files

In the WINDOW Frame Library, import the THERM T2W files for the Head, Sill, and any other needed cross sections that were modeled. 
7. The results for the whole product can now be area-weighted using a spreadsheet in accordance with NFRC 100, or using WINDOW. If using WINDOW, follow the steps listed below.

Using the center-of-glass U-factor results from THERM, as shown in Figures 8-69 and 8-70, make a new glazing system in WINDOW to represent this U-factor.

The following steps explain how to make this generic glazing system in WINDOW with the results from the THERM simulation:

- Obtain the center-of-glass U-factor from THERM (from the U-factor Tag of Center)

- In this example, the center-of-glass U-factor $=0.4994$ for both the Head and Sill simulations. If these values are different, an average value shall be used.

- Obtain the inside glazing system film coefficients from THERM by looking at the boundary condition (named with the glazing system name prepended to U-factor Inside Film) that came in with the WINDOW glazing system.

- In this example, Double Clear U-factor Inside Film $=1.398 \mathrm{Btu} / \mathrm{hr}-\mathrm{ft}^{2}-\mathrm{o} F$

- The exterior surface film coefficient $=5.112 \mathrm{Btu} / \mathrm{hr}-\mathrm{ft}^{2}-\mathrm{oF}$

- Obtain the R-value for the glazing system by taking the inverse of the U-factor, and then subtract the inside and outside film coefficients from the THERM center-of-glass U-factor, which results in the R-

$$
\begin{aligned}
R \quad & =\frac{1}{U_{c}}-\left(\frac{1}{h_{i}}+\frac{1}{h_{o}}\right) \\
& =\frac{1}{0.4994}-\left(\frac{1}{1.398}+\frac{1}{5.112}\right) \\
& =2.00-(0.715+0.196) \\
& =1.091
\end{aligned}
$$

value for just the glazing system.

- For this example, the values are the following:

- Obtain the total width of the glazing system in THERM (using the tape measure or by looking in the Glazing System Library). For IP units, convert this value from inches to feet; for SI units, convert it from millimeters to meters.

- In this example, the overall glazing system width $=0.6680 "$. This value must be converted to feet (divide by 12$)=0.6680 / 12=0.0557$ feet

- Calculate the effective conductivity of the glazing system with the following equation:

$$
\text { Keff }=\frac{d}{R}
$$

where:

Keff $=$ effective conductivity of the glazing system

$\mathrm{D}=\quad$ overall thickness of the glazing system

$\mathrm{R}=\quad \mathrm{R}$-value of the glazing system

- In this example, the Keff would be calculated as:

$$
\begin{aligned}
\text { Keff } & =\frac{0.0557}{1.091} \\
& =0.0510
\end{aligned}
$$


- Notice that in this particular example, using the radiation model does not significantly change the

$\mathrm{U}_{\mathrm{c}}$. However, in other cases with less insulating components, the imapct will be greater.

- Calculate the reflectance and transmittance values from the original WINDOW glazing system inserted into THERM, as shown in Table 8-2.

Table 8-2 Interior convection-only frame film coefficients for radiation enclosures based on frame type.

\begin{tabular}{|l|l|l|}
\hline Glass Library Variable & $\begin{array}{l}\text { Value to use from Original Glazing } \\
\text { System imported into THERM }\end{array}$ & Example value \\
\hline Tsol & SHGCc & 0.76 \\
\hline Rsol 1 \& Rsol 2 & $1-$ SHGCc & $1-0.76=0.24$ \\
\hline Tvis & Vtc & 0.81 \\
\hline Rvis 1\&2 & $1-$ Vtc & $1-0.81=0.19$ \\
\hline Tir & 0 & 0 \\
\hline
\end{tabular}

In WINDOW, define a new glass layer in the Glass Library with:

- $\quad$ Thick $=\mathrm{d}$ (inches for IP, $\mathrm{mm}$ for SI)

(0.6680" in this example)

- $\quad$ Emis1 and Emis2 = 0.84

- $\quad$ Cond $=$ Keff

(0.0510 Btu/hr-ft-o F in this example)

Figure 8-71 shows the glass layer for this example.

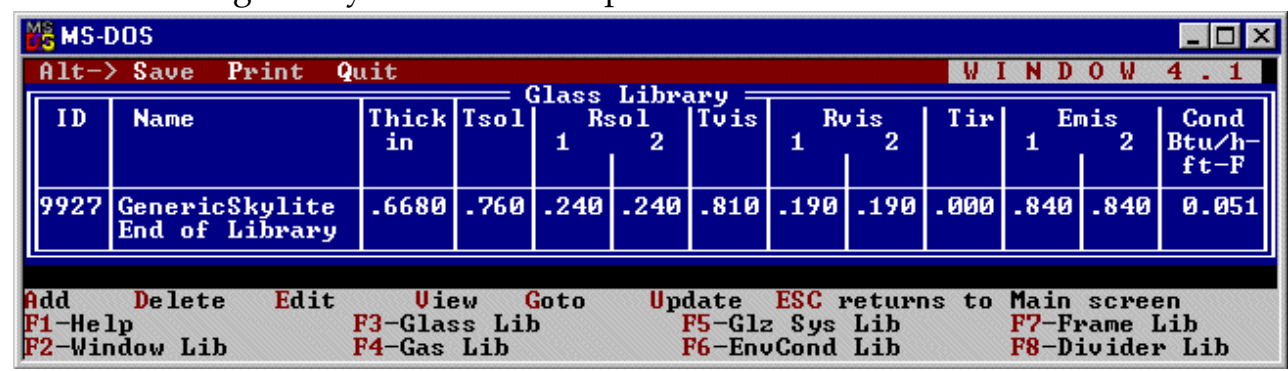

Figure 8-71 Generic skylight glass layer in WINDOW with properties calculated from THERM results.

- In the WINDOW glazing system library, create a new glazing system using the new glass as the only glass layer.

Figure 8-72 shows the glass layer for this example.

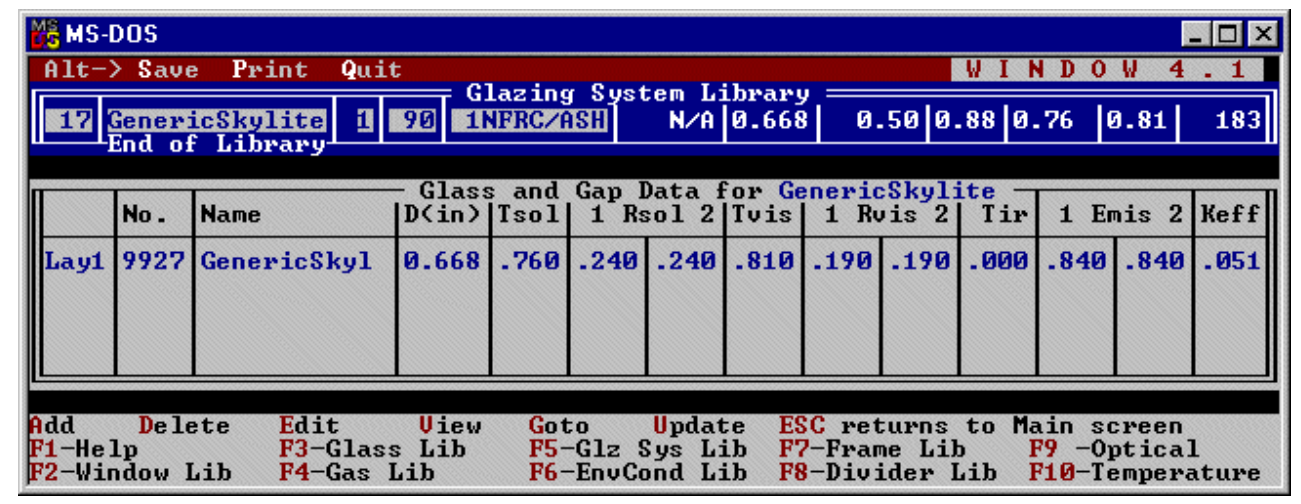

Figure 8-72 Generic skylight glazing system in WINDOW with properties calculated from THERM results. 
8. In WINDOW, use the new generic glazing system and the THERM T2W files in the Frame Library to create the total product U-factor, as shown in Figure 8-73.

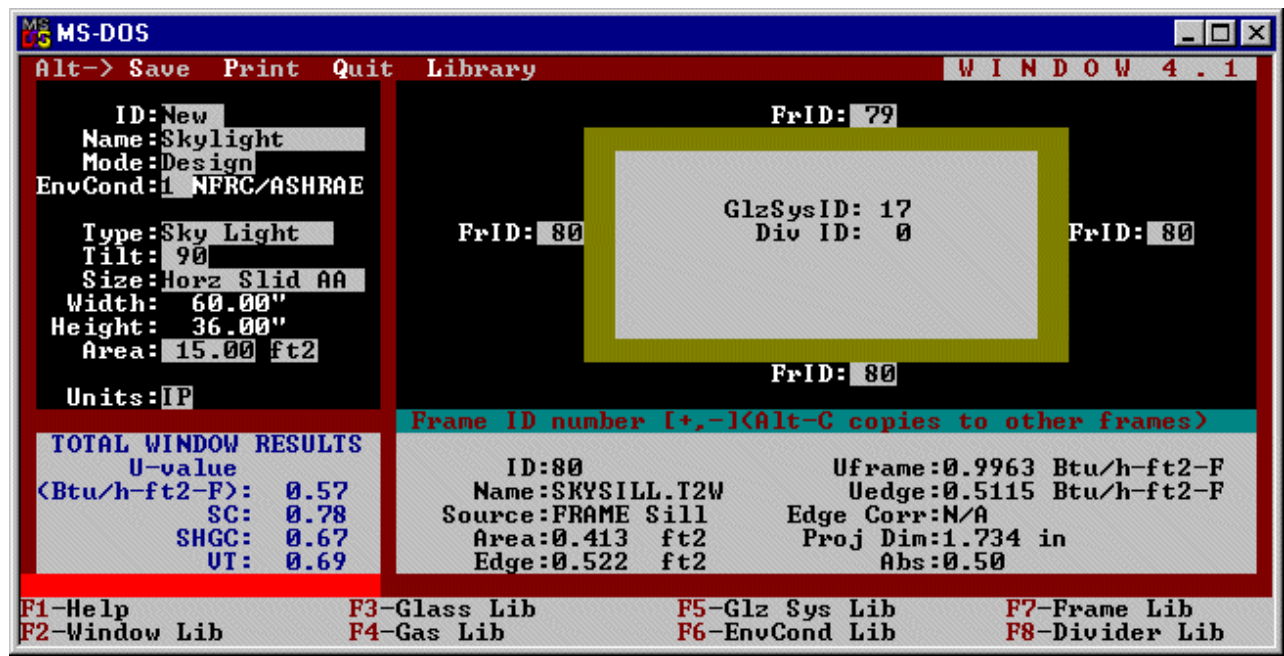

Figure 8-73 WINDOW total product U-factor, SHGC, and VT for the skylight. 


\subsection{Doors}

Swinging entry doors are modeled differently than window products because there are more opaque sections to be modeled in THERM. A separate document NFRC 100: Section B: Procedure for Determining Door System Product Thermal Properties (Currently Limited to U-values) covers the modeling of entry doors. NFRC 100 Section B should be reviewed in detail before modeling any entry door systems.

\subsubsection{Modeling Doors}

One main differences between modeling window products and entry doors is that NFRC 100 Section $B$ defines the door slab size rather than the overall product size. Therefore the overall dimension of door systems will vary slightly from one product to another.

NFRC has defined nine regions within a door that need to be modeled. These regions include:

- Door Frame Area

- Door-Edge Area

- Edge-of-Lite Area

- Door Core Area

- Panel Core Area

- Panel Area

- Door Lite Frame Area

- Center-of-Lite Area

- Edge-of-Panel Area

Six THERM models and one WINDOW model (for the center-of-lite area) are required to model the nine regions in the door. Figure 8-62 indicates the location of the six door sections to be modeled. The door core modeling is included in one of the door frame/edge models, and the panel core modeling is included in the panel model.

In THERM, the U-factor Surface Tags can have any name and as many U-factor Surface Tags can be defined as are needed to accurately describe the model. (See Section 6.2.4, "Define U-factor Surface Tags in the THERM User's Manual). When using WINDOW to area-weight whole product values, the U-factor Surface Tags must be Frame and Edge. However, when using a spreadsheet for area-weighting, as is the case with doors (because the current version of WINDOW does not area-weight doors), define as many U-factor Surface Tags as needed and name them descriptively, such as the names listed in Table 8-3.

The door lite sizes in NFRC 100 Section B have been specified as the dimensions of the overall door lite, not the frame sightline-to-sightline dimension. The difference in these dimensions must be accounted for when area weighting the component values to determine the overall door system U-factor. NFRC 100 Section B requires the U-Factor, SHGC, and VT values be determined for four door lite sizes ( $1 / 4$ glass, $1 / 2$ Glass, 3/4 Glass, and full glass) as well as no glass.

Non-continuous door hardware (such as hinges, locks, and door knobs) is not typically modeled. Any hardware that is continuous in any one section of the door must be modeled in that section.

\subsubsection{Door Cross Sections}

Figure 8-74 indicates the door sections that need to be modeled in THERM. The letters on each of the sections indicate the U-factor surface tags that will be determined in each of the THERM models. Table 8-3 lists the cross-section and the U-factors for the sections. 
Table 8-3 Door Cross Sections

\begin{tabular}{|l|l|l|l|}
\hline Cross Section & \multicolumn{3}{|c|}{ U-Factor Determined } \\
\hline Top Rail & A - Top Rail Frame & B - Top Rail Edge & C - Door Core \\
\hline Door Lite & D - Door Lite Frame & E - Edge-of-Lite & \\
\hline Hinge Stile & F - Hinge Stile Frame & G - Hinge Stile Edge & \\
\hline Lock Stile & H - Lock Stile Frame & I - Lock Stile Edge & \\
\hline Door Panel & J - Door Panel Frame & K - Door Panel Edge & L - Panel Core \\
\hline Bottom Rail & M - Bottom Rail Frame & N - Bottom Rail Edge & \\
\hline
\end{tabular}

When modeling the door-edge region the distance varies depending on the type of door being modeled. Stile and rail doors include $2 \frac{1}{2 \prime}$ " of the door in the model and steel doors include $21 / 2$ " or a distance 1 " from the material transition edge, which ever is greater (see NFRC 100, Section B, Figures B2 and B4).

The panel core model is included in the Door Panel model. THERM allows multiple U-Factor surfaces to be defined. A panel core U-factor Surface Tag can be added to the U-factor Names library and used to calculate the panel core U-factor in the door panel model. The door core model can be added to any of the door frame and edge models, but for consistency the core model should be added to the top rail model and a door core U-factor surface added to the library to be used to calculate the door core U-factor. In both the top rail and door panel models add $2 \frac{1}{2}{ }^{\prime \prime}$ of core material to determined the door core or panel core U-factor.

\subsubsection{Stile and Rail, and Embossed Door Glazing Options}

The glass sizes for stile and rail, and embossed door glazing options are detemined by removing panels and intermediate stiles and railes. The sizes are determined by removing the panel combinations shown in Table $8-4$.

Table 8-4. Glazing option sizes based on panel combinations

\begin{tabular}{|l|l|}
\hline $1 / 4$ Glass & Remove upper two panels and intermediate stiles and rails. \\
\hline $1 / 2$ Glass & Remove upper four panels and intermediate stiles and rails. \\
\hline $3 / 4$ Glass & Remove lower four panels and intermediate stiles and rails. \\
\hline Full Glass & Remove all six panels and intermediate stiles and rails. \\
\hline
\end{tabular}




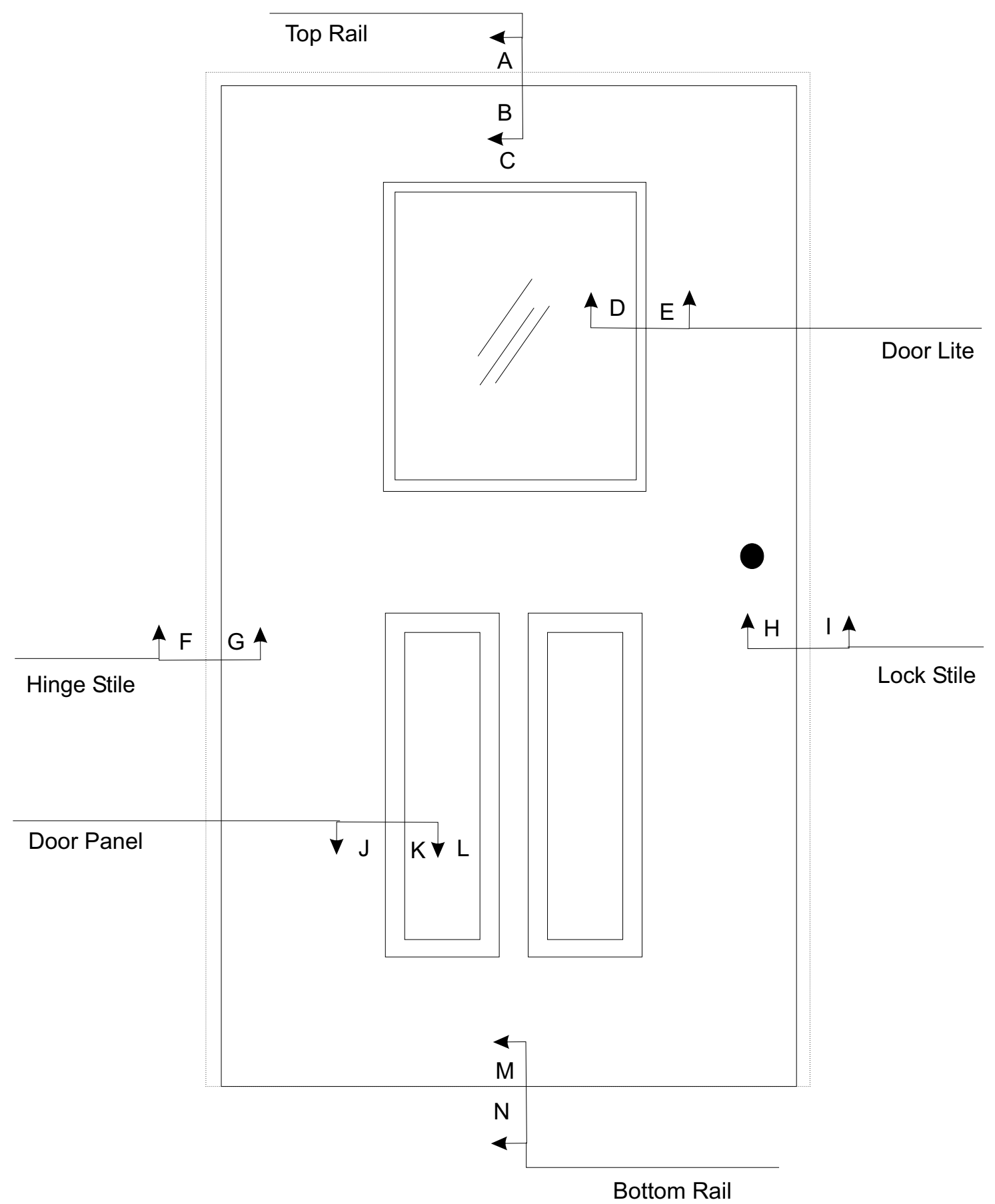

Figure 8-74. Cross Sections for Door Simulations. 


\subsection{Spacers}

\subsubsection{Overview}

THERM has the capability to model spacers in great detail, so that modeling of spacer effective conductivity is no longer needed. Spacer models can be easily reshaped in THERM, and the program's cut and paste feature allows spacers to be copied into each cross section as needed. A library of spacer models can be produced for each spacer type. See the THERM User's Manual, Section 3.5, "Adding a Custom Spacer". A sample spacer, spacer.thm, is included on the THERM installation CD.

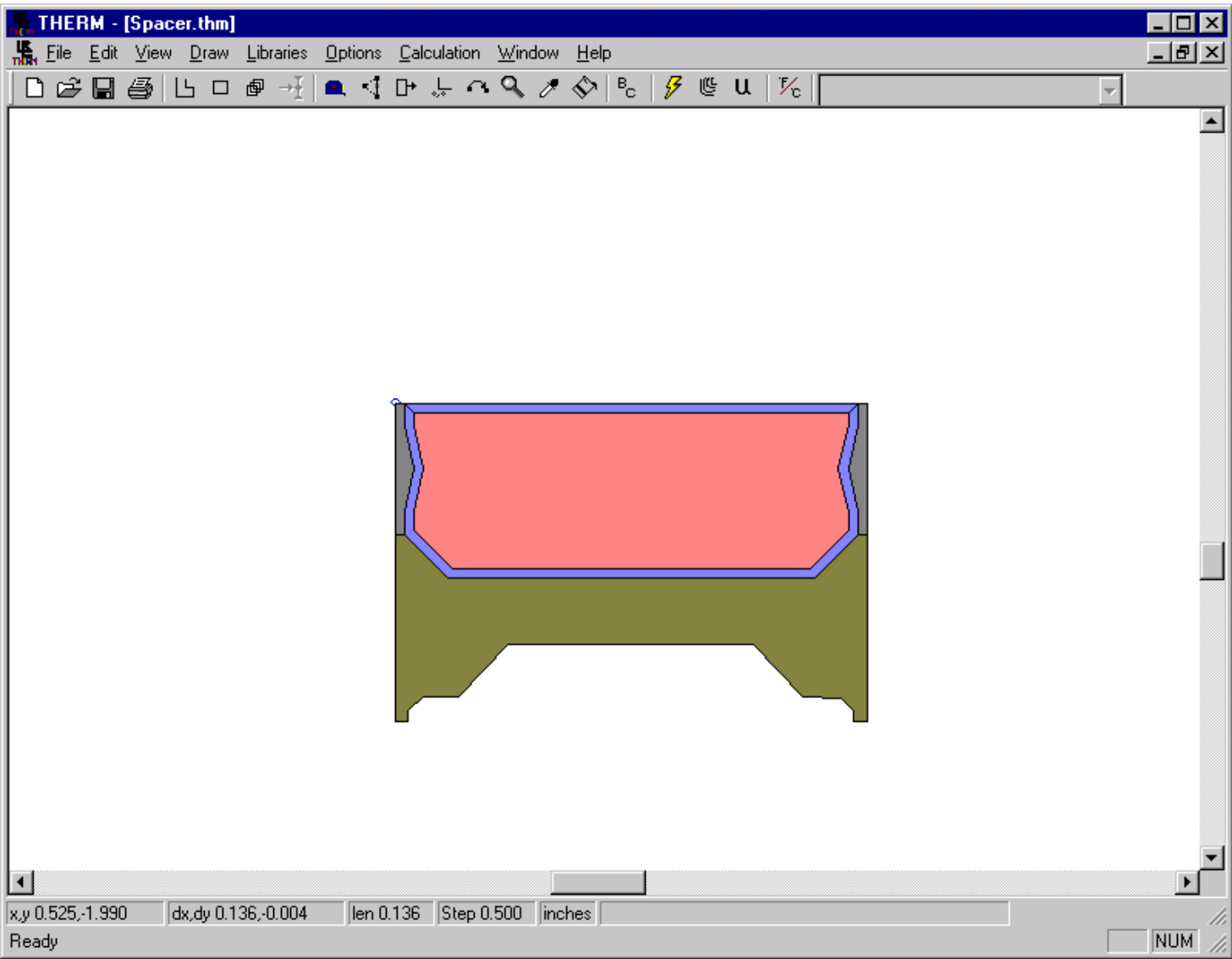

Figure 8-75. spacer.thm sample file. 


\subsubsection{Modifying Glazing Cavities (imported from WINDOW) for Open Spacers}

Glazing cavities can be modified to accommodate open spacers -- check Options/Preferences/Drawing Options/Allow Editing of IG polygons, and then edit the points in the glazing cavity, as shown in Figure 877. It is also possible to leave the glazing cavity unedited, and instead make a new material in the THERM Material Library that is equal to the Keff of the glazing cavity (this can be determined from WINDOW), and then using the Fill Void tool in THERM to fill the open spacer area with this new material, as shown in Figure 8-78.

When using the multiple glazing calculation option, THERM will automatically use the glazing system cavity properties for each glazing option for the entire cavity geometry, including the edited area extended into the open spacer.
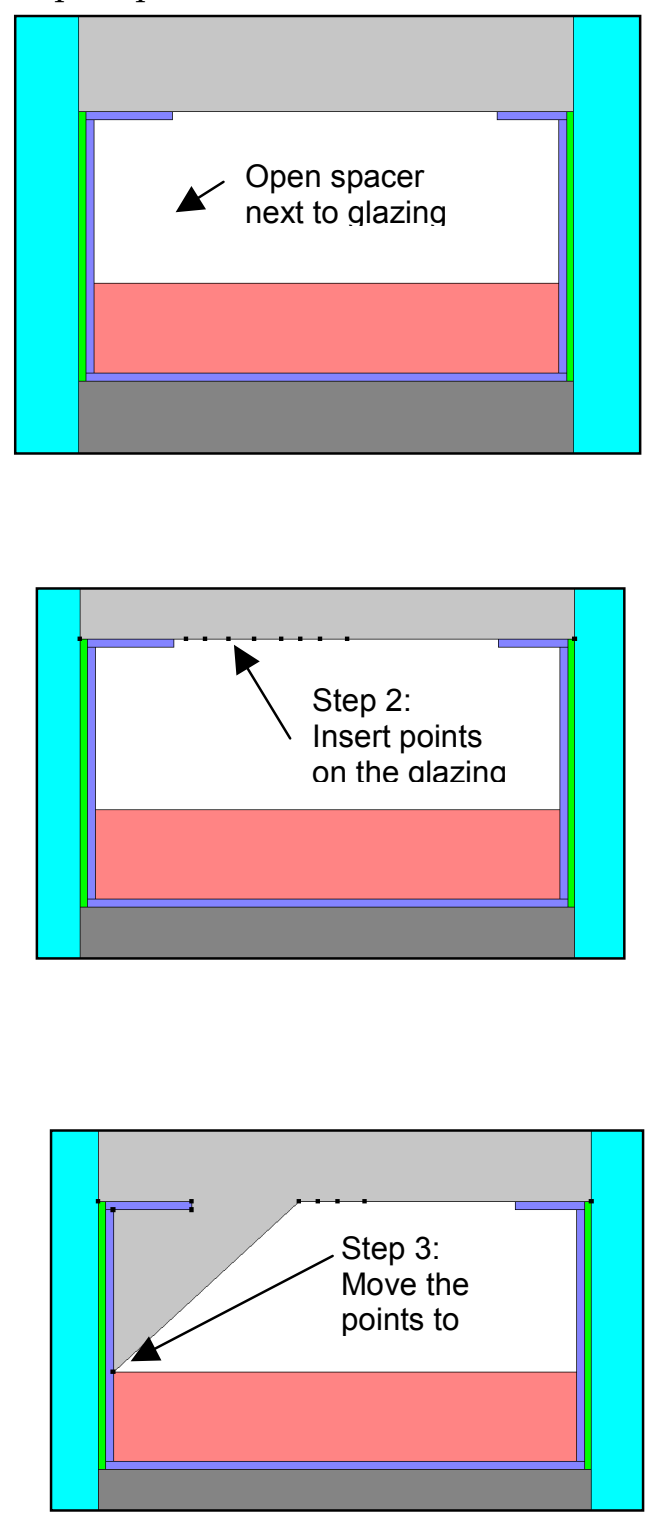

Figure 8-76. Technique for bringing a glazing cavity into a spacer.

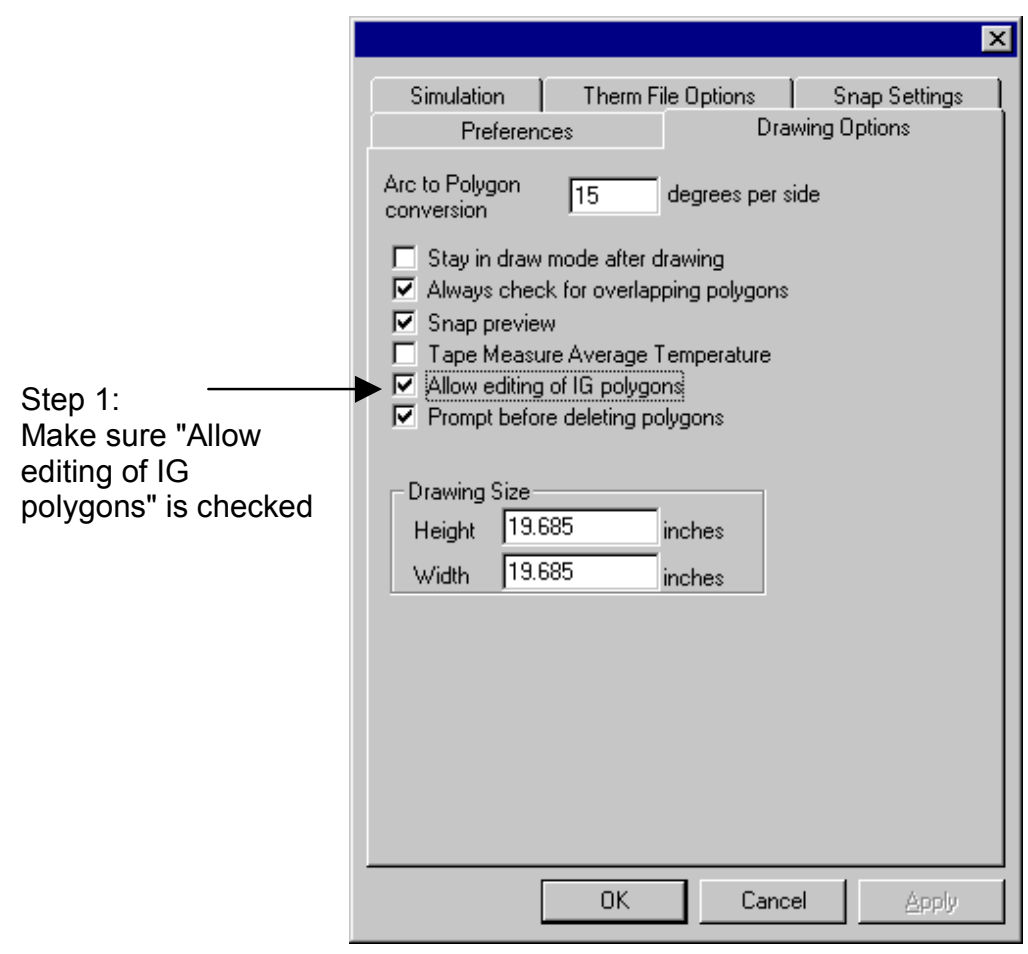

Step 4:

This technique fills the spacer with the glazing

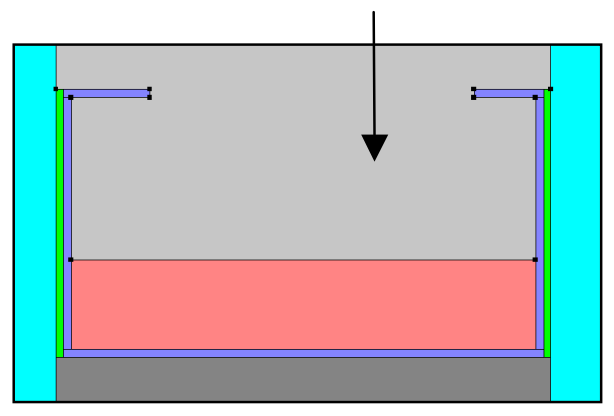


THERM - [open-spacer-glass.thm]

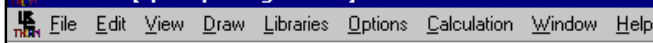

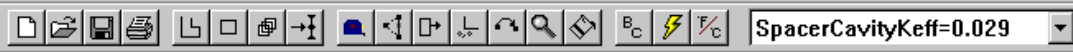

Step 1:

From WINDOW, determine the Keff value of the glazing system cavity.

Step 2:

Define a material with that Keff value, and use the Fill Void tool to fill the onen spacer with this material.
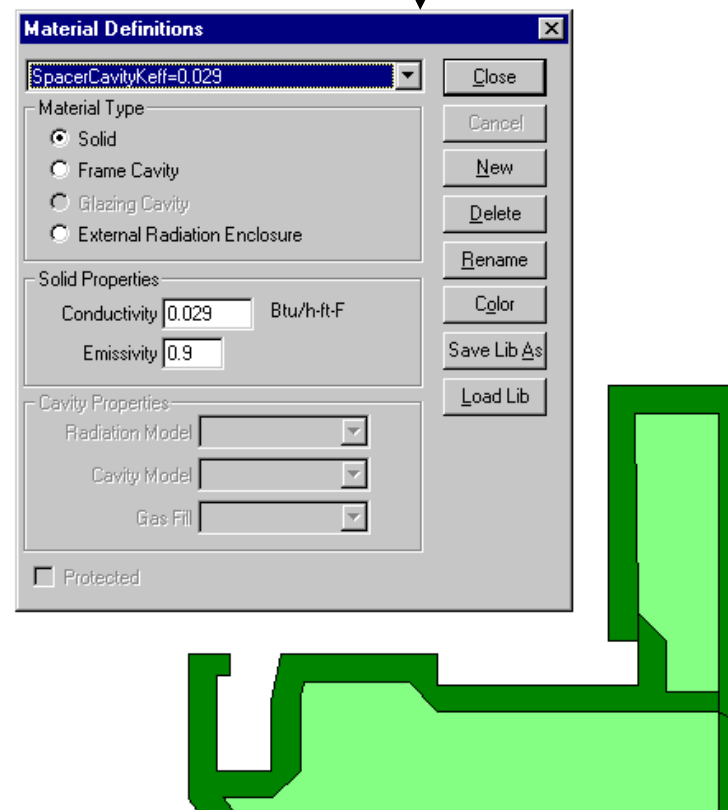

Figure 8-77. Define a material in THERM with the Keff value of the glazing system and use the Fill Void tool to fill the spacer with this material. 


\section{SAMPLE PROBLEMS}

\subsection{Overview}

There are four sample problems:

- Problem 1: Vinyl fixed window

page 9-2

- Problem 2: Aluminum horizontal sliding window

page 9-11

- Problem 3: Skylight

page 9-42

- Problem 4: Door:

page 9-50

These sample problems may contain boundary conditions, frame cavity conditions and modeling techniques which do not conform to the NFRC modeling rules. If this is the case, the NFRC modeling rules always take precedence over what is shown in these example problems. 


\subsection{Problem 1: Vinyl Fixed}

For this fixed vinyl window product, calculate the U-factor, SHGC and VT values.

\subsubsection{Description}

Window Type Fixed picture window

Frame Material PVC frame; one profile is used on all four sides of the windows. The wall thickness is $0.125 "$

Glazing System Double glazing, 0.750" overall I.G. thickness. The outboard lite is double-strength clear glass (0.129" thick), the inboard lite is double-strength clear glass with a PPG Sungate100 low-E coating on surface three, and the glazing cavity (0.492") is air filled.

Spacer type Swiggle Seal ${ }^{\mathrm{TM}}$ by Tremco

Glazing Tape Foam rubber tape, 0.125 " thick

Dividers Aluminum grille pattern, painted white, between the glass. The grille pattern for the window produced at the AA size is 3 by 3 .

Cross Sections $\quad$ See Section 9.2.7 for drawings of this product.

\subsubsection{Glazing Matrix}

The window is offered by the manufacturer both with and without dividers. The drawings indicate that there is less than 1/8" between the glass and the divider, dividers, so the glazing matrix must include both a case with and without the dividers. For the SHGC and VT calculations, the $6 \mathrm{~mm}$ cases must also be calculated.

Table 9-1. Vinyl window glazing matrix

\begin{tabular}{|l|c|c|}
\hline & $\begin{array}{c}\text { Residential } \\
\text { (AA) }\end{array}$ & $\begin{array}{c}\text { Non-Residential } \\
\text { (BB) }\end{array}$ \\
\hline Low-e glazing system(clear, air, low-E) without dividers & & \\
\hline Low-e glazing system (clear, air, low-E) with dividers & & \\
\hline
\end{tabular}

\subsubsection{Center-of-Glass Modeling (WINDOW)}

Model the glazing system in WINDOW with double strength glass, a 0.492" air space, air filled, and Sungate100 Low-e.

Figure 9-1 shows the WINDOW Glazing System Library for this glazing system. 


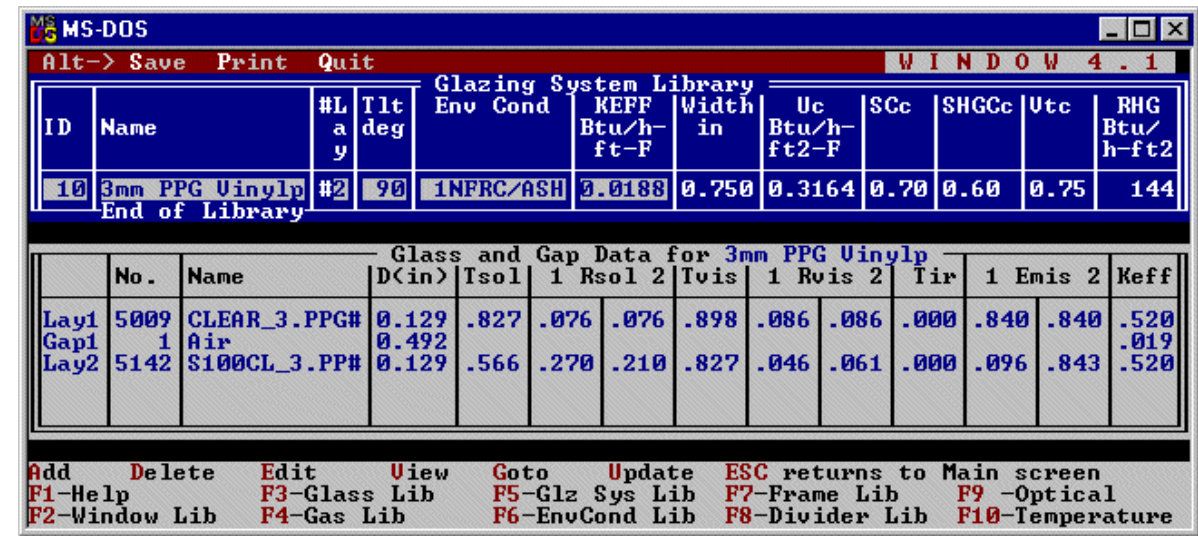

Figure 9-1. WINDOW 4.1 Glazing System Library for vinyl window

The results for the center-of-glass U-factor $(\mathrm{Uc})$ are shown in the following table:

Table 9-2. Center-of-glass U-factor results from WINDOW

\begin{tabular}{|c|c|c|c|}
\hline & \multicolumn{2}{|c|}{$\begin{array}{c}\text { Center-of-glass U-factor } \\
\left(U_{c}\right)\end{array}$} \\
\hline & $\begin{array}{c}\text { Glazing Options }(0.75 " \text { overall } \\
\text { thickness) }\end{array}$ & $\left(\mathrm{Btu} / \mathrm{hr}-\mathrm{ft}^{2}-{ }^{\circ} \mathrm{F}\right)$ & $\mathrm{W} / \mathrm{m}^{2}-{ }^{\circ} \mathrm{C}$ \\
\hline 1 & Clear (3 mm), Air, Low-e (3mm) & 0.3164 & 1.7969 \\
\hline
\end{tabular}

This glazing system will be used in THERM to calculate the edge-of-glass and frame U-factors, and also in WINDOW to calculate the overall product U-factor in Section 9.3.5.

\subsubsection{Edge-of-Glass and Frame Modeling (THERM)}

Because this is a fixed window, the head, sill and jamb have the same geometry, so there is only one crosssection which must be modeled.

Table 9-3 shows the files that are on the CD for this example.

Table 9-3. Files associated with the vinyl window example

\begin{tabular}{|l|l|l|}
\hline Cross Section & DXF Filename & \multicolumn{1}{c|}{ THERM file } \\
\hline Sill, Head \& Jamb with $3 \mathrm{~mm}$ glass & Vinylp.dxf & Vinyl-3mm.thm \\
\hline Sill, Head \& Jamb with $6 \mathrm{~mm}$ glass & & Vinyl-6mm.thm \\
\hline
\end{tabular}

Table 9-4 shows the resulting U-factors the vinyl frame and the divider cross sections.

Table 9-4. THERM results for vinyl window cross sections

\begin{tabular}{|c|c|c|c|c|}
\hline \multirow[b]{2}{*}{ Cross Section } & \multicolumn{2}{|c|}{ Frame U-factor } & \multicolumn{2}{|c|}{ Edge U-factor } \\
\hline & $\begin{array}{l}\text { (Btu/hr-ft }{ }^{2}- \\
\left.{ }^{\circ} \mathrm{F}\right)\end{array}$ & $\begin{array}{l}\left(\mathrm{W} / \mathrm{m}^{2}-\right. \\
\left.{ }^{\circ} \mathrm{C}\right)\end{array}$ & $\begin{array}{l}\text { (Btu/hr-ft }{ }^{2}- \\
\left.{ }^{\circ} \mathrm{F}\right)\end{array}$ & $\begin{array}{l}\left(\mathrm{W} / \mathrm{m}^{2}-\right. \\
\left.{ }^{\circ} \mathrm{C}\right)\end{array}$ \\
\hline Sill, Head \& Jamb, 3mm & 0.3265 & 1.8539 & 0.3953 & 2.2449 \\
\hline Sill, Head \& Jamb, $6 \mathrm{~mm}$ & 0.3278 & 1.8613 & 0.4711 & 2.6750 \\
\hline Divider & 0.4713 & 2.6761 & 0.3542 & 2.0114 \\
\hline
\end{tabular}

Figures 9-2 and 9-3 show the THERM cross sections and U-factor results for this window. 


\section{Head, Sill and Jamb, $3 \mathrm{~mm}$ glazing}

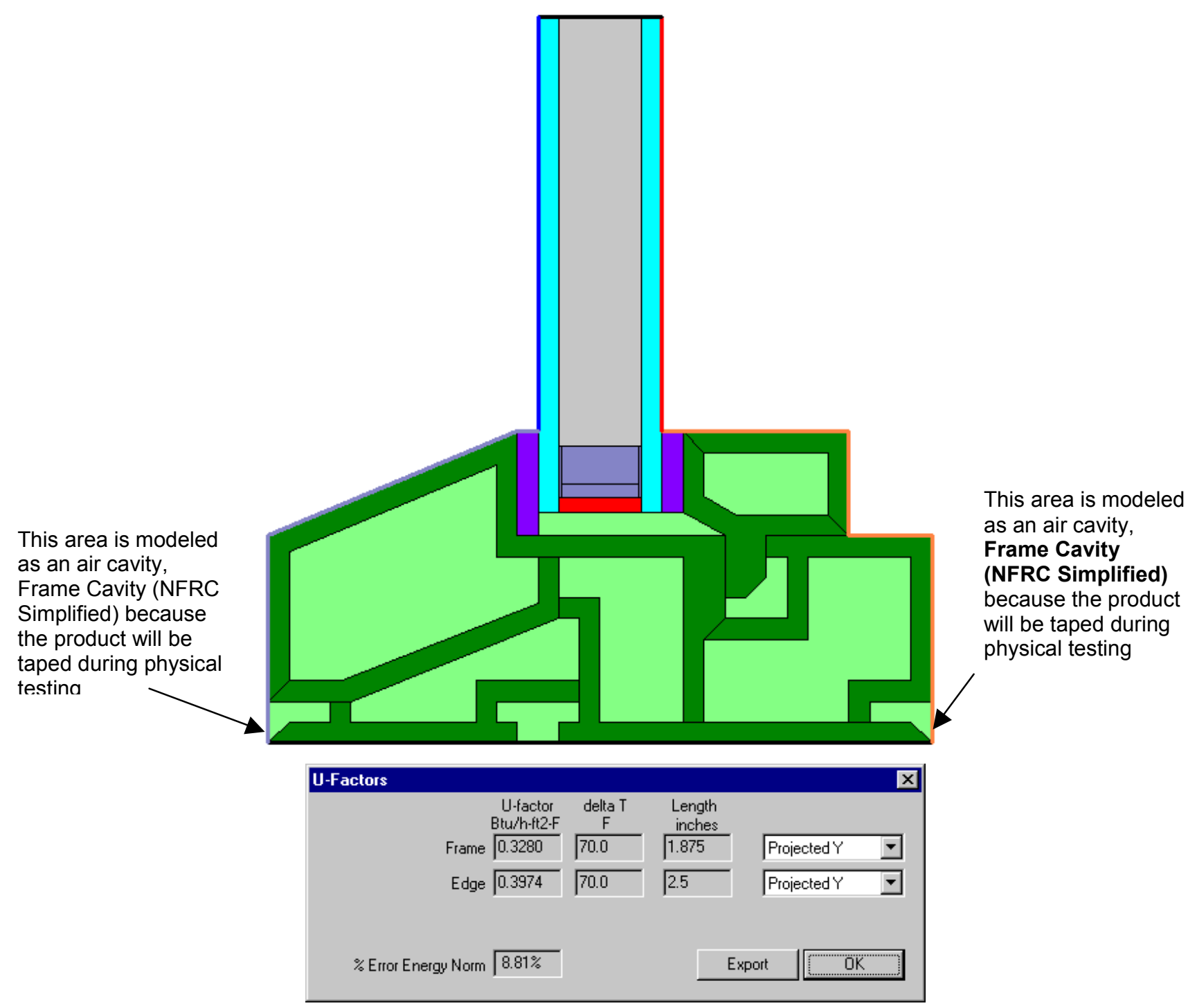

Figure 9-2. THERM cross section and U-factor for the vinyl fixed window head, sill and jamb with $3 \mathrm{~mm}$ glass. 
Head, Sill and Jamb, $6 \mathrm{~mm}$ glazing

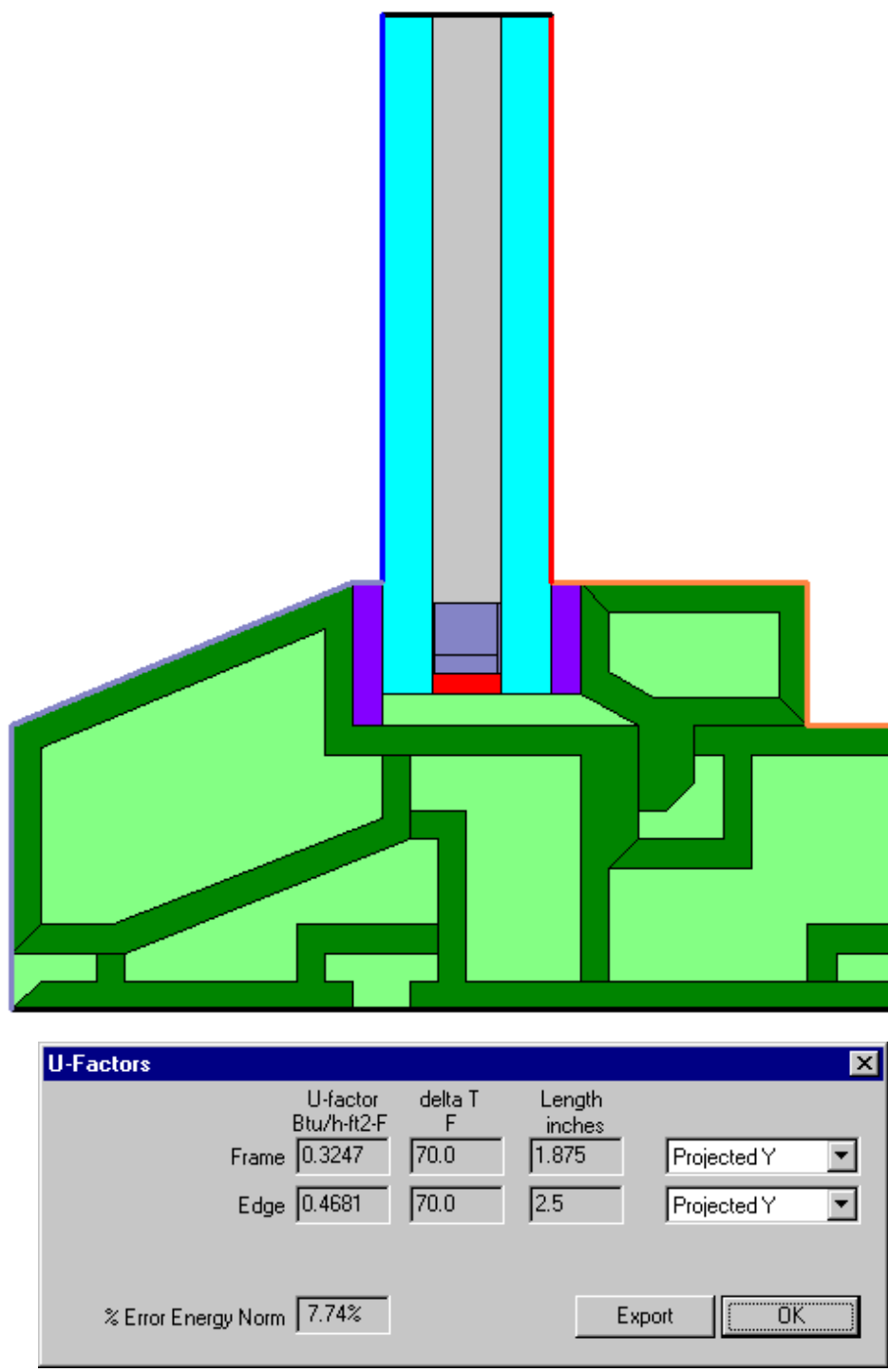

Figure 9-3. THERM cross section and U-factor for the vinyl fixed window head, sill and jamb with $6 \mathrm{~mm}$ glass. 


\section{Divider}
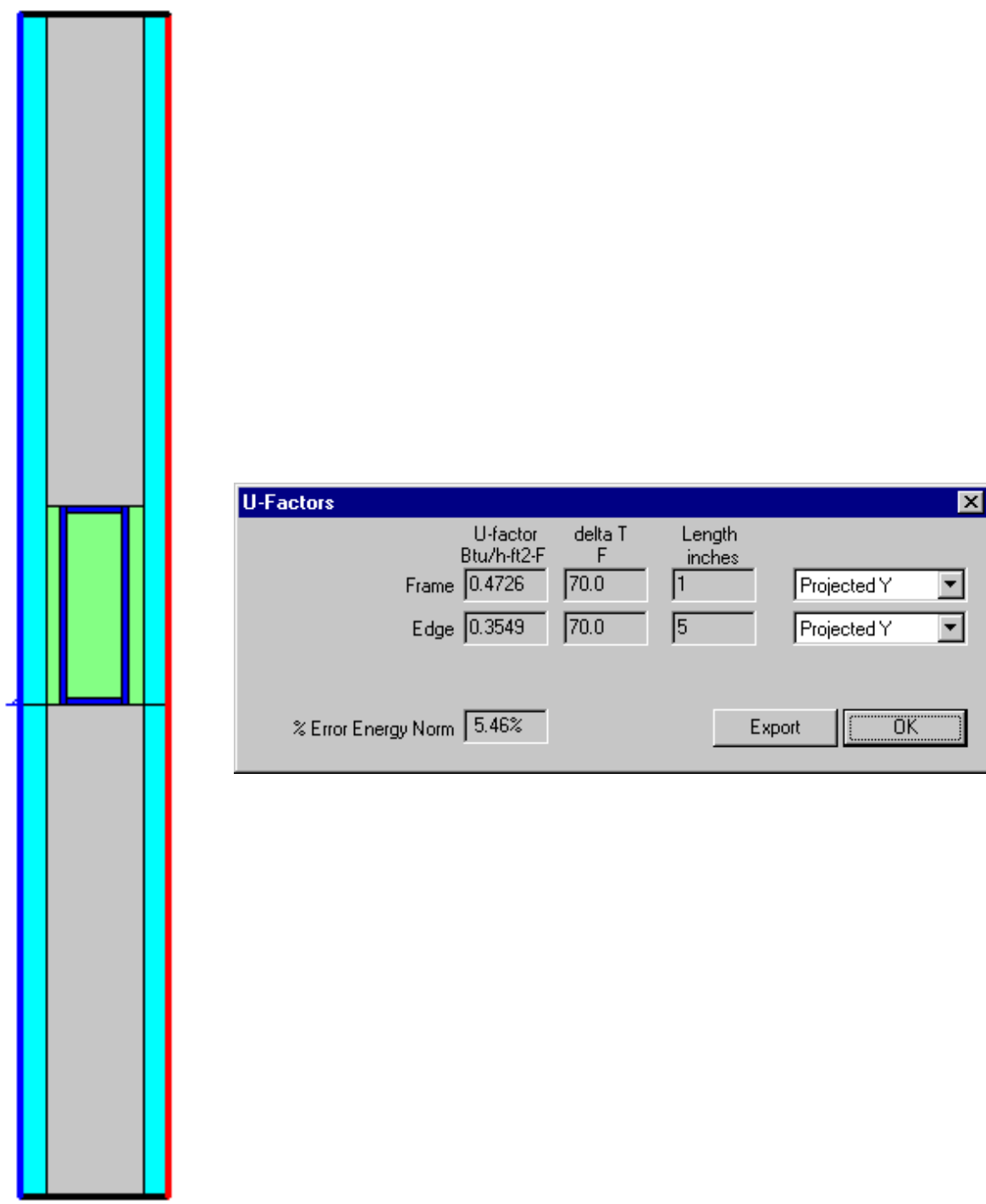

Figure 9-4. THERM cross section and $U$-factor for the vinyl fixed window divider. 


\subsubsection{Total Product U-Factor}

In WINDOW, a fixed AA window is modeled with and without a divider, and a BB window, is modeled without a divider.

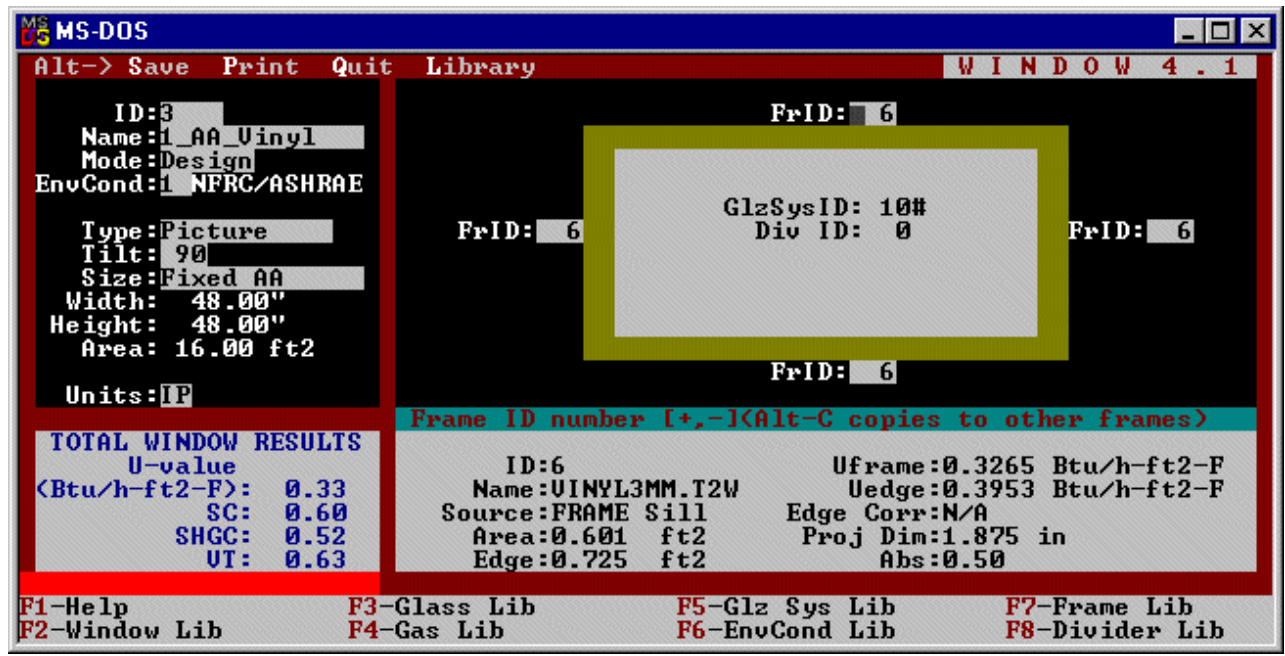

Figure 9-5. WINDOW total product $U$-factor calculation without dividers

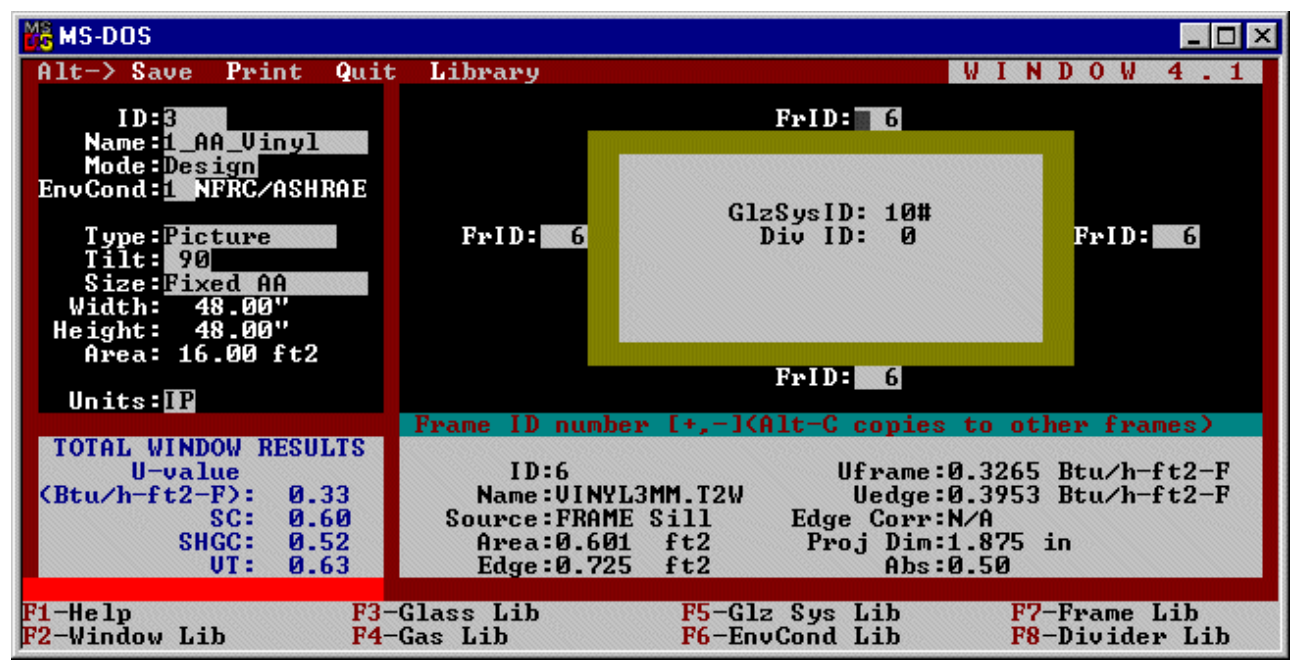

Figure 9-6. WINDOW total product $U$-factor calculation with dividers

Table 9-5 shows the overall product U-factor, from WINDOW, with and without dividers.

Table 9-5. Total Product U-factors

\begin{tabular}{|c|c|c|c|c|c|}
\hline & \multirow{2}{*}{$\begin{array}{c}\text { Glazing Options } \\
\text { (1.0" overall thickness) }\end{array}$} & \multicolumn{2}{|c|}{$\begin{array}{l}\text { Residential (AA) } 3 \mathrm{~mm} \\
\text { Total Product U-factor }\end{array}$} & \multicolumn{2}{|c|}{$\begin{array}{l}\text { Non-residential (BB) } 6 \\
\text { mm } \\
\text { Total Product U-factor }\end{array}$} \\
\hline & & $\begin{array}{l}\left(B t u / h r-\mathrm{ft}^{2}-\right. \\
\left.{ }^{\circ} \mathrm{F}\right)\end{array}$ & $\begin{array}{l}\left(\mathrm{W} / \mathrm{m}^{2}-\right. \\
\left.{ }^{\circ} \mathrm{C}\right)\end{array}$ & $\begin{array}{l}(\text { Btu/hr-ft } \\
\left.{ }^{\circ} \mathrm{F}\right)\end{array}$ & $\begin{array}{l}\left(\mathrm{W} / \mathrm{m}^{2}-\right. \\
\left.{ }^{\circ} \mathrm{C}\right)\end{array}$ \\
\hline 1 & $\begin{array}{l}\text { Clear, Air, Low-e } \\
\text { Without dividers }\end{array}$ & 0.332 & 1.887 & 0.363 & 1.942 \\
\hline 2 & $\begin{array}{l}\text { Clear, Air, Low-e } \\
\text { With dividers }\end{array}$ & 0.342 & 2.064 & N/A & N/A \\
\hline
\end{tabular}




\subsubsection{Total Product Solar Heat Gain Coefficient (SHGC) and Visible Transmittance (VT)}

The methodology for determining the Solar Heat Gain Coefficient for the product is outlined in NFRC 200, and the methodology for determining the Visible Transmittance is outlined in NFRC 300. WINDOW automatically calculates these values, as shown in Figures 9-5 and 9-6. The results for the glazing options are shown in Table 9-6, and the specialty products table is shown in Table 9-7.

Table 9-6. Total Product Solar Heat Gain Coefficients and Visible Transmittance

\begin{tabular}{|c|l|c|c|c|c|}
\hline \multicolumn{2}{|c|}{$\begin{array}{c}\text { Glazing Options } \\
\text { (0.75" overall thickness) }\end{array}$} & \multicolumn{2}{c|}{ Residential (AA) 3 mm } & \multicolumn{2}{c|}{$\begin{array}{c}\text { Non-residential (BB) } 6 \\
\text { mm }\end{array}$} \\
\cline { 2 - 5 } & SHGC & VT & SHGC & VT \\
\hline 1 & $\begin{array}{l}\text { Clear, Air, Low-e, } \\
\text { Without dividers }\end{array}$ & 0.52 & 0.63 & 0.53 & 0.65 \\
\hline 2 & $\begin{array}{l}\text { Clear, Air, Low-e } \\
\text { With dividers }\end{array}$ & 0.46 & 0.55 & N/A & N/A \\
\hline
\end{tabular}

Table 9-7. Specialty Products Table

\begin{tabular}{|c|l|l|l|l|}
\hline & \multicolumn{2}{|c|}{ No Dividers } & \multicolumn{2}{c|}{$\geq 1$ " Dividers } \\
\cline { 2 - 5 } & $\begin{array}{c}\text { Residenti } \\
\text { al(AA) }\end{array}$ & $\begin{array}{c}\text { Non- } \\
\text { residentia } \\
\text { I (BB) }\end{array}$ & $\begin{array}{c}\text { Residenti } \\
\text { al (AA) }\end{array}$ & $\begin{array}{c}\text { Non- } \\
\text { residential } \\
\text { (BB) }\end{array}$ \\
\hline SHGC $_{\mathbf{0}}$ & 0.01 & 0.01 & 0.02 & $\mathrm{~N} / \mathrm{A}$ \\
\hline SHGC $_{\mathbf{1}}$ & 0.86 & 0.88 & 0.76 & $\mathrm{~N} / \mathrm{A}$ \\
\hline $\mathbf{V T}_{\mathbf{0}}$ & 0.00 & 0.00 & 0.00 & $\mathrm{~N} / \mathrm{A}$ \\
\hline $\mathbf{V T}_{\mathbf{1}}$ & 0.85 & 0.87 & 0.74 & $\mathrm{~N} / \mathrm{A}$ \\
\hline
\end{tabular}

Note: It is also necessary to provide a specialty product table that has values for SHGC with SCGC values varying from 0.00 to 1.00 in 0.01 increments and $\mathrm{VT}$ with $\mathrm{VT}_{\mathrm{c}}$ values varying from 0.00 to 1.00 in 0.01 increments. 


\subsubsection{Drawings Vinyl Fixed Window}

The following pages contain detailed drawings for this window.
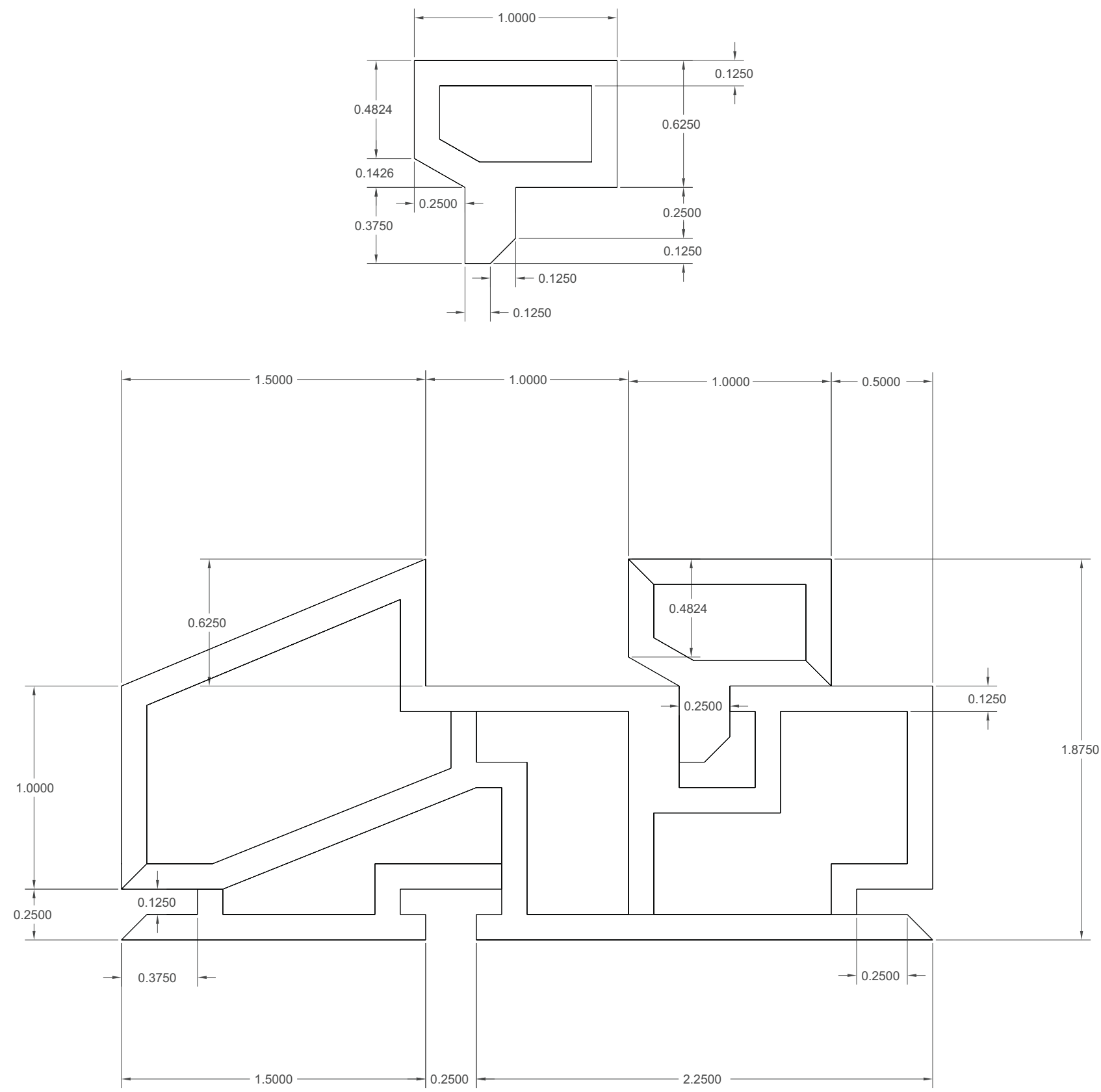

Head, Sill and Jamb

Figure 9-7. Dimensioned drawing for vinyl fixed window. 
Spacer

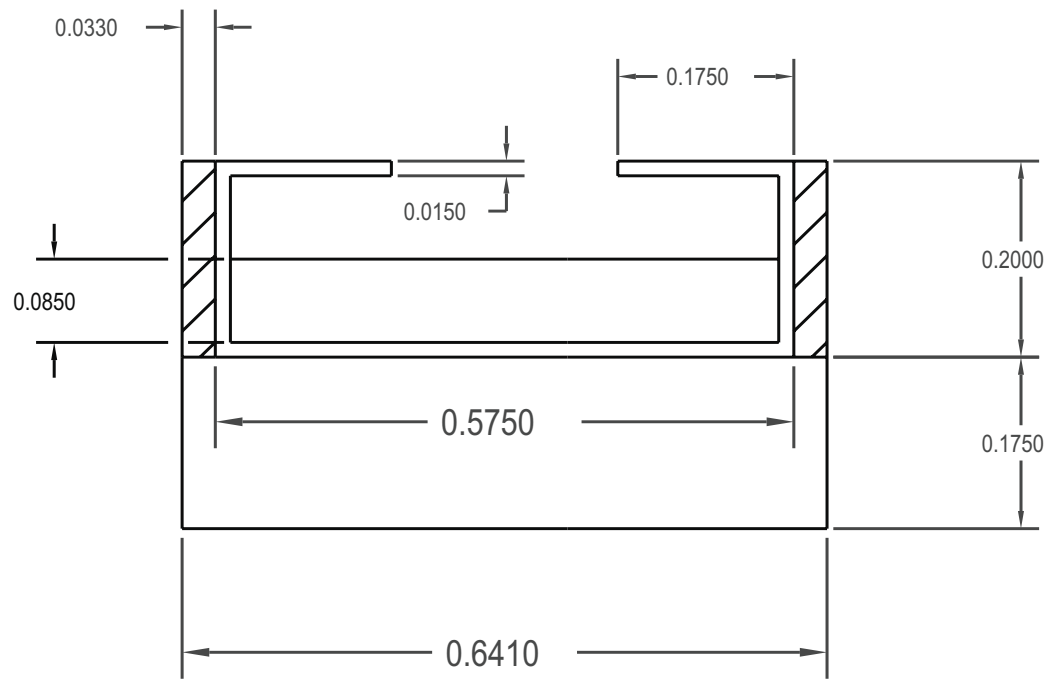

Figure 9-8. Dimensioned drawing for spacer.

\section{Divider}

\section{0-A}

\section{Page 21}

\section{Divider type A}

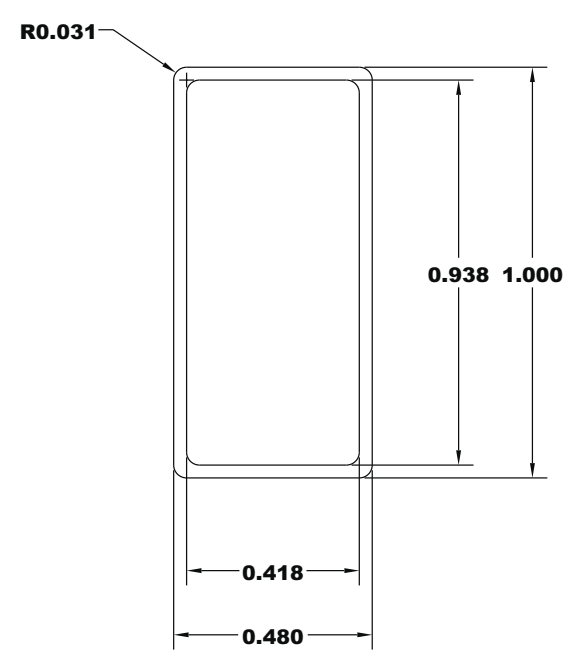

\section{Aluminum Metal white}

Figure 9-9. Dimensioned drawing for divider. 


\subsection{Problem 2: Aluminum Horizontal Slider Window}

For this example problem, there are 4 glazing options. Calculate the total product U-factor, SHGC, VT and the specialty products table.

\subsubsection{Description}
Window Type
Horizontal Slider
Frame Material
Aluminum painted white. Thermal breaks as indicated in the drawing assembly. The manufacturer indicated that the de-bridge width is 0.250 " for all the cross sections. Thermal break material is poured in place polyurethane.
Overall Size
Spacer type
Weather Strip
Dividers
(1) Residential: Width $=60 "$; Height $=36^{\prime \prime}$
(2) Non-Residential: Width $=72^{\prime \prime}$; Height $=48^{\prime \prime}$
See drawings in Section 9.3.7.
See drawings in Section 9.3.7.
Cross Sections
Section 9.3.7 contains the drawings for this example.
Aluminum painted white. See drawing for dimensions.
Manufacturer provides standard 12" on center or less horizontal and vertical grid pattern for his products.
Glazing system The manufacturer uses the following glass type options depending on the market availability and price factor. The manufacturer uses clear and Low-e coated glass from the same manufacturer.
- All clear glass having nominal thickness of $3 \mathrm{~mm}, 4 \mathrm{~mm}, 5 \mathrm{~mm}$, and $6 \mathrm{~mm}$ from PPG or CIG.
- Low-e coated clear glass having emissivity range from 0.100-0.096. Thicknesses are nominal $3 \mathrm{~mm}, 4 \mathrm{~mm}, 5 \mathrm{~mm}$ and $6 \mathrm{~mm}$ from PPG (SUNGATE 100, clear glass) or CIG (LoE SUN45 coating, clear glass)

\subsubsection{Glazing Matrix}

The following table shows the glazing matrix that is to be simulated for this window. However, for this example, only glazing options 1 (the $3 \mathrm{~mm}$ case), and 4 (the $6 \mathrm{~mm}$ case) will be modeled using CIG glass.

Table 9-8. Matrix of Glazing Options for Problem 2

\begin{tabular}{|c|c|c|c|}
\hline & $\begin{array}{c}\text { Glazing Options (1.0" overall } \\
\text { thickness) }\end{array}$ & Grid Option & $\underset{r}{\text { Manufacture }}$ \\
\hline${ }^{*} 1$ & Clear $(3 \mathrm{~mm})$, Argon $(95 \%)$, Low-e $(3 \mathrm{~mm})$ & With and without grids & CIG, PPG \\
\hline 2 & Clear (4 mm), Argon (95\%), Low-e (3 mm) & With and without grids & CIG, PPG \\
\hline 3 & Clear (5 mm), Argon (95\%), Low-e (3 mm) & No grids & CIG, PPG \\
\hline$* 4$ & Clear $(6 \mathrm{~mm})$, Argon $(95 \%)$, Low-e $(6 \mathrm{~mm})$ & No grids & CIG, PPG \\
\hline
\end{tabular}




\subsubsection{Center-of-Glass Modeling (WINDOW)}

In WINDOW, create the glazing systems needed for the Glazing Matrix in Section 9.3.2. An example of the four glazing systems is shown in Figure 9-10. These glazing systems display the center-of-glass U-factor in the $\mathrm{U}_{\mathrm{c}}$ column.

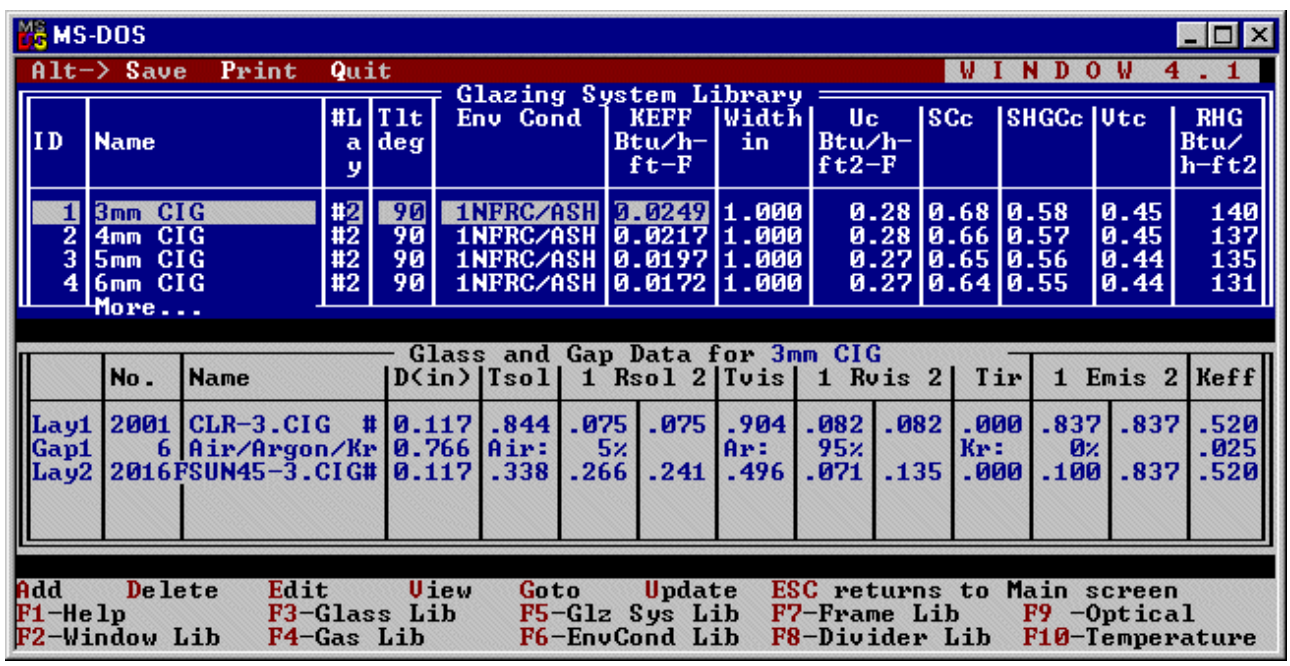

Figure 9-10. WINDOW 4.1 Glazing System Library for the Glazing Options

The results for the center-of-glass $U$-factor $\left(U_{c}\right)$ are shown in the following table:

Table 9-9. Center-of-glass U-factor Results for the Matrix of Glazing Options

\begin{tabular}{|c|c|c|c|}
\hline & \multicolumn{2}{|c|}{$\begin{array}{c}\text { Center-of-glass U-factor } \\
\left(\mathbf{U}_{c}\right)\end{array}$} \\
\hline & $\begin{array}{c}\text { Glazing Options (1.0" overall } \\
\text { thickness) }\end{array}$ & (Btu/hr-ft $\left.{ }^{2}{ }^{\circ} \mathrm{F}\right)$ & $\mathrm{W} / \mathrm{m}^{2}-{ }^{\circ} \mathrm{C}$ \\
\hline 1 & Clear (3 mm), Argon (95\%), Low-e (3mm) & 0.2822 & 1.6025 \\
\hline 4 & Clear (6 mm), Argon (95\%), Low-e (6mm) & 0.2696 & 1.5309 \\
\hline
\end{tabular}

These glazing systems will be used in THERM to calculate the frame and edge-of-glass U-factors, and also in WINDOW to calculate the overall product U-factor as discussed in Section 9.3.5. 


\subsubsection{Edge-of-Glass and Frame Modeling (THERM)}

There are 8 cross-sections which must be modeled for this product, listed in Table 9-10. The THERM files listed in this table can be found on the CD.

Table 9-10. Cross sections to be modeled with their associated $d x f$ and THERM files

\begin{tabular}{|l|l|l|l|}
\hline Cross Section & DXF Filename & THERM file for $\mathbf{3} \mathbf{~ m m}$ & THERM file for $\mathbf{6} \mathbf{m m}$ \\
\hline Sill Vented & 530vtsl.dxf & sv_01.thm & sv_02.thm \\
\hline Sill Fixed & 530fxsl.dxf & sf_01.thm & sf_02.thm \\
\hline Jamb Vented & 530vtjb.dxf & jv_01.thm & jv_02.thm \\
\hline Jamb Fixed & 530fxjb.dxf & jf_01.thm & jf_02.thm \\
\hline Head Vented & 530vthd.dxf & hv_01.thm & hv_02.thm \\
\hline Head Fixed & 530fxhd.dxf & hf_01.thm & hf_02.thm \\
\hline Meeting Rail & 530mtrl.dxf & mr_01.thm & mr_02.thm \\
\hline Grid (for 3 mm case) & N/A & divider_01.thm & N/A \\
\hline Spacer & Cig30.dxf & Spacer-3mm.thm & Spacer-6mm.thm \\
\hline
\end{tabular}

In addition to the DXF files provided on $\mathrm{CD}$, detailed drawings of these cross sections are in Section 9.3 .7 of this manual.

Make a THERM file for each of the cross sections using the following steps:

- Use the DXF files as an underlay for each file. The files were not created in a manner that THERM can autoconvert, but the underlay can be used to trace the cross sections. (See Chapter 5, "Drawing CrossSection Geometry" in the THERM User's Manual).

- As the polygons for each cross section are being drawn, assign the correct material properties to them. (See Chapter 5, "Drawing Cross-Section Geometry" in the THERM User's Manual.)

- Insert the glazing system created in WINDOW 4.1. (See Section 5.9, "Inserting a Glazing System" in the THERM User's Manual.) Notice that the spacer is open to the glazing cavity, so use the technique discussed in Section 8.7.3, "Modifying Glazing Cavities (imported from WINDOW) for Open Spacers."

- Assign the boundary conditions to the entire cross section. (See Chapter 6, "Defining Boundary Conditions" in the THERM User's Manual.)

- Calculate the U-factor for the cross section. (See Chapter 7, "Calculating Results" in the THERM User's Manual.)

- Make a THERM model of the divider grid for both the $3 \mathrm{~mm}$ and $6 \mathrm{~mm}$ glazing options, using the method discussed in Section 8.3, "Modeling Internal Dividers (Suspended Grills)". 
- Table 9-11 shows the resulting U-factors from each cross section for the $3 \mathrm{~mm}$ glazing case. Note the result for the Grid case, which will be imported into the WINDOW Divider Library.

Table 9-11. Frame and Edge THERM U-factor Results for Glazing Option 1: $3 \mathrm{~mm}$

\begin{tabular}{|c|c|c|c|c|}
\hline \multirow[b]{2}{*}{ Cross Section } & \multicolumn{2}{|c|}{ Frame U-factor } & \multicolumn{2}{|c|}{ Edge U-factor } \\
\hline & $\begin{array}{l}\text { (Btu/hr-ft' } \\
\left.{ }^{\circ} \mathrm{F}\right)\end{array}$ & \begin{tabular}{|l}
$\left(\mathrm{W} / \mathrm{m}^{2}-\right.$ \\
$\left.{ }^{\circ} \mathrm{C}\right)$
\end{tabular} & $\begin{array}{l}\text { (Btu/hr-ft' } \\
\left.{ }^{\circ} \mathrm{F}\right)\end{array}$ & $\begin{array}{l}\left(\mathrm{W} / \mathrm{m}^{2}-\right. \\
\left.{ }^{\circ} \mathrm{C}\right)\end{array}$ \\
\hline Sill Vented & 1.1233 & 6.3789 & 0.3894 & 2.2114 \\
\hline Sill Fixed & 1.9316 & 10.9687 & 0.3669 & 2.0837 \\
\hline Jamb Vented & 0.8996 & 5.1082 & 0.3681 & 2.0902 \\
\hline Jamb Fixed & 1.6820 & 9.5516 & 0.3677 & 2.0879 \\
\hline Head Vented & 0.9062 & 5.1457 & 0.3741 & 2.1245 \\
\hline Head Fixed & 1.5134 & 8.5938 & 0.3749 & 2.1291 \\
\hline Meeting Rail & 1.2683 & 7.2019 & 0.3470 & 1.9707 \\
\hline Grid & 0.3366 & 1.9112 & 0.3002 & 1.7049 \\
\hline
\end{tabular}

- Table 9-12 shows the resulting U-factors from each cross-section for the $6 \mathrm{~mm}$ glazing case.

Table 9-12. Frame and Edge THERM U-factor Results for Glazing Option 2: $6 \mathrm{~mm}$

\begin{tabular}{|l|l|l|l|l|}
\hline & \multicolumn{2}{|c|}{ Frame U-factor } & \multicolumn{2}{c|}{ Edge U-factor } \\
\hline Cross Section & $\begin{array}{l}\left(\text { Btu/hr-ft }{ }^{2}-\right. \\
\left.{ }^{\circ} \mathbf{F}\right)\end{array}$ & $\begin{array}{l}\left(\mathbf{W} / \mathbf{m}^{2}-\right. \\
\left.{ }^{\circ} \mathbf{C}\right)\end{array}$ & $\begin{array}{l}\text { (Btu/hr-ft } \\
\left.{ }^{\mathbf{o}} \mathbf{F}\right)\end{array}$ & $\begin{array}{l}\mathbf{( W / m ^ { 2 }} \\
\left.{ }^{\mathbf{o}} \mathbf{C}\right)\end{array}$ \\
\hline Sill Vented & 1.1179 & 6.3481 & 0.4161 & 2.3628 \\
\hline Sill Fixed & 1.9262 & 10.9379 & 0.3879 & 2.2028 \\
\hline Jamb Vented & 0.9124 & 5.1809 & 0.3974 & 2.2567 \\
\hline Jamb Fixed & 1.6945 & 9.6226 & 0.3957 & 2.2470 \\
\hline Head Vented & 0.8996 & 5.1087 & 0.3962 & 2.2497 \\
\hline Head Fixed & 1.5065 & 8.5549 & 0.3976 & 2.2576 \\
\hline Meeting Rail & 1.2532 & 7.1162 & 0.3695 & 2.0982 \\
\hline Grid & 0.5941 & 3.3739 & 0.3740 & 2.1237 \\
\hline
\end{tabular}

Figures 9-11 through 9-18 on the following pages show THERM files and U-factor results screens for each of the cross-sections with the $3 \mathrm{~mm}$ glazing system. 
Sill Vented Cross Section with Glazing Option 1 (3mm)

(sv_01.thm)
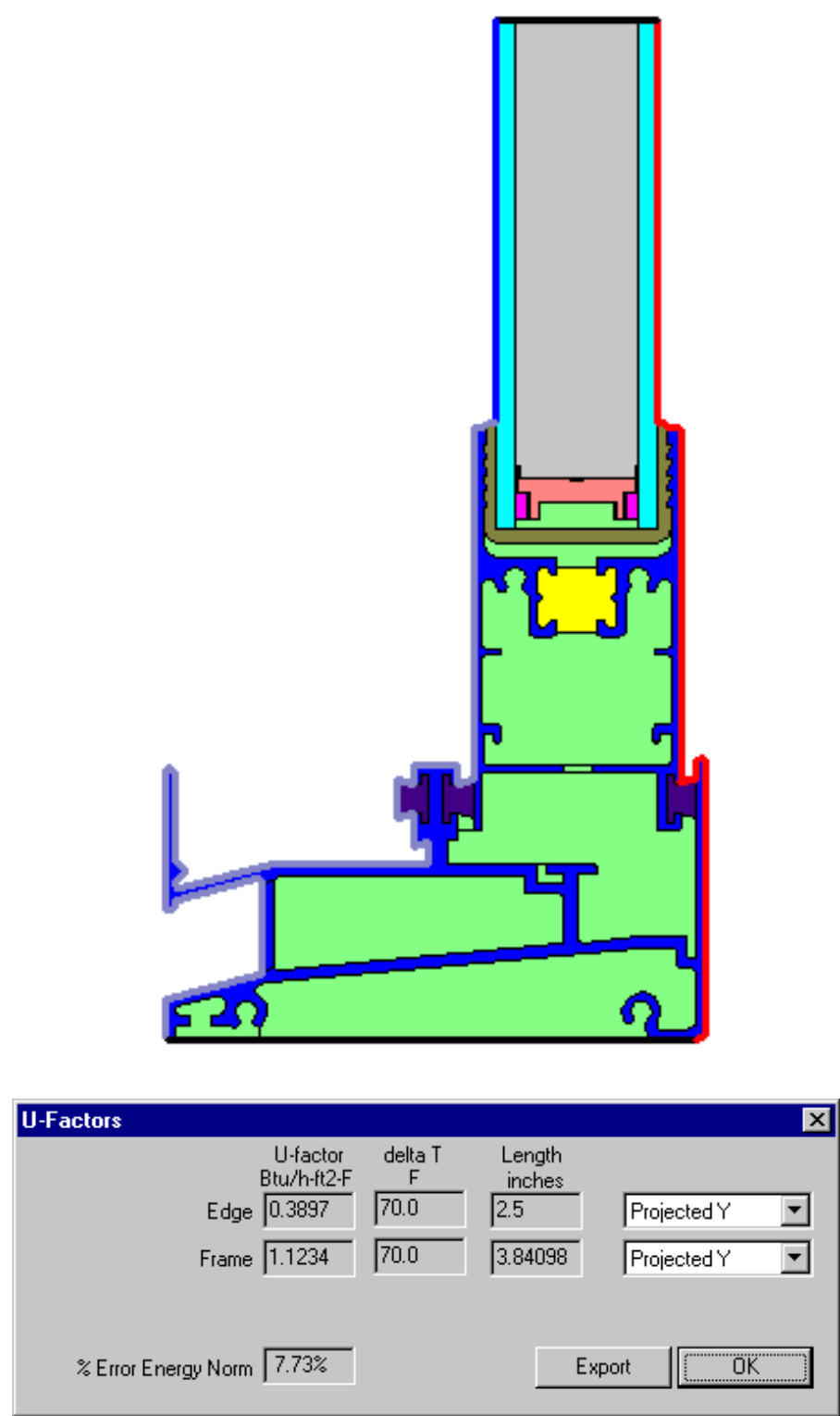

Figure 9-11. THERM file and U-factors for Sill Vented Cross Section with Glazing Option 1. 
Sill Fixed Cross Section with Glazing Option 1 (3mm)

(sf_01.thm)
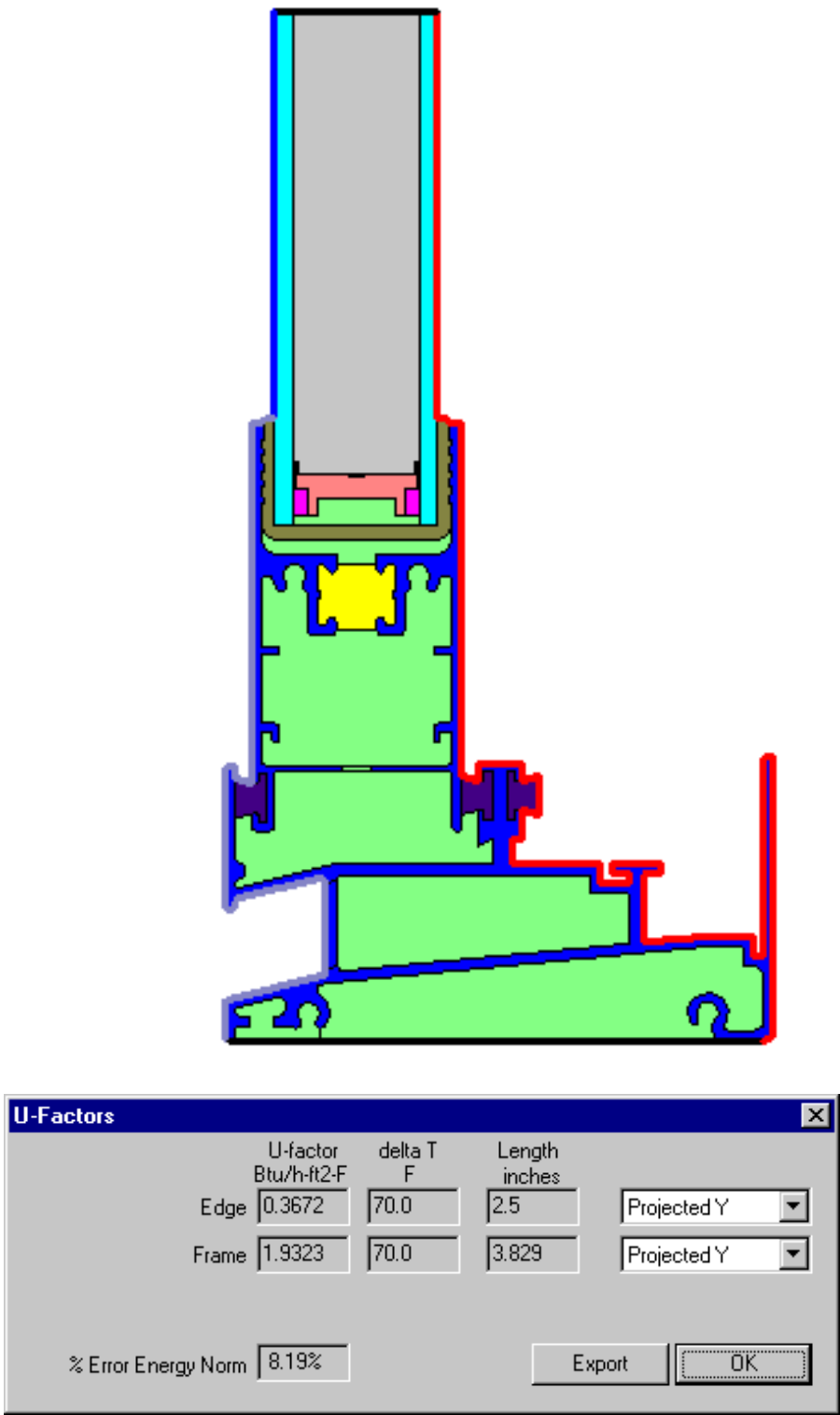

Figure 9-12. THERM file and U-factors for Sill Fixed Cross Section with Glazing Option 1. 


\section{Jamb Vented Cross Section with Glazing Option 1 (3mm)}

(jv_01.thm)
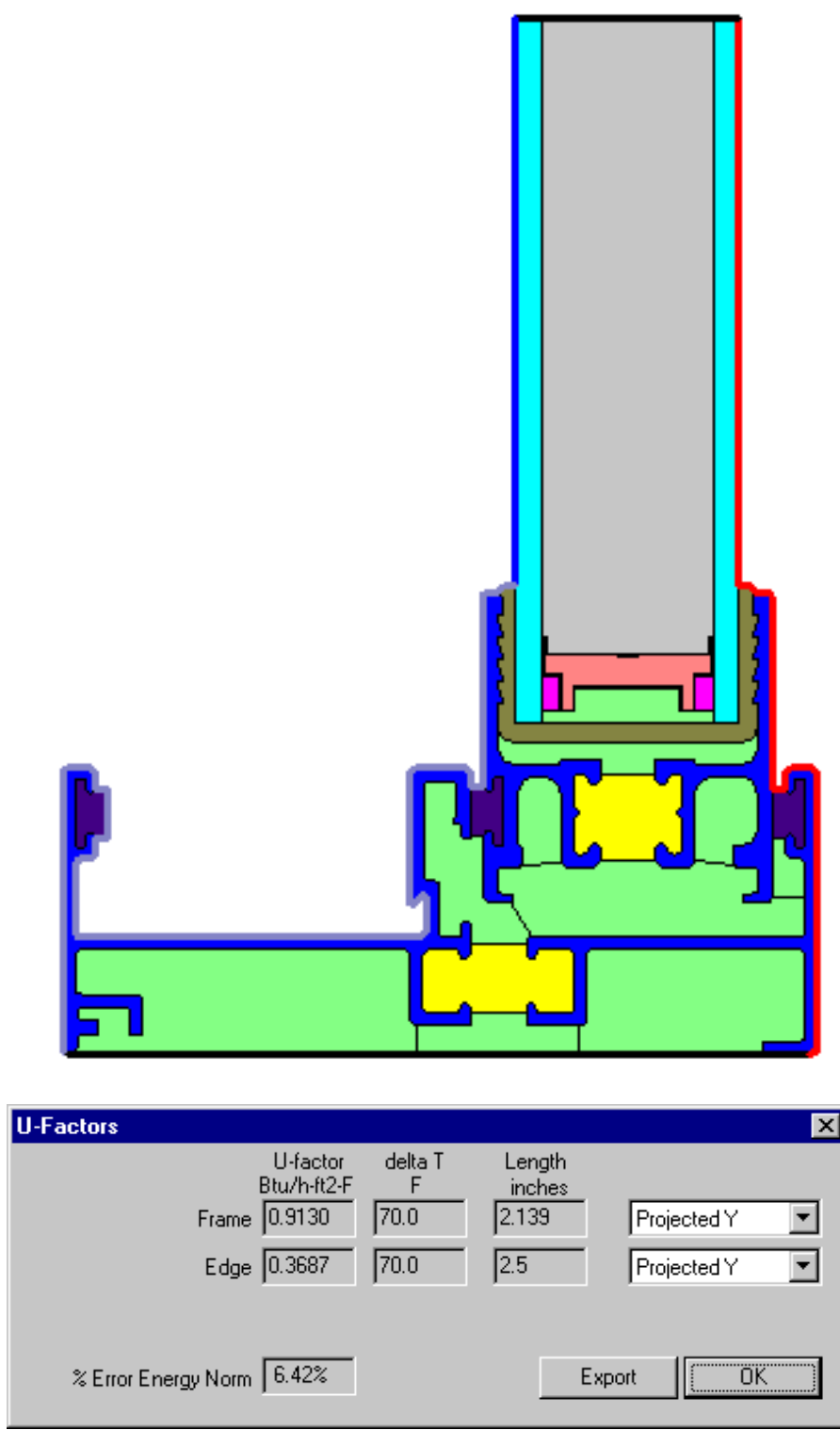

Figure 9-13. THERM file and U-factors for Jamb Vented Cross Section with Glazing Option 1. 


\section{Jamb Fixed Cross Section with Glazing Option 1 (3mm) (jf_01.thm)}
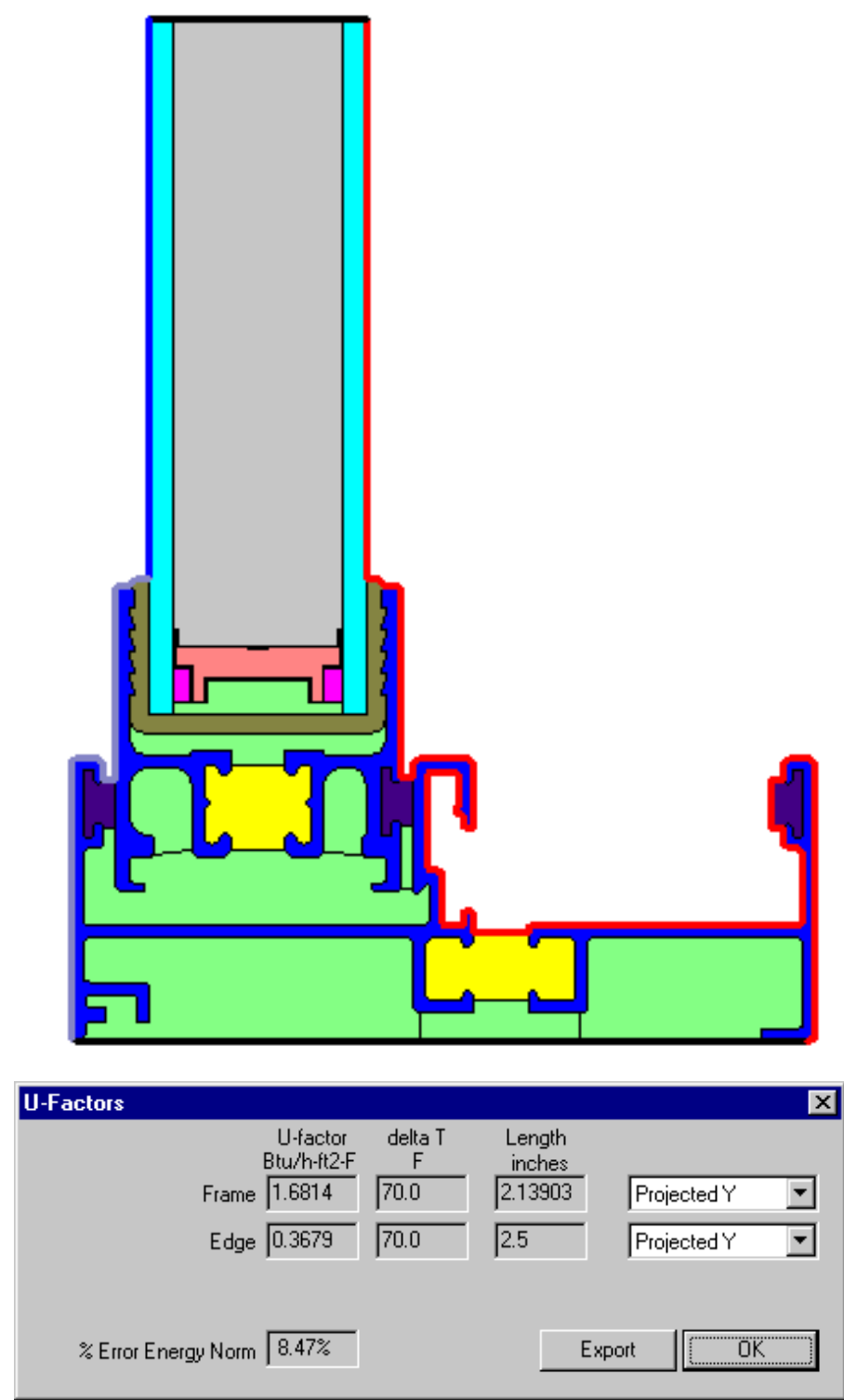

Figure 9-14. THERM file and U-factors for Jamb Fixed Cross Section with Glazing Option 1. 


\section{Head Vented Cross Section with Glazing Option 1 (3mm)}

(hv_01.thm)
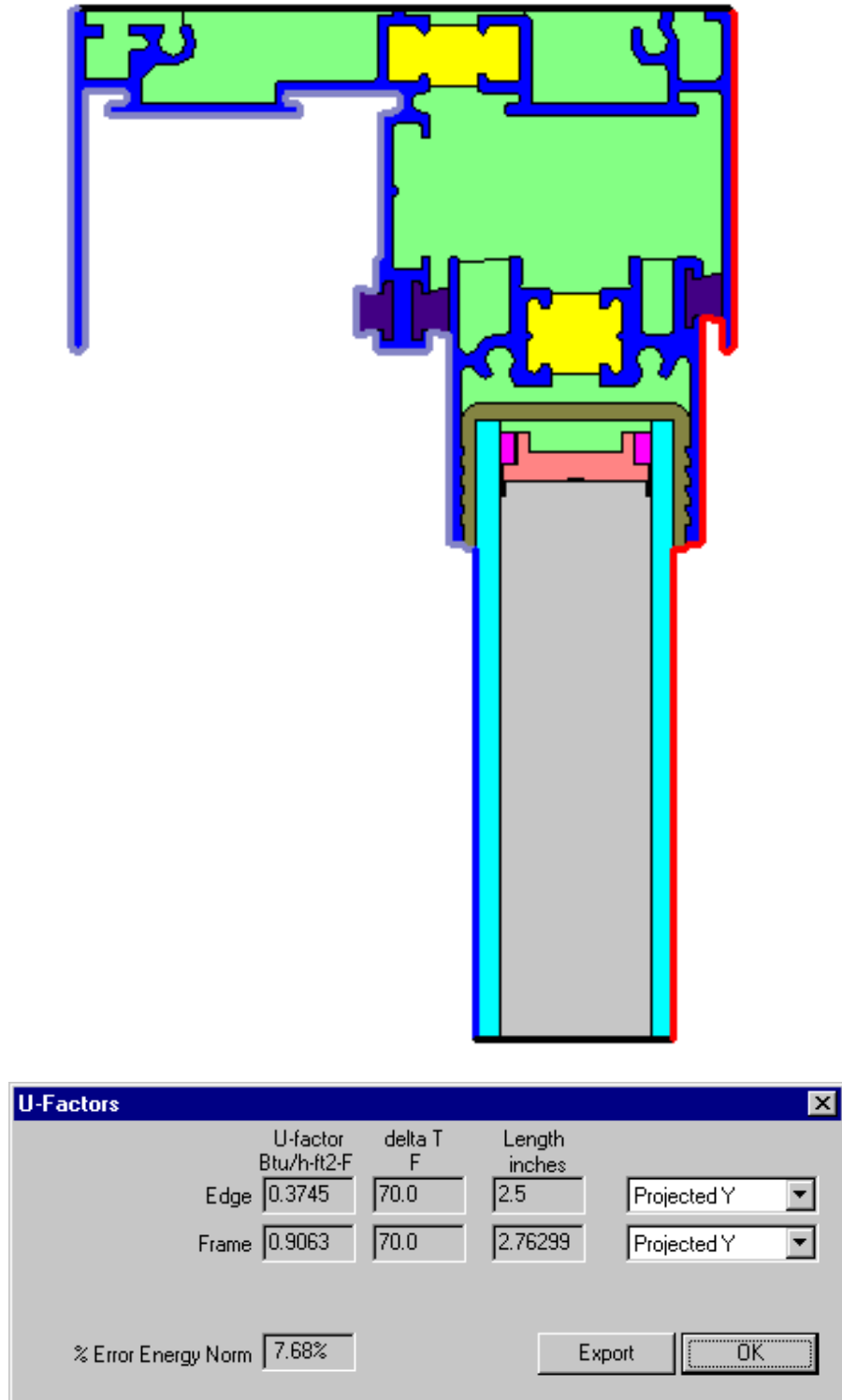

Figure 9-15. THERM file and U-factors for HEad Vented Cross Section with Glazing Option 1. 
Head Fixed Cross Section with Glazing Option 1 (3mm)

(hf_01.thm)
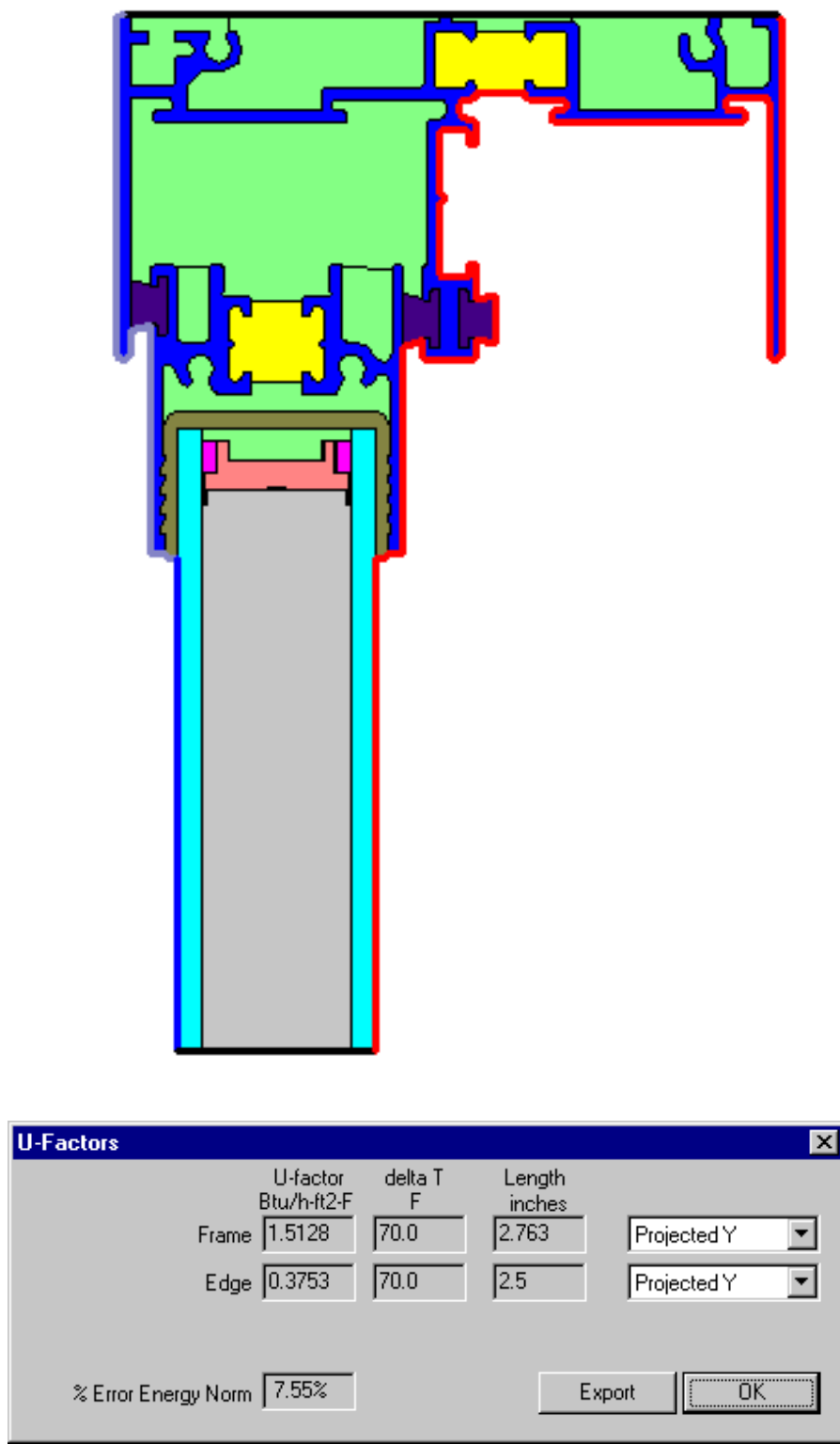

Figure 9-16. THERM file and U-factors for Head Fixed Cross Section with Glazing Option 1. 


\section{Meeting Rail Cross Section with Glazing Option $1(3 \mathrm{~mm})$}

(mr_01.thm)

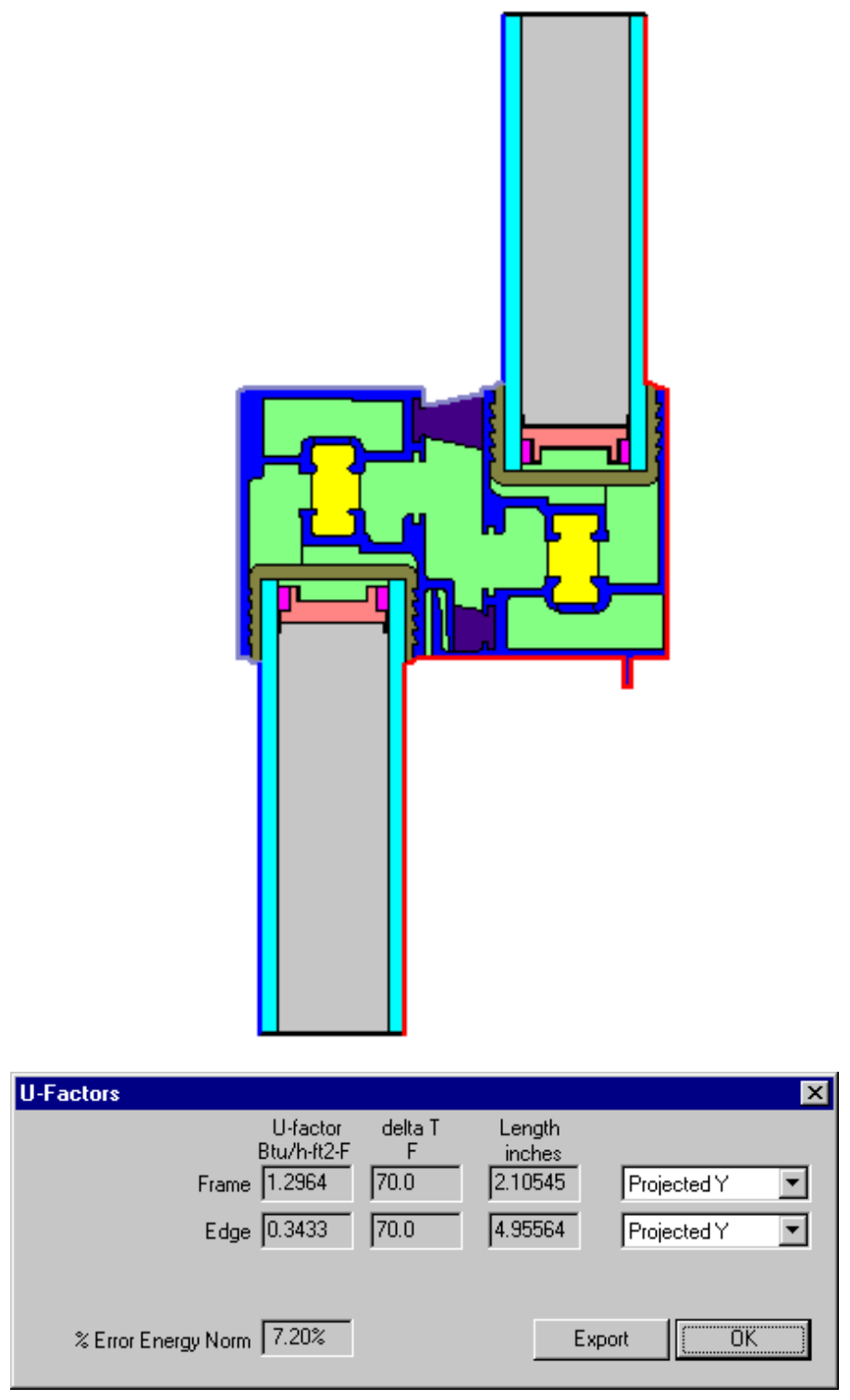

Figure 9-17. THERM file and U-factors for Meeting Rail Cross Section with Glazing Option 1. 
Grid Cross Section with Glazing Option 1 (3mm)
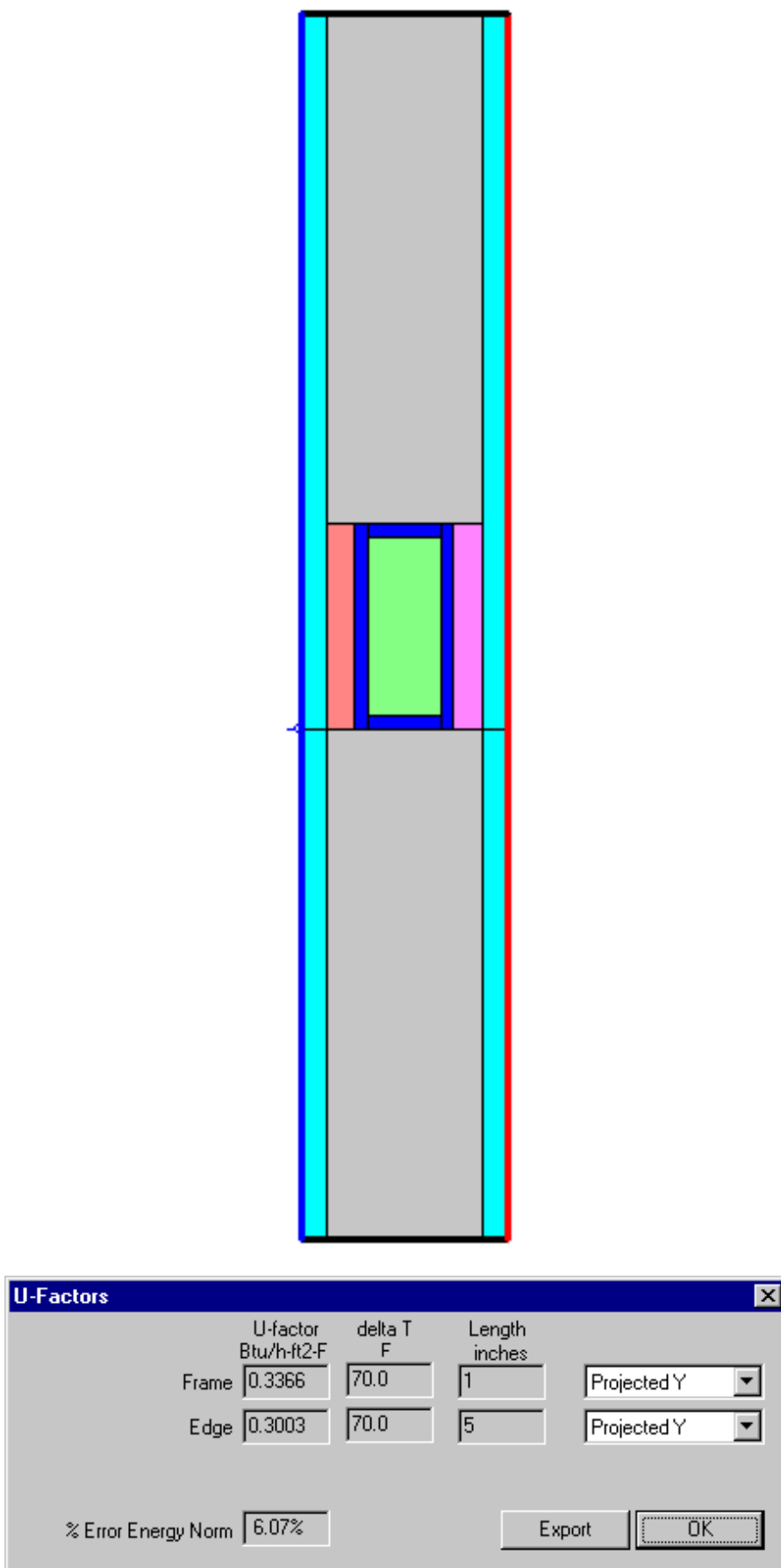

Figure 9-18. THERM file and U-factors for Grid Cross Section with $3 \mathrm{~mm}$ glass. 


\subsubsection{Total Product U-Factor}

To calculate the total product U-factor, an area-weighted value must be calculated from each of the THERM cross sections and the center-of-glass U-factor. This methodology is outlined in NFRC 100, and WINDOW can be used to do these calculation with the following steps:

\section{In THERM}

- Use File/Export to save each THERM cross section to a T2W file that WINDOW can read. Put the T2W files in the FRAMEDAT directory of the WINDOW program. (See Section 7.2 of this manual).

In WINDOW

- Add the frame T2W files to the Frame Library and the grid T2W file to the Divider Library. (See Section 7.2 of this manual).

Head Fixed Head Vented

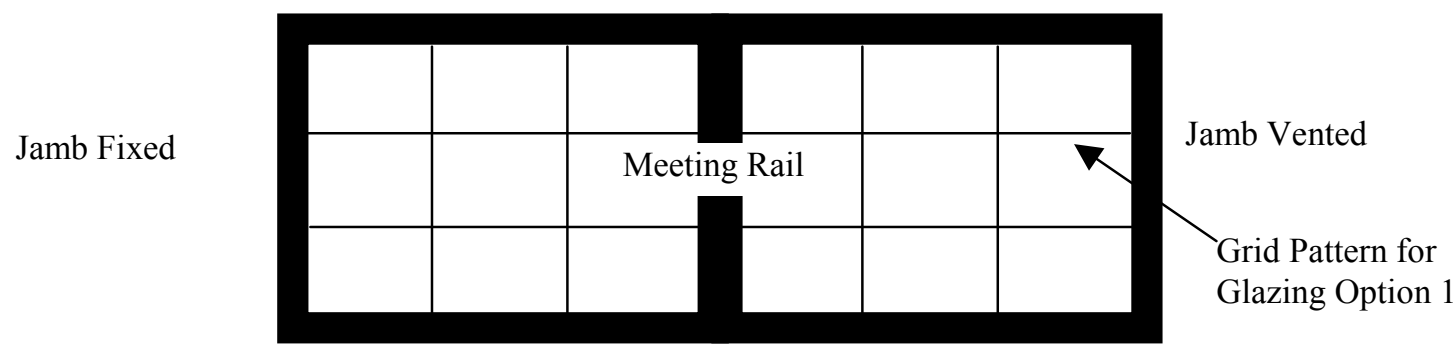

Sill Fixed Sill Vented

Figure 9-19. Frame and Divider configuration for total window product calculation in WINDOW.

- In the WINDOW main screen (press Esc from the libraries to get there), make a new window with the Type set to Horz Slider, the Size set to Horz Slid AA and BB for the two sets of runs, and specify the appropriate $\mathbf{T} \mathbf{2} \mathbf{W}$ cross section file for each of the frames and dividers, as shown in Figure 9-20.

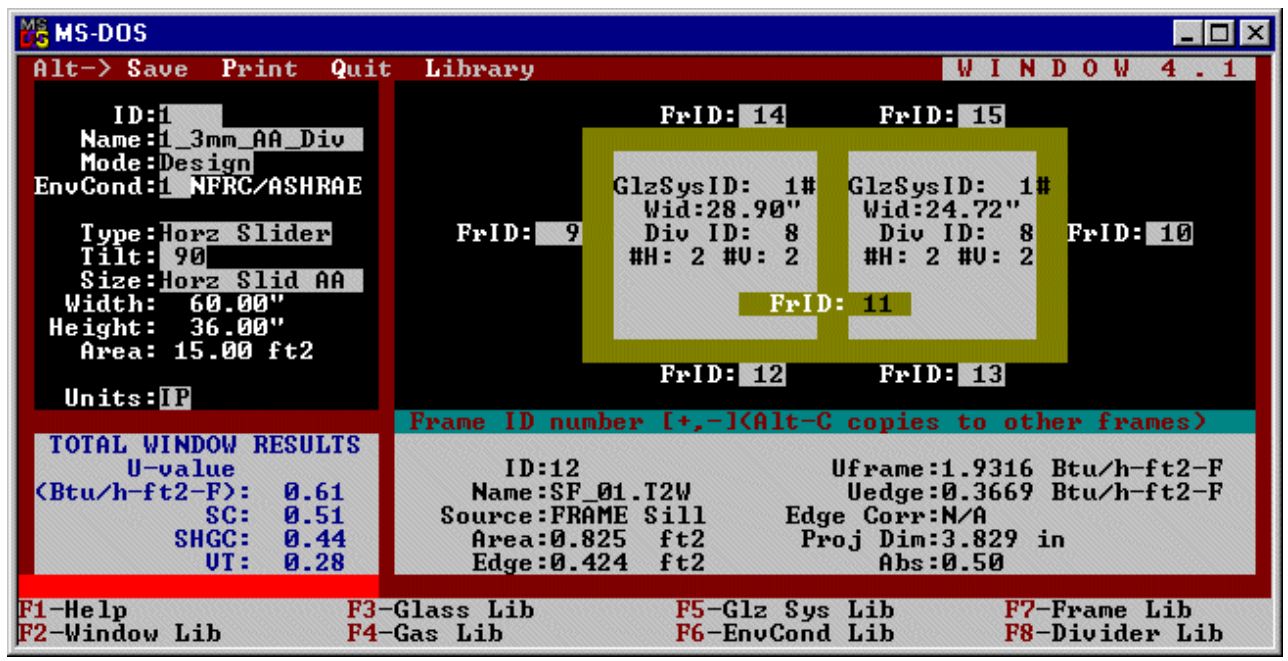

Figure 9-20. WINDOW main screen with total product U-factor calculated for Glazing Option 1, AA size window. 


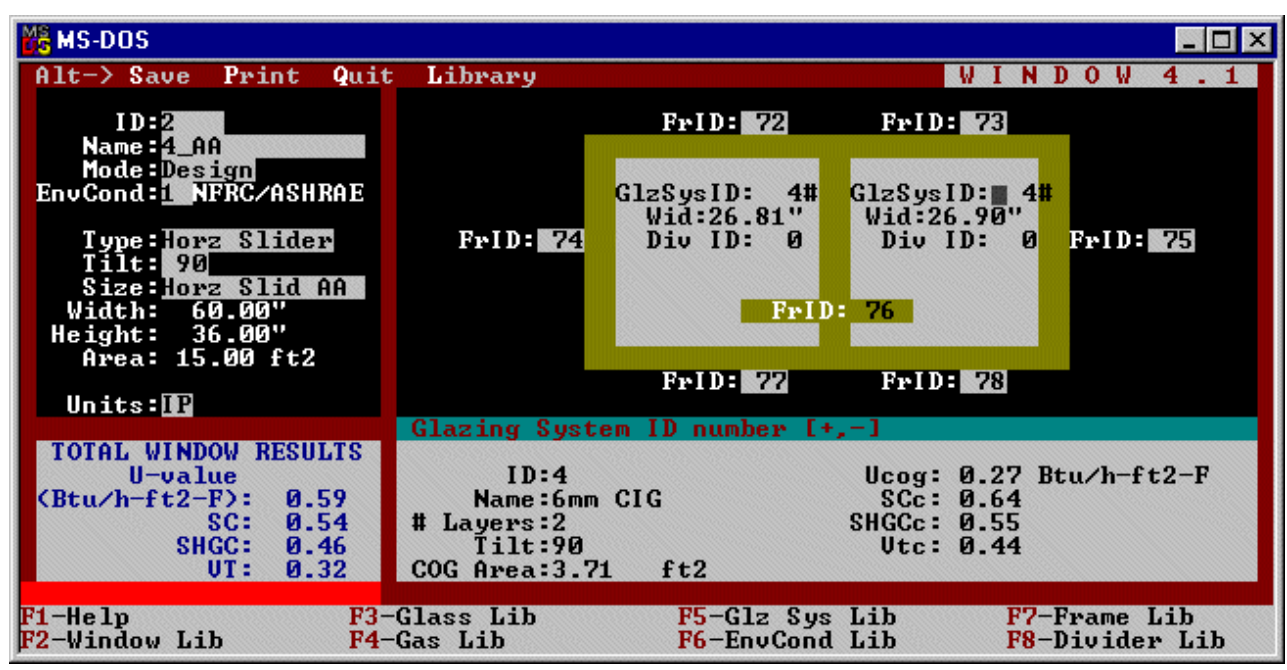

Figure 9-21. WINDOW main screen with total product U-factor calculated for Glazing Option 4, AA size window.

Table 9-13 shows the overall product U-factor, from WINDOW, for Glazing Options 1 and 4. The other glazing options are not calculated in this example.

Table 9-13. Total Product U-factors

\begin{tabular}{|c|c|c|c|c|}
\hline \multirow{2}{*}{$\begin{array}{c}\text { Glazing Options } \\
\text { (1.0" overall thickness) }\end{array}$} & \multicolumn{2}{|c|}{$\begin{array}{l}\text { Residential (AA) } 3 \mathrm{~mm} \\
\text { Total Product U-factor }\end{array}$} & \multicolumn{2}{|c|}{$\begin{array}{l}\text { Non-residential (BB) } \\
6 \mathrm{~mm} \\
\text { Total Product U-facto }\end{array}$} \\
\hline & $\begin{array}{l}\text { (Btu/hr-ft' } \\
\left.{ }^{\circ} \mathrm{F}\right)\end{array}$ & $\begin{array}{l}\left(\mathrm{W} / \mathrm{m}^{2}-\right. \\
\left.{ }^{\circ} \mathrm{C}\right)\end{array}$ & $\begin{array}{l}\text { (Btu/hr-ft' } \\
\left.{ }^{\circ} \mathrm{F}\right)\end{array}$ & $\begin{array}{l}\left(\mathrm{W} / \mathrm{m}^{2}-\right. \\
\left.{ }^{\circ} \mathrm{C}\right)\end{array}$ \\
\hline $\begin{array}{l}\text { Clear, Argon (95\%), Low-e } \\
\text { No Dividers }\end{array}$ & 0.597 & 3.389 & 0.521 & 2.956 \\
\hline $\begin{array}{l}\text { Clear Argon (95\%), Low-e } \\
\text { With Dividers }\end{array}$ & 0.606 & 3.442 & 0.592 & 3.363 \\
\hline
\end{tabular}




\subsubsection{Total Product Solar Heat Gain Coefficient (SHGC) and Visible Transmittance (VT)}

The methodology for determining the Solar Heat Gain Coefficient for the product is outlined in NFRC 200, and the methodology for determining the Visible Transmittance is outlined in NFRC 300. WINDOW automatically calculates these values, as shown in Figure 9-21. The results for Glazing Options 1 and 4 are shown in Table 9-14. The specialty products table is shown in Table 9-15.

Table 9-14. Total Product Solar Heat Gain Coefficients and Visible Transmittance

\begin{tabular}{|c|l|l|l|l|c|}
\hline \multicolumn{2}{|c|}{$\begin{array}{c}\text { Glazing Options } \\
\text { (1.0" overall thickness) }\end{array}$} & \multicolumn{2}{c|}{ Residential (AA) 3mm } & \multicolumn{2}{c|}{ Non-residential (BB) 6mm } \\
\cline { 2 - 5 } & SHGC & VT & SHGC & VT \\
\hline 1 & $\begin{array}{l}\text { Clear, Argon (95\%), Low-e } \\
\text { No Dividers }\end{array}$ & 0.49 & 0.33 & 0.48 & 0.34 \\
\hline 1 & $\begin{array}{l}\text { Clear, Argon (95\%), Low-e } \\
\text { With Dividers }\end{array}$ & 0.44 & 0.28 & 0.43 & 0.30 \\
\hline
\end{tabular}

Table 9-15. Specialty Products Table

\begin{tabular}{|c|l|l|l|l|}
\hline & \multicolumn{2}{|c|}{ No Dividers } & \multicolumn{2}{c|}{$>1$ 1" Dividers } \\
\cline { 2 - 5 } & $\begin{array}{c}\text { Residenti } \\
\text { al(AA) }\end{array}$ & $\begin{array}{c}\text { Non- } \\
\text { residential } \\
\text { (BB) }\end{array}$ & $\begin{array}{c}\text { Residentia } \\
\text { I (AA) }\end{array}$ & $\begin{array}{c}\text { Non-residential } \\
\text { (BB) }\end{array}$ \\
\hline SHGC $_{\mathbf{0}}$ & 0.06 & 0.05 & 0.07 & 0.05 \\
\hline $\mathbf{S H G C}_{\mathbf{1}}$ & 0.79 & 0.84 & 0.70 & 0.76 \\
\hline $\mathbf{V T}_{\mathbf{0}}$ & 0.00 & 0.00 & 0.00 & 0.00 \\
\hline $\mathbf{V T}_{\mathbf{1}}$ & 0.73 & 0.79 & 0.63 & 0.70 \\
\hline
\end{tabular}

Note: It is also necessary to provide a specialty product table that has values for SHGC with SCGC $_{\mathrm{c}}$ values varying from 0.00 to 1.00 in 0.01 increments and $V T$ with $\mathrm{VT}_{\mathrm{c}}$ values varying from 0.00 to 1.00 in 0.01 increments.

\subsubsection{Drawings for Aluminum Horizontal Slider Window}

The following pages contain detailed drawings for this window: 
Figure 9-22. Vented Head Drawing for Horizontal Aluminum Slider.

\section{Sliding Head} (NFRC-VT-HD)

Page 1

\begin{tabular}{|c|c|}
\hline $\begin{array}{l}\text { Glass } \\
\text { Thickness }\end{array}$ & $A$ \\
\hline $3 \mathrm{~mm}$ & $0.120_{-0.005}^{+0.010}$ \\
\hline $4 \mathrm{~mm}$ & $0.155_{-0.005}^{+0.005}$ \\
\hline $5 \mathrm{~mm}$ & $0.185_{-0.005}^{+0.005}$ \\
\hline $6 \mathrm{~mm}$ & $0.225_{-0.005}^{+0.005}$ \\
\hline
\end{tabular}

Note : All Weather Strippings are Type-I

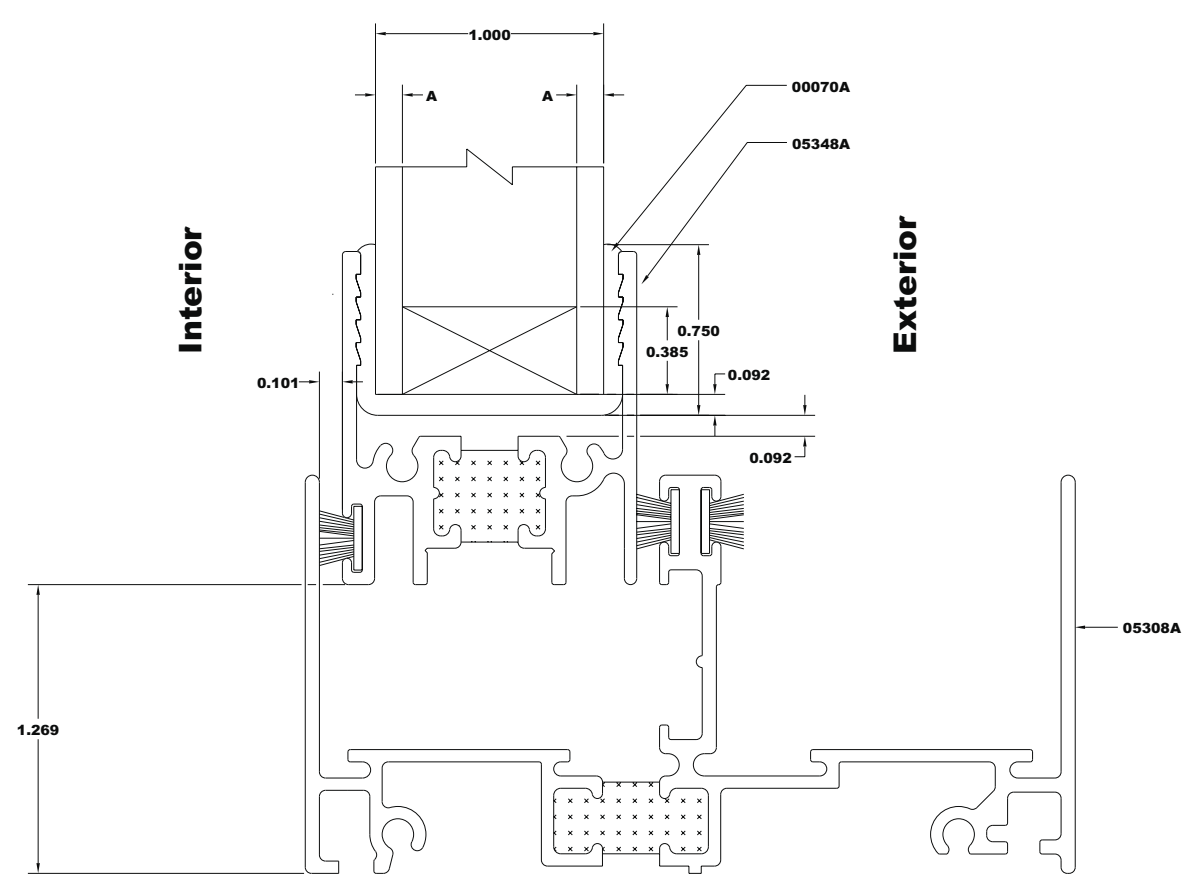


Page 2

\section{Fix Jamb}

\begin{tabular}{|l|c|}
\hline $\begin{array}{l}\text { Glass } \\
\text { Thickness }\end{array}$ & $A$ \\
\hline $3 \mathrm{~mm}$ & $0.120_{-0.005}^{+0.010}$ \\
\hline $4 \mathrm{~mm}$ & $0.155_{-0.005}^{+0.005}$ \\
\hline $5 \mathrm{~mm}$ & $0.185_{-0.005}^{+0.005}$ \\
\hline $6 \mathrm{~mm}$ & $0.225_{-0.005}^{+0.005}$ \\
\hline
\end{tabular}

(NFRC-FX-JB)

Note: All Weather Strippings are Type-I

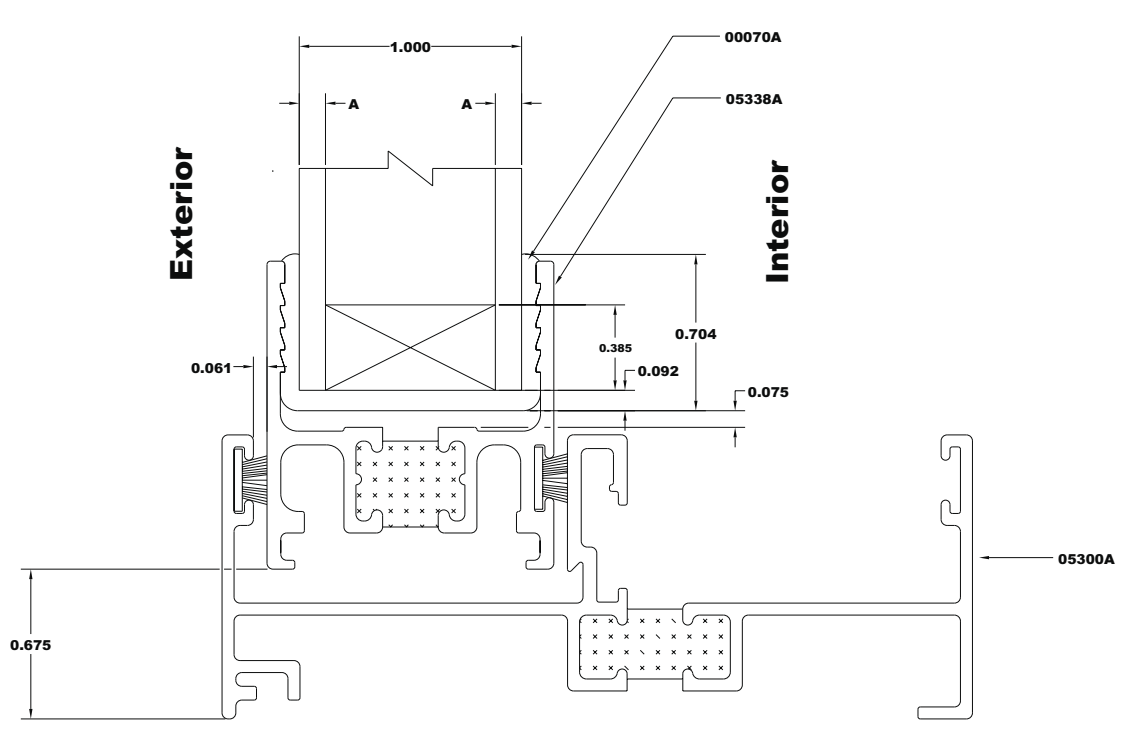

Figure 9-23. Fixed Jamb Drawing for Horizontal Aluminum Slider. 
Sliding Sill

(NFRC-VT-SL)

\begin{tabular}{|c|c|}
\hline $\begin{array}{c}\text { Glass } \\
\text { Thickness }\end{array}$ & $A$ \\
\hline $3 \mathrm{~mm}$ & $0.120_{-0.005}^{+0.010}$ \\
\hline $4 \mathrm{~mm}$ & $0.155_{-0.005}^{+0.005}$ \\
\hline $5 \mathrm{~mm}$ & $0.185_{-0.005}^{+0.005}$ \\
\hline $6 \mathrm{~mm}$ & $0.225_{-0.005}^{+0.005}$ \\
\hline
\end{tabular}

Note : All Weather Strippings are Type-I
Page 3

은

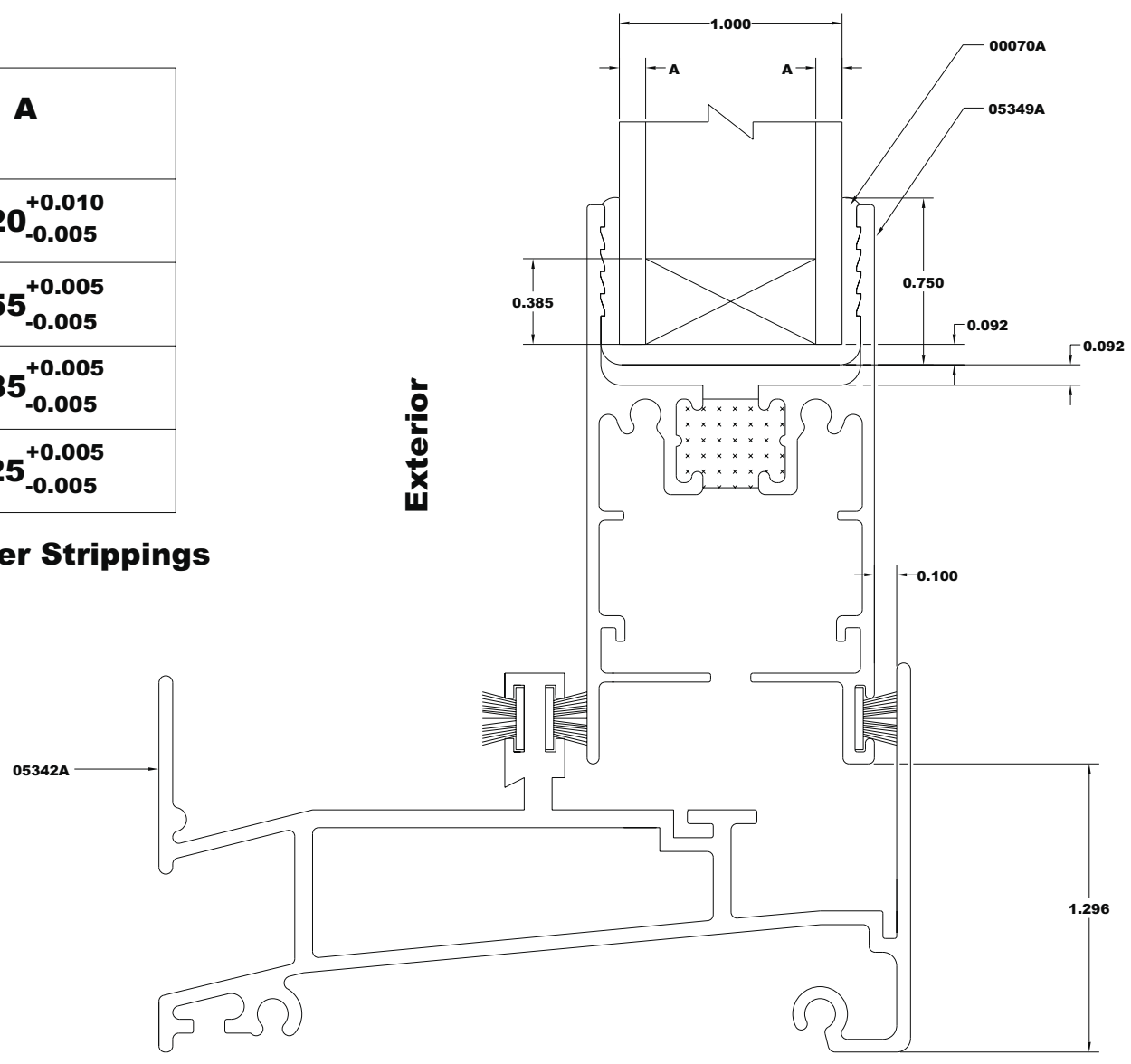

Figure 9-24. Vented Sill Drawing for Horizontal Aluminum Slider. 
Fix Head

(NFRC-FX-HD)

\begin{tabular}{|c|c|}
\hline $\begin{array}{l}\text { Glass } \\
\text { Thickness }\end{array}$ & $A$ \\
\hline $3 \mathrm{~mm}$ & $0.120_{-0.005}^{+0.010}$ \\
\hline $4 \mathrm{~mm}$ & $0.155_{-0.005}^{+0.005}$ \\
\hline $5 \mathrm{~mm}$ & $0.185_{-0.005}^{+0.005}$ \\
\hline $6 \mathrm{~mm}$ & $0.225_{-0.005}^{+0.005}$ \\
\hline
\end{tabular}

Note : All Weather Strippings are Type-I
Page 4

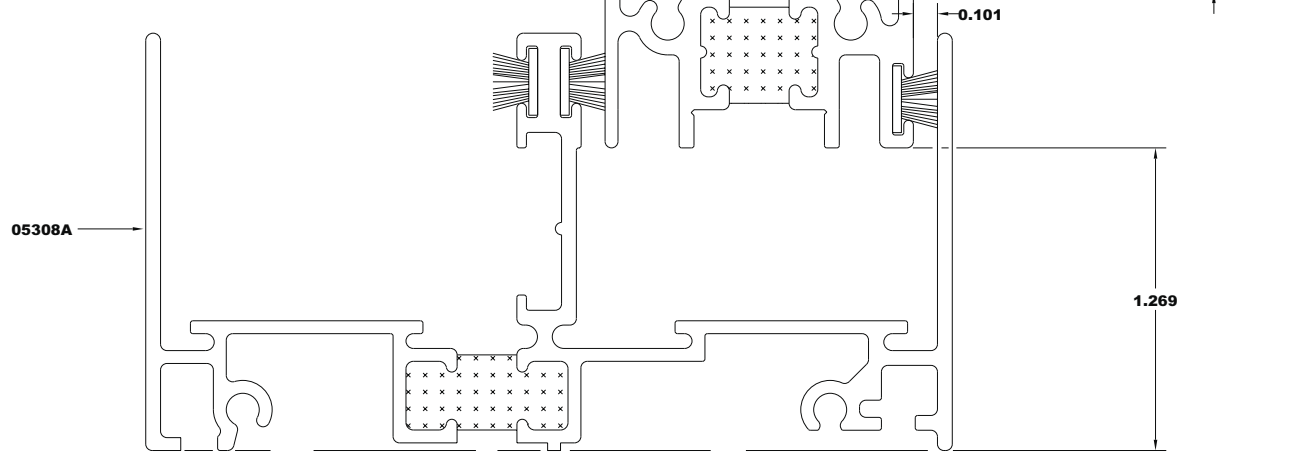

Figure 9-25. Fixed Head Drawing for Horizontal Aluminum Slider 


\section{Page 5}

\section{Sliding Jamb}

(NFRC-VT-JB)

\begin{tabular}{|l|c|}
\hline $\begin{array}{l}\text { Glass } \\
\text { Thickness }\end{array}$ & $A$ \\
\hline $3 \mathrm{~mm}$ & $0.120_{-0.005}^{+0.010}$ \\
\hline $4 \mathrm{~mm}$ & $0.155_{-0.005}^{+0.005}$ \\
\hline $5 \mathrm{~mm}$ & $0.185_{-0.005}^{+0.005}$ \\
\hline $6 \mathrm{~mm}$ & $0.225_{-0.005}^{+0.005}$ \\
\hline
\end{tabular}

Note : All Weather Strippings are Type-I

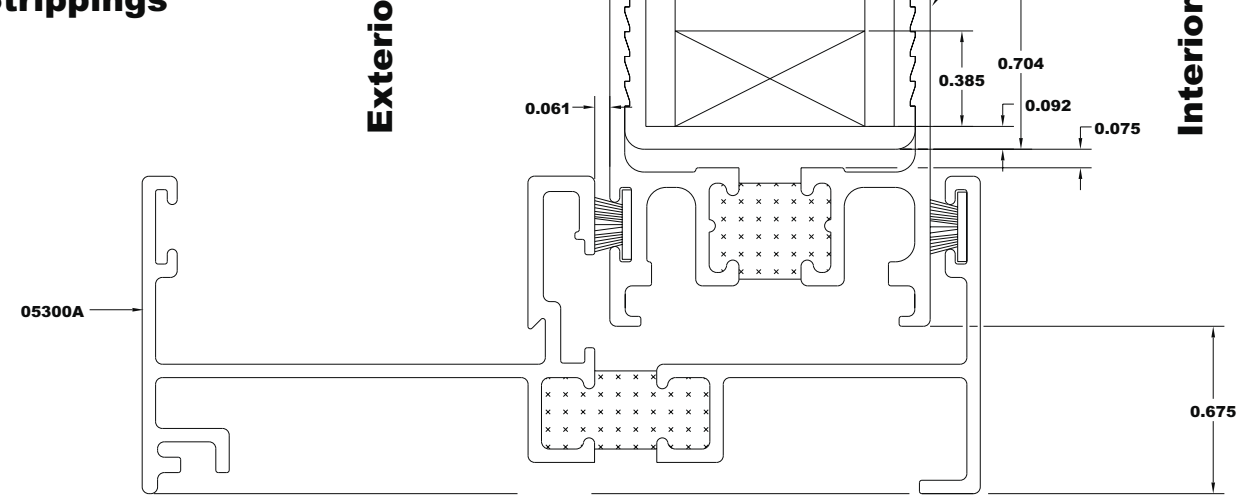

Figure 9-26. Vented Jamb Drawing for Horizontal Aluminum Slider. 
Fix Sill

Page 6

(NFRC-FX-SL)

\begin{tabular}{|c|c|}
\hline $\begin{array}{l}\text { Glass } \\
\text { Thickness }\end{array}$ & $A$ \\
\hline $3 \mathrm{~mm}$ & $0.120_{-0.005}^{+0.010}$ \\
\hline $4 \mathrm{~mm}$ & $0.155_{-0.005}^{+0.005}$ \\
\hline $5 \mathrm{~mm}$ & $0.185_{-0.005}^{+0.005}$ \\
\hline $6 \mathrm{~mm}$ & $0.225_{-0.005}^{+0.005}$ \\
\hline
\end{tabular}

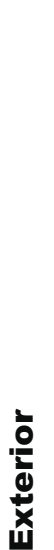

Note : All Weather Strippings are Type-I

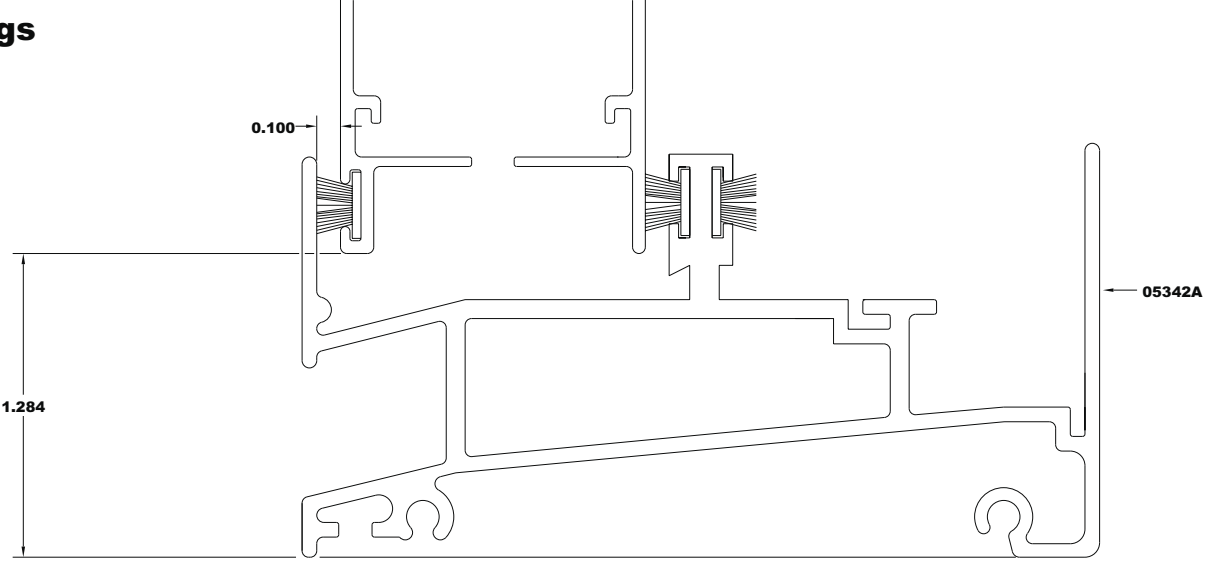

Figure 9-27. Fixed Sill Drawing for Horizontal Aluminum Slider. 
Page 7

\section{Meeting Rail}

\begin{tabular}{|c|c|}
\hline $\begin{array}{l}\text { Glass } \\
\text { Thickness }\end{array}$ & $A$ \\
\hline $3 \mathrm{~mm}$ & $0.120_{-0.005}^{+0.010}$ \\
\hline $4 \mathrm{~mm}$ & $0.155_{-0.005}^{+0.005}$ \\
\hline $5 \mathrm{~mm}$ & $0.185_{-0.005}^{+0.005}$ \\
\hline $6 \mathrm{~mm}$ & $0.225_{-0.005}^{+0.005}$ \\
\hline
\end{tabular}

\section{(NFRC-MTG)}

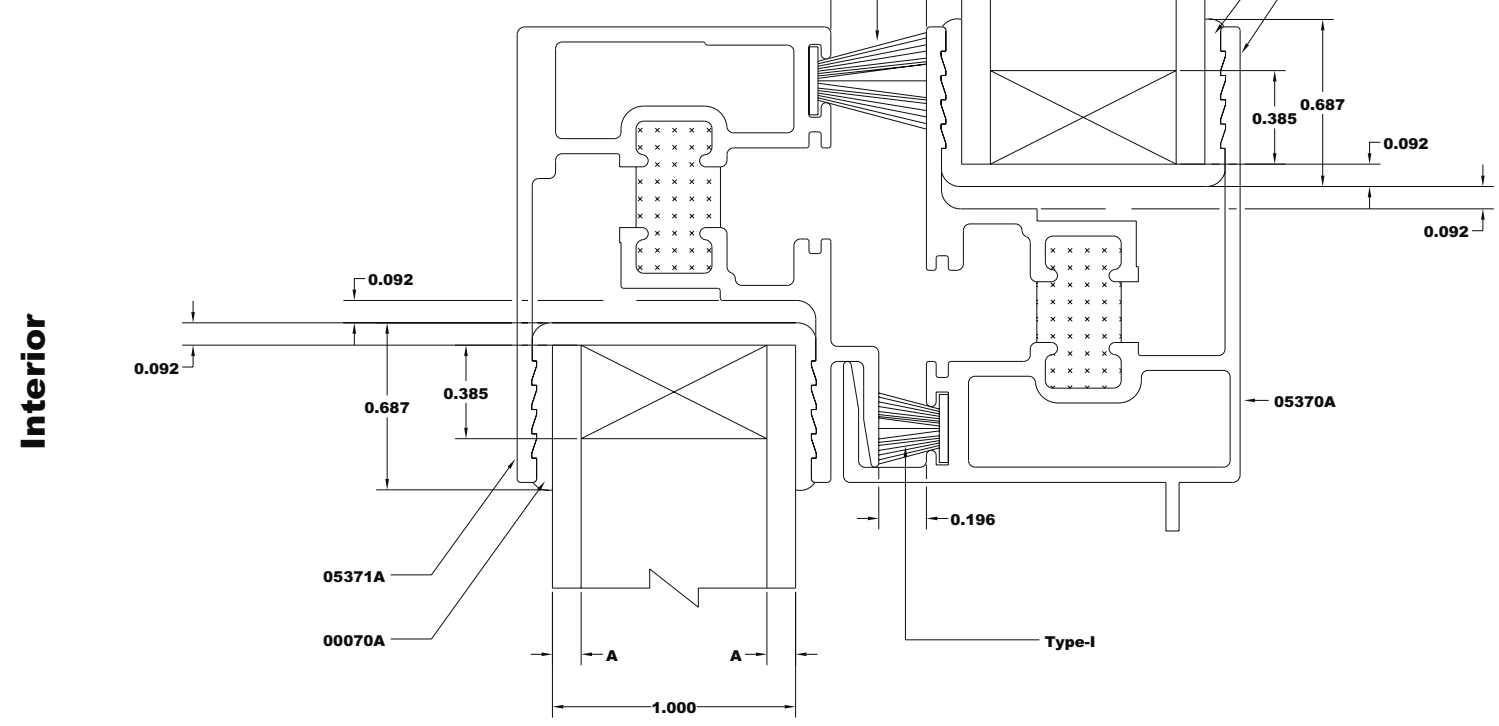

Figure 9-28. Meeting Rail Drawing for Horizontal Aluminum Slider. 


\section{0-A}

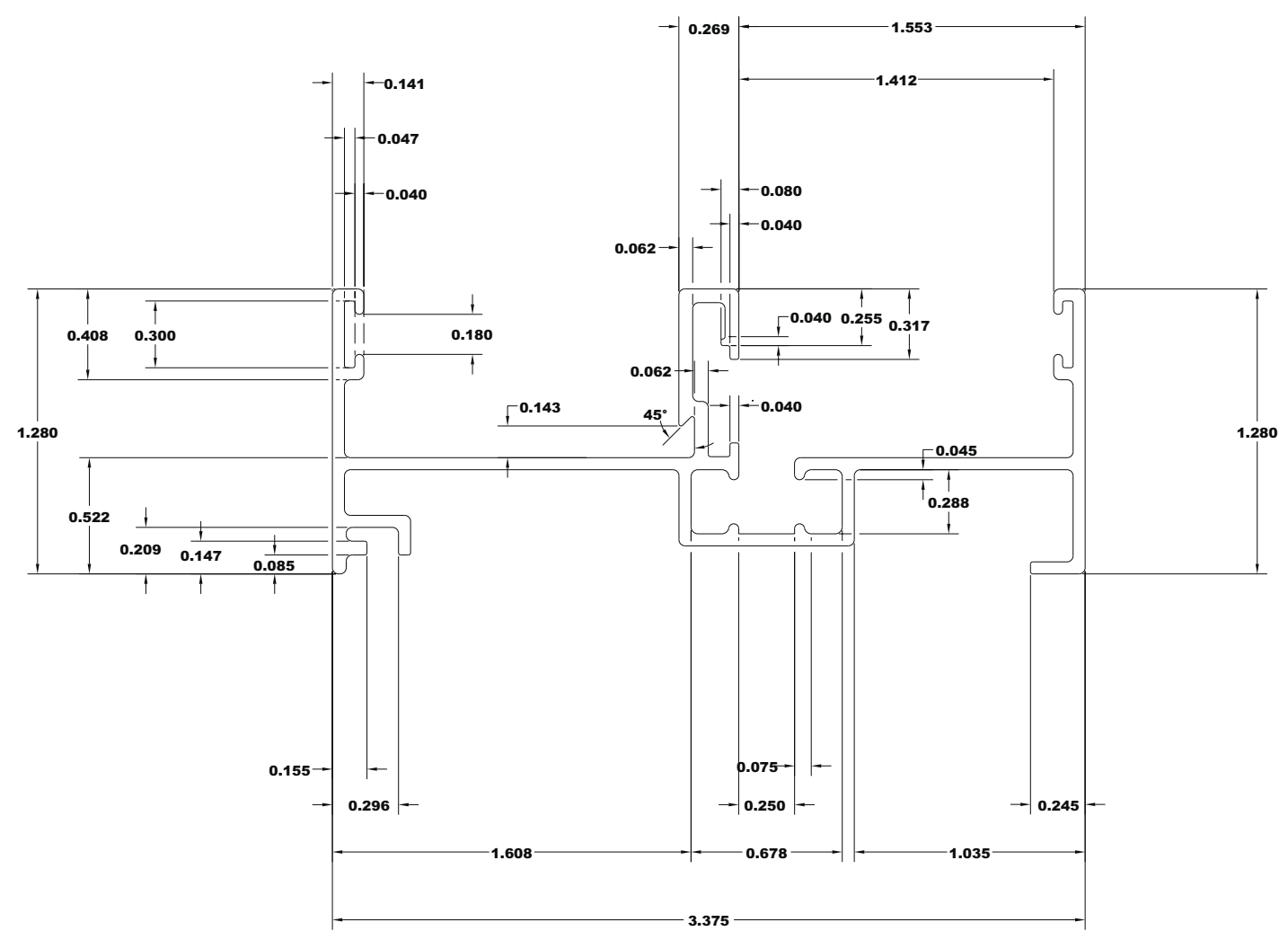

Figure 9-29. Fixed Jamb Drawing for Horizontal Aluminum Slider. 


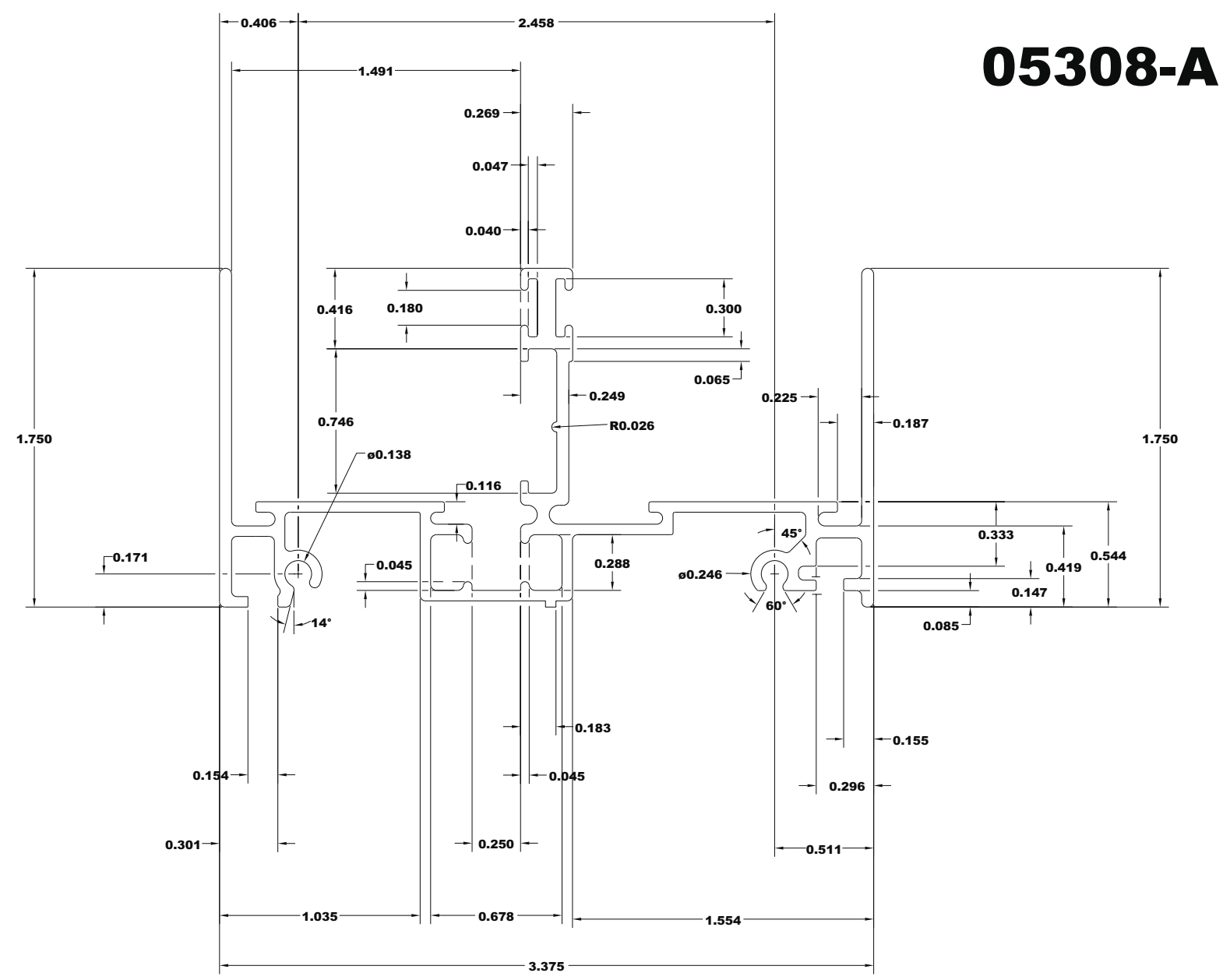

Figure 9-30. Head Drawing for Horizontal Aluminum Slider. 


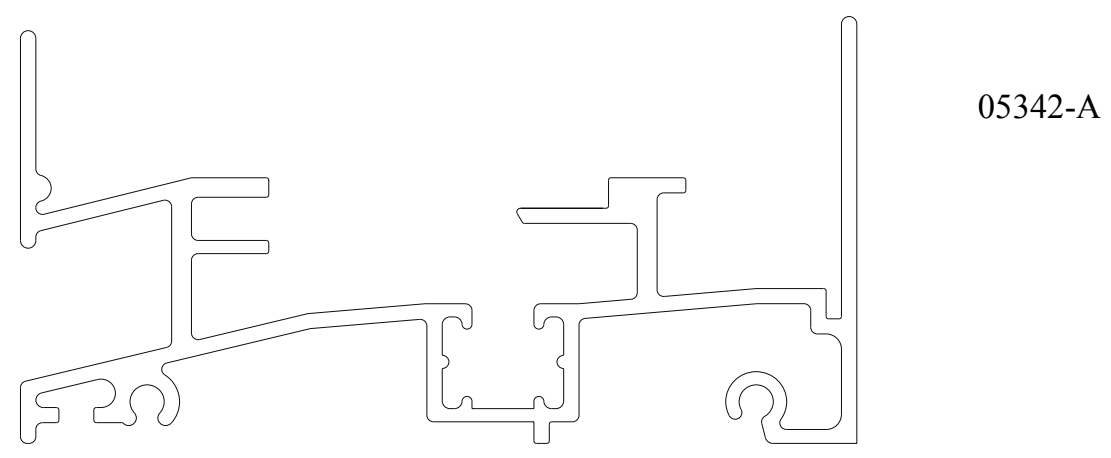

Figure 9-31. Sill Drawing for Horizontal Aluminum Slider.

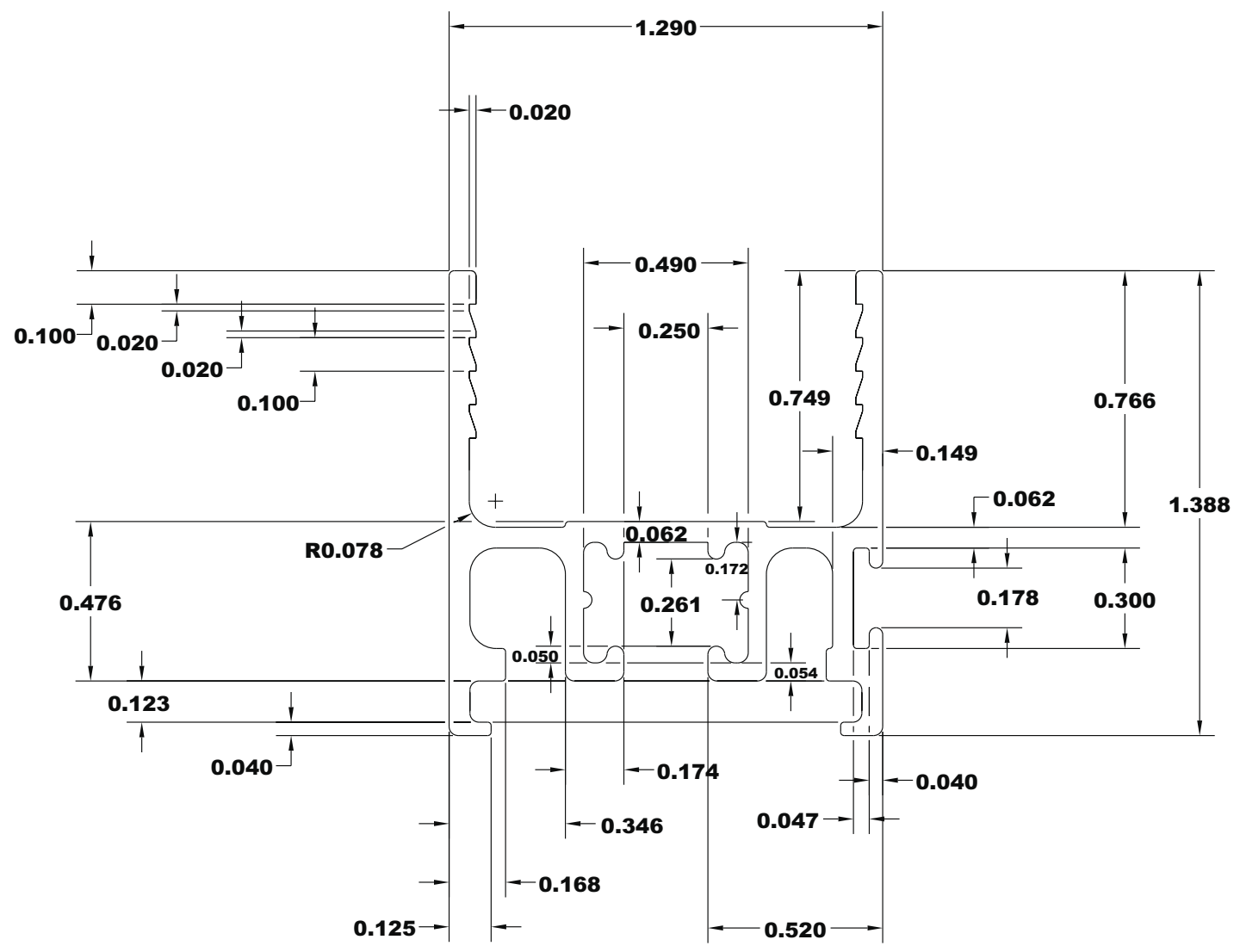

Figure 9-32. Stile-Fixed Drawing for Horizontal Aluminum Slider. 


\section{8-A}

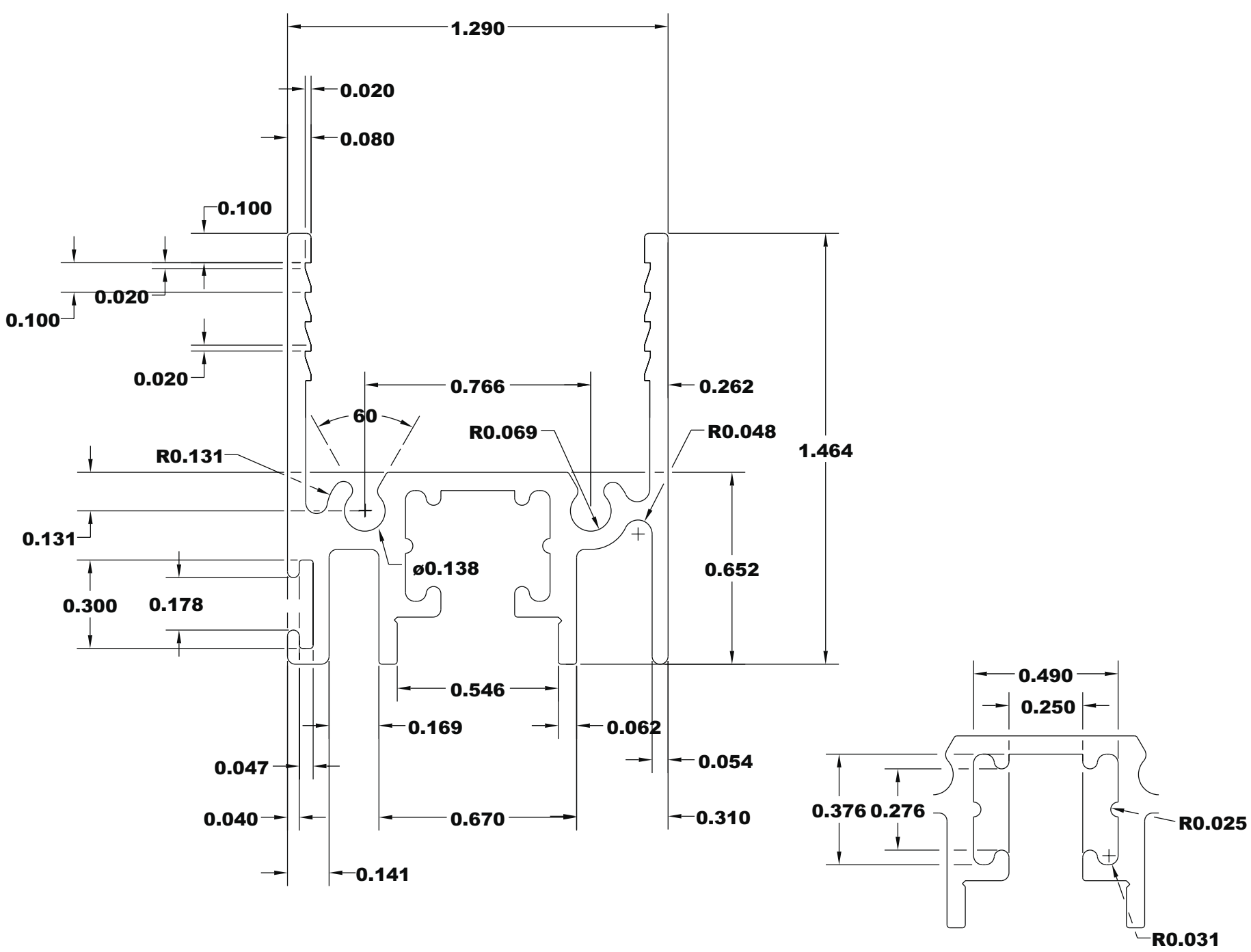

Figure 9-33. Stile-Fixed Drawing for Horizontal Aluminum Slider. 


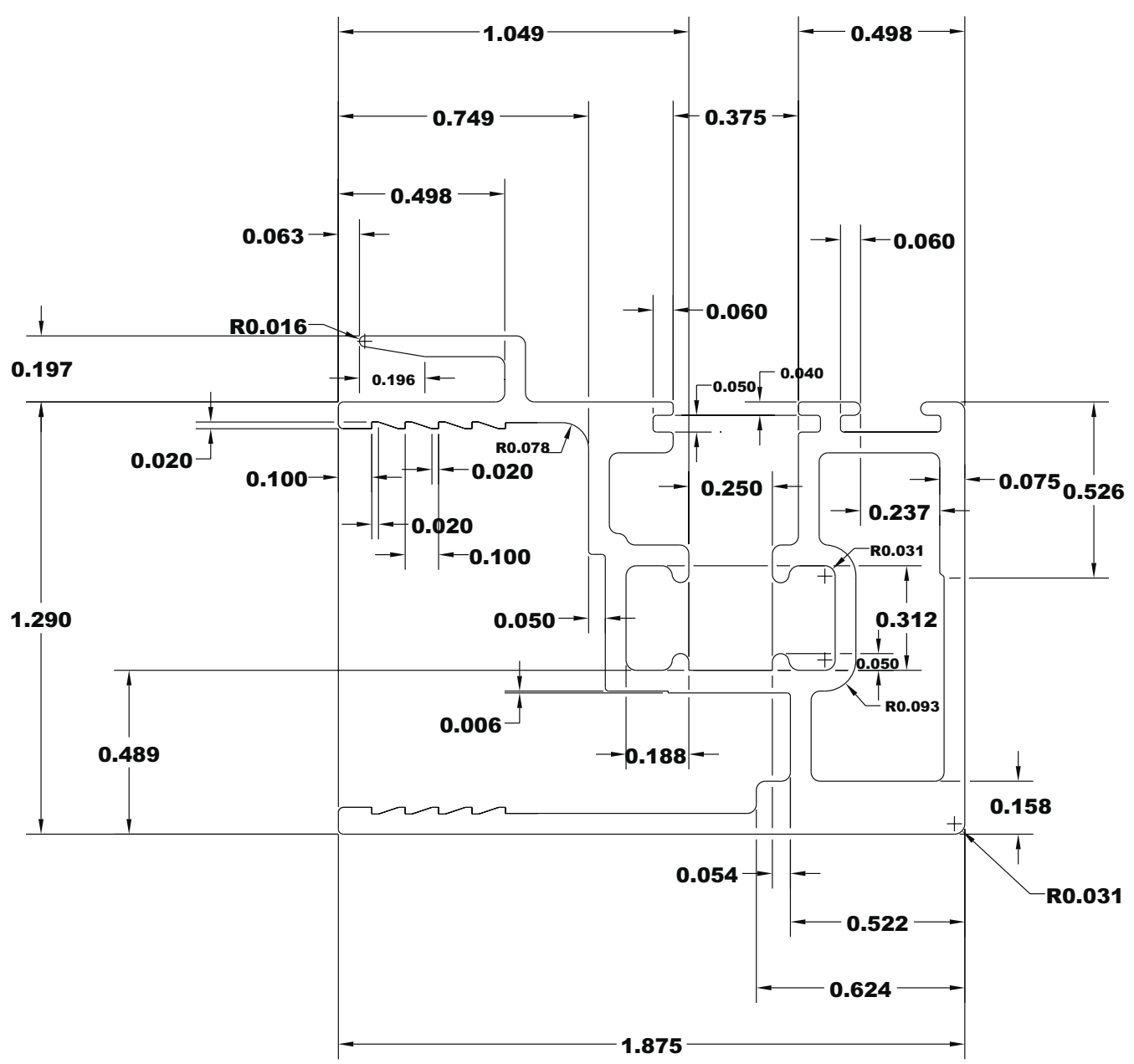

Figure 9-34. Stile-Slide / Silt - Fix Drawing for Horizontal Aluminum Slider. 


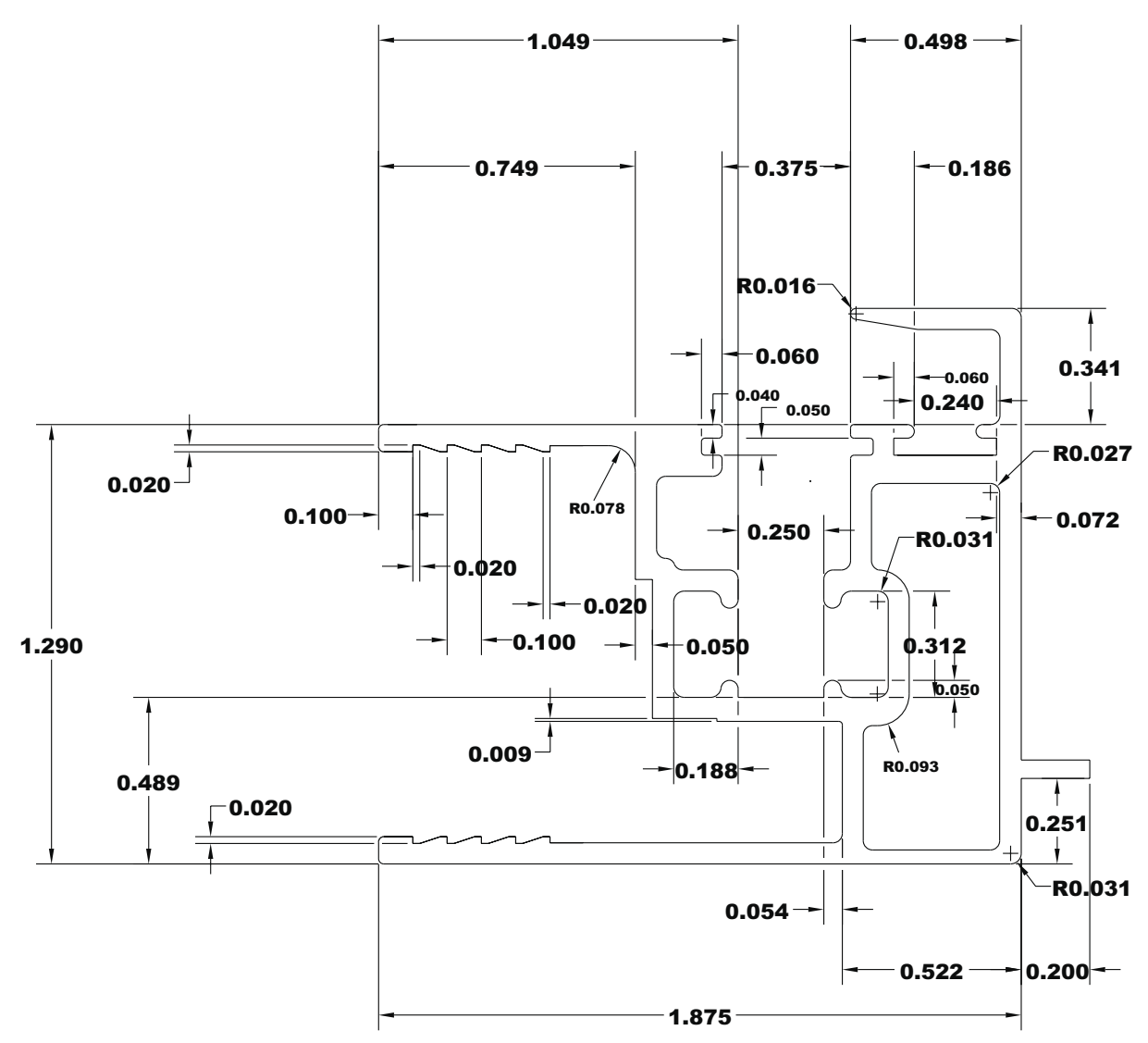

5370-A

Figure 9-35. Stile-Fixed Drawing for Horizontal Aluminum Slider. 


\section{0-A}

Page 16
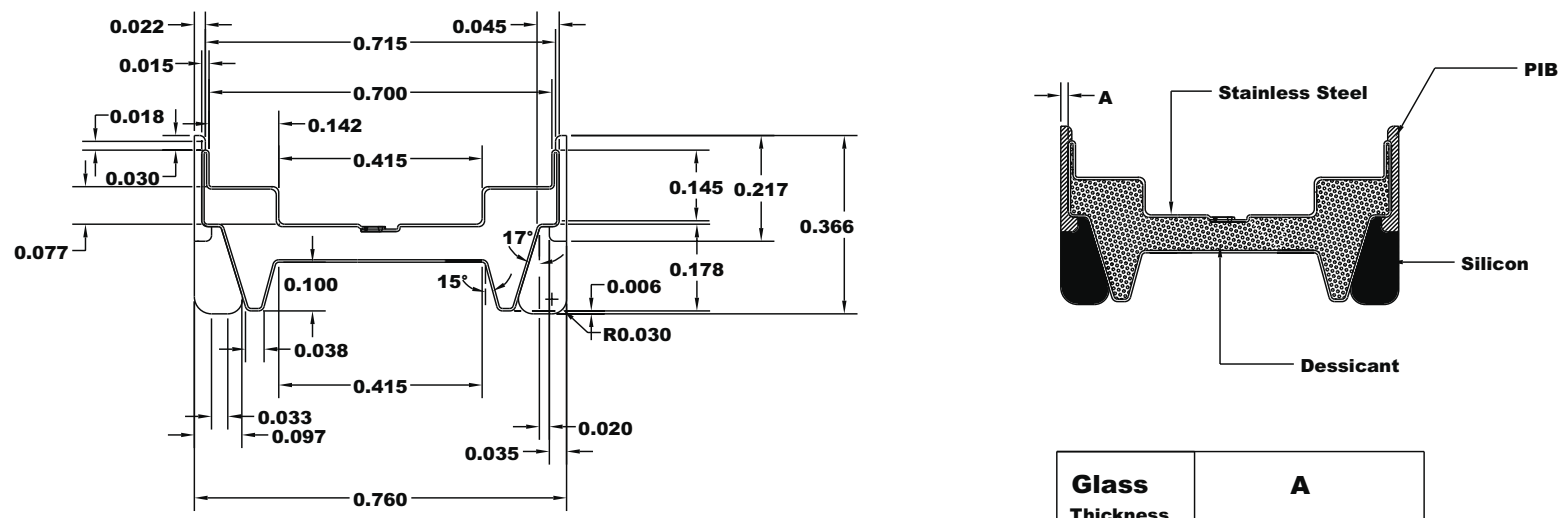

\begin{tabular}{|c|c|}
\hline $\begin{array}{l}\text { Glass } \\
\text { Thickness }\end{array}$ & A \\
\hline $3 \mathrm{~mm}$ & $0.015_{-0.005}^{+0.010}$ \\
\hline $4 \mathrm{~mm}$ & $0.015_{-0.005}^{+0.005}$ \\
\hline $5 \mathrm{~mm}$ & $0.015_{-0.005}^{+0.005}$ \\
\hline $6 \mathrm{~mm}$ & $0.015_{-0.005}^{+0.005}$ \\
\hline
\end{tabular}

Figure 9-36. Spacer Horizontal Aluminum Slider.

\section{0-A}
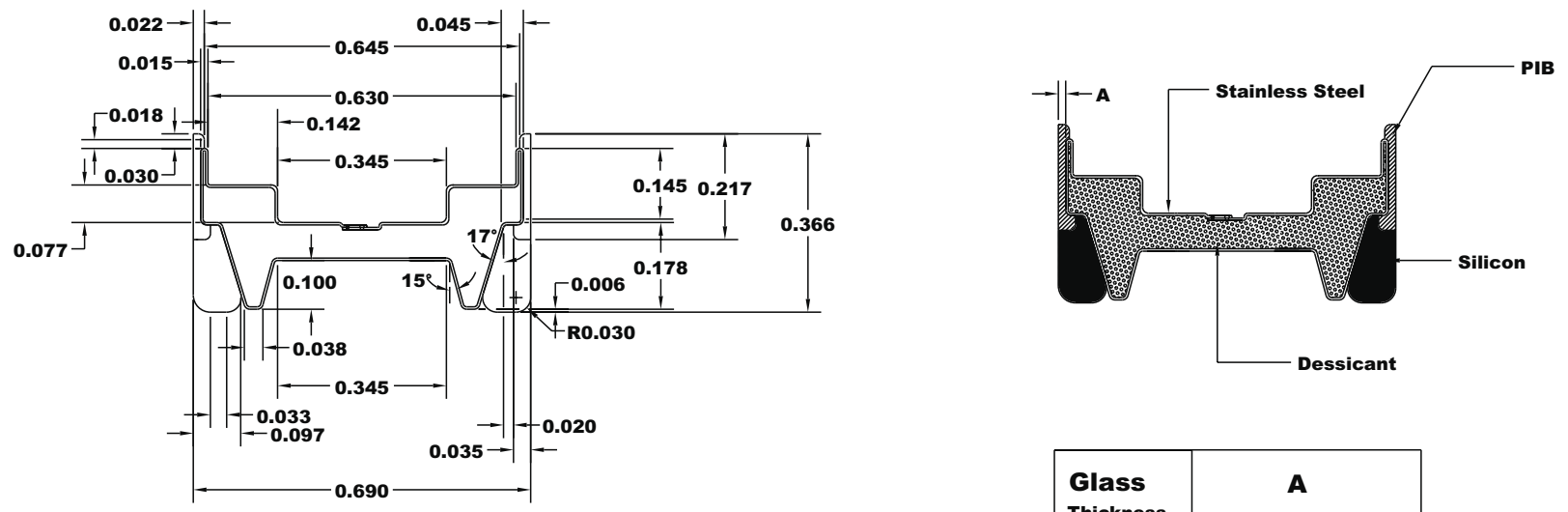

\begin{tabular}{|l|c|}
\hline $\begin{array}{l}\text { Glass } \\
\text { Thickness }\end{array}$ & $A$ \\
\hline $3 \mathrm{~mm}$ & $0.015_{-0.005}^{+0.010}$ \\
\hline $4 \mathrm{~mm}$ & $0.015_{-0.005}^{+0.005}$ \\
\hline $5 \mathrm{~mm}$ & $0.015_{-0.005}^{+0.005}$ \\
\hline $6 \mathrm{~mm}$ & $0.015_{-0.005}^{+0.005}$ \\
\hline
\end{tabular}

Figure 9-37. Spacer for Horizontal Aluminum Slider. 


\section{0-A}

Page 18
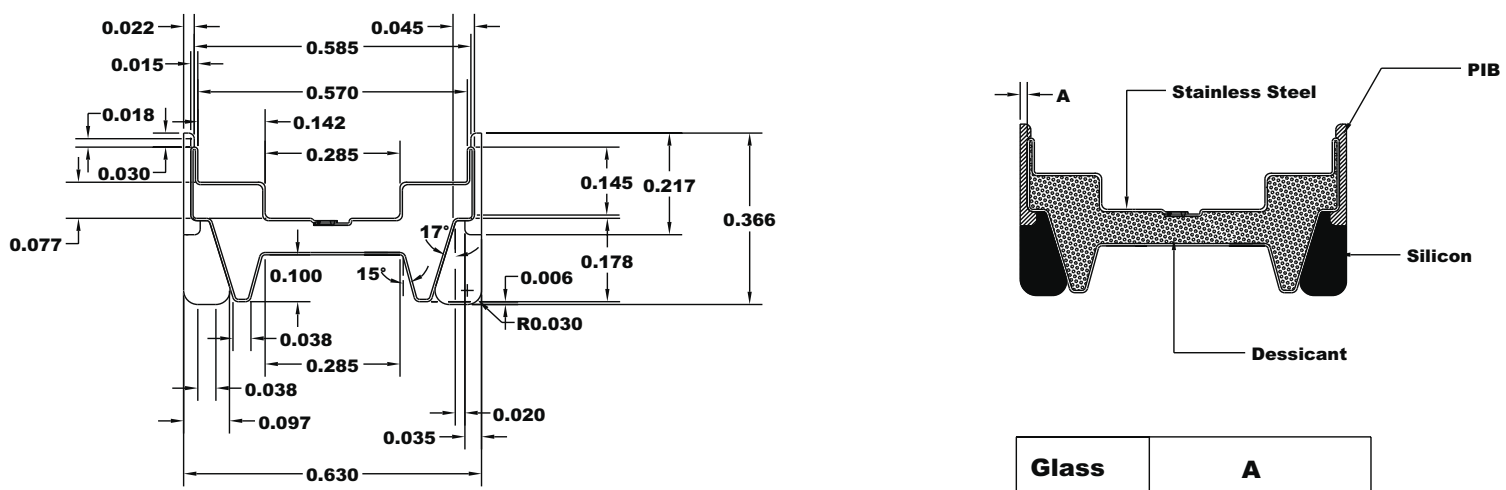

\begin{tabular}{|c|c|}
\hline $\begin{array}{l}\text { Glass } \\
\text { Thickness }\end{array}$ & $A$ \\
\hline $3 \mathrm{~mm}$ & $0.015_{-0.005}^{+0.010}$ \\
\hline $4 \mathrm{~mm}$ & $0.015_{-0.005}^{+0.005}$ \\
\hline $5 \mathrm{~mm}$ & $0.015_{-0.005}^{+0.005}$ \\
\hline $6 \mathrm{~mm}$ & $0.015_{-0.005}^{+0.005}$ \\
\hline
\end{tabular}

Figure 9-38. Spacer Horizontal Aluminum Slider.

\section{0-A}

Page 19
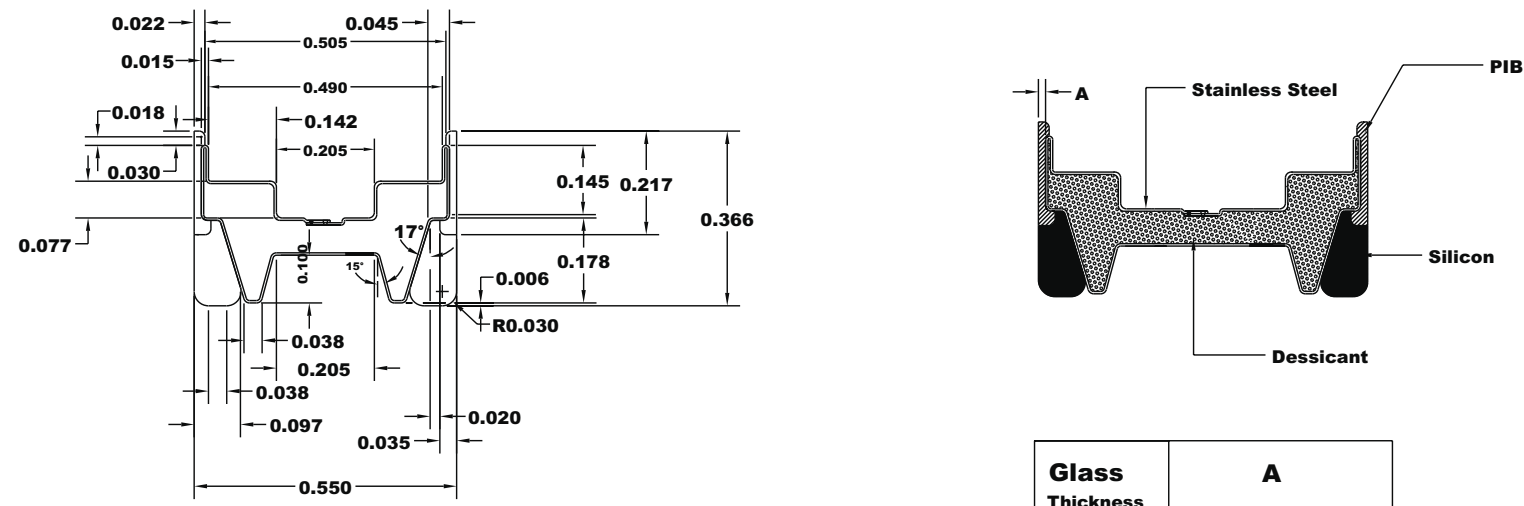

\begin{tabular}{|l|c|}
\hline $\begin{array}{c}\text { Glass } \\
\text { Thickness }\end{array}$ & A \\
\hline $3 \mathrm{~mm}$ & $0.015_{-0.005}^{+0.010}$ \\
\hline $4 \mathrm{~mm}$ & $0.015_{-0.005}^{+0.005}$ \\
\hline $5 \mathrm{~mm}$ & $0.015_{-0.005}^{+0.005}$ \\
\hline $6 \mathrm{~mm}$ & $0.015_{-0.005}^{+0.005}$ \\
\hline
\end{tabular}

Figure 9-39. Spacer Horizontal Aluminum Slider. 


\section{0-A}

Page 21

Divider type A

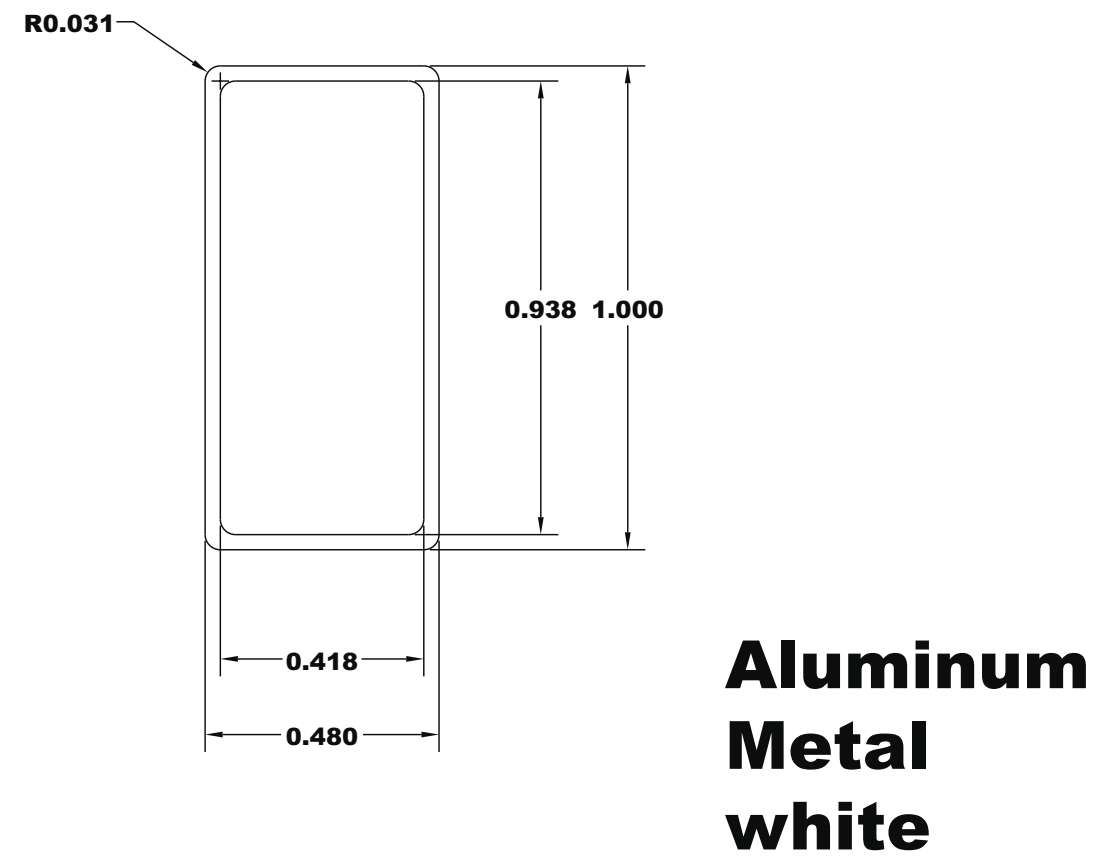

Figure 9-40Divider for Horizontal Slider

\section{0-A}

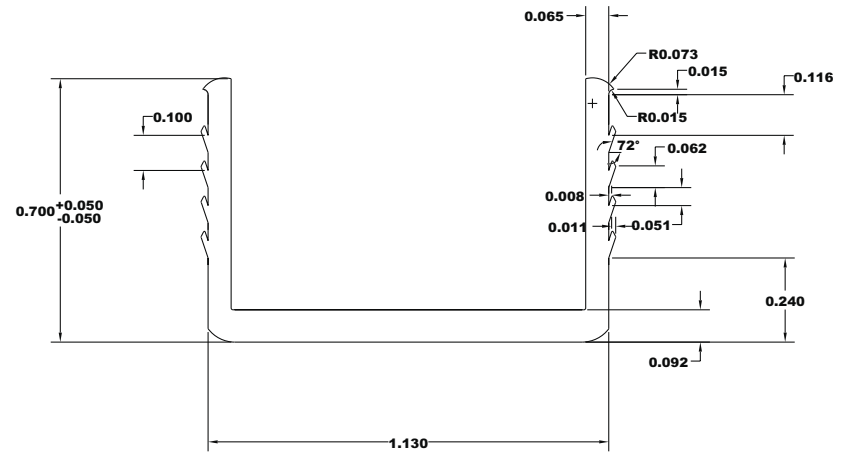

U-Chanel
Figure 9-41. U-channel for Horizontal Slider 


\subsection{Problem 3: Skylight}

\subsubsection{Description}

Window Type Skylight

Frame Material Wood

Overall Size $\quad 46.5^{\prime \prime}$ by $46.5^{\prime \prime}$

Spacer type Aluminum folded spacer.

Weather strip Not applicable.

Cross Sections Full-height head and sill; full-height jambs - the jambs are each the same so only one needs to be modeled, but the full-height cross section is modeled to account for the radiation exchange.

Dividers Not applicable

Glazing System See the glazing matrix in Table 9-16.

\subsubsection{Glazing Matrix}

The following table shows the glazing matrix that is to be simulated for this window.

Table 9-16. Center-of-glass U-factor Results for the Matrix of Glazing Options

\begin{tabular}{|c|c|c|c|}
\hline & $\begin{array}{c}\text { Glazing Options (1.0" overall } \\
\text { thickness) }\end{array}$ & $\begin{array}{c}\text { Grid } \\
\text { Option }\end{array}$ & $\begin{array}{c}\text { Manufacture } \\
\mathbf{r}\end{array}$ \\
\hline 1 & Clear $(3 \mathrm{~mm})$, Air, Clear $(3 \mathrm{~mm})$ & N/A & Generic \\
\hline
\end{tabular}




\subsubsection{Center-of-Glass Modeling (WINDOW)}

In WINDOW, create the glazing system(s) needed for the Glazing Matrix listed above. The center-of-glass Ufactor is displayed in the $U_{c}$ column of the WINDOW Glazing System Library. This glazing system will be brought into the THERM cross-sections.

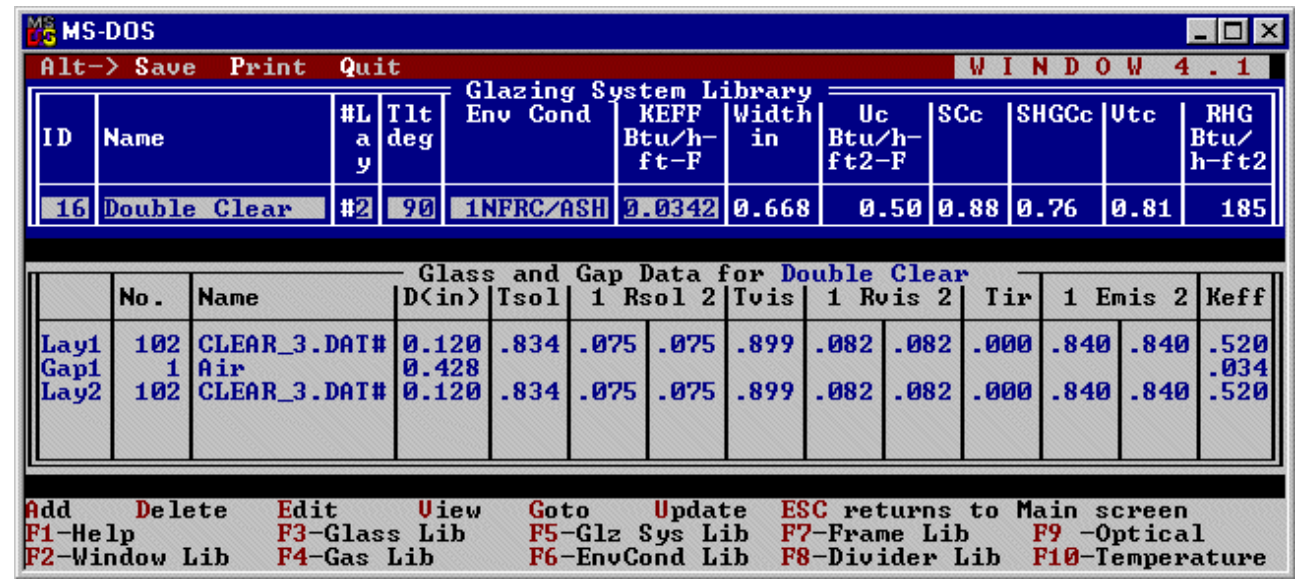

Figure 9-42. WINDOW Glazing System Library for the glazing option.

The results for the WINDOW center-of-glass $U$-factor $\left(U_{c}\right)$ are shown in the following table.

Table 9-17. WINDOW Center-of-glass U-factor Results for the Matrix of Glazing Options

\begin{tabular}{|c|c|c|c|}
\cline { 3 - 3 } \multicolumn{2}{c|}{} & \multicolumn{2}{c|}{$\begin{array}{c}\text { Center-of-glass U-factor } \\
\left(\mathbf{U}_{\mathbf{c}}\right)\end{array}$} \\
\hline & $\begin{array}{c}\text { Glazing Options (1.0" overall } \\
\text { thickness) }\end{array}$ & $\mathbf{( B t u / h r - \mathrm { ft } ^ { 2 } - { } ^ { \circ } \mathbf { F } )}$ & $\mathrm{W} / \mathrm{m}^{2}{ }^{-}{ }^{\circ} \mathrm{C}$ \\
\hline 1 & Clear (3 mm), Air, Clear (3mm) & 0.50 & 2.85 \\
\hline
\end{tabular}




\subsubsection{Edge-of-Glass and Frame Modeling (THERM)}

When modeling skylights, model each cross section as a full-height product, in order to include radiation enclosure modeling. See Section 8-5 of this manual for detailed instructions about modeling skylights.

Table 9-18 lists the cross sections to be modeled, and any associated files (DXF and THERM files) that are found on the CD.

Table 9-18. Cross sections to be modeled with their associated $d x f$ and THERM files

\begin{tabular}{|l|l|l|l|}
\hline Cross Section & DXF Filename & \multicolumn{1}{|c|}{ THERM file for $\mathbf{3} \mathbf{~ m m}$} & THERM file for $\mathbf{6} \mathbf{m m}$ \\
\hline Full-height Sill/Head & N/A & Skylight example head\&sill.thm & N/A \\
\hline Full-height Jamb & N/A & Skylight example jambs.thm & N/A \\
\hline
\end{tabular}

Table 9-19 shows the resulting U-factors from each cross section for the $3 \mathrm{~mm}$ glazing case.

Table 9-19. Frame, Edge and Center-of-Glass THERM U-factor Results for Glazing Option 1: $3 \mathrm{~mm}$

\begin{tabular}{|c|c|c|c|c|c|c|}
\hline \multirow[b]{2}{*}{ Cross Section } & \multicolumn{2}{|c|}{ Frame U-factor } & \multicolumn{2}{|c|}{ Edge U-factor } & \multicolumn{2}{|c|}{ Center-of-glass U-factor } \\
\hline & (Btu/hr-ft $\left.{ }^{2}{ }^{\circ} \mathrm{F}\right)$ & $\left(W / m^{2}-{ }^{\circ} \mathrm{C}\right)$ & (Btu/hr-ft $\left.{ }^{2}{ }^{\circ} \mathrm{F}\right)$ & $\left(\mathrm{W} / \mathrm{m}^{2}{ }^{\circ} \mathrm{C}\right)$ & (Btu/hr-ft $\left.{ }^{2}{ }^{\circ} \mathrm{F}\right)$ & $\left(\mathrm{W} / \mathrm{m}^{2}-{ }^{\circ} \mathrm{C}\right)$ \\
\hline Head & 0.8857 & 5.0296 & 0.5748 & 3.2639 & 0.4989 & 2.8329 \\
\hline Sill & 0.9434 & 5.3575 & 0.5095 & 2.8934 & 0.4989 & 2.8329 \\
\hline Jamb & 0.8890 & 5.0480 & 0.5795 & 3.2910 & 0.4989 & 2.8332 \\
\hline
\end{tabular}

Figures 9-43 and 9-44 on the following pages show THERM files and U-factor results screens for each of the cross sections with $3 \mathrm{~mm}$ glass. 
Full-height Head / Sill Cross Section

With Radiation Enclosure
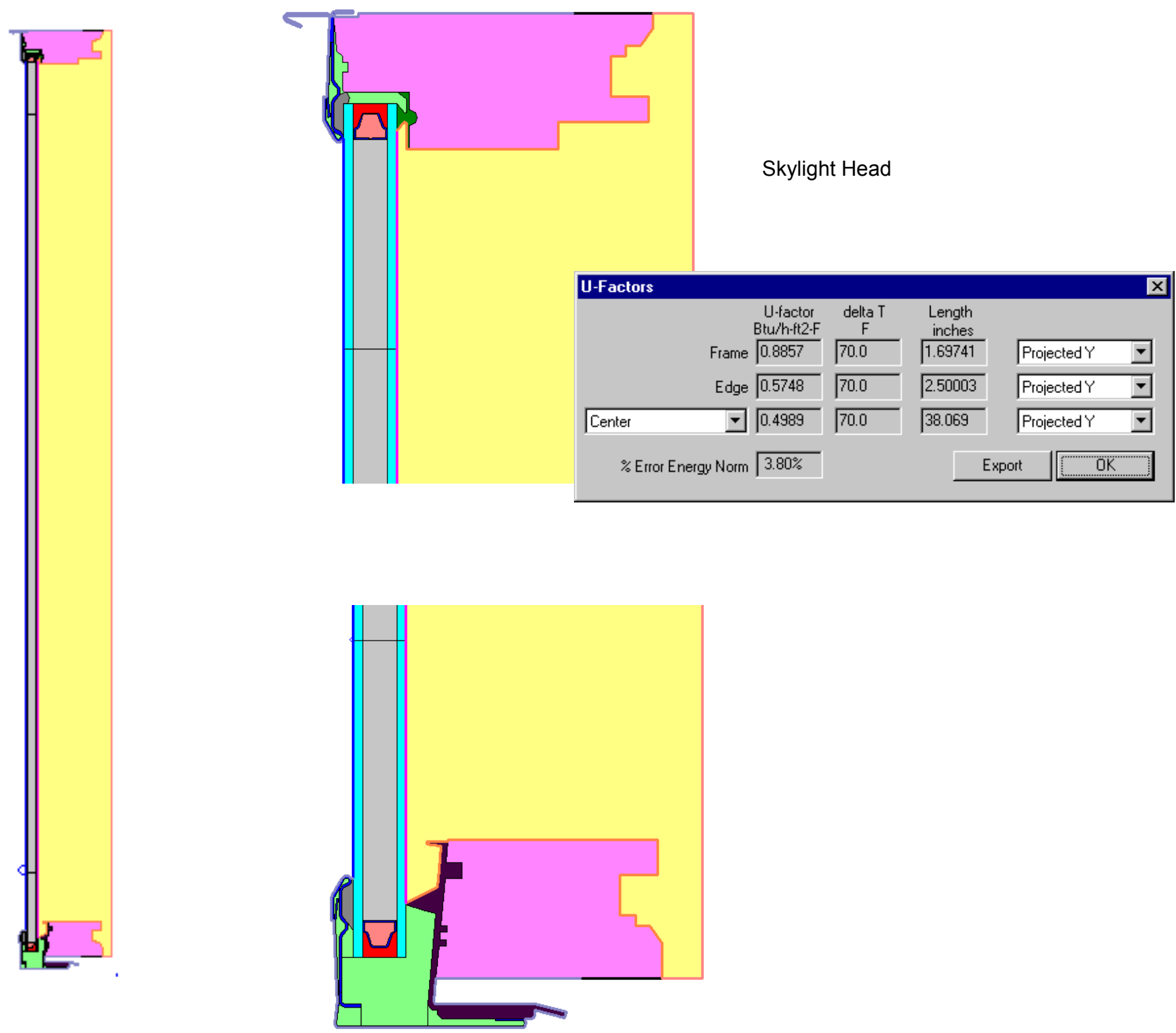

Figure 9-43. THERM file for Head and Sill full-height inset mount skylight cross section. 
Full-height Jamb Cross Section

With Radiation Enclosure
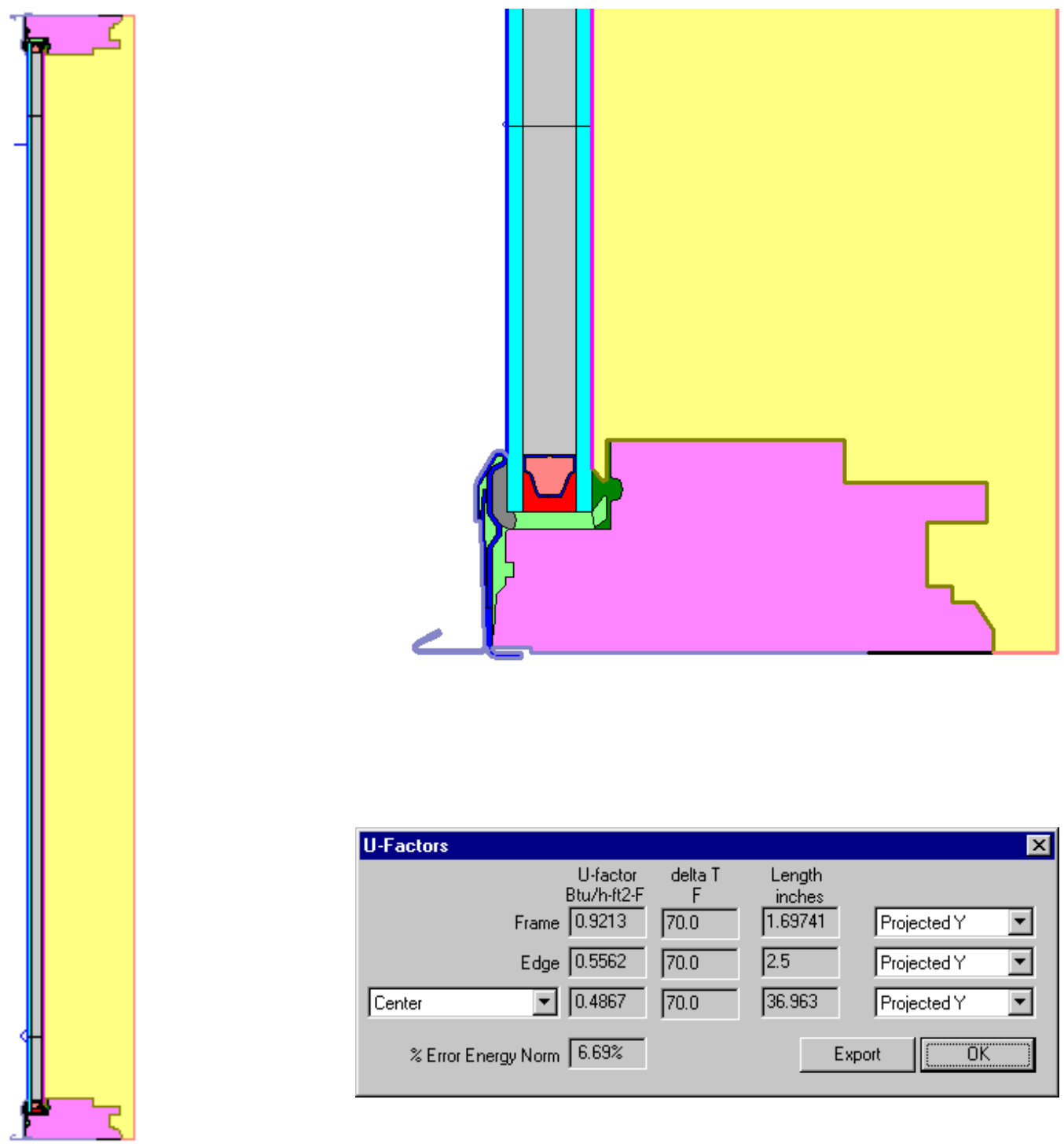

Figure 9-44. THERM file for Jamb full-height inset mount skylight cross section. 


\subsubsection{Total Product U-Factor}

To calculate the total product U-factor, an area-weighted value must be calculated from each of the THERM cross sections and the center-of-glass U-factor. This can be done either in WINDOW or in a spreadsheet. In this example, the area-weighting is done in WINDOW.

For projecting products such as skylights that are modeled in THERM with a radiation enclosure, the centerof-glass (COG) U-factor is calculated based on the COG U-factor results from the THERM calculation (while for non-projecting products the COG value from WINDOW is used). This procedure is explained in detail in this manual in Section 8.5, "Projecting Products". The new WINDOW "generic" glass layer and resulting glazing system from the THERM calculation are shown in Figures 9-45 and 9-46.

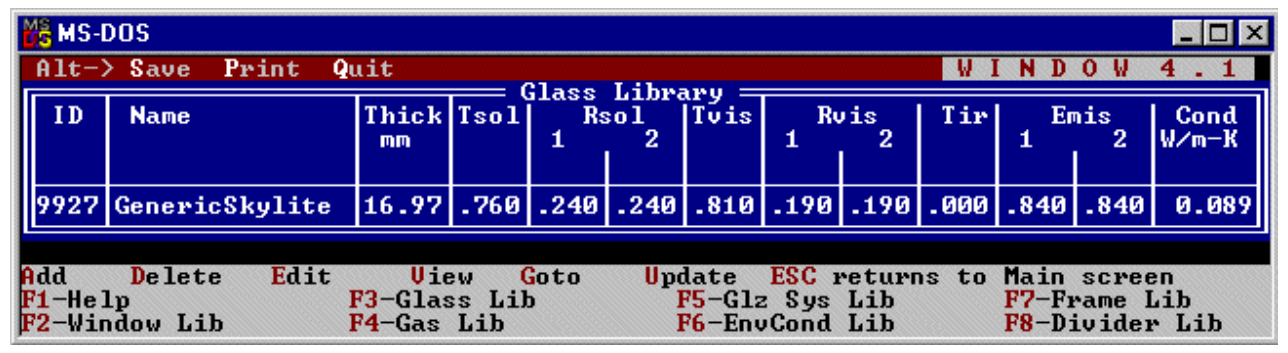

Figure 9-45. WINDOW generic glass layer using results from COG values in THERM.

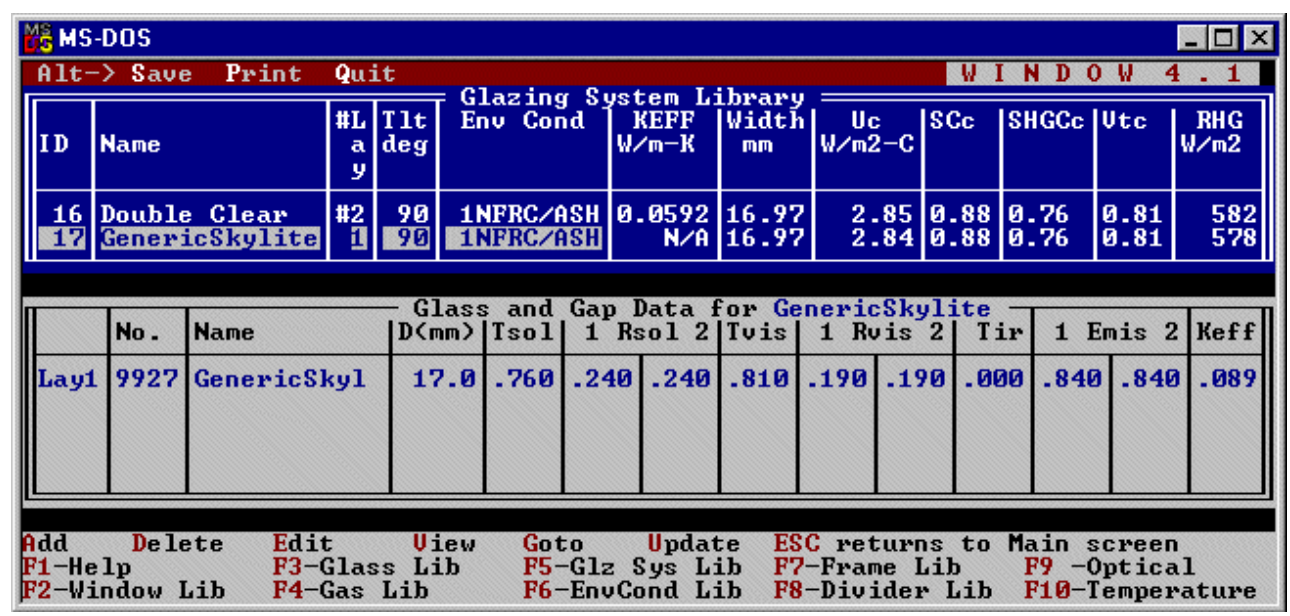

Figure 9-46. WINDOW generic glazing system library using generic glass layer based on results from COG values in THERM.

In the example in Figure 9-46, the glazing system named GenericSkylite from the WINDOW Glazing System Library is then used in the WINDOW Main Screen to calculate the total product U-factor, as shown in Figure 9-47. 


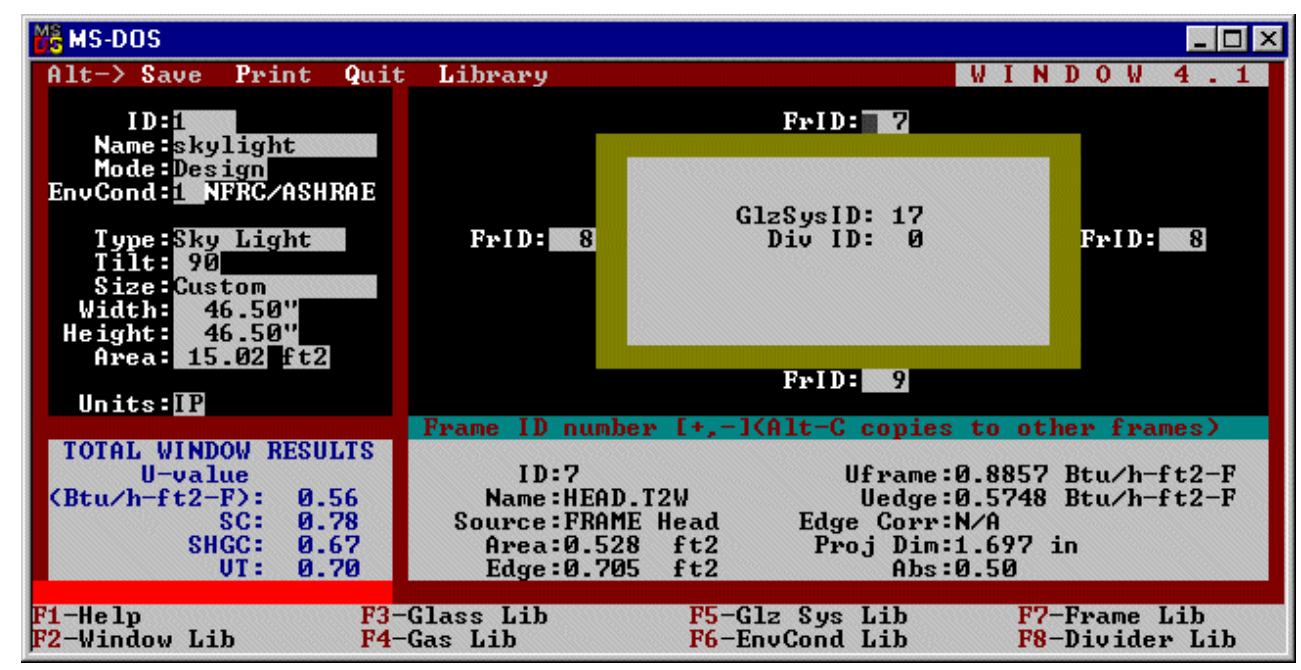

Figure 9-47. Total product U-factor calculation in WINDOW.

Table 9-20 shows the overall product U-factor, from WINDOW, for Glazing Options 1 and 4. The other glazing options are not calculated in this example.

Table 9-20. Total Product U-factors for skylight

\begin{tabular}{|c|c|c|c|}
\hline \multicolumn{2}{|c|}{$\begin{array}{c}\text { Glazing Options } \\
\text { (1.0" overall thickness) }\end{array}$} & \multicolumn{2}{c|}{$\begin{array}{c}\text { Custom Size (46.5"x46.5") } \\
\text { Total Product U-factor }\end{array}$} \\
\cline { 3 - 4 } & $\begin{array}{c}\text { (Btu/hr-ft }{ }^{2}- \\
{ }^{\circ} \text { F) }\end{array}$ & (W/m ${ }^{2}{ }^{\circ}$ C) \\
\hline 1 & $\begin{array}{l}\text { Clear (3 mm), Air, Clear (3mm) } \\
\text { No Dividers }\end{array}$ & 0.56 & 3.16 \\
\hline
\end{tabular}

\subsubsection{Total Product Solar Heat Gain Coefficient (SHGC) and Visible Transmittance (VT)}

The methodology for determining the Solar Heat Gain Coefficient (SHGC) for the product is outlined in NFRC 200, and the methodology for determining the Visible Transmittance is outlined in NFRC 300.

WINDOW automatically calculates these values, as shown in Figure 9-47. The results for glazing option 1 are shown in Table 9-21.

Table 9-21. Total Product SHGC and Visible Transmittance (VT)

\begin{tabular}{|c|l|l|l|l|l|}
\hline \multicolumn{2}{|c|}{$\begin{array}{c}\text { Glazing Options } \\
(1.0 " \text { overall thickness) }\end{array}$} & \multicolumn{2}{c|}{ Residential (AA) 3mm } & \multicolumn{2}{c|}{ Non-residential (BB) 6mm } \\
\cline { 3 - 6 } & SHGC & VT & SHGC & VT \\
\hline 1 & $\begin{array}{l}\text { Clear, Argon (95\%), Low-e } \\
\text { No Dividers }\end{array}$ & 0.67 & 0.70 & N/A & N/A \\
\hline
\end{tabular}


Finally, the SHGC and VLT Specialty Products table values must be determined. These are the $\mathrm{SHGC}_{0}, \mathrm{SHC}_{1}$, $\mathrm{VLT}_{0}$, and $\mathrm{VLT}_{1}$ values. These values are calculated for the one glazing option at $3 \mathrm{~mm}$ and $6 \mathrm{~mm}$ glass thicknesses. The skylight does not have dividers, so that part of the table is not applicable.

Table 9-22. Specialty Products Table Values

\begin{tabular}{|l|l|l|}
\hline & Residential (3mm) & $\begin{array}{l}\text { Non-Residential } \\
(\mathbf{6 m m})\end{array}$ \\
\hline SHGC $_{\mathbf{0}}$ & 0.02 & N/A \\
\hline SHGC $_{\mathbf{1}}$ & 0.88 & N/A \\
\hline VLT $_{\mathbf{0}}$ & 0.00 & N/A \\
\hline VLT $_{\mathbf{1}}$ & 0.86 & N/A \\
\hline
\end{tabular}

\subsubsection{Skylight Drawings.}

Drawings for the skylight are included on the following pages. (Not currently available, but to be supplied) 


\subsection{Problem 4: Door}

For this wood stile and rail door, calculate the U-factor, SHGC and VT.

\subsubsection{Description}

Door Type

Frame Material

Overall Size

Glazing System

Spacer Type

Glazing Method

Dividers

Drawings
Wood stile and rail door

Wood with a thermal-break aluminum sill

36 "x 80 " door slab

Double glazing, $0.500 "$ overall IG thickness. There are four individual glass options all with two layers of double-strength PPG glass (0.129" thickness). The first option is two layers of clear glass air filled, the second is two layers of clear glass argon filled $(90 \%$ two probe filling), the third is two layers of clear glass with a Sungate 1000 on surface 2 and air filled, the fourth option is two layers of clear glass with a Sungate 1000 on surface 2 and argon filled.

Intercept ${ }^{\mathrm{TM}}$ tin-plate with butyl sealant on three sides

Wet glazed with Silicone on both sides

There are no dividers in this product

There are detailed drawings for this door shown in Section 9.5.7. Also included on the $\mathrm{CD}$ are the DXF files of the assembly drawings for this door.

\subsubsection{Glazing Matrix}

\begin{tabular}{|l|l|l|}
\hline Glass Option & \multicolumn{1}{|c|}{$\begin{array}{c}\text { Residential } \\
\text { (AA) }\end{array}$} & $\begin{array}{c}\text { Non-Residential } \\
\text { (BB) }\end{array}$ \\
\hline 1. Clear_Air_Clear & & Not applicable for entry doors \\
\hline 2. Clear_Argon (90\%)_Clear & & Not applicable for entry doors \\
\hline 3. Low-e_Air_Clear & & Not applicable for entry doors \\
\hline 4. Low-e_Argon (90\%)_Clear & & Not applicable for entry doors \\
\hline
\end{tabular}




\subsubsection{Center-of-Glass Modeling (WINDOW)}

The four IG units were modeled in WINDOW with double-strength glass, 0.242 " air space, $90 \%$ argon (where appropriate), and Sungate 1000 Low-e (where appropriate).

Figure 9-48 shows the WINDOW Glazing System Library with all four IG units.

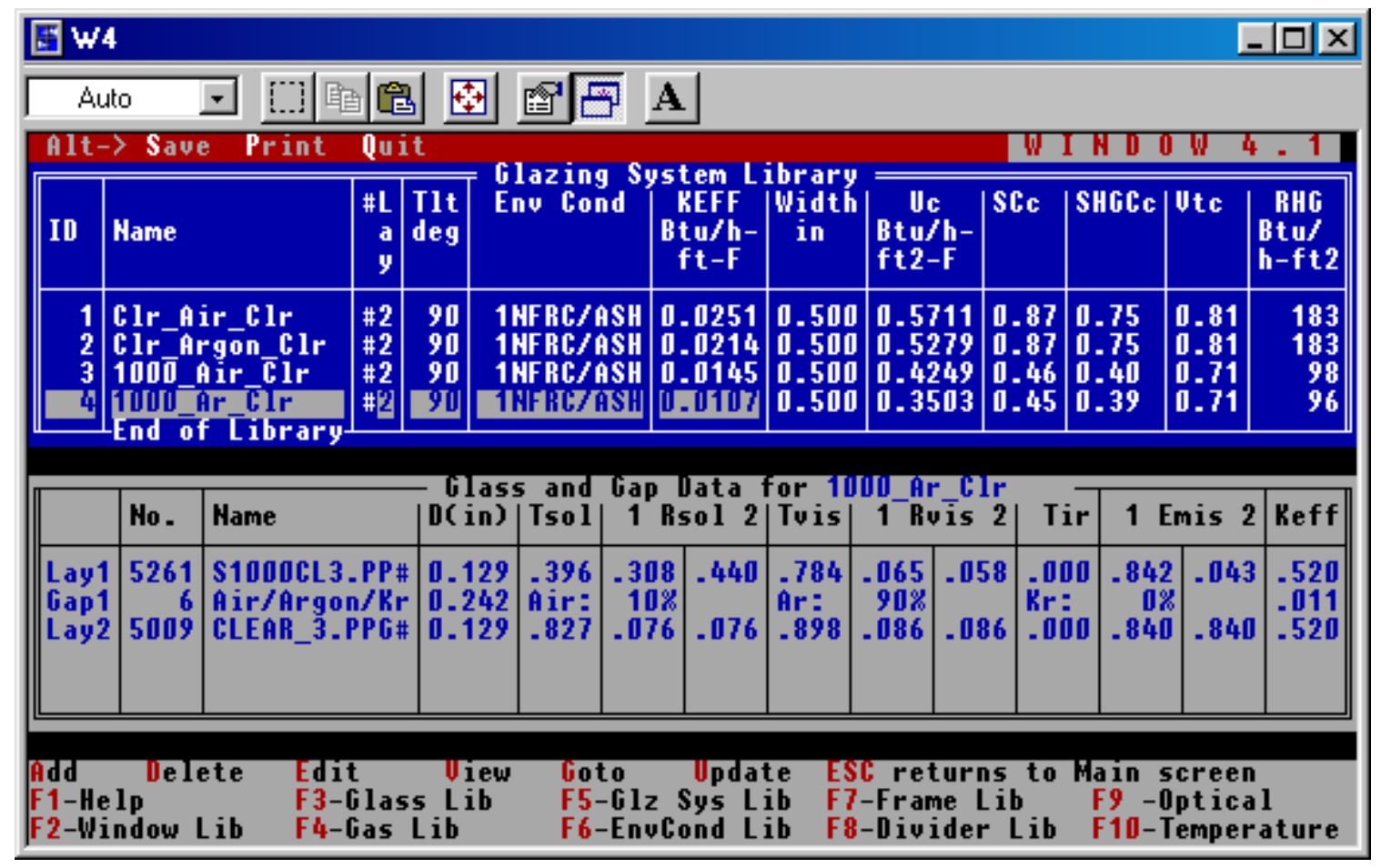

Figure 9-48. WINDOW 4.1 Glazing System Library for vinyl window

The results for the center-of-glass $U$-factor $\left(U_{c}\right)$ are shown in the following table:

Table 9-23. Center-of-glass U-factor results from WINDOW

\begin{tabular}{|c|c|c|c|}
\hline \multirow{2}{*}{\multicolumn{2}{|c|}{\begin{tabular}{|c|} 
Glazing Options \\
(1.0" overall thickness)
\end{tabular}}} & \multicolumn{2}{|c|}{ Center-of-glass U-factor $\left(U_{c}\right)$} \\
\hline & & \multirow{2}{*}{$\frac{\left(B t u / h r-f^{2}{ }^{\circ}{ }^{\circ} \mathbf{)}\right)}{0.5711}$} & \multirow{2}{*}{$\frac{\left(\mathrm{W} / \mathrm{m}^{\mathbf{2}}{ }^{\circ} \mathrm{C}\right)}{3.2429}$} \\
\hline 1 & Clear $\left(0.129^{\prime \prime}\right)$, Air, Clear $\left(0.129^{\prime \prime}\right)$ & & \\
\hline 2 & Clear $\left(0.129^{\prime \prime}\right)$, Argon $(90 \%)$, Clear $\left(0.129^{\prime \prime}\right)$ & 0.5279 & 2.9977 \\
\hline 3 & Low-e $\left(0.129^{\prime \prime}\right)$, Air, Clear $\left(0.129^{\prime \prime}\right)$ & 0.4249 & 2.4126 \\
\hline 4 & Low-e (0.129"), Argon (90\%), Clear (0.129") & 0.3503 & 1.9894 \\
\hline
\end{tabular}

These U-factors will be used with the edge-of-glass and frame values from THERM to calculate the overall Ufactor for the window in Section 9.5.6 (note that four significant digits have been included as these values will have to be entered into a spreadsheet to calculate the overall U-factors). 


\subsubsection{Edge-of-Glass and Frame Modeling (THERM)}

An entry door requires eight THERM models to completely model the heat flow through the door. The eight models are:

- Head

- Lock jamb

- Hinge jamb

- Sill

- Panel

- Door lite

- Panel core

- Door core

This door design has identical sections for the head, lock jamb, and hinge jamb so only one THERM model will be required to represent those sections. The door core model has been added to the head model and the panel core model has been added to the panel model. Four door lite models are required, one for each glass option.

Table 9-24 shows the files that are included on the CD for this example problem.

Table 9-24. Files associated with the wood door example

\begin{tabular}{|l|l|}
\hline Cross Section & \multicolumn{1}{c|}{ THERM file } \\
\hline Head, Lock Jamb, Hinge Jamb & head.thm \\
\hline Sill & sill.thm \\
\hline Panel & panel-edge.thm \\
\hline Door Lite Option 1 & lite_01.thm \\
\hline Door Lite Option 2 & lite_02.thm \\
\hline Door Lite Option 3 & lite_03.thm \\
\hline Door Lite Option 4 & lite_04.thm \\
\hline
\end{tabular}

Table 9-25 shows the resulting U-factors from each cross section for the $3 \mathrm{~mm}$ (with dividers) glazing case.

Table 9-25. THERM results for vinyl window cross sections

\begin{tabular}{|l|l|l|l|l|}
\hline Cross Section & $\begin{array}{l}\text { Frame U- } \\
\text { factor } \\
\left.\text { (Btu/hr-ft }{ }^{2}{ }^{-} \mathbf{F}\right)\end{array}$ & $\begin{array}{l}\text { Edge U- } \\
\text { factor } \\
\mathbf{( B t u / h r - \mathbf { f t } ^ { 2 } -} \\
\left.\mathbf{}^{\mathbf{0}} \mathbf{F}\right)\end{array}$ & $\begin{array}{l}\text { Frame } \\
\text { Height } \\
\text { (in) }\end{array}$ & $\begin{array}{l}\text { Edge } \\
\text { Height (in) }\end{array}$ \\
\hline Head, Lock Jamb, Hinge Jamb & 0.3001 & 0.3440 & 0.875 & 2.500 \\
\hline Door Core (from the head model) & 0.3632 & $\mathrm{n} / \mathrm{a}$ & $\mathrm{n} / \mathrm{a}$ & $\mathrm{n} / \mathrm{a}$ \\
\hline Sill & 1.1359 & 0.3393 & 1.563 & 2.500 \\
\hline Panel & 0.3429 & 0.5533 & 1.000 & 3.016 \\
\hline Panel Core (from the panel model) & 0.4616 & $\mathrm{n} / \mathrm{a}$ & $\mathrm{n} / \mathrm{a}$ & $\mathrm{n} / \mathrm{a}$ \\
\hline Door Lite Option 1 & 0.5120 & 0.6022 & 1.656 & 2.500 \\
\hline Door Lite Option 2 & 0.5083 & 0.5664 & 1.656 & 2.500 \\
\hline Door Lite Option 3 & 0.5000 & 0.4823 & 1.656 & 2.500 \\
\hline
\end{tabular}




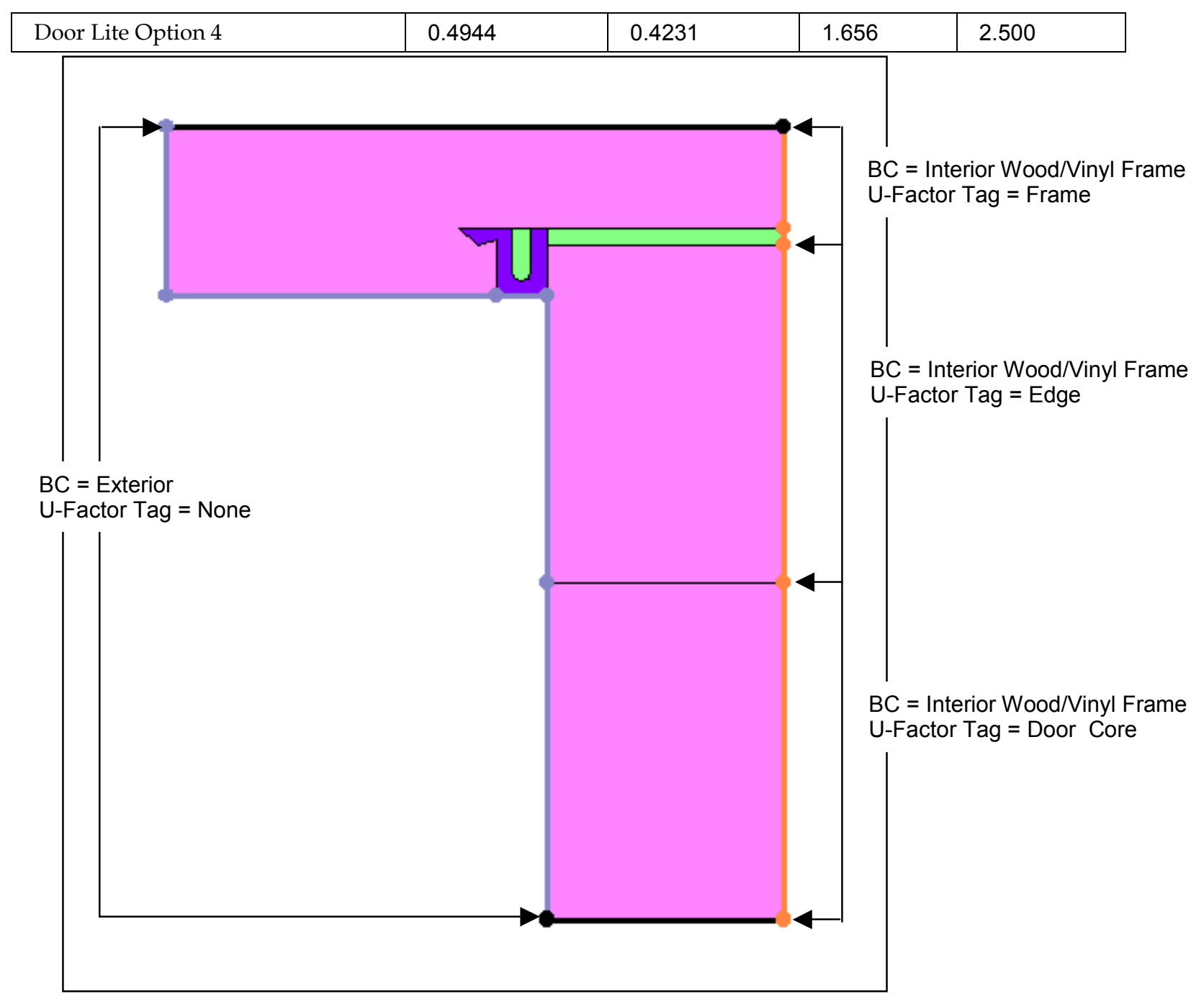

Figure 9-49. Door Head THERM file. 


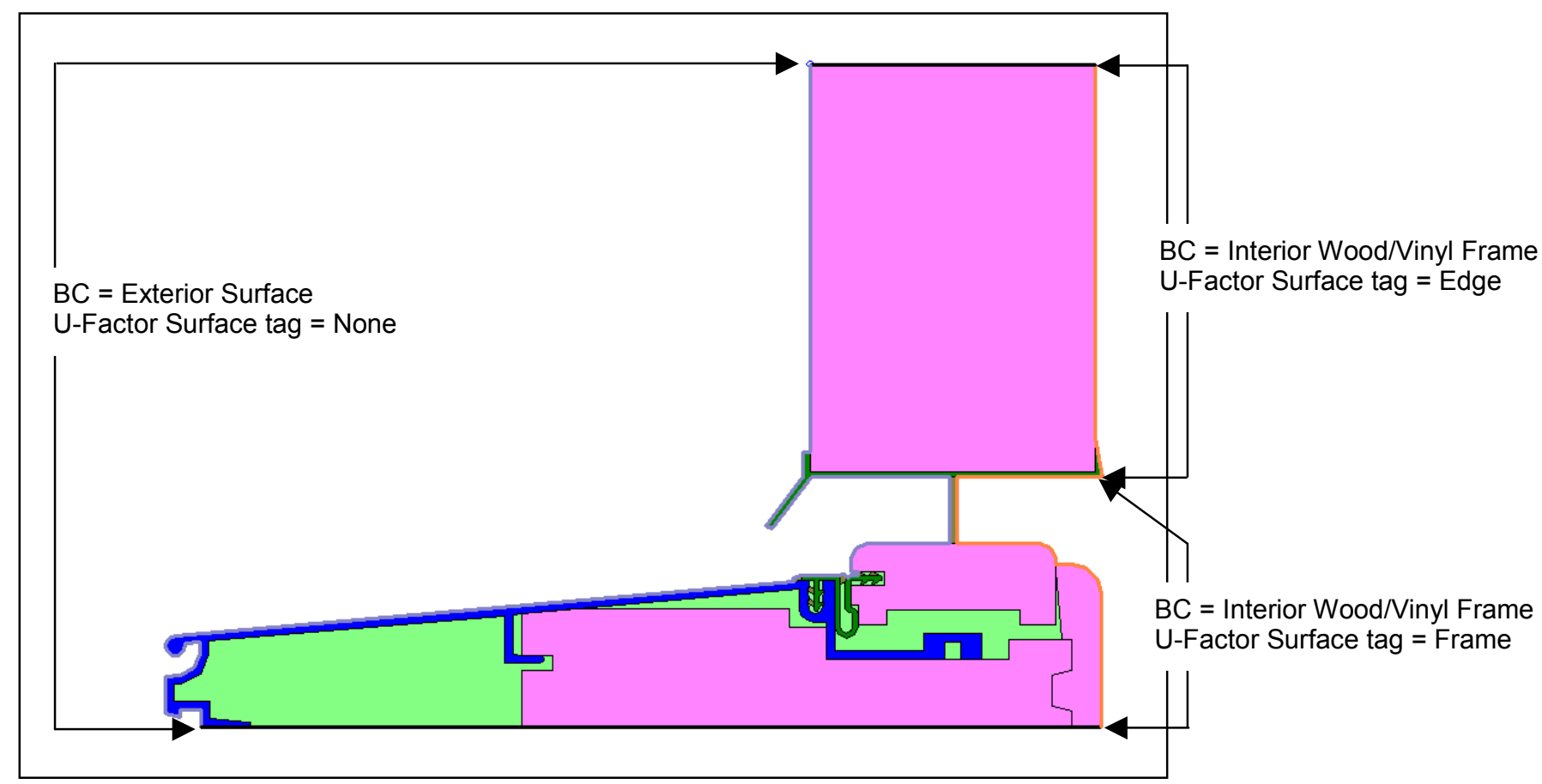

Figure 9-50. Door Sill THERM file. 


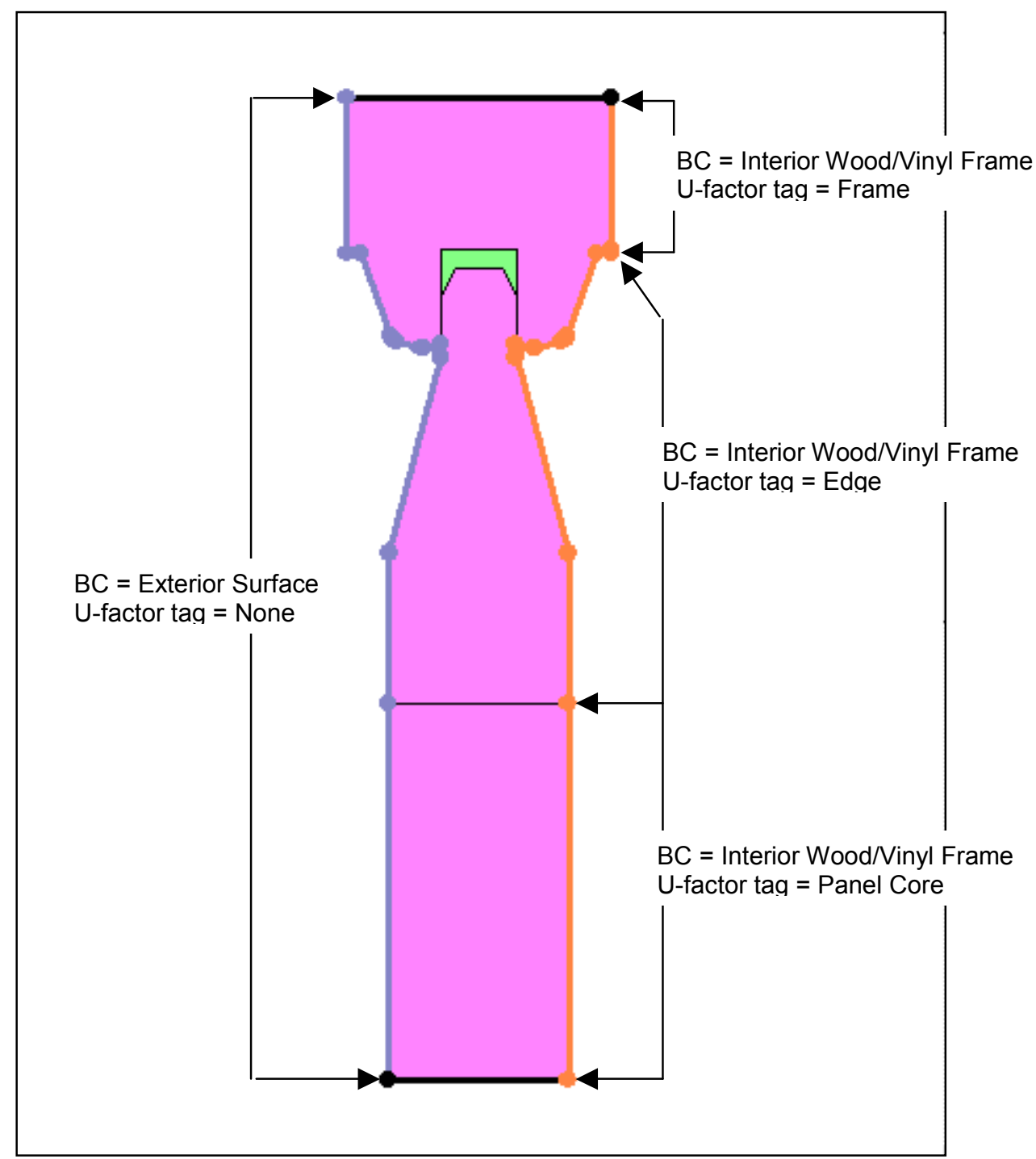

Figure 9-51. Door Panel Edge THERM file. 


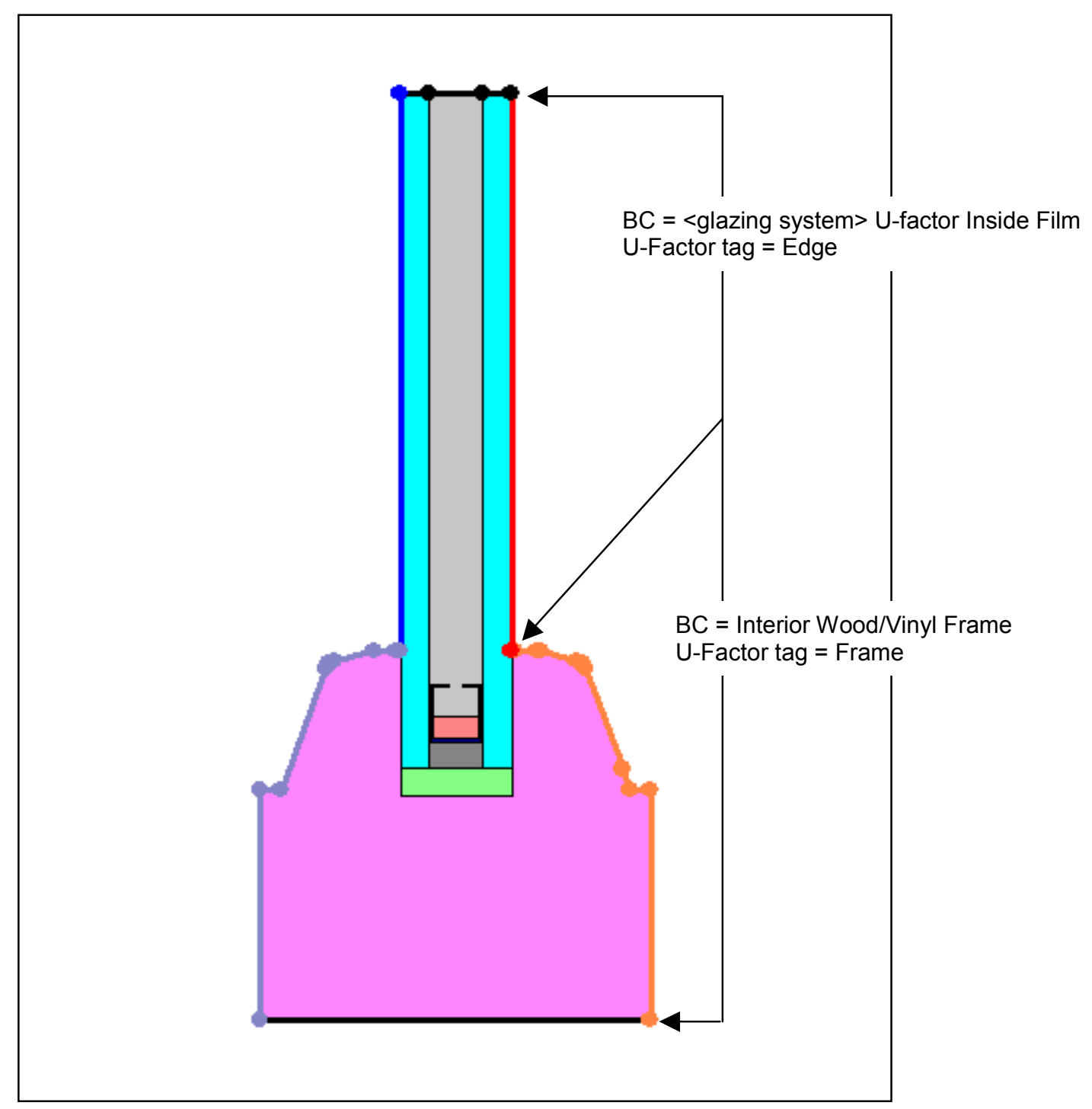

Figure 9-52. Door Lite THERM file.

\subsubsection{Total Product U-Factor}

The four glass options were area weighted using the door spreadsheet (6-panel door.xls) supplied on the CD. The options were only area weighted for the residential size, as the non-residential size is not applicable for entry doors. The spreadsheet will handle upto four glass options.

The data to be entered into the 6-panel door spreadsheet includes the following:

- Overall door dimensions

- WINDOW and THERM data

- Area Calculation data 


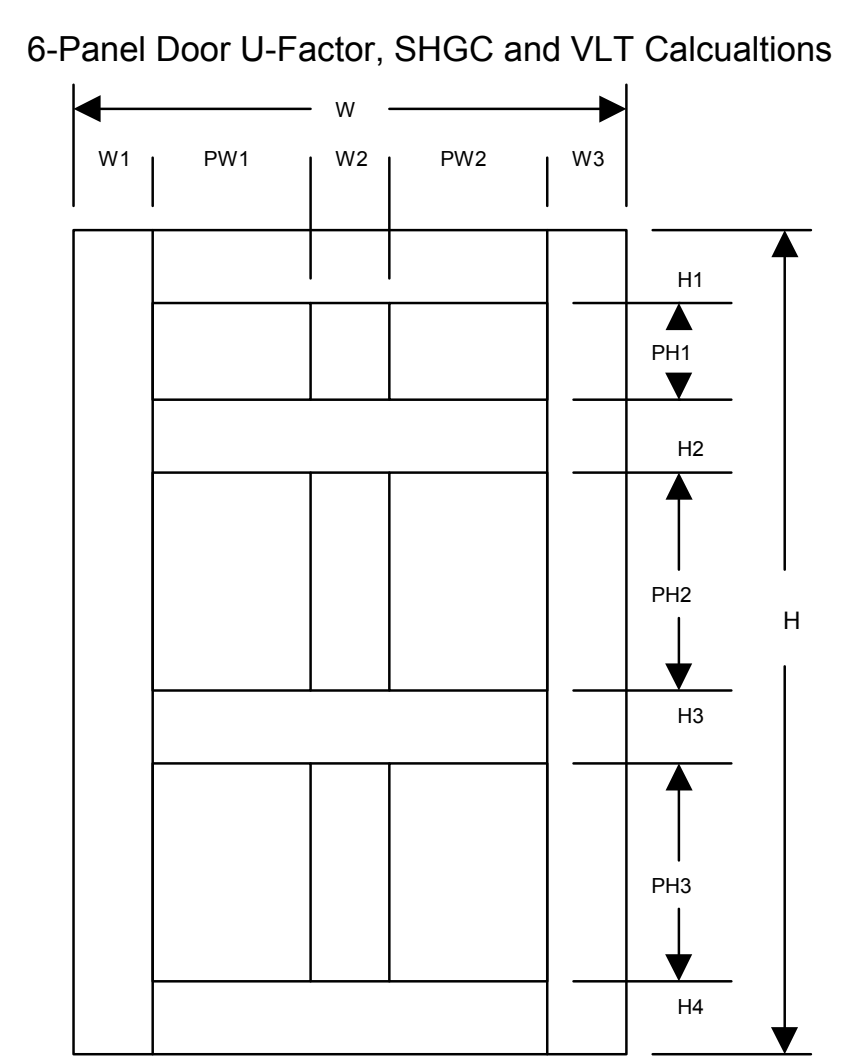

Width (W)

36

Height $(\mathrm{H})$

Hinge Stile Width (W1)

4.5

Center Stile Width (W2)

4.5

Lock Stile Width (W3)

4.5

Left Panel Width (PW1)

11.25

Right Panel Width (PW2)

11.25

Top Rail Height (H1)

4.5

Upper Middle Rail Height (H2)

4.5

Lower Middle Rail Height (H3)

7.5

Bottom Rail Height (H4)

9.25

Upper Panel Height (PH1)

8.25

Middle Panel Height (PH2)

Lower Panel Height (PH3)

Note: The Panel width and height dimensions represent the daylight opening for the panel.

Figure 9-53. Door Dimensions for area-weighting.

The spreadsheet has a data entry page for the overall door dimension data, a page for the WINDOW and THERM data, and a page for the Area Calculation data.

Note the only entries on the Area Calculation page in the spreadsheet are the door lite glass sizes. The door lite glass sizes are normally the standard values from NFRC 100 Section B (see NFRC 100 Section B, Section 5.0 ), but in the case of a stile and rail door the value will have to be determined based on the dimension of the stiles and rails. The glass sizes are determined by removing panels and intermediate stiles and rails according to Table 8-4. 
This door has the following glass lite sizes:

Table 9-26. Glass lite sizes for example door.

\begin{tabular}{|l|l|l|l|l|}
\cline { 2 - 5 } \multicolumn{1}{c|}{} & \multicolumn{4}{c|}{ Glass Sizes (inches) } \\
\hline & Width & Height & Glass Area & For Doors with \\
\hline $1 / 4$ Glass & 28.062 & 9.312 & 261.3 & 262 sq. In. or less \\
\hline $1 / 2$ Glass & 28.062 & 36.812 & 1089.1 & $262-1089$ sq. in. \\
\hline $3 / 4$ Glass & 28.062 & 54.562 & 1531.1 & $1089-1531$ sq. in. \\
\hline Full Glass & 28.062 & 67.312 & 1888.9 & 1531 sq. in. or more \\
\hline
\end{tabular}

Note that the glass size includes the total glass unit. The dimension from the door lite frame sight line to the bottom of the glass must be measured in the door lite model and entered into the spreadsheet to determine the correct area weighting.

Table 9-28 shows the overall product U-factor for Glazing Options 1 through 4.

Table 9-27. Total Product U-factors

\begin{tabular}{|c|c|c|c|c|c|c|}
\hline & \multirow[b]{2}{*}{ Glazing Options } & \multicolumn{5}{|c|}{$\begin{array}{l}\text { Residential AA Total Product U-factor } \\
\left(\text { Btu/hr- } \mathrm{ft}^{2}{ }^{\circ} \mathrm{F}\right)\end{array}$} \\
\hline & & $\begin{array}{l}\text { No } \\
\text { Glass }\end{array}$ & $1 / 4$ & $\begin{array}{l}1 / 2 \\
\text { Glass }\end{array}$ & $\begin{array}{l}3 / 4 \\
\text { Glass }\end{array}$ & $\begin{array}{l}\text { Full } \\
\text { Glass }\end{array}$ \\
\hline 1 & Clear $\left(0.129^{\prime \prime}\right)$, Air, Clear $\left(0.129^{\prime \prime}\right)$ & 0.43 & 0.44 & 0.47 & 0.49 & 0.51 \\
\hline 2 & Clear $\left(0.129^{\prime \prime}\right)$, Argon $(90 \%)$, Clear $\left(0.129^{\prime \prime}\right)$ & 0.43 & 0.44 & 0.46 & 0.47 & 0.49 \\
\hline 3 & Low-e $\left(0.129^{\prime \prime}\right)$, Air, Clear $\left(0.129^{\prime \prime}\right)$ & 0.43 & 0.43 & 0.43 & 0.43 & 0.43 \\
\hline 4 & Low-e $\left(0.129^{\prime \prime}\right)$, Argon $(90 \%)$, Clear $\left(0.129^{\prime \prime}\right)$ & 0.43 & 0.43 & 0.41 & 0.39 & 0.39 \\
\hline
\end{tabular}

\subsubsection{Total Product Solar Heat Gain Coefficient (SHGC) and Visible Light Transmittance (VLT)}

The methodology for determining the Solar Heat Gain Coefficient and Visible Transmittance for the product is outlined in NFRC 200 and NFRC 300. WINDOW does not calculate these values for entry doors. These calculations have been included in the 6-Panel Door spreadsheet. Table 9-29 shows the SHGC values for the four glass options with no glass, $1 / 4$ glass, $1 / 2$ glass, $3 / 4$ glass and full glass. Table 9-30 shows the VLT values for the four glass options with no glass, $1 / 4$ glass, $1 / 2$ glass, $3 / 4$ glass and full glass.

Table 9-28. Total Product SHGC

\begin{tabular}{|l|l|c|c|c|c|c|}
\hline \multirow{2}{*}{} & \multicolumn{1}{|c|}{ Glazing Options } & \multicolumn{4}{c|}{ Residential AA Total Product SHGC } \\
\cline { 3 - 7 } & \multicolumn{1}{|c|}{ No Glass } & $1 / 4$ Glass & $1 / 2$ Glass & $3 / 4$ Glass & $\begin{array}{c}\text { Full } \\
\text { Glass }\end{array}$ \\
\hline 1 & Clear $\left(0.129^{\prime \prime}\right)$, Air, Clear $\left(0.129^{\prime \prime}\right)$ & 0.07 & 0.12 & 0.28 & 0.39 & 0.46 \\
\hline 2 & Clear $\left(0.129^{\prime \prime}\right)$, Argon $(90 \%)$, Clear $\left(0.129^{\prime \prime}\right)$ & 0.07 & 0.12 & 0.28 & 0.39 & 0.46 \\
\hline 3 & Low-e $\left(0.129^{\prime \prime}\right)$, Air, Clear $\left(0.129^{\prime \prime}\right)$ & 0.07 & 0.10 & 0.17 & 0.22 & 0.26 \\
\hline 4 & $\begin{array}{l}\text { Low-e }\left(0.129^{\prime \prime}\right), \text { Argon }(90 \%), \text { Clear } \\
\left(0.129^{\prime \prime}\right)\end{array}$ & 0.07 & 0.10 & 0.17 & 0.22 & 0.25 \\
\hline
\end{tabular}


Table 9-29. Total Product Visible Light Transmittance (VT)

\begin{tabular}{|l|c|c|c|c|c|c|}
\hline \multirow{2}{*}{} & Glazing Options & \multicolumn{5}{c|}{ Residential AA Total Product VT } \\
\cline { 3 - 7 } & & $\begin{array}{l}\text { No } \\
\text { Glass }\end{array}$ & $1 / 4$ Glass & $\begin{array}{l}1 / 2 \\
\text { Glass }\end{array}$ & $\begin{array}{l}\text { 3/4 } \\
\text { Glass }\end{array}$ & $\begin{array}{l}\text { Full } \\
\text { Glass }\end{array}$ \\
\hline 1 & Clear $\left(0.129^{\prime \prime}\right)$, Air, Clear $\left(0.129^{\prime \prime}\right)$ & 0.00 & 0.06 & 0.25 & 0.38 & 0.47 \\
\hline 2 & Clear $\left(0.129^{\prime \prime}\right)$, Argon $(90 \%)$, Clear $\left(0.129^{\prime \prime}\right)$ & 0.00 & 0.06 & 0.25 & 0.38 & 0.47 \\
\hline 3 & Low-e $\left(0.129^{\prime \prime}\right)$, Air, Clear $\left(0.129^{\prime \prime}\right)$ & 0.00 & 0.05 & 0.22 & 0.33 & 0.41 \\
\hline 4 & Low-e $\left(0.129^{\prime \prime}\right)$, Argon $(90 \%)$, Clear $\left(0.129^{\prime \prime}\right)$ & 0.00 & 0.05 & 0.22 & 0.33 & 0.41 \\
\hline
\end{tabular}

Finally, the SHGC and VT Specialty Products table values must be determined. These are the SHGC $\mathrm{SHGC}_{1}$, $\mathrm{VT}_{0}$, and $\mathrm{VT}_{1}$ values. These values will have to be determined for the no glass, $1 / 4$ glass, $1 / 2$ glass, $3 / 4$ glass and full glass options. If this door included any dividers the Specialty Products Table values would also have to be determined for dividers $<1$ " and dividers $\geq 1$ ". The 6-Panel Door spreadsheet determines the Specialty Products table values for the door without dividers. Table 9-31 shows these values. The specialty products table for this door has been included in the 6-Panel Door spreadsheet, but not printed here.

Table 9-30. Specialty Products Table Values

\begin{tabular}{|l|l|l|l|l|}
\hline & $\mathbf{S H G C}_{\mathbf{0}}$ & $\mathbf{S H G C}_{\mathbf{1}}$ & $\mathbf{V T}_{\mathbf{0}}$ & $\mathbf{V T}_{\mathbf{1}}$ \\
\hline No Glass & 0.073 & 0.073 & 0.000 & 0.000 \\
\hline 1/4 Glass & 0.068 & 0.140 & 0.000 & 0.072 \\
\hline 1/2 Glass & 0.050 & 0.360 & 0.000 & 0.310 \\
\hline 3/4 Glass & 0.038 & 0.502 & 0.000 & 0.464 \\
\hline Full Glass & 0.030 & 0.605 & 0.000 & 0.575 \\
\hline
\end{tabular}

\subsubsection{Wood Stile and Rail Door Drawings.}

Note that some of the drawings provided (indicated with an asterisk on the following pages) are patented drawings and shall not be used by anyone without the written consent from NFRC. 


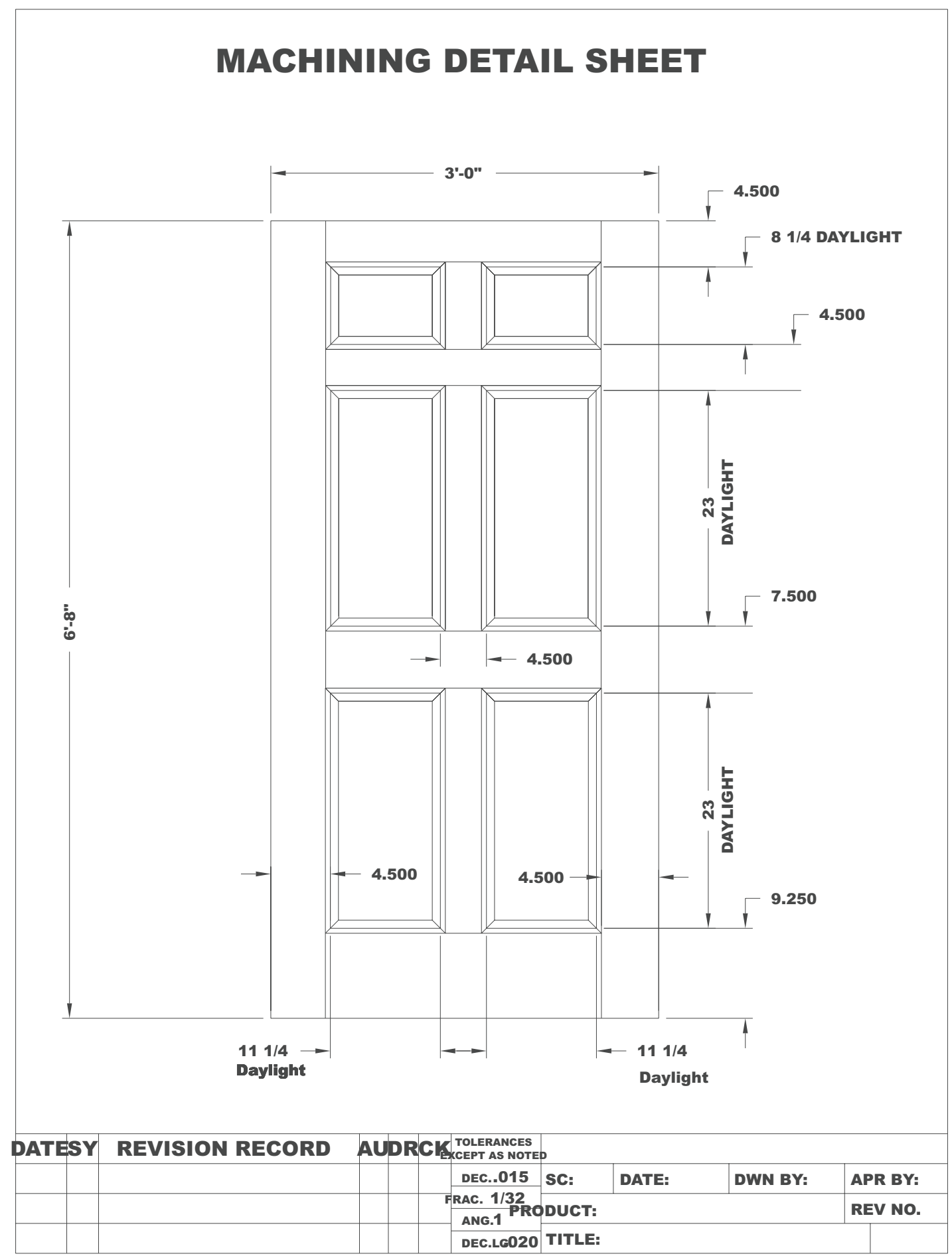

Figure 9-54. 6-Panel Panel Layout.dwg 


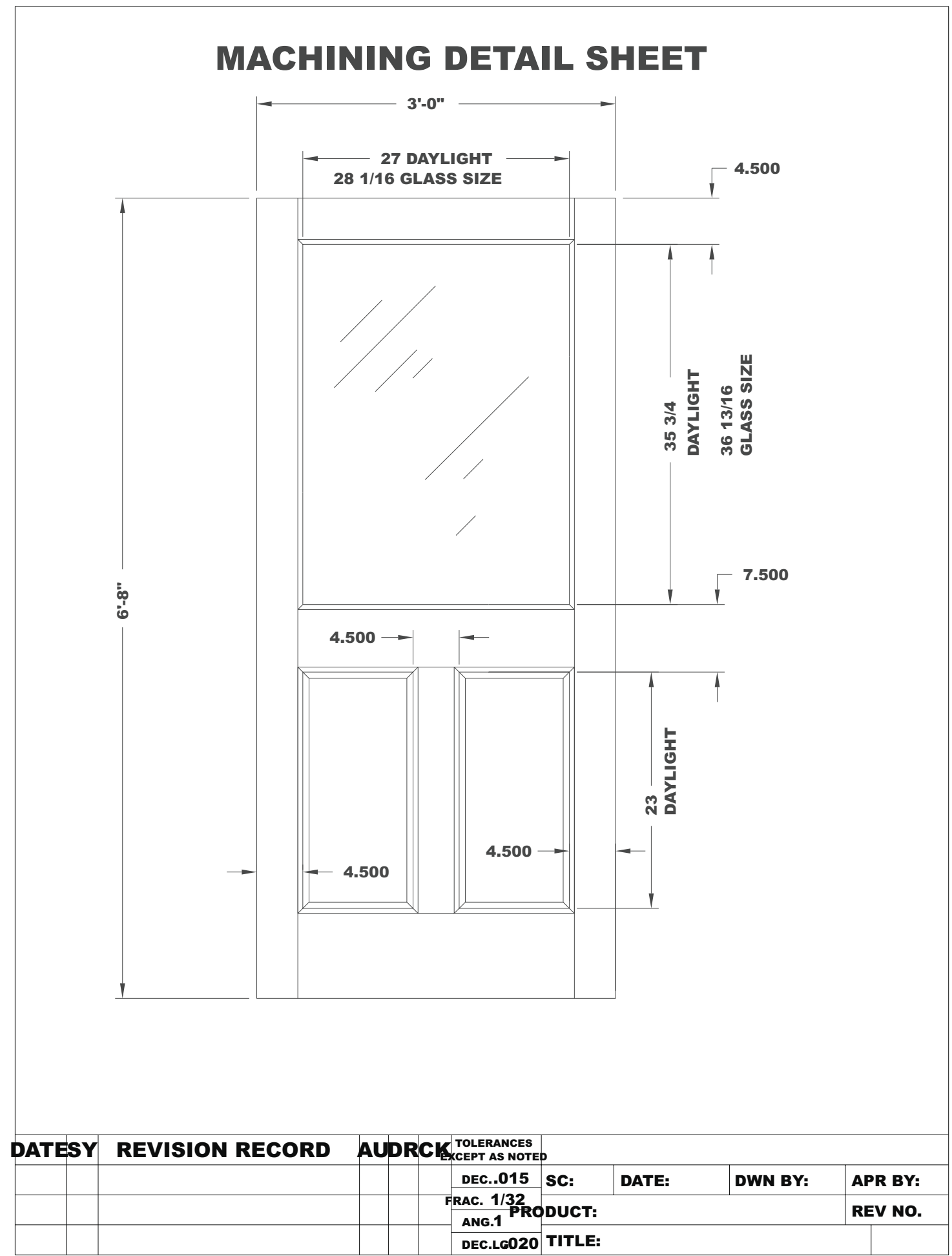

Figure 9-55. Half Panel with Glass Layout.dwg 


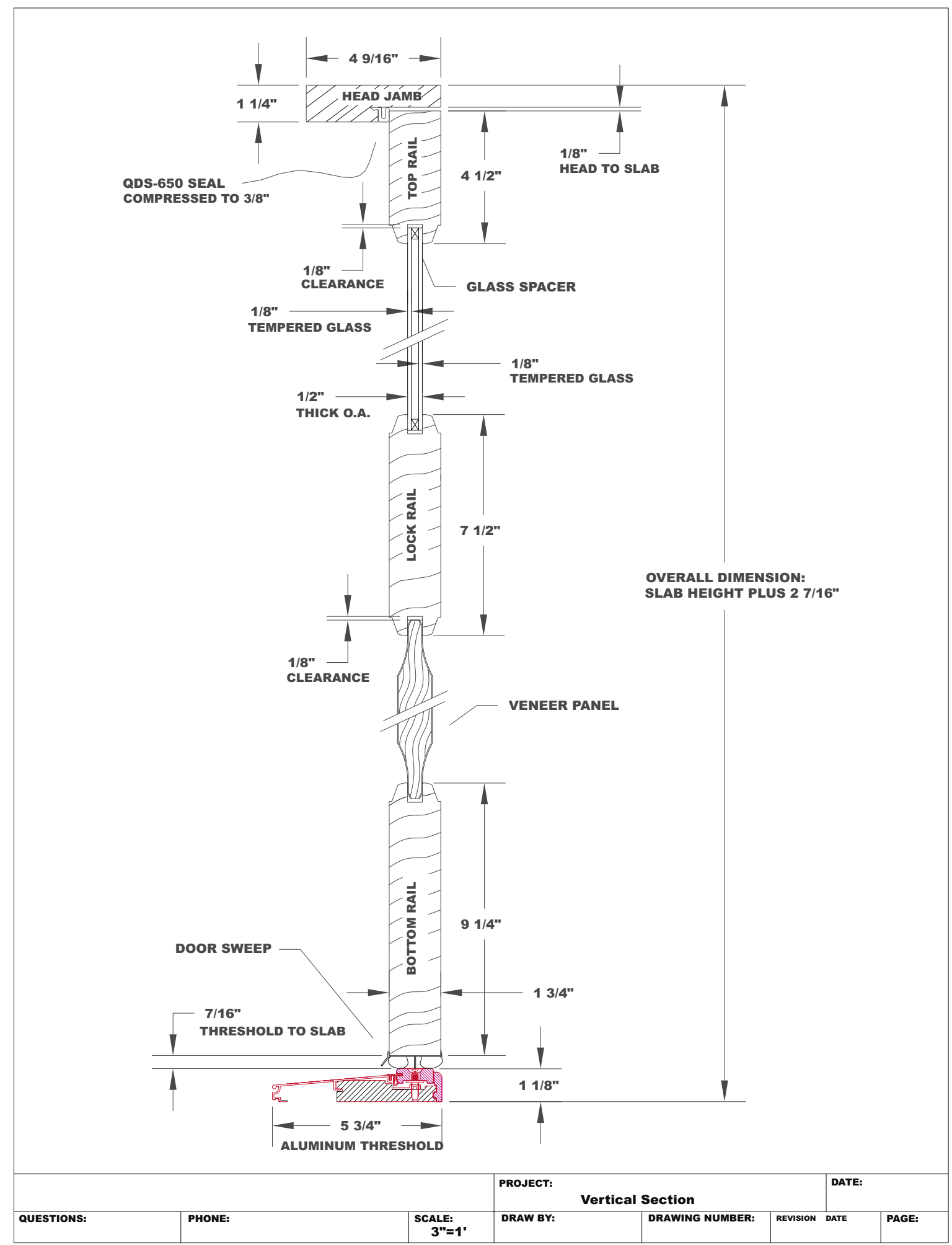

Figure 9-56. Half Panel with Glass Vert Sec.dwg 


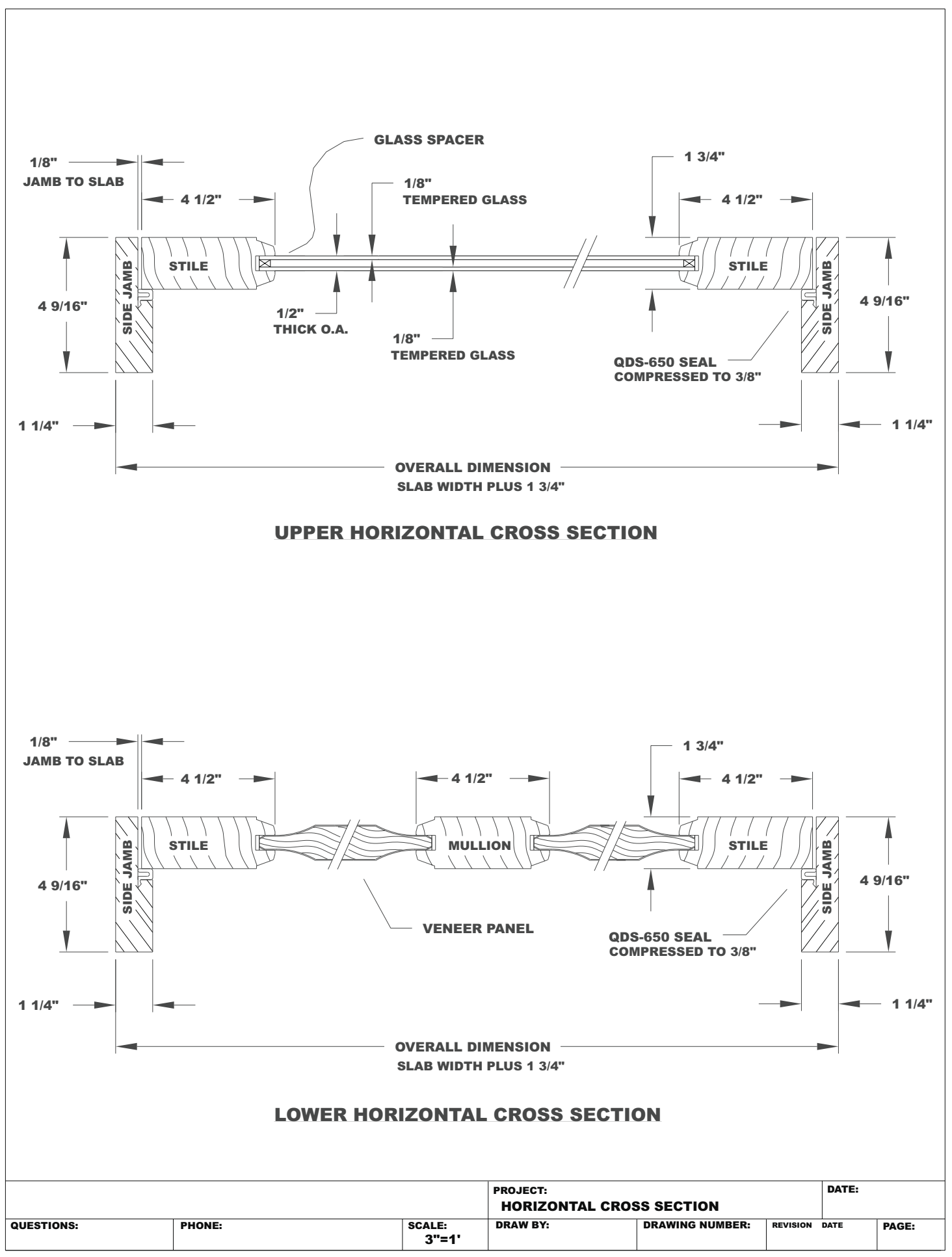

Figure 9-57. Half Panel with Glass Horz Sec.dwg 


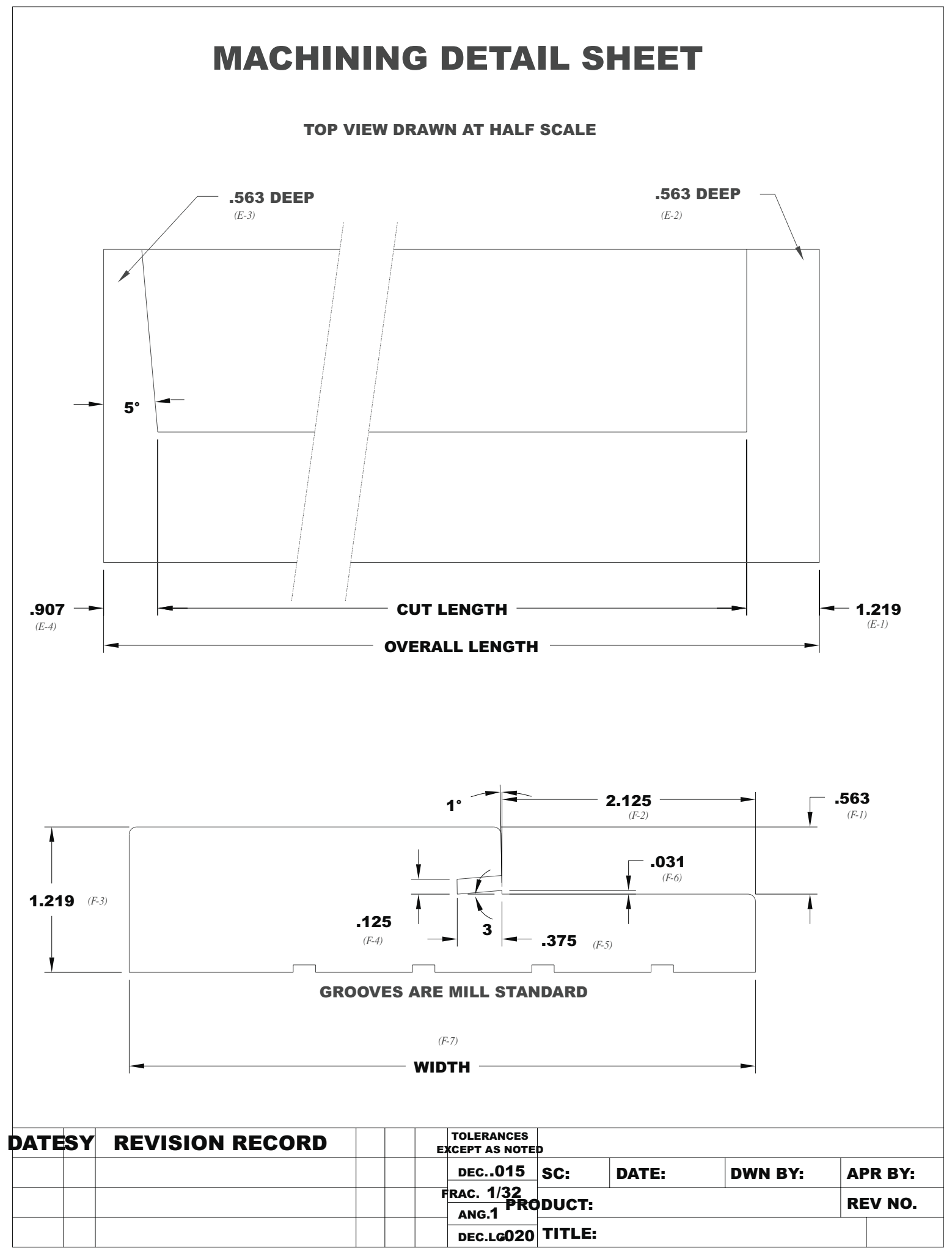

Figure 9-58. 6-Panel Jamb.dwg 


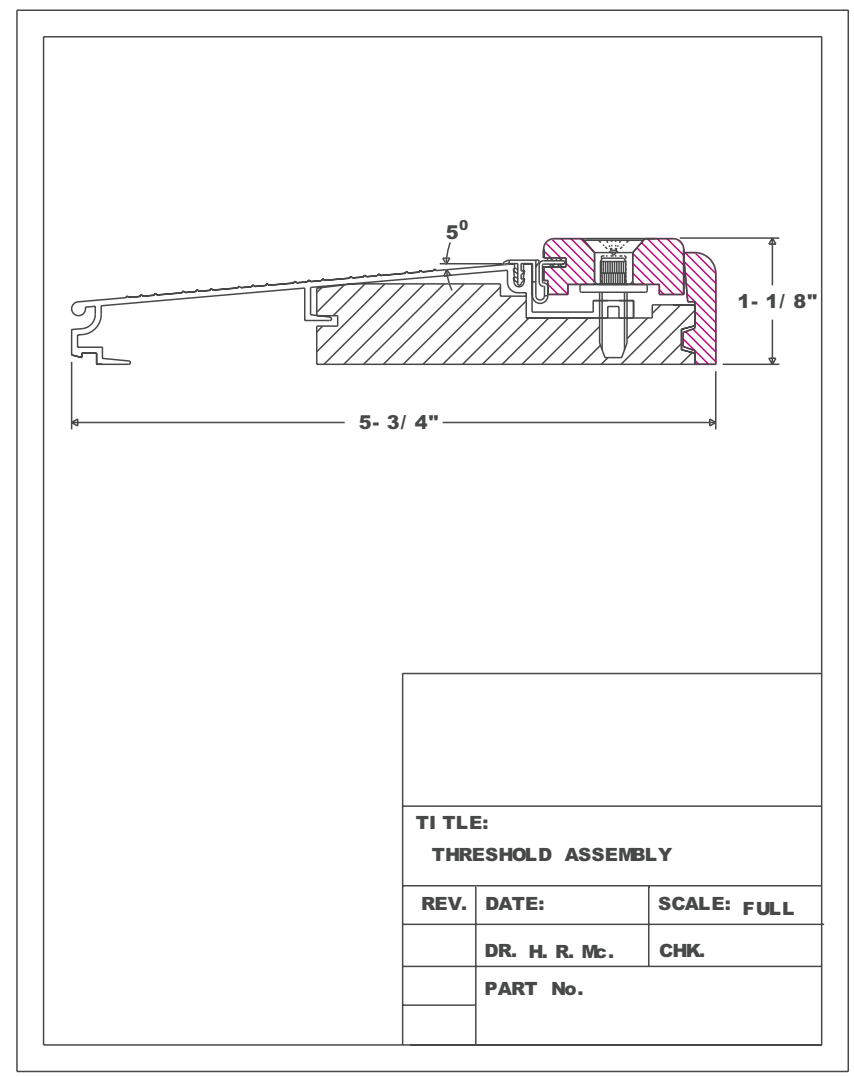

Figure 9-59. 6-Panel Sill.dwg (* PATENTED DRAWING)

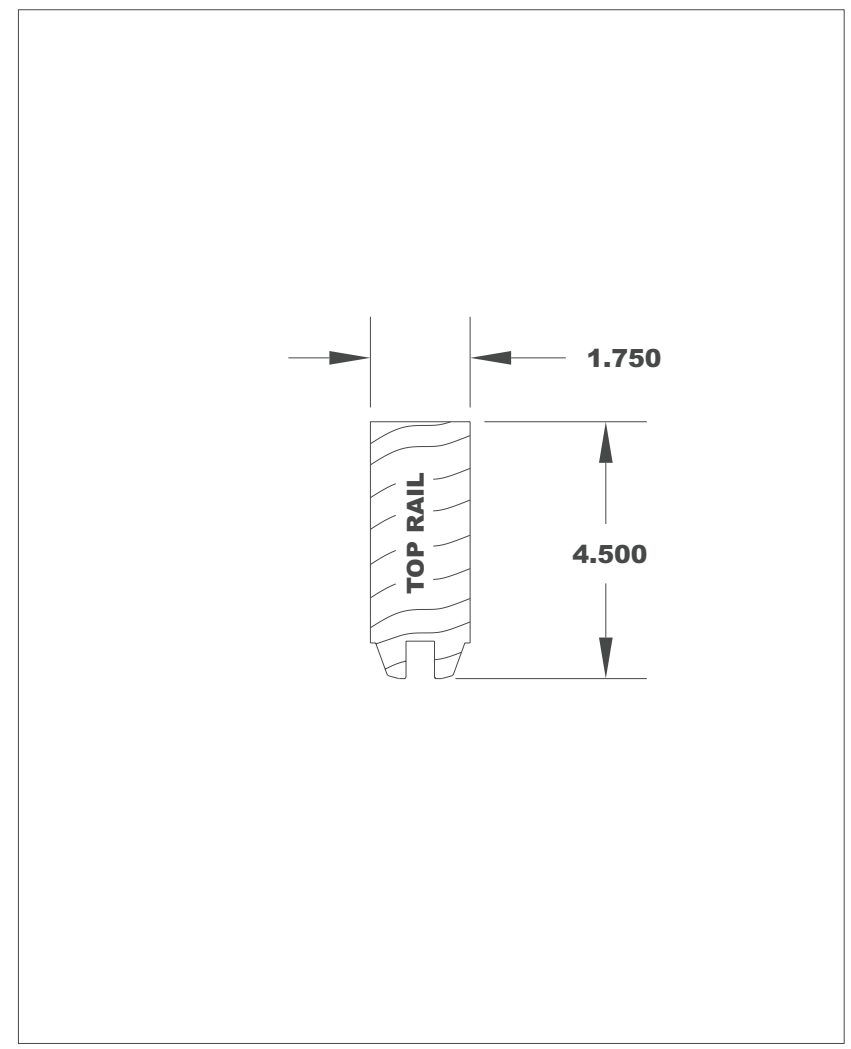

Figure 9-60. 6-Panel Top Rail.dwg 


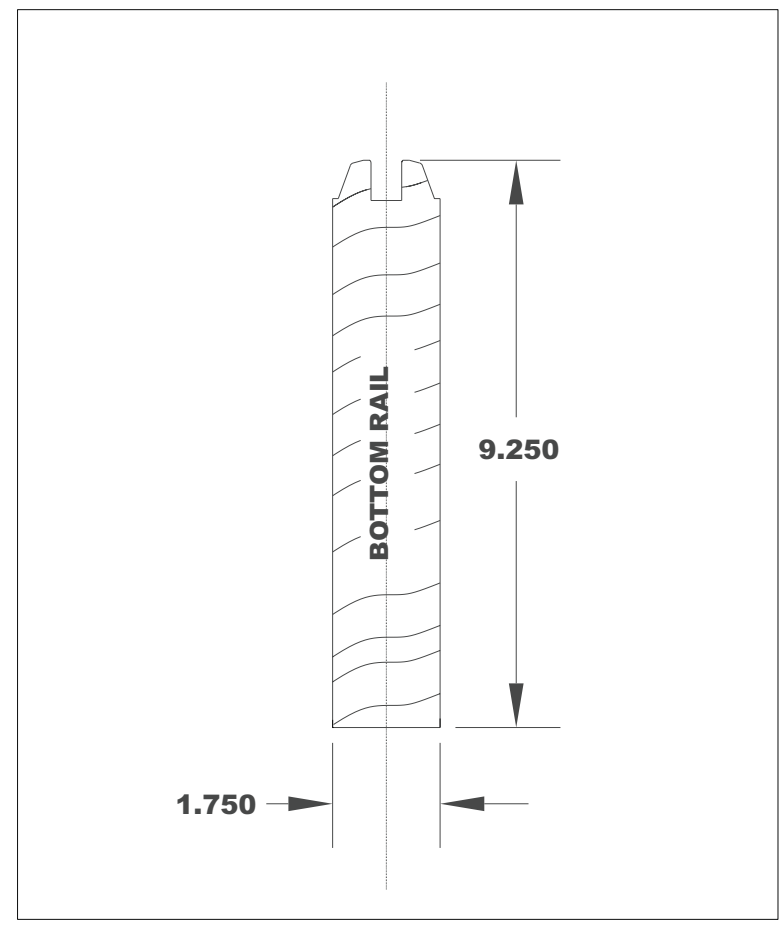

Figure 9-61. 6-Panel Bottom Rail.dwg

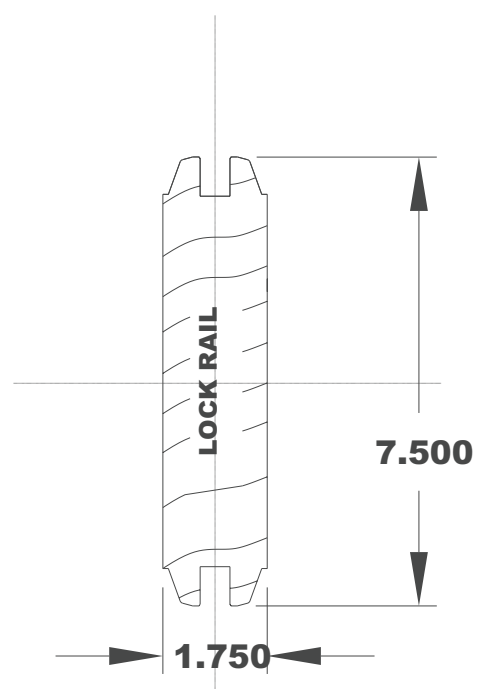

Figure 9-62. 6-Panel Lock Rail.dwg 


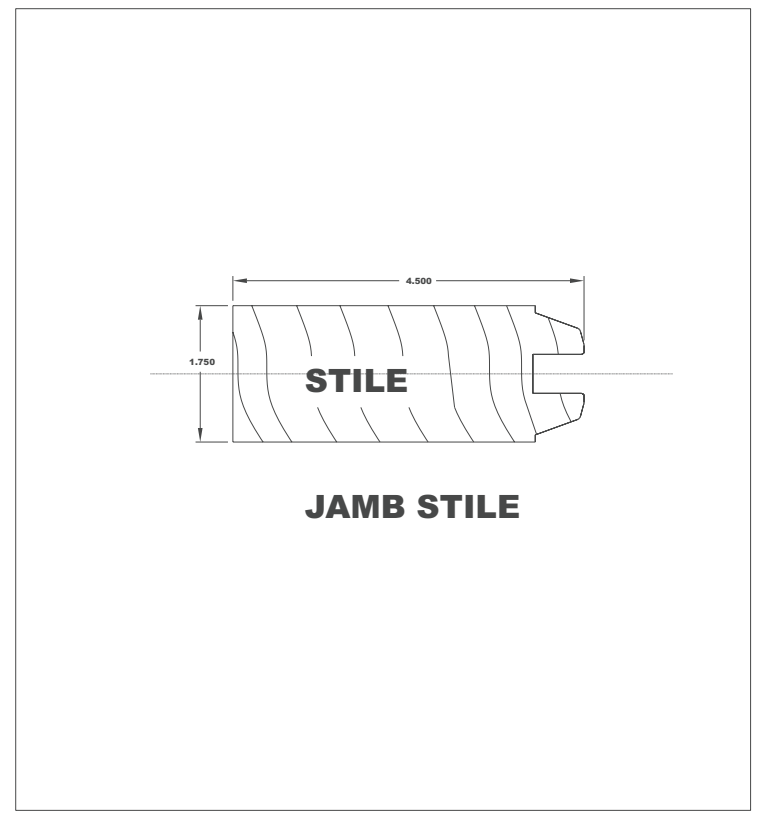

Figure 9-63. 6-Panel Stile.dwg

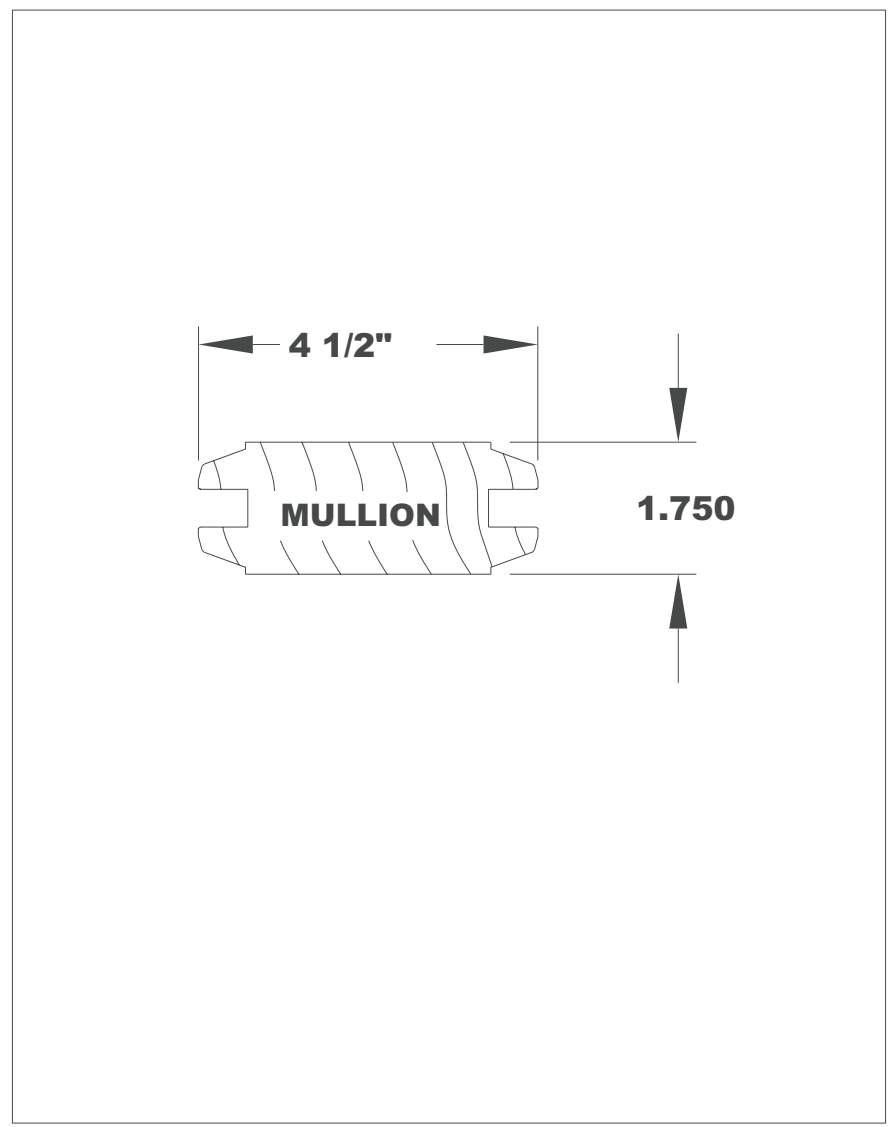

Figure 9-64. 6-Panel Mullion.dwg 


\section{MACHINING DETAIL SHEET}

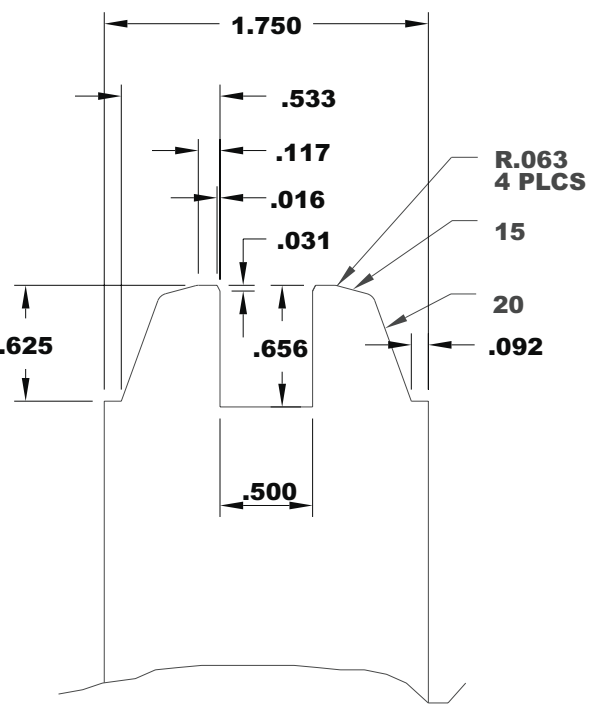

\begin{tabular}{|c|c|c|c|c|c|c|}
\hline DATESY & REVISION RECORD & AUDRCK & & & & \\
\hline & & DEC..015 & sc: & DATE: & DWN BY: & APR BY: \\
\hline & & $\begin{array}{l}\text { FAC. } 1 / 32 \\
\text { ANG.1 PR }\end{array}$ & PDUCT: & Milling & & REV NO. \\
\hline & & DEC.LG02 & TITLE: & & & \\
\hline
\end{tabular}

Figure 9-65. 6-Panel Milling Detail.dwg 


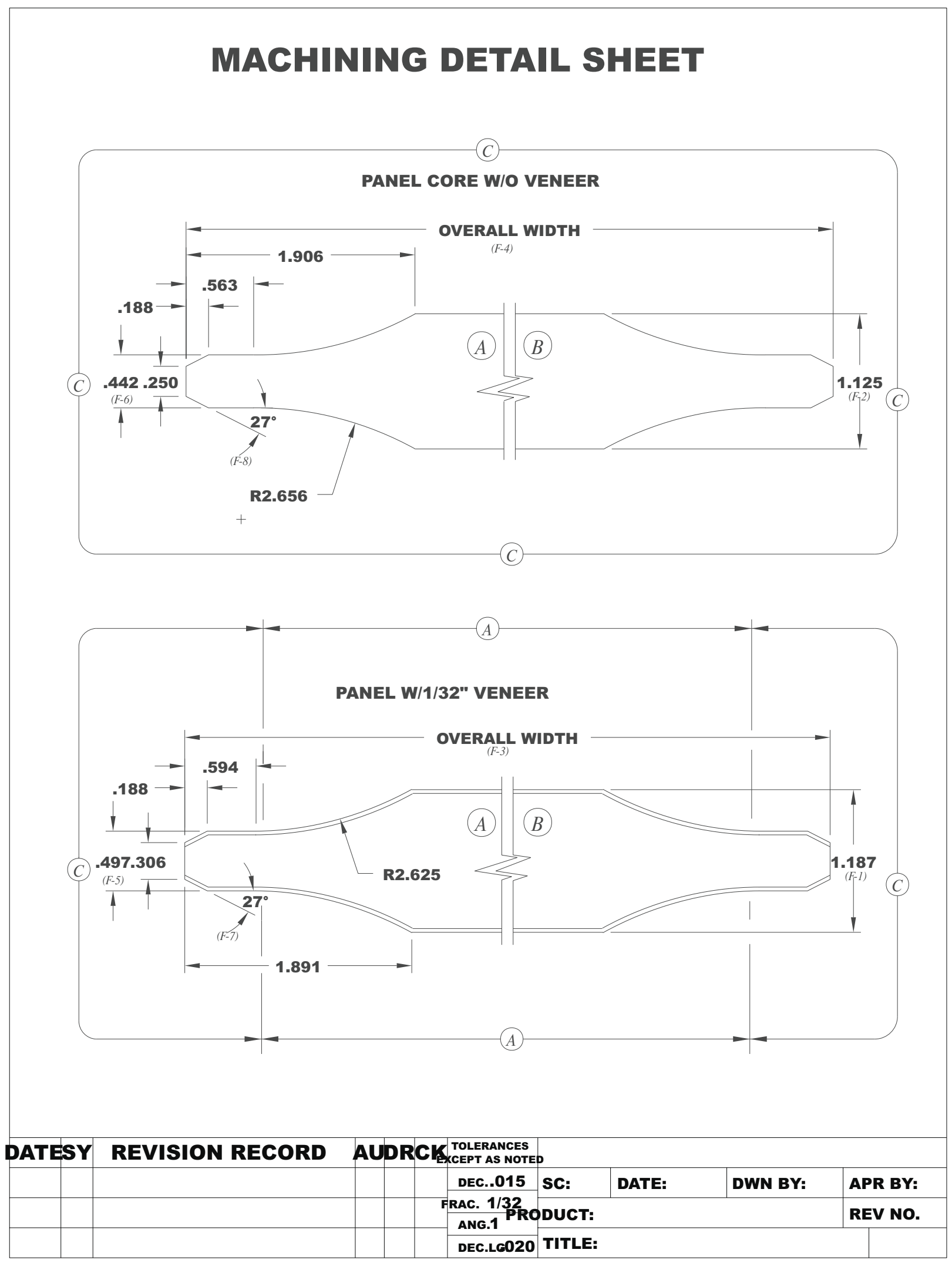

Figure 9-66. 6-Panel Panel.dwg 


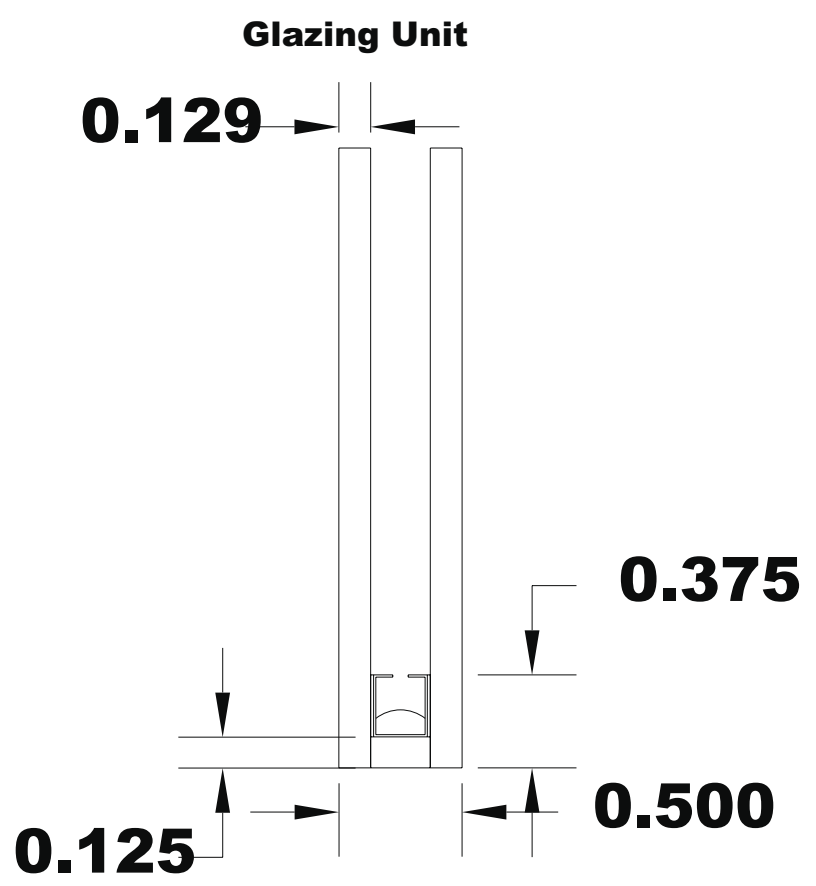

Figure 9-67. 6-Panel Glazing.dwg

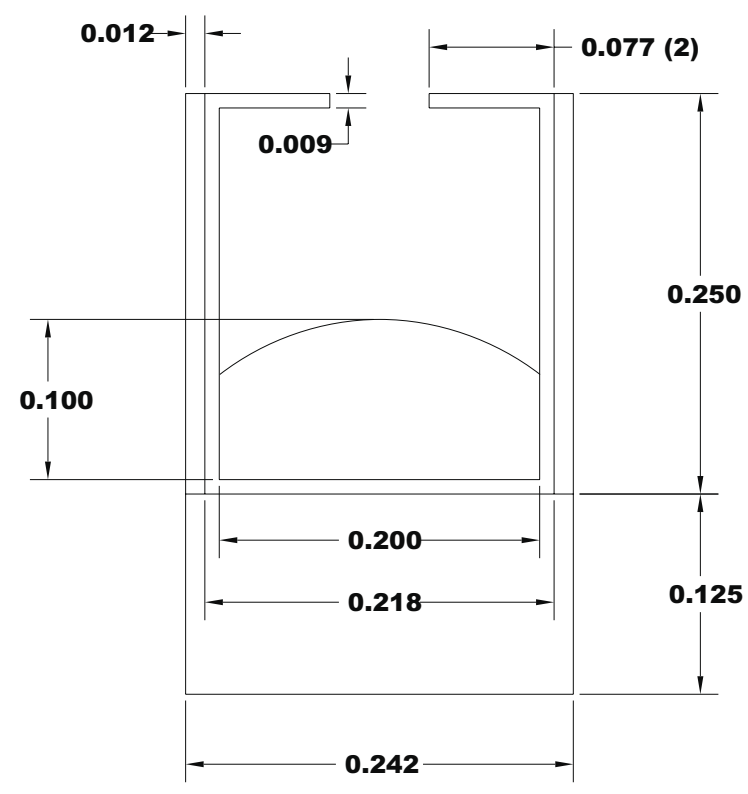

\section{Intercept Spacer}

Figure 9-68. 6-Panel Spacer.dwg 


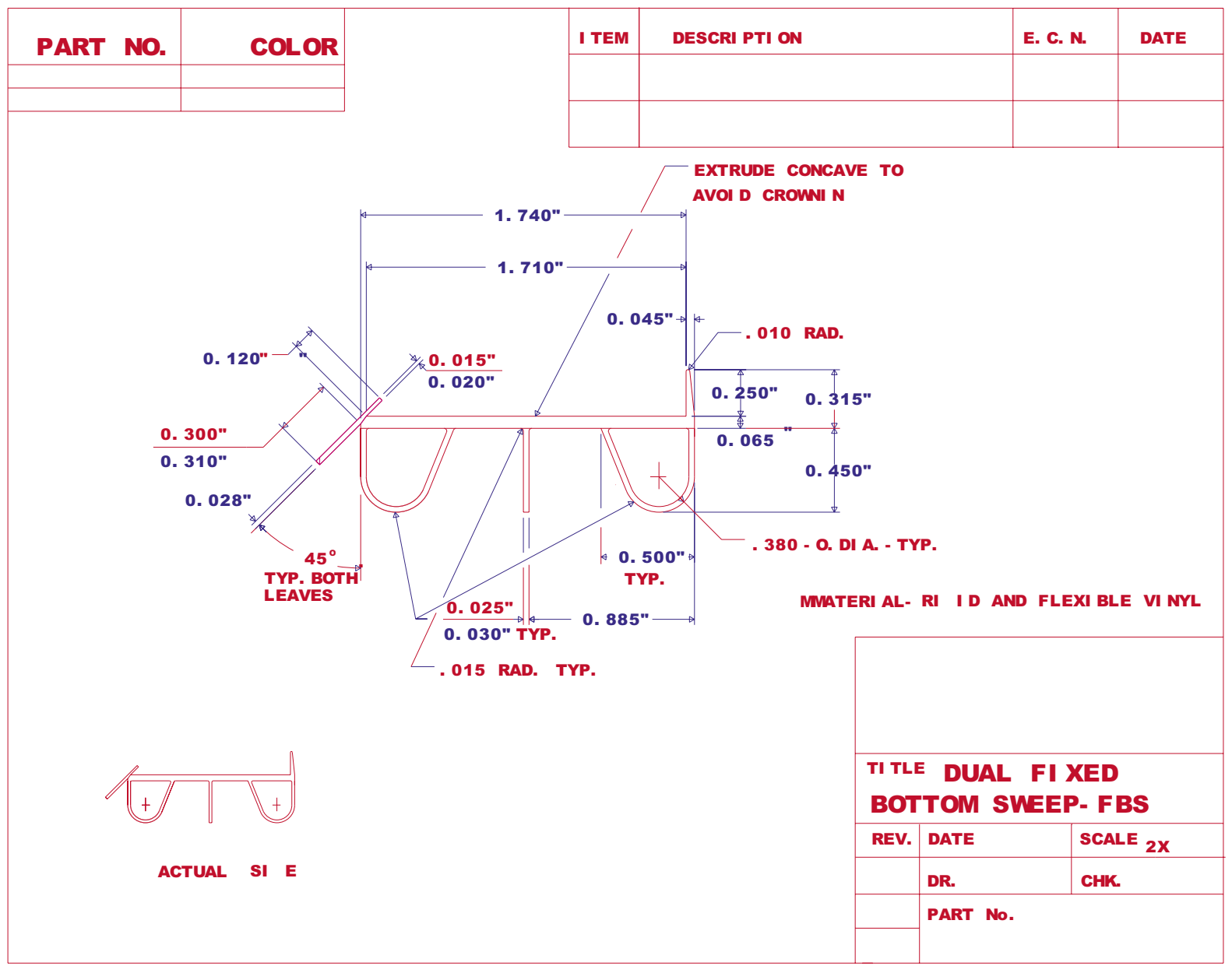

Figure 9-69. 6-Panel Bottom Sweep.dwg ( ${ }^{*}$ PATENTED DRAWING) 


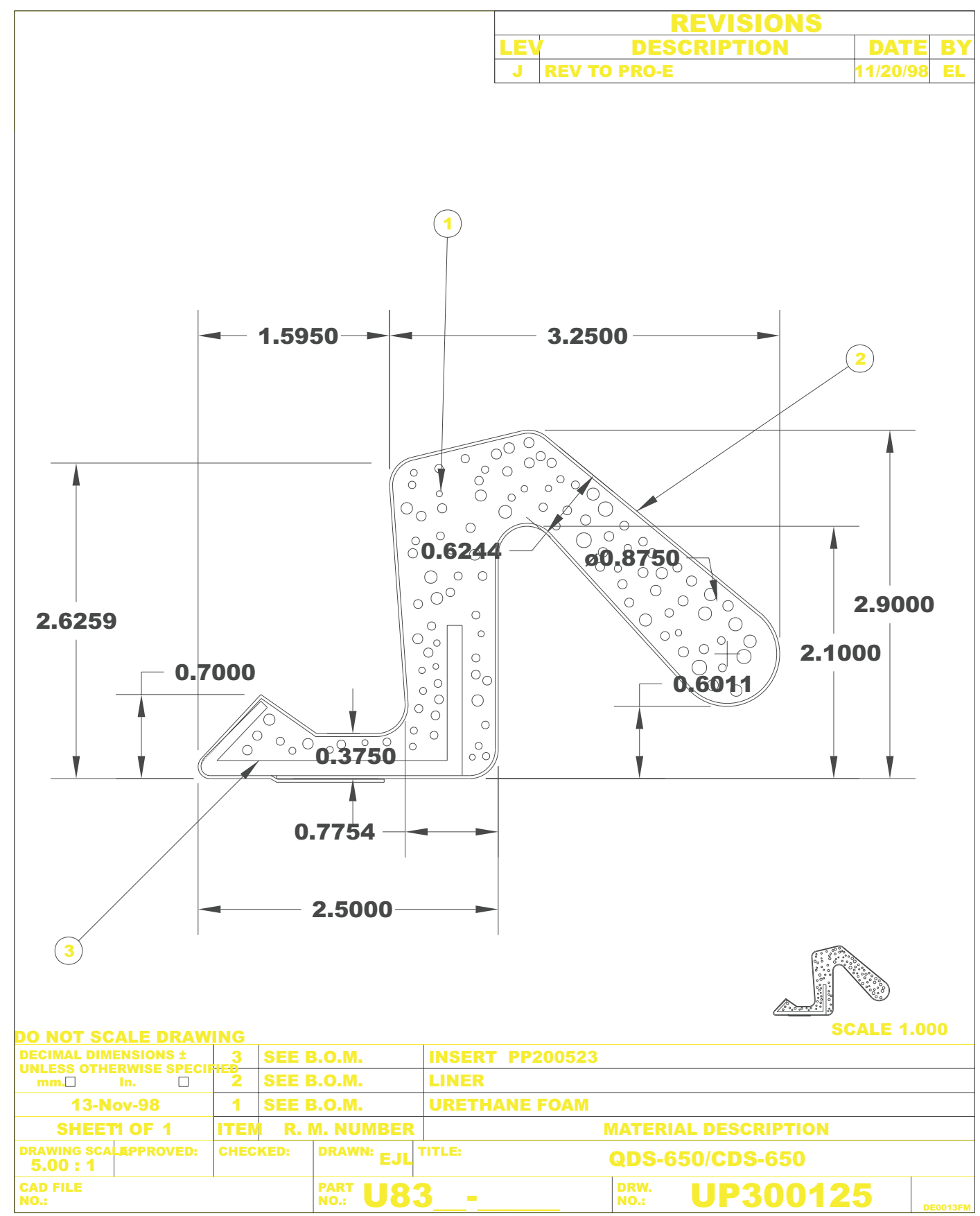

Figure 9-70. 6-Panel Weather Strip.dwg ( ${ }^{*}$ PATENTED DRAWING) 


\subsection{Drawing Tips}

The THERM User's Manual describes in detail how to draw cross-sections in THERM. This section of the NFRC Simualation Training Manual presents some additional key points which may help draw the models.

\subsubsection{Drawing Methods}

There are a few different ways that cross sections can be created in THERM. They are:

- Draw the cross sections from dimensioned drawings

- Draw the cross sections by tracing a DXF file used as an underlay

- Use the autoconvert feature to convert a DXF file into a THERM drawing

Each method has it's advantages, and the method you choose will depend primarily on the type of information you have about the product to be analyzed. The THERM User's Manual has detailed instructions about each of these methods. The following sections contain specific drawing tips that are particularly useful when drawing fenestration cross sections.

\subsubsection{DXF Files}

THERM can interpret the polygons in DXF files, if the files are created correctly, and thus save much drawing time. There is extensive discussion of DXF files in the THERM User's Manual (Section 5); the following add to, reiterate, or clarify some of the information in that manual:

- DXF files are imported using the File/Underlay command (See THERM User's Manual, Section 5.2)

- The Arc to Polygon Conversion command under Options/Preferences/Drawing options determines how many straight line segments will be used to approximate curves in the DXF files. The value can be set from 1 to 45 degrees. The program is conservative in its determination of how many segments are needed to represent the curve. For example if Arc to Polygon Conversion is set to 45 degrees, and the angle is $\geq 90$ degrees, the curve will be divided into a minimum of 3 segments. The circles. $d x f$ file on the $\mathrm{CD}$ can be used to discover how changing the arc to polygon value changes the way the DXF file is imported. (See THERM User's Manual, Section 5.3.2)

- The horizontal and vertical scaling factors are only used with DXF files that are drawn at a scale other than 1:1. (See THERM User's Manual, Section 5, pg. 5-3)

- The choice of units is a scaling factor for DXF files that were originally drawn in SI units; by default THERM assumes that DXF files are drawn in IP units. This unit setting is different than the overall units setting for THERM. (See THERM User's Manual, Section 5, pg. 5-3)

- Note you will need to re-import the DXF file for changes in the arc conversion or scaling settings to take effect. 
- By default, the THERM origin and DXF origin are the same. You can use the origin setting to change this if needed. (See THERM User's Manual, Section 5, pg. 5-4)

- Only one DXF file at a time can be used as an underlay. In a file that already has an underlay, you can pick a new underlay, but it will supercede the previous one.

- In THERM, the base unit is millimeters when drawing in SI units, and inches when drawing in IP units.

\subsubsection{Representing Curves with Line Segments}

THERM can draw straight lines at any angle but it currently must approximate curves with a series of straight line segments. The guidelines for geometric representation of curved surfaces are still being discussed by the ASHRAE committee charged with coming up with a standard for calculating heat transfer in windows (SPC 142P). Some parameters to consider in evaluating the accuracy of the geometric representation are path length, displacement, and area.

For NFRC simulations, the model must be within $5 \mathrm{~mm}(0.2 \mathrm{in})$ of the actual product. In most cases, using either 30 or 45 degrees per side for the Arc to Polygon Conversion parameter will create a drawing that is within 0.2 inches $(5 \mathrm{~mm}$ ) of the product and eliminate unnecessary complexity in the drawing. A drawing with many points very close together may result in problems when creating the boundary conditions or during the simulation when the mesh is being generated by THERM.

\section{Interpreting Arcs in DXF Files}

The Arc to Polygon conversion setting in the Options/Preferences menu option, Drawing Options tab controls how many line segments THERM will use to translate an arc in a DXF file. THERM treats every curve the same regardless of radius. The circles.dxf file, which contains many arcs, can be used to illustrate how using the Arc to Polygon conversion setting when autoconverting a DXF file can affect the resulting THERM polygons. If you decide to model curves in detail setting the Snap to Angle value to the value chosen to divide up the arc will make drawing easier. The lowhoriz.dxf on the accompanying CD can also be used to learn how THERM interprets arcs when autoconverting a DXF file.

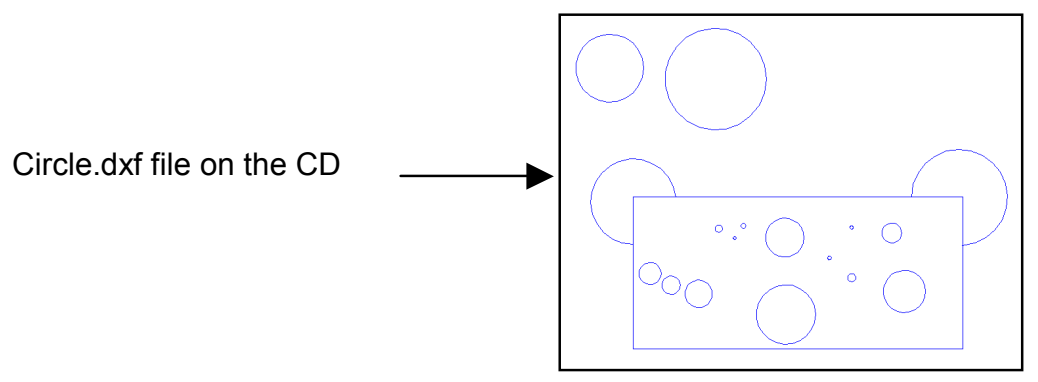

Figure 10-1. Circle.dxf sample file. 


\section{DXF Filter Settings}

The DXF Filter settings in the DXF Underlay also control how the $\mathrm{dxf}$ file is translated by THERM. This can be seen using the circles.dxf file.

1:

When the Arc to Polygon conversion setting is 45 degrees per side, the autoconverted polygon for circles.dxf looks like this. Setting this value to 30 or 45 is recommended because it results in sufficient detail for proper modeling and eliminates unnecessary complexity which may result in problems with boundary conditions or the mesh generation.
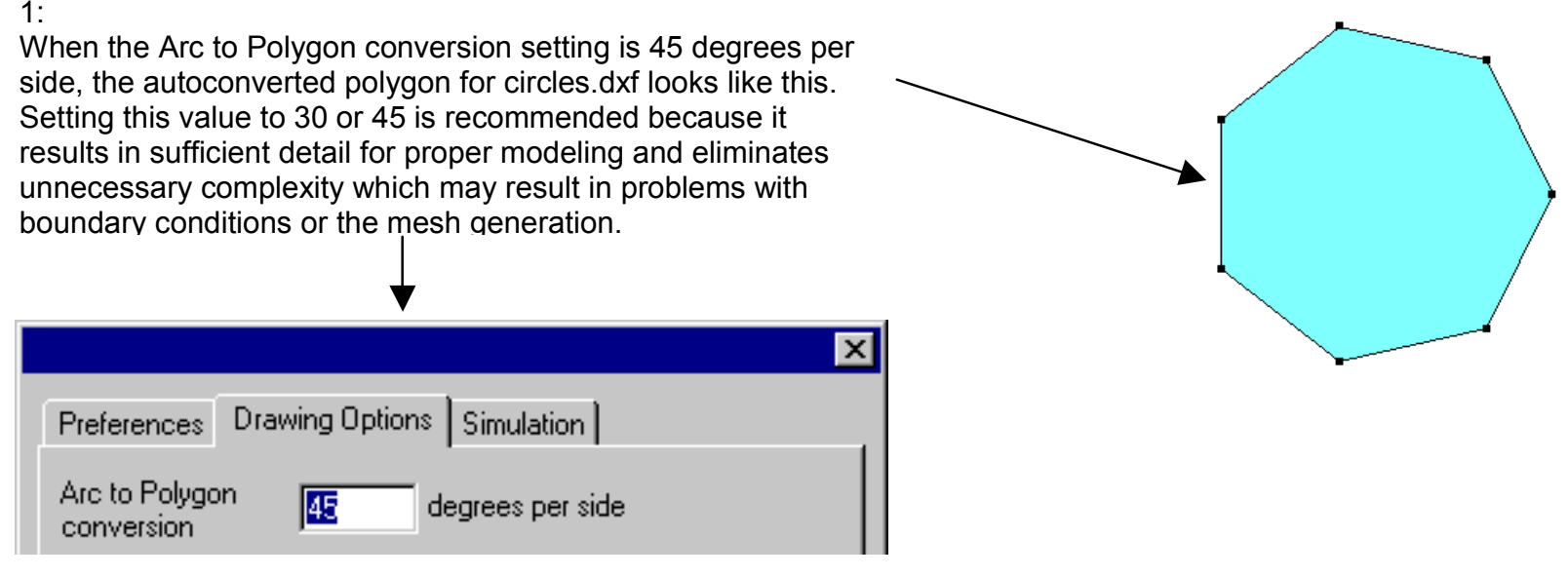

2:

When the Arc to Polygon conversion setting is 15 degrees per side, the autoconverted polygon for circles.dxf looks like this. There are many more line segments representing the arc. This level of detail is usually unnecessary and may lead to problems when generating boundary conditions or during simulation meshing.

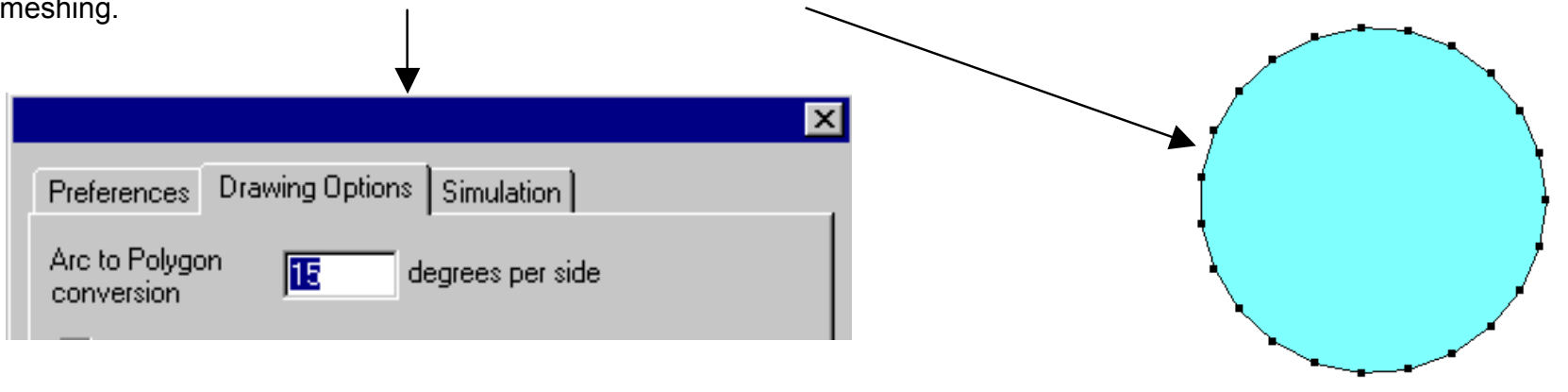

Figure 10-2. Arc to Polygon Conversion in THERM. 


\subsubsection{DXF Files -- AutoConvert}

- For Autoconvert to work the DXF file needs to be created with closed polylines and curves. A polyline is a shape created in a CAD program made up of joined line segments and curves. (See THERM User's Manual, Section 5.2.3, pg. 5-6)

- Autoconvert may create overlapping polygons if polylines are defined multiple times in the CAD program and if the polylines in the CAD files overlap. Use the View/Show Voids and Overlaps (See THERM User's Manual, Section 5.3.5, pg 5-11) to check if this has happened. A single overlapping region will show as an overlap but if yet another region exists it will look like there is no overlap. A further check can be made by deleting (or moving) one of the polygons. If there is nothing underneath it the polygon can be restored using the Edit/Undo feature.

- Extrusions are often drawn using polylines. The external profile is one polyline and each of the cavities is a separate polyline. (See discussion about "Drawing Extrusions from DXF Files", and look at the auto. $d x f$ file on the CD.)

- Import the DXF file auto. $d x f$ (from the CD) using the Autoconvert feature. Check for overlapping polygons. Delete any overlapping polygons. Use the Undo feature to restore any inadvertently deleted polygons.

- Import auto. $d x f$ (from the CD) with only polylines checked using the DXF filter. The rounded corners of the extrusion are chopped off, which greatly simplifies the model. 

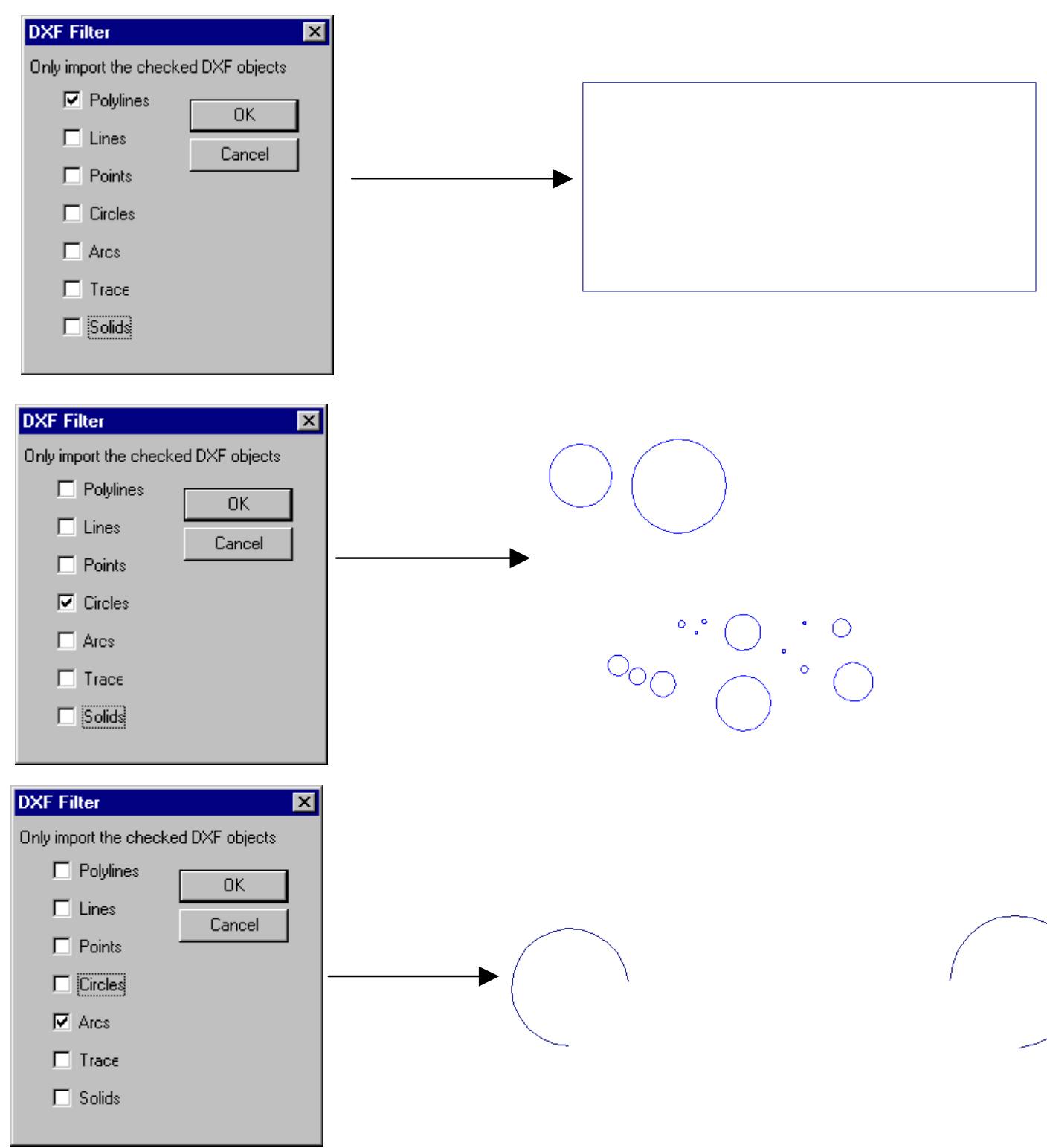

\section{]}

Only import the checked DXF objects

\begin{tabular}{|c|c|}
\hline Г Polylines & OK \\
\hline$\Gamma$ Lines & Cancel \\
\hline Г Points & \\
\hline ГCircles & \\
\hline V Arcs & \\
\hline Г Trace & \\
\hline$\Gamma$ Solids & \\
\hline
\end{tabular}

Figure 10-3. DXF Filter settings. 


\subsubsection{Sticky Distance and Snapping}

- Drawing in THERM is facilitated by the fact that the cursor will jump to a DXF file vertex, polygon vertex or polygon line, if the cursor is within the "sticky distance" from those objects.

- The " sticky distance" is equal to one half of one side of the drawing cross-hair. This is equal to $3 \mathrm{~mm}$ if the drawing is viewed at 100\%. (See THERM User's Manual, Section 5.3.1, pg. 5-7)

- When zooming in on the drawing, the sticky distance is still equal to one half of one side of the drawing cross hair, but the drawing has become more detailed. If there are 2 points next to each other that are both within the sticky distance of the cursor at $100 \%$, so it is not possible to control which point the cursor sticks to, zoom in to that area of the drawing and the the cursor will stick to the desired point.

- While drawing, if an error message appears saying that a point can not be placed on top of another or that overlapping polygons cannot be created, the sticky distance may be causing the cursor to stick to points that are not desired, such as a point that is within the sticky distance of the previously drawn point. In this case, zoom in to the drawing and it is usually possible to get to the desired points.

- There are several snap features that can help with drawing. Snap to Vertical and Horizontal and Snap to Underlay are the defaults. Snap to Angle is helpful for drawing detailed curves. Smart Snap extends the edges of existing polygons and helps to line up polygons that aren't adjacent. The Snap features can be easily turned on and off through the toolbar. (See THERM User's Manual, Section 5.3.3, "Snap Settings", pgs 5-8 through 5-10)

\subsubsection{Drawing Tips}

- Moving points and lines: Press the Modify Points button on the toolbar to be able to modify points. A double headed arrow is the cursor for moving points. (See THERM User's Manual, Section 5.6.4, pgs. 5-21 through 5-22). A four headed arrow is the cursor for moving lines. (See THERM User's Manual, Section 5.6.5, pgs. 5-22 through 5-23). Holding down the shift key will constrain the movement to the horizontal and vertical directions. Sometimes line move has trouble sticking to adjacent polygons and it is possible to draw overlapping polygon errors when moving.

- Space Bar to snap: Use the space bar to snap to a point (and also to DXF file vertices) without changing the model. This is useful for determining the start of a line when measuring, and to move or measure relative to a certain point.

- Copying and Pasting: When using the Edit/Copy and Edit/Paste features within the same drawing, it is possible to create overlapping polygons. Make sure when pasting a polygon that the Locator (Shift F2) is moved before the paste is performed, or that the pasted polygon is moved after pasting if it is located directly over the copied polygon.

- Scooting: While drawing, Zoom Out (Shift right click) to see a point that is off the drawing area, move the cursor to that point, then Zoom In (right click) to actually draw the point. This is the best way to move the cursor large distances while drawing.

- Scroll Bars: The Scroll Bars can be accessed while drawing a polygon (a much better method than pushing the drawing cursor against the edge of the drawing window, which can result in display problems.)

- Switching Units: - The ${ }^{\circ} \mathrm{F} /{ }^{\circ} \mathrm{C}$ toolbar button switches between IP/SI units. 
- Display clean-up: Sometimes when deleting points from a polygon there is residue left on the screen. Press Escape to force the screen to redraw and the extraneous images will go away.

Scrolling up and down makes the labels of the isotherms get bunched together. Escape will redraw them with the labels positioned correctly.

- Editing Points on Multiple Adjacent Polygons: Move, add or delete the same point on all adjacent polygons (by selecting all the affected polygons) at the same time -- moving them separately may cause voids and overlaps.

- Windows ${ }^{\mathrm{TM}}$ Operating System: Make sure the Windows95 ${ }^{\mathrm{TM}}$ option is unchecked if you are using WindowsNTTM (Options/Preferences).

- Select Material/BC toolbar button: The Select Material/BC toolbar button $\square$ can be used to set the Material Library or Boundary Condition Library choice. To use this feature, click on the Select Material/BC toolbar button, move the cursor to the material or boundary condition to be selected, and click the left mouse button. This sets the value of the object selected. The cursor will the material or boundary condition to object (polygon, void or boundary segment) to be set to the selected material or boundary condition, and click the left mouse button.

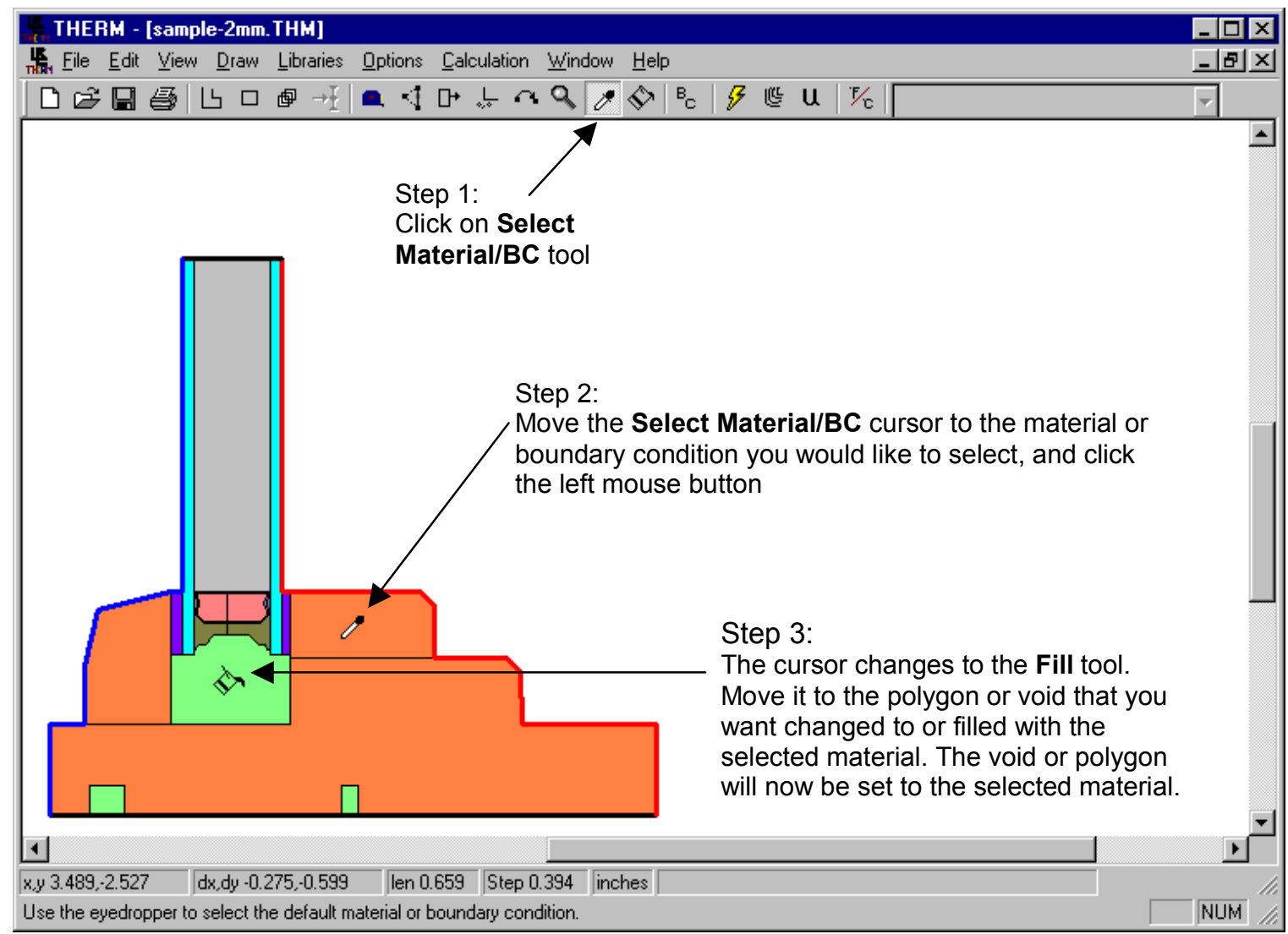

Figure 10-4. Steps for using the Select Material/BC toolbar button. 
- Fill toolbar button: The Fill toolbar button $\otimes$

can be used to either fill a void with a selected material, or change a polygon from one material to another. To use this feature, click on the Fill toolbar button, select the desired choice from the Material list, and move the Fill cursor to the void or polygon to be set to the selected material. Click the left mouse button, and the void or polygon will be set to the selected material.

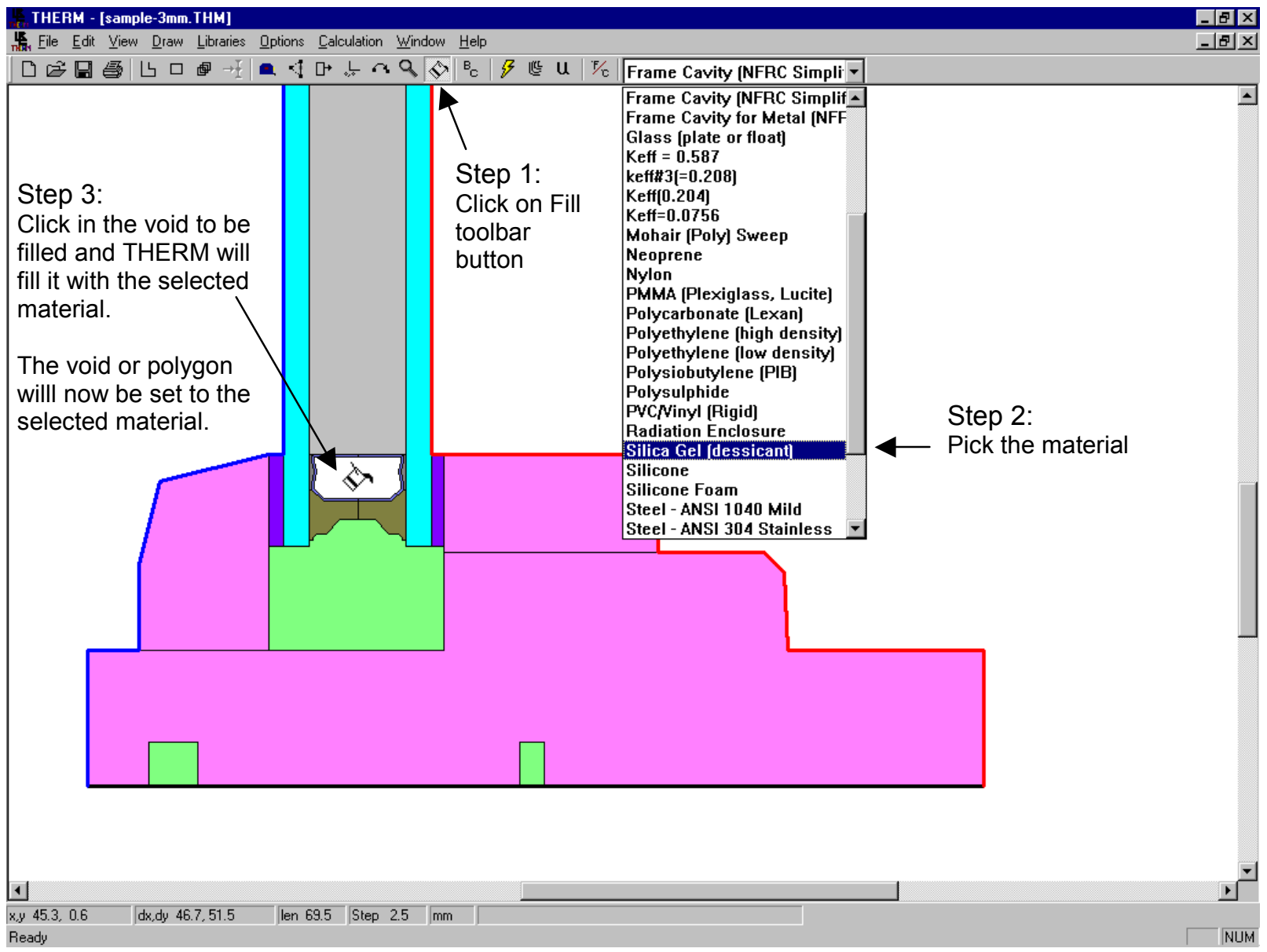

Figure 10-5. Steps for using the Fill toolbar button. 


\subsubsection{Drawing with the Keyboard}

- Typing a number will bring up the Step Size box, and set the Step Size. Then the arrow keys will move the distance set in the Step Size. Rectangles and polygons can be drawn entering dimension values into the step size box and moving the arrow keys in the appropriate direction. See Section 5.5, "Drawing Using the Keyboard (Numeric Cursor Positioning)" in the THERM User's Manual for more

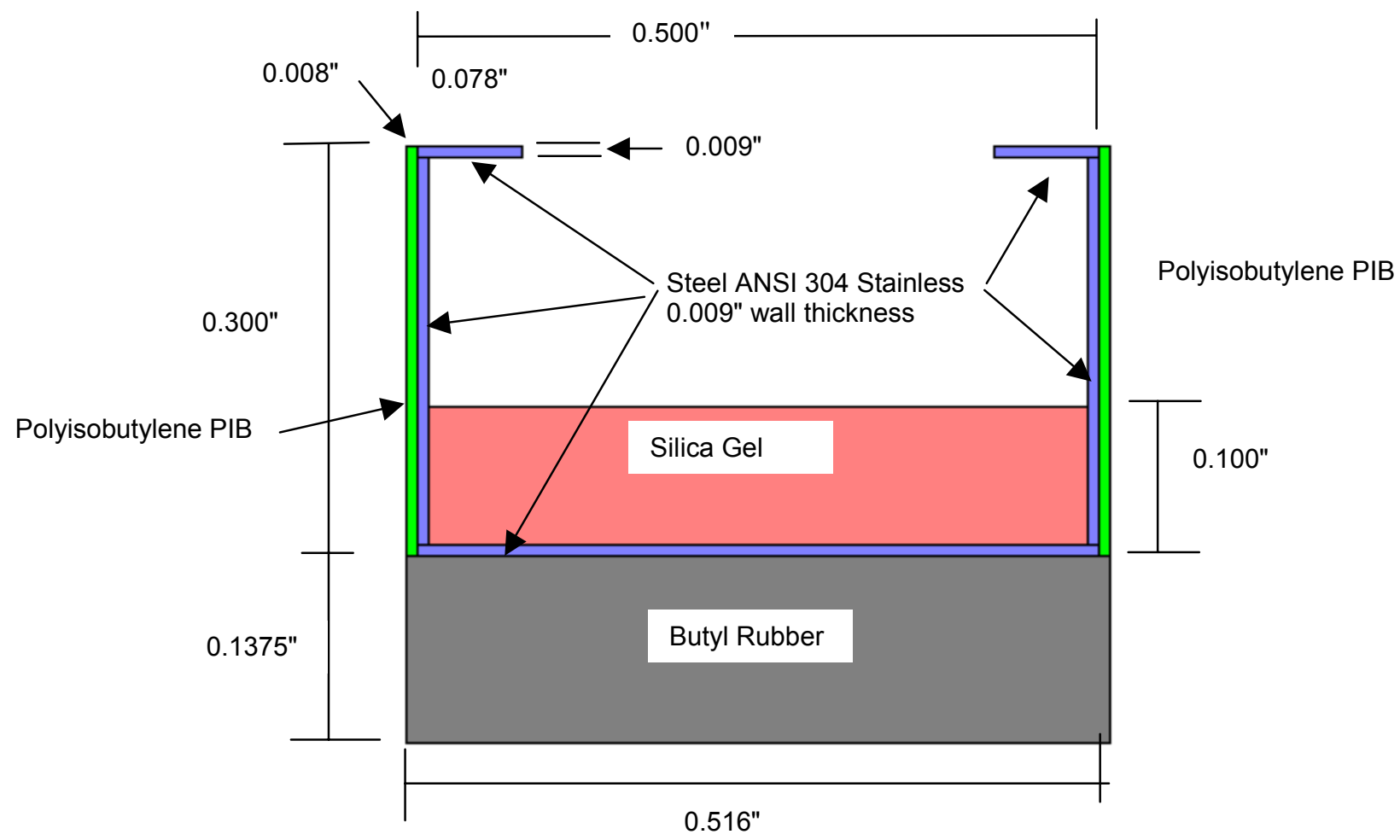

information about drawing with the keyboard.

Figure 10-6. A sample spacer to use for drawing with the keyboard from a dimensioned drawing. 


\subsubsection{Donuts}

- A region that connects back onto itself, such as a "donut", must be broken into multiple polygons in THERM

- When breaking up the "donut" regions, try not to create angles that are extremely acute. (There are many examples of extremely acute angles in Bad4.thm on the CD).

\section{"Donut"}

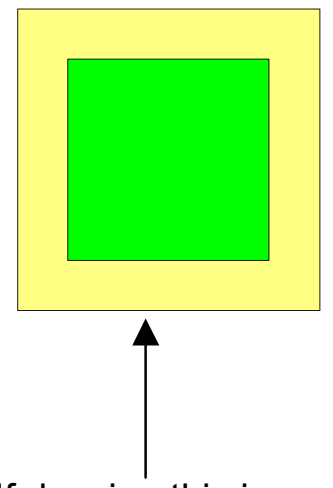

If drawing this in THERM, when closing the polygon an error message will appear saying that "Overlapping polygons are not allowed".

\section{Methods for breaking up "donut" in THERM}
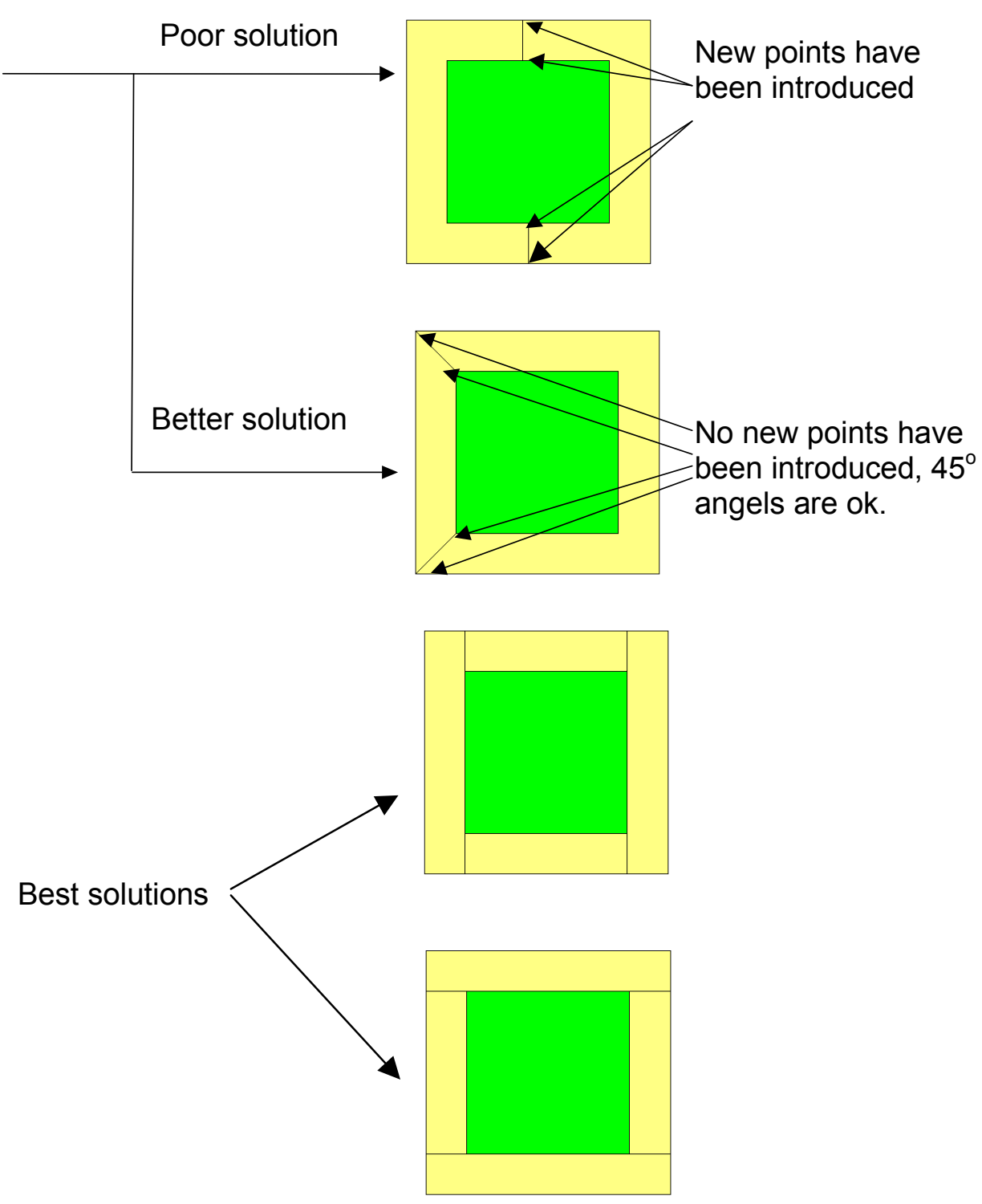

Figure 10-7. Breaking up "donut" regions in a cross section. 


\subsubsection{Breaking Up Extrusions into Polygons}

- THERM currently does not allow polygons that come back on themselves such as "donuts".

- When working with a CAD drawing it is usually necessary to break extrusions into polygons that THERM can handle.

- THERM can handle very complicated polygons. The method for breaking up the extrusion is a matter of personal working style, although for generating boundary conditions and the mesh during simulation, it is usually better to have more simple polygons that just a few very complicated one:

- Make many simple rectangles and polygons OR

- Make fewer, more complicated polygons

- Basic rules in breaking up extrusions

- Try not to create new points-- use points (vertices) that are already there

- Try not to create extremely acute angles (less than $5^{\circ}$ )

- If an extrusion cannot be broken up without creating an extremely acute angle, then introduce a point.

- Its easy to get lost while making very complicated polygons, which is one reason to make smaller, more simple polygons.

- The cen1.dxf, cen2.dxf, autofill.dxf and autofill2.dxf underlays (on the CD) can be used to practice breaking up extrusions. 


\section{Cen2.dxf}

The Cen2.dxf file found on the CD contains donuts which must be broken up when drawing the extrusion.

Draw a few well-placed rectangles to break up the donuts in the extrusion dxf file

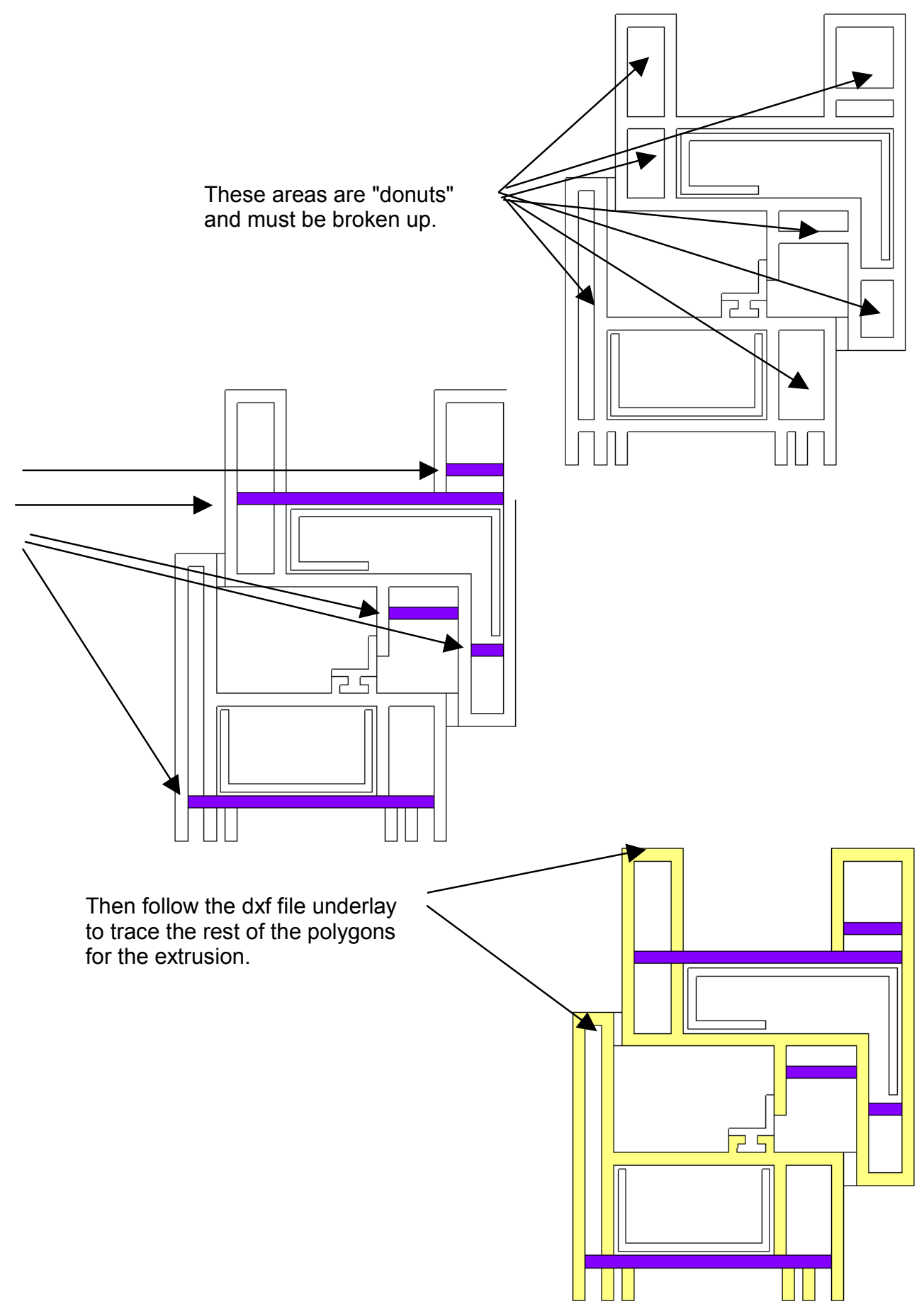

Figure 10-8. Breaking up "donut" regions in a cross section. 


\subsubsection{Drawing Extrusions from DXF Files -- False Die Mold Method}

- Many DXF files of extrusions are drawn as polylines, with the external profile as one polyline and
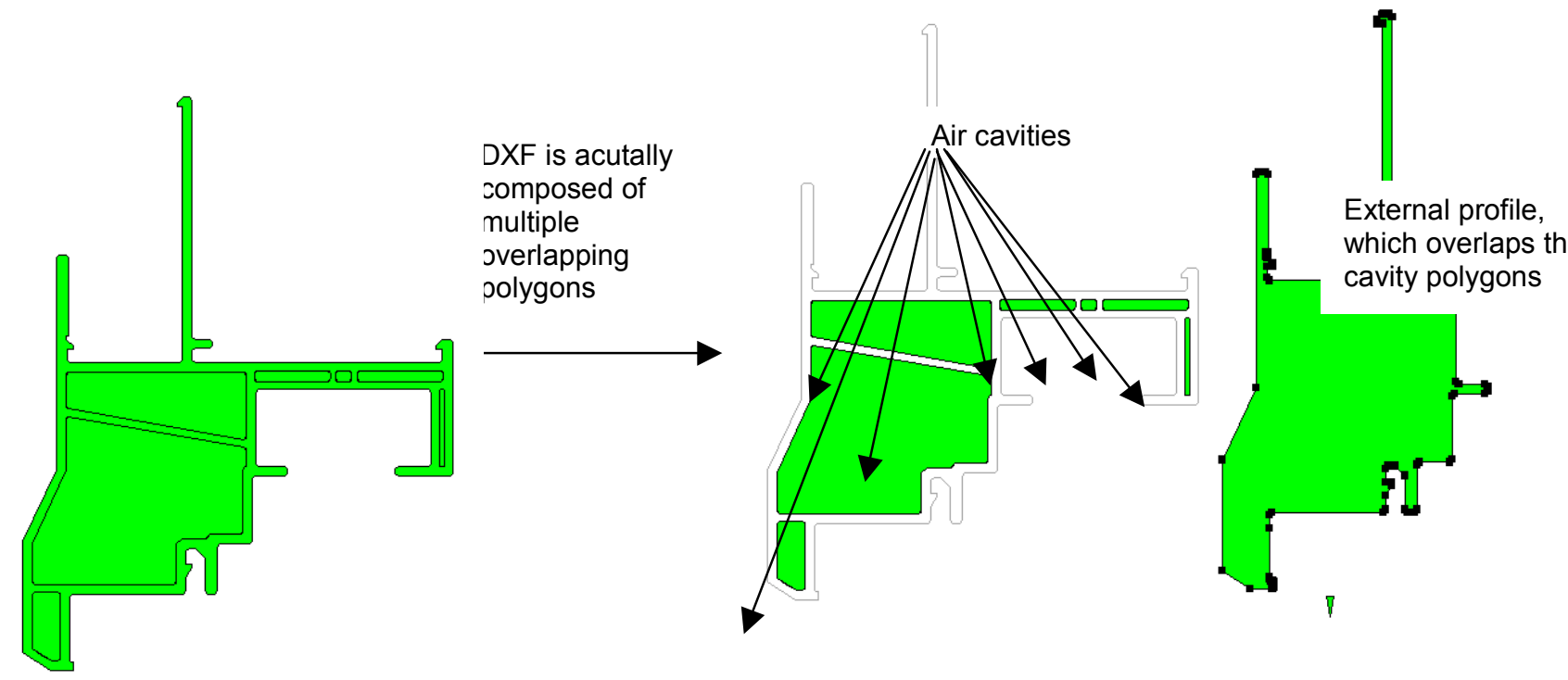

each of the air cavities is a separate polyline -- auto.dxf is an example of this.

Figure 10-9. Auto.dxf has multiple polygons on top of each other.

- Keeping the external profile polygon in place overlapping the cavity polygons, create a series of polygons that enclose the external profile (remember that you can't draw donuts).

- Use Fill Void to fill in the areas between the enclosing polygons and the external extrusion profile 
Figure 10-10. Method for getting the external extrusion shape.

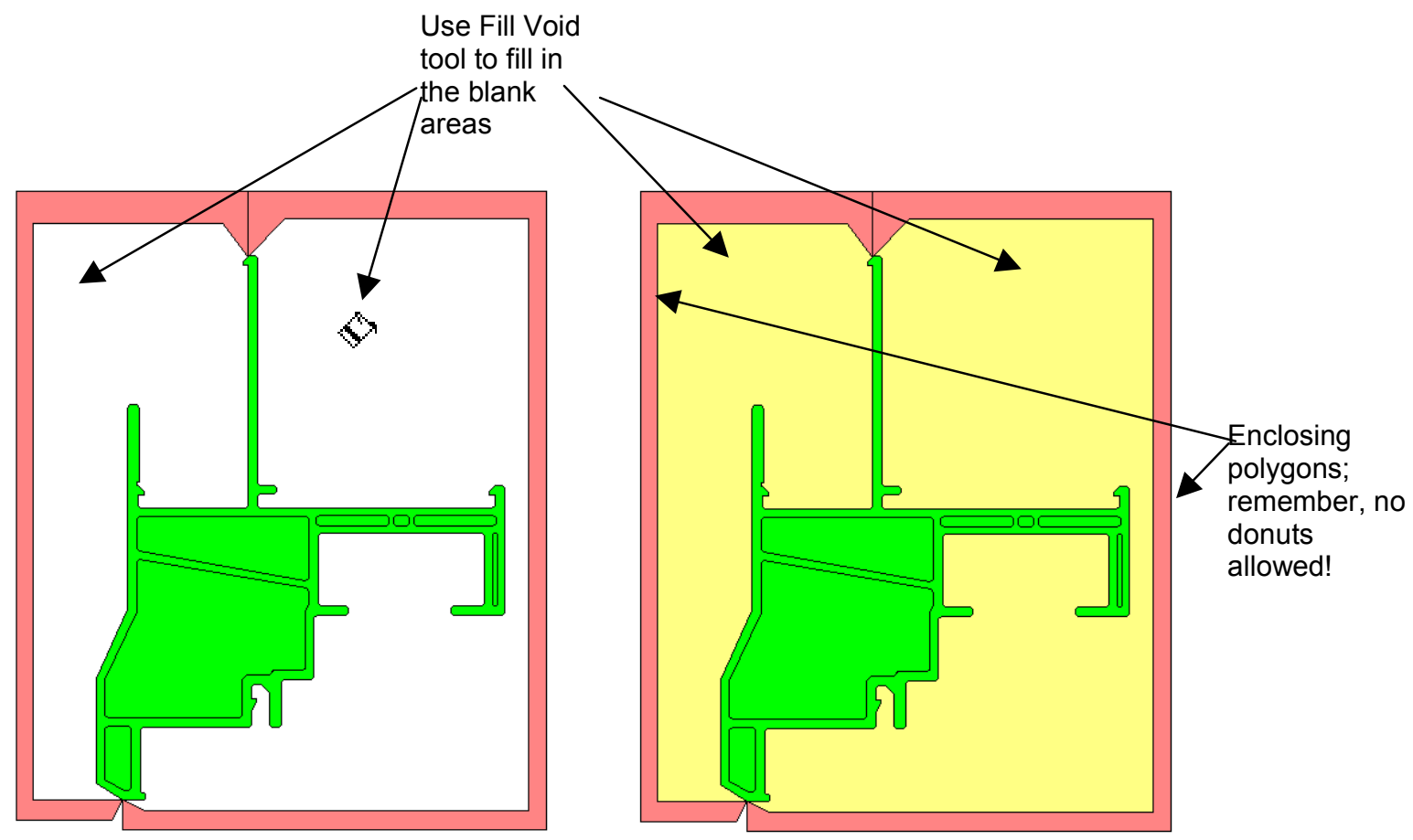




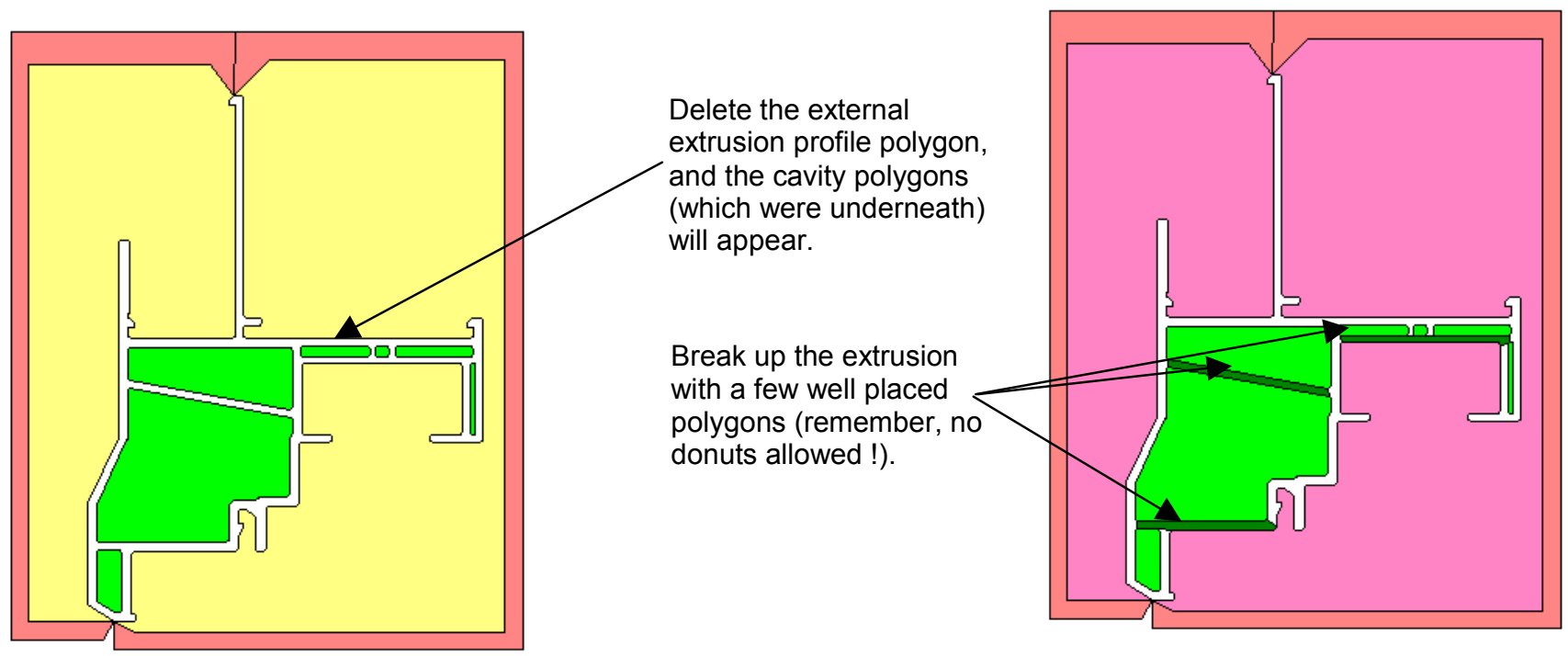

Figure 10-11. Get the extrusion profile and break up the extrusion so there are no "donuts".

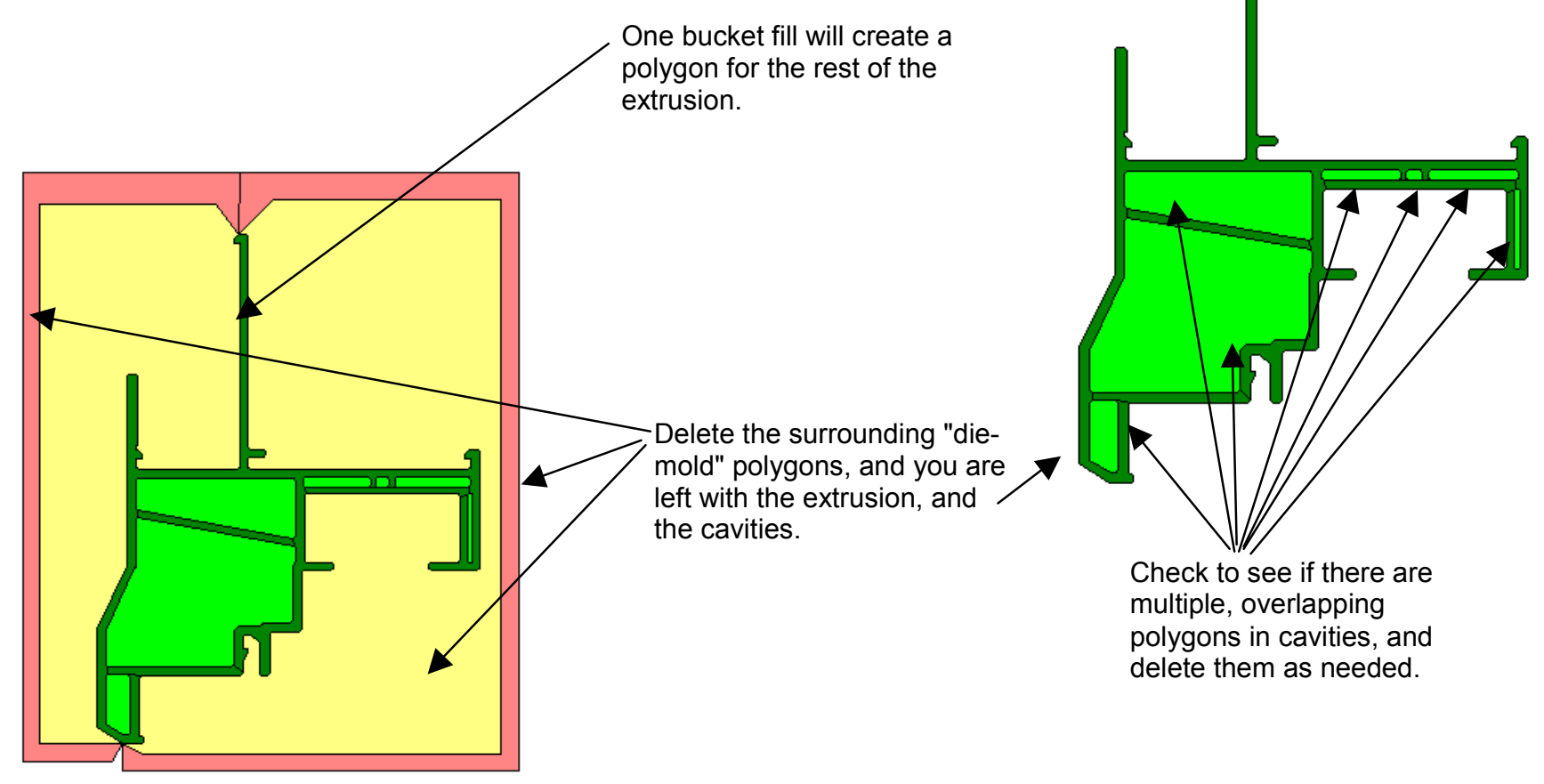

Figure 10-12. Finish creating the extrusion profile.

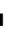




\subsubsection{Cuttins, Relocating, Flipping and Rotating Discussion}

- There are several ways to select polygons in THERM.

- Edit/Special Select

- The Drag Box

- Mouse clicks, shift clicks, tabs

- There are two ways to move polygon

- The Draw/Move Polygon command

- Using the Draw Locator - this is recommended for precise moves

- THERM has Flip and Rotate features but they rotate and flip everything in the drawing so you need to Cut and Paste the polygons that need to be manipulated to a temporary file, perform the operation and paste them back into the original drawing.

- The Cut and Paste feature in THERM eliminates unnecessary redrawing of parts that are exactly duplicated in multiple cross sections. You can make libraries of spacers and other components in THERM files to be used in many cross sections.

- The files spacerhalf.thm and cutEpaste.thm can be used to practices these features.

The cutEpaste.thm file can be used to practice these concepts. 


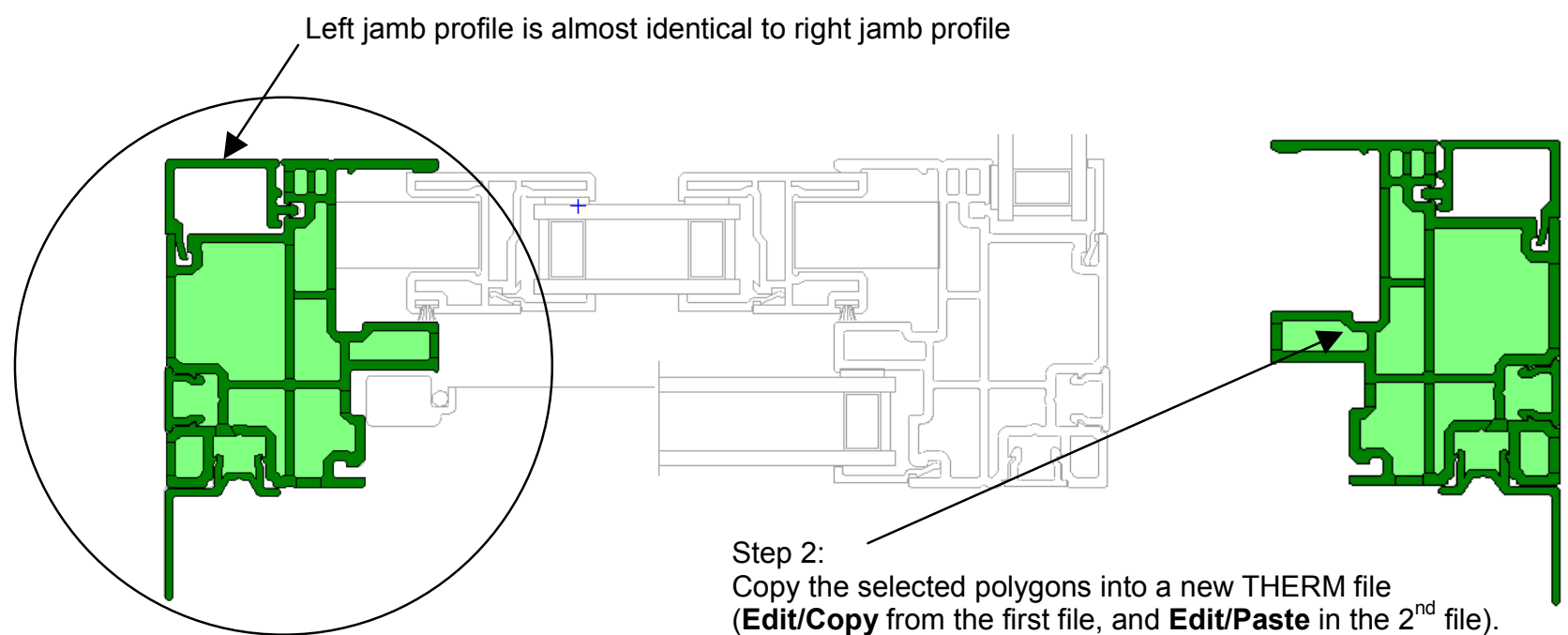

Step 1:

Select all the

polygons in the left iamh

Then in the $2^{\text {nd }}$ THERM file, flip the drawing horizontally (Draw/Flip/Horizontal).

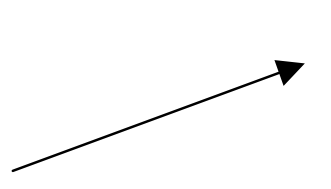

Step 3: Still in the $2^{\text {nd }}$ THERM file:

1. Delete unwanted polygons.

2. Set the Locator (Shift F2) to a clean point that exists in both files.

3. Select all polygons and copy them (Edit/Copy).

Step 4:

In the first THERM file, paste the polygons from the $2^{\text {nd }}$ file (Edit/Paste).

Because the Locators for both files are set to the same point, the new polygons will come in at the correct loctation

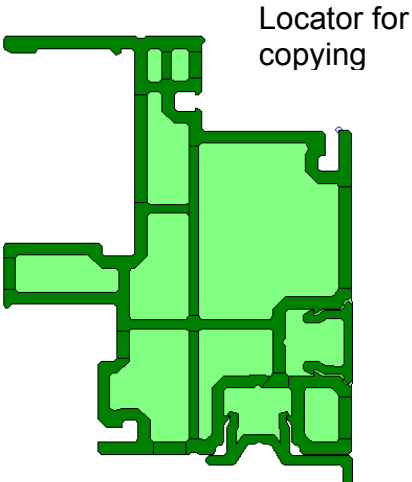

Locator for

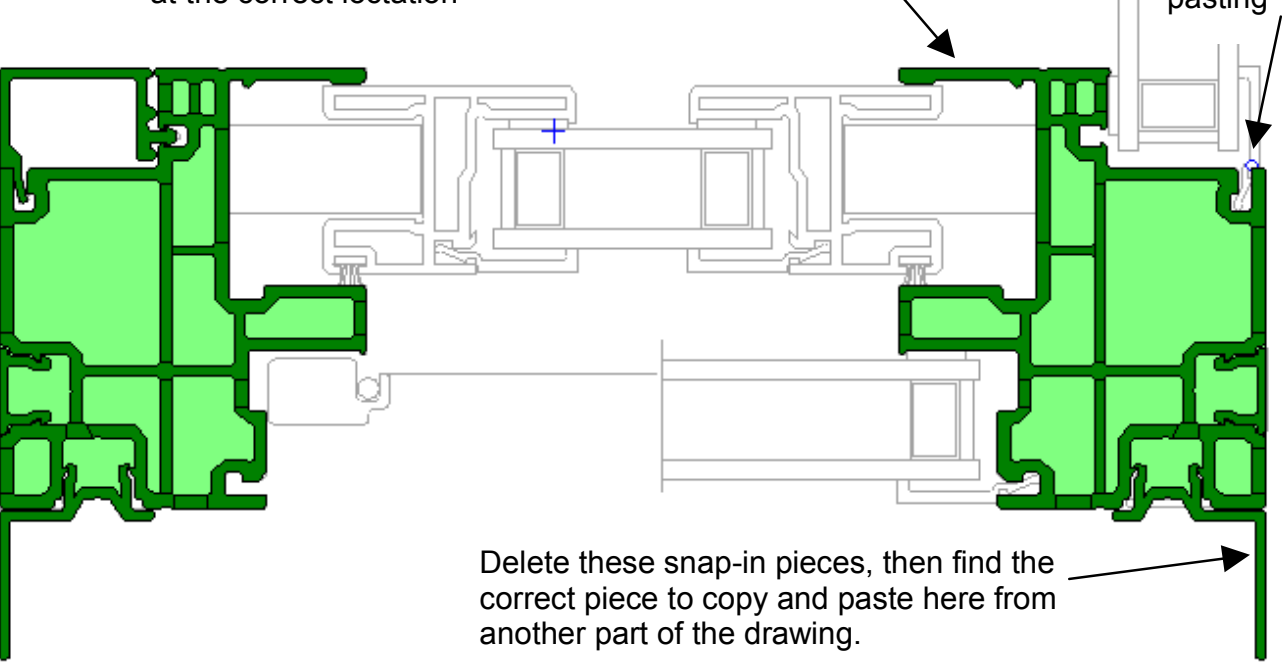

Figure 10-13. Use Edit/Cut and Edit/Paste to copy similar components within a drawing or to another THERM file 


\section{ACKNOWLEDGEMENTS}

\section{Acknowledgements}

This work was supported by the Assistant Secretary for Energy Efficiency and Renewable Energy, Office of Building Technology, State and Community Programs, Office of Building Systems of the U.S. Department of Energy under Contract No. DE-AC03-76SF00098. The program authors wish to thank the window manufacturers who supplied data and drawings on their products for use in this manual; Marcia Falke, Randy Van Voorst, and Bipin Shah for their review of draft versions; the many members of the NFRC community who contributed suggestions and ideas; and Pat Ross for all her many daily contributions including the final production of the program and manual. 


\section{Appendix A: THERM Material Library}

The THERM program comes with a default Material Library, containing a set of protected records. The values of this default library are listed below.

\begin{tabular}{|c|c|c|}
\hline Name & $\begin{array}{l}\text { Solid Properties } \\
\text { Conductivity } \\
\text { (Btu/h-ft-o } \mathrm{F})\end{array}$ & Emissivity \\
\hline Aluminum Alloys & 92.448 & 0.2 \\
\hline Butyl Rubber & 0.138 & 0.9 \\
\hline Fiberglass (PE Resin) & 0.173 & 0.9 \\
\hline Foam Rubber & 0.017 & 0.9 \\
\hline Foam Weatherstripping & 0.017 & 0.9 \\
\hline Glass (plate or float) & 0.577 & 0.84 \\
\hline Mohair (Poly) Sweep & 0.080 & 0.9 \\
\hline Neoprene & 0.109 & 0.9 \\
\hline Nylon & 0.144 & 0.9 \\
\hline PMMA (Plexiglass, Lucite) & 0.115 & 0.9 \\
\hline Polycarbonate (Lexan) & 0.109 & 0.9 \\
\hline Polyethylene (high density) & 0.300 & 0.9 \\
\hline Polyethylene (low density) & 0.190 & 0.9 \\
\hline Polyisobutylene (PIB) & 0.138 & 0.9 \\
\hline Polysulphide & 0.109 & 0.9 \\
\hline PVC/Vinyl (Rigid) & 0.098 & 0.9 \\
\hline Silica Gel (desiccant) & 0.017 & 0.9 \\
\hline Silicone & 0.208 & 0.9 \\
\hline Silicone Foam & 0.098 & 0.9 \\
\hline Steel - ANSI 1040 Mild & 27.734 & 0.2 \\
\hline Steel - ANSI 304 Stainless & 8.262 & 0.2 \\
\hline Urethane (liquid) & 0.179 & 0.9 \\
\hline Vinyl (flexible) & 0.069 & 0.9 \\
\hline Wood & 0.060 & 0.9 \\
\hline Wood (Cedar or Redwood) & 0.063 & 0.9 \\
\hline Wood (hardwood: maple) & 0.092 & 0.9 \\
\hline Wood (Pine, Spruce, Fir) & 0.080 & 0.9 \\
\hline
\end{tabular}


